\title{
Zur Bedeutung diskreter Arbeitsweisen im Mathematikunterricht
}

\author{
Inaugural-Dissertation \\ zur \\ Erlangung des Doktorgrades \\ der Naturwissenschaftlichen Fachbereiche \\ - Fachbereich Mathematik und Informatik, Physik, Geographie - \\ der Justus-Liebig-Universität Gießen \\ vorgelegt von \\ Silke Thies
}

Gießen, April 2002 


\section{Inhaltsverzeichnis}

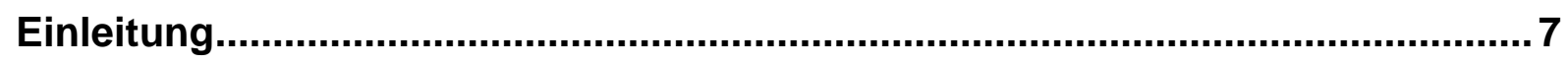

1 Zur Entwicklung Diskreter Mathematik.........................................................12

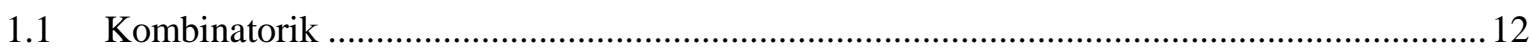

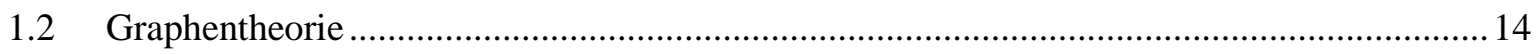

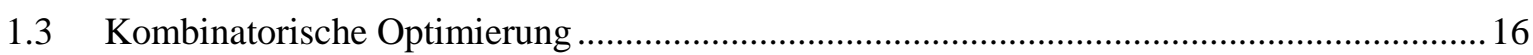

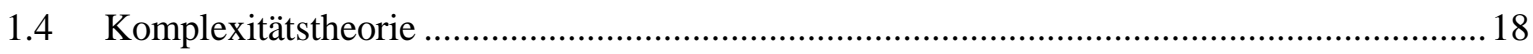

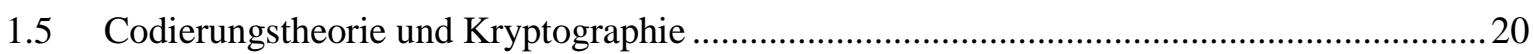

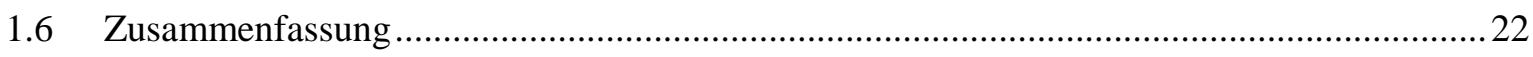

2 Diskrete Mathematik im Mathematikunterricht .............................................24

2.1 Rückblick auf die Entwicklung diskreter Mathematik im Mathematikunterricht ..................24

2.1.1 Kombinatorik als (eigenständiger) Unterrichtsgegenstand ......................................2 24

2.1.2 Graphentheoretische Elemente im Mathematikunterricht .......................................2 27

2.1.3 Codierungstheorie und Kryptographie als Gegenstand des Mathematik-

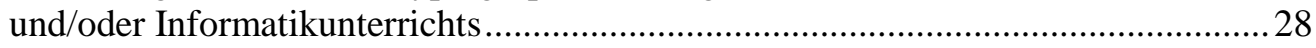

2.1.4 Zur Beziehung „Diskrete Mathematik - Kontinuierliche Mathematik“..................... 30

2.1.4.1 Zunehmende Bedeutung des algorithmischen Aspekts.............................. 30

2.1.4.2 Klassische Analysis versus diskrete Analysis ............................................ 32

2.1.4.3 Der NCTM-Standard „Diskrete Mathematik“ ............................................ 35

2.1.5 Sichtweisen auf diskrete Mathematik im Mathematikunterricht..................................36

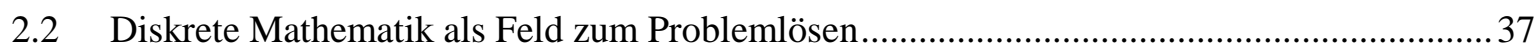

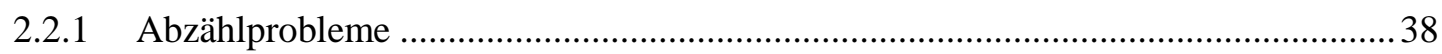

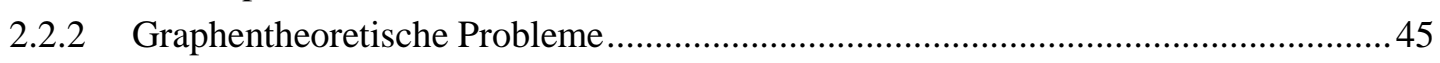

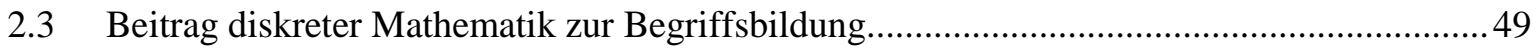

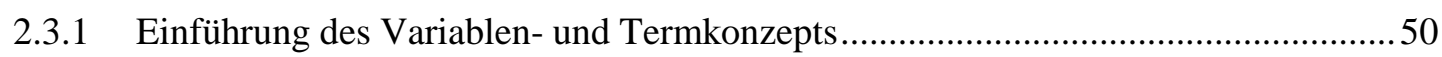

2.3.2 Beitrag zur Entwicklung des Funktionsbegriffs ....................................................53

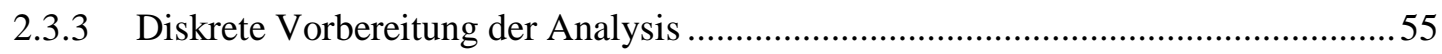

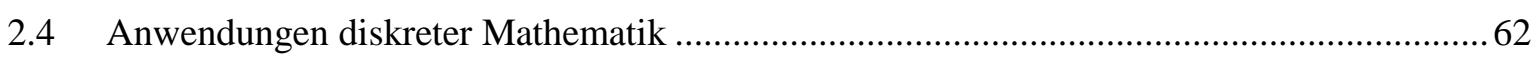

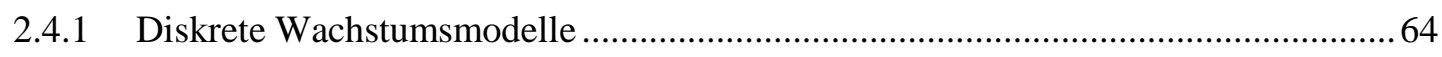

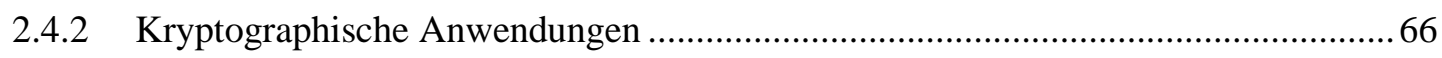

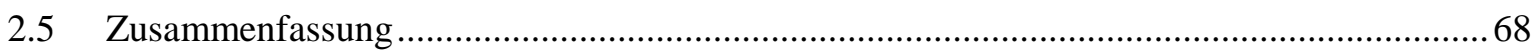

\section{Zur Bedeutung von Handlungen im Lernprozess .........................................70}

3.1 Entwicklung mathematischen Denkens aus Handlungen ...................................................... 70

3.1.1 Genetische Erkenntnistheorie von Jean Piaget ...................................................... 70

3.1.2 Hans Aebli: Verinnerlichung und operatives Durcharbeiten..................................... 71

3.1.3 Theorie der Darstellungsebenen nach Jerome S. Bruner.........................................73

3.1.4 Der Verinnerlichungs- und Verallgemeinerungsprozess aus der Sicht des Klagenfurter EFQUIM-Projekts .... 


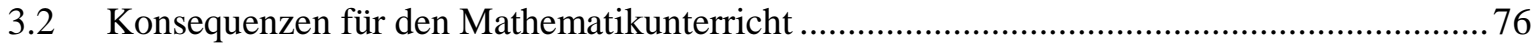

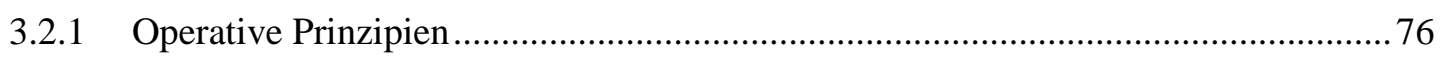

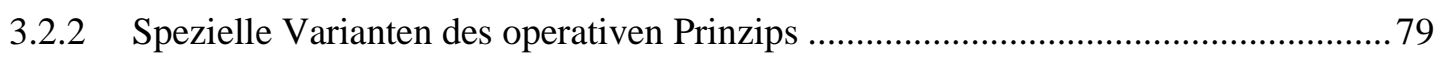

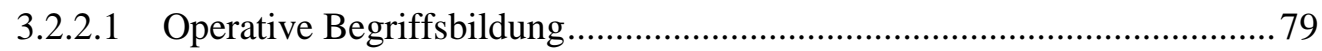

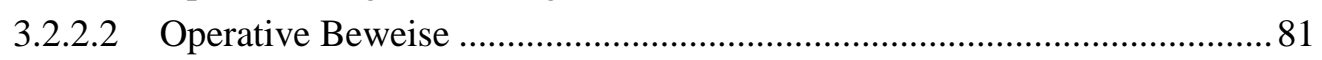

3.2.3 Kritische Bemerkungen zum Einsatz operativer Prinzipien ...................................... 85

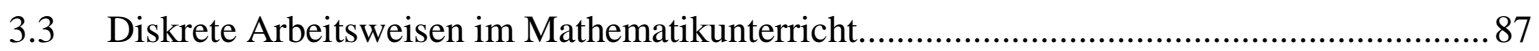

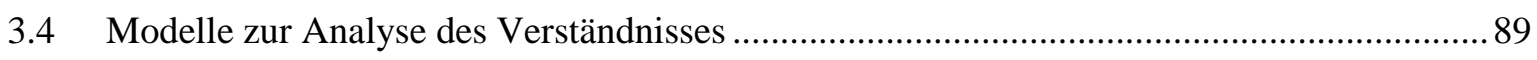

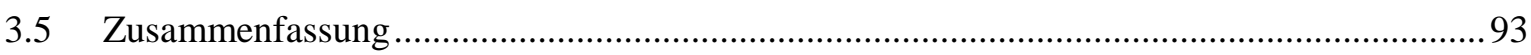

4 Tabellenkalkulationsprogramme:

Didaktisch-methodische Möglichkeiten - konkrete Arbeitsweisen ................94

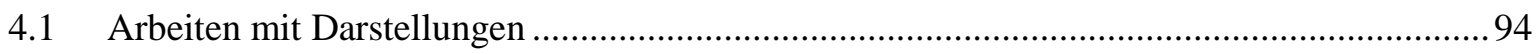

4.1.1 Generalisieren und Formalisieren von Gesetzmäßigkeiten .......................................94

4.1.2 Beitrag zur Entwicklung des Folgenbegriffs .............................................................97

4.1.3 Entdecken und Beweisen kombinatorischer Beziehungen .......................................98

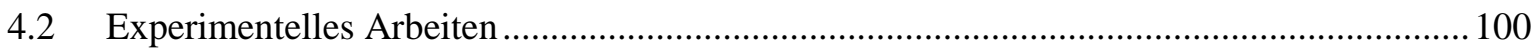

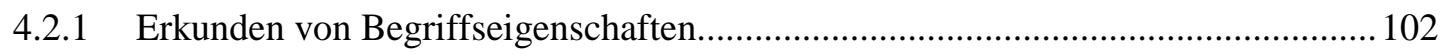

4.2.2 Experimentelles Arbeiten im Problemlöseprozess .................................................. 107

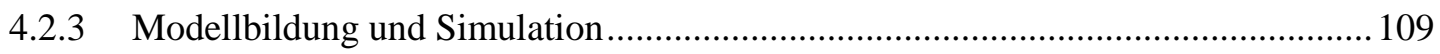

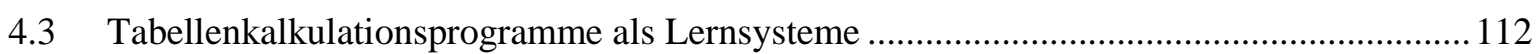

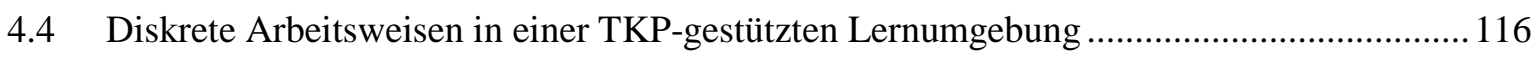

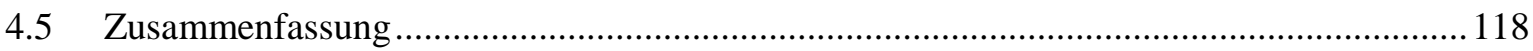

5 Planung und Durchführung der empirischen Untersuchung..........................119

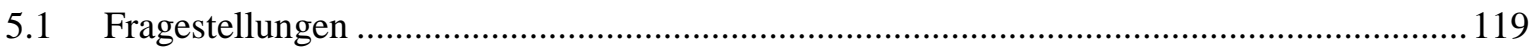

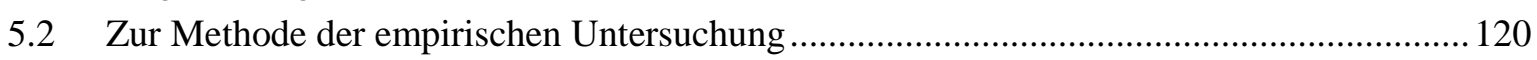

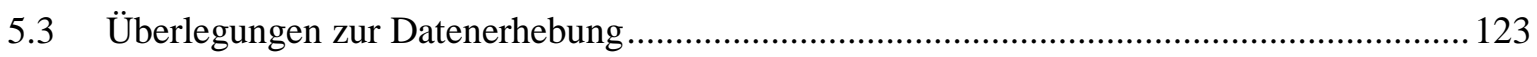

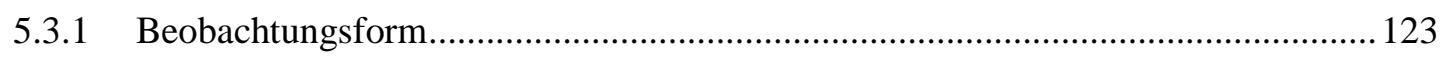

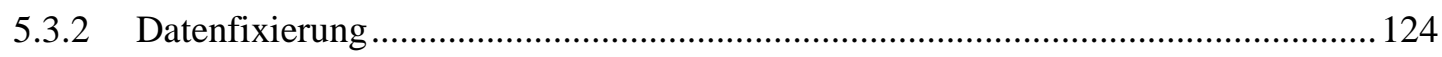

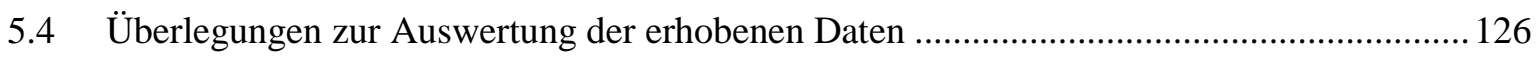

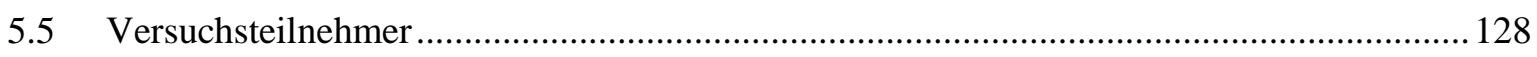

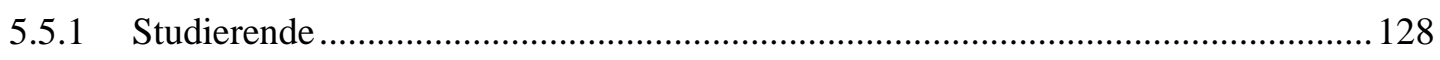

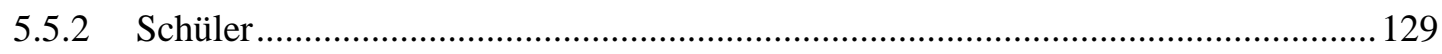

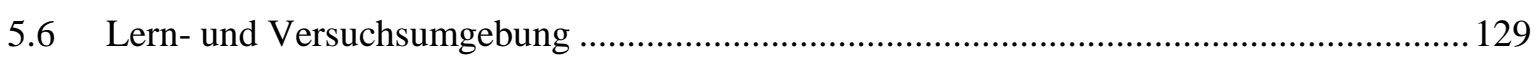

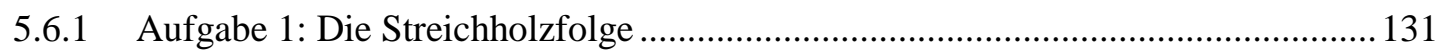

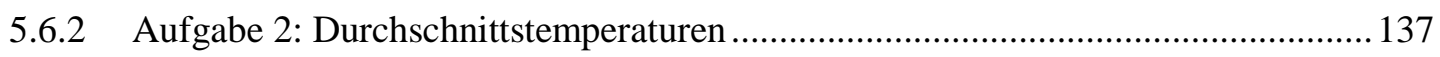

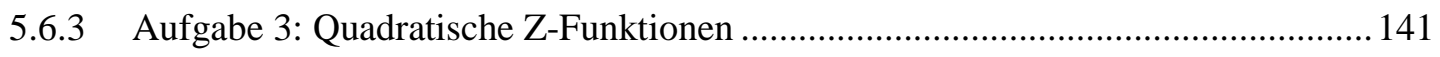

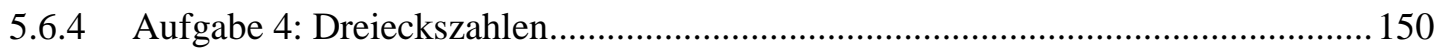

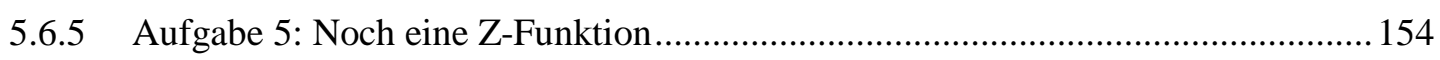

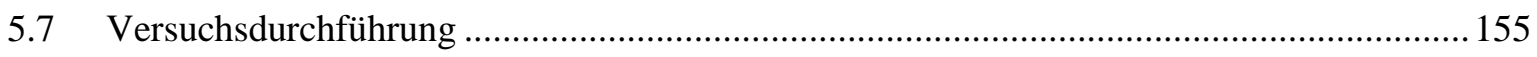

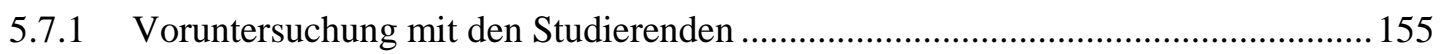

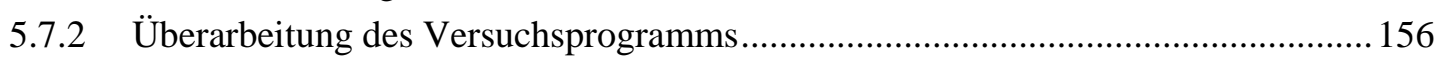




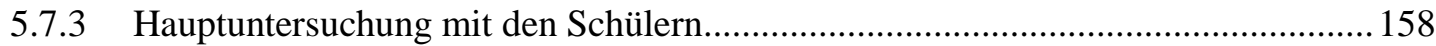

5.7.4 Vergleich der drei Testversionen: Übersicht ..................................................... 160

6 Ergebnisse der empirischen Untersuchung............................................161

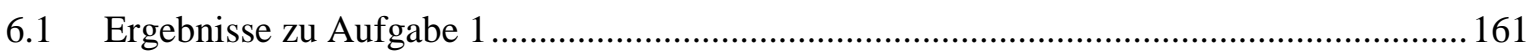

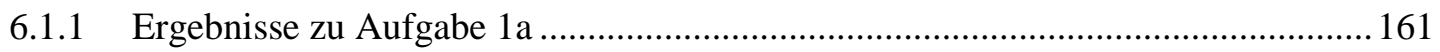

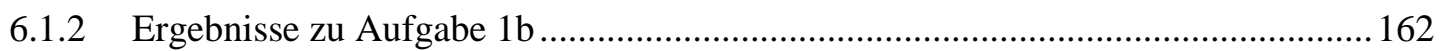

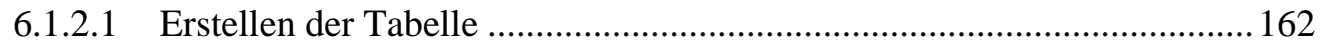

6.1.2.2 Inhaltliche/technische Probleme …............................................................ 163

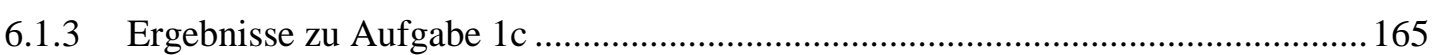

6.1.3.1 Lesen der Tabellendarstellung ............................................................. 165

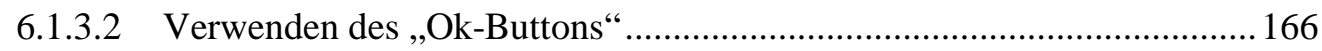

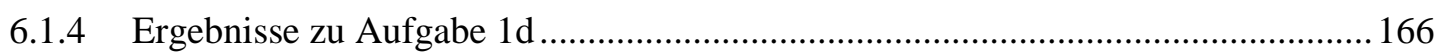

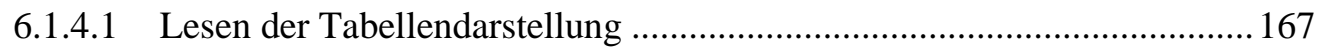

6.1.4.2 Nutzen des ,Ok-Buttons““..................................................................... 168

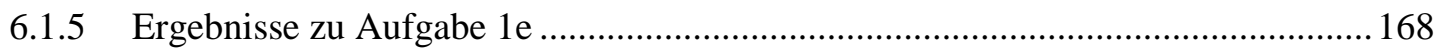

6.1.5.1 Erstellen der Tabelle ............................................................................... 168

6.1.5.2 Inhaltliche/technische Probleme ……..................................................... 169

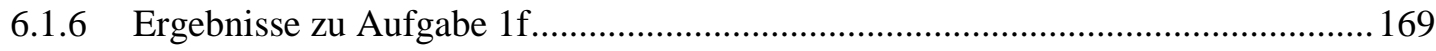

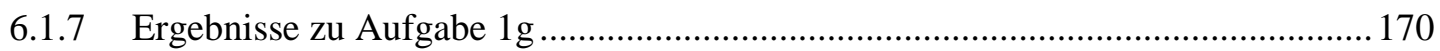

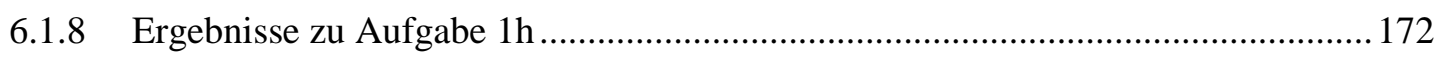

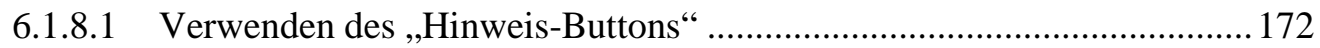

6.1.8.2 Inhaltliche/technische Probleme ............................................................. 173

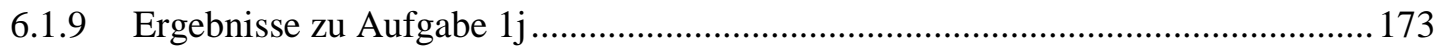

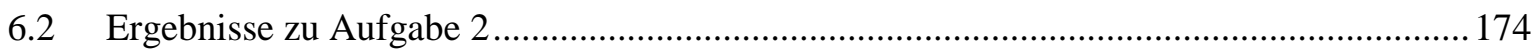

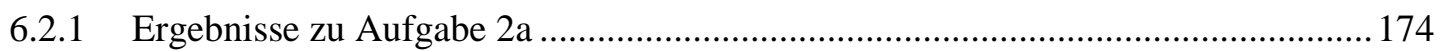

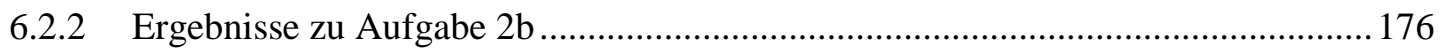

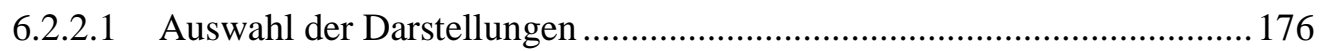

6.2.2.2 Strategien beim Arbeiten mit Darstellungen ............................................ 177

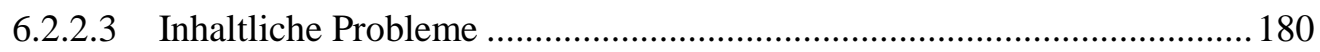

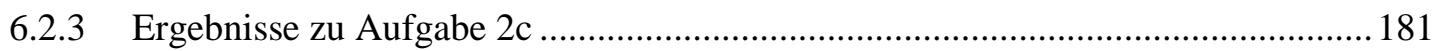

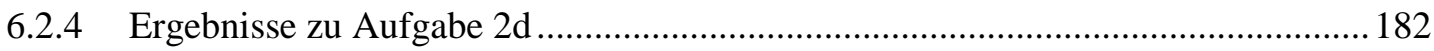

6.2.4.1 Arbeiten mit dem Graph................................................................... 182

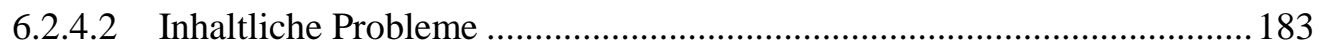

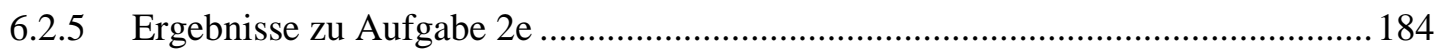

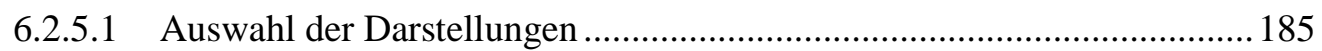

6.2.5.2 Strategien beim Arbeiten mit Darstellungen .......................................... 185

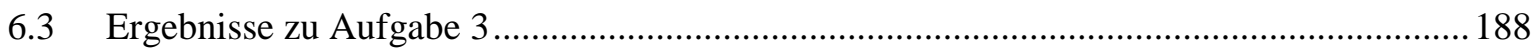

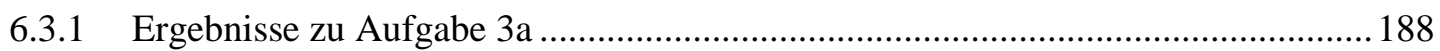

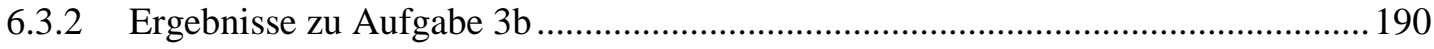

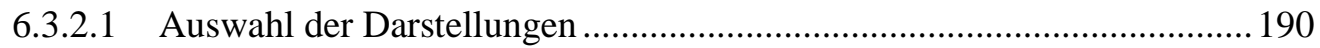

6.3.2.2 Strategien beim Arbeiten mit Darstellungen ............................................. 191

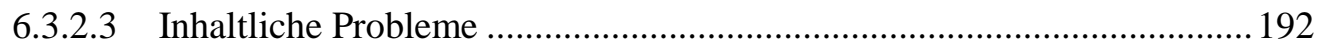




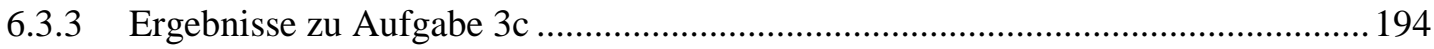

6.3.3.1 Arbeiten mit der Tabelle ........................................................................... 194

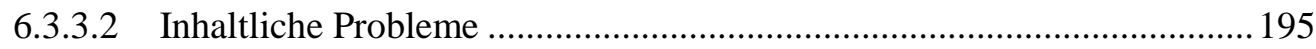

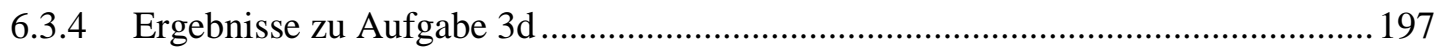

6.3.4.1 Verbale Beschreibung der Differenzenfunktion........................................ 197

6.3.4.2 Begründung der Eigenschaften der Differenzenfunktion.......................... 198

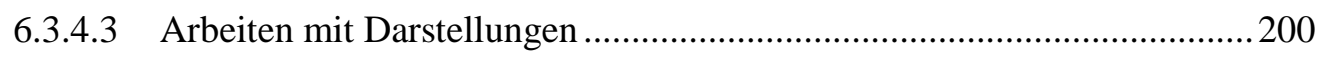

6.3.4.4 Beziehung: Arbeiten mit Darstellungen - Argumentationen .................... 201

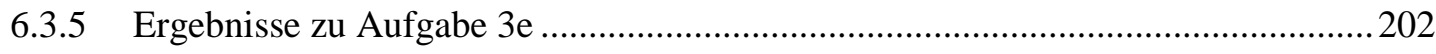

6.3.5.1 Aufgabenteil 1: Lesen von Darstellungen ..............................................202

6.3.5.2 Aufgabenteil 2: Argumentieren über Eigenschaften ...............................2203

6.3.5.3 Strategien beim Arbeiten mit Darstellungen .........................................205

6.3.5.4 Einfluss der Darstellungswahl auf das Lösen der Aufgabe...................... 206

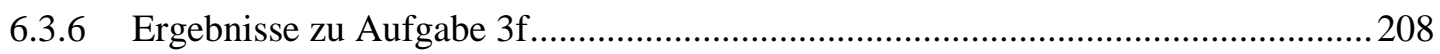

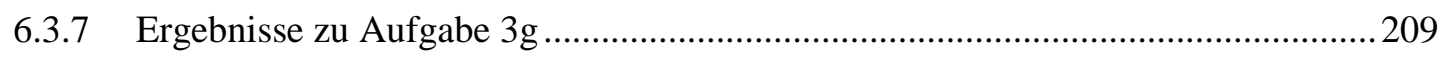

6.3.7.1 Verbale Beschreibung der Differenzenfunktion......................................209

6.3.7.2 Begründung der Eigenschaften der Differenzenfunktion.........................2 211

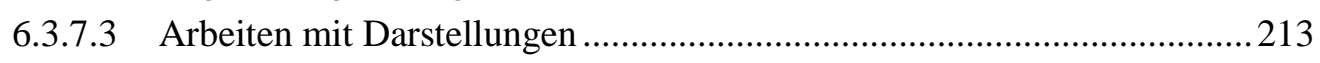

6.3.7.4 Beziehung: Arbeiten mit Darstellungen - Argumentationen..................... 214

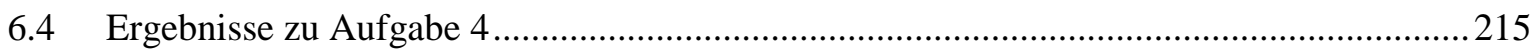

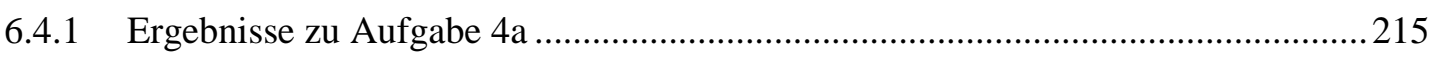

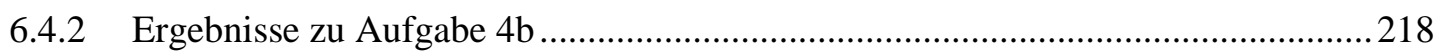

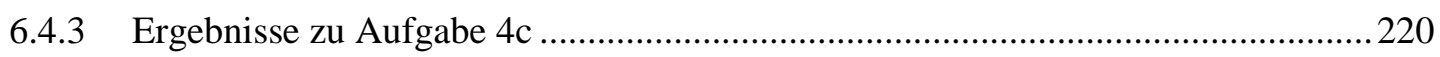

6.4.3.1 Inhaltliche/technische Probleme ...........................................................220

6.4.3.2 Vergleich: Aufgabe 4b - Aufgabe 4c.....................................................222

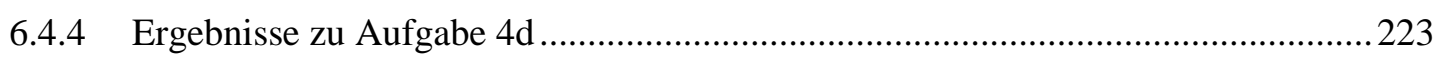

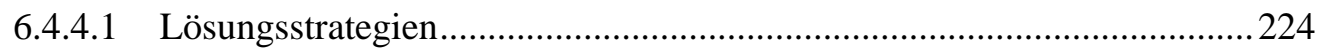

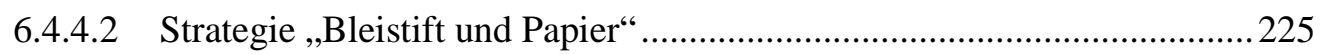

6.4.4.3 Strategie ,Differenzenfolge“ “...................................................................226

6.4.4.4 Experimentelles Problemlösen...........................................................227

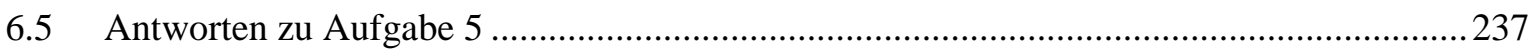

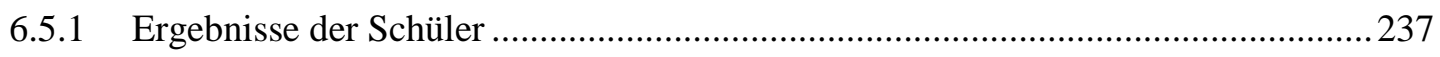

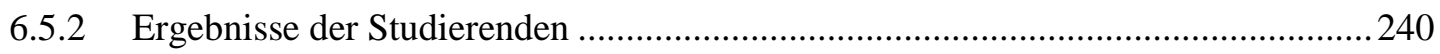

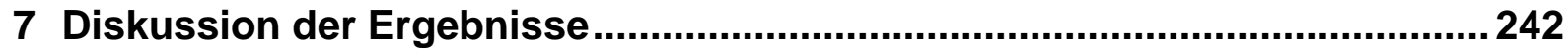

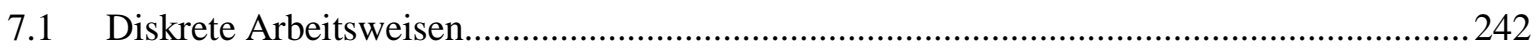

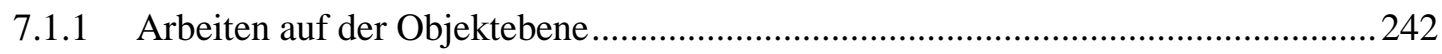

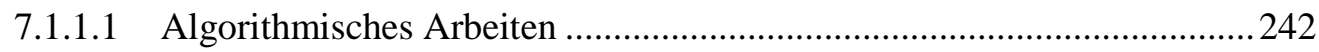

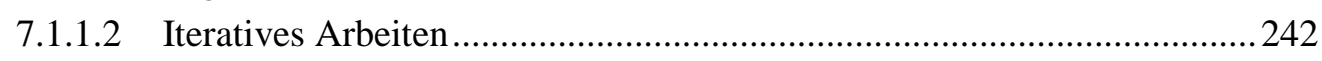

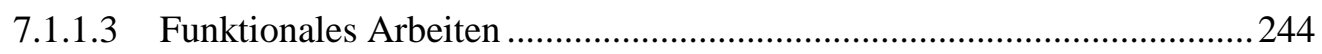

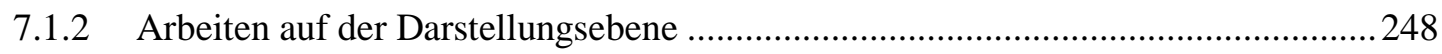

7.1.2.1 Arbeiten mit nicht-dynamisierten Darstellungen .................................... 248

7.1.2.2 Arbeiten mit dynamisierten Darstellungen..............................................249 


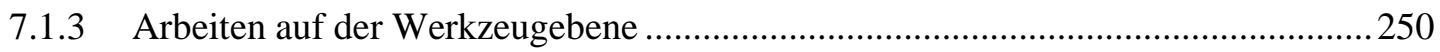

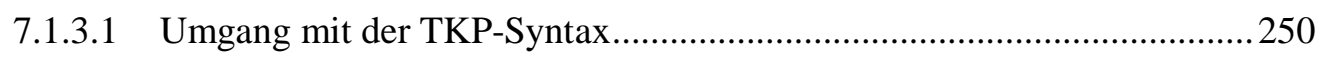

7.1.3.2 Bedienung und Steuerung des Lernprogramms ......................................251

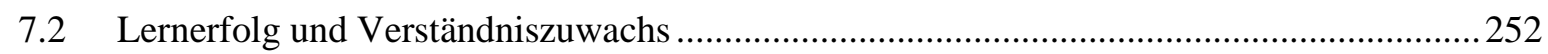

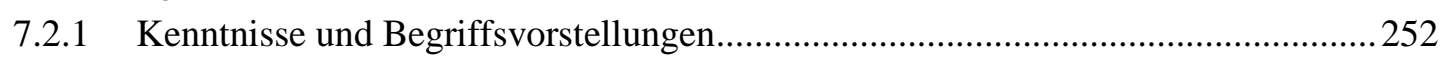

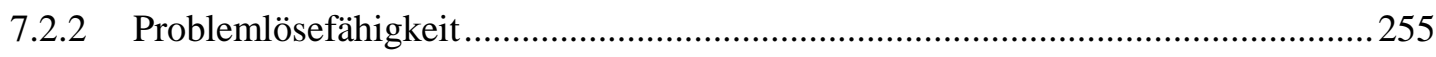

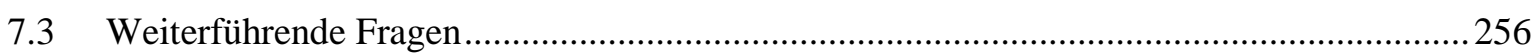

Literatur 


\section{Einleitung}

Diskrete Mathematik stellt ein relativ junges Teilgebiet der Mathematik dar, welches sich inhaltlich nicht eindeutig eingrenzen lässt. Es handelt sich vielmehr um einen Zusammenschluss verschiedener Themengebiete, wie etwa Kombinatorik, Graphentheorie, Komplexitätstheorie und Kryptographie, die sich in wechselseitiger Beziehung zueinander entwickelt haben. Gemeinsam ist ihnen die Beschäftigung mit endlichen bzw. diskreten Mengen und Strukturen. Obwohl viele diskrete Fragestellungen bis weit ins Altertum reichen, haben der Aufschwung der elektronischen Datenverarbeitung und die Einführung der ersten schnellen Rechner die Entwicklung der Diskreten Mathematik in den letzten 50 Jahren stark vorangetrieben. Seit Beginn der 70er Jahre wurde immer wieder dafür plädiert, diskrete Inhalte ${ }^{1}$ auch verstärkt in den Mathematikunterricht zu integrieren. Es gab Empfehlungen zur Behandlung graphentheoretischer und informationstheoretischer Themen und es wurde gefordert, den Mathematikunterricht stärker an einem ,algorithmischen Strang“ zu orientieren. Vereinzelt wurde bereits von einer „Legitimationskrise“ einer klassisch kontinuierlichen Disziplin, der Analysis, gesprochen. Trotz zahlreicher unterrichtspraktischer Vorschläge für den Mathematikunterricht liegt eine umfassende Analyse des Themengebiets „Diskrete Mathematik“ bisher noch nicht vor. In der vorliegenden Arbeit soll die Bedeutung diskreter Inhalte für den Mathematikunterricht untersucht werden und es soll analysiert werden, welche Möglichkeiten neue Technologien - insbesondere Tabellenkalkulationsprogramme (TKP) - beim Lehren und Lernen von (diskreten) Begriffen sowie zur Behandlung diskreter Problemstellungen im Unterricht eröffnen. Die zentrale Fragestellung wird es dabei sein, was unter diskreten Arbeitsweisen verstanden wird und in welcher Beziehung diese zum Verständnis diskreter Begriffe stehen.

Das erste Kapitel vermittelt einen Einblick in die wichtigsten Bereiche Diskreter Mathematik und deren historische Entwicklung. Dabei werden Wurzeln diskreter Begriffe und Beziehungen zu anderen Teilgebieten der Mathematik aufgezeigt. Drei Aspekte werden dabei im Hinblick auf eine mögliche Thematisierung diskreter Inhalte im Mathematikunterricht besonders herausgestellt. Erstens wurde die Entwicklung der Diskreten Mathematik durch zahlreiche reizvolle (innermathematische) Problemstellungen vorangetrieben, die man historisch der Beschäftigung mit natürlichen Zahlen zuordnen kann und die offensichtlich für viele Mathematiker herausfordernd waren. Zum Zweiten hat die Beschäftigung mit diskreten Fragestellungen die Entwicklung anderer mathematischer Gebiete, wie etwa der Wahrscheinlichkeitsrechnung, der Analysis, der Zahlentheorie und der endlichen Geometrie, unterstützt und vo-

\footnotetext{
${ }^{1}$ Im Rahmen dieser Arbeit werden mit Ausdrücken wie „diskrete Inhalte“, „diskrete Begriffe“, „diskrete Probleme“ Inhalte, Begriffe und Problemstellungen aus dem Bereich der diskreten Mathematik bezeichnet. Die Verwendung des kleingeschriebenen „diskret“ soll dabei auf eine weitere Fassung dieses Begriffs hindeuten. Diskre-
} 
rangetrieben. ${ }^{2}$ Gleichzeitig und in Wechselwirkung mit diesen Bereichen erhielt die Diskrete Mathematik wichtige Impulse zur Etablierung als eigenständiges Gebiet. Darüber hinaus und zum Dritten gewann die Diskrete Mathematik als Werkzeug zur Modellierung außermathematischer Situationen an Bedeutung, vor allem bedingt durch die fortschreitende Technologisierung der Gesellschaft.

Im zweiten Kapitel wird das didaktische Potenzial diskreter Themen für den Mathematikunterricht untersucht. Hierzu werden zunächst bestehende fachdidaktische Vorschläge für die einzelnen Teilgebiete der Diskreten Mathematik analysiert. Dabei wird insbesondere auch auf die amerikanische Diskussion um die Bedeutung und Stellung Diskreter Mathematik in der College- und Highschool-Ausbildung eingegangen, die mit Beginn der 90er Jahre zur Verankerung Diskreter Mathematik in den Lehrplänen des National Council of Teachers of Mathematics (NCTM) und damit zu zahlreichen unterrichtspraktischen Vorschlägen führte.

Im Rahmen dieser Analyse kristallisieren sich drei für den Mathematikunterricht wesentliche Aspekte heraus, die anhand von Beispielen illustriert werden. So bieten diskrete Fragestellungen die Möglichkeit, verschiedene Problemlösestrategien zu initiieren (Problemlöseaspekt). Zum anderen können zahlreiche Anwendungsmöglichkeiten aufgezeigt und der Modellbildungsprozess betont werden (Anwendungsaspekt). Schließlich ermöglichen diskrete Begriffe und Problemstellungen einen elementaren Zugang zu wichtigen Begriffen des Mathematikunterrichts, wie Variable, Term, Folge und Funktion, und bieten eine Chance für eine diskrete Vorbereitung der Analysis (Begriffsbildungsaspekt). Es wird deutlich, dass sich viele diskrete Begriffe und die damit verbundenen Operationen handelnd auf der enaktiven oder/und auf einer inhaltlich-anschaulichen Ebene erschließen lassen. Dadurch rücken Handlungen bzw. Arbeitsweisen in den Mittelpunkt des Interesses. Bei der Behandlung diskreter Fragestellungen im Mathematikunterricht - insbesondere bei Themen aus dem Bereich der Kombinatorik, der Differenzengleichungen oder der „diskreten Analysis“ - ergeben sich häufig umfangreiche, wenngleich elementare Berechnungen, die mit einem zeitaufwändigen Anfertigen entsprechender Darstellungen verbunden sind. Damit ergibt sich die Frage, inwieweit neue Technologien diese Tätigkeiten übernehmen bzw. unterstützen können und welcher Beitrag dabei zur Förderung der oben genannten drei Aspekte geleistet werden kann. An Computerprogrammen bieten sich dabei insbesondere Tabellenkalkulationsprogramme an, da der zellenorientierte diskrete Aufbau eine unmittelbare Darstellung diskreter Begriffe und Algorithmen erlaubt.

Zur Beantwortung der im zweiten Kapitel aufgeworfenen Fragen wird im dritten Kapitel zunächst auf die Entwicklungs- bzw. Lerntheorien Piagets, Bruners und Aeblis sowie auf Er-

\footnotetext{
te Begriffe und Inhalte können in diesem Sinne auch in Grenzbereichen bzw. außerhalb der „eigentlichen“ fachwissenschaftlichen Disziplin der „Diskreten Mathematik“ bzw. der oben genannten Teilgebiete liegen.

${ }^{2}$ Zum Teil werden Zahlentheorie und endliche Geometrie zur Diskreten Mathematik (im engeren Sinne) gezählt. Wir beschränken uns jedoch in den folgenden Analysen auf die eingangs aufgeführten Disziplinen.
} 
gebnisse eines Klagenfurter Forschungsprojekts - des sogenannten EFQUIUM-Projekts ${ }^{3}$ eingegangen. Die aus diesen theoretischen Ansätzen abgeleiteten „operativen Prinzipien“ sowie die Theorie der Prototypen betonen die Bedeutung des Handelns und Operierens für die kognitive Entwicklung und geben Hinweise zur Gestaltung von Lernprozessen, bei denen das Arbeiten mit (prototypischen) Darstellungen eine wichtige Rolle spielt. Wie anhand von Beispielen aufgezeigt wird, eignen sich viele diskrete Inhalte in besonderem Maße zur Realisierung wesentlicher Aspekte der „operativen Prinzipien“. Indem das Handeln und Operieren als ein zentrales Element des Lernprozesses herausgestellt wird, ergibt sich jedoch auch die Gefahr, dass Tätigkeiten von Schülern und Schülerinnen ${ }^{4}$ in einen oberflächlichen Aktivismus ausarten und der Bezug zu theoretischen Überlegungen und mathematischem Hintergrundwissen verloren geht sowie intendierte Vorstellungen und Prototypen nicht ausgebildet werden. Nun ist über das tatsächliche Schülerhandeln und das erreichte Verständnis im Allgemeinen wenig bekannt. Um Rückschlüsse über die Qualität von traditionellen und insbesondere auch computerunterstützten Lernumgebungen sowie über den Umgang mit Darstellungen bzw. den dargestellten mathematischen Begriffen und das damit erzielte Schülerverständnis zu erhalten, ist es daher erforderlich, die individuellen Arbeitsweisen der Schüler sowie deren Begriffsvorstellungen näher zu analysieren. Zu diesem Zweck wird ein Konzept entwickelt, das es ermöglicht, beobachtbare Tätigkeiten und Handlungen der Schüler am Rechner genauer zu beleuchten. Diese Arbeitsweisen werden auf verschiedenen Handlungsebenen analysiert, der Ebene der mathematischen Objekte, der Darstellungsebene und der Werkzeugebene.

Im vierten Kapitel werden unter Berücksichtigung der skizzierten Lerntheorien didaktischmethodische Möglichkeiten aufgezeigt, die sich durch den Einsatz eines Tabellenkalkulationsprogramms bei der Behandlung diskreter Inhalte eröffnen. So ermöglicht ein TKP neben der Reduktion manuell-algorithmischer Tätigkeiten das Erzeugen vielfältiger Darstellungen auf „Knopfdruck“, was insbesondere das Arbeiten auf einer „,vorformalen“ numerischen und graphischen Ebene fördert. Darüber hinaus gewinnt die experimentelle Analyse von Begriffseigenschaften und das experimentelle Problemlösen bei inner- und außermathematischen Fragestellungen an Bedeutung, und es lassen sich Lernumgebungen entwickeln, die dem Lernenden ein hohes Maß an Eigentätigkeit belassen. Ob und wie Schüler diese Möglichkeiten nutzen, welches Verständnis und welche Vorstellungen sie entwickeln, lässt sich letztendlich nur empirisch beantworten.

Im fünften Kapitel wird die im Rahmen dieser Arbeit durchgeführte empirische Untersuchung vorgestellt. Ziel der Untersuchung war es, die Arbeitsweisen von Schülern einer

\footnotetext{
${ }^{3}$ Die Abkürzung EFQUIM steht für das Projekt „Entwicklung formaler Qualifikationen im Mathematikunterricht".

${ }^{4}$ Aus pragmatischen Gründen wird im Folgenden bei Personenangaben in der Regel die maskuline Form verwendet.
} 
11. Jahrgangsstufe sowie einer Gruppe von Studierenden im Umgang mit den Begriffen „Folge und Differenzenfolge“ bzw. „Z-Funktion ${ }^{5}$ und Differenzenfunktion“ zu erfassen und hinsichtlich des zugrunde liegenden bzw. erreichten Verständnisses zu analysieren. Die Probanden hatten die Aufgabe, im Rahmen eines zu diesem Zweck auf der Basis des TKP Excel konzipierten Lern- und Versuchsprogramms lokale und globale Eigenschaften von Folgen und Differenzenfolgen bzw. Z-Funktionen und Differenzenfunktionen zu untersuchen und zu beschreiben sowie diskrete Probleme zu lösen. Durch die Wahl dieses Lerninhalts war es möglich, sowohl den Problemlöseaspekt als auch - im Hinblick auf die Bedeutung von Differenzenfunktionen bei einem elementaren Zugang zur Analysis - den Begriffsbildungsaspekt diskreter Mathematik zu betonen. Die Aufzeichnung der Arbeitsweisen erfolgte über ein im Hintergrund mitlaufendes Programm, das sämtliche Aktivitäten der Probanden am Rechner in Form eines „Computerprotokolls“ in Echtzeit festhielt.

Das erhobene Datenmaterial wird im sechsten Kapitel qualitativ und quantitativ ausgewertet. Die Analyse soll darüber Aufschluss geben, ob die Schüler die durch das Werkzeug angebotenen neuen Möglichkeiten nutzen und welche Probleme sich dabei ergeben. Weiterhin soll untersucht werden, wie Schüler mit den Darstellungen arbeiten, welche Strategien in Problemlöseprozessen erkennbar sind und inwiefern typische diskrete Arbeitsweisen beim Umgang mit Folgen, Differenzenfolgen, Z-Funktionen und Differenzenfunktionen durch das Werkzeug unterstützt werden. Darüber hinaus soll festgestellt werden, welche Fähigkeiten die Probanden beim Umgang mit Folgen und Differenzenfolgen im Problemlöseprozess besitzen und welche Kenntnisse und Vorstellungen sie über Eigenschaften von Differenzenfolgen und Differenzenfunktionen während der Bearbeitung des Lernprogramms erwerben. Da die Schüler im Vorfeld der Untersuchung noch keine Kenntnisse der Differenzialrechnung erworben hatten, interessiert insbesondere ein Vergleich mit den Arbeitsweisen und dem Verständnis von Studierenden, also Probanden, die sich bereits mit der Infinitesimalrechnung beschäftigt haben.

Im siebten Kapitel werden die Ergebnisse der empirischen Untersuchung unter Berücksichtigung der drei Arbeitsebenen Objekt, Darstellung und Werkzeug sowie im Hinblick auf den Lernerfolg und den Verständniszuwachs zusammengefasst und diskutiert. Die Analyse der Arbeitsweisen auf der Objektebene macht deutlich, dass der Themenkomplex „Differenzenfunktionen“ elementar zugänglich ist, wenngleich dessen Behandlung mit manchen Schwierigkeiten verbunden ist. Diese Probleme sind zum einen in formalen Begriffsbestimmungen begründet, zum anderen lassen sie sich auf eine noch unzureichende Vertrautheit mit den Darstellungen zurückführen. Es zeigt sich jedoch auch, dass die Möglichkeit des einfachen Wechselns zwischen den Darstellungen Tabelle und Graph sowie die Möglichkeit des dyna-

\footnotetext{
${ }^{5}$ Mit Z-Funktionen werden polynomiale Funktionen bezeichnet, die auf den ganzen Zahlen definiert sind. Das Konzept „Z-Funktionen und Differenzenfunktionen“ kann als Erweiterung des Konzepts „Folge und Differenzenfolge" angesehen werden.
} 
mischen Visualisierens zur Entwicklung von Begriffsvorstellungen, insbesondere von Vorstellungen von Differenzenfunktionen als spezielle Änderungs(raten)funktionen, beitragen kann. Weiterhin wird deutlich, dass das Werkzeug TKP einen experimentellen Zugang zu Begriffsbildungsprozessen und Problemlösungen unterstützt, wobei jedoch das stete InBeziehung-Setzen zu theoretischen Überlegungen unbedingt erforderlich ist. Dabei zeigt sich auch, dass eine sinnvolle und effektive Auseinandersetzung mit den mathematischen Inhalten eine grundlegende Einführung in die technischen Bedienungselemente eines TKP erfordert. Wie die Untersuchung darüber hinaus deutlich macht, kann beim Einsatz eines TKP ein inhaltliches Verständnis für die Begriffe Differenzenfolge bzw. Differenzenfunktion entwickelt und die Problemlösekompetenz gefördert werden. Inwiefern dieses Vorverständnis dann im Rahmen des späteren Analysisunterrichts gewinnbringend aufgegriffen werden kann und wie das Zusammenspiel experimenteller Methoden und theoretischer Reflexion im Rahmen von Problemlöseprozessen über einen längeren Zeitraum hinweg initiiert und aufrechterhalten werden kann, ist im Rahmen weiterer Untersuchungen zu klären. 


\section{Zur Entwicklung Diskreter Mathematik}

Diskrete Mathematik umfasst aus heutiger Sicht verschiedene, eigenständige Themengebiete bzw. Forschungsrichtungen. Ihnen gemeinsam ist die Beschäftigung mit „diskreten Strukturen“, insbesondere mit endlichen Mengen und darauf vereinbarten Relationen oder algebraischen Strukturen. Im Folgenden wird die historische Genese der wichtigsten Bereiche der Diskreten Mathematik kurz skizziert. Dabei sollen Wurzeln diskreter Begriffe herausgearbeitet und ihre Bedeutung im Rahmen ihrer Entwicklungsgeschichte sowie Beziehungen zwischen den einzelnen Teildisziplinen aufgezeigt werden.

\subsection{Kombinatorik}

Die Anfänge der Kombinatorik - man versteht darunter im weiteren Sinne die Beschäftigung mit natürlichen Zahlen oder endlichen Mengen - waren zunächst eng mit zahlentheoretischen Fragestellungen verbunden. ${ }^{6}$ Hierzu zählen etwa die „,magischen Quadrate“7 , deren Wurzeln im China der Zeit 2200 v. Chr. vermutet werden, sowie die Polygonalzahlen ${ }^{8}$ und deren Veranschaulichungen als Punktemuster (figurierte Zahlen), mit denen sich bereits die Pythagoräer 500-400 v. Chr. beschäftigten. Kombinatorische Betrachtungen wurden in der Folgezeit nur vereinzelt angestellt und sind häufig dem Bereich der „Zahlenspielerei“ zuzuordnen. So befasste sich Leonardo von Pisa in seinem Werk „Liber Abacci“ (1202) mit einem speziellen kombinatorischen Zählproblem, der „Kaninchenaufgabe“" ${ }^{\text {} 9}$. Die Lösung des Problems, die so genannte Fibonacci-Folge, kann als eine der ersten rekursiv definierten Zahlenfolgen angesehen werden. Mit der Berechnung von Summen von Gliedern endlicher Folgen, wie etwa der Quadrat- und Kubikzahlen, oder von arithmetischen Reihen höherer Ordnung ${ }^{10}$ beschäftigten sich im 15. und 16. Jahrhundert z. B. die italienischen Mathematiker Luca Pacioli und Niccolo Tartaglia. Auch die magischen Quadrate waren immer wieder Gegenstand kombinatorischer Untersuchungen. Systematische Untersuchungen begannen erst im 17. Jahrhundert und

\footnotetext{
${ }^{6}$ Einen Überblick über die historische Entwicklung der Kombinatorik gibt Flachsmeyer 1972, S. 215 ff.

${ }^{7}$ Magische Quadrate sind Zahlenquadrate, deren Zeilen-, Spalten-, und Diagonalsummen denselben Wert liefern.

${ }^{8}$ Polygonalzahlen entstehen als Folge der Partialsummen einer Zahlenfolge, deren Glieder den gleichen „Abstand" haben. Durch Betrachten der Summe $1+2+3+4+\ldots$ erhält man auf diese Weise die Dreieckszahlen, $1+3+5+7 \ldots$ ergibt die Quadratzahlen usw.

${ }^{9}$ Bei der „Kaninchenaufgabe“ geht man von der folgenden Fragestellung aus: Kaninchenpaare bringen ab dem 2. Monat ihres Lebens jeden Monat ein neues Kaninchenpaar hervor. Wie viele Kaninchenpaare gibt es dann im $n$-ten Monat, wenn man davon aus geht, dass alle Kaninchen ewig leben? ( $\mathrm{Zu}$ einer alternativen Formulierung dieses Problems vgl. Kap. 2.2.1).

${ }^{10}$ Als arithmetische Reihe höherer (bzw. $k$-ter) Ordnung bezeichnet man die Summenfolge einer arithmetischen Folge höherer (bzw. $k$-ter) Ordnung. Arithmetische Folgen $k$-ter Ordnung sind Folgen, deren $k$-te Differenzenfolge konstant und deren ( $k-1)$-te Differenzenfolge nicht konstant ist. (Zum Begriff der Differenzenfolge vgl. auch die Ausführungen im Rahmen von Kap. 2.2.1.)
} 
waren eng mit der Begründung der Wahrscheinlichkeitstheorie verbunden. Das Abzählen von Konfigurationen, wie Permutationen, Variationen und Kombinationen, stellte hier ein Hilfsmittel zur Lösung praktischer Probleme dar, so etwa bei Fragen der Gewinnwahrscheinlichkeit bei Glücksspielen. Pascals Abhandlung „Traité du triangle arithmétique“ (1654), in der er die Binomialkoeffizienten ${ }^{11}$ beschrieb und diese in dem nach ihm benannten Pascal'schen Dreieck anordnete, erwies sich in diesem Zusammenhang bei der Abzählung von Kombinationen als hilfreich. In Verbindung und in Wechselwirkung mit wahrscheinlichkeitstheoretischen Überlegungen entstanden Werke wie die „Dissertatio de arte combinatoria“12 von Leibniz (1666) oder Jakob Bernoullis „Ars conjectandi“ aus dem Jahr 1713.

Kombinatorische Überlegungen hatten im 17. und 18. Jahrhundert auch Einfluss auf die Begründung und Entwicklung der Infinitesimalrechnung. So beschäftigte sich Leibniz in seiner „Dissertatio de arte combinatoria“ mit der inversen Beziehung zwischen Summen- und Differenzenbildung bei Zahlenfolgen. Die von ihm analysierten Differenzenfolgen entstehen durch das Bilden der Differenz benachbarter Folgenglieder, umgekehrt erhält man die Ausgangsfolge bei Kenntnis des Anfangsfolgenglieds durch Summenbildung zurück. Diese an Zahlenfolgen gemachten Beobachtungen übertrug Leibniz auf die Ordinaten einer Kurve, wobei er annahm, dass die Differenzen unendlich klein seien (vgl. Heuser 1990, S. 671 f.). Die arithmetische Beschäftigung mit Differenzen von Folgen gab Leibniz - seinen eigenen Angaben nach entscheidende Anregungen für die Infinitesimalrechnung. ${ }^{13}$ Später gewannen kombinatorische Betrachtungen in Verbindung mit der Algebraischen Analysis erneut an Bedeutung. Die Algebraische Analysis, die auf Euler zurückgeht, kann als Versuch beschrieben werden, die Analysis möglichst umfassend auf das Rechnen mit Potenzreihen zurückzuführen (vgl. Volkert 1988, S. 147). Im 18. Jahrhundert wurde diese Idee von der weitgehend in Deutschland vertretenen kombinatorischen Schule um Hindenburg aufgegriffen. Ausgehend von der Annahme, dass alle (wichtigen) Funktionen in endliche oder unendliche Reihen entwickelt werden können, sahen die Vertreter dieser Schule ihre Hauptaufgabe in der Beschreibung algebraischer Operationen mit kombinatorischen Mitteln. ${ }^{14}$ Damit rückte der polynomische Satz ${ }^{15}$

\footnotetext{
${ }^{11}$ Eine Tabelle der Binomialkoeffizienten sowie ein rekursives Bildungsgesetz dieser Zahlen findet sich jedoch bereits in Stifels „Arithmetica integra“" aus dem Jahr 1544.

${ }^{12}$ Auf dieses Werk geht auch der Begriff „Kombinatorik“ zurück.

${ }^{13}$ Eine geometrische Vorstellung vom Differenzialquotient erwarb Leibniz dabei durch die Betrachtung des charakteristischen Dreiecks, das er in einem nicht veröffentlichten Manuskript mit dem Titel „Historie et origio“ als zweite Quelle für seine Entdeckung der Infinitesimalrechnung angab (vgl. Volkert 1987, S. 94).

${ }^{14}$ Dieser Ansatz kann als Umkehrung der von Euler und Laplace entwickelten so genannten „Methode der erzeugenden Funktionen“ betrachtet werden. Hierbei werden Beziehungen zwischen Potenzreihen genutzt, um kombinatorisch interpretierbare Gleichungen zu beweisen oder kombinatorische Identitäten zu gewinnen (vgl. Jahnke 1999, S. 168).

${ }^{15}$ Der allgemeine polynomische Satz gibt eine Auswertung einer beliebigen Potenz eines mehrgliedrigen Summenausdrucks $a_{1}+a_{2}+\ldots+a_{n}$ an. Er kann als Verallgemeinerung des allgemeinen binomischen Lehrsatzes angesehen werden, welcher besagt: Für alle rationalen und negativen Zahlen $m$ gilt:
} 
in den Mittelpunkt ihrer Untersuchungen. Hindenburg bemühte sich um einen rein kombinatorischen Beweis dieses Satzes und lieferte dabei die erste explizite Formel für die Potenzierung beliebiger Reihen mit beliebigen Exponenten. ${ }^{16}$ Neben dem Versuch, die algebraische Sicht der Analysis weiterzuentwickeln und mit kombinatorischen Mitteln zu systematisieren, leisteten die Arbeiten der kombinatorischen Schule auch einen wichtigen Beitrag zur Etablierung einer eigenständigen Disziplin Kombinatorik (vgl. Jahnke 1999, S. 161 ff).

Im Jahr 1901 erschien das erste umfassende Lehrbuch zur Kombinatorik (Netto 1901). Die darin vertretene Auffassung von Kombinatorik als das Gebiet, ,welches sich mit Stellung, Anordnung und Auswahl von Elementen aus endlichen Mengen beschäftigt" (Netto 1901, S. 1), das also im Wesentlichen den Fokus auf Zählprobleme richtet, änderte sich in den folgenden 30 Jahren kaum. Erst ab Mitte des 20. Jahrhunderts interessierte man sich zunehmend auch für andere kombinatorische Fragestellungen, wie Existenz- und Optimierungsaufgaben. Diese Entwicklung stand in engem Zusammenhang mit der Entwicklung der modernen Algebra, Logik und Geometrie. ${ }^{17}$

\subsection{Graphentheorie}

Die Entwicklung der Graphentheorie begann mit einer Arbeit Eulers aus dem Jahr 1736 über das so genannte Königsberger Brückenproblem. Er ging darin der Frage nach, ob es einen Spaziergang durch Königsberg gibt, bei dem man jede der sieben Königsberger Brücken über den Pregel genau einmal überquert und zum Ausgangspunkt zurückkehrt. Euler erstellte ein abstraktes Wegenetz, indem er verschiedene Landgebiete durch Punkte (Ecken) und die jeweiligen Brückenverbindungen durch Linien (Kanten) darstellte. Anhand des so entstandenen Graphen ${ }^{18}$ bewies Euler die Unlösbarkeit des Problems und gab auch notwendige und hinreichende Bedingungen für die Existenz derartiger Rundwege (eulersche Wege) bei beliebigen Graphen an. Graphentheoretische Fragestellungen wurden zunächst nur unsystematisch und sporadisch weiterverfolgt. Anwendungen fanden sich u. a. in der Physik und in der Chemie. So verwendete Kirchhoff in einer Arbeit aus dem Jahr 1878 zum Fluss des elektrischen Stroms durch verzweigte Leiter (Kirchhoff'sche Regeln) graphentheoretisch-kombinatorische Mittel (vgl. Dieudonné 1985, S. 644). Diagramme wurden außerdem zur Darstellung von Mo-

$$
(1+x)^{m}=1+\left(\begin{array}{c}
m \\
1
\end{array}\right) x+\left(\begin{array}{c}
m \\
2
\end{array}\right) x^{2}+\left(\begin{array}{c}
m \\
3
\end{array}\right) x^{3}+\ldots=\sum\left(\begin{array}{l}
m \\
k
\end{array}\right) x^{k} .
$$

${ }^{16}$ Frühere Untersuchungen zum polynomischen Satz, wie etwa durch Leibniz, Jakob Bernoulli oder Euler, beschränkten sich zum Teil auf natürliche Exponenten oder benutzten zum Beweis Mittel der Differenzialrechnung (vgl. Jahnke 1990, S. 189).

${ }^{17}$ Zur Entwicklung der Kombinatorik in jüngerer Zeit vgl. Aigner 1990, S. 85 ff.

${ }^{18}$ Unter einem Graph versteht man ein Paar $G=(V, E)$, wobei $V$ eine beliebige Menge ist und $E$ eine Menge zweielementiger Teilmengen von $V$ ist. Die Elemente von $V$ heißen Ecken und die Elemente von $E$ Kanten von 
lekülen verwendet, mit deren Hilfe Fragen nach der Anzahl und Art bestimmter chemischer Verbindungen untersucht wurden. ${ }^{19}$ Andere Anwendungen waren dagegen eher der Unterhaltungsmathematik zuzuordnen, wie etwa das 1859 von Hamilton vorgestellte Gesellschaftsspiel „Around the World“. Das Problem bestand darin, in einem speziellen Graph ${ }^{20}$ einen geschlossenen Weg zu finden, der jede Ecke genau einmal durchläuft. Graphen, die einen derartigen Kreis besitzen, werden heute als „hamiltonsch“ bezeichnet. Im Gegensatz zu den eulerschen Graphen ist bis heute keine befriedigende Charakterisierung hamiltonscher Graphen bekannt.

Das Bemühen um eine Lösung des Vierfarbenproblems ${ }^{21}$, das 1878 von Cayley veröffentlicht wurde, hat wesentlich zur Entwicklung der Graphentheorie beigetragen und kennzeichnete die Epoche der Graphentheorie bis 1930. ${ }^{22}$ Heawood zeigte 1890, dass zur Färbung einer Landkarte fünf Farben auf jeden Fall ausreichen. Ob eine Färbung auch mit vier Farben möglich ist, war lange Zeit ein offenes Problem. Erst im Jahre 1977 gaben Appel, Haken und Koch einen Beweis an, der aufgrund äußerst umfangreicher Fallunterscheidungen bisher nur auf dem Computer bearbeitet werden konnte. Das Überprüfen der Korrektheit des Beweises führte immer wieder zur Aufdeckung (und Behebung) kleinerer Fehler im verwendeten Computerprogramm. Bis heute ist die Frage offen, ob es einen „einfachen“, vollständig von der Hand überprüfbaren Beweis gibt.

Im Jahre 1936 erschien mit dem Lehrbuch von König eine erste umfassende Darstellung der Graphentheorie für den deutschen Sprachraum. Die Entwicklung der Graphentheorie in den 30er Jahren war wesentlich durch das Interesse an Eigenschaften mathematischer Strukturen bzw. deren Existenz unter vorgegebenen Bedingungen geprägt. Bedeutende Arbeiten aus diesem Zeitraum stammen von Topologen und Algebraikern, wie etwa Withney und Hall. Während Withney eine neue Charakterisierung planarer (d. h. in die Ebene einbettbarer) Graphen mit Hilfe des Begriffs Matroid angab, untersuchte Hall Bedingungen für die Existenz von Matchings bipartiter Graphen ${ }^{23}$. Das von Hall 1935 angegebene Ergebnis, das als „Heirats-

G. Graphen veranschaulicht man durch Diagramme (vgl. Ihringer 1994, S. 12). Diese Diagramme entsprechen den von Euler verwendeten „Wegenetzen“.

${ }^{19}$ Die Darstellung von Molekülen durch Strukturdiagramme geht auf F. A. Kekulé zurück, der die Theorie der chemischen Verbindungen begründet hat. In diesem Zusammenhang wird im Jahr 1878 auch der Name „Graph“ erstmalig in der Literatur benutzt (vgl. Nägler \& Stopp 1996, S. 9).

${ }^{20}$ Es handelte sich hierbei um das Schlegeldiagramm eines regulären Dodekaeders.

${ }^{21}$ Dieses Problem wird irrtümlicherweise englischen Kartographen zugeschrieben, geht aber auf Francis Guthrie, einen Juristen, Botaniker und Mathematiker, zurück. Guthrie stellte beim Färben einer Landkarte die Vermutung auf, dass eine beliebige Landkarte so mit vier Farben gefärbt werden kann, dass benachbarte Länder immer verschiedene Farben haben. In der graphentheoretischen Formulierung des Problems werden Landkarten als Diagramme planarer Graphen aufgefasst. Zur Geschichte des Vierfarbensatzes vgl. die ausführliche Darstellung von Fritsch \& Fritsch 1994.

${ }^{22}$ Vgl. Aigner 1990, der einen Überblick über die historische Entwicklung der Graphentheorie gibt. Zur fachwissenschaftlichen Behandlung der Graphentheorie vgl. z. B. Aigner 1996, Jungnickel 1994.

${ }^{23}$ Bipartite Graphen sind Graphen, deren Eckenmengen in zwei disjunkte Teilmengen zerlegt werden können, so dass alle Kanten ihren einen Eckpunkt in der einen Teilmenge und den anderen Eckpunkt in der anderen Teil- 
satz" in die Literatur eingegangen ist, ${ }^{24}$ wurde in den folgenden Jahren von bipartiten Graphen auf allgemeine Strukturen erweitert (vgl. Aigner 1990, S. 98 ff.).

\subsection{Kombinatorische Optimierung}

Durch die Entwicklung leistungsfähiger Computer in der Mitte des 20. Jahrhunderts erhielt die Beschäftigung mit diskreten Strukturen eine neue Bedeutung. Waren diskrete Modelle und Rechenverfahren bzw. Algorithmen ${ }^{25}$ bislang hauptsächlich von theoretischem Interesse, so konnten nun die damit verbundenen umfangreichen Berechnungen bzw. Datenverarbeitungen von einem Computer übernommen werden und zur Lösung praktischer Probleme in Wirtschaft und Technik eingesetzt werden. Insbesondere Optimierungsfragen rückten damit in den Mittelpunkt des Forschungsinteresses. ${ }^{26}$ Einige Beispiele sollen dies verdeutlichen.

\section{- Kürzeste Wege}

Graphen werden im Alltag häufig zur Darstellung von Verkehrs- und Kommunikationsnetzen eingesetzt. Beispiele hierzu sind etwa Übersichtskarten der Autobahnen oder das Streckennetz von U- und S-Bahnlinien bzw. einer Fluggesellschaft. In diesem Zusammenhang interessiert häufig der ,günstigste Weg“ von einer Stelle $a$ zu einer Stelle $b$, d. h. - je nach Fragestellung die kürzeste, schnellste oder billigste Verbindung. Zur Modellierung dieser Netze werden den Kanten eines Graphen „Längen“ (bzw. „Gewichte“) zugeordnet, man erhält so genannte Netzwerke. Dijkstra gab 1959 einen Algorithmus an, der die Abstände zweier Punkte des Graphen bestimmte, d. h. das Minimum der Längen aller Kantenzüge mit Anfangspunkt $a$ und Endpunkt $b$ angab. Bei leichter Abänderung kann der Algorithmus von Dijkstra auch zur Konstruktion solcher kürzester Wege eingesetzt werden.

\section{- Maximale Flüsse}

In der Flusstheorie werden die Längen in den Netzwerken als Kapazitäten interpretiert. Die entsprechenden „Kapazitätsnetzwerke“ werden zur Modellierung von Pipeline- oder Bewässerungssystemen, Stromversorgungsnetzen oder Verkehrssystemen eingesetzt. Es ergibt sich die Frage, welche Menge (Wasser, Strom, Autos) maximal von einer „Quelle“ zu einer „Sen-

\footnotetext{
menge haben. Unter einem Matching eines bipartiten Graphen $G$ versteht man eine Menge von Kanten von $G$, so dass keine zwei Kanten eine Ecke gemeinsam haben (vgl. Ihringer 1994, S. 46).

${ }^{24}$ In einer ,anschaulichen“ Formulierung des Heiratssatzes werden die beiden Eckenteilmengen $S$ und $T$ des bipartiten Graphen als Menge von Damen bzw. Menge von Herren interpretiert. Eine Verbindung durch eine Kante kennzeichnet, dass das entsprechende Paar einer Heirat nicht abgeneigt ist. Der Satz gibt nun eine notwendige und hinreichende Bedingung dafür an, dass alle Männer eine Partnerin finden, d. h. der Graph ein Matching der Mächtigkeit $|T|$ besitzt.

${ }^{25}$ Unter einem Algorithmus verstehen wir hier ein allgemeines Verfahren, das nach endlich vielen Einzelschritten die Lösung eines Problems liefert. Die Reihenfolge der Lösungsschritte ist dabei eindeutig festgelegt.

${ }^{26}$ Einen guten Einblick in die Probleme der Kombinatorischen Optimierung und die entsprechenden Algorithmen geben z. B. Ihringer 1994, Jungnickel 1994.
} 
ke“ transportiert werden kann, so dass zum einen die Kapazität der Kanten (Wasserleitungen, Stromleitungen, Straßen) nicht überschritten wird und zum anderen die Knotenregel erfüllt ist (d. h. aus jeder Ecke, mit Ausnahme von Quelle und Senke, fließt genauso viel hinein wie hinaus). Ein Algorithmus zum Finden eines „maximalen Flusses“ wurde 1956 von Ford und Fulkerson angegeben.

\section{- Minimal aufspannende Bäume ${ }^{27}$}

Bei der Konstruktion von Straßensystemen oder Elektrizitätsnetzwerken sollen häufig möglichst ökonomische, kostengünstige Lösungen gefunden werden, indem überflüssige Wege ${ }^{28}$ vermieden werden, aber dennoch alle Knotenpunkte (wenn auch möglicherweise über Umwege) miteinander verbunden bleiben. Zur Modellierung des Problems werden zunächst alle in Frage kommenden Verbindungen sowie die damit verbundenen Kosten durch ein Netzwerk dargestellt. Gesucht ist nun ein minimal aufspannender Baum, d. h. ein kreisfreier, zusammenhängender Graph mit minimalem Gesamtgewicht. Eine Lösung des Problems liefert der Algorithmus von Kruskal aus dem Jahr 1956. Es handelt sich hierbei um einen Sonderfall des so genannten Greedy-Algorithmus. Die dahinter stehende Strategie ist insofern einfach, als immer das momentan am besten erscheinende Element (in diesem Fall eine zulässige Kante mit kleinstem Gewicht) ausgewählt und zur Lösung hinzugefügt wird („Gierige Strategie“).

\section{- Ablaufplanung}

Die Ablaufplanung ist ein Spezialgebiet der Kombinatorischen Optimierung, deren Probleme sich zum großen Teil aus Aufgabenstellungen der Projektplanung oder der industriellen Fertigung ergeben, d. h. dort wo Teilaufgaben koordiniert bzw. die Reihenfolge ihrer Durchführung optimal geplant werden müssen. Häufig wird dabei vorausgesetzt, dass bestimmte Aufgaben erst nach der Beendigung anderer Aufgaben begonnen werden können. Von Interesse sind die kürzestmögliche Gesamtdauer des Projekts sowie die Zeitpunkte, zu denen die einzelnen Teilaufgaben begonnen werden sollten. In einfachen Fällen geht man zusätzlich davon aus, dass zu jedem Zeitpunkt die nötigen Arbeitskräfte bzw. das nötige Material zur Verfügung gestellt werden können. Dieses Problem führt zur Bestimmung längster Wege in kreisfreien gerichteten Graphen, das mit der 1956/57 entwickelten „Critical Path Method“ (vgl. Jungnickel 1994, S. 104 ff.) gelöst werden kann. ${ }^{29}$ Komplexere Situationen entstehen, wenn die Anzahl der verwendeten Arbeitskräfte oder Maschinen beschränkt ist. Auch hierfür sind

\footnotetext{
${ }^{27}$ Anstelle des Begriffs ,,minimal aufspannender Baum“ wird in der fachwissenschaftlichen Literatur auch häufig der Begriff „minimal erzeugender Baum“ verwendet.

${ }^{28}$ Hierzu zählen in erster Linie „Rundwege“.

${ }^{29}$ Bei einem gerichteten Graphen wird den einzelnen Kanten eine „Richtung“ zugeordnet, d. h. man betrachtet eine Menge $V$ von Punkten und eine Menge $E$ von geordneten Paaren, deren Elemente aus $V$ stammen. Die Ablaufplanung kann auch mengentheoretisch formuliert werden. Anstelle kreisfreier gerichteter Graphen betrachtet
} 
Teillösungen bekannt, wie etwa für Spezialfälle des so genannten 2-Maschinenmodells. ${ }^{30} \mathrm{Un}$ ter der Voraussetzung, dass die Dauer für die Bearbeitung aller Teilaufgaben gleich ist, berechnet der Algorithmus von Coffman und Graham aus dem Jahr 1972 einen geeigneten Ablaufplan.

Bei den vorstehenden Beispielen handelte es sich ausschließlich um graphentheoretisch formulierte Beispiele der kombinatorischen Optimierung. Solche oder verwandte Probleme, wie etwa das Angebot-Nachfrage-Problem ${ }^{31}$, das Job-Zuordnungsproblem ${ }^{32}$ oder das TravelingSalesman-Problem ${ }^{33}$, lassen sich zum Teil auf das Problem zurückführen, ein lineares Funktional unter vorgegebenen Nebenbedingungen zu maximieren oder minimieren. Mit der allgemeinen Behandlung solcher Probleme beschäftigt sich die Theorie der linearen Optimierung, die auch als Teilgebiet der Diskreten Mathematik verstanden werden kann. ${ }^{34}$ Fundamentale Resultate dieser Theorie stellen der auf v. Neumann zurückgehende Dualitätssatz und der von Danzig entwickelte Simplex-Algorithmus aus dem Jahr 1947 dar.

\subsection{Komplexitätstheorie}

Durch die Möglichkeiten der elektronischen Datenverarbeitung gewann die Entwicklung von Lösungsverfahren zur Konstruktion optimaler Konfigurationen an Bedeutung. Trotz immer schnellerer Computer war jedoch der Aufwand zur Lösung bestimmter Probleme immer noch unverhältnismäßig groß und damit für die Praxis nur von untergeordneter Relevanz. Damit rückte in den 60er Jahren die Suche nach „guten“, d. h. insbesondere zeiteffizienten Algorithmen sowie nach einer Möglichkeit zur Beurteilung der Qualität von Algorithmen in das Zentrum des Forschungsinteresses und es entstand das Gebiet der Komplexitätstheorie. In der

man hierzu endliche geordnete Mengen (vgl. Ihringer 1994, S. 209). Unter einer Ordnung versteht man hierbei eine Relation auf einer Menge, welche die Bedingungen Reflexivität, Antisymmetrie und Transitivität erfüllt.

${ }^{30}$ Für das allgemeine 2-Maschinenproblem ist bisher keine Lösung bekannt. Es handelt es sich um ein NPvollständiges Problem (vgl. Kap. 1.4).

${ }^{31}$ Das Angebot-Nachfrage-Problem lautet wie folgt: Von einer bestimmten Anzahl von Produktionsstätten sollen Waren zu einer bestimmten Anzahl von Abnehmerstätten transportiert werden. Produktions- und Abnehmerstätte sind über ein Straßensystem verbunden, welches gewissen Kapazitätsbeschränkungen unterliegt und dessen Benutzung mit Kosten verbunden ist. Jede Produktionsstätte besitzt ein gewisses Angebot (an Waren), und in jeder Abnehmerstätte besteht eine bestimmte Nachfrage nach diesem Produkt. Das Problem besteht in der Konstruktion eines minimalen (kostengünstigsten) (Waren-)Flusses, der nicht mehr Waren aus den einzelnen Produktionsstätten hinausführt als dort angeboten werden und mindestens die jeweils von den Abnehmerstätten angeforderten Warenmengen anliefert.

${ }^{32}$ Es handelt sich hierbei um eine andere Formulierung des Heiratsproblems (vgl. Kap. 1.2), wobei jedoch die Konstruktion eines maximalen Matchings im Vordergrund steht.

${ }^{33}$ Das Traveling-Salesman-Problem geht von der folgenden Fragestellung aus: Ein Handlungsreisender plant eine Rundreise durch verschiedene Städte; dabei möchte er am Ende der Reise wieder am Ausgangspunkt ankommen. In welcher Reihenfolge muss er die Städte besuchen, so dass seine Reise eine möglichst geringe Gesamtlänge hat?

${ }^{34}$ Eine Einführung in die Theorie der linearen Optimierung gibt Aigner 1996. Insbesondere wird hier ein Bezug zu zuvor behandelten graphentheoretisch formulierten Problemstellungen hergestellt. 
Komplexitätstheorie wird die Qualität eines Algorithmus anhand des Zeitaufwands zur Bearbeitung eines Problems und des dafür benötigten Speicherplatzes bestimmt. Sowohl Zeit- als auch Speicherplatzaufwand hängen von der Anzahl der Eingabeparameter ab. Zur Abschätzung des Zeitaufwands definiert man die Zeitkomplexität als eine Funktion $f(n)$, welche die Anzahl der (Rechen-)Schritte angibt, die der Algorithmus zur Lösung eines Problems vom Umfang $n$ maximal benötigt. Dabei werden unter einem Rechenschritt z. B. arithmetische Operationen, Zugriffe zu Feldern oder Vergleichsoperationen verstanden (vgl. Jungnickel 1994, S. 69) ${ }^{35}$ Unter praktischen Gesichtspunkten hält man im Allgemeinen nur polynomiale Algorithmen für gut, d. h. Algorithmen der Komplexität $\boldsymbol{O}\left(n^{k}\right)$, wenngleich es auch schnell arbeitende Algorithmen exponentieller Komplexität gibt, wie etwa den SimplexAlgorithmus. ${ }^{36}$

Mit der Klassifikation von Problemen ${ }^{37}$ ähnlicher Komplexität beschäftigte sich Cook in seiner Arbeit „The Complexity of Theorem Proving Procedures“ aus dem Jahr 1971 (vgl. Aigner 1990, S. 109 f.). Er unterschied die Klassen $P$ und $N P$. In die Klasse $P$ (für „polynomial“) fallen diejenigen Probleme, die mit polynomialem Zeitaufwand lösbar sind. Dagegen wird bei der Klasse NP (für „nicht-deterministisch polynomial“) der Aufwand zur Verifizierung einer gegebenen Lösung betrachtet. Die Klasse NP besteht aus Problemen, bei denen eine Verifizierung einer gegebenen Lösung mit polynomialem Zeitaufwand möglich ist. Die Klasse $P$ ist in der Klasse NP enthalten; die Klasse NP enthält darüber hinaus auch Probleme, zu denen bisher kein polynomialer Lösungsalgorithmus bekannt ist, wie etwa das Hamiltonian-PathProblem $^{38}$. Die Frage, ob es für solche Probleme einen guten Algorithmus gibt, ob also $P=N P$ gilt, ist eine der großen offenen Fragen der Mathematik. Die Frage kann dann positiv beantwortet werden, wenn es gelingt einen polynomialen Lösungsalgorithmus $\mathrm{zu}$,,besonders schwierigen" Problemen in $N P$ zu finden. Diese so genannten $N P$-vollständigen Probleme können als Repräsentanten der Klasse $N P$ angesehen werden: Aus der polynomialen Lösbarkeit eines $N P$-vollständigen Problems folgt die polynomiale Lösbarkeit aller NP-Probleme. Cook gab 1971 mit dem so genannten Satisfiability-Problem für Bool'sche Ausdrücke das erste Beispiel eines $N P$-vollständigen Problems an. Heute kennt man zahlreiche solcher $N P$ -

\footnotetext{
${ }^{35}$ Die Begriffe Algorithmus, Eingabeparameter, Zeit- und Platzbedarf, Komplexität werden hier zur Übersicht nur informell dargestellt. Eine Präzisierung kann durch den Begriff der Turing-Maschine geleistet werden.

${ }^{36}$ Dies liegt daran, dass die Komplexität stets für den ungünstigsten Fall eines Problems einer Problemklasse berechnet wird.

${ }^{37}$ In der Theorie der Komplexitätsklassen werden nur „Entscheidungsprobleme“ behandelt, d. h. Probleme, deren Lösung nur eine Antwort ,ja“ oder „,nein“ liefert. Optimierungsprobleme können durch Angabe einer zusätzlichen Schranke in Entscheidungsprobleme umgewandelt werden. Die Lösung dieses Problems ist dabei nicht schwerer als die des zugehörigen Optimierungsproblems.

${ }^{38}$ Es handelt sich hierbei um das Entscheidungsproblem, ob ein gegebener Graph einen hamiltonschen Kreis besitzt. Dieses Problem gehört zur Klasse NP, denn die Behauptung, ein bestimmter Weg in einem Graph sei ein hamiltonscher Kreis, lässt sich mit polynomialem Zeitaufwand überprüfen.
} 
Probleme, zu denen auch bedeutende Probleme, wie etwa das Traveling-Salesman-Problem oder das Hamiltonian-Path-Problem, zählen.

\subsection{Codierungstheorie und Kryptographie}

Durch die zunehmende Technologisierung und elektronische Vernetzung unserer Gesellschaft im Laufe des 20. Jahrhunderts wurden und werden in ständig wachsendem Umfang alle Arten von Daten auf elektronischem Weg gespeichert, übertragen und verarbeitet. Bei der Datenübertragung können sich dabei verschiedene Probleme ergeben. So kann der Übertragungskanal zufälligen Störungen, wie etwa einem allgemeinen „Leitungsrauschen“ oder einem Blitzeinschlag, ausgesetzt sein, so dass die Daten fehlerhaft verändert werden. Hier sind Methoden von Bedeutung, die Übertragungsfehler erkennen und korrigieren. Neben der Sicherung der Daten gegenüber zufälligen Störungen ist man insbesondere bei vertraulichen Informationen daran interessiert, dass kein unbefugter Dritter diese Nachrichten erhalten oder verändern kann. Fragen der Datensicherheit führen somit zu der Aufgabe, Nachrichten so zu gestalten, dass Außenstehenden der Zugriff verwehrt bleibt bzw. dass die Echtheit der Daten nachgewiesen werden kann. Ausgehend von diesen Problemstellungen entwickelten sich Mitte des 20. Jahrhunderts zwei weitere Bereiche der Diskreten Mathematik: die Codierungstheorie und die Kryptographie.

\section{Codierungstheorie}

Einen wesentlichen Beitrag zur Begründung der Codierungstheorie leistete Shannon mit seiner Arbeit „A mathematical theory of information“ aus dem Jahr 1984. Die Hauptergebnisse dieser Arbeit, die ersten und zweiten Hauptsätze der Informationstheorie, waren sowohl für das Problem der Quellencodierung als auch für die Sicherung codierter Nachrichten gegenüber zufälligen Störungen von großer Bedeutung. ${ }^{39}$ Bei der Quellencodierung werden die (verbalen) Nachrichten zum Zwecke der Datenübertragung zunächst in eine 0-1-Folge oder in ein anderes vorgegebenes System codiert. ${ }^{40}$ Diese Codierung soll zum einen eine eindeutige Entschlüsselung ermöglichen; zum anderen soll die durchschnittliche Codewortlänge - als Maß für die Datenkompression - möglichst klein sein, damit die Nachrichten möglichst effizient codiert und decodiert werden können. Mit Hilfe des ersten Hauptsatzes der Informationstheorie kann nun die optimale durchschnittliche Codewortlänge bis auf einen Fehler kleiner 1 bestimmt werden. Ausgangspunkt von Shannons Überlegungen zur Sicherung von Datenübertragungen war die Idee der Redundanz: Durch das Anhängen von Korrekturzeichen

\footnotetext{
${ }^{39}$ Vgl. Aigner 1990. Eine Einführung in die fachwissenschaftlichen Grundlagen der Codierungstheorie geben Aigner 1996, Ihringer 1994.

${ }^{40} \mathrm{Zu}$ diesem Zweck wird jedem Buchstaben des der Originalnachricht zugrunde liegenden Alphabets ein 0-1Wort zugeordnet. Diese Codewörter können unterschiedlich lang sein. Durch Aneinanderreihung entsprechender Codewörter kann die Originalnachricht codiert werden.
} 
an die gesendeten Worte sollte es dem Empfänger möglich sein, Übertragungsfehler zu korrigieren oder zumindest zu entdecken. Dabei sollte die Informationsrate ${ }^{41}$ möglichst groß und die Fehlerwahrscheinlichkeit bei der Decodierung möglichst klein sein. Mit dem zweiten Hauptsatz der Informationstheorie wird die Existenz eines „,bestmöglichen“ Codes bewiesen. Das Problem, wie ein derartiger Code oder „gute“ Codes konstruiert werden können, und wie man sie effizient implementiert, wurde von Hamming aufgegriffen. Es entstand die algebraische Codierungstheorie, in der die Codierung und Decodierung mittels Matrizenoperationen erklärt wurde. In diesem Zusammenhang konnten Fragen zur Fehlerentdeckung und Fehlerkorrektur angegangen und befriedigend gelöst werden.

\section{Kryptographie}

Der älteste Zweig der Kryptographie beschäftigt sich mit der Geheimhaltung von Nachrichten durch Verschlüsselung und diente lange Zeit hauptsächlich militärischen Zwecken. So wandten bereits die Spartaner (um 500 v. Chr.) kryptographische Techniken an, indem sie die Stellung von Buchstaben einer Nachricht mit Hilfe eines zylindrischen Stabs, der so genannten Skytale, veränderten. Julius Cäsar (100-44 v. Chr.) nutzte zur Verschlüsselung seiner Nachrichten Verschiebechiffren, deren Grundlage zwei gegeneinander verschobene Alphabete waren. Derartige Verschiebechiffren sind ein Spezialfall monoalphabetischer Kryptosysteme ${ }^{42}$, die - sofern ihnen die gesprochene Sprache zugrunde liegt - von einem „Angreifer“ mit statistischen Methoden leicht zu „brechen“43 sind. Diese Sicherheitsmängel wurden durch die Entwicklung der Vigenère-Verschlüsselung, die 1586 von dem französischen Diplomaten Blaise de Vigenère vorgestellt wurde, zunächst behoben. Die Vigenère-Verschlüsselung, bei der verschiedene monoalphabetische Chiffrierungen im Wechsel benutzt wurden, galt noch bis 1917 als „unbrechbar“. ${ }^{44}$ Im 20. Jahrhundert ergaben sich durch die Verbreitung von Rechnern neue Aufgabenfelder für die Kryptographie, die über die ursprünglichen militärischen Anwendungen hinausreichen. So ist heute der Schutz vertraulicher Nachrichten in Form von Telefongesprächen oder elektronischer Nachrichten (Emails) ein Anliegen der gesamten Kommunikationsgesellschaft. Im Jahr 1976 stellten Diffie und Hellmann das Public-KeySystem $^{45}$ vor, ein asymmetrisches Verschlüsselungsverfahren, bei dem ein weiteres großes

\footnotetext{
${ }^{41}$ Geht man von Worten der gleichen Länge $k$ sowie $r$ Kontrollsymbolen pro Wort aus, so gibt die Informationsrate das Verhältnis der Anzahl der tatsächlichen Informationssymbole zu der Anzahl der Symbole des gesamten Codeworts an, also $k:(k+r)$.

${ }^{42}$ Bei monoalphabetischen Kryptosystemen wird jeder Buchstabe des Klartextalphabets stets zu demselben Geheimtextbuchstaben verschlüsselt.

${ }^{43}$ Der Ausdruck „,brechen“ soll signalisieren, dass es sich hierbei um den Angriff einer nichtautorisierten Person handelt, welcher der Entschlüsselungsalgorithmus zunächst nicht bekannt ist.

${ }^{44}$ Vgl. Beutelspacher 1994, der eine Einführung in die elementaren Methoden der Kryptographie unter Berücksichtigung historischer Aspekte gibt.

${ }^{45}$ Beim Public-Key-System besitzt jeder Benutzer einen öffentlichen, allgemein zugänglichen Schlüssel und einen geheimen Schlüssel, der nicht ausgetauscht wird. Das berühmteste Public-Key-System ist das 1978 erfun-
} 
Sicherheitsproblem bisheriger Kryptosysteme - das des Schlüsselaustauschs zwischen Sender und Empfänger - gelöst wurde. Dies kann als der Beginn der modernen Kryptographie angesehen werden (vgl. Beutelspacher, Schwenk \& Wolfenstetter 1995). Der Aspekt der Geheimhaltung von Nachrichten ist jedoch nur ein Untersuchungsgegenstand der modernen Kryptographie. Weitere Problembereiche, die in den letzten Jahren eine zunehmende Bedeutung erlangten, sind Fragen der Authentikation und der Anonymität. Während es bei der Anonymität u. a. um die Geheimhaltung einer oder beider am Nachrichtenaustausch beteiligten Personen geht, ${ }^{46}$ ist man bei der Authentikation an der Sicherung von Nachrichten gegenüber unbefugten Änderungen interessiert. Ziel ist es hier, Methoden bereitzustellen, die es erlauben, Veränderungen von Nachrichten zu erkennen bzw. zu entscheiden, ob eine Nachricht tatsächlich vom angegebenen Absender stammt. ${ }^{47}$

Im Rahmen der oben aufgeführten Grundprobleme der Kryptographie fanden viele Ergebnisse der Zahlentheorie und der endlichen Geometrie eine Anwendung. Dabei wurden diese beiden mathematischen Teildisziplinen durch das wachsende Interesse an komplexen Sicherheitssystemen in ihrer Entwicklung vorangetrieben.

\subsection{Zusammenfassung}

Diskrete Fragestellungen reichen bis weit ins Altertum. In der Entwicklungsgeschichte der Diskreten Mathematik betrachtete man diskrete Begriffe oder diskrete Strukturen einerseits als eigenständige Objekte, an deren Eigenschaften man interessiert war. Andererseits dienten diese Begriffen als Hilfsmittel zum Lösen von Problemen bzw. für die Entwicklung anderer Begriffe. In diesem Zusammenhang lassen sich gewisse Schwerpunktverschiebungen feststellen, wobei drei Anknüpfungspunkte für fachdidaktische Untersuchungen deutlich werden:

- Die Beschäftigung mit diskreten Begriffen, wie etwa magischen Quadraten, figurierten Zahlen oder Graphen war zunächst vielfach intrinsisch motiviert und konnte häufig dem Bereich der „Unterhaltungsmathematik“ zugeordnet werden. Die Beschäftigung mit diesen reizvollen (innermathematischer) diskreten Problemstellungen hat die Entwicklung der Diskreten Mathematik vorangetrieben.

- Mit der Behandlung diskreter Fragestellungen ergaben sich Beziehungen zur Entwicklung anderer mathematischer Gebiete. So gab die Beschäftigung mit Differenzenfolgen Impulse zur Begründung der Infinitesimalrechnung und kombinatorische Überlegungen beein-

\footnotetext{
dene RSA-System, benannt nach seinen Entdeckern Rivest, Shamir und Adleman. Die Sicherheit dieses System beruht auf der Primfaktorzerlegung großer Zahlen.

${ }^{46}$ Anwendungen finden sich etwa bei Methoden des elektronischen Bezahlens oder bei Modellen elektronisch durchgeführter Wahlen.

${ }^{47}$ Man spricht hier von Nachrichtenauthentikation. Ein weiteres Problem besteht im Nachweis der Identität einer Person gegenüber einer Maschine wie etwa einem Bankautomaten. Es handelt sich hierbei um das Problem der Teilnehmerauthentikation.
} 
flussten sowohl die Entwicklung der Wahrscheinlichkeitsrechnung als auch die der algebraischen Analysis. In jüngerer Zeit zeigen sich insbesondere zwischen verschiedenen Teilgebieten der Diskreten Mathematik zahlreiche Wechselwirkungen, so etwa zwischen Kryptographie, endlicher Geometrie und Zahlentheorie.

- Durch die Einführung der ersten schnellen Rechner und den damit verbundenen Möglichkeiten einer effektiven Datenverarbeitung gewannen diskrete Begriffe und Methoden bei der Modellierung außermathematischer Situationen an Bedeutung, und es eröffneten sich vielfältige neue Anwendungsbereiche in Wirtschaft und Technik.

Im Hinblick auf diese Aspekte soll im Folgenden untersucht werden, welche Bedeutung diskrete Problemstellungen, Begriffe und Anwendungen im Mathematikunterricht haben können und inwiefern diskrete Inhalte im Sinne des genetisch-historischen Prinzips in den Unterricht integriert werden sollten. 


\section{Diskrete Mathematik im Mathematikunterricht}

Diskrete Mathematik stellt kein „Standardthema“ des derzeitigen Mathematikunterrichts dar. Dennoch waren diskrete Inhalte und Themen immer wieder Gegenstand der didaktischen Diskussion. Im Rahmen dieses Kapitels sollen zunächst Ansätze dieser Diskussion vorgestellt und die wesentlichen Argumentationslinien aufgezeigt werden. Dabei wird wie im ersten Kapitel eine inhaltliche, fachgebundene Perspektive eingenommen. Zur Analyse der didaktischen Bedeutung diskreter Mathematik im Mathematikunterricht wird daran anschließend ein Sichtwechsel vorgenommen. Ausgehend von den im Rahmen der mathematikdidaktischen Diskussion immer wieder herausgestellten Aspekten Problemlösen, Begriffsbilden und Anwenden, werden ausgewählte diskrete Themen unter Berücksichtigung bestehender Vorschläge genauer untersucht.

\subsection{Rückblick auf die Entwicklung diskreter Mathematik im Mathematik- unterricht}

Das didaktische Potenzial diskreter Themengebiete wie Kombinatorik, Graphentheorie und Kryptographie wurden in der Vergangenheit im Allgemeinen fachgebunden diskutiert. Eine breitere Sicht auf das gesamte Spektrum der Diskreten Mathematik zeigte sich Ende der 80er Jahre insbesondere in den USA. Den Hintergründen für diese Entwicklung und den Zielen, die mit einer möglichen Integration diskreter Themen in den Mathematikunterricht verbundenen wurden, soll im Folgenden nachgegangen werden.

\subsubsection{Kombinatorik als (eigenständiger) Unterrichtsgegenstand}

In der didaktischen Diskussion um die Bedeutung und Einordnung kombinatorischer Fragestellungen lassen sich zwei Sichtweisen unterscheiden. Zum einen wird Kombinatorik unter Berücksichtigung der historischen Genese als Teil der Wahrscheinlichkeitsrechnung verstanden, zum anderen betrachtet man sie als eigenständigen Unterrichtsgegenstand, womit man stärker ihrer aktuellen Bedeutung gerecht wird.

Die Kombinatorik als Basis der Wahrscheinlichkeitsrechnung wird bereits in den Gymnasien des 19. Jahrhunderts behandelt (vgl. Scheid 1984, S. 6), sie findet jedoch in verschiedenen Empfehlungen und Richtlinien Anfang des 20. Jahrhunderts nur zum Teil eine Berücksichtigung. Der Wahrscheinlichkeitsrechnung selbst wird nach einer Phase der Neuorientierung des Mathematikunterrichts nach dem zweiten Weltkrieg mit Beginn der 60er Jahre eine stärkere Bedeutung beigemessen, und sie wird in der Folgezeit sowohl in Richtlinien und Lehrplänen für die Primarstufe als auch für die Sekundarstufe I und II aufgenommen (vgl. Kütting 1994, S. 146 f.). Die Frage, in welchem Umfang kombinatorische Inhalte im Rahmen eines Stochastikunterrichts der Sekundarstufe II behandelt werden sollen, war immer wieder Gegen- 
stand der didaktischen Diskussion. Eine eindeutige Beantwortung dieser Frage ist nicht möglich; es muss in jedem Fall die Zielsetzung des Unterrichts berücksichtigt werden. Nach einer Resolution zum Mathematikunterricht in Grundkursen aus dem Jahr 1983 werden u. a. folgende Schwerpunktsetzungen vorgeschlagen (vgl. Kroll 1983, S. 220):

1. Beurteilende Statistik als Leitvorstellung.

2. Verstärkte Berechnung von Wahrscheinlichkeiten unter Zuhilfenahme kombinatorischer Methoden.

Unterrichtspraktische Vorschläge, die der zweiten Zielsetzung zugeordnet werden können, sind in dem MU-Themenheft „Kombinatorik in der Wahrscheinlichkeitsrechung“ aus dem Jahr 1984 aufgeführt (vgl. Fricke 1984a). Scheid (1984) zeigt hier ausgehend von den kombinatorischen Grundformeln eine Fülle interessanter kombinatorischer Anwendungsprobleme aus dem Bereich der Glücksspiele sowie aus dem Gebiet der Physik auf. Daneben wird anhand von Beispielen beschrieben, wie kombinatorische Überlegungen in die Konstruktion parameterfreier Tests einfließen können. Fricke (1984b, 1984c) betrachtet ein Irrfahrtenproblem und das Problem der vollständigen Serie, die beide mit weiterführenden kombinatorischen Überlegungen behandelt werden können.

Aufgrund der wachsenden Bedeutung statistischer Fragestellungen wird heute jedoch verstärkt gefordert, den kombinatorischen „Vorlauf“ innerhalb der Stochastik der Sekundarstufe II auf ein Minimum zu beschränken und der beurteilenden Statistik eine größere Beachtung zu schenken. ${ }^{48}$ So fordert der aktuelle hessische Lehrplan Mathematik für die gymnasiale Oberstufe, dass ,Zählverfahren [...] nur so weit behandelt werden [sollten], wie sie für das Verstehen der nachfolgenden Fragestellungen nötig sind“, so etwa zur Behandlung spezieller Wahrscheinlichkeitsverteilungen oder zum Testen von Hypothesen (Hessisches Kultusministerium 2000, S. 26). Diese Forderung steht in Einklang mit der oben genannten ersten Schwerpunktsetzung.

Nun gibt es seit einiger Zeit Empfehlungen, Elemente der Stochastik und damit auch kombinatorische Inhalte nicht nur in den Sekundarstufen zu behandeln, sondern auch in die Grundschule zu integrieren. ${ }^{49}$ Empirische Untersuchungen weisen darauf hin, dass bereits Grundschüler ein Verständnis für den Inhalt kombinatorischer Aufgaben und für elementare Zählprinzipien aufbringen. ${ }^{50}$ Durch einen kombinatorischen Vorkurs und ein Wiederaufgreifen und Vertiefen bestimmter Inhalte in höheren Schulstufen kann im Sinne des Bruner'schen

\footnotetext{
${ }^{48}$ Die beurteilende Statistik ist bereits seit einiger Zeit verpflichtender Lehrstoff des Stochastikunterrichts. In der Praxis des realen Mathematikunterrichts wurde die Stochastik jedoch häufig mit einem zum Teil isoliert zu anderen Inhalten stehenden Bereich „Kombinatorik“ unterrichtet und Fragen der beurteilenden Statistik nicht oder nur kurz angesprochen.

${ }^{49}$ Vgl. Kütting 1994, S. 149 ff.

${ }^{50}$ Gegenstand der Untersuchungen sind vor allem einfache Permutationsaufgaben, die in eine die Kinder ansprechende Situation eingekleidet wurden. Vgl. hierzu die Erfahrungsberichte von Winter 1976, Malmendier, N. \& Kaeseler, P. 1985. Über erste intuitive Zugänge und Strategien von Grundschulkindern im Umgang mit kombinatorischen Aufgaben berichtet Neubert 1998.
} 
Spiralprinzips gehandelt und eine Propädeutik zum Erlernen stochastischer Grundbegriffe geleistet werden. Wenn bereits frühzeitig geeignete Hilfsmittel für die elementare Wahrscheinlichkeitsrechung bereitgestellt würden, wäre in der Oberstufe mehr Raum für kombinatorische Aktivitäten. Auf diese Weise könnte man beide der oben genannten Zielsetzungen angemessen berücksichtigen. Darüber hinaus wäre eine stärkere Betonung der Kombinatorik in der Grundschule und in der Sekundarstufe I auch im Sinne einer Konzeption, die Kombinatorik als eigenständigen Unterrichtsgegenstand auffasst. Die wahrscheinlichkeitspropädeutische Funktion steht hier nicht im Zentrum; es werden vielmehr allgemeine Lernziele betont. Ansätze einer solchen Sichtweise zeigen sich bereits in den 70er Jahren. So weist Jeger darauf hin, „dass die Kombinatorik einen relativ breiten Elementarbereich aufweist, also Probleme und Lösungsmethoden, die leicht verständlich sind“ (Jeger 1973, S. 5). Daneben hebt er die zahlreichen Anwendungsmöglichkeiten der modernen Kombinatorik hervor. Ähnlich argumentiert Kapur (1970), der darüber hinaus Beziehungen zu mengentheoretischen und algebraischen Begriffen wie Abbildung, Relation, Funktion, Äquivalenzklassen und Isomorphismus sieht. ${ }^{51}$ In den 80er Jahren sprechen sich Danckwerts und Vogel (1983a) für die Behandlung der Kombinatorik als eigenständigen Unterrichtsgegenstand aus und geben in dem MUThemenheft „Kombinatorik“ zahlreiche Beispiele, die sowohl dem Abzählaspekt als auch dem Existenz- und Optimierungsaspekt Rechnung tragen. Abzählprobleme werden dabei unter einem neuen didaktischen Blickwinkel betrachtet: So kann etwa durch ein Thematisieren der Binomialentwicklung

$$
(1+x)^{n}=\left(\begin{array}{l}
n \\
0
\end{array}\right)+\left(\begin{array}{l}
n \\
1
\end{array}\right) x+\left(\begin{array}{l}
n \\
2
\end{array}\right) x^{2}+\ldots+\left(\begin{array}{l}
n \\
n
\end{array}\right) x^{n}
$$

bereits in der Mittelstufe eine Verknüpfung von Kombinatorik und Algebra aufgezeigt werden. ${ }^{52}$ Anhand der Methode der erzeugenden Funktionen lässt sich diese Beziehung in der Sekundarstufe II formal weiterführen und vertiefen sowie die Bedeutung der Algebra beim Lösen kombinatorischer Probleme verdeutlichen. ${ }^{53}$ Damit kann einer „einseitigen Ausrich-

\footnotetext{
${ }^{51}$ Diese Sicht und die damit verbundenen Beispiele (vgl. etwa Claus 1973) war noch von der Reformbewegung der „Neuen Mathematik“ geprägt, die im Anschluss an die von Bourbaki aufgestellte „Architektur der Mathematik" versuchte, den Unterricht an Strukturbegriffen wie Menge, Relation, Verknüpfung, Abbildung zu orientieren. Die Begriffe Abbildung und Funktion gehören auch heute noch zu den fundamentalen Begriffen des Mathematikunterrichts. Welchen Beitrag kombinatorische Aktivitäten zu deren Entwicklung leisten können, wird in Kapitel 2.3.2 aufgezeigt.
}

${ }^{52}$ Eine Verknüpfung besteht insofern, als sich die Binomialkoeffizienten $\left(\begin{array}{l}n \\ k\end{array}\right)$ und damit das kombinatorische Problem „Anzahl der $k$-elementigen Teilmengen einer $n$-Menge“ durch Ausmultiplizieren von $(1+x)^{n}$ und Zusammenfassen der entstehenden Terme rein algebraisch bestimmen lassen.

${ }^{53}$ Erzeugende Funktionen sind formale Potenzreihen, bei denen Konvergenzfragen unberücksichtigt bleiben. Sie stellen nicht nur bei Zahlzerlegungsproblemen ein wichtiges Werkzeug dar, sondern dienen auch dazu, lineare Rekursionen zu lösen und eine Vielzahl kombinatorisch interpretierbarer Gleichungen über Binomialkoeffizienten rein algebraisch zu beweisen (vgl. Danckwerts \& Vogel 1983b). 
tung der Algebra als Instrument zum Lösen von Gleichungen“ entgegengewirkt und die „Universalität der formalen algebraischen Methode“ betont werden (vgl. Danckwerts, Deubner \& Vogel 1983, S. 15)..$^{54}$

\subsubsection{Graphentheoretische Elemente im Mathematikunterricht}

In Zusammenhang mit Bestrebungen, Elemente der Geometrie im Grundschulunterricht zu etablieren, wird von der deutschen Mathematikdidaktik mit Beginn der 70er Jahre verstärkt auch für eine Integration graphentheoretischer Inhalte in den Mathematikunterricht der Grundschule plädiert. Im Rahmen einer propädeutischen Behandlung geometrischer, graphentheoretischer bzw. topologischer ${ }^{55}$ Themen sollen Schüler angeleitet werden, die Umwelt zu erschließen, allgemeinere „Gesetze des Raums“ zu entdecken sowie „,räumliche Beziehungen und deren Gesetzmäßigkeiten in bestimmten realen Phänomenbereichen“ zu erfassen (vgl. Winter 1971). ${ }^{56}$ Einen bedeutenden Impuls gaben in diesem Zusammenhang die psychologischen Untersuchungen der Genfer Schule um Jean Piaget. Unter dem Einfluss von Bourbaki ${ }^{57}$ stellte Piaget ausgehend von Versuchsergebnissen zur Raumerfassung von Kindern eine Theorie auf, nach der Kinder zunächst topologische und erst danach projektive und euklidische Beziehungen erkennen (vgl. Müller \& Wittmann 1984, S. 226).

Graphentheoretische Inhalte werden zunehmend jedoch nicht nur in Verbindung mit einer geometrischen Propädeutik, sondern auch im Hinblick auf allgemeine Lernziele diskutiert. So kann nach Winter die Beschäftigung mit Netzen (Graphen) „,kreatives, kombinatorisches und argumentierendes Denken“ fördern (Winter 1971, S. 58). Bigalke betont bei einer Behandlung der Graphentheorie insbesondere die ,,immense Anwendungsfreudigkeit“, die „große Anschaulichkeit“ und die „Problemfreudigkeit auf jedem beliebigen Niveau“ (Bigalke 1974, S. 6). Mit „Problemfreudigkeit“ verbindet Bigalke u. a. die Möglichkeit, leichtverständliche innermathematische Sätze über Wege und Färbungen zu thematisieren und zu beweisen. Beispiele für Anwendungen könnten seiner Meinung nach im Bereich der Netzplantechnik und in Zusammenhang mit Netzwerkflüssen und Rundreiseproblemen gefunden werden. Darüber

\footnotetext{
${ }^{54}$ Der binomische Lehrsatz und dessen Beziehung zur Kombinatorik war bereits im 19. Jahrhundert Gegenstand des Gymnasialunterrichts. Diese Inhalte waren jedoch in das Konzept der Algebraischen bzw. Kombinatorischen Analysis eingebettet. Vgl. hierzu Jahnke 1990, S. 323 ff.

${ }^{55}$ Die mathematische Disziplin der Topologie beschäftigt sich (informell betrachtet) mit Lage-, Nachbarschafts-, Umgebungs- und Zusammenhangsbeziehungen. Die Graphentheorie kann als ein Teilgebiet der kombinatorischen Topologie aufgefasst werden.

${ }^{56}$ Als Beispiel für einen solchen realen Phänomenbereich gibt Winter (1971) eine Straßenkreuzung und deren Ampelschaltung an. Bei der Analyse dieser Situation können topologische Begriffsbildungen initiiert werden.

${ }^{57}$ Bourbaki ist ein Pseudonym für eine Gruppe französischer Mathematiker, die es sich seit Ende der 30er Jahre zum Ziel gesetzt hatten, das Gebiet der reinen Mathematik aus der Mengenlehre mit Hilfe dreier Mutterstrukturen (algebraische Strukturen, Ordnungsstrukturen, topologische Strukturen) zu ordnen und hierarchisch aufzubauen. Der Einfluss von Bourbaki äußerte sich in Deutschland in den 60er Jahren in Form der ,Neuen Mathematik“ und drang bis in die allgemeinbildenden Schulen vor (vgl. hierzu auch Führer 1997, S. 73 ff.).
} 
hinaus seien Graphen ein Mittel sowohl zur Veranschaulichung innermathematischer Relationen als auch zur Mathematisierung bzw. Darstellung außermathematischer Situationen.

Heute finden sich kaum noch graphentheoretische bzw. topologische Elemente im Mathematikunterricht. Die Lehrpläne der Grundschulen sehen diese im Rahmen einer geometrischen Propädeutik nicht mehr vor, da ihre Behandlung weder aus entwicklungspsychologischer noch aus fachwissenschaftlicher Sicht gerechtfertigt erscheint. Topologischen Inhalten wird jedoch im Hinblick auf allgemeine Ziele des Mathematikunterrichts immer noch eine Bedeutung beigemessen (vgl. Franke 1999, S. 3). In den beiden Sekundarstufen wurden in der Vergangenheit - möglicherweise auch in Ermangelung überzeugender Vorschläge seitens der Fachdidaktik $^{58}$ - nur wenige der für die Grundschule vorgeschlagenen topologischen und graphentheoretischen Inhalte aufgegriffen und weitergeführt. Dennoch wird gerade in letzter Zeit im Hinblick auf die fundamentalen Ideen „Optimieren“ und „Algorithmisieren“ und in $\mathrm{Zu}$ sammenhang mit dem Informatikunterricht wieder verstärkt auf die Bedeutung von Fragestellungen der kombinatorischen Optimierung hingewiesen. ${ }^{59}$

\subsubsection{Codierungstheorie und Kryptographie als Gegenstand des Mathematik- und/oder Informatikunterrichts}

Erste Vorschläge zur Integration der Informationstheorie bzw. der Codierungstheorie in den Mathematikunterricht gibt es bereits Anfang der 70er Jahre. Unter Bezug auf die wachsende Bedeutung dieses mathematischen Themengebiets in vielen Anwendungsbereichen und im Hinblick auf Bestrebungen, die Informatik als Schulstoff zu institutionalisieren, hat als einer der ersten Wefelscheid für die Behandlung der Informationstheorie in der gymnasialen Oberstufe plädiert. Das von ihm herausgegebene MU-Themenheft „Informationstheorie“ führt zunächst in die wesentlichen Begriffe dieser Theorie ein und zeigt anschließend für die Schule relevante Aspekte auf. Dabei werden Bezüge zur Linearen Algebra, Statistik und Graphentheorie hergestellt (vgl. Wefelscheid 1974).

In den 80er Jahren werden - vor dem Hintergrund der wachsenden gesellschaftlichen Bedeutung des Computers bzw. der „Informations- und Kommunikationsmedien (IuK)“ - Forderungen nach einer zeitgemäßen Allgemeinbildung laut, die insbesondere eine ,informationstechnische Grundbildung“ einschließen. In diesem Zusammenhang werden von der BundLänder-Kommission für Bildungsplanung und Forschungsförderung die folgenden Inhaltsbe-

\footnotetext{
${ }^{58}$ Das heißt nicht, dass es keine entsprechenden fachdidaktischen Beiträge gab. So schlugen Rinkens \& Schrage (1974) eine spiralförmige Behandlung der Topologie vor, bei der - im Hinblick auf den Themenbereich der Analysis - auf den Begriff der homöomorphen Abbildung hingearbeitet wird. Anregungen zur Behandlung von hamiltonschen Wegen und Färbungsproblemen finden sich im MU-Themenheft „Graphentheorie III“ (vgl. Klose 1978). Mit Fragestellungen der kombinatorischen Optimierung beschäftigten sich u. a. Vogel 1977, Floer 1977, Baptist 1979, Kießwetter \& Rosenkranz 1982. Offensichtlich hatten diese Beiträge aber nur einen geringen Einfluss auf die Praxis des Mathematikunterrichts.

${ }^{59}$ Vgl. hierzu Jäger \& Schupp 1997, Schupp 1997, Malle 1999b, Schuster 2001.
} 
reiche genannt, die für alle Schüler der Sekundarstufe I verbindlich sein sollten (vgl. Koerber \& Peters 1998, S. 21):

- Aufarbeitung und Einordnung der individuellen Erfahrungen mit Informationstechniken;

- Vermittlung von Grundtechniken und Grundbegriffen, die für die Informationstechniken von Bedeutung sind;

- Einführung in die Handhabung eines Computers und dessen Peripherie;

- Vermittlung von Kenntnissen über die Einsatzmöglichkeiten und die Kontrolle der Informationstechniken;

- Einführung in die Darstellung von Problemlösungen in algorithmischer Form;

- Gewinnung eines Einblicks in die Entwicklung der elektronischen Datenverarbeitung;

- Schaffung des Bewusstseins für die sozialen und wirtschaftlichen Auswirkungen, die mit der Verbreitung der Mikroelektronik verbunden sind;

- Darstellung der Chancen und Risiken der Informationstechniken sowie Aufbau eines rationalen Verhältnisses zu diesen;

- Einführung in Probleme des Persönlichkeits- und Datenschutzes.

Auf der Basis dieses Bildungskonzepts erhalten Codierungstheorie und auch Kryptographie neue Aktualität, sowohl für den Mathematik- als auch für den Informatikunterricht. ${ }^{60}$ Vorschläge für den Informatikunterricht zielen dabei darauf ab, Programmiertechniken weiterzuentwickeln, indem etwa kryptographische Verfahren implementiert werden, oder bestimmte Fragen der Informationssicherheit vertieft zu behandeln. ${ }^{61}$ Im Mathematikunterricht sieht man demgegenüber in der Behandlung von Kryptographie und Codierungstheorie eine Möglichkeit, allgemeine Lernziele wie Modellbilden und Problemlösen zu betonen, sowie viele traditionelle Lerninhalte wie Stellenwertsysteme, Primzahlen, Teilerfremdheit, Prozentrechnung und Statistik anwendungsorientiert zu üben. ${ }^{62}$ Dabei ist das Vermitteln einer informatorischen Grundbildung an keine durchgängige Computernutzung gebunden, wie Herget (1989) herausstellt. In der von ihm vorgestellten Unterrichtseinheit zum Thema „Prüfziffern und Strichcode“ in einer 7. Klasse ,geht es nicht um das Programmieren verschiedener Prüfzifferverfahren oder gar um das Erstellen eines Programms zum Ausdruck von Strichcodes, sondern um das Verständnis des weiten, von den neuen Techniken geprägten außermathematischen Problemfeldes und der Rolle, die dabei die verschiedenen mathematischen Lösungsvorschläge spielen“ (a. a. O., S. 19). Damit werden Inhalte der Codierungstheorie insbesondere für einen anwendungsorientierten Mathematikunterricht bedeutsam.

\footnotetext{
${ }^{60}$ Vgl. hierzu z. B. das MU-Themenheft „Codieren und Chiffrieren“ (Beutelspacher \& Schulz 1987) bzw. das LOG IN Heft zum Thema „Kryptographie und Sicherheit in Netzen“ (Witten 1996).

${ }^{61}$ Vgl. hierzu z. B. Uhl 1987, Baumann 1996. Uhl berichtet über eine Unterrichtsreihe mit dem Thema Kanalcodierung, die er in einem Informatikkurs der Jahrgangsstufe 13 gehalten hat. Baumann möchte mit seinem Aufsatz einen Weg zur Behandlung des Themas „Informationssicherheit durch kryptologische Verfahren“ aufzeigen. Im Zentrum des Unterrichts sollten seiner Meinung nach kryptographische Protokolle und Informatiksysteme stehen.
}

${ }^{62}$ Vgl. hierzu z. B. die unterrichtspraktischen Vorschläge von Biernot 1992, Failing 1998, Waimer 1997. 


\subsubsection{Zur Beziehung „Diskrete Mathematik - Kontinuierliche Mathematik“}

Zwischen diskreter und kontinuierlicher Mathematik ${ }^{63}$ gibt es viele Beziehungen. Diskrete (numerische) Verfahren können Näherungslösungen für kontinuierliche Probleme, wie etwa aus dem Bereich der Algebra und Analysis, liefern. Darüber hinaus gibt es für zahlreiche kontinuierliche Konzepte (struktur)ähnliche, vergleichbare diskrete Begriffe und Verfahren. So bestehen Verbindungen oder sogar Analogien zwischen der diskreten und kontinuierlichen Wahrscheinlichkeitsrechnung, zwischen der Differenzen- und der Differentialrechnung und zwischen der Theorie der Differenzengleichungen und der Differenzialgleichungen. Mit der zunehmenden Bedeutung des Computers in vielen gesellschaftlichen Bereichen kam es Ende der 70er Jahre zu einer Diskussion um den Stellenwert diskreter und kontinuierlicher Konzepte im Mathematikunterricht. Im Zentrum dieser Diskussion stand eine „klassische“ kontinuierliche Disziplin, die Analysis. Auf einige der Hintergründe soll im Folgenden näher eingegangen werden, und es sollen Bestrebungen, Diskrete Mathematik als eigenständigen Unterrichtsgegenstand zu etablieren, aufgezeigt werden.

\subsubsection{Zunehmende Bedeutung des algorithmischen Aspekts}

Durch die wachsende Bedeutung elektronischer Rechengeräte in Wissenschaft, Technik und Wirtschaft und die Verbreitung von Taschenrechnern und Computern wird in den 70er Jahren dem ,algorithmischen Aspekt“ der Mathematik eine größere Aufmerksamkeit geschenkt. Damit einher gehen Forderungen, algorithmisches Arbeiten nicht nur auf das Ausführen von Algorithmen zu beschränken, sondern dem Entwickeln und Analysieren von Algorithmen eine größere Bedeutung beizumessen. ${ }^{64}$ Mit der Behandlung von Algorithmen verbindet man die Hoffnung, dass Schüler - wie Engel (1979) im Vorwort seines Lehrbuchs „Elementarmathematik vom algorithmischen Standpunkt" hervorhebt - vertiefte Einsichten in traditionelle Gebiete des Mathematikunterrichts gewinnen:

„Manche Themen der Schulmathematik gewinnen außerordentlich, wenn man sie vom algorithmischen Standpunkt aufbaut. [...] Die Algorithmik liefert einen wichtigen Beitrag zur Konkretisierung, Vereinfachung und Vertiefung des Schulstoffes.“ (Engel 1979, S. 3)

Mit der Betonung der algorithmischen Sicht wird der Blick stärker von kontinuierlichen zu diskreten Inhalten und Methoden gelenkt. In diesem Zusammenhang zeigen sich in der deutschen Mathematikdidaktik auch erste Ansätze einer integrierenden Sicht auf das gesamte Spektrum der Diskreten Mathematik, wohingegen zuvor diskrete Themenbereiche hauptsächlich ,isoliert“ thematisiert wurden. So plädieren Danckwerts, Deubner und Vogel (1983, S. 13f.)

\footnotetext{
${ }^{63}$ Mit „kontinuierlicher Mathematik“ bezeichnen wir im Rahmen dieser Arbeit Themengebiete, die sich - in Abgrenzung zur diskreten Mathematik - in einem weiteren Sinne mit nicht abzählbaren Mengen, insbesondere reellen Zahlen, beschäftigen.

${ }^{64}$ Der Begriff des Algorithmus wird in der fachdidaktischen Literatur zu den „fundamentalen Ideen“ im Sinne Bruners gezählt (vgl. etwa Schreiber 1979, Schweiger 1992, Führer 1997).
} 
in dem MU-Themenheft „Kombinatorik“ (vgl. Danckwerts \& Vogel 1983) für eine Einführung in die Methoden der finiten Mathematik, die u. a. die Themengebiete Kombinatorik, Graphentheorie, endliche Stochastik und theoretische Informatik umfasst. ${ }^{65}$ Der algorithmische Aspekt, der - wie bereits Ralston (1981) hervorgehoben hatte - als verbindendendes Element der ansonsten recht unterschiedlichen Themenbereiche der Diskreten Mathematik verstanden werden kann, ${ }^{66}$ wird in diesem Heft zum Anlass genommen, insbesondere algorithmische Elemente in Kombinatorik und Graphentheorie offen zu legen, denn - so betonen Bovermann, Danckwerts und Vogel:

„In den kombinatorischen Aktivitäten Abzählen, Aufzählen, Optimieren ist der algorithmische Aspekt angelegt.“ (Bovermann, Danckwerts \& Vogel 1983, S. 66)

Die Idee, die verschiedenen Themengebiete der Diskreten Mathematik in einem größeren Zusammenhang zu betrachten, wird in der Folgezeit in der deutschen Mathematikdidaktik zunächst nicht weiterverfolgt. Stattdessen wird, wenngleich diskrete Inhalte aus den Bereichen Kombinatorik, Graphentheorie und Kryptographie immer wieder Gegenstand der didaktischen Diskussion sind, mit Nachdruck für die Behandlung diskreter (bzw. numerischer) Methoden zur Vertiefung traditioneller Themen aus dem Bereich der Algebra und der Analysis plädiert. ${ }^{67}$ In diesem Zusammenhang wird insbesondere einem zentralen diskreten Begriff - dem Begriff der Folge - als eigenständigem Unterrichtsgegenstand mehr Bedeutung beigemessen. So bemerken Blum und Törner:

„Durch den ständig steigenden Einsatz von Computern sind Folgen in Zusammenhang mit Iterationsverfahren als ein universelles Werkzeug in inner- sowie außermathematischen Anwendungssituationen eher aufgewertet worden. Diesem Trend kann sich auch der Unterricht nicht verschließen, nicht allein nur um der hohen mathematischen und historisch begründeten Bedeutung des Folgenbegriffs wegen, sondern insbesondere aufgrund des didaktischen Impulses durch den algorithmischen Aspekt.“ (Blum \& Törner 1983, S. 67)

Im Mittelpunkt des Interesses stehen dabei rekursiv definierte Folgen, die nicht nur in Verbindung mit numerischen Näherungsverfahren eine wichtige Rolle spielen, sondern auch ein

\footnotetext{
${ }^{65}$ Der Begriff „,finit“ bedeutet „endlich“. Grundlage der finiten Mathematik sind somit endliche Mengen. Auch die Diskrete Mathematik (im engeren Sinne) beschäftigt sich mit endlichen Mengen. Insbesondere können die von Danckwerts, Deubner und Vogel genannten Themengebiete auch der Diskreten Mathematik zugeordnet werden.

${ }^{66}$ So betont Ralston (1981) im Hinblick auf einen Lehrgangsentwurf zur Diskreten Mathematik für Studierende der Informatik: „The ,glue“ which should be used [...] to hold together an (otherwise) disparate set of topics is the notion of an algorithm and the analysis of algorithms." (S. 479)

${ }^{67}$ So können etwa Gleichungen numerisch gelöst, die Zahl Pi oder bestimmte Quadratwurzeln approximativ bestimmt werden, aber auch Elemente der Zahlentheorie, wie z B. der euklidische Algorithmus, thematisiert werden. Vgl. hierzu Engel 1977.
} 
Hilfsmittel beim Lösen innermathematischer Probleme ${ }^{68}$, bei der Einführung des Grenzwertbegriffs ${ }^{69}$ oder beim Mathematisieren dynamischer Prozesse ${ }^{70}$ darstellen.

\subsubsection{Klassische Analysis versus diskrete Analysis}

Die didaktische Diskussion der 70er Jahre um eine algorithmische Durchsetzung des klassischen Schulstoffs ist im Zusammenhang mit der Analysis besonders ausgeprägt. So setzen sich Engel (1977) und Klingen (1981) für eine Behandlung numerischer Algorithmen, wie etwa Verfahren zur Interpolation, zur Bestimmung von Nullstellen und Extremwerten, zur numerischen Integration und zum numerischen Lösen von Differentialgleichungen, ein. Winkelmann ist sogar der Ansicht, dass sich „manche klassischen Motivationen für die schulische Behandlung der begrifflich aufwändigen Analysis nicht mehr ohne weiteres aufrecht erhalten“ lassen, da die meisten dieser numerischen Verfahren leicht zu programmieren sind, keine expliziten Kenntnisse der klassischen Analysis erfordern und darüber hinaus bei praktischen Anwendungen von großer Bedeutung sind (Winkelmann 1982, S. 25). Dies bedinge „eine Legitimationskrise des traditionellen Analysisunterrichts an der Schule“ und es sei „deutlich $\mathrm{zu}$ machen [ist], worin heutzutage noch der Sinn einer Beschäftigung mit kontinuierlicher Mathematik liegen soll“ (a. a. O., S. 25). Die Diskussion um einen zeitgemäßen Analysisunterricht und den Stellenwert diskreter Begriffe und Verfahren ist auch heute noch aktuell, wenngleich die Bedeutung der klassischen Analysis unumstritten ist. So sind kontinuierliche Begriffe wichtig bei der Modellierung von Anwendungssituationen und bei der theoretischen Absicherung von Näherungsverfahren. Darüber hinaus stellen sie eine bedeutende kulturhistorische Errungenschaft dar (vgl. Blum 1991, S. 78). Durch den Computer kommt es jedoch zu Verschiebungen in den Zielsetzungen. Da z. B. Computeralgebrasysteme wie DERIVE nicht nur numerische sondern auch symbolische Berechnungen übernehmen können, verliert das Ausführen des Differenzialkalküls als eigenständig von Hand auszuführende algorithmische Tätigkeit an Bedeutung. In diesem Sinne äußert sich auch G. Schmidt auf einer Podiumsdiskussion zur MNU-Hauptversammlung:

„Mit der zunehmenden Verfügbarkeit des Computers auch im Mathematikunterricht stehen Werkzeuge zur Verfügung, die einmal den hohen Kalkülanteil des Analysisunterrichts (insbesondere der Prüfungen), entlarven', zum anderen auch die im Mittelpunkt stehenden Verfahren der schematisierten Kurvendiskussion, der Extremwertbestimmung und der Berechnung von Flächeninhalten und Volumina als Legitimation für Analysis in Frage stellen.“(vgl. Danckwerts \& Vogel 1992, S. 372)

\footnotetext{
${ }^{68}$ Zahlreiche Aufgaben, die durch rekursive Verfahren gelöst werden können, finden sich in Stowasser \& Mohry 1978.

${ }^{69}$ Eine Analyse verschiedener Konzeptionen zur Einführung des Konvergenz- und Grenzwertbegriffs findet sich in Weigand 1989, S. 51 ff. Zur Bedeutung des Folgenbegriffs für das Verständnis des Grenzwertbegriffs vgl. Weigand 1993, S. $80 \mathrm{ff}$.

${ }^{70}$ Vgl. hierzu z. B. Dürr \& Ziegenbalg 1989.
} 
Ein größerer Schwerpunkt kann daher auf das Erlernen und das Verständnis der zentralen kontinuierlichen Konzepte wie Grenzwert und Ableitung gelegt werden.

„Im Analysisunterricht sollte - beim Begriffsbilden wie beim Beweisen - mehr präformal gearbeitet werden, ohne dabei Abstriche an der Strenge zu machen. Schüler sollten angemessene Grundvorstellungen insbesondere vom Funktions-, Ableitungs- und Integralbegriff erwerben.“ (Blum 2000, S. 8)

Hier könnte eine neue Bedeutung diskreter Begriffe und Methoden liegen. Aufgrund der „Elementarität“ dieser Konzepte kann ein „,diskreter Zugang zur Analysis“ bereits früher als erst in der Sekundarstufe II erfolgen. So vertreten auch Hartmann und Streule die Ansicht:

„Die den einfachen numerischen Verfahren der Analysis zugrunde liegenden Ideen sind elementarer Natur. Der diskrete Zugang zur Analysis erlaubt es deshalb, diese Themen schon viel früher im Unterricht zu behandeln. Erst später wird die kontinuierliche Sichtweise nach und nach zum Zuge kommen und neben den graphischen und numerischen Verfahren auch vermehrt symbolische Methoden thematisiert werden." (Hartmann \& Streule 2000, S. 217)

Eine Verschiebung der Bedeutung kontinuierlicher Mathematik (insbesondere „kontinuierlicher“ Analysis) zugunsten diskreter Mathematik (insbesondere „diskreter Analysis“) zeigt sich auch in anderen Ländern. In den USA fordert als einer der ersten Ralston (1979), die ersten beiden Studienjahre an Colleges unter besonderer Berücksichtigung diskreter Themen neu zu strukturieren, wobei er zwischen der „diskreten Analysis“ und Themengebieten, die zur Diskreten Mathematik (im engeren Sinne) gezählt werden können, unterscheidet:

„Before proceeding further it would be well for me to define what I take the term 'discrete mathematics' to encompass. On one hand it implies an emphasis on discrete analysis rather than the traditional emphasis on the analysis of continuous functions. But additionally this term is used here to cover various other branches of mathematics which focus mainly or entirely on discrete objects such as Combinatorics, Graph Theory, Abstract Algebra, Linear Algebra, Number Theory, Set Theory, Discrete Probability.“ (a. a. O., S. 50)

Von der Behandlung dieser Themen erhofft sich Ralston eine adäquate und zeitgemäße Vorbereitung der angehenden Wirtschaftswissenschaftler, Informatiker und Mathematiker auf ihr späteres Berufsleben in Wirtschaft und Industrie (vgl. Ralston 1979, S. 50 f.). Ralstons Vorschlag führt zu einer intensiven Diskussion über eine geeignete Stellung Diskreter Mathematik im College-Curriculum, die sich im Wesentlichen darauf konzentriert, ob Diskrete Mathematik vor oder nach Analysis behandelt werden sollte und wie die beiden Disziplinen gewichtet werden sollten (vgl. Ralston \& Young 1983). Dabei orientiert man sich an den Bedürfnissen der jeweiligen Studienrichtungen ${ }^{71}$ und an den Lehrplänen anderer Institutionen wie Schule und Universität. In diesem Zusammenhang werden neben eigenständigen Kursen zu Diskreter Mathematik auch neue Konzepte für das Lehren der Analysis entwickelt. Im Zentrum eines anwendungsbezogenen Analysisunterrichts sollten dabei nach Ralston sowohl

\footnotetext{
${ }^{71}$ Hinsichtlich der Studienrichtungen Informatik, Wirtschaftswissenschaften, Mathematik und Informatik äußert sich Ralston hierzu im Jahr 1992 wie folgt: „For those courses aimed at specific student populations, the balance might be weighted more in the direction of the discrete for informatics and social and management science students, might be equal for mathematics students themselves and surely should be weighted more toward traditional continuous mathematics for physical science and engineering students." (Ralston 1992, S. 20)
} 
kontinuierliche als auch diskrete Funktionen bzw. Folgen stehen, deren Eigenschaften mit Hilfe des Differenzial- bzw. Differenzenkalküls untersucht werden können.

„If functional behaviour and representation are to be the focus of the calculus course, then continuous functions and discrete functions (i. e. sequences) must be emphasized and motivated by a wide variety of mathematical models. [...] An important theme in calculus courses should be the contrast between the local and global behaviour of functions. Local behaviour is, of course, derived by studying the derivative for continuous functions (and the difference operator for discrete functions). And similarly the integral (and summation) operators are used to derive global information about functions." (Ralston 1992, S. 22)

Auf die Bedeutung des endlichen Differenzen- und Summenkalküls für einen „diskreten Zugang zur Analysis“ hat bereits Gordon im Jahr 1979 hingewiesen. ${ }^{72}$ Er macht zum einen deutlich, dass zwischen diskreten und kontinuierlichen Konzepten zahlreichen Analogien bestehen (vgl. Tab. 1).

\begin{tabular}{|c|c|}
\hline Differenzialoperator $\frac{d}{d x}$ & Differenzenoperator $\Delta$ \\
$\frac{d}{d x} f(x)=\lim _{h \rightarrow 0} \frac{f(x+h)-f(h)}{h}$ & $\Delta f(n)=f(n+1)-f(n)$ \\
\hline$f(x)=a_{k} x^{k}+a_{k-1} x^{k-1}+\ldots+a_{1} x+a_{0}$ & $f(n)=a_{k} n^{k}+a_{k-1} n^{k-1}+\ldots+a_{1} n+a_{0}$ \\
$\Rightarrow \frac{d^{n}}{d x^{n}} f(x) \equiv$ const & $\Rightarrow \Delta^{n} f(n) \equiv$ const \\
\hline Stammfunktion F & Diskrete Stammfunktion $F$ \\
$F^{\prime}(x)=f(x)$ & $\Delta F(n)=F(n+1)-F(n)=f(n)$ \\
\hline Hauptsatz der Differenzial- und & Hauptsatz der Differenzen- und \\
Integralrechnung & Summenrechnung \\
$\int_{a}^{b} f(x) d x=F(b)-F(a)$ & $\sum_{k=a}^{b} f(k)=F(b+1)-F(a)$ \\
\hline
\end{tabular}

Tab.1: Analogien zwischen Konzepten kontinuierlicher und diskreter Analysis

Weiterhin hinaus hebt er hervor, dass im diskreten Fall nur Kenntnisse in Arithmetik und Algebra erforderlich seien, so dass bereits auf einer elementaren Ebene Erfahrungen mit den zentralen Ideen der Infinitesimalrechnung möglich seien.

„By beginning each topic with finite difference and sum analogues which require only manipulative ability in arithmetic and algebra, the student would develop an understanding of, and familiarity with, the concepts and methods at a reasonably simple level. The infinitesimal calculus results can then be quickly and naturally introduced by passing to the limit as the step goes to zero in the corresponding discrete results. All the continuous results would thus become a natural dual to the discrete results [...]. The net effect would be to give the student a firmer grasp of both the theory and applications of calculus.“ (Gordon 1979, S. 26)

\footnotetext{
${ }^{72}$ Hier werden Beziehungen zur Idee Leibniz’ beim Zugang zur Infinitesimalrechung deutlich (vgl. Kap. 1.1).
} 
Damit werden auch hier diskrete Begriffe und Methoden nicht in Konkurrenz zu kontinuierlichen Konzepten gesehen, sondern eher als Bereicherung bzw. als Hilfsmittel zum Erlernen der entsprechenden kontinuierlichen Begriffe und Methoden.

\subsubsection{Der NCTM-Standard ,Diskrete Mathematik“6}

Die Diskussion, diskrete Themen in der Mathematikausbildung an Colleges angemessen zu berücksichtigen, hat auch Auswirkungen auf den Mathematikunterricht der amerikanischen Highschools. Verschiedene Empfehlungen zur Integration Diskreter Mathematik in den Mathematikunterricht (vgl. Maurer 1983, Fey 1984, Ralston 1985, Sandefur 1985) führen im Jahr 1989 zur Aufnahme dieses Themengebiets in die „Curriculum and evaluation standards for school mathematics (K 9-12)“ des National Council of Teachers of Mathematics (NCTM) ${ }^{73}$. In der Einleitung des 12. Standards „Discrete Mathematics“ heißt es:

„In grades 9-12, the mathematics curriculum should include topics from discrete mathematics, so that all students can -

- $\quad$ represent problem situations using discrete structures such as finite graphs, matrices, sequences, and recurrance relations;

represent and analyze finite graphs using matrices;

develop and analyze algorithms;

solve enumeration and finite probability problems;

and so that, in addition, college-intending students can -

represent and solve problems using linear programming and difference equations;

investigate problem situations that arise in connection with computer validation and the application of algorithms.“(NCTM 1989, S. 176)

Auf der Basis dieses Standards werden von der NCTM Konzepte zur Integration Diskreter Mathematik in das Curriculum ausgearbeitet, und es wird ein eigener Kurs für höhere Klassen mit fundierten Algebrakenntnissen entwickelt. ${ }^{74}$ Zahlreiche Unterrichtsvorschläge in der amerikanischen Zeitschrift „Mathematics Teacher“, einem eigens dem Thema „Diskrete Mathematik“ gewidmeten Jahrbuch der NCTM (vgl. Kenney \& Hirsch 1991) und viele Diskussionsbeiträge (vgl. Rosenstein, Franzblau \& Roberts 1997) zeugen von der Bedeutung, die diesem Thema in den 90er Jahren beigemessen wird.

In den aktuellen „Principles and Standards“ findet sich zwar kein eigenständiger Punkt „Diskrete Mathematik“ mehr, es wird aber darauf hingewiesen, dass diskrete Themen das gesamte Curriculum durchziehen und in Verbindung mit traditionellen Inhalten gesehen werden sollten:

„In Principles and Standards, the main topics of discrete mathematics are included, but they are distributed across the standards, instead of receiving separate treatment, and they span the years from prekindergarten

\footnotetext{
${ }^{73}$ Die NCTM ist eine große amerikanische Lehrervereinigung. Die von ihr beschlossenen „Standards“ sind als Richtlinien bzw. Empfehlungen zur Gestaltung des Mathematikunterrichts anzusehen; sie besitzen aber nicht den verbindlichen Charakter deutscher Lehrpläne.

${ }^{74}$ Einen Überblick über diese Entwicklung gibt Dossey 1991.
} 
through grade 12 . As an active branch of contemporary mathematics that is widely used in business and industry, discrete mathematics should be an intgral part of the school mathematics curriculum, and these topics naturally occur throughout the other strands of mathematics." (NCTM 2000, S. 31)

\subsubsection{Sichtweisen auf diskrete Mathematik im Mathematikunterricht}

In den vorstehend skizzierten Begründungsansätzen zur Behandlung bestimmter diskreter Inhalte zeigen sich viele Beziehungen zu Inhalten und Zielen des gegenwärtigen Mathematikunterrichts. Betrachtet man die didaktische Diskussion, erscheinen drei Sichtweisen oder Aspekte besonders bedeutsam, die in Beziehung zur (wechselnden) Rolle diskreter Mathematik in der historischen Entwicklung gesehen werden können.

- Problemlöseaspekt

Diskrete Mathematik bietet zahlreiche elementare, anschauliche und auch unter historischen Aspekten interessante Problemstellungen.

- Begriffsbildungsaspekt

Diskrete (und damit „elementare“) Begriffe und Methoden können im Rahmen des Mathematikunterrichts zur Vorbereitung bzw. Vertiefung traditioneller (kontinuierlicher) Konzepte aus dem Bereich der Algebra und Analysis beitragen.

- Anwendungsaspekt

Diskrete Mathematik besitzt vielfältige Anwendungen, die von großer gesellschaftlicher Bedeutung sind. Mit der Behandlung diskreter Modelle im Mathematikunterricht können einige dieser Anwendungen aufgezeigt sowie Modellbildungsaktivitäten initiiert werden.

Wenngleich es sicher möglich ist, eigenständige Kurse zur Diskreten Mathematik zu entwickeln, wie es das Beispiel USA deutlich macht, erscheint es unter Berücksichtigung der oben genannten Aspekte als eine wichtige Alternative, Elemente diskreter Mathematik in das bestehende Curriculum zu integrieren. Zur Verdeutlichung dieser Auffassung sollen im Folgenden einige diskrete Inhalte detailliert im Hinblick auf die Ziele eines problemorientierten Mathematikunterrichts, eines anwendungsorientierten Mathematikunterrichts sowie hinsichtlich ihres Beitrags zum Lernen für den Mathematikunterricht bedeutsamer Begriffe analysiert werden. 


\subsection{Diskrete Mathematik als Feld zum Problemlösen}

Die Mathematik als systematische, deduktive Wissenschaft entwickelt sich durch einen Prozess des Experimentierens, Vermutens und Widerlegens ständig weiter. ${ }^{75}$ Forderungen, den induktiven Aspekt bzw. den Prozesscharakter der Mathematik im Mathematikunterricht stärker zu betonen, wurden schon häufig erhoben und spiegelten sich auch in den verschiedenen Unterrichtskonzeptionen wider, wie etwa dem „Genetischen Unterricht“ (vgl. Wagenschein 1965, Wittenberg 1963), der „Nacherfindung unter (heuristischer) Führung“ (vgl. Freudenthal 1973) und - in neuerer Zeit - dem Konzept des „Entdeckenden Lernens“ (vgl. Winter 1984, 1991). ${ }^{76}$ Dem liegt eine Auffassung zugrunde, dass Lernen um so wirkungsvoller ist, wenn Schüler die Möglichkeit haben, ihr Wissen aktiv selbst zu konstruieren und Mathematik nicht als Fertigprodukt im Sinne von Faktenwissen präsentiert wird. Diese Vorstellung von Mathematikunterricht geht einher mit Forderungen, auch dem Problemlösen im Mathematikunterricht eine stärkere Bedeutung beizumessen. ${ }^{77}$

Das Problemlösen besitzt vielfältige didaktische Funktionen. So lassen sich bereits erworbene Kenntnisse problemorientiert üben ${ }^{78}$ bzw. vertiefen, und es können Beziehungen zwischen verschiedenen, vorher getrennt behandelten Themen hergestellt werden. Weiterhin stellen Probleme auch häufig den Ausgangspunkt von Begriffsbildungen dar (vgl. auch Kap. 2.3.1). Darüber hinaus verfolgt man mit einem problemorientierten Mathematikunterricht das Ziel, allgemeine Problemlösefähigkeit und heuristische Strategien zu fördern. Heuristische Strategien steuern ein gezieltes Herangehen an Aufgaben, für die kein Lösungsalgorithmus bekannt ist. ${ }^{79}$ Indem sie vorhandenes Wissen umstrukturieren bzw. verändern oder auch neues Wissen schaffen, ermöglichen sie letztendlich die Lösung eines Problems. ${ }^{80}$

Trotz eines breiten Konsenses hinsichtlich der Bedeutung des Problemlösens stellen die Ergebnisse der TIMSS-Studien (vgl. Baumert u. a. 1997) dem deutschen Mathematikunterricht ein schlechtes Zeugnis aus. Die unbefriedigenden Ergebnisse der deutschen Schüler können

\footnotetext{
${ }^{75}$ Am Beispiel des Euler'schen Polyedersatzes hat Lakatos (1979) diesen Aspekt ausführlich erläutert.

${ }^{76}$ Einen Überblick über diese Unterrichtskonzeptionen gibt z. B. Führer 1997, S. 46 ff.

${ }^{77}$ Vgl. Schoenfeld 1985, NCTM 1989, 2000, Zimmermann 1983, 1991.

${ }^{78}$ Vgl. Winter 1984. Winter setzt sich für eine kreative Übungspraxis im Rahmen eines entdeckenden Unterrichts ein. In diesem Zusammenhang unterscheidet er vier Aspekte des Übens: problemorientiert, operativ, produktiv, anwendungsorientiert.

${ }^{79}$ Neben den von Polya (1949) ausführlich besprochenen Strategien Induktion, Verallgemeinern, Spezialisieren und Analogisieren finden sich in der mathematikdidaktischen Literatur u. a. folgende Strategien: Organisation von Material, Variation der Aufgabe, Anfertigen einer Zeichnung, Einführen von Hilfselementen, Bearbeiten von Hilfsproblemen, Ausnutzen von Symmetrien, Rückwärtsarbeiten, Vorwärtsarbeiten, Aufstellen einer Gleichung, Widerspruchsbeweis, Schubfachprinzip (vgl. Polya 1949, Schoenfeld 1985, Zimmermann 1991).

${ }^{80}$ In den kognitionspsychologischen Modellen zur Beschreibung menschlichen Wissens wird in diesem Zusammenhang von zwei verschiedenen Wissensstrukturen ausgegangen, die in einer Wechselbeziehung zueinander stehen. Dörner (1976) unterscheidet hier die ,epistemische Stuktur“", die das fachspezifische Wissen über Begriffe sowie (algorithmisches) Handlungswissen enthält, und die „heuristische Struktur“, in der allgemeine Verfahren für Problemlösungen gespeichert sind.
} 
u. a. darauf zurückgeführt werden, dass der vorherrschende Unterricht zu sehr auf das Lernen von Fakten, Algorithmen sowie das Bearbeiten von Standardaufgaben ausgerichtet ist und zu wenig Wert auf ein selbstständiges, aktives, entdeckendes Lernen gelegt wird (vgl. Blum 1998). Dadurch gewinnen Vorschläge und Konzepte für einen problemorientierten Mathematikunterricht an Aktualität. An geeignete Problemstellungen werden dabei verschiedene Bedingungen gestellt. So sollte die Problemstellung für den Schüler gut verständlich sein und an Vorkenntnisse, Vorerfahrungen und Interessen des Schülers anknüpfen. Insbesondere sollten sich das Problem oder Teile davon anschaulich-graphisch oder enaktiv darstellen und erfassen lassen. Im Hinblick auf die didaktischen Funktionen des Problemlösens, sollte sich der Problemkontext darüber hinaus in ein vielfältiges Beziehungsnetz einbetten lassen sowie sich zur Förderung heuristischer Strategien eignen. ${ }^{81}$ Viele diskrete Problemstellungen erfüllen diese Anforderungen. Dies soll im Folgenden anhand von zwei diskreten Problemklassen exemplarisch aufgezeigt werden.

\subsubsection{Abzählprobleme}

Bei Abzählproblemen geht es um das Zählen gewisser Objekte und um das Finden und Aufstellen einer Zählfolge in expliziter, rekursiver oder Summendarstellung. Abgezählt werden dabei Punktemuster (z. B. figurierte Zahlen), Geradenmuster (z. B. Diagonalen in Polygonen), Wegemuster (z. B. Anzahl der Wege zwischen zwei Punkten in einem Gitternetz) und dergleichen mehr. ${ }^{82}$ Bei einfachen Zählproblemen wie der folgenden „Streichholzfolge“ ${ }^{\text {83 }}$ liegt ein linearer Zusammenhang zwischen der Zahlvariablen $n$ und der Anzahl der Hölzer $A(n)$ zugrunde.

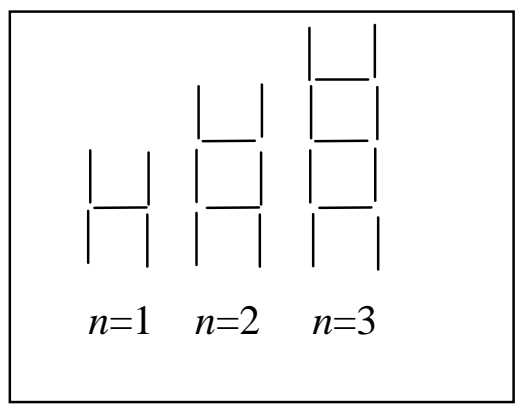

Frage:

a) Wie viele Streichhölzer braucht man für eine Leiter mit $n=5,10,15$ Sprossen?

b) Geben Sie eine Formel an, mit deren Hilfe man die Anzahl der Streichhölzer der Stufe $n$ berechnen kann.

Abb. 1: Die Streichholzfolge

\footnotetext{
${ }^{81}$ Vgl. hierzu auch Tietze, Klika \& Wolpers 1997, S. 96 und Schmidt 1981, S. 80.

${ }^{82}$ Zahlreiche Beispiele finden sich in Stowasser \& Mohry 1978 und in Conway \& Guy 1997. Conway \& Guy behandeln u. a. ausführlich die figurierten Zahlen - auch unter Berücksichtigung der dritten (räumlichen) Dimension - sowie die Fibonacci-Zahlen, die hinsichtlich ihrer Bedeutung in der Botanik analysiert werden.

${ }^{83}$ Das Zählproblem „Streichholzfolge“ stellt einen Teil des im Rahmen dieser Arbeit entwickelten Lern- und Versuchsprogramms dar (vgl. Kap. 5).
} 
Viele Zählprobleme führen zu arithmetischen Folgen höherer Ordnung, so z. B. die Dreieckszahlen (vgl. Abb. 2), oder sogar zu exponentiellen Zusammenhängen, wie bei der Folge der Fibonacci-Zahlen, hier dargestellt als Zählproblem bei der Vermehrung von Hasen $(\operatorname{vgl} \text { Abb. 3) })^{84}$ :

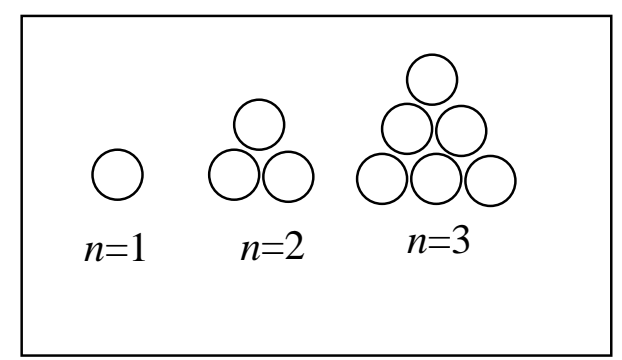

Abb. 2: Die Dreieckszahlen

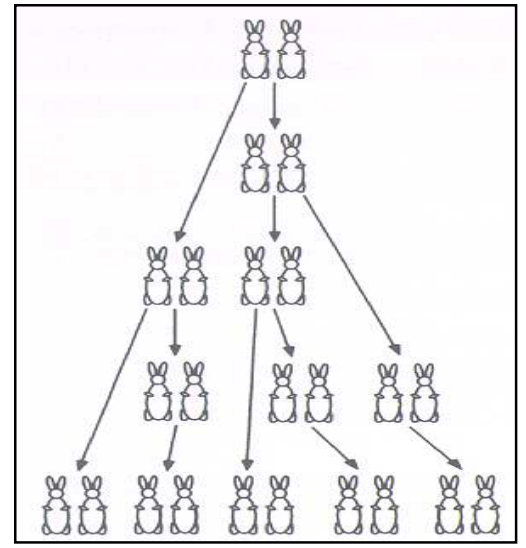

Abb. 3: Die Fibonacci-Folge (aus Conway \& Guy 1997, S. 126)

Zählprobleme - insbesondere die figurierten Zahlen - besitzen häufig eine ansprechende geometrische Veranschaulichung und werden in der Literatur unter vielfältigen inhaltlichen Gründen vorgeschlagen. Glaser (1978) hat in diesem Zusammenhang Unterrichtsvorschläge analysiert und klassifiziert. Das Beziehungsnetz um den Themenkreis „Zählprobleme“ umfasst nach seiner Darstellung dabei u. a. die folgenden Punkte:

- Bereicherung des Arithmetikunterrichts;

- Beispiele für Folgen und Reihen in der gymnasialen Oberstufe;

- Übungsbeispiele zur vollständigen Induktion;

- Überraschende Anwendungen und Verallgemeinerungen der Binomialkoeffizienten;

- Berechnung von Potenzsummen in Verbindung mit der (approximativen) Integration von Parabeln;

- Erzeugende Polynome von Zahlenfolgen;

- Lineare Differenzengleichungen;

- Anwendungen der Linearen Algebra.

Neben der Betonung dieser inhaltlichen Aspekten, ermöglichen Zählprobleme eine Vielzahl strategischer Aktivitäten und sind daher geeignet, heuristisches Wissen zu entwickeln, wie die folgenden beiden Beispiele verdeutlichen.

\footnotetext{
${ }^{84}$ Hintergrund des hier dargestellten Zählproblems ist eine alternative Formulierung der „Kaninchenaufgabe“ (vgl. Kap. 1.1): „Angenommen Kaninchenpaare bringen in der folgenden und in der darauf folgenden Generation jeweils ein neues Kaninchenpaar hervor und sterben danach. Wie viele Paare von Kaninchen werden dann in der $n$-ten Generation geboren, wenn zu Anfang genau ein Paar vorhanden ist?““
} 


\section{Beispiel: Kreisaufgabe ${ }^{85}$}

Wie viele Sehnen lassen sich zu n Punkten auf einem Kreisrand ziehen?

Im Mittelpunkt dieser Aufgabe steht eine arithmetische Folge zweiten Grades. Zum Aufstellen eines allgemeinen rekursiven Bildungsgesetzes ist es zweckmäßig, das Änderungsverhalten der ersten 4 bis 5 Folgenglieder zu analysieren. Indem man die Folgenglieder auf einer numerischen Ebene schrittweise vergleicht, kann man die Differenzen aufeinander folgender Folgenglieder als (iteratives) Muster identifizieren. So erkennt man, dass sich die Anzahl der Sehnen mit wachsendem $n$ um $+2,+3,+4, \ldots$ erhöht.

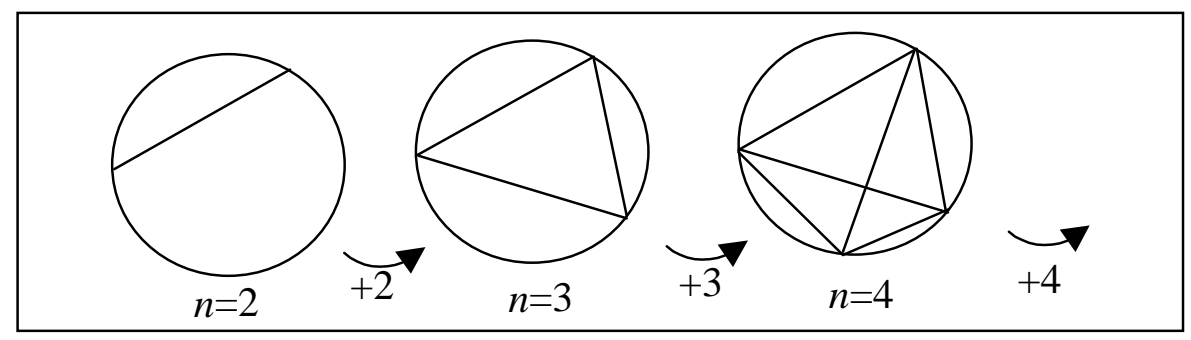

Abb. 4: Die ,Kreisfolge“6

Induktives Schließen führt dann auf die rekursive Folge $K(n+1)=K(n)+n$ mit dem Anfangsfolgenglied $K(2)=1$, deren Allgemeingültigkeit jedoch noch begründet werden muss. Hier hilft rekursives Argumentieren weiter, indem man die Anzahl der Sehnen zu $n+1$ Punkten auf einem Kreisrand dadurch bestimmt, dass man zunächst die Anzahl der Sehnen zu $n$ Punkten feststellt und dann die Anzahl der Sehnen, die beim Zufügen eines weiteren Kreispunkts dazukommen, hinzuaddiert. ${ }^{86}$

- Strategie I (Summenformel)

Eine Summenformel erhält man, indem die einzelnen Änderungen beginnend mit dem Anfangsfolgenglied $K(2)=1$ sukzessive aufsummiert werden:

$$
K(n)=\sum_{i=1}^{n-1} i
$$

\section{- Strategie II (explizite Formel)}

Zum Finden einer expliziten Formel können verschiedene Strategien zum Ziel führen. Geht man von der obigen Summenformel aus, kann eine Zeichnung weiterhelfen (vgl. Abb. 5). Die

\footnotetext{
${ }^{85}$ Die Bezeichnung „Kreisaufgabe“ wird von Hass (2000, S. 18 ff.) im Rahmen einer anderen Problemstellung verwendet. Es geht dort darum, die Anzahl der Gebiete zu zählen, in die der Kreis durch $n$ Sehnen zerlegt wird. Bei der Suche nach einer expliziten Formel führt dabei ein empirisch-experimentelles Vorgehen nicht zum Ziel bzw. verleitet zu einem fehlerhaften induktiven Schluss. Statt dessen bieten sich - u. a. durch Ausnutzung des Extremalprinzips - eine Fülle anderer Strategien zur Lösung des Problems an.

${ }^{86}$ Man führt somit das Problem auf ein ähnliches Teilproblem zurück.
} 
Summe wird als treppenstufiges Gebilde dargestellt und mit einer zweiten „Treppenstufe“ zu einem Rechteck ergänzt. Ausgehend von der Flächenformel für das Rechteck erhält man die Formel

$$
K(n)=\frac{n(n-1)}{2} \cdot 87
$$

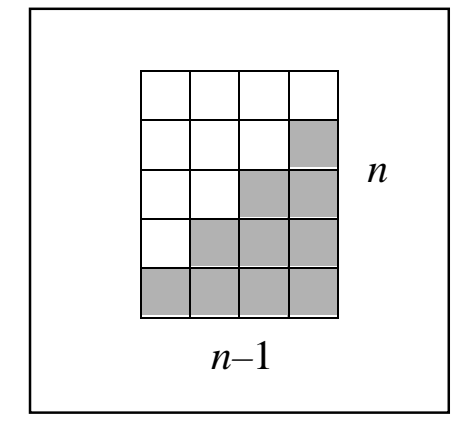

Abb. 5: Inhaltlich-anschaulicher Beweis

\section{- Strategie III (explizite Formel)}

Eine andere Termdarstellung erhält man, indem man die Sichtweise auf das Zählproblem ändert (das Problem „umstrukturiert“) und als Kombinationsproblem (ohne Wiederholung) identifiziert. Man zählt die Anzahl der Möglichkeiten, aus $n$ Kreispunkten jeweils zwei Punkte „herauszugreifen“ und durch eine Sehne zu verbinden. Dabei spielt es keine Rolle, welcher der beiden Punkte zuerst „gewählt“"wird. Man erhält somit die Darstellung

$$
K(n)=\left(\begin{array}{l}
n \\
2
\end{array}\right) .
$$

\section{- Strategie IV (explizite Formel)}

Ein weiterer Lösungsweg erschließt sich durch das Betrachten der Differenzenfolge. ${ }^{88}$ Eine Differenzenfolge entsteht, indem man die Differenzen benachbarter Folgenglieder bildet und die dabei entstehenden Terme als Funktionswerte einer neuen Folge interpretiert. Dieses Verfahren kann man wiederholen und erhält dadurch die zweite, dritte, ... Differenzenfolge. Handelt es sich bei der Ausgangsfolge um eine arithmetische Folge $n$-ter Ordnung, so ist die $(n-1)$-te Differenzenfolge nicht konstant und die $n$-te Differenzenfolge konstant. Die Umkehrung dieser Aussage gilt ebenfalls. Bei diesem Beispiel ist die 1. Differenzenfolge nicht konstant und die 2. Differenzenfolge konstant (vgl. Abb. 6) ${ }^{89}$; es handelt sich bei der Ausgangsfolge somit um eine Folge quadratischen Typs.

\footnotetext{
${ }^{87}$ In der didaktischen Literatur spricht man in diesem Zusammenhang von inhaltlich-anschaulichen Beweisen (vgl. auch Kap. 3.2.2.2) oder von „Proofs without words“ (vgl. Nelsen 1993).

${ }^{88}$ Das Betrachten der Differenzenfolge ist eine wichtige Strategie bei der Analyse von Zahlenmustern im Allgemeinen und von figurierten Zahlen im Besonderen. Viele Übungsaufgaben finden sich hierzu u.a. in Stowasser \& Mohry 1978. Insbesondere in England wurden im Rahmen von Projekten zur Verbesserung des Mathematikunterrichts Unterrichtsmaterialien erstellt, die zahlreiche Beispiele zu Zahlenmustern, die auf Zahlenfolgen mit konstanten Differenzen basieren, beinhalten. Vgl. hierzu etwa die Materialien des Shell Center for Mathematical Education (1982) und den Bericht von Burghes (1997), der explizit auf die Diskussion um TIMSS eingeht.
}

89 Aus Gründen der besseren Übersicht beginnt bei dieser Darstellung die Folge $K(n)$ mit dem Folgenglied $K(1)=0$. 


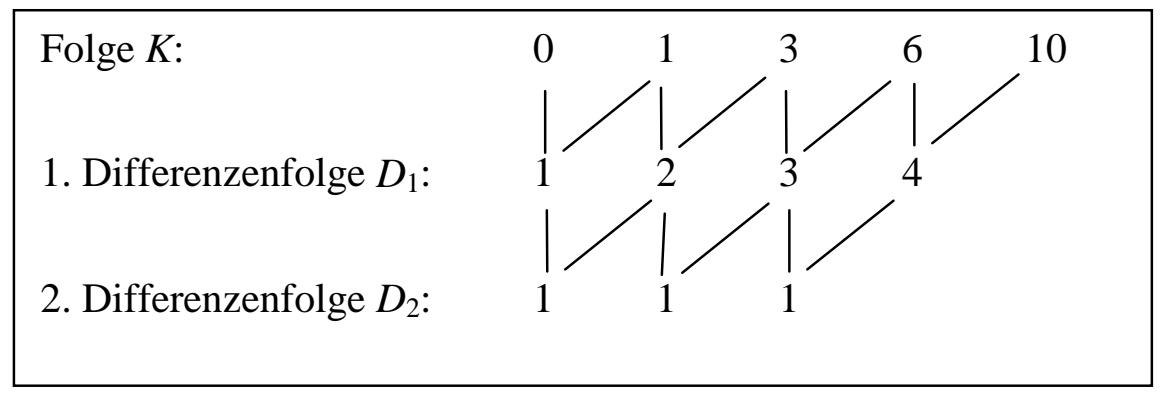

Abb. 6: 1. und 2. Differenzenfolge der ,Kreisfolge“

Die erste Differenzenfolge der allgemeinen quadratischen Folge $f$ mit $f(n)=a n^{2}+b n+c$ ist nun aber $D_{1}(n)=f(n+1)-f(n)=2 a n+a+b$. Weiterhin erkennt man, dass sich die erste Differenzenfolge der Kreisfolge durch die Formel $D_{1}(n)=n$ darstellen lässt. Damit können die Parameter $a$ und $b$ der Ausgangsfolge durch das Lösen eines linearen Gleichungssystems eindeutig bestimmt werden. Der Parameter $c$ ist mit der Nebenbedingung $K(1)=0$ Null. Man erhält die gesuchte Formel

$$
K(n)=0,5 n^{2}-0,5 n
$$

\section{Beispiel: Tetraederzahlen}

Tennisbälle werden in Form eines gleichseitigen Dreiecks angeordnet, so dass sich darauf eine Pyramide aufbauen lässt. Die nebenstehende Abbildung zeigt die ersten vier Stufen der Tennisballpyramide. Wie viele Tennisbälle benötigt man für eine fünfzigstufige (n-stufige) Pyramide? ${ }^{90}$

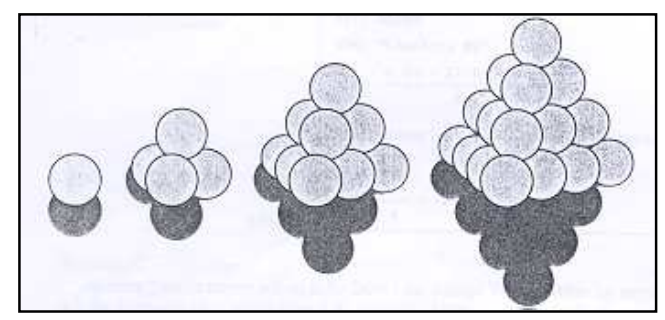

Abb. 7: Die Tetraederzahlen (aus Schmidt 1997, S. 38)

Im Mittelpunkt dieser Aufgabe steht eine arithmetische Folge dritten Grades. Ihre Behandlung ermöglicht die Verzahnung von inhaltlichem und strategischem Wissen. So lassen sich die Tetraederzahlen in einen größeren Kontext „Figurierte Zahlen“ einbetten. Dabei können zum einen mathematische Inhalte wie Funktionen und Gleichungssysteme der Mittelstufe wieder aufgegriffen und problemorientiert geübt sowie neue Begriffe wie Zahlenfolge, Summe, Rekursion und Iteration eingeführt und das Beweisverfahren der vollständigen Induktion motiviert werden. Zum anderen bietet die Suche nach einer allgemeinen Gesetzmäßigkeit - insbesondere auch durch den Einsatz eines Computeralgebrasystems (CAS) - viel Raum für heuristisches und kreatives Vorgehen, wie im Folgenden verdeutlicht wird.

\footnotetext{
${ }^{90}$ Die Formulierung der Aufgabe sowie die nachfolgenden Überlegungen basieren auf einem Vorschlag von Schmidt (1997). Schmidt betrachtet im Rahmen dieser Aufgabe insbesondere Lösungsmöglichkeiten, die sich beim Einsatz eines Computeralgebrasystems ergeben.
} 
- Strategie I (rekursive Formel)

Die rekursive Formel wird mit Hilfe einer geeigneten Visualisierung gefunden (vgl. Abb. 8).

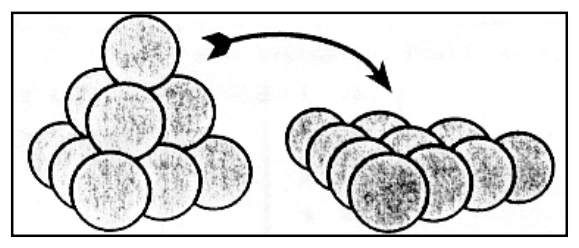

Abb. 8: Inhaltlich-anschauliches Erschließen einer rekursiven Formel (aus Schmidt 1997, S. 39)

Man erhält

$$
T(n)=T(n-1)+(1+2+3+\ldots+n) .
$$

- Strategie II (Summenformel)

$\mathrm{Zu}$ einer Summenformel führt eine genauere Analyse der ersten empirisch ermittelten Folgenglieder und eine geringfügig geänderte Sicht auf das dadurch neu gewonnene Zahlenmaterial. Hierzu werden pro Stufe die Kugeln jeder Ebene zunächst getrennt gezählt.

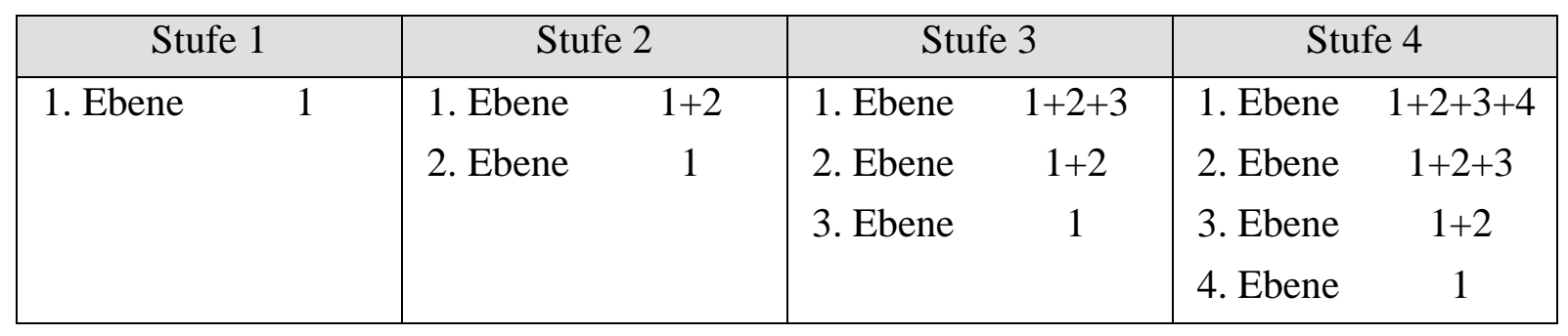

Tab. 2: Induktives Erschließen der Summenformel

Die Anzahl der Kugeln pro Stufe erhält man dann durch Summieren der einzelnen Partialsummen. Indem man wiederholte Additionen derselben Zahl durch eine Multiplikation ersetzt, erhält man:

$$
\begin{aligned}
& T(1)=1 \\
& T(2)=1 \cdot 2+2 \cdot 1 \\
& T(3)=1 \cdot 3+2 \cdot 2+3 \cdot 1 \\
& T(4)=1 \cdot 4+2 \cdot 3+3 \cdot 2+4 \cdot 1
\end{aligned}
$$

Durch induktives Schließen findet man:

$$
T(n)=1 \cdot n+2 \cdot(n-1)+3 \cdot(n-2)+\ldots+(n-1) \cdot 2+n \cdot 1=\sum_{i=1}^{n} i \cdot(n-i+1) .
$$

Diese Summenformel kann auch mit Hilfe einer geeigneten Visualisierung erschlossen werden. Die nachfolgende Darstellung der 3. Tetraederzahl (vgl. Abb. 9) lenkt das Augenmerk auf die Terme $1 \cdot 3,2 \cdot 2$ und $3 \cdot 1$. Damit ergibt sich $T(3)=1 \cdot 3+2 \cdot 2+3 \cdot 1$ und durch Verallgemeinerung die allgemeine Summenformel. Da sich hierbei die Lösung des allgemeinen 
Falls unmittelbar aus der Lösung eines Spezialfalls ergibt, spricht man auch von einem „repräsentativen Spezialfall“.

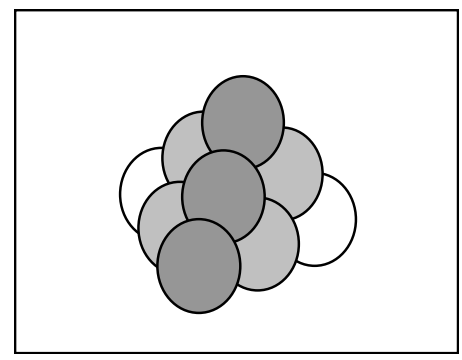

Abb. 9: Inhaltlich-anschauliches Erschließen einer Summenformel

\section{- Strategie III (Summenformel)}

Eine weitere Lösungsstrategie, die zu einer Summenformel führt, setzt bei der expliziten Formel für die Folge der Dreieckszahlen $D$ an, die mit Hilfe eines CAS einfach berechnet werden kann:

$$
D(n)=\sum_{i=1}^{n} i=\frac{n(n+1)}{2} .
$$

Die Folge der Tetraederzahlen erhält man, indem man die Partialsummen der Folge der Dreieckszahlen bestimmt:

$$
T(n)=\sum_{i=1}^{n} D(i)=\sum_{i=1}^{n} \frac{i(i+1)}{2}
$$

- $\quad$ Strategie IV (explizite Formel) $)^{11}$

Eine explizite Formel kann mit einem CAS aus den Summendarstellungen direkt gewonnen werden. Neben dieser Lösung ,auf Knopfdruck“ sind jedoch auch andere Lösungswege denkbar. So können die ersten empirisch ermittelten Folgenglieder zunächst graphisch dargestellt werden. Aufgrund der parabelförmigen Gestalt des Graphen kann vermutet werden, dass der Funktion ein polynomialer Zusammenhang zugrunde liegt. Da ein Ansatz mit einem quadratischen Polynom nicht zum Ziel führt, liegt es nahe, von einem Polynom dritten Grades auszugehen. Der Ansatz $T(n)=a n^{3}+b n^{2}+c n+d$ führt unter Berücksichtigung der ersten drei Fol-

\footnotetext{
${ }^{91}$ Steht - wie bei dem Unterrichtsvorschlag von Schmidt (1997) - ein CAS zur Verfügung, können mit Hilfe einer rekursiven Formel oder einer Summenformel einzelne aber auch mehrere Folgenglieder sehr leicht berechnet werden. Aus rein pragmatischen Gründen ist dann eine explizite Darstellung nicht notwendig. Es sind vielmehr theoretische Gesichtspunkte, die eine Formel in geschlossener Darstellung interessant erscheinen lassen. So können die Eigenschaften der Tetraederzahlen analysiert und mit denen anderer figurierter Zahlen verglichen werden. Auf diese Weise sind Klassifikationen hinsichtlich des Funktionstyps möglich. So lassen sich die Polygonalzahlen durch ein Polynom 2. Grades darstellen. Zur Beschreibung figurierter Zahlen unter Berücksichtigung der dritten Dimension (Bsp.: Tetraederzahlen, quadratische Pyramidalzahlen) benötigt man ein Polynom 3. Grades.
} 
genglieder $T(1)=1, T(2)=4$ und $T(3)=10$ zu einem Gleichungssystem mit drei Gleichungen und drei Unbekannten:

$$
\begin{aligned}
& a+b+c+d=1 \\
& 8 a+4 b+2 c+d=4 \\
& 27 a+9 b+3 c+d=10 .
\end{aligned}
$$

Dieses Gleichungssystem kann mit einem CAS auf Knopfdruck gelöst werden und man erhält

$$
T(n)=\frac{n^{3}}{6}+\frac{n^{2}}{2}+\frac{n}{3}
$$

Um die Allgemeingültigkeit dieser auf induktivem Wege gefundenen Formel zu beweisen, bietet sich das Beweisverfahren der vollständigen Induktion an.

\section{- Strategie V (explizite Formel)}

Wie bei der Kreisaufgabe kann auch hier die explizite Formel durch die Analyse der Differenzenfolgen gewonnen werden. Da die dritte Differenzenfolge der Tetraederzahlen - wie sich numerisch leicht nachprüfen lässt - konstant ist, kann geschlossen werden, dass sich diese Zahlen durch ein Polynom dritten Grades beschreiben lassen. Zur Bestimmung der Koeffizienten des Polynoms kann wie in Strategie IV beschrieben vorgegangen werden. Es ist jedoch auch möglich, die erste, zweite und dritte Differenzenfolge mit Hilfe des CAS symbolisch zu berechnen. Durch Betrachten geeigneter Folgenglieder erhält man wieder ein Gleichungssystem mit drei Gleichungen und drei Unbekannten, welches mit einem CAS gelöst werden kann.

\subsubsection{Graphentheoretische Probleme}

Graphentheoretische Fragestellungen lassen sich häufig in interessante Anwendungskontexte einbetten bzw. daraus herauslösen. Dabei sind in vielen Fällen nur wenige Vorkenntnisse erforderlich oder die benötigten Begriffe (Ecke, Kante, Graph, Kreis, Baum) ergeben sich zwangsläufig im Rahmen der Bearbeitung. ${ }^{92}$ Im Folgenden wird zunächst eine Übersicht über inhaltlich leicht zugängliche Problemstellungen mit exemplarischen Verweisen auf deren Behandlung in der didaktischen Literatur gegeben. Anhand eines Beispiels sollen im Anschluss verschiedene strategische Herangehensweisen an ein graphentheoretisches Problem verdeutlicht und Beziehungen zum Algorithmusbegriff sowie Möglichkeiten zur Erweiterung des Problemkreises aufgezeigt werden.

\footnotetext{
${ }^{92}$ Kießwetter \& Rosenkranz (1982) schlagen in diesem Zusammenhang vor, die Begriffsbezeichnungen den Schülern zunächst selbst zu überlassen. So seien Bezeichnungen wie Knoten, Netz, Gerüst häufig näher liegend als die entsprechenden ,ungewohnten“"Fachtermini Ecke, Graph, Baum.
} 
Die vielfältigen Vorschläge zur Graphentheorie lassen sich in zwei Klassen einteilen: ${ }^{93}$

\section{- Konstruktionsprobleme}

Als Konstruktionsprobleme werden Probleme bezeichnet, die durch einen Algorithmus gelöst werden können. Ziel ist es, diesen Algorithmus zu finden bzw. zu konstruieren. Bedeutsame Problemklassen sind hierbei: ${ }^{94}$

- Minimal aufspannende Bäume

Vgl. z. B. Kießwetter \& Rosenkranz 1982, Bovermann, Danckwerts \& Vogel 1983, Malle 1999b.

- Kürzeste Wege / Maximale Flüsse

Vgl. Floer 1977, Vogel 1977, Danckwerts \& Vogel 1983, Baptist 1979.

- Traveling-Salesman-Problem

Vgl. Jäger \& Schupp 1997.

- Netzplantechnik

Vgl. Bigalke 1974.

\section{- Beweisprobleme}

Beweisprobleme umfassen graphentheoretische Aussagen, deren Allgemeingültigkeit nachgewiesen werden soll. Hinsichtlich der zu beweisenden Aussagen kann man unterscheiden:

- Nachweis der Korrektheit eines Algorithmus

Führt ein gefundener Algorithmus in jedem Fall zu einer Lösung? (Vgl. die oben genannten Literaturangaben zu „Konstruktionsproblemen“.)

- Sätze über Eigenschaften von Graphen

Sätze über Zusammenhänge zwischen Eckengraden und Eckenanzahlen (vgl. z. B. Kirsch 1979), Euler'scher Polyedersatz (vgl. z. B. Rinkens \& Schrage 1974).

- Existenzsätze

Charakterisierung der eulerschen Graphen (vgl. z. B. Kirsch 1979), einfache Sätze über Flächenfärbungen (vgl. z. B. Nussbaum \& Stowasser 1970, Henn 1979).

\footnotetext{
${ }^{93}$ Auch andere, differenziertere Klassifikationen sind möglich. So unterscheidet Bigalke (1974):

- Modelle für Probleme, die eine algorithmische Lösung erfordern;

- $\quad$ Lösung von Problemen mit Hilfe graphentheoretischer Sätze;

- Veranschaulichung von (eventuell komplizierten) Zusammenhängen;

- Leichtverständliche Probleme mit beliebig hohem Schwierigkeitsgrad.

Da eine genauere Analyse graphentheoretischer Fragestellungen nicht Gegenstand dieser Arbeit ist und die Einteilung in Beweis- und Konstruktionsprobleme unseren Anforderungen genügt, soll auf diese Klassifizierung nicht weiter eingegangen werden.

${ }^{94}$ Vgl. auch Kap. 1.3.
} 


\section{Beispiel: Minimal aufspannende Bäume}

In einem abgelegenen und unbesiedelten Gebiet befinden sich sechs Forschungsstationen A, B, C, D, E, F, die durch das in Abb. 10 dargestellte Wegenetz miteinander verbunden sind. Die bei den einzelnen Wegen stehenden Zahlen geben die Entfernungen in $\mathrm{km}$ an. Da die Instandhaltung der Wege aufwändig ist, wird beschlossen, möglichst viele Wege aufzugeben, aber nur so viele, dass man immer noch von jeder Forschungsstation zu jeder anderen gelangen kann. Welche Wege sollen erhalten bleiben, damit das verbleibende Netz minimal ist $?^{95}$

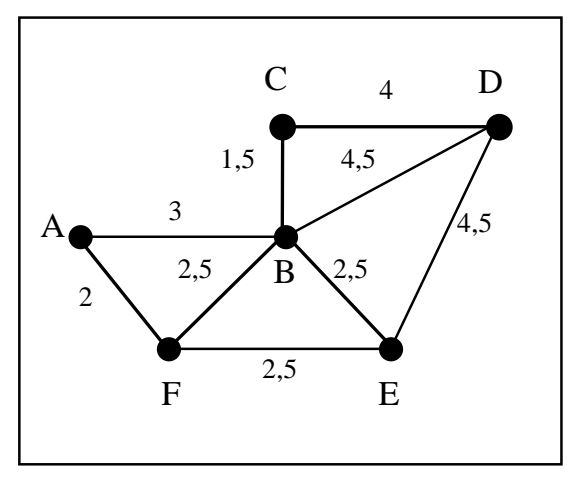

Abb. 10: Wegenetz

Bei diesem Beispiel handelt es sich um ein Konstruktionsproblem, an das sich ein Beweisproblem anschließt. Zur Lösung des Konstruktionsproblems sind verschiedene Herangehensweisen denkbar. So kann der minimal aufspannende Baum durch Probieren gefunden werden, indem zunächst alle möglichen Bäume aufgelistet und die Summe der Kantenlängen berechnet werden sowie das Minimum bestimmt wird. Dieser Lösungsweg ist jedoch recht umständlich, wenn die Struktur des Netzes komplexer bzw. die Anzahl der Kanten größer ist. Ein effektiveres Verfahren stellt der von dem Mathematiker Kruskal 1956 gefundene gleichnamige Algorithmus dar (vgl. auch Kap. 1.3):

\section{- Algorithmus von Kruskal}

Bei diesem Algorithmus geht man von einer Kante mit möglichst geringem Gewicht aus. Dann wählt man die nächste Kante mit möglichst geringer Bewertung, aber so, dass sie mit den bereits gewählten Kanten keinen Kreis bildet. Man wiederholt das Verfahren so oft, bis die Wahl einer neuen Kante zu einem Kreis führen würde. Je nach Auswahl der Kanten kann man auf diese Weise verschiedene minimal spannende Bäume erhalten (vgl. Abb. 11).
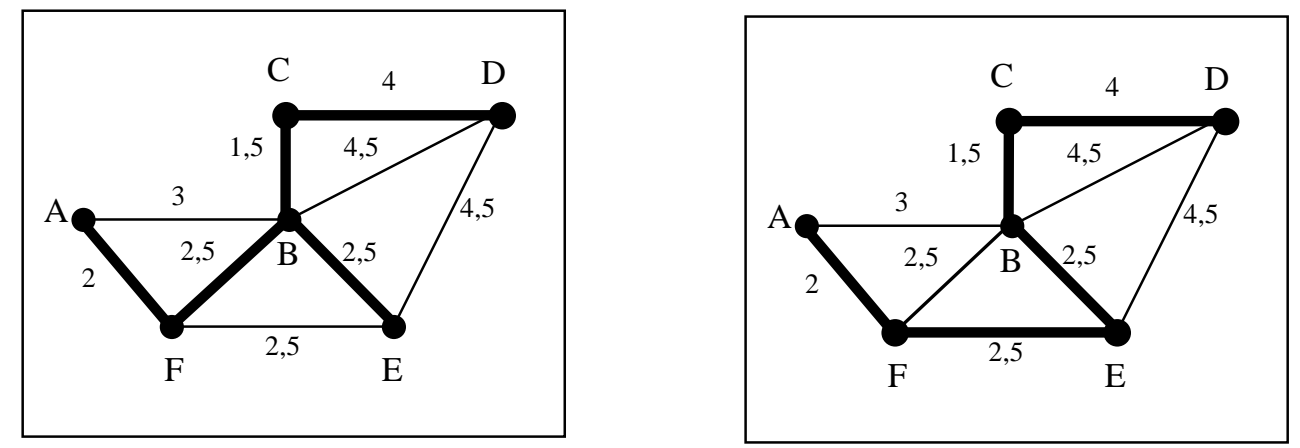

Abb. 11: Zwei verschiedene minimal aufspannende Bäume

\footnotetext{
${ }^{95}$ Die Formulierung dieser Aufgabe geht zurück auf einen Vorschlag von Malle 1999b.
} 
Wie Kießwetter \& Rosenkranz (1982) berichten, ist das Finden dieses von ihnen als ,abstraktaufbauend“ bezeichneten Verfahrens durch die Schüler mit einer starken Steuerung durch den Lehrer verbunden. ${ }^{96}$ Werden Schüler bzw. Studierende aufgefordert, eigene Algorithmen zu entdecken, sind nach ihrer Erfahrung folgende Strategien naheliegender:

\section{- Anschaulich-abbauendes Verfahren}

Die vorhandenen Kreise des Wegenetzes werden möglichst günstig aufgelöst. Hierzu beginnt man bei irgendeinem Kreis und entfernt dort die längste Kante. Man fährt so lange fort, bis alle Kreise aufgelöst sind.

\section{- Anschaulich-aufbauendes Verfahren}

Ausgehend von einem beliebigen Knoten des Wegenetzes bestimmt man die kürzeste Kante, die von diesem Knoten wegführt und ergänzt diese durch ihren Endpunkt. Ausgehend von dieser Kante bestimmt man wiederum die kürzeste Kante, die von einem ihrer Knoten ausgeht und fügt diese zur Ausgangskante hinzu. Dieses Verfahren wird wiederholt, wobei darauf zu achten ist, dass die jeweils zum Teilgraph hinzugefügte Kante mit diesem keinen Kreis bildet.

\section{- Abstrakt-abbauendes Verfahren}

Beim abstrakt-abbauenden Verfahren werden die Kanten des Wegenetzes zunächst der Größe nach geordnet. Anschließend wird Schritt für Schritt die jeweils größte Kante entfernt, die Teil eines Kreises ist. Diese Verfahren stellt gewissermaßen den „dualen Algorithmus“ zum Algorithmus von Kruskal dar. ${ }^{97}$

Das Finden und Formulieren von Algorithmen kann einen wichtigen Beitrag zum Verständnis des Algorithmenbegriffs leisten. Zur Vertiefung können sich im Mathematikunterricht eine Beurteilung der Effizienz der verschiedenen Algorithmen und das Überprüfen von deren Korrektheit anschließen. Die Frage nach der Korrektheit der Algorithmen führt auf ein Beweisproblem. $\mathrm{Ob}$ und wie dieses Beweisproblem behandelt wird, hängt von der entsprechenden Lerngruppe ab. So hält Malle (1999b) den von Kruskal angegebenen Beweis des nach ihm benannten Verfahrens für mathematisch-interessierte Schüler der 9. bzw. 10. Jahrgangsstufe

\footnotetext{
${ }^{96}$ Kießwetter \& Rosenkranz beschreiben in diesem Zusammenhang ein „Spiel“ als methodische Hilfe: „Zwei Parteien A und B spielen gegeneinander. Partei A hat die Aufgabe, ein minimales Gerüst zu konstruieren, Partei B soll dies verhindern. Partei A hat gewonnen, wenn sie ein minimales Gerüst konstruiert hat; sonst gewinnt Partei B. Der Lehrer ist Schiedsrichter. Er kennt das minimale Gerüst. A und B dürfen aus dem Liniennetz Linien wählen. A soll solche Linien wählen, die zum minimalen Gerüst gehören. B versucht dies durch Belegung von Linien, die dann durch A nicht mehr gewählt werden dürfen, zu verhindern. Allerdings hat A unmittelbar nach der Wahl von B Einspruchsrecht, muss aber dann selbst die von B angestrebte Linie wählen. B beginnt, nach jeder Wahl von A zieht B.“ (S. 13)

${ }^{97}$ Während (ältere) Schüler in der Regel das anschaulich-abbauende und das anschaulich-aufbauende Verfahren entdecken, finden Studierende nach dem Bericht von Kießwetter \& Rosenkranz häufig das abstrakt-abbauende Verfahren. Auch in einer von der Autorin im Sommersemester 2000 durchgeführten Veranstaltung „Diskrete Mathematik“ mit Studierenden des Lehramts für Haupt- und Realschulen wurde von den meisten Teilnehmern das abstrakt-abbauende Verfahren selbstständig gefunden.
} 
für zugänglich. Es handelt sich hierbei um einen indirekten Beweis, der „kurz, einfach und ästhetisch ansprechend“ (a. a. O., S. 22) ist. Der Vorschlag von Kießwetter \& Rosenkranz zielt auf einen Beweis der Korrektheit einer ganzen Algorithmenklasse ab, welche die vorstehend beschriebenen vier Verfahren enthält. Teile dieses Beweises basieren auf dem Verfahren der vollständigen Induktion, so dass eine Behandlung erst in der Oberstufe sinnvoll erscheint. Eine weitere Thematisierung und Modifikation der gefundenen Algorithmen ist im Rahmen vielfältiger Erweiterungen des Themenkreises „Minimal aufspannende Bäume“ möglich (vgl. Kießwetter \& Rosenkranz 1982, S. 24 ff.). Es ergeben sich auch Anknüpfungspunkte für andere - nicht nur graphentheoretische - Fragestellungen. So stellt insbesondere das dem Algorithmus von Kruskal zugrunde liegende Prinzip, immer die „bestmögliche“ Kante zu wählen, ein allgemeines heuristisches Prinzip dar, das auf zahlreiche Optimierungsaufgaben auch außerhalb der Graphentheorie angewendet werden kann. Diese so genannte „Greedy-Strategie“ liefert jedoch nicht immer die optimale Lösung, sondern häufig nur eine Näherungslösung, wie etwa im Fall des Traveling-Salesman-Problems ${ }^{98}$. Sie kann aber einen Ausgangspunkt zur Entwicklung leistungsfähigerer Algorithmen darstellen (vgl. Jäger \& Schupp 1997).

\subsection{Beitrag diskreter Mathematik zur Begriffsbildung}

Die meisten grundlegenden mathematischen Begriffe haben eine lange Geschichte. Einen Anstoß zur Bildung von Begriffen liefern in der Regel konkrete Probleme, die mit Hilfe des neuen Begriffs gelöst werden können. In Verbindung mit Bemühungen, die neuen Begriffe und die entwickelten Methoden im Umgang mit ihnen zu rechtfertigen, werden die Begriffe selbst in der Folgezeit zum Gegenstand der Untersuchung. Dabei werden Eigenschaften und Beziehungen zu anderen mathematischen Inhalten analysiert und auftretende Widersprüche geklärt. Im Rahmen dieses Prozesses der Klärung und Formalisierung wird der Begriff immer weiter präzisiert bzw. „exaktifiziert“, wobei jedoch manche Aspekte des ursprünglichen Begriffs verloren gehen (vgl. Fischer \& Malle 1985, S. 150 ff., Vollrath 1984, S. 194 ff.). ${ }^{99}$

Auch im Unterricht sollte die historische Entwicklung der Begriffe berücksichtigt werden. So ist es nicht sinnvoll, formale Begriffe und Axiomensysteme an den Anfang einer Unterrichtseinheit zu stellen. Exakte Begriffe sind zwar „klarer“ und „,deutlicher“, d. h. sie können zu einer Verbesserung der Kommunikation unter Experten beitragen. Da sie jedoch in der Regel abstrakt und unanschaulich sind, sind sie für einen Nichtmathematiker, insbesondere für einen Schüler nur schwer verständlich. Im Mathematikunterricht sind daher ,,mathematische Inhalte so zu vereinfachen, dass sie für den Schüler erfassbar werden. Dabei soll Wesentliches nicht

\footnotetext{
${ }^{98}$ Der entsprechende Algorithmus wird dort als Nearest-Neighbour-Verfahren bezeichnet.

${ }^{99}$ Fischer \& Malle (1985, S. 156 f.) führen als Beispiel u. a. den Diriclet'schen Funktionsbegriff an, bei dem der Aspekt der „Glattheit“ gegenüber früheren Vorstellungen von Funktionen (wie etwa bei Euler) verloren gegangen ist. Um diesen Aspekt als besondere Eigenschaft (wieder) hervorzuheben, waren Zusatzbegriffe wie ,Stetigkeit“, „Integrierbarkeit“ oder „Differenzierbarkeit“ erforderlich.
} 
verfälscht und ein späterer Übergang zu einer exakteren Darstellung nicht unnötig erschwert werden“ (Tietze, Klika \& Wolpers 1997, S. 64). Unterricht sollte daher bei elementaren Begriffen und Methoden ansetzen. In diesem Sinne kann auch das folgende Zitat Wittenbergs verstanden werden:

„Im Unterricht muss sich für den Schüler eine gültige Begegnung mit der Mathematik, mit deren Tragweite, mit deren Beziehungsreichtum, vollziehen; es muss ihm am Elementaren ein echtes Erlebnis dieser Wissenschaft erschlossen werden. Der Unterricht muss dem gerecht werden, was Mathematik wirklich ist.“ (Wittenberg 1990, S. 50 f.)

Im Zuge einer solchen Elementarisierung empfiehlt es sich, zunächst auf einer inhaltlichkonkreten Ebene zu arbeiten, sowie an Vorwissen und Vorerfahrungen der Schüler anzuschließen. Ein wichtiges Anliegen von Unterricht sollte es dabei sein, angemessene Grundvorstellungen der zentralen mathematischen Begriffe aufzubauen. Grundvorstellungen beschreiben nach v. Hofe „Beziehungen zwischen mathematischen Strukturen, individuellpsychologischen Prozessen und realen Sachzusammenhängen oder kurz: Beziehungen zwischen Mathematik, Individuum und Realität" (v. Hofe 1996, S. 259). Dabei wird der mathematische Begriff auf eine für den Lernenden verständliche Art konkretisiert und einer inhaltlich-anschaulichen, nicht unbedingt mathematikspezifischen Vorstellung untergeordnet. Im Rahmen eines sinnvoll fortschreitenden Exaktifizierungsprozesses kann man sich dann allmählich von konkreten Vorstellungen und Vorerfahrungen lösen und eine stärkere Formalisierung anstreben. Dieses Vorgehen lässt sich auch durch lernpsychologische Theorien stützen (vgl. Kap. 3) und steht im Einklang mit der genetischen Methode (vgl. Wittmann 1981, S. $130 \mathrm{f}$.).

Im Folgenden soll aufgezeigt werden, welchen Beitrag diskrete Begriffe und Methoden zur Elementarisierung von bzw. beim Zugang zu zentralen Begriffen der Algebra und Analysis leisten können und welche Begriffsaspekte und -vorstellungen damit besonders betont werden.

\subsubsection{Einführung des Variablen- und Termkonzepts}

Gegenstand der elementaren Algebra ist das Erlernen der Formelsprache, d. h. der Umgang mit dem Variablen- und Termbegriff. Es handelt sich hierbei um einen langfristigen Prozess, der mit einer Phase intuitiven Gebrauchs der Sprache beginnt, in der die Sprachelemente hauptsächlich als Werkzeug zur Beschreibung von Problemsituationen verwendet werden. In einer zweiten Phase werden die bisher betrachteten Begriffe und ihre Verwendungsmöglichkeiten reflektiert, und es werden geeignete Bezeichnungen eingeführt. Ist der Umgang mit Variablen und Termen in den ersten beiden Phasen noch von inhaltlichen Überlegungen geprägt, so rücken anschließend zunehmend syntaktische Aspekte in den Vordergrund. In diesem Zusammenhang werden Regeln für Termumformungen erarbeitet und die Formelsprache wird genutzt, um mathematische Probleme zu lösen (vgl. Vollrath 1994, S. 92 f.). 
Die verschiedenen Verwendungsbereiche von Variablen und die Art, wie mit Variablen gearbeitet wird bzw. welche Vorstellungen damit verbunden sind, legen es nahe, bestimmte Aspekte des Variablenbegriffs zu unterscheiden. Malle (1993) betrachtet in diesem Zusammenhang die folgenden drei Aspekte ${ }^{100}$ :

- Gegenstandsaspekt: Variable als unbekannte oder nicht näher bestimmte Zahl;

- Einsetzungsaspekt: Variable als Platzhalter für Zahlen;

- Kalkülaspekt: Variable als bedeutungsloses Zeichen, mit dem nach bestimmten Regeln operiert werden darf.

Auch wenn alle Aspekte beim praktischen Arbeiten in enger Wechselwirkung zueinander stehen, sollten im Sinne eines inhaltlichen Zugangs zur Algebra zunächst nur die ersten beiden Aspekte berücksichtigt werden. Malle geht sogar noch einen Schritt weiter, indem er - im Hinblick auf eine zunächst auf inhaltlichen Überlegungen basierende Gleichungslehre - für eine anfängliche Betonung des Gegenstandsaspekts plädiert. ${ }^{101}$

Will man im Mathematikunterricht dem Gegenstandsaspekt gerecht werden, sollten Variablen und Terme in bedeutungsvollen Sachsituationen eingeführt und geeignete Aufgaben zum Aufstellen und Interpretieren von Formeln gestellt werden. Hierzu bieten sich zunächst Aufgaben zum formelmäßigen Erfassen von Alltagssituationen an. Daneben ist ein weiterer Bereich in der didaktischen Literatur zunehmend bedeutsam geworden: Das Formulieren von Gesetzmäßigkeiten in Verbindung mit diskreten geometrischen oder numerischen Mustern. Solche Abzählprobleme werden unter dem Stichwort „Generalization activities“ insbesondere in England als ein wichtiger Zugang zur Algebra angesehen (vgl. Stacey 1989, Lee 1996, Mason 1996, Radfort 1996), aber auch in der deutschen Mathematikdidaktik gibt es entsprechende Forderungen und Vorschläge (vgl. Wellstein 1978, Sauer 1997). Indem Schüler zunächst ein einfaches Muster (vgl. Abb. 12) untersuchen, es numerisch fortführen, eine verbale Regel formulieren und abschließend die Situation unter Ausnutzung von Variablen verallgemeinern, wird der Abstraktionsgrad stufenweise gesteigert.

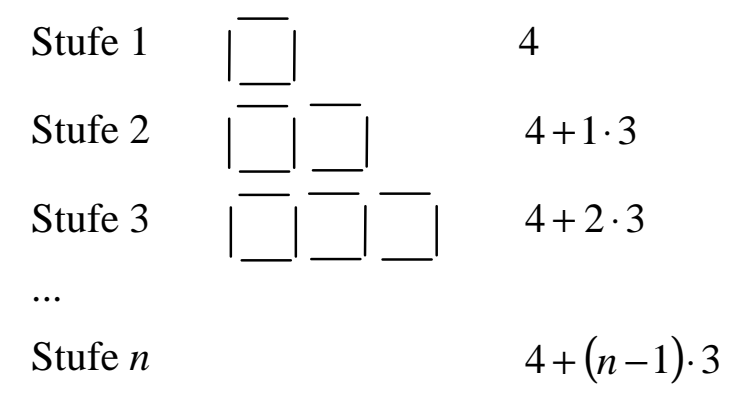

Abb. 12: Formalisieren einer Gesetzmäßigkeit

\footnotetext{
${ }^{100}$ Die genannten Aspekte lassen sich auch auf Terme und Gleichungen übertragen.

101 So könne man beim inhaltlichen Lösen einer Gleichung unter Betonung des Gegenstandsaspekts an dem vertrauten „Zahlenrechnen“ anknüpfen, und es seien keine speziellen Begriffe wie „Platzhalter“, „Aussageform“, „Äquivalenz“ oder „Lösungsmenge“ erforderlich (vgl. Malle 1993, S. 51).
} 
Der Gegenstandsaspekt wird hierbei insofern betont, als die Variable $n$ im Rahmen der Formalisierung, d. h. der Angabe der für eine beliebige Stufe $n$ benötigten Streichhölzer, als eine nicht näher bestimmte natürliche Zahl gedacht bzw. verwendet wird. Indem die Formel im Anschluss mit konkretem Zahlenmaterial empirisch überprüft bzw. zur Berechnung eines visuell unzugänglichen Folgenglieds ausgenutzt wird, also konkrete Zahlen eingesetzt werden, wird darüber hinaus auch der Einsetzungsaspekt berücksichtigt.

Viele Muster legen verschiedene Abzählstrategien nahe bzw. lassen sich durch mehr als eine allgemeine Regel beschreiben, wie etwa das folgende „T-Muster“ (vgl. Mason 1996, S. 84 ff.):
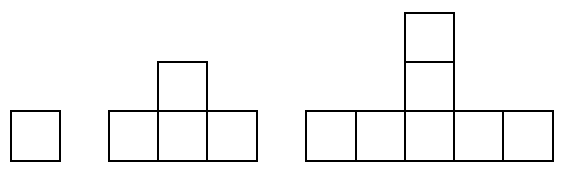

Abb. 13: ,T-Muster“6

Hier kann die Anzahl der Quadrate der Figur in der n-ten Stufe durch verschiedene äquivalenten Terme beschrieben werden (vgl. Abb. 14):
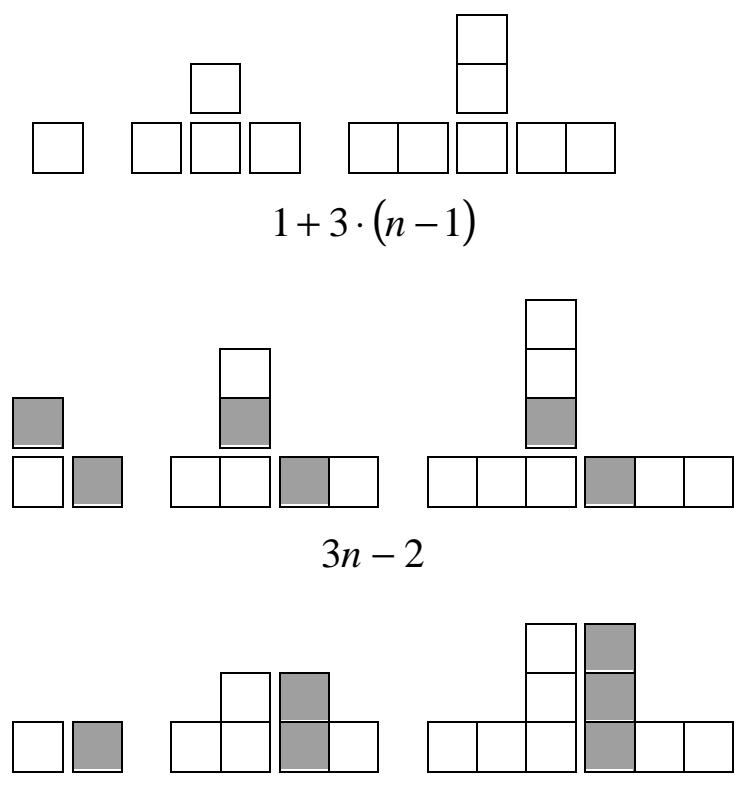

$$
2 \cdot(2 n-1)-n
$$

Abb. 14: Verschiedene Terme zur Beschreibung des ,T-Musters“

Durch die Frage, ob denn alle Lösungen richtig sind und welches die „einfachste“ Form ist, können Termumformungen motiviert werden (vgl. Vollrath 1994). Daneben können solche Muster aber auch den Anstoß zu einer (verbalen) Formulierung allgemeiner rekursiver $\mathrm{Zu}$ sammenhänge geben, indem erkannt wird, dass in jeder Stufe drei Quadrate zu der Anzahl der Quadrate der vorhergehenden Stufe hinzukommen. 


\subsubsection{Beitrag zur Entwicklung des Funktionsbegriffs}

Der Funktionsbegriff ist ein zentraler Begriff der Mathematik und besitzt in vielen außermathematischen Anwendungsgebieten eine große Bedeutung. Funktionen werden hier als Werkzeug bei der Modellbildung verwendet: Wird zwischen Größen eine Abhängigkeit vermutet, dann wird eine Funktion gesucht, die diesen Zusammenhang möglichst gut beschreibt. Umgekehrt werden mit Funktionen, wie z. B. Ware-Preis-Funktionen, auch Zusammenhänge gesetzt, nach denen sich eine Gesellschaft zu richten hat. Auch im Mathematikunterricht sollten Funktionen als Werkzeug zum Mathematisieren angemessen berücksichtigt werden. So plädiert Vollrath für einen Algebraunterricht, der bei Umweltsituationen ,ansetzt und bei dem auf unterschiedlichen Niveaus mathematisch gearbeitet wird“ (Vollrath 1982, S. 6). Dabei sei es sinnvoll, solche Situationen zu untersuchen, die weniger fachspezifisch sind, sondern vielmehr „elementar zum Erfahrungsschatz“ des Schülers gehören (a. a. O., S. 6). Im Rahmen einer solchen Konzeption können Abhängigkeiten zwischen Größen, wie etwa der Länge eines zurückgelegten Wegs und der dafür benötigten Zeit, verbal formuliert und dann mit Variablen bzw. Termen erfasst werden. Während es sich in diesem Fall um Größenbereiche handelt, die mit Hilfe „stetiger“ Variablen bzw. Funktionen auf $\mathbf{Q}^{+}$oder $\mathbf{R}^{+}$beschrieben werden, gibt es zahlreiche Sachsituationen (z. B. Anzahl-Gewicht-, Anzahl-Preis-Beziehungen), die durch diskrete Funktionen bzw. Folgen adäquater beschrieben werden können. Nach Ansicht von Vollrath sind diese Probleme Schülern sogar einfacher zugänglich: „Quantity-costproblems are easier to handle than distance-time problems" (Vollrath 1992, S. 232). Es bietet sich daher an, diskreten Umweltsituationen im Hinblick auf die Einführung des Funktionsbegriffs eine größere Beachtung zu schenken. Eine solche „diskrete Sicht“ auf Funktionen findet sich auch in Empfehlungen, die - wie etwa die Standards der NCTM (1989) - im Erkennen und Erfassen von ,patterns“ in Phänomenen und Situationen eine entscheidende Propädeutik des Funktionsbegriffs sehen. „Dabei kann man ,pattern“ als ,Muster“ im geometrischen und übertragenen Sinne, aber auch spezifischer je nach Phänomen als Regelmäßigkeit, Regel, Bildungsgesetz, Aufbauprinzip etc. übersetzen“ (Löthe 1997, S. 328). Damit gewinnen nicht nur diskrete Umweltsituationen, sondern auch elementare Abzählprobleme beim Zugang zum Funktionsbegriff an Bedeutung. Hier kann an die Überlegungen zur Einführung des Variablenbegriffs (vgl. Kap. 2.3.1) angeknüpft werden.

Funktionen sind im weiteren Verlauf der Sekundarstufe I nicht nur ein Werkzeug zur Beschreibung von Zusammenhängen, sondern werden zunehmend auch selbst Gegenstand der Untersuchung. Die Schüler sollen in diesem Zusammenhang Eigenschaften des Begriffs kennen lernen und diese zum Lösen von Problemen einsetzen sowie den Begriff in ein Begriffsnetz einordnen. Im Umgang mit dem Funktionsbegriff und dessen Darstellungen wird hierbei 
ein Beitrag zur Entwicklung „funktionalen Denkens“ geleistet. Vollrath (1989) unterscheidet in diesem Zusammenhang drei Aspekte: ${ }^{102}$

- Zuordnungsaspekt: Durch Funktionen stiftet man Zusammenhänge zwischen Größen: Eine Größe ist dann einer anderen zugeordnet, so dass die eine Größe als abhängig von der anderen gesehen wird.

- Änderungsaspekt: Durch eine Funktion erfasst man, wie sich Änderungen einer Größe auf eine andere auswirken.

- Ganzheitliche Sicht: Mit Funktionen betrachtet man einen gegebenen oder gestifteten $\mathrm{Zu}$ sammenhang als Ganzes.

Alle Aspekte sollten im Unterricht angemessen berïcksichtigt werden. Vollraths Analysen von Forschungsarbeiten zum funktionalen Denken weisen jedoch darauf hin, dass im Umgang mit Wertetabellen die Betrachtung ,horizontaler“ Zusammenhänge überwiegt und der Änderungsaspekt nur unzureichend beachtet wird. Insbesondere haben Schüler offensichtlich „erhebliche Schwierigkeiten mit endlichen und diskreten Definitionsbereichen. Funktionales Denken ist vielfach auf stetige Veränderungen eingeschränkt“ (a. a. O., S. 31). Durch die Behandlung von Zählproblemen kann diesem Defizit entgegengewirkt und der (diskrete) Änderungsaspekt im Umgang mit Funktionen betont werden. So stellt die Analyse des Änderungsverhaltens häufig einen Ausgangspunkt für das Finden und Aufstellen der Termdarstellung der Zählfolge in rekursiver oder expliziter Form dar. Da ein rekursiver Ansatz vielfach einfacher ist, bezeichnen Stowasser und Mohry dieses Vorgehen auch als eine „mächtige Methode der lokalen Beschreibung funktionaler Zusammenhänge“" (Stowasser \& Mohry 1996, S. 6). Zählprobleme können weiterhin hinsichtlich des Änderungsverhaltens der mit ihnen verbundenen Zählfolgen klassifiziert werden. Während viele einfache „Streichholzfiguren“ auf arithmetische Folgen führen, d. h. die Änderung von Stufe zu Stufe konstant ist, stellen die figurierten Zahlen quadratische Folgen bzw. arithmetische Folgen zweiter Ordnung dar, deren Änderung von Stufe zu Stufe konstant zunimmt. Während es sich bei diesen Problemklassen empfiehlt, die Differenzen benachbarter Folgenglieder zu analysieren, ist es bei Zählfolgen, denen ein exponentieller Zusammenhang zugrunde liegt, sinnvoll, die Quotienten benachbarter Folgenglieder zu betrachten, um zu einer rekursiven Darstellung zu gelangen. Dennoch kann auch hier die Analyse der Differenzenfolge - die, wie im Fall der „Türme von Hanoi““103 wieder eine geometrische Folge darstellt (vgl. Tab. 3) - einen wichtigen Einblick in die Cha-

\footnotetext{
${ }^{102}$ Diese Sicht betont - wie Vollrath hervorhebt - den methodologischen Aspekt des Mathematiklernens. Nach Krüger (2000) wird damit der Bedeutung des Begriffs „funktionales Denken“ im ursprünglichen Sinne der Meraner Reform, d. h. als umfassendes didaktisches Prinzip, nur bedingt gerecht. Da hier jedoch der Funktionsbegriff im Mittelpunkt der Analyse steht, beschränken wir uns auf die Charakterisierung von Vollrath.

${ }^{103}$ Bei den Türmen von Hanoi handelt es sich um das folgende Zählproblem: Auf einem von drei Stiften sind $n$ kreisförmige Scheiben der Größe nach gestapelt. Dieser Scheibenturm soll auf einen anderen Stift umgebaut werden. Dabei müssen die Scheiben einzeln umgelegt werden, ohne dass eine größere Scheibe auf eine kleinere
} 
rakteristika exponentiellen Wachstums und dessen Abgrenzung zu polynomialem Wachstum geben.

\begin{tabular}{|l|c|c|c|c|c|c|c|c|}
\hline Anzahl der Scheiben & $n$ & 1 & 2 & 3 & 4 & 5 & 6 & 7 \\
\hline $\begin{array}{l}\text { Anzahl der Umlegungen beim } \\
\text { Umbau des Turms von Hanoi }\end{array}$ & $M(n)$ & 1 & 3 & 7 & 15 & 31 & 63 & 127 \\
\hline Differenzenfolge & $D(n)$ & 2 & 4 & 8 & 16 & 32 & 64 & 126 \\
\hline
\end{tabular}

Tab. 3: Folge und Differenzenfolge beim Zählproblem ,Türme von Hanoi“‘

Eine weitere Möglichkeit zur Betonung des Änderungsaspekts ergibt sich im Rahmen einer vorbereitenden oder auch ergänzenden diskreten Modellierung außermathematischer (stetiger) Situationen. Indem zeitabhängige Umweltsituationen mit (rekursiven) Folgen beschrieben werden, können darüber hinaus spezifische (diskrete) Charakteristika deutlich gemacht werden. Dies betrifft nicht nur Prozesse, denen ein linearer und exponentieller Zusammenhang zugrunde liegt. Betrachtet man z. B. das physikalische Fallgesetz aus einer diskreten Sicht, d. h. in Verbindung mit einer Modellierung mit quadratischen Folgen, erhält man durch die Interpretation des (diskreten) Änderungsverhaltens eine tiefere Einsicht in das dahinter stehende Umweltphänomen. So sieht Wagenschein gerade in der Gesetzmäßigkeit, dass ein Stein, der in einer Zeiteinheit eine bestimmte Strecke fällt, ,,in der nächsten, der 2. Zeiteinheit, das - nein nicht 2fache, sondern - 3fache dieser Strecke, in der dann wieder nächsten, dritten, das 5fache, dann das 7 fache, das 9fache und so fort" (Wagenschein 1970, S. 128) durchläuft, einen wichtigen „Bildungseffekt des physikalischen Verstehens“. Bei einer diskreten Modellierung außermathematischer Situationen ist jedoch zu berücksichtigen, dass die verwendeten Folgen auch in ein Beziehungsnetz zu den entsprechenden kontinuierlichen Funktionen eingebettet werden, denn - so beklagte Kirsch bereits 1976 - ,Zinseszinsrechnung, Exponentialfunktion und geometrische Folge werden (wenn überhaupt) weitgehend ohne Beziehung zueinander gelehrt [...], insbesondere ohne Bezug zu relevanten Wachstumsprozessen“ (Kirsch 1976, S. 258). In diesem Zusammenhang ist vor allem darauf zu achten, dass sowohl die rekursive als auch die entsprechende explizite Darstellung angemessen berücksichtigt wird (vgl. Weigand 1993, S. 179).

\subsubsection{Diskrete Vorbereitung der Analysis}

Ein zentrales Anliegen des Analysisunterrichts ist die Entwicklung des Ableitungsbegriffs. Dabei werden im Wesentlichen die Konzepte Grenzwert des Differenzenquotienten, stetige Fortsetzung der Differenzenquotientenfunktion und lineare Approximation unterschieden (vgl. Tietze, Klika \& Wolpers 1997, S. 191). Eng verbunden mit dem ersten Konzept ist die Vorstellung der Ableitung als lokale Änderungsrate und ihre geometrische Veranschauli-

Scheibe gelegt werden darf. Es ist die minimale Anzahl $M(n)$ der Umlegungen gesucht (vgl. Stowasser \& Mohry 1978, S. 11 ff.). 
chung als Tangentensteigung. Die Änderungsrate, insbesondere in Form der mittleren Änderungsrate, kann zur Beschreibung und Erklärung vieler Umweltsituationen herangezogen werden, in denen zwei Größen voneinander abhängen und man sich nicht für die Werte dieser Größen selbst, sondern deren Änderung interessiert, so etwa bei Geschwindigkeiten, Temperaturveränderungen, Preissteigerungsraten und Steigungen. Bei einer Orientierung an diesem Konzept kann an Vorerfahrungen der Schüler angeknüpft werden. Dabei ist jedoch zu berücksichtigen, dass auch eine angemessene Vorstellung zur absoluten Differenz bzw. zum Differenzenquotienten als absolute Änderung bzw. mittlere Änderungsrate entwickelt wird. Malle (1999a, S. 70 f.) nennt in diesem Zusammenhang sechs verschiedene Grundvorstellungen, die im Sinne des Grundvorstellungskonzepts v. Hofes (1996) als normative didaktische Kategorien für den Mathematikunterricht verstanden werden können:

1. Differenzenquotient als Verhältnis: Der Differenzenquotient ist gleich dem Verhältnis der Änderung der Funktionswerte zur Änderung der Argumente.

2. Differenzenquotient als mittlere Änderung der Funktionswerte pro Argumenteneinheit.

3. Differenzenquotient als Faktor: Der Betrag des Differenzenquotienten gibt an, wie viel mal stärker sich die Funktionswerte ändern als die Argumente.

4. Vorzeichen des Differenzenquotienten: Ist der Differenzenquotient der Funktion $f$ in $[\mathrm{a}, \mathrm{b}]$ positiv, so steigt $f$ insgesamt in $[a, b]$; ist er negativ so fällt $f$ insgesamt in diesem Intervall. Ist der Differenzenquotient der Funktion $f$ in $[a, b]$ gleich 0 , so ist $f$ insgesamt in $[a, b]$ weder steigend noch fallend.

5. Der Differenzenquotient einer linearen Funktion ist in jedem Intervall auch gleich der Steigung der linearen Funktion.

6. Differenzenquotient einer Funktion f als Steigung der zugehörigen Sekantenfunktion.

Bei der Entwicklung von Vorstellungen zur mittleren und lokalen Änderungsrate kommt der Analyse des Änderungsverhaltens von Funktionen mit Hilfe des Differenzenquotienten eine große Bedeutung zu. Dabei können lokale oder globale Betrachtungen im Vordergrund stehen.

\section{Lokale Funktionsuntersuchungen mit dem Differenzenquotienten}

Mit Hilfe des Differenzenquotienten sind lokale Funktionsuntersuchungen ${ }^{104}$ auf einer elementaren Ebene möglich. So kann man etwa das Änderungsverhalten einer Funktion an verschiedenen Stellen vergleichen, indem man die entsprechenden Differenzenquotienten bestimmt und die jeweiligen Werte interpretiert. Durch die Verwendung des Differenzenquotienten, d. h. durch das schrittweise Berechnen des Quotienten aus der Änderung der Funktionswerte und der Änderung der Argumente, können die zugrunde

\footnotetext{
${ }^{104}$ Es geht hierbei um Funktionsuntersuchungen in der „Umgebung“ ausgezeichneter Elemente des Definitionsbereichs.
} 
liegenden Fragestellungen zum Änderungsverhalten "transparenter" gemacht werden und möglicherweise einsichtiger beantwortet werden als in Verbindung mit einem (verfrühten) algorithmischen Berechnen der Ableitungsfunktion und dem Bestimmen der Ableitung an dem interessierenden Punkt. Darüber hinaus kann ein Beitrag zur Entwicklung der 1. und 4. Grundvorstellung zur mittleren Änderungsrate im Sinnne Malles geleistet werden. Es ist jedoch zu berücksichtigen, dass diese diskrete Analysemethode mit einem großen operativen Aufwand verbunden ist. Wird das lokale Änderungsverhalten einer Funktion an verschiedenen Stellen untersucht, sind zum Berechnen der entsprechenden Differenzenquotienten zwar nur elementare Rechenoperationen, dafür aber ein Vielfaches an Rechenschritten als bei Verwendung des Differenzialkalküls erforderlich. Weiterhin lassen sich mit Hilfe des Ableitungskalküls bei einer ,theoretischen“ Analyse einer Funktion, so etwa bei der Bestimmung von Extremstellen, Rechnungen „vereinfachen“ sowie "eleganter" und “übersichtlicher" dargestellen. (vgl. Blum 1991, S. 78, Fischer \& Malle 1985, S. 169, Schneider 2000, S. 19 ff.). Diskrete Methoden der Funktionsuntersuchung sollen daher hier und auch im Folgenden nicht als Alternative, sondern als Möglichkeit zur Vorbereitung und Ergänzung kontinuierlicher Konzepte betrachtet werden.

Wie empirische Untersuchungen zeigen, scheint jedoch das Arbeiten mit diskreten Begriffen trotz ihrer „Elementarität“ und „Transparenz“ für einen Schüler nicht unbedingt einfacher zu sein als das Arbeiten mit den entsprechenden kontinuierlichen Begriffen. So weisen die Ergebnisse von Springnagel (2000) darauf hin, dass Schüler beim Umgang mit dem Differenzenquotienten größere Schwierigkeiten haben als beim Umgang mit dem Differenzialquotienten und dass Vorstellungen zum Differenzenquotienten (aber auch zum Differenzialquotienten) nur unzureichend ausgeprägt sind. Da diese Untersuchung mit den traditionellen Mitteln „Bleistift und Papier“ durchgeführt wurde, ergibt sich die Frage, inwiefern neue Technologien, welche die aufwändigen Berechnungen übernehmen können, das Erlernen dieser Begriffe unterstützen können. Wie Aspetsberger (2000) und Schneider (2000), die sich vorrangig mit den didaktischen Möglichkeiten von Computeralgbrasystemen (CAS) beschäftigen, herausstellen, ergibt sich durch den Einsatz von Computern eine neue Chance zur „Elementarisierung“, d. h. sie machen die Verwendung elementarer (diskreter) Verfahren und Methoden - insbesondere in Verbindung mit lokalen Funktionsuntersuchungen - operativ handhabbar. Dabei kann zum einen der Zugang zum Differenzialquotienten als Grenzwert von Differenzenquotienten durch einen schrittweisen Übergang vom Differenzen- zum Differenzialquotienten anschaulicher gestaltet werden. Zum anderen erhält das Arbeiten mit dem Differenzenquotienten selbst eine eigenständige Bedeutung.

„Die Reduktion des operativen Aufwands durch CAS macht die Verwendung von Differenzenquotienten bei Problemlösungen einfacher und kann auch so - und nicht nur bei der Einführung des Differentialquotienten sinnvoll genutzt werden. Differenzenquotienten können als eine geeignete Modellierung von Änderungsraten 
erfahren werden, die durchaus angemessen sein kann, solange es vorrangig um Berechnungen und Deutungen einzelner Werte geht" (Schneider 2000, S. 202). ${ }^{105}$

Dass diskrete Begriffe auch bei der globalen Analyse des Änderungsverhaltens einer Funktion hilfreich sein können, soll im Folgenden aufgezeigt werden.

\section{Lokale und globale Funktionsuntersuchungen mit Differenzenfunktionen}

Das globale Änderungsverhalten von Folgen bzw. Funktionen kann mit Hilfe der Differenzenfolge bzw. der Änderungsratenfunktion „diskret“ analysiert werden. Wie bereits in den 70er Jahren betont wurde, lassen sich damit entsprechende inifinitesimale Begriffe und Methoden vorbereiten. So sieht Fischer in der Behandlung von Differenzenfolgen eine „Vorübung für die Ableitungsfunktion“ (Fischer 1976, S. 189), und Gordon (1979) betrachtet das Differenzen- und Summenkalkül als wichtiges Instrument bei dem von ihm beschriebenen „diskreten Zugang zur Analysis“ (vgl. Kap. 2.1.4.2). Durch die Möglichkeiten des Computers erhalten in jüngerer Zeit diskrete, globale Funktionsanalysen eine neue Bedeutung. So skizziert Henn (2000) einen Unterrichtsgang, in dem Änderungsraten bzw. die Kumulation von Änderungsraten als Zugang zu den Begriffen Ableitung und Integral verwendet werden. Dabei spielen graphische und numerische Lösungs- und Analysemethoden sowie der Einsatz von CAS von Beginn an eine wichtige Rolle. Indem Schüler die Beziehung zwischen der Änderungsratenfunktion (als Näherung für die Ableitungsfunktion) und der Ausgangsfunktion untersuchen, können sie nach Henns Erfahrungen die zentrale Aussage des Hauptsatzes der Differenzial- und Integralrechnung auf einer elementaren Ebene erschließen.

Im Rahmen dieser Arbeit soll der von Gordon (1979) vorgeschlagene „,diskrete Zugang zur Analysis“ mit Hilfe des Differenzen- und Summenkalküls und die von ihm betonten Analogien zwischen diskreten und kontinuierlichen Begriffen aufgegriffen und auf einer inhaltlichen Ebene weiterentwickelt werden. ${ }^{106}$ Hierzu werden so genannte Z-Funktionen und deren Differenzenfunktionen betrachtet. Mit Z-Funktionen bezeichnen wir polynomiale Funktionen, die auf den ganzen Zahlen $\mathbf{Z}$ definiert sind, so z. B. die Funktion $f$ mit $f(n)=n^{2}-2 n+3, n \in \boldsymbol{Z}$. Analog zum Begriff der Differenzenfolge bilden wir in diesem Zusammenhang den Begriff der Differenzenfunktion $D$ mit $D(n)=f(n+1)-f(n)$. Mit dem Konzept der Z-Funktionen ergibt sich die Möglichkeit, kalkülhafte und inhaltlichanschauliche Aspekte miteinander zu verknüpfen. So kann man Schülern einerseits die Aufgabe stellen, Differenzenfunktionen numerisch (und formal) zu berechnen. Andererseits sind Fragestellungen möglich, die stärker auf das Entwickeln von (Grund-)Vorstellungen abzielen:

\footnotetext{
105 Ähnliche Argumente können auch für ein TKP angeführt werden.

${ }^{106}$ Gordons Vorschläge sind auf das Arbeiten auf einer formalen Ebene ausgerichtet und damit zum Aufbau von inhaltlichen Vorstellungen nur bedingt geeignet.
} 
- Untersuchung lokaler Beziehungen

Bei lokalen Fragestellungen geht es darum, zu bestimmen, wie sich z. B. eine Funktion $f: n \mapsto f(n)$ in der „Umgebung“ eines diskreten $n$-Werts und insbesondere beim Übergang von einem diskreten $n$-Wert zum nächsten verhält:

- Wie ändern sich die Funktionswerte von $f$ mit $f(n)=n^{2}-4 n-13$ beim Übergang von $n=4$ nach $n=5$ ? Was bedeutet $D(-4)$ ?

- Geben Sie möglichst genau den Bereich an, in dem die Differenzenfunktion $D$ ihr Vorzeichen wechselt. Welche Bedeutung hat dies für die Funktion $f$ ?

Zur Beantwortung solcher Fragestellungen können die graphischen Darstellungen von $f$ und $D$ hinzugezogen werden (vgl. Abb. 15). Dabei müssen der Betrag und das Vorzeichen bestimmter Funktionswerte der Differenzenfunktion im Hinblick auf die Ausgangsfunktion interpretiert werden. Damit kann ein Beitrag zur Entwicklung angemessener Vorstellungen der Differenz $f(n+1)-f(n)$ als absolute Änderung bzw. - da den Z-Funktionen ein äquidistanter Definitionsbereich zugrunde liegt - als mittlere Änderungsrate im Intervall $[n, n+1]$ geleistet werden. ${ }^{107}$
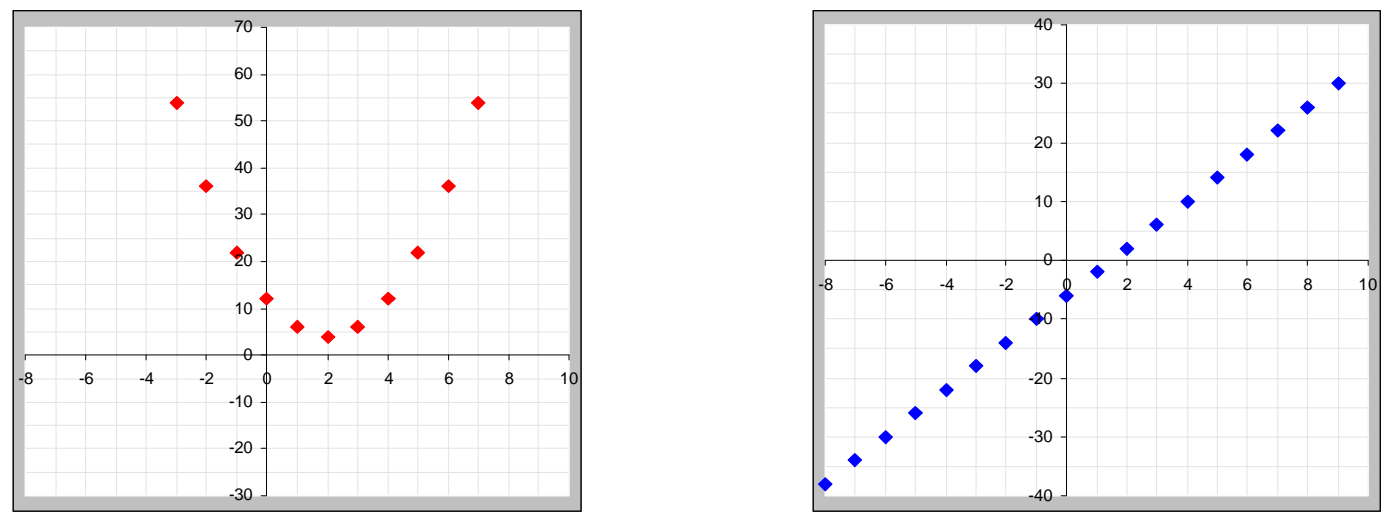

Abb. 15: Quadratische Z-Funktion und Differenzenfunktion

\section{- Graphisches Differenzenbilden}

Beim „graphischen Differenzenbilden“ als Analogie zum graphischen bzw. numerischen Differenzieren wird auf einer inhaltlich-anschaulichen Ebene von Eigenschaften der Z- Funktion $f$ auf Eigenschaften der Differenzenfunktion $D$ geschlossen. Man kann dabei zum einen qualitativ vorgehen, indem man zunächst die Abschnitte, in denen der Graph von $f$ fällt ( $\Rightarrow D$ negativ) oder steigt ( $\Rightarrow D$ positiv) sowie die zugehörigen Übergänge (d. h. die Extremwerte von f) markiert. Anschließend kann dann auf dem Gitter der ganzen Zahlen die entsprechende diskrete Differenzenfunktion ,skizziert“ werden. Eine andere Möglichkeit besteht darin, die

\footnotetext{
${ }^{107}$ Bei diesen Fragestellungen wird im Sinne der 4. Grundvorstellung von Malle (1999) insbesondere die Bedeutung des Vorzeichens der Differenzen bzw. der Funktionswerte der Differenzenfunktion betont.
} 
Funktionswerte $f(n)$ am Graph abzulesen, schrittweise die Differenzen $f(n+1)-f(n)$ zu berechnen und diese dann der Differenzenfunktion zuzuordnen.

\section{- Untersuchung von Funktionenklassen}

Neben dem „graphischen Differenzenbilden“ stellt die Analyse des Änderungsverhaltens von Funktionenklassen eine weitere Methode dar, globale Aspekte zu betonen. Dabei kann man nach dem Änderungsverhalten der Funktion als Ganzes fragen oder die Aufgabe stellen, invariante Eigenschaften einer Funktionenklasse zu analysieren. Verändert man etwa den Parameter $c$ in $f(n)=2 n^{2}-8 n+c$, so führt dies zu einer „Verschiebung“ der „Parabel“ in Richtung der positiven bzw. negativen $y$-Achse, wohingegen der Graph der Differenzenfunktion unverändert bleibt (vgl. Abb. 16 und 17). ${ }^{108}$
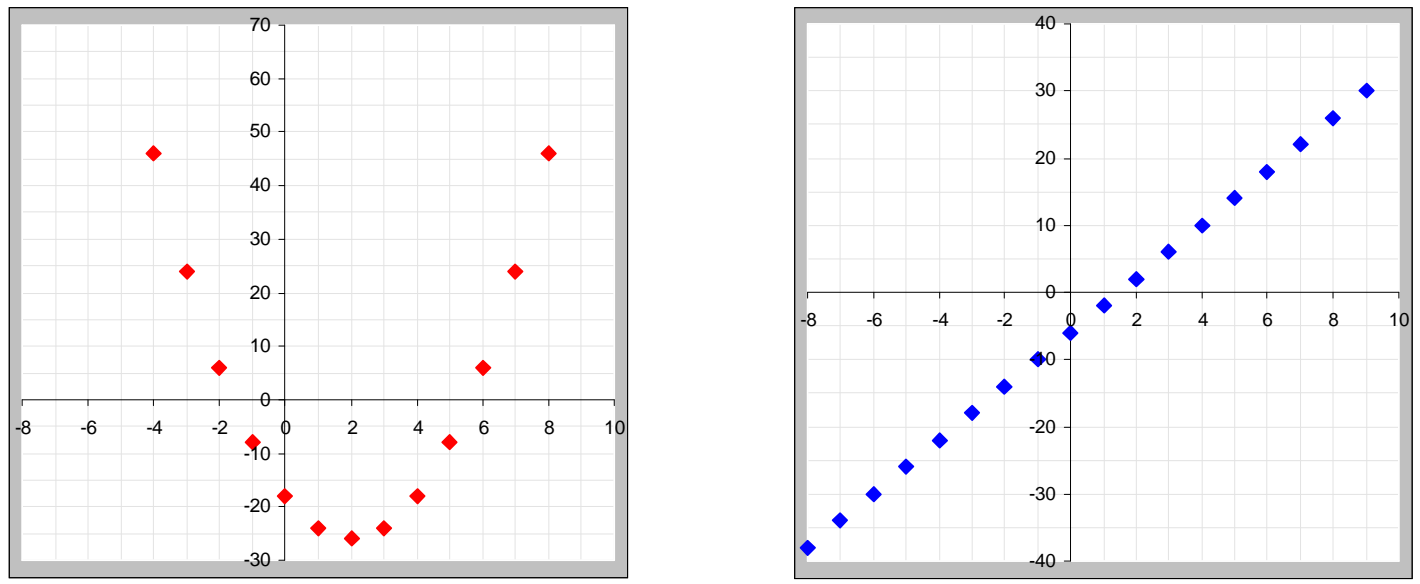

Abb. 16: Die Z-Funktion $f(n)=2 n^{2}-8 n-18$ und deren Differenzenfunktion
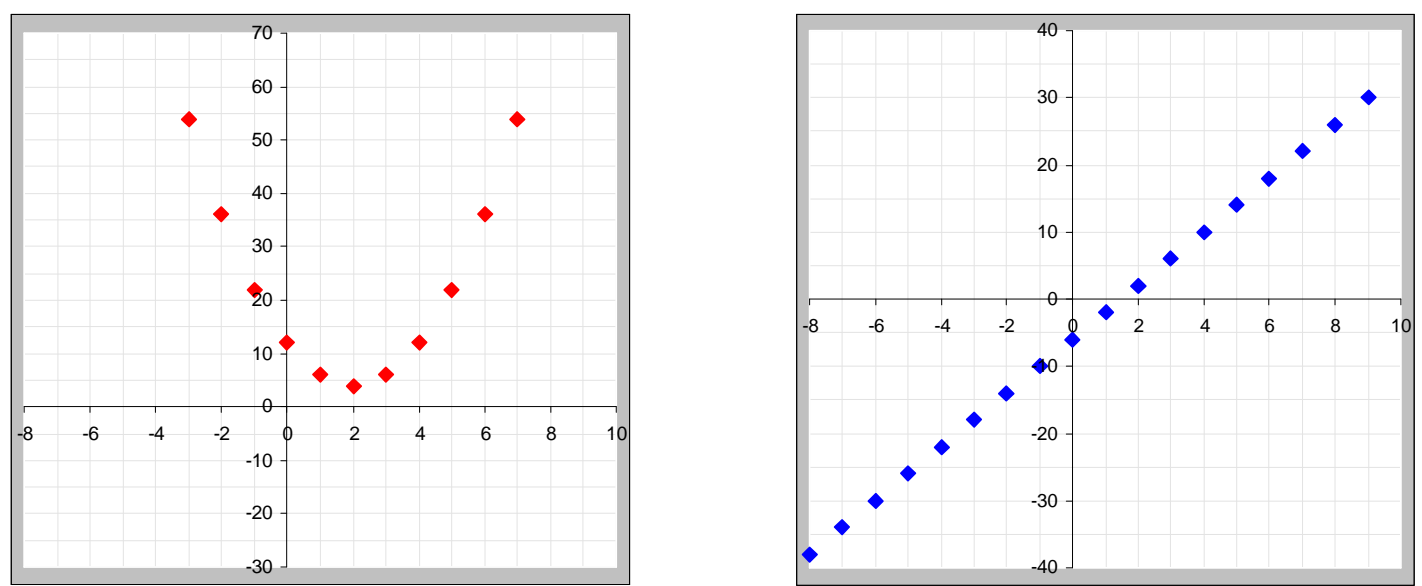

Abb. 17: Die Z-Funktion $f(n)=2 n^{2}-8 n+12$ und deren Differenzenfunktion

108 Das Verändern des Parameters $c$ sowie das Erstellen der entsprechenden Graphiken ist mit einem großen operativen Aufwand verbunden. Hier können Tabellenkalkulationsprogramme neue Möglichkeiten eröffnen (vgl. Kap. 4.2). 
Man kann diese Beobachtung grundsätzlich auf zwei verschiedene (elementare) Weisen begründen. Im Rahmen einer formalen Begründung wird die Differenz $D(n)=f(n+1)-f(n)$ algebraisch bestimmt. Man erkennt, dass die Differenzenfunktion vom Parameter $c$ unabhängig ist. Bei einer geometrischen Begründung wird hervorgehoben, dass ein Verändern des Parameters $c$ nur eine Verschiebung der Parabel als Ganzes in $y$-Richtung bewirkt, wobei die „Abstände“ bzw. Differenzen benachbarter Funktionswerte konstant bleiben. Damit ist aber die Differenzenfunktion (als Funktion der Differenzen) konstant.

Diese rein diskrete Sicht auf die Funktionen erweist sich beim geometrischen Begründen des Einflusses des Parameters $b$ auf die Differenzenfunktion der Z-Funktion $f$ mit $f(n)=a n^{2}+b n+c$ (mit $a$ und $c$ fest, aber beliebig) jedoch als hinderlich. Nicht die diskrete Z-Funktion, sondern deren kontinuierliches Pendant wird durch das Verändern des Parameters $b$ im Koordinatensystem im geometrischen Sinne „verschoben”. Betrachtet man beispielsweise die Z-Funktion $f$ mit $f(n)=n^{2}$ (also $a=1$ und $b=c=0$ ), so gilt $f(0)=0$. Wir bezeichnen diesen Punkt mit $P$, also $P(0 ; 0)$. Verändert man $b$ und setzt etwa $b=2,6$, so erhält man den gegenüber der Normalparabel im Koordinatensystem um 1,3 Einheiten nach links und 1,69 Einheiten nach unten verschobenen Graph der Funktion $g$ mit $g(n)=n^{2}+2,6 n=(n+1,3)^{2}-1,69$ (vgl. Abb. 18). Im Rahmen dieser "Verschiebung" wird der Punkt $P$ auf den Punkt $Q(-1,3 ;-1,69)$ abgebildet. Dieser Punkt ist aber kein Element des Graphen von $g$.
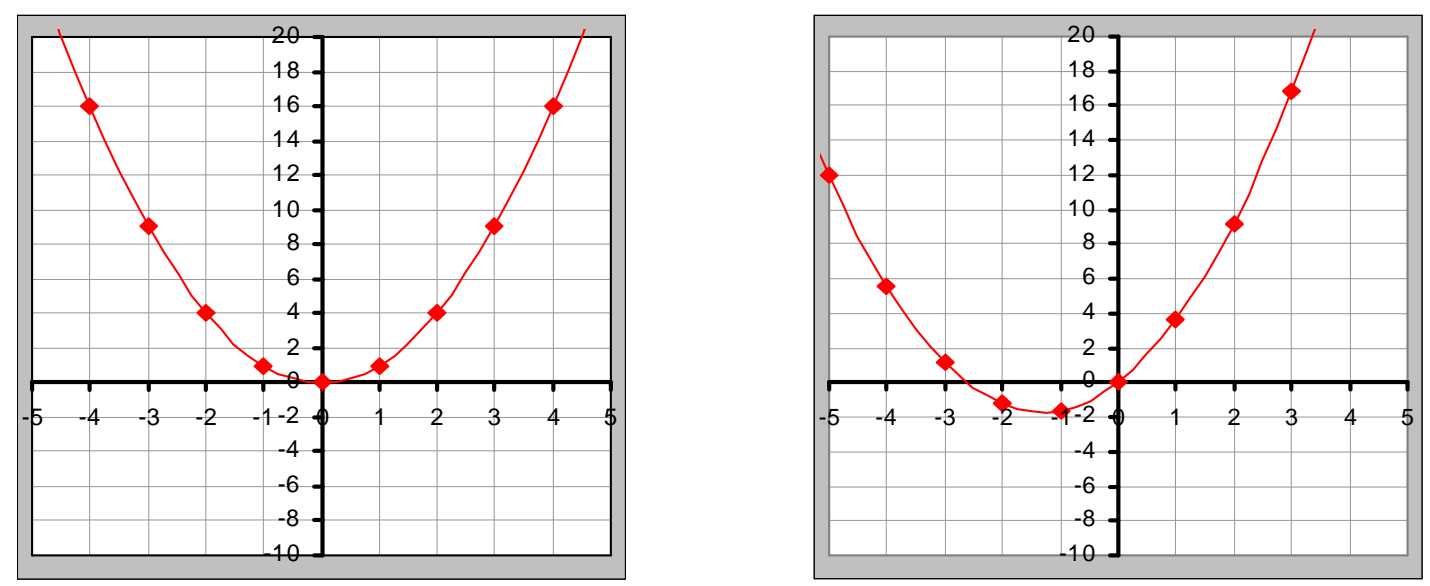

Abb. 18: Variation des Parameters $b$ in $f(n)=n^{2}+b n .^{109}$

Damit ist auch das Verhalten der Differenzenfunktion nicht quantitativ über „,konstant bleibende Abstände, die nur verschoben werden" erklärbar, sondern nur qualitativ über das Argument des ,gleichen Änderungsverhaltens”. Hier zeigen sich Grenzen des Arbeitens mit diskreten Funktionen und die Bedeutung einer kontinuierlichen Sichtweise.

\footnotetext{
109 Zur besseren Verdeutlichung der „Verschiebung“ wurden die diskreten Funktionswerte bei der graphischen Darstellung interpoliert.
} 


\subsection{Anwendungen diskreter Mathematik}

In den letzten 30 Jahren lässt sich in der mathematikdidaktischen Diskussion als Gegentendenz zu den Bestrebungen um die „Neue Mathematik“ ein Trend zu einer stärkeren Betonung von Anwendungen im Mathematikunterricht beobachten (vgl. Blum 1996). Dabei besteht zunehmend Konsens darüber, sich im Mathematikunterricht nicht nur auf das Vorführen von Standardanwendungen zu beschränken, sondern die Schüler zu einem bewussten Erarbeiten von und Arbeiten mit Modellen anzuleiten, d. h. dem Modellbildungsprozess (vgl. Abb. 19) ein größeres Gewicht beizumessen.

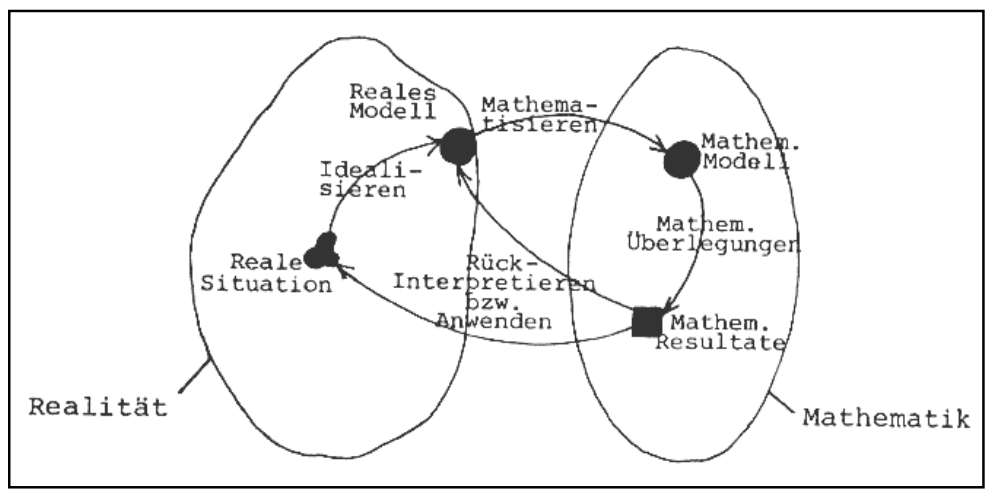

\section{Abb. 19: Der Modellbildungsprozess} (aus Blum \& Törner 1983. S. 248)

Ausgangspunkt eines solchen Modellbildungsprozesses ${ }^{110}$ ist eine problemhaltige Umweltsituation. Im Rahmen einer „Komplexitätsreduktion“ werden die vorhandenen Informationen strukturiert, vereinfacht bzw. auf einige wesentliche Merkmale eingeschränkt, wobei ein ,reales Modell“" der Ausgangssituation entsteht. In einem zweiten Schritt wird das noch umgangssprachlich formulierte reale Modell mathematisiert, d. h. unter Ausnutzung der mathematischen Fachsprache in ein formales mathematisches Modell übersetzt. Die durch mathematische Überlegungen gewonnenen Resultate müssen in einem weiteren Schritt wieder in die reale Ausgangssituation zurückübersetzt und interpretiert werden. Dabei ist zu prüfen, inwiefern die eingangs gestellten Fragen auf der Basis des verwendeten Modells hinreichend gut beantwortet werden. Häufig kommt es zu Diskrepanzen, so dass das Modell im Nachhinein modifiziert oder sogar durch ein neues Modell ersetzt werden muss. ${ }^{111}$ Dies führt zu einem wiederholten Durchlaufen des Modellbildungsprozesses.

Mit der stärkeren Betonung des Modellbildungsprozesses lässt sich nach Blum in der mathematikdidaktischen Diskussion auch eine breitere Sicht auf schulrelevante Anwendungsgebiete

\footnotetext{
${ }^{110}$ Zur Modellauffassung für mathematische Anwendungen vgl. etwa Fischer \& Malle 1985, Blum 1985.

${ }^{111}$ So kann zum einen die Ausgangssituation unzulässig vereinfacht worden sein. Zum anderen ist zu berücksichtigen, dass die meisten Situationen durch verschiedene Modelle beschrieben werden können, wobei nicht
} 
feststellen. So sei zu beobachten, dass neben den „klassischen“ Gebieten wie der Physik, die häufig mit den Mitteln der Algebra und Analysis behandelt werden können, zunehmend auch andere Anwendungsfelder berücksichtigt werden, wie etwa Ökologie, Sport, Kunst, Sozialwissenschaften. Dies gehe einher mit einer „Verbreiterung der , angewandten“ mathematischen Themengebiete“, die auch neuere Disziplinen einschließen wie Stochastik und Diskrete Mathematik (vgl. Blum 1996, S. 20). In der Tat gibt es zur „diskreten“ Behandlung dynamischer Systeme in der Ökologie oder in den Sozialwissenschaften mittlerweile eine umfangreiche Literatur und entsprechende unterrichtspraktische Vorschläge. ${ }^{112}$ Zur Mathematisierung werden hier Differenzengleichungen - das diskrete Analogon zu Differenzialgleichungen eingesetzt. Unter Anwendungsaspekten wurde auch für die Berücksichtigung anderer diskreter Themengebiete wie Graphentheorie und Kryptographie - wie der Rückblick in Kapitel 2.1 zeigte - immer wieder plädiert. In jüngerer Zeit findet man jedoch hierzu nur wenige Vorschläge und Anregungen. ${ }^{113}$ Dies ist wenig verständlich, denn mit der Integration von Anwendungsgebieten der diskreten Mathematik kann man in besonderem Maße den Zielsetzungen eines anwendungsorientierten Mathematikunterrichts gerecht werden, wie im Folgenden anhand von Beispielen noch weiter ausgeführt wird. Bei der Analyse der Beispiele beziehen wir uns auf die von Blum (1996, S. 20 ff.) genannten Hauptaspekte von Anwendungsbezügen im Mathematikunterricht:

- Pragmatische Aspekte: Schülern soll beim Verstehen und Bewältigen von Umweltsituationen geholfen werden.

- Formale Aspekte: Schüler sollen im Mathematikunterricht allgemeine Qualifikationen wie z. B. Problemlösen, Argumentieren sowie Modellbildungsqualifikationen erwerben.

- Kulturbezogene Aspekte: Der Mathematikunterricht soll Schülern ein möglichst ausgewogenes Bild von Mathematik als kulturelles und gesellschaftliches Gesamtphänomen vermitteln. Dazu gehören sowohl das Erkennen ,,verborgener Mathematik“ in der technologisierten Gesellschaft als auch das Erfahren von Grenzen und Gefahren beim Gebrauch bzw. Missbrauch von Mathematik.

- Lernpsychologische Aspekte: Anwendungsbezüge können dazu beitragen, dass mathematische Begriffe und Verfahren besser verstanden werden. Insbesondere können mathematische Inhalte anwendungsorientiert geübt werden.

\footnotetext{
immer sofort ersichtlich ist, welche Modellierung die relevanten Merkmale besser erfasst und damit zu zweckmäßigeren Aussagen führt.

${ }^{112}$ Vgl. etwa Dürr \& Ziegenbalg 1989, Ossimitz 1990.

113 Anders ist es beispielsweise in England. Hier misst man Anwendungen im Mathematikunterricht eine sehr hohe Bedeutung bei. Insbesondere wurde in den 80er Jahren von der Spode-Gruppe umfangreiches Unterrichtsmaterial unter besonderer Berücksichtigung diskreter Themen wie Graphentheorie, Codierungstheorie, Differenzengleichungen, Lineare Optimierung etc. entwickelt. Mittlerweile ist Diskrete Mathematik als Teil des Mathematikkurses „Angewandte Mathematik“ in den Lehrplan der Oberstufe integriert (vgl. hierzu Burghes 1995 sowie das ML-Themenheft „Anregungen aus England“, hier insbesondere die Beiträge von Burghes 1997, Green 1997, Kaiser 1997).
} 


\subsubsection{Diskrete Wachstumsmodelle}

Der Modellierung von Wachstumsprozessen wird im Mathematikunterricht eine große Bedeutung beigemessen. Bei der Behandlung populationsdynamischer Fragestellungen aus den Bereichen Natur, Gesellschaft oder Wirtschaft kann in der Regel an alltägliche Erfahrungen der Schüler angeknüpft werden. Zur Beschreibung vieler komplexer Systeme dienen die Standardbausteine des linearen, exponentiellen, begrenzten und logistischen Wachstums. Welcher dieser Bausteine zur Modellierung am besten geeignet ist, hängt von der entsprechenden Umweltsituation bzw. dem real beobachtbaren Wachstumsprozess ab und muss gegebenenfalls im Rahmen des Modellbildungsprozesses (mehrfach) kritisch überprüft und abgeändert werden (formaler Aspekt).

Die meisten Wachstumsprozesse lassen sich sowohl diskret als auch kontinuierlich modellieren. So kann etwa ein Abkühlvorgang unter der Annahme exponentiellen Wachstums als Differenzengleichung $N_{n+1}=N_{n}+r \cdot N_{n}$ bzw. als Folge $N_{n}=N_{0} \cdot(1+r)^{n}$ oder aber als Differenzialgleichung $N^{\prime}(t)=r \cdot N(t)$ bzw. als reelle Funktion $N(t)=N_{0} \cdot e^{r t}$ mathematisiert werden. Entsprechende Alternativen gibt es auch bei der Annahme linearen, begrenzten oder logistischen Wachstums und bei komplexeren Systemen. ${ }^{114}$ Der Vorteil eines kontinuierlichen Modells liegt darin begründet, dass Funktionsuntersuchungen, wie das Bestimmen von Extrempunkten, das Untersuchen auf Monotonie oder das Bestimmen einer expliziten Darstellung, auf der formalen Ebene „einfacher“ anzustellen sind als bei einem entsprechenden diskreten Modell. Die Komplexität verringert sich mit der Reduktion der Variablen (vgl. Fischer \& Malle 1985, S.169). Zu berücksichtigen ist jedoch, dass das erforderliche analytische Werkzeug (Grenzwertbetrachtungen, Differenziations- und Integrationsmethoden) in der Schule frühestens in der Sekundarstufe II zur Verfügung steht und es hierzu in der Regel umfangreicher theoretischer Überlegungen bedarf. Der Zugang zu Wachstumsprozessen mit Differenzengleichungen ist dagegen schon früh möglich, da zum Aufstellen und Untersuchen des Modells nur elementare Rechenoperationen notwendig sind. ${ }^{115}$ Der Themenkreis „Modellbildung mit Differenzengleichungen“ kann dann im Rahmen eines spiralig aufgebauten Lehrgangs in der Sekundarstufe II wieder aufgegriffen und vertieft werden. So kann ausgehend von einer Klasse formal ,ähnlicher“ Modelle die Frage nach der Lösungsgesamtheit von Differenzengleichungen eines bestimmten Typs aufgeworfen werden, oder es lassen sich Bedingungen für stabiles oder chaotisches Verhalten analytisch untersuchen. Begriffe und Techniken der Linearen Algebra und der Analysis werden in diesem Zusammenhang anwendungs-

\footnotetext{
${ }^{114}$ Einen Überblick über die Möglichkeiten der diskreten Behandlung dynamischer Systeme mit Hilfe von Differenzengleichungen bieten etwa Keidel \& Lorbeer 1988, Dürr \& Ziegenbalg 1989, Körner 2000. Zur Behandlung elementarer Wachstumsprozesse mit Differenzialgleichungen vgl. z. B. Racke \& Stein 1995, Winter 1994.

115 Diese elementaren aber in der Regel aufwändigen Rechenoperationen können von einem Computer übernommen werden (vgl. auch Kap. 4.2.3).
} 
orientiert geübt und vertieft (lernpsychologischer Aspekt). Im Rahmen des Analysisunterrichts können weiterhin auch Differenzialgleichungen als alternative Möglichkeit zur Mathematisierung von Wachstumsprozessen thematisiert werden. Dadurch lassen sich Beziehungen zwischen Algebra und Analysis aufzeigen (vgl. Waits \& Demana 1997).

Durch eine Behandlung diskreter Wachstumsmodelle und den Vergleich mit den entsprechenden kontinuierlichen Modellen lassen sich darüber hinaus - wie bereits bei der Entscheidung für ein lineares, exponentielles oder logistisches Modell $-{ }^{116}$ grundsätzliche Fragen der Modellbildung aufwerfen (formaler Aspekt): Welches Modell gibt die Realität besser wieder? Welches Modell besitzt die größere Aussagekraft? Von besonderer Bedeutung sind diese Fragen, wenn Modellierungen durch Differenzen- oder Differenzialgleichungen zu qualitativ unterschiedlichen Ergebnissen führen, wie z. B. beim logistischen Wachstum. Bei einer stetigen Modellierung besitzt die graphische Darstellung der Lösung der logistischen Differenzialgleichung stets einen s-förmigen Verlauf. Wird dagegen diskret modelliert, erhält man neben der typischen s-förmigen Annäherung auch Fixpunkte mit alternierender Annäherung, periodische Zyklen oder einen chaotischen Verlauf (vgl. Tietze, Klika \& Wolpers 1997, S. 315 ff.).

\section{Beispiel: Räuber-Beute-System}

Eine Gegenüberstellung von kontinuierlichem und diskretem Modell unter Berücksichtigung formaler Aspekte bietet sich insbesondere im Fall des so genannten Räuber-Beute-Systems an. Es handelt sich hierbei um ein System, bei dem zwei Populationen in Wechselwirkung zueinander stehen. Dabei begünstigt die Population der Beute das Wachstum der Räuber, wohingegen die Räuber das Wachstum der Beute behindern. Geht man von einem exponentiellen Wachstum der Beutepopulation $B$ in Abwesenheit der Räuber $R$ sowie von einer exponentiellen Abnahme einer isolierten Räuberpopulation aus, und nimmt man an, dass die Reduzierung der Beutetiere und der Zuwachs der Räuberpopulation jeweils proportional zum Produkt $B \cdot R$ (der Anzahl der möglichen „Begegnungen“) sind, erhält man ein System gekoppelter Differenzengleichungen:

$$
\begin{aligned}
& B_{k+1}=B_{k}+a B_{k}-b B_{k} \cdot R_{k} \\
& R_{k+1}=R_{k}-c R_{k}+d B_{k} \cdot R_{k},
\end{aligned}
$$

bzw. ein System gekoppelter Differenzialgleichungen:

$$
\begin{aligned}
& B^{\prime}(t)=a B(t)-b B(t) \cdot R(t) \\
& R^{\prime}(t)=-c R(t)+d B(t) \cdot R(t) .
\end{aligned}
$$

Die Bestandsentwicklung in Abhängigkeit von der Zeit liefert in beiden Fällen gegeneinander phasenverschobene, periodisch schwankende Lösungskurven. Während jedoch das System

\footnotetext{
${ }^{116}$ Diese Wahl, die zunächst unabhängig von dem favorisierten mathematischen Mittel ist, wird unter Berücksichtigung des allgemeinen Wachstumsverhaltens getroffen.
} 
der Differenzialgleichungen stabil ist, schaukeln sich die Schwankungen der Lösungskurven des Differenzengleichungssystems immer weiter auf und führen letztendlich zum Aussterben einer bzw. beider Populationen. Um den Zusammenbruch des diskreten Systems zu vermeiden, müsste man die Zeitintervalle kürzer wählen sowie die Parameter entsprechend abändern. ${ }^{117}$ Welches der angesprochenen Modelle den Wachstumsprozess am besten beschreibt, ist vor der Modellierung oder auch im Rahmen der Validierungsphase durch eine genauere Analyse der außermathematischen Situation zu klären. Vermehrt sich eine Population sehr häufig bzw. innerhalb sehr kurzer Zeitabstände, so dass von einer stetigen Entwicklung ausgegangen werden kann, ist eine kontinuierliche Modellierung naheliegend. Betrachtet man andererseits Populationen, bei denen sich nur in festen, vergleichsweise „großen“ Zeitabständen Nachwuchs einstellt, ist möglicherweise ein diskretes Modell angemessener, das ein unter Umständen auch chaotisches Verhalten des Systems besser beschreiben kann. ${ }^{118}$

\subsubsection{Kryptographische Anwendungen}

In der heutigen Kommunikationsgesellschaft sind kryptographische Verfahren von großer Bedeutung, so etwa bei der Verschlüsselung von Telefongesprächen per Mobiltelefon oder von elektronischer Post (Emails), beim Pay-TV, bei elektronischen Geldüberweisungen oder bei der Überprüfung der Unversehrtheit von Nachrichten (Nachrichtenintegrität) z. B. mit Hilfe von Prüfziffern. Unter kulturbezogenen Gesichtspunkten kann man - indem man kryptographische Themen zum Gegenstand des Mathematikunterrichts macht - an alltäglichen Erfahrungen der Schüler anknüpfen und den für sie zunächst „,verborgenen“ mathematischen Gehalt sichtbar machen. Durch das Aufdecken dieser Mathematik lernen die Schüler einen Teil ihrer Erfahrungswelt besser verstehen, und es werden die prinzipiellen Möglichkeiten, Grenzen und Gefahren ${ }^{119}$ kryptographischer Anwendungen deutlich. Weiterhin ist es im Rahmen solcher Themen möglich, Schülern das Modellieren als wichtige mathematische Tätigkeit bewusst zu machen. So stellen kryptographische Verfahren im Gegensatz zu Modellen, die zur Beschreibung von Wachstumsprozessen genutzt werden, normative Modelle dar. „Diese Modelle setzen Normen, schaffen dadurch eine Situation, determinieren eine diffuse Situation oder legen eine variable Situation fest" (Fischer \& Malle 1985, S. 104). Häufig sind normative Modelle von begrenzter Gültigkeitsdauer, abhängig von der aktuellen gesellschaftlichen Situation und - wie im Fall der Kryptographie - auch von der technologischen Entwicklung. So steigt mit wachsender Leistungsfähigkeit der Rechner die Wahrscheinlichkeit,

\footnotetext{
${ }^{117}$ Eine andere Möglichkeit wäre es, von einem logistischen Beutewachstum auszugehen. Hier unterscheiden sich diskrete und stetige Beschreibungen qualitativ nicht.

118 Tietze, Klika \& Wolpers (1997, S. 315 f.) führen in diesem Zusammenhang als Beispiel eine Bakterien- und eine Schmetterlingspopulation an. Die Bakterienpopulation vermehrt sich häufig und zeitlich unabhängig, wohingegen die Schmetterlingspopulation eine sprunghafte Entwicklung zeigt.

${ }^{119}$ So etwa können besonders sichere Verschlüsselungsverfahren - sofern sie in die falschen Hände gelangen auch eine Bedrohung für die Gesellschaft darstellen.
} 
dass bestehende Systeme gebrochen werden; dies erfordert wiederum die Entwicklung komplexerer mathematischer Algorithmen bzw. kryptographischer Verfahren.

Neben kulturbezogenen Gesichtspunkten können durch die Behandlung kryptographischer Anwendungen auch andere Aspekte eines anwendungsorientierten Mathematikunterrichts hervorgehoben werden, wie die folgenden beiden Beispiele verdeutlichen.

\section{Beispiel: Elementare Verschlüsselungsverfahren}

Verschiedene unterrichtspraktische Vorschläge ${ }^{120}$ haben elementare Verschlüsselungsverfahren zum Gegenstand. Wird wie in der Unterrichtsreihe von Failing (1998) die geschichtliche Entwicklung der klassischen Kryptographie im Unterricht nachvollzogen, wird der Modellbildungsprozess mehrfach durchlaufen (formaler Aspekt): Beginnend beim monoalphabetischen System schlüpft der Schüler beim Validieren des Modells in die Rolle des Kryptoanalytikers, indem er die Schwachstellen eines Verfahrens untersucht bzw. versucht, ein System ohne Kenntnis des entsprechenden Schlüssels zu brechen. Dadurch wird das polyalphabetische Vigenère-System motiviert. Auch dieses System besitzt Sicherheitsmängel, die der Schüler in einer erneuten Validierungsphase aufdecken kann. Der prinzipielle Nachteil des Schlüsselaustauschs bei symmetrischen Systemen kann dann die Frage nach einem unbrechbaren System aufwerfen. Hier kann mit der Idee der asymmetrischen Systeme angeknüpft werden. Unter lernpsychologischen Aspekten können bekannte mathematische Inhalte wie Prozentrechnung, Statistik, Zahldarstellungen, Teilbarkeit, Primzahlen oder Zuordnungen anwendungsorientiert geübt bzw. neue Inhalte aus dem Bereich der modularen Arithmetik und der Zahlentheorie direkt in einen Anwendungskontext gestellt werden. Darüber hinaus werden Schüler durch die Behandlung der Kryptographie im Allgemeinen und elementarer Verschlüsselungsverfahren im Besonderen zu einem bewussteren Umgang mit vertraulichen Informationen, etwa beim Versenden von Emails, angeleitet (pragmatischer Aspekt).

\section{Beispiel: EAN- und ISBN-Code}

Die meisten der in Europa auf dem Markt befindlichen käuflichen Produkte besitzen eine 13stellige normierte Artikelnummer, die so genannte Europäische Artikel-Nummer (EAN). Sie dient zur eindeutigen Beschreibung des jeweiligen Produkts und macht die Überwachung eines Warenbestands oder das Bestellen neuer Ware (elektronisch) möglich. Beim Eingeben von Artikelnummern über eine Computertastatur kann es jedoch zu Fehlern kommen, die mit Hilfe einer adäquaten Prüfziffer erkannt werden können. Herget (1994) beschreibt in diesem Zusammenhang einen Unterrichtsgang in einer 7. Klasse, bei dem zunächst die Artikelnummern auf Lebensmittelpackungen analysiert, die Idee der Prüfziffer erarbeitet und am speziellen EAN-Code verdeutlicht wird. Daran anschließend wird das dem Code zugrunde liegende mathematische Modell analysiert, und es werden andere mögliche Modelle hinsichtlich ihrer

\footnotetext{
${ }^{120}$ Vgl. etwa die Unterrichtsreihen von Biernoth 1992, Waimer 1997, Failing 1998.
} 
Stärken und Schwächen bei der Beschreibung von Produkten verglichen. So handelt es sich beim EAN-Code um einen so genannten Zehnercode mit den Gewichten 1 und 3, ${ }^{121}$ der alle Einzelfehler, jedoch nicht alle Vertauschungsfehler (,Zahlendreher“) erkennt. Ein Übergang zu einer (einfacheren) Prüfziffer, die mit Hilfe der Quersumme der einzelnen Ziffer bestimmt wird, würde insofern eine Verschlechterung des Modells bedeuten, als kein einziger Vertauschungsfehler erkannt würde. Dagegen liefert ein Zehnercode mit den Gewichten 1 und 2 keine Verbesserung: Er erkennt alle Vertauschungsfehler aber nicht alle Einzelfehler, die statistisch jedoch am häufigsten vorkommen. Indem Schüler nun selbst solche oder auch andere alternative Modelle vorschlagen und analysieren, wird der Modellbildungsprozess (mehrfach) aktiv durchlaufen (formaler Aspekt) und viele traditionelle Lerninhalte wie Stellenwertsysteme, Primzahlen, Teilerfremdheit, Prozentrechnung und Statistik anwendungsorientiert geübt (lernspychologischer Aspekt). Dass eine Entscheidung für ein bestimmtes Prüfsystem nicht allein aufgrund mathematischer Gütekriterien getroffen wird, sondern auch wirtschaftliche und gesellschaftliche Einflüsse eine Rolle spielen (kulturbezogener Aspekt), zeigt eine Analyse des zu jeder EAN gehörenden Strichcodes. Strichcodes werden aufgedruckt, damit die EAN von einem speziellen Lesegerät einer Computerkasse eingelesen werden kann. Die Strich-Codierung erfolgt im Wesentlichen ziffernweise, wobei jedoch nach einem bestimmten Muster in Abhängigkeit von der ersten Ziffer zwischen drei Codes gewechselt wird. Diese umständliche Codierung ist erforderlich, damit europäische Strichcode-Scanner auch die in den USA verwendeten Strichcodes verarbeiten können. Die Frage nach einer besseren oder bestmöglichen Prüfziffer, kann die Behandlung der 10-stelligen Internationalen StandardBuchnummer (ISBN) motivieren. Der entsprechende ISBN-Prüfcode erkennt im Vergleich zum EAN-Code alle Vertauschungs- und Einzelfehler; er erfordert jedoch eine Erweiterung der Prüfziffern um das Zeichen $\mathrm{X}$ und somit das Verlassen des Nummernbereichs auf der Tastatur eines Eingabegeräts. Dies kann möglicherweise eine schnelle (Erst-)Erfassung einer ganzen „Produktpalette“ behindern. Damit werden Grenzen der Mathematisierbarkeit deutlich (kulturbezogener Aspekt).

\subsection{Zusammenfassung}

Wie in diesem Kapitel anhand zahlreicher Beispiele herausgestellt wurde, können diskrete Inhalte zu wichtigen Zielen des Mathematikunterrichts einen Beitrag leisten. So lassen sich durch diskrete Problemstellungen verschiedene heuristische Strategien fördern, aber auch vielfältige Inhalte problemorientiert erarbeiten, wiederholen und integriert üben (Problemlöseaspekt). Weiterhin ermöglichen diskrete Methoden und Begriffe einen elementaren Zugang zu zentralen Begriffen des Mathematikunterrichts, so etwa zum Variablen-, Term- und Funk-

\footnotetext{
${ }^{121}$ Dabei werden die ersten 12 Ziffern der EAN abwechselnd mit 1 und 3 multipliziert und die Produkte addiert. Die Prüfziffer erhält man, indem man diese Summe zur nächsten Zehnerzahl ergänzt.
} 
tionsbegriff sowie - insbesondere in Verbindung mit dem Konzept der Differenzenfunktionen - zum Ableitungsbegriff (Begriffsbildungsaspekt). Darüber hinaus wird der Anwendungsaspekt betont, indem nicht nur die zahlreichen Anwendungsmöglichkeiten diskreter Mathematik aufgezeigt, sondern Schüler auch selbst zum Modellbilden angeleitet werden (Anwendungsaspekt).

Zur Behandlung diskreter Themen sind - wie auch in der didaktischen Diskussion immer wieder herausgestellt wurde - nur wenige Vorkenntnisse erforderlich, häufig ist ein Zugang auf einer konkret handelnden bzw. inhaltlich-anschaulichen Ebene möglich. Welche Bedeutung dieser Aspekt vor dem Hintergrund lernpsychologischer Untersuchungen und Theorien besitzt, ist Gegenstand des nächsten Kapitels. 


\section{Zur Bedeutung von Handlungen im Lernprozess}

Schon vielfach wurde in der didaktischen und psychologischen Literatur auf die Bedeutung von Handlungen im Lernprozess hingewiesen. Im Folgenden werden zunächst einige Theorien zur Denkentwicklung vorgestellt, die bei Handlungen ansetzen. Dabei sollen sowohl der Begriff des Handelns und Operierens als auch der Begriff der „Handlungselemente“ (Darstellungen, Prototypen) näher beleuchtet werden. Aus diesen theoretischen Ansätzen lassen sich didaktische Prinzipien und Hinweise zur Gestaltung von Lernprozessen ableiten, die unter dem Begriff „operative Prinzipien“ zusammengefasst werden können. Einige spezielle „Anwendungen“ bzw. Aspekte dieser operativen Prinzipien lassen sich in besonderer Weise im Rahmen der Behandlung diskreter Inhalte verwirklichen. Dies soll im zweiten Abschnitt anhand von Beispielen illustriert werden. Um Aussagen über geeignete Darstellungen und Lernumgebungen machen zu können, ist es notwendig, die tatsächlich beobachtbaren Handlungen der Schüler in Verbindung mit deren Begriffsverständnis und -vorstellungen zu untersuchen. Der Begriff der „tatsächlich beobachtbaren Handlungen“ führt zum Begriff der (diskreten) „Arbeitsweisen“, der abschließend erläutert wird und in Beziehung zu Verständnismodellen gesetzt wird.

\subsection{Entwicklung mathematischen Denkens aus Handlungen}

Behavioristische Lerntheorien, wie etwa die Theorien von B. F. Skinner oder R. M. Gagné , betrachten Lernen als ein Phänomen, das durch Reiz-Reaktions-Modelle erklärt werden kann. Diese Ansätze beschränken sich streng auf das Beschreiben beobachtbaren Verhaltens von Lebewesen und lehnen die Annahme innerer Prozesse als unzulässig ab. ${ }^{122}$ Im Gegensatz dazu stehen Lerntheorien, wie etwa die genetische Erkenntnistheorie von J. Piaget oder die Theorie von J. S. Bruner, die Denk- und Aneignungsprozessen eine große Beachtung schenken, wobei insbesondere Handlungen eine wichtige Rolle spielen. Diese Theorien sowie daran anschließende Arbeiten stellen eine psychologische Grundlage des heutigen Mathematikunterrichts dar. Auf einige der wesentlichen Inhalte wird im Folgenden eingegangen.

\subsubsection{Genetische Erkenntnistheorie von Jean Piaget}

Nach der genetischen Erkenntnistheorie des Entwicklungspsychologen Jean Piaget entwickelt sich die Intelligenz etappenweise in Wechselwirkung zwischen Mensch und Umwelt. ${ }^{123} \mathrm{Im}$ Anfangsstadium stellen Handlungen grundlegende Mittel der Wechselwirkung dar. Diese

\footnotetext{
122 Einen Überblick über verschiedene behavioristische Theorien des Lernens, insbesondere über Skinners „operante“ Konditionierung, geben Hilgard \& Bower 1970.

${ }^{123}$ Eine Einführung in die genetische Erkenntnistheorie von Piaget findet sich in Wittmann 1981, S. 59 ff.
} 
Handlungen werden in späteren Entwicklungsstadien weiterentwickelt und zu Operationen verinnerlicht. Dabei bildet sich Denken in Form von immer besser organisierten und flexibleren Strukturen oder „kognitiven Schemata“ aus, die die Aktivitäten des Einzelnen steuern. Hinsichtlich der Ausbildung dieser kognitiven Schemata und deren Organisationsgrad unterscheidet Piaget im Wesentlichen vier Stadien:

Im sensomotorischen Stadium (ca. 0-2 Jahre) erwirbt das Kind zahlreiche einfache Handlungsschemata und Vorstellungen, die jedoch stark an die Wahrnehmung gebunden sind. Es ist noch nicht fähig, Sprache, Bilder und Symbole zu gebrauchen. Dies erfolgt während des präoperativen Stadiums (ca. 2-7 Jahre), in dem das Kind zunehmend auch lernt, Handlungen gedanklich auszuführen (,Verinnerlichung“). Hinsichtlich ihrer Koordination gibt es jedoch Einschränkungen. So können Handlungen im Allgemeinen nicht in Gedanken umgekehrt werden, d. h. sie sind nicht reversibel. ${ }^{124}$ Im Stadium des konkret-operativen Denkens (ca. 711 Jahre) löst sich diese Beschränkung auf, indem sich die Denkhandlungen (,Operationen“) in so genannten Operationssystemen (Gruppierungen) organisieren, d. h. sie werden „kompositionsfähig“ (sie können zu komplexeren Operationen zusammengesetzt werden), ,,assoziativ“" (sie können auf verschiedenen Wegen zu einer neuen Operation zusammengesetzt werden) und „reversibel“ (sie können umgekehrt werden). Das Denken ist dabei jedoch immer noch an die unmittelbare Anschauung bzw. an die kurz zuvor gemachte Erfahrung mit konkretem Material gebunden. Erst im Stadium der formalen Operationen (ab ca. 11 Jahren) werden die Kinder zunehmend fähig, abstrakte Beweise und algebraische Umformungen zu verstehen, indem sie rein formal und unabhängig von konkreten Vorstellungen mit mathematischen Begriffen operieren.

\subsubsection{Hans Aebli: Verinnerlichung und operatives Durcharbeiten}

Während Piaget die kognitive Entwicklung im Wesentlichen als altersspezifischen „Reifeprozess" darstellt, betont Hans Aebli, der in den 60er Jahren an die Arbeiten von Piaget anknüpfte, stärker die Bedeutung von Erziehung und damit auch von Unterricht. Ziel von Unterricht sollte es sein, ausgehend von konkreten Handlungen „bewegliche Operationen“ auszubilden. Dabei versteht Aebli unter einer Operation in Anlehnung an Piaget eine abstrakte Handlung, d. h. ,eine effektive, vorgestellte (innere) oder in ein Zeichensystem übersetzte Handlung, bei deren Ausführung der Handelnde seine Aufmerksamkeit ausschließlich auf die entstehende Struktur richtet“ (Aebli 1983, S. 209). Es fällt auf, dass damit eine Operation nicht durch ihre „Innerlichkeit“" gekennzeichnet ist.

„Es ist falsch zu sagen, eine Operation sei eine ,interiorisierte Handlung‘. Wenn der Schüler eine Handlung effektiv ausführt, indem er sich die Gegebenheiten wahrnehmungsmäßig vergegenwärtigt, so ist das genauso

\footnotetext{
${ }^{124}$ So berichtet Piaget (1974, S. 183 ff.), dass Kinder nur beschränkt in der Lage sind, zu erkennen, dass eine bestimmte Anzahl von Perlen beim Umfüllen von einem breiten Glas in ein schmales Glas unverändert bleibt (Volumenexperiment). Die vollzogene Handlung kann offensichtlich nicht in Gedanken umgekehrt werden.
} 
eine Operation, wie wenn er sich diese Gegebenheiten als Zeichen vergegenwärtigt. Entscheidend ist nicht die Art der Vergegenwärtigung der Gegebenheiten; entscheidend ist das Bewusstsein der Beziehungen, die durch die Operation erzeugt oder verändert werden.“" (a. a. O., S. 220)

Im Rahmen des Mathematikunterrichts sollte jedoch die Verinnerlichung von Operationen angestrebt werden, d. h. der Schüler sollte lernen, Operationen innerlich und symbolisch auszuführen. Dieser Vorgang vollzieht sich nach Aebli in drei Hauptsstufen:

„Am Anfang steht der effektive Vollzug am konkreten Gegenstand. Auf der zweiten Stufe wird der Gegenstand bildlich dargestellt, und der Schüler stellt sich die Operation aufgrund dieser Gegebenheit vor. Auf der dritten Stufe bedient sich der Schüler bei ihrem Vollzug ausschließlich der Zeichen, welche Gegenstand und Operation vertreten.“ (Aebli 1961, zitiert nach Zech 1996, S. 95)

Die drei Hauptstufen sind dabei auf zwei Arten miteinander verbunden. Zum einen gibt es Übergangsphasen, in denen zwei aufeinander folgende Darstellungsebenen miteinander in Verbindung gebracht werden. „Das Ziel ist, dass sich die symbolischere ${ }^{125}$ Darstellung mit der Bedeutung auflädt, die die konkrete Darstellung schon besitzt“ (Aebli 1983, S. 238). Zum anderen wird die Verinnerlichung der Operation auf allen Stufen durch einen sprachlichen Kommentar begleitet und unterstützt.

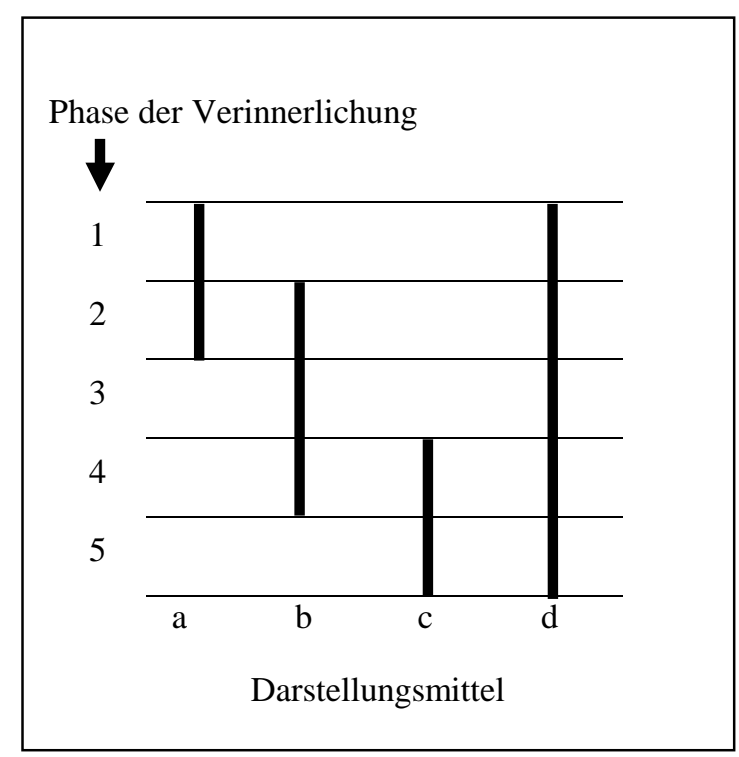
Verinnerlichung einer Operation
„Sie ist durch den Fortschritt vom Handeln mit wirklichen Gegenständen (a), zum Durchdenken der Operation aufgrund ihrer Bilder (b) und zum Operieren mit Zeichen (c) gekennzeichnet. Die Phasen 1, 3 und 5 des Vorgangs sind durch die bloße Verwendung eines der drei Darstellungs- mittel gekennzeichnet, die Phasen 2 und 4 sind Übergangsphasen, in denen die Bedeutung vom vorangehenden Darstellungsmittel auf das neue übertragen wird. Auf jeder Stufe werden die erarbeiteten Zusammenhänge auch mündlich (d) formuliert.“ (Aebli 1983, S. 239)

\section{Abb. 20: Die schrittweise Verinnerlichung einer Operation (aus Aebli 1983, S. 239).}

Mit der Definition des Begriffs „Operation“ hat Aebli bewusst und in Abgrenzung zu Piaget noch nichts über die Beweglichkeit von Operationen ausgesagt. Die Beweglichkeit von Operationen muss erst im Rahmen des operativen Durcharbeitens ausgebildet werden. ${ }^{126}$ Damit bezeichnet Aebli eine Art sinnbezogenes Üben, das der Vertiefung des Verständnisses dient, und dabei noch nicht auf eine Automatisierung ausgerichtet ist. Aufgabenstellungen, die das

\footnotetext{
${ }^{125}$ Die Bezeichnung ,symbolischer“ hat hier die Bedeutung von „,wirklichkeitsferner“.

${ }^{126}$ Dabei lassen sich die Phase der Verinnerlichung und die des operativen Durcharbeitens nicht scharf trennen.
} 
operative Durcharbeiten einer Operation zum Ziel haben, sind also in der Regel nicht auf einer rein symbolischen Ebene (,Bereich der Zeichen“) anzusiedeln, sondern auf oder in Verbindung mit den konkreteren Darstellungsebenen (,Bereich der Bedeutungen“).

„Während die Übung mit den Symbolen operiert, in die die Operation übersetzt worden ist, erfolgt das Durcharbeiten noch mit den Objektmengen oder ihren konkreten Symbolen, oder mit den räumlichen Körpern oder ihrer anschaulichen Abbildung, nicht bloß mit der Zeichenfolge, die beim Üben zum Automatismus eingeschliffen wird.“(Aebli 1983, S. 319)

\subsubsection{Theorie der Darstellungsebenen nach Jerome S. Bruner}

Nach Bruner (1971b) gibt es drei Darstellungsebenen oder Repräsentationsmodi von Wissen: enaktiv (durch Handlungen), ikonisch (durch Bilder ${ }^{127}$ ), symbolisch (durch Verwendung eines Zeichensystems oder Sprache). Diese Ebenen sind von den Stufen des Verinnerlichungsprozesses nach Piaget zu unterscheiden und bilden sich nach- und nebeneinander aus. Zuerst dominiert die enaktive Darstellung. Mit der Zeit treten die ikonische und symbolische Darstellung hinzu, bis ein Kind mit fortschreitender Entwicklung auf alle drei Formen gleichzeitig zugreifen kann. Die verschiedenen Darstellungsebenen stehen in starker Wechselbeziehung zueinander. Intellektueller Fortschritt zeichnet sich dabei durch die Fähigkeit aus, flexibel zwischen den verschiedenen Darstellungsebenen wechseln zu können.

„Jede dieser drei Darstellungsebenen, die handlungsmäßige, die bildhafte und die symbolische, hat ihre eigene Art, Vorgänge zu repräsentieren. Jede prägt das geistige Leben des Menschen in verschiedenen Altersstufen, und die Wechselwirkung ihrer Anwendungen bleibt ein Hauptmerkmal des intellektuellen Lebens des Erwachsenen.“ (Bruner, 1971b, S. 21)

Indem Fragestellungen aus der Umwelt enaktiv, ikonisch oder symbolisch dargestellt werden bzw. zwischen den Darstellungsebenen gewechselt wird, lassen sich Probleme lösen. Andererseits können Diskrepanzen zwischen verschiedenen Darstellungsebenen kognitive Konflikte auslösen, welche die geistige Entwicklung vorantreiben können. ${ }^{128}$

\footnotetext{
${ }^{127}$ Hiermit sind auch ,innere“ Vorstellungsbilder gemeint.

${ }^{128}$ So konnte Bruner (1971a) nachweisen, dass 5-7-jährige Kinder durchaus in der Lage sind, im Rahmen von Volumenexperimenten die Invarianz von Flüssigkeitsmengen beim Umfüllen in verschiedene Gefäße zu erkennen. Hierzu schirmt man die Gefäße während des Umfüllvorgangs und damit die dimensionale Veränderung der Flüssigkeitssäule zunächst ab und fordert die Kinder auf, die Situation sprachlich darzustellen (Volumenexperiment mit Abschirmtechnik). Anschließend wird das Experiment ohne Schirm wiederholt. Das Erkennen der Invarianz lässt sich hierbei weniger auf das Verständnis von Reversibilität (also das gedankliche Zurückschütten der Flüssigkeit) zurückführen. Vielmehr ist das Volumenexperiment mit Abschirmtechnik ein Beispiel für das Nichtübereinstimmen der sprachlich-symbolischen und der ikonischen Darstellungsweise. Der damit verbundene kognitive Konflikt fördert die Denkentwicklung. Vgl. hierzu auch Steiner 1973, S. 176 ff.
} 


\title{
3.1.4 Der Verinnerlichungs- und Verallgemeinerungsprozess aus der Sicht des Klagenfurter EFQUIM-Projekts ${ }^{129}$
}

Nach Piaget und Aebli sind Handlungen der Ursprung mathematischen Denkens. Auch Dörfler (1988a) sieht in gegenständlichen Handlungen ein wesentliches Mittel zur kognitiven Konstruktion mathematischer Begriffe und Operationen. Sein Interesse gilt jedoch der genaueren Analyse der Übergangsphase, d. h. dem Verinnerlichungs- und Abstraktionsprozess, den er als bisher nur ungenügend erfasst ansieht. Insbesondere vertritt er die Auffassung, dass Handlungen nicht allein und automatisch die intendierten kognitiven Prozesse steuern. Zur Konstruktion mathematischer Objekte ${ }^{130}$ und Operationen ist es für den Lernenden notwendig, seine Aufmerksamkeit auf bestimmte Stadien und Elemente seiner Handlung zu fokussieren.

\begin{abstract}
„Mathematische Objekte können durch Handlungen konstruiert werden und zwar als Beziehungen oder Systeme von Beziehungen zwischen bzw. an Handlungselementen, Handlungszuständen oder Handlungsprodukten. Diese Beziehungen sind Beziehungen wieder zwischen bereits früher kognitiv konstruierten mathematischen Objekten, die mit den genannten Elementen, Zuständen oder Produkten der konstituierenden Handlungen assoziiert werden. Für die kognitive Konstituierung dieser Beziehungen ist eine Fokussierung von Aufmerksamkeit auf die relevanten Elemente, Zustände und Produkte der Handlungen und die mit ihnen zu assoziierenden mathematischen Objekte erforderlich.“ (Dörfler 1988a, S. 66)
\end{abstract}

Indem die Aufmerksamkeit nicht nur auf die Elemente der Handlung und auf gewisse Zwischenstadien, sondern auch auf die Veränderung der Handlungselemente im Verlauf der Handlung gerichtet wird, werden mathematische Operationen konstruiert. Ein so verstandener Wissenserwerb betont die konstruktive, individuelle Aufbauleistung des Lernenden, die jedoch zusätzlich einer Steuerung von außen bzw. einer Anleitung durch den Lehrer erfordert. Die Bedeutung der Aufmerksamkeitsfokussierung zeigt sich z. B. in einer von Malle (1986) geschilderten Unterrichtssituation, die die Einführung des Differenzenquotienten zum Gegenstand hatte. Die Schüler hatten hierbei die Aufgabe, anhand einer in einer Tabelle dargestellten „Temperaturfunktion“ relative und absolute Temperaturänderungen zu berechnen. Ihr Interesse war dabei auf das numerische Endergebnis gerichtet; sie sahen offensichtlich keine

\footnotetext{
${ }^{129}$ Das in den Jahren 1982-86 am Institut für Mathematik der Universität Klagenfurt durchgeführte EFQUIMProjekt (Entwicklung formaler Qualifikationen im Mathematikunterricht) hatte zum Ziel, allgemeine und von bestimmten mathematischen Inhalten unabhängige Fähigkeiten zu identifizieren und zu charakterisieren. Aufbauend auf Piagets ,abstraction réfléchissante“ (d. h. der Abstraktion ausgehend von Handlungen) und den Abstraktions- und Verallgemeinerungstheorien von Rubinstein und Dawydow wurden Abstraktion und Verallgemeinerung als zentrale Grundformen mathematischen Denkens betrachtet und als eigenes theoretisches Konzept weiterentwickelt (vgl. Peschek 1989). Das Abstraktions- und Verallgemeinerungskonzept dient nicht nur zur Erklärung des Begriffsbildungsprozesses. Spezifische Ausprägungen von Abstraktion und Verallgemeinerung lassen sich auch beim Problemlösen und Beweisen herausarbeiten (vgl. Dörfler 1988a, S. 94 ff.).

${ }^{130}$ Der Terminus „Objekt“ wird nach Dörfler (1988a, S. 64) enger gefasst als der Terminus „Begriff“. Ein mathematisches Objekt zeichnet sich durch eine „kognitive Abgeschlossenheit“ aus, wohingegen ein mathematischer Begriff eher als offenes System angesehen wird, das aus verschiedenen mathematischen Objekten bestehen kann.
} 
Notwendigkeit in dem Notieren des vom Lehrer angestrebten Terms $\frac{f(b)-f(a)}{b-a}$. In diesem Fall hätte es vorab oder in Verbindung mit den Aktivitäten der Schüler einer Instruktion oder Erläuterung seitens des Lehrers bedurft, mit Hilfe derer die Bedeutung eines allgemeinen Terms herausgestellt worden wäre.

Neben einer Steuerung der Aufmerksamkeit bedarf es auch des Einsatzes geeigneter Darstellungen. Dörfler (1989, S. 4) bezeichnet Darstellungen oder Repräsentanten, die ,paradigmatisch für die oder besser für einen Aspekt der Bedeutung eines Begriffs“" sind, als Prototypen. Dabei ist es jedoch die Beziehung des Individuums zum Gegenstand, die diesen zum Prototypen macht:

„Damit ein Gegenstand (materiell oder vorgestellt) für ein Individuum als Prototyp wirksam wird, muss dieses eine bestimmte Sichtweise auf diesen Gegenstand entwickeln. Dies kann kurz als Fähigkeit bezeichnet werden, den Gegenstand eben nicht als solchen, sondern als prototypischen, paradigmatischen Vertreter der jeweils relevanten Klasse anzusehen.“(Dörfler 1991, S. 66)

Prototypen sind im Allgemeinen subjektiv, d. h. jedes Individuum wird zunächst seine eigenen, individuellen Prototypen konstruieren. Aufgrund der Vielfalt der Darstellungsmöglichkeiten eines mathematischen Begriffs ist es möglich, dass zu einem Begriff verschiedene Prototypen entwickelt werden, die jeweils unterschiedliche Eigenschaften und Beziehungen in den Vordergrund rücken. Dörfler (1988b) unterscheidet in diesem Zusammenhang verschiedene Arten von Prototypen: figurale, operative und symbolische Prototypen. Anhand figuraler Prototypen lässt sich die jeweilige Relation in besonders klarer Weise „,ablesen“ bzw. rekonstruieren.

„Hier wird der Gegenstand (materiell oder mental) aufgrund seiner Erscheinungsform, seiner wahrnehmbaren (oder vorgestellten) ,äußeren’ Eigenschaft zum Prototyp eines gewissen Begriffs.“ (Dörfler 1989b, S. 6)

Operative Prototypen haben nach Dörfler die Eigenschaft, dass an ihnen gegenständliche Handlungen besonders leicht und durchsichtig ausführbar bzw. vorstellbar sind. Diese Handlungen entsprechen den mit den repräsentierten mathematischen Objekten intendierten Operationen. Um Handlungen schematisch-algorithmisch ausführen zu können, sind Symbole oder symbolische Prototypen von besonderer Bedeutung.

Dörfler (1988a, S. 89 f.) illustriert die Konstruktion eines mathematischen Objekts bzw. deren Prototypen u. a. am Beispiel der Exponentialfunktion. Das Objekt „Exponentialfunktion“ besteht für Dörfler aus dem Beziehungssystem, welches in symbolischer Form als $\Delta y=\alpha \cdot y \cdot \Delta x$ angegeben werden kann. Zur Konstruktion dieses Beziehungssystems kann man einen Wachstumsprozess betrachten, der durch Messhandlungen „interpunktiert“ wird. Mit den beobachteten Zuständen des Prozesses werden zunächst Zahlen bzw. Größen assoziiert. Indem man nun die Aufmerksamkeit auf äquidistante Stadien sowie die damit assoziierten Größen und deren Verhältnis zueinander richtet, kann die exponentielle Beziehung konstruktiv festgestellt werden. Als Prototyp für das mathematische Objekt nennt Dörfler den Graph der Exponentialfunktion, da an ihm die genannten Handlungen besonders einfach 
durchführbar seien. Zum Aufdecken konstitutiver Eigenschaften eignet sich jedoch auch in besonderer Weise die Tabelle, in der die Quotienten jeweils aufeinander folgender Funktionswerte dargestellt und als konstant erkannt werden können.

Während sich der Prozess der Verinnerlichung bzw. der Abstraktion durch bestimmte Akte der Aufmerksamkeitsfokussierung, der Wahrnehmung und der kognitiven Konstruktion beschreiben lässt, werden im Prozess der Verallgemeinerung die Handlungs- und Beziehungsstruktur auf die Ausgangshandlung zurückbezogen und dabei ihr Einsatzbereich erweitert (vgl. Peschek 1989, 246 ff.). Diese Erweiterung des Einsatzbereichs bedeutet, dass zum einen die gegenständlichen Handlungselemente, zum anderen die mathematischen Objekte selbst als austauschbar erkannt werden. In diesem Zusammenhang werden so genannte ,generative Schemata“ (Dörfler 1988a, S. 112) entwickelt. Solche Schemata enthalten variable Teile, die zunächst durch Prototypen der ,einsetzbaren“ Elemente repräsentiert und bei Aufruf des Schemas dann mit spezifischen Elementen belegt werden.

„Das Belegen der Variablen im generativen Schema ist dann kognitiv ein Ersetzen der Prototypen durch handlungsäquivalente Gegenstände. Die Aktualisierung des Schemas in einem bestimmten Kontext liefert dann das spezifische mathematische Objekt bzw. die spezifische Ausprägung der mathematischen Operation.“(Dörfler, 1988a, S. 112 f.)

Prototypen repräsentieren somit für das Individuum das Allgemeine im Besonderen (vgl. Dörfler 1991, S.66).

\subsection{Konsequenzen für den Mathematikunterricht}

Im Anschluss an die oben skizzierten Handlungstheorien entstanden verschiedene Konzepte zur Realisierung eines „operativen“ Mathematikunterrichts, die sich an einigen Stellen zu didaktischen Prinzipien verdichten lassen. Diese Prinzipien können - wie im Folgenden aufgezeigt wird - nicht nur bei Begriffsbildungsprozessen, sondern auch beim Beweisen zur Anwendung kommen.

\subsubsection{Operative Prinzipien}

In den 70er Jahren schloss Fricke (1970) an die Überlegungen Piagets und Aeblis an und formulierte die so genannte „operative Methode“ für den Rechenunterricht der Grundschulen. Palzkill und Schwirtz (1971) übertrugen das Konzept auf den Geometrieunterricht, und Müller (1979) erweiterte es auf die Lineare Algebra. Ergebnisse von Untersuchungen mit dem Ziel, den auf Piaget zurückgehenden Begriff der Gruppierung von formalen Widersprüchen zu befreien, flossen in eine „Erweiterung des operativen Prinzips“ durch Wittmann ein:

„Ein vertieftes Verständnis der operativen Methode und eine erweiterte Fassung des operativen Prinzips werden sich im Folgenden dadurch ergeben, dass nicht nur die Verknüpfung von Operationen, sondern auch die ,Wirkung' von Operationen auf Eigenschaften bzw. Relationen zwischen Zuständen betrachtet werden, was innerhalb der operativen Methode allenfalls implizit und ansatzweise praktiziert wurde." (Wittmann 1976, S. 167) 
In der Folgezeit wurden die definitorischen Eigenschaften einer Gruppierung, die „Kompositionsfähigkeit“, „Reversibilität“, „Assoziativität“ zunehmend als unnötige Einschränkung des Operationsbegriffs empfunden. So sollten nach Aebli (1985) weniger die formale Fassung des Gruppierungsbegriffs als vielmehr Konzepte zur Förderung der Beweglichkeit mathematischer Begriffe Gegenstand didaktischer Untersuchungen sein. In diesem Zusammenhang formulierte Wittmann das folgende ,operative Prinzip der Mathematikdidaktik“:

„Objekte erfassen bedeutet, zu erforschen, wie sie konstruiert sind und wie sie sich verhalten, wenn auf sie Operationen (Transformationen, Handlungen, ...) ausgeübt werden. Daher muss man im Lern- und Erkenntnisprozess in systematischer Weise

1. untersuchen, welche Operationen ausführbar und wie sie miteinander verknüpft sind,

2. herausfinden, welche Eigenschaften und Beziehungen den Objekten durch Konstruktion aufgeprägt werden,

3. beobachten, welche Wirkungen Operationen auf Eigenschaften und Beziehungen der Objekte haben (Was geschieht mit ..., wenn ...?).“(Wittmann 1985b, S. 9)

Indem man wie Aebli das operative Prinzip als einen allgemeineren „Satz von innerlich zusammenhängenden Regeln auffasst, die auf bestimmten Vorstellungen über den Ursprung, das Wesen und das Ziel menschlichen Denkens beruhen“ (Aebli 1985, S. 4), kann man - wie Zech (1996, S. 115 f.) - anstelle des operativen Prinzips auch von mehreren „operativen Prinzipien“" sprechen, die sich aus den Theorien Bruners, Aeblis und Piagets ableiten lassen. Zech unterscheidet in diesem Zusammenhang:

\section{- Prinzip der Stufengemäßheit}

Das Prinzip der Stufengemäßheit folgt aus den Überlegungen Piagets und fordert eine Berücksichtigung der unterschiedlichen Stadien der Denkentwicklung. So sollten Grundschulkinder mathematische Begriffe und Operationen unter Ausnutzung geeigneten Materials zunächst konkret-handelnd aufbauen. Mit Aebli sollte jedoch berücksichtigt werden, dass auch ältere Schüler bei neuen Themenbereichen im Allgemeinen eine anschauliche Erfahrungsbasis benötigen.

\section{- Prinzip der Verinnerlichung und der Verzahnung von Darstellungsebenen}

Anschließend an die Überlegungen Aeblis sollte „der Mathematikunterricht aller Stufen [...] von einer konkret-anschaulichen Darstellung [...] allmählich zu einer abstrakt-symbolischen Darstellung [...] übergehen - mit dem Ziel, dass der Schüler mit der abstrakten Formulierung immer noch eine konkrete Vorstellung verbinden kann“ (Zech 1996, S. 116 f.). Dabei sollten die Verinnerlichungsstufen nicht isoliert voneinander durchlaufen werden, sondern eng miteinander verzahnt und durch einen sprachlichen Kommentar begleitet werden. Zech nennt als weitere Unterprinzipien:

Rückschaltprinzip: Bei Schwierigkeiten auf einer abstrakteren Stufe sollte ein Wechsel auf eine konkretere Stufe möglich sein.

Zwischenschaltprinzip: Anstelle die Handlung begleitend zu kommentieren, ist es auch möglich, sie vorab oder nachträglich zu verbalisieren. In bestimmten Situationen kann es 
darüber hinaus hilfreich sein, dass die eigene Handlung zuvor durch den Lehrer demonstriert wird.

Abschirmprinzip: Wie bereits Bruner aufgezeigt hat, kann die Verinnerlichung durch Abschirmung der ,unmittelbaren“ Anschauung und durch Operieren mit dem zuvor wahrgenommenen Objekt in der Vorstellung gefördert werden.

\section{- Prinzip der operativen Durcharbeitung}

Zech formuliert hier drei Unterprinzipien, die alle den Aspekt des Variierens betonen. Das Prinzip der Variation der Darstellungsebenen geht auf Bruner zurück und empfiehlt einen vielfältigen Wechsel zwischen enaktiver, ikonischer und symbolischer Darstellung. Dabei sollten insbesondere „konkretisierende“ Übergänge angemessen berücksichtigt werden. Nach dem Prinzip der Variation des Unwesentlichen sollten zum einen ein mathematischer Unterrichtsgegenstand auf mehrere Weisen veranschaulicht werden, zum anderen sollten mathematische Variablen, die für einen Begriff nebensächlich sind, variiert werden. Weiterhin sollten auch unterschiedliche Anwendungskontexte berücksichtigt werden.

Mit dem Prinzip des operativen Durcharbeitens (im engeren Sinne) werden die ursprünglich mit der Beweglichkeit von Begriffen und Operationen verbundenen Aspekte der Assoziativität, der Reversibilität und der Kompositionsfähigkeit angesprochen. Es geht hier um die Variation von Lösungswegen, die Variation der gesuchten Größe und das systematische Verändern von Ausgangssituationen in Verbindung mit der Untersuchung, was diese Veränderungen bewirken.

Hinter der Idee der operativen Methode oder der operativen Prinzipien steht eine Vorstellung von Unterricht, der entgegen dem „Prinzip der kleinen und allerkleinsten Schritte“ und dem „Grundsatz der Isolierung der Schwierigkeiten“ geeignet voneinander abgegrenzte Einheiten als Ganzes behandelt und von Anfang an den Beziehungscharakter mathematischer Inhalte betont (vgl. Fricke 1970). Ein charakteristisches Merkmal der Einstiegs- und Erarbeitungsphase ist dabei das heuristisch-forschende Arbeiten. So betont Fricke, dass

„nach Konfrontierung mit einem echten Problem stets eine breit angelegte Phase des spielerischen Umgangs, des experimentierenden Erprobens und Erforschens [steht], auch mit bewusst unzureichenden Mitteln, mit dem Ziel, Erfahrungen zu sammeln, Schwierigkeiten zu erkennen und das mathematische Denken zu aktivieren.“ (Fricke 1970, S. 97)

Damit wird den beiden Grundideen des operativen Prinzips - dem Aufbau von Operationen aus Handlungen und der Gruppierung von Handlungen bzw. Operationen zu beweglichen Gesamtsystemen - eine weitere Idee zur Seite gestellt: die aktiv-entdeckende Auseinandersetzung mit dem Lernmaterial (vgl. hierzu auch Bauer 1993). 


\subsubsection{Spezielle Varianten des operativen Prinzips}

Durch mehrere Arbeiten von Hering ${ }^{131}$ wurde in den 80er Jahren das operative Prinzip auch auf den Sekundarstufenunterricht übertragen. Neben dem Aspekt des Verinnerlichungsprozesses thematisierte Hering insbesondere die „steuernden Einflüsse“ des operativen Prinzips bei heuristischen Aktivitäten und beim Beweisen. Dieses so genannte „operative Beweisen“ bezeichnet Wittmann (1985b, S. 11) genauso wie die „operative Begriffsbildung“ als „Spezialfall des operativen Prinzips““. ${ }^{132}$ Aufgrund ihrer besonderen Bedeutung für diskrete Inhalte sollen diese beiden Spezialfälle im Folgenden noch einmal genauer analysiert und anhand von Beispielen verdeutlicht werden.

\subsubsection{Operative Begriffsbildung}

Zur Einführung eines Begriffs gibt es häufig verschiedene Möglichkeiten, die in der Regel eng mit den verschiedenen Arten der Begriffsbestimmung - oder der Begriffsbildung ${ }^{133}$ verknüpft sind. Man kann unterscheiden ${ }^{134}$ :

- Begriffsbildung durch intensionale Abstraktion ${ }^{135}$ (ausgehend von Beispielen);

- Begriffsbildung durch Spezifikation ${ }^{136}$ (ausgehend von bekannten Oberbegriffen);

- Begriffsbildung durch Idealisierung (hineinsehen von Eigenschaften);

- Begriffsbildung durch Handlung oder Konstruktionsvorschrift;

- implizit-axiomatische Begriffsbildung (Kennzeichnung von Grundbegriffen einer Theorie);

- Begriffserwerb durch extensionale Abstraktion (mit Hilfe von Äquivalenzrelationen).

Mathematische Begriffe lassen sich im Allgemeinen nicht wie empirische (Alltags-)Begriffe durch (sinnlich) wahrnehmbare Eigenschaften erfassen, sondern sie ,repräsentieren vielmehr Beziehungen und Beziehungssysteme zwischen Objekten oder auch zwischen deren Eigenschaften“ (Peschek 1989, S. 246), die durch Handlungen erst hergestellt werden müssen. Dies

\footnotetext{
${ }^{131}$ Vgl. etwa Hering 1985, 1986, 1988.

${ }^{132}$ Daneben nennt Wittmann noch weitere Varianten des operativen Prinzips: das „funktionale Denken“, das „operative Üben“ und das „,heuristische Prinzip der Geometrie“.

${ }^{133}$ Die Bezeichnung „Begriffsbildung“ wird in der didaktischen Literatur in unterschiedlichen Kontexten verwendet. Sie kann die historische Entwicklung eines Begriffs bezeichnen oder psychologische Aspekte betonen. Weiterhin kann sie - wie in diesem Zusammenhang - als „Handlungsabsicht des Lehrers im Unterricht“ verstanden werden (vgl. hierzu Klika, Tietze \& Wolpers 1997, S. 56).

${ }^{134}$ Vgl. Winter 1983, Holland 1975.

${ }^{135}$ Winter (1983) spricht hier von exemplarischer Begriffsbestimmung.

${ }^{136}$ Winter (1983) spricht hier von explizit-definitorischer Begriffsbestimmung.
} 
betont die besondere Bedeutung des konstruktiven Begriffserwerbs. In der Literatur wird dieser Weg auch als operative Begriffsbildung bezeichnet (vgl. Wittmann, 1981, S. 81). ${ }^{137}$

Beim operativen Begriffserwerb werden Begriffe ausgehend von Handlungen oder Konstruktionen gebildet. „Der angestrebte Begriff wird dadurch repräsentiert, dass man ein generelles Verfahren ausführen und beschreiben kann, das die Dinge, die unter den Begriff fallen, herstellt“" (Winter 1983, S. 189). Wird die Begriffsbildung in einen Problemlöseprozess eingebettet, so ergibt sich der Begriff häufig als Lösung eines Problems. Das Handeln kann jedoch auch ein „Nachmachen“ sein, bei dem der Lehrer zunächst demonstriert, wie eine bestimmte Operation auszuführen ist. Grundsätzlich ist darauf zu achten, dass die Schüler im Umgang mit dem Begriff die wesentlichen, konstitutiven Eigenschaften erfahren (vgl. Vollrath 1984, S. 103 ff.). Viele Begriffe aus dem Bereich der kombinatorischen Optimierung bzw. der Graphentheorie lassen sich konstruktiv bestimmen, so etwa „minimale Netze“ oder „kürzeste Wege“. Indem der Schüler das entsprechende Konstruktionsverfahren (den Algorithmus) durchführt, erwirbt er ein „operativ-instrumentelles Verständnis“138 des Begriffs. Die Eigenschaften des jeweiligen Begriffs treten jedoch deutlicher hervor, wenn das Verfahren vom Schüler selbst entdeckt und anschließend systematisch analysiert wird.

Winter (1983, S. 189) nennt als spezielle Form konstruktiver Begriffsbildung die rekursive Begriffsbildung. Bei der rekursiven Begriffsbildung geht man von einem bestimmten Anfangsobjekt und einer Berechnungsvorschrift aus. Mit Hilfe der Berechnungsvorschrift lassen sich alle folgenden Objekte aus bereits konstruierten Objekten entwickeln. Zahlreiche Begriffe aus dem Bereich der Kombinatorik lassen sich auf diese Weise „herstellen“. So können etwa Zahlenfolgen rekursiv erfasst werden, indem man die Schüler eine allgemeine Regel entdecken lässt, mit der sie aus bereits gegebenen Gliedern die folgenden bestimmen können. Das kann in eine rekursive Definition münden. Geeignete Darstellungen oder auch konkrete Materialien können den Lernprozess unterstützen. So beschreiben Abramovich und Pieper (1996), wie mit Hilfe verschiedenfarbiger Scheiben ein rekursiver Zugang zu Permutationen und Kombinationen im Rahmen eines Problemlöseprozesses auf „,natürliche Weise“ ermöglicht werden kann. Zur Bestimmung der Anzahl der insgesamt möglichen Permutationen von $n$ Objekten ist hierbei der Blick auf die unterschiedlichen Möglichkeiten zur Anordnung der Scheiben zu lenken. Im Vordergrund steht dabei die Frage nach einem effektiven Verfahren, mit dem ausgehend von bereits bekannten Permutationen von $n$ Scheiben eine Permutation von $n+1$ Scheiben erzeugt werden kann. Man geht hierzu zunächst von einem Objekt aus, das sich auf genau eine Art anordnen lässt. Auch die zwei verschiedenen Anordnungen zweier

\footnotetext{
${ }^{137}$ Das heißt nicht, dass grundsätzlich alle Begriffe operativ eingeführt werden sollten, sondern betont lediglich die besondere Bedeutung von Handlungen im Begriffsbildungsprozess. Da die verschiedenen Formen der Begriffsbildung in der Regel miteinander verknüpft sind, ist es somit sinnvoll und notwendig, z. B. auch nach der Einführung eines Begriffs durch „Beispiele und Gegenbeispiele“ mit diesen Begriffen zu handeln.

${ }^{138}$ Mit „operativ- instrumentellem Verständnis“ bezeichnet Winter (1983, S. 182) ein Begriffsverständnis, welches sich in Form von Fertigkeiten im Umgang mit diesem Begriff äußert.
} 
Scheiben sind unmittelbar ersichtlich. Zur Konstruktion einer 3er-Permutationen kann sowohl die erste als auch die zweite bereits konstruierte 2er-Permutationen verwendet werden. In beiden Fälle kann die dritte (weiße) Scheibe vor, zwischen bzw. hinter die zwei gemusterten Scheiben platziert werden. Man erhält damit für die Anzahl aller 3er-Permutationen: $P(3)=3 \cdot P(2)$ (vgl. Abb. 21). Durch Verallgemeinern erhält man die allgemeine Formel $P(n)=n \cdot P(n-1)$.

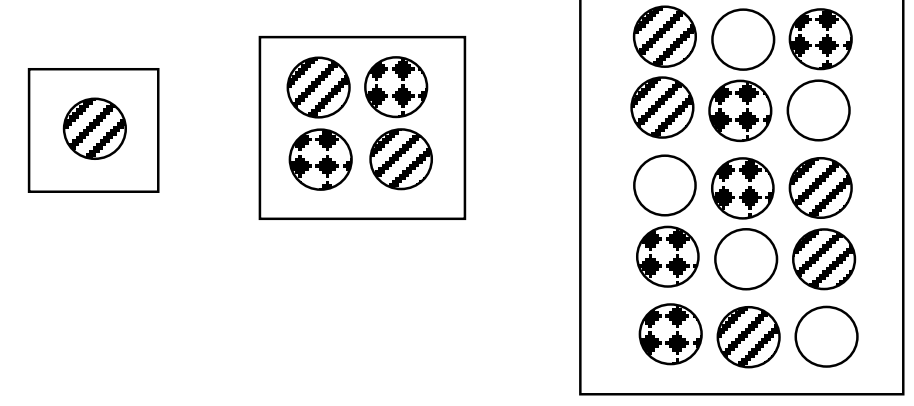

Abb. 21: Erschließen einer rekursiven Formel für die Anzahl der Permutationen einer $\boldsymbol{n}$-elementigen Menge

Die rekursive Begriffsbildung setzt abzählbare Objekte voraus. Man kann daher die rekursive Begriffsbildung als typisch für viele diskrete Objekte bezeichnen.

\subsubsection{Operative Beweise}

Unter „operativen Beweisen“ versteht Wittmann (1985b, S. 11) „Beweise, bei denen die den Objekten durch Konstruktion aufgeprägten Eigenschaften und Beziehungen sowie deren Verhalten bei Operationen explizit ausgenutzt werden [...]“. Im Rahmen dieser Beweise wird das von Wittmann formulierte erweiterte operative Prinzip in der Kurzform „Was geschieht mit ..., wenn ...?“ als heuristisches Prinzip verstanden (vgl. hierzu auch Hering 1988). Indem mit den betrachteten Objekten operiert wird, sollen „Verträglichkeiten zwischen Zustandseigenschaften, -relationen, -funktionen und den Operationen“ (vgl. Wittmann 1976, S. 170) aufgedeckt werden. Dabei stellen „Verträglichkeiten“ nach Wittmann nichttriviale, allgemeine Beziehungen, insbesondere Invarianzen dar. Das operative Prinzip steuert hier also weniger Lern- bzw. Verinnerlichungsprozesse, sondern Handlungen werden - wie Dörfler (1985, S. 95) formuliert - zu „Untersuchungsmitteln für (meist vorgegebene) Begriffe und heuristische Mittel zur Problemlösung“.

„Somit sind unter dem Operativen Prinzip strukturell sehr unterschiedliche Ansätze subsumiert: Einerseits steht die Schematisierung (Verinnerlichung) des Prozesses (der Ausführung) von Handlungen im Zentrum, andererseits werden nicht die Handlungen entwickelt, sondern mit den Handlungen werden Resultate und Beziehungen hergestellt.“ (a. a. O., S. 95)

Die folgenden Beispiele sollen verdeutlichen, wie das operative Prinzip bei diskreten Problemstellungen den Problemlöseprozess steuern und zu operativen Beweisen führen kann. 


\section{Beispiel: Euler'scher Polyedersatz}

Der Euler'sche Polyedersatz stellt einen Zusammenhang zwischen der Anzahl der Ecken, Kanten und Flächen (Länder) eines beliebigen zusammenhängenden ebenen Graphen (einer zusammenhängenden Landkarte) her. Zu den Flächen wird hierbei auch das ,äußere Gebiet“ einer Landkarte gezählt. Der Satz lautet wie folgt: Sind $e, k$ und $f$ die Anzahlen der Ecken, Kanten und Flächen eines zusammenhängenden ebenen Graphen, dann gilt $e-k+f=2 .{ }^{139}$

Der Euler'sche Polyedersatz lässt sich im Rahmen eines operativen Vorgehens auf der enaktiven Ebene beweisen. In diesem Zusammenhang stellt man die Kanten z. B. in Form von Drahtstïcken dar und erweitert eine triviale Ausgangskarte, die nur aus einer Ecke und einer Fläche besteht, schrittweise durch sukzessives Hinzufügen neuer Kanten bzw. Lötstellen. Hierbei gibt es vier elementare Erweiterungen (vgl. hierzu Wittmann 1976, S. 169):

(1) Man fügt an eine vorhandene Ecke eine neue Kante mit einem freien Ende an.

(2) Man fügt eine neue Kante an, indem man ihre beiden Enden an eine schon vorhandene Ecke anhängt.

(3) Man fügt eine neue Kante an, indem man ihre beiden Enden an zwei verschiedene, schon vorhandene Ecken anhängt.

(4) Man zerlegt eine bereits vorhanden Kante durch das Einfügen einer Ecke in zwei Kanten.

Damit ändern sich die Anzahlen von $e, f$ und $k$ wie folgt:
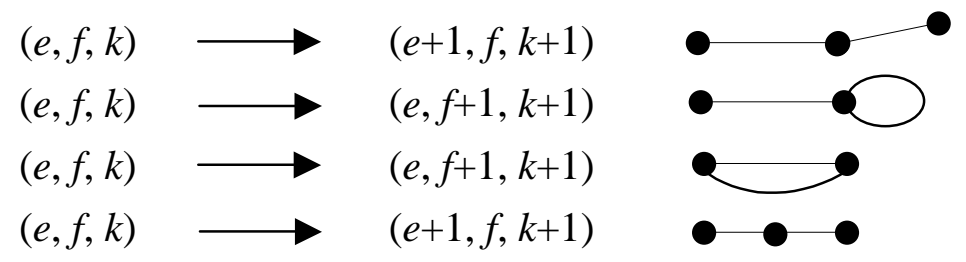

Bei jedem Schritt stellt man fest, dass die Summe $e-k+f$ jeweils konstant bleibt. Da diese Konstante bei der trivialen Karte bestehend aus nur einer Ecke den Wert 2 besitzt, gilt die Beziehung $e-k+f=2$ allgemein.

\section{Beispiel: Quadratzahlen}

Auch die arithmetische Beziehung $1+3+5+\ldots+(2 n-1)=n^{2}$ kann im Rahmen eines operativen Vorgehens bewiesen werden: Man stellt die Folge der Quadratzahlen durch eine Figur aus zusammengesetzten Quadraten gleicher Größe dar (vgl. Abb. 22). Dabei erkennt man, dass jede Quadratzahl aus der vorhergehenden durch Hinzufügen eines „Rands“ entsteht.

\footnotetext{
${ }^{139}$ Mit der Bezeichnung „Polyedersatz“ wird deutlich gemacht, dass eine Beziehung zu einem Satz über Polyeder, also räumlichen Gebilden, besteht. So lassen sich alle konvexen Polyeder zu zusammenhängenden Landkarten „plätten“, die genauso viele Ecken, Kanten und Flächen besitzen wie der entsprechende Ausgangskörper. Auf diese Landkarten kann der obige Satz angewendet werden. Damit gibt der Polyedersatz auch Auskunft über den Zusammenhang der Anzahl der Ecken, Kanten und Flächen eines beliebigen konvexen Polyeders.
} 
Beim Übergang von $n^{2}$ nach $(n+1)^{2}$ beträgt die Anzahl der hinzugefügten Quadrate $2 n+1$. Indem man die Folge der Partialsummen der Folge der ungeraden Zahlen bestimmt, also immer eine weitere ungerade Zahl (dargestellt durch einen „Rand“) hinzuaddiert, erhält man die Quadratzahlen (vgl. Wittmann 1985b).

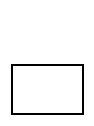

1

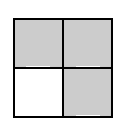

4

$1+3$

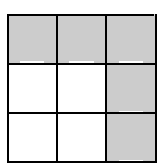

9

$1+3+5$

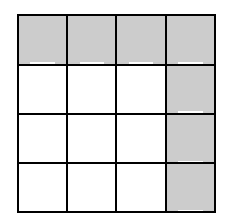

16

$1+3+5+7$

Abb. 22: Operatives Vorgehen beim Beweis der arithmetischen Beziehung $1+3+5+\ldots+(2 n-1)=n^{2}$

Viele weitere diskrete Problemstellungen legen einen Zugang im Sinne des erweiterten operativen Prinzips nahe, wie etwa der Zwei-Farbensatz (vgl. Besuden 1979), das Sperner'sche Lemma (für die Ebene) (vgl. Hering 1985, Wittmann 1985a) oder die zahlentheoretische Beziehung $\operatorname{ggT}(a, b) \cdot \operatorname{kgV}(a, b)=a \cdot b$ (vgl. Hering 1988).

Im Allgemeinen erfolgen die Handlungen bei den operativen Beweisen im Sinne Wittmanns auf einem festen Schematisierungs- und Abstraktionsniveau (vgl. Dörfler 1985, S. 95); insbesondere umfassen operative Beweise auch Beweise auf der formalen Ebene. ${ }^{140}$ Dagegen werden bei den von Semadeni (o. J.) und Kirsch (1979) als prämathematisch bezeichneten Beweisformen explizit die enaktive Darstellungsebene angesprochen sowie der Verinnerlichungs- und Abstraktionsprozess betont.

„Nach Semadeni [...] besteht ein prämathematischer Beweis grob gesagt aus gewissen konkreten Handlungen (Operationen im Sinne von J. Piaget, aber dieser wird großzügig interpretiert). Diese Handlungen werden zuerst wirklich ausgeführt, dann nur vorgestellt (verinnerlicht). Sie müssen korrekten mathematischen Argumenten entsprechen, die in ihrer psychologisch natürlichen Ordnung aufeinander folgen [...].“ (Kirsch 1979, S. 261 f.)

Bei prämathematischen und auch bei so genannten inhaltlich-anschaulichen Beweisen, die auf der ikonischen Ebene bzw. in einem Realkontext präsentiert bzw. interpretiert werden, tritt die steuernde Wirkung des operativen Prinzips im Sinne der Frage „Was passiert mit ..., wenn ...?" im Allgemeinen nicht so deutlich hervor. Dennoch handelt es sich auch hierbei um „,vollgültige“ Beweise, bei denen sich alle Handlungen und Schlüsse ausgehend von einem Spezialfall direkt verallgemeinern und in korrekte formal-mathematische Argumente übersetzen lassen. ${ }^{141}$ Beiden Konzepten kommt daher im Hinblick auf die vorher skizzierten operativen Prinzipien „Stufengemäßheit“ und „Verinnerlichung“ eine wesentliche Bedeutung zu.

\footnotetext{
${ }^{140}$ Vgl. hierzu etwa den Beweis der Ungleichung vom geometrischen und arithmetischen Mittel in Hering 1986.

${ }^{141}$ Blum \& Kirsch $(1989,1991)$ fassen beide Beweisgedanken unter dem Konzept des präformalen Beweisens zusammen.
} 
Die folgenden zwei Beispiele verdeutlichen, dass sich insbesondere kombinatorische und graphentheoretische Problemstellungen für eine Behandlung im Sinne des Konzepts des prämathematischen bzw. inhaltlich-anschaulichen Beweisens eignen.

\section{Beispiel: Binomialkoeffizienten}

Eine wichtige Beziehung zwischen Binomialkoeffizienten drückt die folgende Gleichung aus:

$$
\left(\begin{array}{l}
n \\
k
\end{array}\right)=\left(\begin{array}{c}
n \\
n-k
\end{array}\right)
$$

Interpretiert man die Binomialkoeffizienten als die Anzahl der $k$-elementigen Teilmengen einer $n$-Menge, so liegt der folgende ,realitätsbezogene Beweis“ nahe (vgl. Biermann \& Blum 1998): Man betrachtet exemplarisch eine Menge von 11 Personen, aus der eine Gruppe von 4 Personen ausgewählt werden soll. Die Anzahl aller möglichen 4er-Gruppen („Komitees“) beträgt $\left(\begin{array}{c}11 \\ 4\end{array}\right)$. Zu jedem (möglichen) 4er-Komitee gehört ein 7er-(Nicht-)Komitee (man betrachtet hierbei die bei der jeweiligen Auswahl übriggebliebenen Personen). Daher gibt es genauso viele 4er-Komitees wie 7er-(Nicht-)Komitees.

Ähnlich begründet man die Gleichung:

Im konkreten Fall

$$
\left(\begin{array}{l}
n \\
k
\end{array}\right)=\left(\begin{array}{c}
n-1 \\
k
\end{array}\right)+\left(\begin{array}{l}
n-1 \\
k-1
\end{array}\right)
$$

$$
\left(\begin{array}{c}
11 \\
4
\end{array}\right)=\left(\begin{array}{c}
10 \\
4
\end{array}\right)+\left(\begin{array}{c}
10 \\
3
\end{array}\right)
$$

isoliert man zunächst eine Person X aus der 11-er Menge und zählt dann getrennt die 4erKomitees, in denen die ausgezeichnete Person Mitglied ist, und jene 4er-Komitees, in denen die ausgezeichnete Person nicht Mitglied ist. Im ersten Fall hat man neben der bereits ins Komitee gewählten Person X noch 3 weitere Personen aus der restlichen 10er-Gruppe zu bestimmen. Im zweiten Fall müssen alle vier Personen aus der 10er-Gruppe gewählt werden, der die Person $\mathrm{X}$ nicht angehört.

\section{Beispiel: Euler'scher Satz}

Der Euler'sche Satz besagt: Wenn ein zusammenhängender Graph sich in einem Zug durchlaufen lässt, dann besitzt er höchstens zwei Ecken mit ungeradem Eckengrad. ${ }^{142}$ Unter der Voraussetzung der „Durchlaufbarkeit“ kann man den entsprechenden Weg im Graph durch das Auflegen eines Fadens darstellen, der jede Kante genau einmal bedeckt. Alle Ecken, in denen nicht der Anfangs- bzw. Endpunkt des Fadens liegt, werden mindestens einmal durch-

\footnotetext{
${ }^{142}$ Es gilt auch die Umkehrung dieses Satzes. Ein prämathematischer Beweis dieser Aussage ist jedoch weniger nahe liegend. Vgl. hierzu jedoch auch Kirsch 1979, S. 267.
} 
laufen. Diese Ecken müssen einen geraden Eckengrad haben. Somit kann es nur zwei ungerade Ecken geben, nämlich die Ecken, in denen der Faden beginnt bzw. endet.

\subsubsection{Kritische Bemerkungen zum Einsatz operativer Prinzipien}

Das operative Prinzip stellt ein zentrales Unterrichtsprinzip zur Organisation von Lern- und Erkenntnisprozessen dar. Dennoch müssen auch dessen Grenzen sowie alternative Vorschläge zur Gestaltung von Unterrichtsabläufen berücksichtigt werden.

\section{- Zur Verwirklichung des aktiv-entdeckenden Lernens}

Wenngleich das aktiv-entdeckende Lernen eine Grundidee des operativen Prinzips ist, werden im Rahmen einer kritische Auseinandersetzung mit diesem Unterrichtsprinzip Grenzen und Gefahren deutlich. ${ }^{143}$ So ist zu beachten, dass sich nicht jeder mathematische Lernstoff für Entdeckungslernen gleich gut geeignet. Ein umfangreiches und komplexes Wissensgebiet lässt sich möglicherweise besser vermitteln, wenn es von dem Lehrer vorstrukturiert und in gegliederten und nach Schwierigkeiten gestuften Abschnitten dargeboten wird. Weiterhin müssen die unterschiedlichen kognitiven Voraussetzungen der Schüler berücksichtigt werden. „Entdeckendes Lernen“ kann weniger neugierige, kreativ-begabte und ausdauernde Schüler überfordern und zu Frustration führen. ${ }^{144}$ Darüber hinaus ist der für ein entdeckendes Lernen erforderliche Material- und Zeitaufwand nicht zu unterschätzen. Damit ist ein durchgängige aktiv-entdeckende Auseinandersetzung mit den im Rahmen des Lehrplans vorgeschriebenen Lerninhalten kaum möglich. Es erscheint somit sinnvoll, das „entdeckende Lernen“ und die „darbietende Unterrichtsmethode“ nicht als zwei „Globalalternativen“ bei der Vermittlung von Lerninhalten anzusehen, sondern beide Methoden im Rahmen des Mathematikunterrichts angemessen zu berücksichtigen. ${ }^{145}$

\section{- Zum Einsatz der Variationsprinzipien}

Ein gedankenloses Anwenden der vielen (operativen) Variationsprinzipien, insbesondere der Variation der Darstellungsebenen bzw. der Veranschaulichungsmittel, birgt verschiedene Gefahren in sich. ${ }^{146}$ So kann das eigentliche Ziel, das Entwickeln eines Begriffs- bzw. Operationsverständnisses, aus den Augen verloren werden, und die Tätigkeiten können in einen blinden Aktionismus ausarten. Weiterhin ist zu berücksichtigen, dass die Stufung ,enaktivikonisch-symbolisch“ nicht immer einer Stufung nach Schwierigkeit entsprechen muss. Ins-

\footnotetext{
${ }^{143}$ Grenzen des aktiv-entdeckenden Lernens wurden bereits von Ausubel (1973) aufgedeckt. Eine Zusammenstellung kritischer Einwände gegen das ,entdeckende Lernen“ findet sich in Führer 1997, S. 61 ff.

${ }^{144}$ Vgl. hierzu auch jedoch auch Wittmann 1990, S. 160. Seiner Ansicht nach werden lernschwache Schüler durch eine Gliederung des Lernstoffs in isolierte Einheiten in ihren Lernprozessen eher behindert. Ein beziehungshaltiges Lernen sei dabei nicht möglich.

${ }^{145}$ Vgl. hierzu auch Bauer 1993, S. 81 und Führer 1997, S. 63 f.

${ }^{146}$ Vgl. hierzu auch Bauer 1993.
} 
besondere die von Zech (1996) geforderten „konkretisierenden Übergänge“ von einer symbolisch-abstrakten zu einer anschauungsgebundenen, inhaltlichen Ebene bereiten Schülern häufig mehr Probleme, als das alleinige Arbeiten im symbolischen Bereich. Darüber hinaus muss beachtet werden, dass Veranschaulichungsmittel auch zusätzlichen Lernstoff darstellen können, der aufgrund der Bereichsspezifität ${ }^{147}$ des Wissens nur bedingt mit bereits vorhandenen „subjektiven Erfahrungsbereichen“ verknüpft werden kann. Sind Vorstellungen und Erfahrungen, die mit den verschiedenen Darstellungsformen verbunden sind, nur unzureichend vernetzt, führt dies häufig zu Schwierigkeiten und Fehlern im Umgang mit dem Begriff, so etwa beim Übersetzen zwischen verschiedenen Repräsentationsebenen. Dennoch macht die Entwicklung allgemeiner Begriffe den Vergleich und die Vernetzung verschiedener bereits existierender Erfahrungsbereiche und damit eine (reflektierte) Variation der Darstellungsebenen erforderlich.

\section{- Zum Einsatz von Darstellungen}

Zur Vermeidung einer unreflektierten Variation von Darstellungen, erscheint eine Beschränkung auf wenige, zentrale Veranschaulichungen (vgl. Bauer 1993, S. 79) sinnvoll und wichtig. Im Sinne Dörflers sollten dabei solche Darstellungen gewählt werden, die besonders gut als gegenständlicher Prototyp eines bestimmten mathematischen Begriffs geeignet sind. Diese Darstellungen betonen bestimmte Aspekte eines Begriffs und sind so strukturiert, dass sie die Aufmerksamkeit der Schüler in besonderer Weise auf relevante Eigenschaften richten und das „Hineinsehen“ bzw. Rekonstruieren von Beziehungen erleichtern. ${ }^{148}$

Nun stellen jedoch Prototypen - wie bereits in Kapitel 3.1.4 herausgestellt - subjektive Konstruktionen des Individuums dar. Daher besteht beim Einsatz von Veranschaulichungsmitteln und bei einer zu starken Konzentration des Lernenden auf deren figurale Gestalt die Gefahr, dass sich Fehlvorstellungen ausbilden bzw. verfestigen oder nur defizitäre Vorstellungen entwickelt werden. ${ }^{149}$ Es besteht weiterhin keine Garantie, dass Schüler den vom Lehrer inten-

\footnotetext{
${ }^{147}$ Die Theorie der „,subjektiven Erfahrungsbereiche“ geht davon aus, dass Begriffe „bereichsspezifisch“ also individuell und gebunden an spezifische Situationen entwickelt und gespeichert werden. Dabei spielen auch soziale und emotionale Aspekte eine wichtige Rolle (vgl. Bauersfeld 1983). Die kognitive Struktur stellt hierbei keine hierarchisch geordnete Gesamtheit dar. Erst mit zunehmenden Lernerfahrungen erfolgt eine gewisse Hierarchisierung, indem die einzelnen Erfahrungsbereiche umstrukturiert, vernetzt bzw. zu neuen kontrollierenden und koordinierenden Bereichen ausgebaut werden.

${ }^{148}$ Vgl. die Ausführungen Dörflers (1989) zu „,figurativen Prototypen“: „Die ausgezeichneten Eigenschaften und Beziehungen können z. B. physikalischer oder geometrischer Natur sein und werden in der Wahrnehmungstätigkeit (re)konstruiert. Diese Wahrnehmungstätigkeit kann jedoch am „Prototyp in besonders ungestörter, rauschfreier Form erfolgen und kann daher an ihm auch in besonders günstiger Weise gelernt werden." (Dörfler 1989, S. 6)

${ }^{149}$ So zeigt eine Untersuchung Pescheks (1989, S. 267 ff.), dass Schüler - als sie nach Begriffseigenschaften einer linearen Funktion gefragt wurden - fast ausschließlich äußere, sinnlich wahrnehmbare, figurative Eigenschaften der mathematischen Objekte bzw. deren Darstellungen benannten (Beschreibung einer linearen Funktion als Gerade, Angabe einer Gleichung $y=a x+b$ ), dass in keiner Antwort jedoch die wesentliche Eigenschaft der konstanten Änderungsrate erwähnt wurde. Dies ist für ihn ein Hinweis auf eine vorwiegend empirische Begriffsbildung, bzw. auf das Vorliegen eines defizitären empirischen Prototyps für das Objekt „lineare Funktion“.
} 
dierten Prototyp entwickeln bzw. beim Arbeiten mit bestimmten Darstellungen eine Sichtweise auf den Begriff einnehmen, die der des Lehrers entspricht. So können bestimmte Eigenschaften, die für den Lehrer in einer für ihn prototypisch wirksamen Darstellung besonders deutlich hervortreten, für den Schüler nicht sichtbar sein. ${ }^{150}$ Damit wird deutlich, dass beim Einsatz von Darstellungen eine Berücksichtigung der individuellen Schülervorstellungen unumgänglich ist.

\subsection{Diskrete Arbeitsweisen im Mathematikunterricht}

Dem Begriff des „Handelns“ kommt im Rahmen der skizzierten Theorien eine besondere Bedeutung zu. Ziel dieser Arbeit ist es, das „Handeln“ oder allgemeiner den „Umgang“ von Schülern mit diskreten Objekten (in einer computergestützten Lernumgebung) näher zu analysieren. Dies macht es erforderlich, den Begriff des Handelns differenzierter zu betrachten. Für Aebli (1980, S. 18 ff.) stellt das Verhalten den weitesten Begriff dar. Der Verhaltensbegriff umfasst willkürliche und unwillkürliche, bewusste und unbewusste Reaktionen des Menschen. Als eine Tätigkeit oder Tun bezeichnet Aebli absichtsvolles, zielgerichtetes Verhalten. Dabei werden jedoch Mittel zum Erreichen dieser Ziele häufig nicht bewusst eingesetzt. Handlungen sind Tätigkeiten mit hochgradiger Bewusstheit und Zielgerichtetheit. Als Operationen bezeichnet Aebli (1980, S. 113 ff.) schließlich Handlungen, die abstrakt und unter strukturellen Gesichtspunkten betrachtet werden.

Will man den Umgang der Schüler mit mathematischen Objekten untersuchen, erscheint eine solche Stufung als zu grob und auch zu unscharf. In der Regel hat man keinen Einblick in unbewusste Vorgänge, so dass eine Unterscheidung zwischen Handlung und Tätigkeit im Einzelfall nur schwer möglich ist. Darüber hinaus berücksichtigt diese Einteilung nicht, worauf sich die Handlungen beziehen, womit also gehandelt wird. So ist zu beachten, dass der Umgang mit mathematischen Objekten auf bestimmten Darstellungsebenen mit Hilfe von Darstellungen oder Veranschaulichungsmitteln erfolgt, die verändert werden können und zwischen denen hin- und hergewechselt werden kann.

Um auszudrücken, dass wir an tatsächlich beobachtbaren Tätigkeiten und Handlungen in Verbindung mit konkreten diskreten Begriffen interessiert sind, wird hier der Begriff Arbeitsweisen gewählt. In Anlehnung an Weigand \& Weller (2001) unterscheiden wir hierbei drei

\footnotetext{
${ }^{150}$ In diesem Zusammenhang sei auf eine empirische Untersuchung von Weigand (1988) zum Entdecken von Funktionseigenschaften verwiesen. Im Rahmen dieser Untersuchung bestätigte sich, dass das Erkennen von Begriffseigenschaften von der Darstellungsform abhängt, dass insbesondere der Funktionsgraph im Allgemeinen die geometrische Sichtweise von Funktionseigenschaften hervorhebt, während bei der Tabellendarstellung eher numerische Aspekte betont werden. Bei manchen Aufgaben zur Tabellendarstellung überwog aber dennoch die geometrische Sichtweise. So argumentierten die Schüler häufig geometrisch, wenn aus den angegebenen tabellarischen Funktionswerten keine einfache Gesetzmäßigkeit abzulesen war, so etwa bei der Symmetrie und beim asymptotischen Verhalten. Die Tabellendarstellung lenkte somit offensichtlich nicht „Zwangsläufig“ den Blick auf numerische Eigenschaften, wie es vom Lehrer intendiert war.
} 
verschiedene Dimensionen dieses Begriffs: die Werkzeugebene, die Darstellungsebene und die Objektebene.

\section{- Arbeiten auf der Werkzeugebene}

Arbeitsweisen lassen sich als eine Folge von Darstellungen und Übergängen zwischen Darstellungen charakterisieren. Die Art und Weise, wie diese Übergänge real oder technisch durchgeführt werden, wird auf der so genannten Werkzeugebene untersucht. Bei den verwendeten Werkzeugen wird hier zunächst nur zwischen traditionellen Werkzeugen wie „Bleistift und Papier" und technischen Werkzeugen wie Taschenrechner und Computer unterschieden. Diese Werkzeuge sowie deren didaktischen und methodischen Möglichkeiten müssen im konkreten Fall genauer benannt und charakterisiert werden. ${ }^{151}$ Bei der Analyse der Arbeitsweisen auf der Werkzeugebene interessiert insbesondere, wie das Potenzial des Werkzeugs genutzt wird und welche Schwierigkeiten sich bei dessen Verwendung ergeben.

\section{- Arbeiten auf der Darstellungsebene}

Alle Arbeitsweisen erfolgen auf bestimmten Darstellungsebenen, die in Beziehung zu dem verwendeten Werkzeug stehen. Geht man von traditionellen Werkzeugen aus, kann man die Ebenen enaktiv, ikonisch, symbolisch unterscheiden. Untersucht man das Arbeiten mit einem Computeralgebrasystem oder einem Tabellenkalkulationsprogramm, betrachtet man in der Regel die Ebenen numerisch, graphisch, symbolisch. Das Arbeiten mit Darstellungen umfasst das Erstellen und das Ändern von Darstellungen sowie den Wechsel zwischen Darstellungen bzw. Darstellungsebenen.

\section{- Arbeiten auf der Objektebene}

Das Arbeiten auf der Darstellungsebene lässt sich beobachten. Um einen Bezug dieses Arbeitens zu mathematischen Objekten herzustellen, müssen die Darstellungen bzw. das Ändern von und das Operieren mit Darstellungen interpretiert werden. Bei einer Beschränkung auf diskrete Objekte lassen sich die Arbeitsweisen dahingehend unterscheiden, in welcher „Form“ mit den mathematischen Objekten (bzw. deren Darstellungen) gehandelt wird. Wir unterscheiden:

\section{- Algorithmisches Arbeiten}

Hierunter verstehen wir die Fähigkeit (Fertigkeit), einen Algorithmus, eine Berechnung(svorschrift) durchführen zu können.

- Iteratives Arbeiten

Beim iterativen Arbeiten wird eine Handlung, Gesetzmäßigkeit oder ein Muster sukzessive wiederholt. Iteratives Arbeiten umfasst das Erkennen und das Aufstellen einer solchen

\footnotetext{
${ }^{151}$ Wir werden uns im Hinblick auf die Ziele der empirischen Untersuchung in Kapitel 4 auf die Möglichkeiten eines TKP konzentrieren.
} 
Gesetzmäßigkeit und deren Fortführung. Hierbei sind Beziehungen zum algorithmischen Arbeiten erkennbar.

\section{Funktionales Arbeiten}

Funktionales Arbeiten beinhaltet, je nachdem welche der folgenden Aspekte betont bzw. angesprochen werden, verschiedene Fähigkeiten im Umgang mit Funktionen. In Anlehnung an Vollrath (1989) (vgl. auch Kap. 2.3.2) unterscheiden wir:

(a) Lokale Aspekte: Hier beschreibt oder stiftet man Zusammenhänge zwischen Größen (Zuordnungsaspekt) bzw. man betrachtet, wie sich Änderungen der einen Größe auf die andere auswirken ( ̈̈nderungsaspekt).

(b) Globale Aspekte: Hier betrachtet man die Menge aller Wertepaare bzw. die Zuordnung als Ganzes (ganzheitlicher Aspekt).

Diese Arbeitsweisen stehen in enger Beziehung zu den Darstellungen der mathematischen Objekte, mit denen gehandelt wird. Darüber hinaus müssen beim Arbeiten mit den mathematischen Objekten auch das jeweilige Werkzeug, d. h. dessen didaktischen Möglichkeiten und dessen Grenzen, berücksichtigt werden. Beim Einsatz eines Computers betrifft dies insbesondere den Umgang mit der in der Regel werkzeugspezifischen Syntax.

\subsection{Modelle zur Analyse des Verständnisses}

Handlungen stellen nach den oben skizzierten Lerntheorien eine Basis für die kognitive Konstruktion von Begriffen dar. Umgekehrt steuern die entsprechenden kognitiven Schemata zielgerichtetes Handeln (vgl. Dörfler 1988, S. 61). Damit ist noch nichts über die Qualität der Begriffsbildung bzw. das Verständnis der Begriffe ausgesagt. Um Begriffsbildungen analysieren zu können und den Lernerfolg abschätzen zu können, muss beschrieben werden, was mit dem „Verstehen“ gemeint ist. Zur Klärung des Verstehensbegriffs sind aus psychologischer und mathematikdidaktischer Sicht verschiedene Modelle entwickelt worden, ${ }^{152}$ wobei an dieser Stelle nur auf die Modelle von Beck und Maier (1993) sowie Vollrath (1984) und Skemp (1979) näher eingegangen werden soll.

Beck und Maier definieren „Verstehen“ als einen „durch äußere Impulse initiierter, zielgerichteten und idiosynkratischen Prozess der Sinnkonstruktion“ (Beck \& Maier 1993, S. 157). Dieser Sicht liegt das so genannte ,interpretative Paradigma“ zugrunde: „Dieses Paradigma unterstellt methodologisch, dass sich Bedeutungen erst im Ablauf der Interaktion [...] ausbilden und wandeln“ (a. a. O. S. 173). Das Interesse eines die Handlungen von Schülern untersuchenden Forschenden liegt damit auf der Rekonstruktion von Bedeutungen, d. h. es wird versucht, die gewonnenen Daten aus der Schülerperspektive zu analysieren und die individuellen und subjektiven Verstehensprozesse zu rekonstruieren. Verständnis wird in diesem Zu-

\footnotetext{
${ }^{152}$ Vgl. etwa Laucken 1984 sowie Beck \& Maier 1993, Skemp 1979, 1982, Herscovics und Bergeron 1983, Vollrath 1984.
} 
sammenhang als deskriptive Kategorie verstanden, insbesondere wird bewusst auf eine Wertung im Sinne einer Abgrenzung von „gutem“ und „,weniger gutem“ Verstehen verzichtet.

Charakteristisch für die Verständnismodelle von Vollrath (1984) und Skemp (1979) ist, dass sie verschiedene Formen oder Stufen des Verstehens unterscheiden und diese durch gewisse nachprüfbare Fähigkeiten und Kenntnisse in Verbindung mit dem Begriff beschreiben. Vollrath (1984, S. 209 f.) führt vier Stufen des Verständnisses an, die auf Kenntnisse bestimmter Aspekte, Eigenschaften und Darstellungen des Begriffs sowie auf die Fähigkeit, ihn im Rahmen von Problemlöseprozessen einsetzen zu können, abzielen.

- Der Begriff als Phänomen (intuitives Begriffsverständnis);

- Der Begriff als Träger von Eigenschaften (inhaltliches Begriffsverständnis);

- Der Begriff als Teil eines Beziehungsnetzes (integriertes Begriffsverständnis);

- Der Begriff als formales Objekt (formales Begriffsverständnis). ${ }^{153}$

Skemp (1979, S. 45) unterscheidet drei Formen des Verstehens, die sich jedoch stärker an beobachtbarem Verhalten orientieren:

Instrumentelles Verständnis zeigt sich in der Fähigkeit, eine erinnerte Regel zur Lösung einer Aufgabe adäquat anwenden zu können, ohne zu wissen, warum die Regel funktioniert.

- Beziehungsvolles (oder relationales) Verständnis zeigt sich in der Fähigkeit, spezifische Regeln oder Verfahren aus allgemeinen mathematischen Zusammenhängen ableiten zu können. Hierbei besitzt der Schüler ein Wissen sowohl darüber, wie eine Regel funktioniert, als auch darüber, warum sie funktioniert.

- Formales (oder logisches) Verständnis zeigt sich in der Fähigkeit, mathematische Symbole und Notationen mit relevanten mathematischen Ideen verbinden zu können und logische Schlüsse durchführen zu können.

Bei der im Rahmen dieser Arbeit durchgeführten Untersuchung (vgl. Kap. 5) ist das Verständnismodell von Skemp von besonderem Interesse, da nicht nur dem Ergebnis eines Lernprozesses und den Lernprodukten Aufmerksamkeit geschenkt werden soll, sondern insbesondere der Lernprozess bzw. die Arbeitsweisen der Schüler genauer beschrieben und analysiert sowie die Beziehung zum zugrunde liegenden bzw. entwickelten Begriffsverständnis untersucht werden sollen. Es wird jedoch auch das Modell von Vollrath hinzugezogen, wobei sich zum Teil Überschneidungen ergeben, wie die folgenden Darstellungen zum Erwerb der Begriffe Folge und Differenzenfolge verdeutlichen:

\footnotetext{
${ }^{153}$ Im Hinblick auf das Lehren des Funktionsbegriffs führt Vollrath (1994, S. 137 ff.) noch eine weitere, 5. Stufe an: das kritische Begriffsverständnis. Schüler, die diese Stufe erreicht haben, können sich kritisch mit dem Begriff auseinander setzen, sie erkennen Beziehungen zum Relationsbegriff, kennen unterschiedliche Möglichkeiten zur Definition und können einfache Beweise führen. Im Rahmen dieser Arbeit soll jedoch nicht zwischen formalem und kritischem Begriffsverständnis unterschieden werden.
} 


\section{Beispiel: Ein Stufenschema zum Erwerb der Begriffe Folge und Differenzenfolge}

\section{- Stufenschema von Vollrath}

\section{Intuitives Verständnis}

- Die Schüler können einfache Folgen durch das Analysieren der Differenzenfolge iterativ fortsetzen.

- Sie verbinden mit dem Begriff der Differenzenfolge Vorstellungen über das Änderungsverhalten einer Ausgangsfolge.

\section{Inhaltliches Verständnis}

- Die Schüler kennen Eigenschaften bestimmter Differenzenfolgen. Insbesondere wissen sie, dass die $n$-te Differenzenfolge einer arithmetischen Folge $n$-ten Grades konstant ist.

- Sie kennen verschiedene Darstellungen einer Folge und deren Differenzenfolge (Tabelle, Graph, Term).

- Sie können Eigenschaften einer Folge mit Hilfe von Eigenschaften ihrer Differenzenfolge beschreiben.

\section{Integriertes Verständnis}

- Die Schüler können das Konzept der Differenzenfolge als heuristische Strategie beim Lösen von Zählproblemen anwenden.

- Sie können das Konzept der Differenzenfolge in das Konzept der Z-Funktionen und deren Differenzenfunktionen einordnen.

- Sie können Beziehungen zwischen Eigenschaften einer Klasse von (quadratischen) Z-Funktionen und deren Differenzenfunktionen beschreiben und begründen.

- Sie kennen Analogien zwischen dem Konzept der Differenzenfunktionen und der Differenzialrechnung.

\section{Formales Verständnis}

- Die Schüler können beweisen, dass die $(n-1)$-te Differenzen einer polynomialen Folge $n$-ten Grades nicht konstant und die $n$-ten Differenzen dieser Folge konstant sind. Sie können weiterhin zeigen, dass auch die Umkehrung dieser Aussage gilt.

- Die Schüler kennen die Begriffe „Differenzenoperator“, „diskrete Stammfunktion“ und die Regel für die partielle Summation.

- Sie kennen die Bedeutung des Differenzenkalküls zur Auswertung von Summen, die sich z. B. im Rahmen von Abzählproblemen ergeben können. Sie finden - ausgehend von der Summendarstellung einer Folge - mit Hilfe des Differenzenkalküls eine explizite Darstellung dieser Folge. 
- Stufenschema von Skemp

Instrumentelles Verständnis

- Die Schüler können die Differenzenfolge einer gegebenen Folge bestimmen.

- Die Schüler können einfache Folgen durch das Analysieren der Differenzenfolge iterativ fortsetzen.

\section{Relationales Verständnis}

- Die Schüler können das Konzept der Differenzenfolgen/-funktionen zum Lösen einfacher (Zähl-)Probleme einsetzen.

- Sie können Beziehungen zwischen Eigenschaften einer Klasse von (quadratischen) Z-Funktionen und deren Differenzenfunktionen beschreiben und begründen.

\section{Formales Verständnis}

- Die Schüler können beweisen, dass die $(n-1)$-te Differenzen einer polynomialen Folge $n$-ten Grades nicht konstant und die $n$-ten Differenzen dieser Folge konstant sind. Sie können weiterhin zeigen, dass auch die Umkehrung dieser Aussage gilt.

- Sie kennen die Bedeutung des Differenzenkalküls zur Auswertung von Summen, die sich z. B. im Rahmen von Abzählproblemen ergeben können. Sie finden - ausgehend von der Summendarstellung einer Folge - mit Hilfe des Differenzenkalküls eine explizite Darstellung dieser Folge.

Die Verstehensmodelle von Vollrath und Skemp drücken gestufte Zielvorstellungen beim Lernen von Mathematik aus. Vertreter des ,interpretativen Paradigmas“ bezeichnen daher dieses Modell in Abgrenzung zum deskriptiven Verstehensbegriff als „,normativ“ oder „defizitorientiert", da das Handeln des Schülers an Verfahren und Ergebnissen gemessen wird, die als richtig oder ideal eingeschätzt werden. Zwischen normativem und deskriptivem Paradigma bestehe eine „entscheidende methodologische Differenz“ (Beck \& Maier 1993, S. 172), sie würden somit grundlegende Orientierungen darstellen, die sich ausschließen. Nach Ansicht von Weigand handelt es sich bei den beschriebenen Verständnismodellen jedoch nicht um Alternativen in der mathematikdidaktischen Forschung, sondern vielmehr um ,verschiedene Arten des Verständnisses [handelt], die beide in der Mathematikdidaktik berücksichtigt und hinsichtlich Möglichkeiten und Grenzen diskutiert werden sollten“ (Weigand 1995, S. 147). Bei der Begriffsentwicklung sollten in diesem Sinne sowohl die Lernziele nicht aus den Augen verloren werden als auch die individuellen Lernprozesse angemessen berücksichtigt werden. Im Rahmen unserer Untersuchung werden wir versuchen, beide Aspekte zu beachten. Um der Individualität der Schüler gerecht zu werden, werden die Arbeitsweisen mit Hilfe eines Aufzeichnungsinstruments dokumentiert. Damit ist eine detaillierte Analyse und Interpretation von Einzelfällen möglich. Insbesondere wird bei der Interpretation des gesamten Datenmaterials darauf geachtet, dass zunächst der Fokus auf einer Beschreibung der Ar- 
beitsweisen liegt und erst abschließend eine Beziehung zu den obigen Stufenschemata hergestellt bzw. eine „Bewertung“ des Begriffsverständnisses vorgenommen wird.

\subsection{Zusammenfassung}

Anschließend an die genetische Erkenntnistheorie Piagets entstanden Lerntheorien, die Handlungen als wesentliche Komponente des Lernprozesses betrachten. In diesem Zusammenhang wurden verschiedene operative Prinzipien für den Mathematikunterricht formuliert. Diese Prinzipien verweisen auf die unterschiedlichen Stadien der Denkentwicklung, auf die sukzessive Verinnerlichung von Begriffen und Operationen ausgehend von konkreten oder inhaltlich-anschaulichen Handlungserfahrungen sowie auf das operative Durcharbeiten von Begriffen auf verschiedenen Darstellungsebenen. Darüber hinaus wird mit Wittmann die steuernde Wirkung des erweiterten operativen Prinzips (,Was passiert mit ..., wenn ...?“) bei Problemlöseprozessen herausgestellt. Wie anhand einiger Beispiele aufgezeigt wurde, können viele diskrete Begriffe und Probleme auf einer konkreten oder inhaltlich-anschaulichen Ebene im Sinne der operativen Prinzipien behandelt werden.

Nicht alle Darstellungen eignen sich gleich gut zur Initiierung und Steuerung von Lernprozessen. Was eine sinnvolle Auswahl an Darstellungen ist und wie Schüler mit bestimmten mathematischen Begriffen umgehen, lässt sich letztendlich nur empirisch klären. Hierbei ist es erforderlich, die individuellen Arbeitsweisen der Schüler sowie deren Begriffsvorstellungen näher zu analysieren. Mit dem Begriff der Arbeitsweisen werden tatsächlich beobachtbare Tätigkeiten und Handlungen der Schüler bezeichnet, wobei drei verschiedene Ebenen unterschieden werden: die Werkzeug-, Darstellungs- und Objektebene.

Durch den Einsatz neuer Technologien ergeben sich nun vielfältige neue Möglichkeiten, um mit diskreten Objekten auf eine neue Art zu operieren. Die didaktisch-methodischen Möglichkeiten, die sich insbesondere bei Tabellenkalkulationsprogrammen unter Berücksichtigung der skizzierten Lerntheorien ergeben, sind Gegenstand des nächsten Kapitels. 


\section{Tabellenkalkulationsprogramme: Didaktisch-methodische Möglichkeiten - konkrete Arbeitsweisen}

Bei vielen diskreten Inhalten insbesondere aus dem Bereich der Kombinatorik, der Differenzengleichungen und der diskreten Analysis fallen häufig zeitaufwändige, aber elementare Berechnungen an. Durch den Einsatz neuer Technologien kann dieser operative Aufwand reduziert und die Aufmerksamkeit des Lernenden stärker auf übergeordnete Fragestellungen gerichtet werden. Während Computeralgebrasysteme (CAS) wie DERIVE vorrangig das Arbeiten auf der symbolischen Ebene unterstützen, ermöglichen Tabellenkalkulationsprogramme (TKP) einen vielfältigen Umgang mit tabellarisch aufbereiteten numerischen Daten. Dabei spiegelt der zellenartige Aufbau der TKP-Arbeitsblätter die mit diskreten Prozessen verbundenen Vorstellungen in besonderer Weise wider (vgl. Weigand 2001a, S. 3).

In diesem Kapitel sollen die didaktische Bedeutung von TKP bei der Gestaltung von Lehrund Lernprozessen näher analysiert und die damit verbundenen neuen Möglichkeiten des Arbeitens und Operierens im Sinne der Klassifizierung von Kapitel 3.3 herausgestellt werden.

\subsection{Arbeiten mit Darstellungen}

Ein reflektierter Einsatz von und Umgang mit Darstellungen ist ein zentrales Anliegen eines operativen Mathematikunterrichts (vgl. Kap. 3.2). Dabei sollten Darstellungen nach Dörfler (1989a, 1991) nicht nur Hilfsmittel am Weg zum „eigentlichen“ Begriff sein, sondern als kognitive Werkzeuge im Sinne mentaler Prototypen ständig verfügbar bleiben.

„Ich halte diese Verfügbarkeit von mentalen Prototypen für mathematische Begriffe und Operationen ganz allgemein für ungemein wichtig, weil das Denken vorzugsweise gegenständlich erfolgt und nicht abstrakt sich nur auf abgelöste Eigenschaften und Relationen beziehen kann. [...] Dies entspricht auch meiner Position, dass didaktische Zugänge zu mathematischen Begriffen nicht nur Mittel zum Zweck sind, sondern als dauerhafte Denkmittel (eben als Prototypen) einsetzbar sein sollen.“(Dörfler 1989a, S. 81 f.)

Wie ein solcher Einsatz und Umgang mit Prototypen unter Ausnutzung eines TKP aussehen kann und zu welchen Aspekten oder Ziele des Mathematikunterrichts dabei ein Beitrag geleistet werden kann, soll im Folgenden exemplarisch verdeutlicht werden.

\subsubsection{Generalisieren und Formalisieren von Gesetzmäßigkeiten}

Wie in Kapitel 2.3.1 aufgezeigt, bieten diskrete Zählprobleme eine Möglichkeit, das Variablenkonzept zu verdeutlichen bzw. das Formalisieren von Sachsituationen zu üben. Dabei können die Zellen eines TKP in Verbindung mit den Operationen, die sich auf diese Felder beziehen, verschiedene Variablenaspekte im Sinne Malles (1993) verdeutlichen. So lässt sich etwa der Einsetzungsaspekt betonen, indem Zellen Werte zugewiesen oder in Zellen Werte verändert werden. Werden Zellen durch die Verwendung von Zelladressen interaktiv miteinander verknüpft, wirkt sich die Veränderung eines Werts in der Ausgangszelle automatisch 
auf den Wert der Ergebniszelle aus. Die Ausgangszelle kann somit als Platzhalter in Formeln fungieren. Darüber hinaus unterstützt ein TKP auch den Gegenstandsaspekt von Variablen, bei dem diese als allgemeine Rechenobjekte betrachtet werden. So wird in der Ergebniszelle bzw. den Formeln, die dieser Zelle zugewiesen werden, nicht nur auf den Wert der Ausgangszelle(n), sondern auf die Zelle(n) selbst, d. h. deren Adresse bzw. Name, Bezug genommen. „Das TKP integriert sozusagen die arithmetische und die algebraische Ebene“ (Dörfler 1989, S. 81).

Das Arbeiten mit Variablen kann im Kontext der TKP-Umgebung als ein Arbeiten mit Zellen oder Zelladressen angesehen werden. Da die Zellen eines TKP am Bildschirm visuell wahrnehmbar sind, bilden sie eine „Vergegenständlichung“ von Variablen und können zur Entwicklung eines (mentalen) Prototypen für das Variablenkonzept beitragen:

„Durch das systematische Arbeiten mit dem TK (=Tabellenkalkulationsprogramm, S.T.) kann sich die Verwendung der Felder zu einem kognitiven Prototyp für das Konzept der Variablen entwickeln, der für den Schüler auch später den Gebrauch von Variablen steuert und anleitet. [...] Das algebraische Denken kann dann vermittelt durch den Prototyp ,TK und Felder" erfolgen, zumindest kann immer wieder darauf rekurriert werden.“ (Dörfler 1989, S. 81)

Ausgehend von diesem Variablenmodell kann sukzessive der Umgang mit der „echten“ algebraischen Syntax entwickelt werden. ${ }^{154}$ Dieses Vorgehen bietet sich insbesondere bei der Formulierung rekursiver Gesetzmäßigkeiten an. So betont Neuwirth:

„In rein algebraischer Schreibweise ist diese Darstellung oft für viele Lernende schwierig, weil der Rekursion in dieser Schreibweise fälschlicherweise das Odium der inkonsistenten Selbstreferenz anhaftet. In einer Tabellendarstellung wird aber klar, dass Rekursion einfach eine Berechnungsmethode ist, die auf , die Zelle darüber“ oder ,die Zelle links darüber“ Bezug nimmt.“ (Neuwirth 2001, S.52)

Durch die Verwendung relativer Zellbezüge und die Möglichkeit des Kopierens von Zellen lassen sich Ausdrücke, wie „Summe der Zelle darüber und der Zelle links darüber“, direkt in mathematische Handlungen umsetzen und Gesetzmäßigkeiten generalisieren.

„Der Vorgang des Kopierens von Formeln ist eine Operationalisierung des Begriffs ,Verallgemeinern“, und die Verwendung von relativen Zellverweisen, in die Zelle darüber' und, in die Zelle daneben“ ist eine Operationalisierung der Rekursion (bzw. der vollständigen Induktion).“ (a. a. O., S. 64)

Dass bereits 12- bis 13-jährige Schüler mit Hilfe eines TKP wertvolle Teilerfahrungen mit rekursiven Zusammenhängen machen können, zeigt der von Hoyles, Sutherland und Healy (1991) beschriebene Unterrichtsversuch zur Behandlung der Polygonalzahlen. Den Schülern standen hierbei ein Aufgabenblatt, auf dem jeweils die ersten vier Glieder der Folgen zusammen mit einer geometrischen Veranschaulichung dargestellt waren, sowie das TKP Excel zur Verfügung. Die Schüler hatten die Aufgabe, mit Hilfe von Excel weitere Folgenglieder zu berechnen und dabei nach verschiedenen Wegen zur Darstellung des allgemeinen Sachver-

${ }^{154}$ Vgl. hierzu jedoch auch die kritischen Anmerkungen von Wynands (1991). Demnach ist der Einsatz eines TKP zur Entwicklung des Variablenbegriffs wenig förderlich, sofern nur (unreflektiert) mit Zahlen hantiert wird. Dies widerspricht aber nicht der Auffassung Dörflers, der insbesondere die Bedeutung der Aufmerksamkeitsfo- 
halts zu suchen. Ausführlich berichten Healy, Hoyles und Sutherland über die Arbeitsweisen der 12-jährigen Schülerinnen Penny und Nadine. Obwohl diese beiden noch keine Erfahrung im Formulieren rekursiver Gesetzmäßigkeiten hatten, legten hier der Sachverhalt und auch das Medium TKP einen rekursiven Zugang bei der Darstellung der Dreiecks- und Fünfeckszahlen nahe. So gelang es den Schülerinnen, den allgemeinen Zusammenhang zunächst durch Gesten, d. h. durch ein Hinzeigen mit den Fingern auf die jeweils in Beziehung stehenden Zellen, und dann durch das Ausnutzen relativer Zellbezüge zu erfassen.

„Her ambiguous verbal description was clarified by accompanying gestures pointing to cells of the spreadsheet array on the computer screen - the cells are ,mathematical objects' and the gestures capture the general relationship between them. Penny's construction illustrates how the medium structures the mathematical discourse - here in a way which made recurrence relations ,natural'." (Hoyles 1993, S. 5 f.)

Auch der umgekehrte Weg ist vorstellbar. Zur Vertiefung des Variablenkonzepts und im Sinne des Prinzips der Variation der Darstellungsebenen kann ein einmal entdeckter und bereits formalisierter Zusammenhang in eine Tabelle eines TKP übertragen werden. Bei einfachen Zählproblemen, wie der „Streichholzfolge“ aus Kapitel 2.2.1, bietet es sich an, die ersten Folgenglieder zunächst numerisch darzustellen. Soll - ausgehend von der expliziten Darstellung $A(n)=3 n+2$ - eine größere Anzahl von Folgengliedern berechnet werden, ist es sinnvoll, die TKP-Syntax zu verwenden und die Tabelle durch Kopieren der entsprechenden Formel zu erzeugen. Das Verbalisieren der Berechnungsmethode durch Verweis auf die verwendeten Zellen (,Multipliziere die Zelle links daneben mit 3 und addiere 2“) bzw. das Verwenden bedeutungsvoller Namen (,Multipliziere die Stufe [Nummer] der Streichholzzahl mit 3 und addiere 2") kann hierbei helfen, den Sachverhalt mit Hilfe des TKP formal bzw. durch Anklicken der entsprechenden Zellen zu erfassen (vgl. Abb. 23). Bei diesem Vorgehen werden im Umgang mit Variablen verschiedene Formalisierungsstufen angesprochen. Ausgehend von der algebraischen Formel wird der gesetzmäßige Zusammenhang zunächst numerisch erfasst und

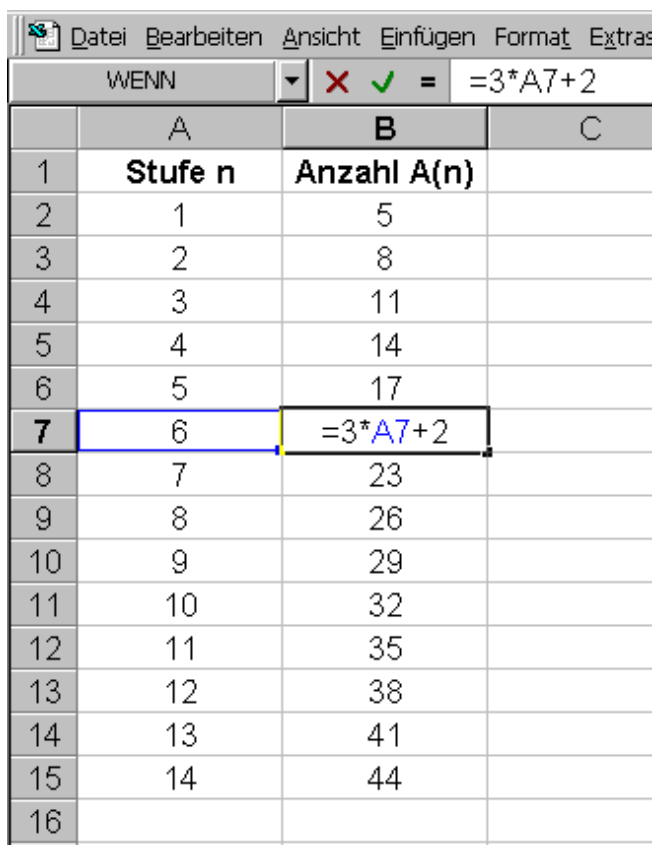
Abb. 23: Erfassen und Berechnen der
„Streichholzfolge“ mit Hilfe eines TKP dann - in Verbindung mit einer sprachlichen Begleitung - in die TKP-Syntax übersetzt. Schornstein (1996) spricht in diesem Zusammenhang von Buchstaben-, Pseudo- und Wortvariablen, die zur Vermeidung von Schwierigkeiten im Umgang mit Variablen angemessen berücksichtigt werden sollten. 


\subsubsection{Beitrag zur Entwicklung des Folgenbegriffs}

Beim Arbeiten mit einem TKP steht zunächst das Tabellenblatt im Vordergrund. Hier können Daten eingegeben, analysiert und anschließend gegebenenfalls graphisch aufbereitet werden. Damit besteht im Hinblick auf die Behandlung des Funktions- und insbesondere des Folgenbegriffs die Möglichkeit, numerische Aspekte bzw. das Arbeiten mit der Darstellung Tabelle stärker zu betonen. Anhand der Tabelle kann die Eindeutigkeit funktionaler Zuordnungen besonders gut verdeutlicht werden. Daneben ermöglicht die tabellarische Darstellung die Analyse „vertikaler Zusammenhänge“ bzw. die Änderung einer Größe auf eine abhängige Größe (vgl. Vollrath 1989, S. 12) und damit auch die Konstruktion operativer (bzw. relationaler) Prototypen im Sinne Dörflers. So können z. B. die Differenzen und Quotienten aufeinander folgender Folgenglieder einer geometrischen Folge in einer Tabellenspalte ohne großen operativen Aufwand berechnet werden, indem zunächst die Differenz bzw. der Quotient des ersten und zweiten Folgenglieds unter Ausnutzung relativer Zellbezüge bestimmt und anschließend diese Formel in die gesamte Spalte kopiert wird. Da nun ein TKP eine Wiederholung derselben Operation an verschiedenen geometrischen Folgen (bzw. deren tabellarischen Darstellungen) auf einfache Weise ermöglicht, kann die Aufmerksamkeit des Lernenden auf bestimmte Eigenschaften gerichtet werden, die bei diesen Manipulationen invariant bleiben und somit die konstitutiven Eigenschaften des Begriffs - in diesem Fall die konstante Wachstumsrate - bilden. Alternativ kann der Schüler eine tabellarische Darstellung einer oder mehrerer rekursiv dargestellter geometrischer Folgen erstellen. Hierbei wird die konstitutive Eigenschaft durch das Kopieren der konstanten Wachstumsrate betont (vgl. Dörfler 1991, S. 70).

Neben den numerischen Möglichkeiten bietet ein TKP auch eine Vielfalt graphischer Darstellungsformen an, die technisch einfach erstellt werden und das Arbeiten auf einer inhaltlichanschaulichen Ebene unterstützen können.

„Der Computer mit seinen vielfältigen graphischen (und symbolischen) Darstellungsmöglichkeiten erscheint mir [...] als ein Medium, das entscheidend das Denken in und mit gegenständlichen Prototypen unterstützen kann. Zum Beispiel erlaubt Computergrafik die Realisierung von ,Gegenständen', die mit materiellen Mitteln nicht oder nur mit großem Aufwand herstellbar sind. Der Computer bietet gewissermaßen Prototypen an, die ohne ihn nicht verfügbar sind.“ (Dörfler 1991, 68 f.)

Darüber hinaus haben Lernende die Möglichkeit, mit verschiedenen Prototypen gleichzeitig zu arbeiten und Beziehungen zwischen diesen herzustellen. So kann etwa unter Ausnutzung der interaktiven Zellverknüpfung die Auswirkung des Parameters $a$ auf die graphische Darstellung der geometrischen Folge $y$ mit $y_{n}=a^{n}$ untersucht werden. Auch hierbei lassen sich bestimmte „Invarianten“ feststellen, die wesentliche Eigenschaften des Begriffs darstellen und damit zur Konstruktion eines operativen Prototyps beitragen können. So erkennt man, dass alle Folgen der Klasse $\left\{y_{n}=a^{n}, a>1\right\}$ monoton steigen. Die Folgen der Klasse 
$\left\{y_{n}=a^{n}, 0<a<1\right\}$ fallen dagegen monoton und nähren sich dabei asymptotisch Null (vgl. Abb. 24).
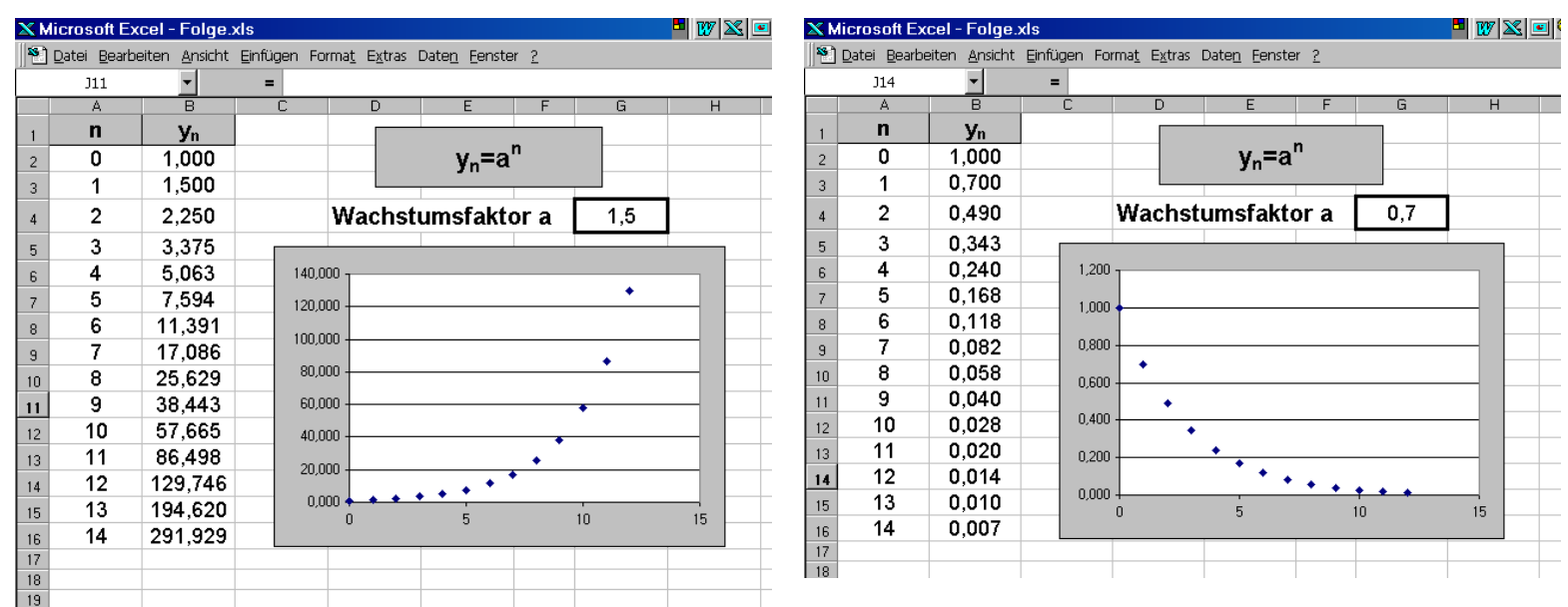

Abb. 24: Graphische Darstellung von zwei geometrischen Folgen des Typs $y_{n}=a^{n}$

Durch das parallele Arbeiten mit verschiedenen Darstellungen und das gleichzeitige Sehen von Eigenschaften von Folgen, kann auch der Transfer zwischen verschiedenen Darstellungsformen erleichtert werden. Im Rahmen einer empirischen Untersuchung zu Übersetzungsqualifikationen von Schülern zwischen den Darstellungsformen Funktionsgleichung, -graph und -tabelle stellte Müller-Philipp (1994) fest, dass ein computerunterstützter Unterricht insbesondere zu einer Verbesserung der Leistungen bei Übersetzungsprozessen Graph-Term und umgekehrt führen kann. Bei dieser Untersuchung wurde ein Funktionenplotter ${ }^{155}$ eingesetzt und der Tabellendarstellung sowohl im Hinblick auf die Auswahl der Software als auch bei der Formulierung der Testfragen eine bewusst geringe Bedeutung beigemessen. Somit bleibt die Frage offen, ob durch den Einsatz eines TKP insbesondere der wechselseitige Transfer zwischen Tabelle und Term verbessert werden kann bzw. eine Beziehung zwischen Tabelle und Term besser erkannt wird.

\subsubsection{Entdecken und Beweisen kombinatorischer Beziehungen}

Die spezielle Struktur des Tabellenblatts eines TKP kann bei der Konstruktion einfacher Beweise für kombinatorische Formeln visuelle Hilfestellungen bieten. Insbesondere ist es möglich, formale algebraische Schlüsse durch inhaltlich-anschauliche Überlegungen zu ersetzen, also einen präformalen Beweis zu führen (vgl. hierzu Neuwirth 1995a, 2001).

\footnotetext{
${ }^{155}$ Es handelt sich hierbei um ein Programm zum Zeichnen von Funktionsgraphen.
} 


\section{Beispiel: Beziehungen am Pascal'schen Dreieck}

Zur Berechnung der ersten Stufen des Pascal'schen Dreiecks mit Hilfe eines TKP kann die rekursive Beziehung

$$
\left(\begin{array}{l}
n \\
k
\end{array}\right)=\left(\begin{array}{l}
n-1 \\
k-1
\end{array}\right)+\left(\begin{array}{c}
n-1 \\
k
\end{array}\right)
$$

genutzt werden. Diese Beziehung spiegelt sich in den Handlungen wider, d. h. dem Anklicken entsprechender Zellen und dem anschließenden Kopieren der jeweiligen Formeln. Darüber hinaus besteht die Möglichkeit, die Struktureigenschaften des Dreiecks mit Hilfe einer speziellen Pfeildarstellung, der so genannten „Detektiv“-Option zu visualisieren. ${ }^{156}$ Dabei stellen „Spurpfeile“ eine Beziehung zwischen - über relative Zellbezüge miteinander verbundenen Zellen her. Hierbei sind zwei Vorgehensweisen möglich. Die Kennzeichnung derjenigen Zellen, deren Inhalt in eine bestimmte ausgezeichnete Zelle einfließen, erfolgt mit der Schaltfläche „Spur zum Vorgänger“. Dabei werden die Pfeile nach der Zielzelle gebündelt. Auf diese Weise lässt sich die obige rekursive Beziehung der Binomialkoeffizienten visualisieren (vgl. Abb. 25).

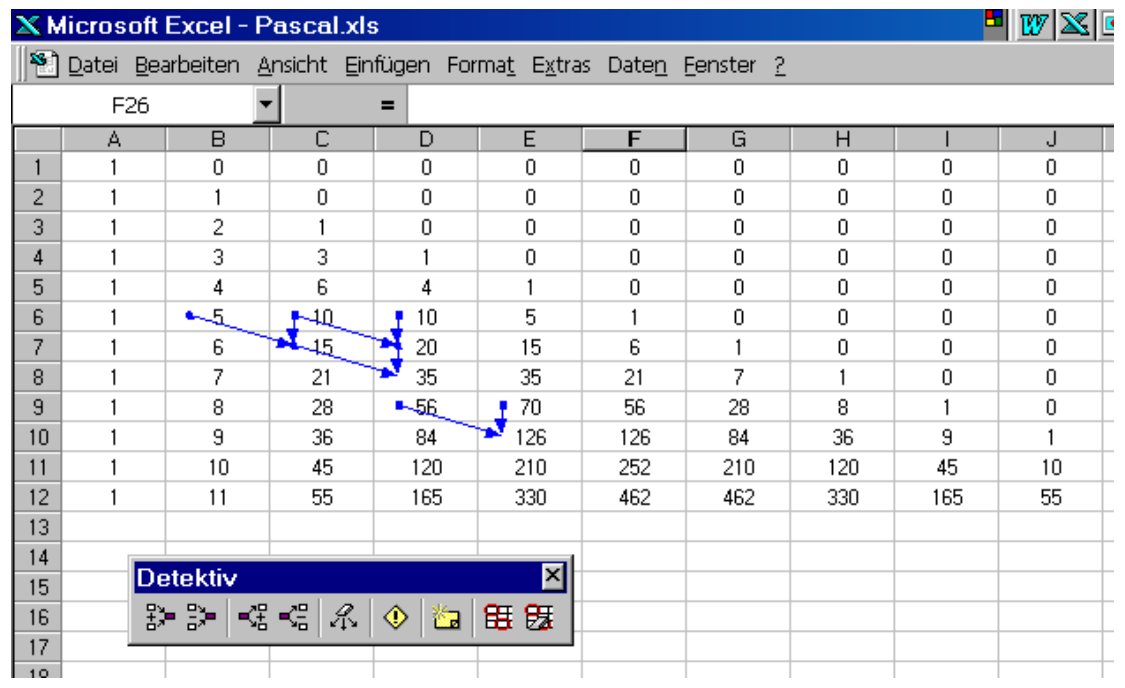

Abb. 25: Detektiv-Option: Visualisieren der rekursiven Struktur des Pascal'schen Dreiecks

Bündelt man dagegen die Pfeile nach der Ausgangszelle bzw. überlegt man sich, in welche Zellen der Inhalt einer bestimmten Zelle unmittelbar eingeht, erhält man das folgende Bild:

\footnotetext{
${ }^{156}$ Neuwirth $(1995 b, 2001)$ verwendet zur Verdeutlichung der Struktureigenschaften des Pascal'schen Dreiecks so genannten „Pfeildiagramme“. Es handelt sich hierbei um eine zeichnerische Darstellung der Tabelle und der in Beziehung stehenden Zellen. Auch wenn die Detektiv-Option von Neuwirth nicht explizit erwähnt wird, kann die „Pfeildarstellung“ als deren Pendant auf der traditionellen „Bleistift und Papier“-Ebene aufgefasst werden.
} 


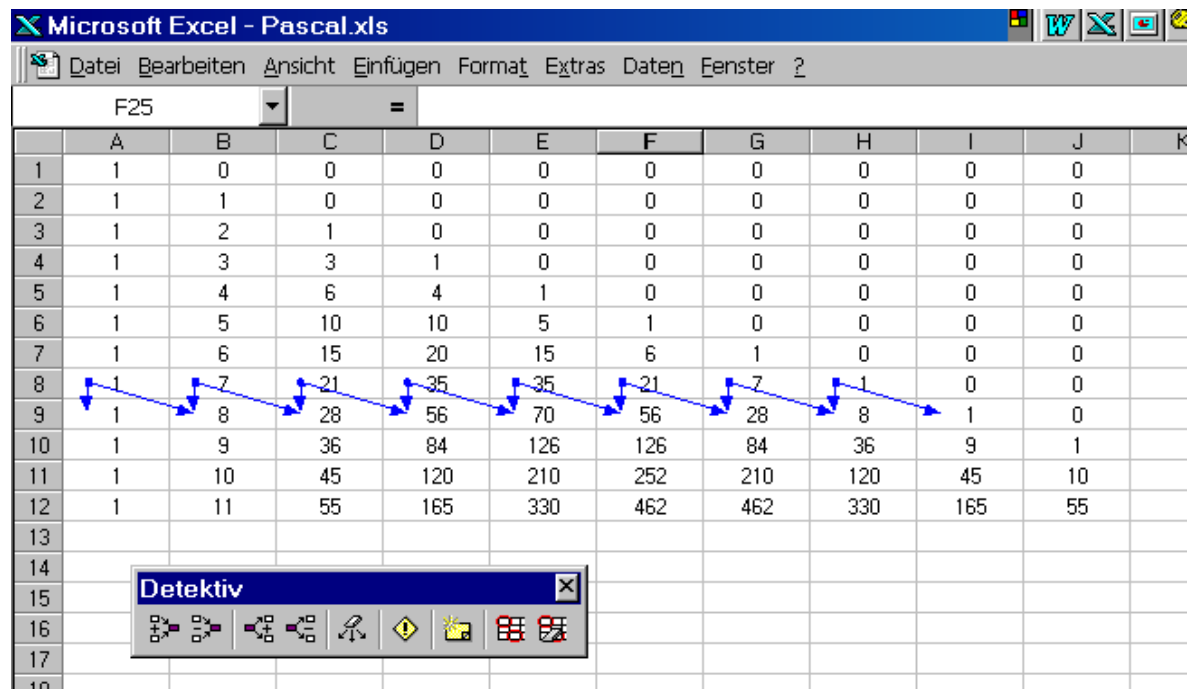

Abb. 26: Detektiv-Option: Bündelung nach der Ausgangszelle

Man erkennt hier, dass der Inhalt jeder Zelle einer bestimmten Zeile in der folgenden Zeile sowohl in der unmittelbar ,darunter liegenden“ Zelle als auch in der Zelle „rechts daneben“ verarbeitet wird. Da die Zellen über die obige Rekursionsbeziehung additiv miteinander verbunden sind, wird ohne weitere algebraischen Umformungen offensichtlich, dass sich die Zeilensumme von Zeile zu Zeile verdoppelt. Da die Zeilensumme der ersten Zeile des Pascal'schen Dreiecks (Tabellenzeile 1) den Wert 1 besitzt, ist die Summe der Zellinhalte in der $n$-ten Zeile $2^{n-1}$. Damit ist die Gleichung $\sum_{k=0}^{n-1}\left(\begin{array}{c}n-1 \\ k\end{array}\right)=2^{n-1}$ bzw. $\sum_{k=0}^{n}\left(\begin{array}{l}n \\ k\end{array}\right)=2^{n}$ präformal und im Sinne eines operativen Vorgehens (vgl. Kap. 3.2.2.2) bewiesen. ${ }^{157}$

„Der Beweis liefert dank seiner Struktur auch mehr Einsicht als der übliche Beweis mittels vollständiger Induktion. In unserer Vorgehensweise wird die vollständige Induktion implizit durch den Bezug auf die vorhergehende Zeile verwendet, aber nicht , algebraisiert"." (Neuwirth 2001, S. 58)

\subsection{Experimentelles Arbeiten}

Experimentelles Arbeiten wird in der didaktischen Literatur häufig auch als entdeckendes, exploratives, heuristisches, heuristisch-experimentelles Arbeiten bezeichnet, bzw. die Begriffe werden synonym ohne genaue Abgrenzung nebeneinander verwendet (vgl. Schneider 2000, S. 163 ff.). Mit der Betonung des „Experimentellen“ soll im Allgemeinen deutlich gemacht werden, dass bei dieser Methode ein zielgerichtetes Untersuchen von konkretem Daten- oder Beispielmaterial im Vordergrund steht, bei dem es um das Aufstellen und Überprüfen von Vermutungen bzw. das Entwickeln von Beweis- und Lösungsstrategien geht. In diesem Sinne

\footnotetext{
${ }^{157}$ Das Problem der Indextransformation kann vermieden werden, indem man die erste TKP-Tabellenzeile als Zeile 0, die zweite Tabellenzeile als Zeile 1 usw. bezeichnet.
} 
kann experimentelles Arbeiten auch als induktives Vorgehen eingeordnet werden (vgl. Pruzina 1989). ${ }^{158}$

Experimentelles Arbeiten steht in zweifacher Hinsicht in Beziehung zur operativen Behandlung eines mathematischen Gegenstands. So betrachtet Fricke - wie bereits in Kapitel 3.2.1 herausgestellt - die heuristisch-erforschende Erarbeitung, d. h. eine „Phase des spielerischen Umgangs, des experimentierenden Erprobens und Erforschens" (Fricke 1970, S. 97) als integralen Bestandteil seiner operativen Methode. Andererseits finden sich im experimentellen Arbeiten selbst Bezüge zum „erweiterten operativen Prinzip“ nach Wittmann. So hebt Kautschitsch (1999, S. 176), neben der Analyse der Situation, der Sammlung von Daten und deren Darstellung in Tabellen und Graphen, auch das systematische Abändern einzelner Bedingungen und das Beobachten der Auswirkungen unter Berücksichtigung des Frageschemas „Was geschieht ..., wenn ...?“ als wichtige Komponenten des experimentellen Arbeitens hervor. Experimentelles Arbeiten - insbesondere in Verbindung mit dem Aspekt der Variation kann somit als ein wesentlicher Bestandteil einer Behandlung eines mathematischen Gegenstands im Sinne des operativen Prinzips angesehen werden.

Durch die Verfügbarkeit technologischer Hilfsmittel erhält das experimentelle Arbeiten im Mathematikunterricht eine neue Bedeutung. Neben CAS können insbesondere TKP als Experimentierwerkzeug genutzt werden. TKP unterstützen in diesem Zusammenhang vor allem die folgenden Tätigkeiten:

- Mit Hilfe eines TKP können numerische Berechungen ohne großen zeitlichen und rechentechnischen Aufwand durchgeführt und wiederholt werden. ${ }^{159}$

- TKP bieten eine Vielfalt an Darstellungsmöglichkeiten, die einfach erstellt und abgeändert werden können. Durch die Integration spezieller Schaltflächen besteht darüber hinaus die Möglichkeit, komfortabel zwischen Tabellenblättern und damit auch zwischen verschiedenen Darstellungsformen zu wechseln (vgl. auch Kap. 4.3).

- Mit Hilfe eines TKP können Variablen- und Parameterbelegungen einfach geändert werden. Da Zellen und auch Darstellungen interaktiv miteinander verknüpft werden können, führt eine Parameteränderung zu einer direkten Aktualisierung der damit gekoppelten numerischen und graphischen Darstellungen. Das Variieren der Parameter kann technisch auf zwei Arten realisiert werden: Zum einen können verschiedene numerische Werte schrittweise in eine Zelle eingegeben werden, zum anderen lassen sich Parameter sehr bequem mit Hilfe eines Rollbalkens verändern. Indem mit dem Rollbalken in kurzer Zeit

\footnotetext{
${ }^{158}$ Pruzina (1989) befasst sich mit einem Spezialfall experimentellen Arbeitens, dem so genannten ,numerischen Experimentieren“. Hierbei steht das zielgerichtete Auswerten von Zahlenmaterial mit Hilfe des Taschenrechners im Vordergrund.

${ }^{159}$ Damit wird insbesondere das numerische Experimentieren unterstützt. Dagegen besitzen CAS ein stärkeres Potenzial beim Arbeiten auf der symbolischen Ebene, was zur selbstständigen Erschließung mathematischer Regeln und Verfahren durch Schüler ausgenutzt werden kann (vgl. Schneider 2000, S. 177 ff.).
} 
sehr viele Parameter verändert werden können, wird der Eindruck des dynamischen Visualisierens verstärkt. ${ }^{160}$

Damit ergibt sich die Möglichkeit zur Realisierung eines „,computerunterstützten operativen Prinzips“ (Kautschitsch, Keune \& Henning 1999, S. 15). Hierbei kann zwischen einem objekt- und eigenschaftsbezogenen operativen Prinzip unterschieden werden: „Bei der ersten Form sucht man nach möglichen und interessanten Operationen an gegebenen Objekten (Konstellationen) und studiert, welche Auswirkungen sie besitzen. Bei der zweiten Form geht man von einer Eigenschaft oder Beziehung zwischen Objekten aus und sucht nach Operationen (Handlungen), die diese invariant lassen“ (a. a. O., S. 15 f.).

Im Folgenden wird anhand einiger Beispiele deutlich gemacht, wie ein von TKP unterstütztes experimentelles Arbeiten einen operativen Umgang mit diskreten Begriffen ermöglicht und als Instrument zur Erkundung und Lösung problemhaltiger Situationen eingesetzt werden kann.

\subsubsection{Erkunden von Begriffseigenschaften}

Durch den Einsatz eines TKP können Eigenschaften ganzer Funktionenklassen hinsichtlich der Einwirkungen von Parametern auf das Monotonie- und Änderungsverhalten oder hinsichtlich bestimmter Eigenschaften, wie Symmetrie und asymptotisches Verhalten, untersucht werden. Die zelluläre Struktur eines TKP unterstützt dabei insbesondere die Analyse diskreter Funktionen bzw. Folgen. Da die symbolische Ebene beim Arbeiten mit einem TKP eine geringere Bedeutung besitzt und lediglich über die Verwendung relativer Zellbezüge in die numerische Ebene integriert ist, erfolgt die experimentelle Analyse in einem Wechselspiel zwischen numerischen und graphischen Darstellungen, also (fast) ausschließlich auf einer präformalen Ebene. Es sollen hier exemplarisch einige Themenkreise beschrieben werden, bei denen ein TKP das Erkunden von Begriffseigenschaften unterstützt.

\section{Beispiel: Untersuchen von Eigenschaften der allgemeinen Tilgungsgleichung}

Neben „klassischen“ Folgen, wie arithmetischen oder polynomialen Folgen, die auf einfache Weise in ein Beziehungsnetz mit reellen linearen und polynomialen Funktionen eingeordnet werden können, lassen sich durch die Verwendung eines TKP auch komplexere bzw. aspektreichere Folgen wie die geometrische Folge oder die allgemeine Tilgungsgleichung $a_{n+1}=A a_{n}+B$ (vgl. Abb. 27) behandeln.

\footnotetext{
${ }^{160}$ Auch CAS ermöglichen die Variation von Variablen- und Parameterbelegungen. Hierbei muss jedoch in der Regel zwischen einer symbolischen und graphischen Ebene per Knopfdruck gewechselt werden. Ein paralleles Ändern von Parametern und gleichzeitiges Beobachten der Auswirkungen auf andere Darstellungen ist bei den meisten CAS nicht möglich. Darüber hinaus steht im Allgemeinen kein mit dem Rollbalken vergleichbares Instrument zur Verfügung.
} 


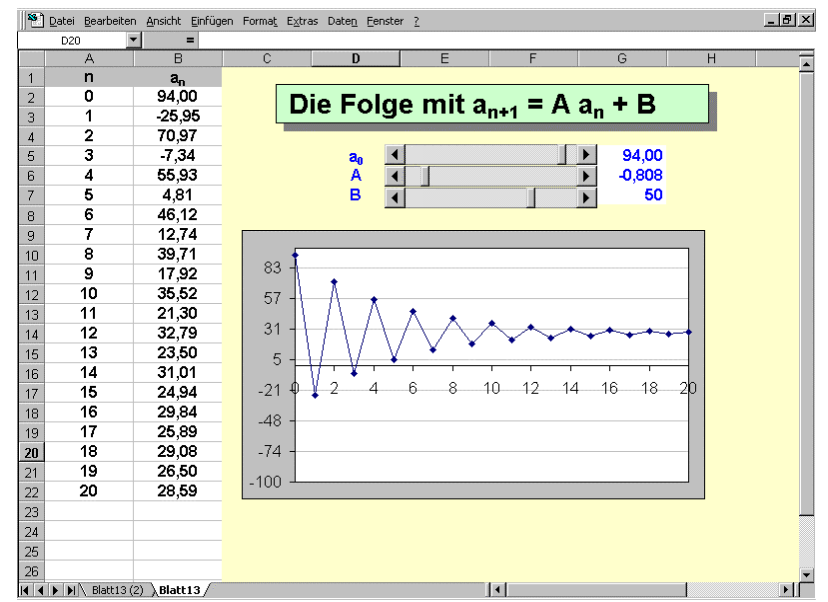

Abb. 27: Experimentelle Analyse der Tilgungsgleichung

Durch die komfortable Möglichkeit des Variierens mit Hilfe des Rollbalkens können interessante bzw. auffällige Eigenschaften der Tilgungsgleichung entdeckt werden. Es besteht jedoch die Gefahr, dass alle drei Parameter $A, B$ und $a_{0}$ gleichzeitig und unreflektiert variiert werden. Ein solches unsystematisches Vorgehen liefert wenig Einblick in funktionale $\mathrm{Zu}$ sammenhänge. Hier ist eine Steuerung seitens des Lehrers bzw. durch geeignete Fragestellungen notwendig, die den Blick des Lernenden zunächst nur auf die Variation eines Parameters und dessen Auswirkungen auf bestimmte Eigenschaften richten. So ist es etwa sinnvoll, zunächst $B=0 \mathrm{zu}$ setzen und den Schülern beispielsweise folgende Fragen zu stellen (vgl. Weigand 2001b, S. 19 ff.):

- Beschreibe die Eigenschaften des Graphen für Wachstumsfaktor A größer bzw. kleiner als 1 .

- Wie verändert sich der Endwert $a_{20}$ bei doppeltem, dreifachem, ... Anfangswert? Experimentiere auch hier mit Wachstumsfaktoren A größer bzw. kleiner als 1 .

- Wie verändert sich der Endwert $a_{20}$ bei schrittweiser Erhöhung des Wachstumsfaktors?

- Wie muss der Wachstumsfaktor geändert werden, wenn - bei gleichem Anfangswert - der Endwert verdoppelt wird?

- Erkläre die unterschiedlichen Auswirkungen der beiden Parameter $a_{0}$ und A auf die Veränderung des Wertes $a_{20}$.

Diese Fragen betonen sowohl objekt- als auch eigenschaftsbezogene Aspekte des operativen Prinzips und regen darüber hinaus theoretische Überlegungen zur Begründung der beobachteten Phänomene an. In ähnlicher Weise lassen sich solche Fragen auch für die allgemeine Tilgungsgleichung stellen:

- Erkläre die Auswirkungen des Veränderns von $a_{0}$ und B auf den Graph, wenn A = 1 gesetzt wird.

- Wir setzen $A=1,1(0,9)$. Erkläre die unterschiedlichen Auswirkungen des Veränderns von $a_{0}$ und B auf die Veränderung des Wertes $a_{20}$.

- Wir setzen $a_{0}=50, A=0,9$. Für $B=0$ fällt der Graph der Folge, für $B=20$ steigt er. Verändere B! Für welchen B-Wert geht der steigende in einen fallenden Graphen über? Erkläre! 


\section{Beispiel: Analyse von Differenzenfunktionen}

Im Rahmen eines TKP-unterstützten Unterrichts kann das Konzept der Z-Funktion und deren Differenzenfunktion (vgl. Kap. 2.3.3) ohne großen operativen Aufwand realisiert werden. Mit Hilfe von Rollbalken, die die Parametervariation unterstützen, können nun insbesondere Beziehungen zwischen Eigenschaften von Funktionenklassen wie $f(n)=2 n^{2}-8 n+c$ und deren Differenzenfunktionen auf einfache Weise sowohl auf einer graphischen als auch einer tabellarischen Ebene analysiert werden (vgl. Abb. 28). ${ }^{161}$ Damit können geometrische (oder präformale) Begründungen initiiert werden, bei denen die „dynamisierten“ Graphen der Z-Funktionen zu den entsprechenden Differenzenfunktionen in Beziehung gesetzt werden. ${ }^{162}$ Eine formale Begründung, bei der zur Erklärung der Invarianz der Differenzenfunktionen der Klasse $f(n)=2 n^{2}-8 n+c$ die Differenz $D(n)=f(n+1)-f(n)$ algebraisch bestimmt wird, würde zwar für eine höhere Stufe des Begriffsverständnisses im Sinne der in Kapitel 3.4 aufgeführten Stufenmodelle sprechen. Jedoch ist im Unterricht zu berücksichtigen, dass - im Sinne der operativen Prinzipien (vgl. Kap. 3.2.1) - auch die anderen Verständnisebenen angesprochen und entwickelt werden sollten.

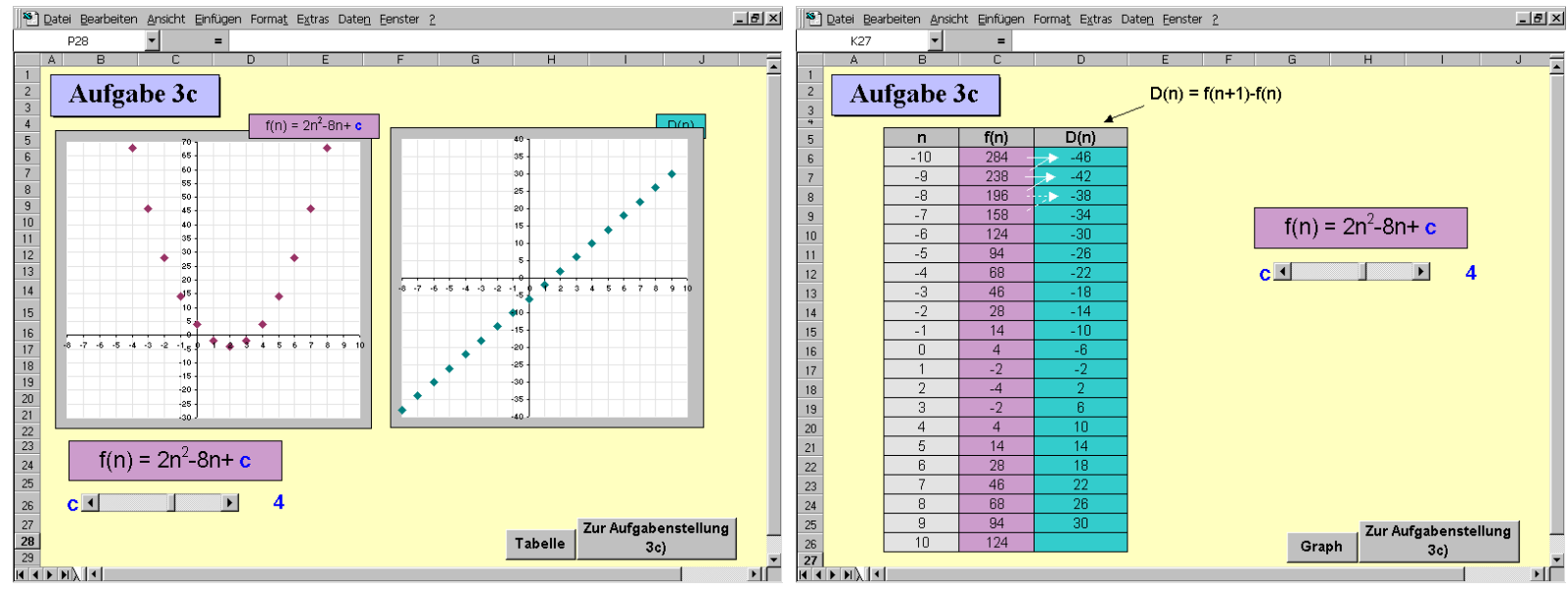

Abb. 28: Experimentelle Analyse von quadratischen Z-Funktionen und deren Differenzenfunktionen

Wie beim vorhergehenden Beispiel ist auch bei diesem Themenkreis eine Realisierung des objekt- und eigenschaftsbezogenen operativen Prinzips möglich. Während quadratische Z-Funktionen im Rahmen der oben beschriebenen Analysetätigkeit Ausgangspunkt einer Problemstellung sind, werden bei der folgenden Aufgabenstellung (vgl. Abb. 29) eine bzw. mehrere Z-Funktionen mit einer ausgezeichneten Eigenschaft gesucht (die Differenzenfunktion soll die Termdarstellung $D(n)=3 n$ bzw. $D(n)=3 n+8$ haben). Dabei stellen

\footnotetext{
${ }^{161}$ Bei der tabellarischen Darstellung wird die (numerische) Beziehung zwischen Funktion und Differenzenfunktion durch „Zuordnungspfeile“ hervorgehoben. Weiterhin ist es möglich, die zugrunde liegende Beziehung durch Anklicken bestimmter Zellen sichtbar zu machen.

${ }^{162}$ Vgl. hierzu den Unterabschnitt „Untersuchung von Funktionenklassen“ in Kap. 2.3.3.
} 
Z-Funktionen das Ergebnis einer Problemstellung dar. Insbesondere wird der Blick auf Operationen gelenkt, welche bestimmte Eigenschaften der Differenzenfunktion invariant lassen. In diesem Fall führt - ist der Parameter $a=1,5$ bestimmt - ein Verändern des Parameters $b$ zu eine Differenzenfunktion mit derselben absoluten Änderung bzw. Änderungsrate 3, aber mit unterschiedlichem Achsenabschnitt. Beim Variieren des Parameters $c$ bleibt die Differenzenfunktion dagegen invariant.
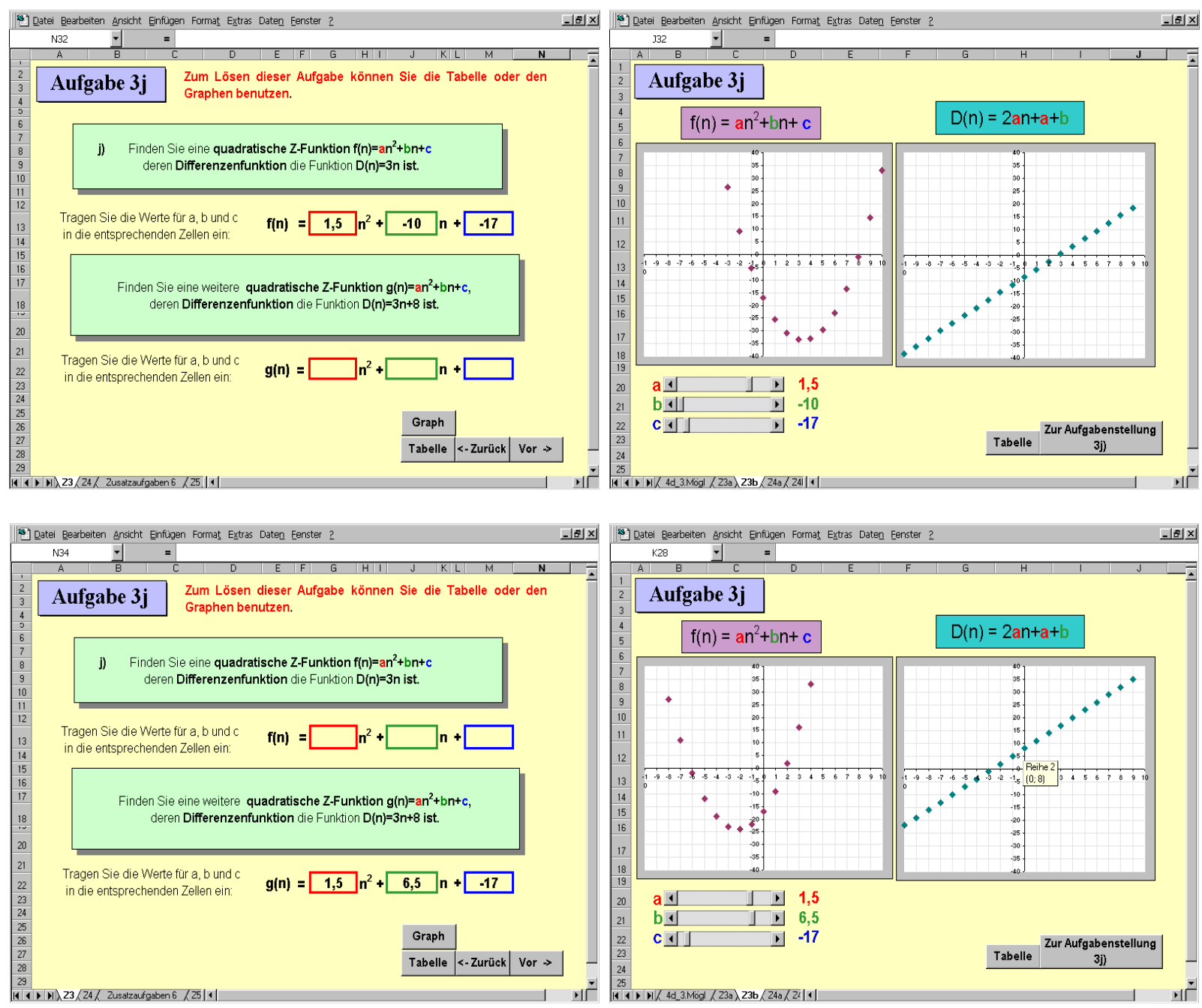

Abb. 29: Finden einer quadratischen Z-Funktion mit ausgezeichneten Eigenschaften 


\section{Beispiel: Kreative Übungen mit Fibonacci-Zahlen}

Ein experimentelles Erkunden von Begriffen und deren Eigenschaften kann auch einen Ausgangspunkt für kreative Übungen darstellen, bei denen Schüler bereits erlernte Inhalte reflektieren, in einem neuen oder ähnlichen Kontext anwenden und dabei für sie subjektiv neues entdecken. ${ }^{163}$ Ein großes Untersuchungsfeld bieten in diesem Zusammenhang die FibonacciZahlen. Mit Hilfe eines TKP lassen sich nahezu beliebig viele Folgenglieder ,,auf Knopfdruck“ erzeugen und es können auf empirischem Wege Vermutungen hinsichtlich des Wachstumsverhaltens aufgestellt, Teilbarkeitseigenschaften entdeckt oder besondere zahlentheoretische Beziehungen zwischen Fibonacci-Zahlen festgestellt werden. ${ }^{164}$

Davon ausgehend können Schüler nun selbständig zum Erfinden und Erkunden ,,verwandter“ Fibonacci-Folgen angeregt werden, indem sie sowohl die beiden Anfangsfolgenglieder als auch die Rekursionsbeziehung abändern. Schon bei einer Beschränkung auf eine lineare Kombination der beiden Anfangsfolgenglieder und dem Iterieren dieser Operation erhält man ein breites Spektrum an Folgen mit interessanten Eigenschaften. ${ }^{165}$ Dabei können die bisher entdeckten Eigenschaften als Orientierungen bei der Suche nach weiteren interessanten Phänomenen dienen. So erkennt man etwa, dass eine unterschiedliche Wahl der Anfangsparameter $f(1)$ und $f(2)$ bei Beibehaltung der Beziehung $f(n+2)=f(n+1)+f(n)$ das Konvergenzverhalten bzw. den Grenzwert des Quotienten $f(n+1) / f(n)$ nicht ändert. Dagegen führt eine Veränderung der Rekursionsbeziehung zu Folgen mit unterschiedlichem Wachstumsverhalten. So erhält man ausgehend von den rekursiven Darstellungen $f(n+2)=f(n+1)+2 f(n)$ oder $f(n+2)=f(n+1)+3 f(n)$ Folgen exponentiellen Typs, deren Quotientenfolge gegen (voneinander verschiedene) Werte größer 1 konvergieren. Dagegen führt die rekursive Beziehung $f(n+2)=f(n+1)-f(n)$ zu einer Folge, die sich - unabhängig von der Wahl der beiden Anfangsparameter - ab dem 7. Glied zyklisch wiederholt. Betrachtet man wiederum die rekursive Beziehung $f(n+2)=2 f(n+1)-f(n)$, stellt man fest, dass die Folgenglieder dem Gesetz einer arithmetischen Folge folgen (vgl. Abb. 30).

\footnotetext{
${ }^{163}$ Vgl. hierzu Weth (1999), der den umfassenden und unscharfen Begriff der Kreativität aus mathematikdidaktischer Sicht analysierte und für den Mathematikunterricht relevante Aspekte herausarbeitete. Demnach soll „ein Produkt (eine Idee) [...] im Mathematikunterricht als kreativ akzeptiert werden, wenn es für die Schülerin bzw. den Schüler subjektiv neu ist oder neuartige Elemente enthält und wenn ein sinnvoller Beitrag zu einer Problemlösung geleistet wird oder selbst Problemstellungen erzeugt werden, welche einen reichhaltigen mathematischen Kontext eröffnen." (Weth 2001, S. 7 f.)

${ }^{164}$ Einen Einblick in die Fibonacci-Zahlen und ihre Eigenschaften gibt Worobjow 1971. Zu unterrichtspraktischen Vorschlägen vgl. z. B. Brockmeyer 1996, Coackley 1992, Dion 1995.

${ }^{165}$ Dieses Vorgehen entspricht den von Weth (1999, S. 67 ff.) formulierten Kreativitätsroutinen des Kombinierens und Iterierens.
} 


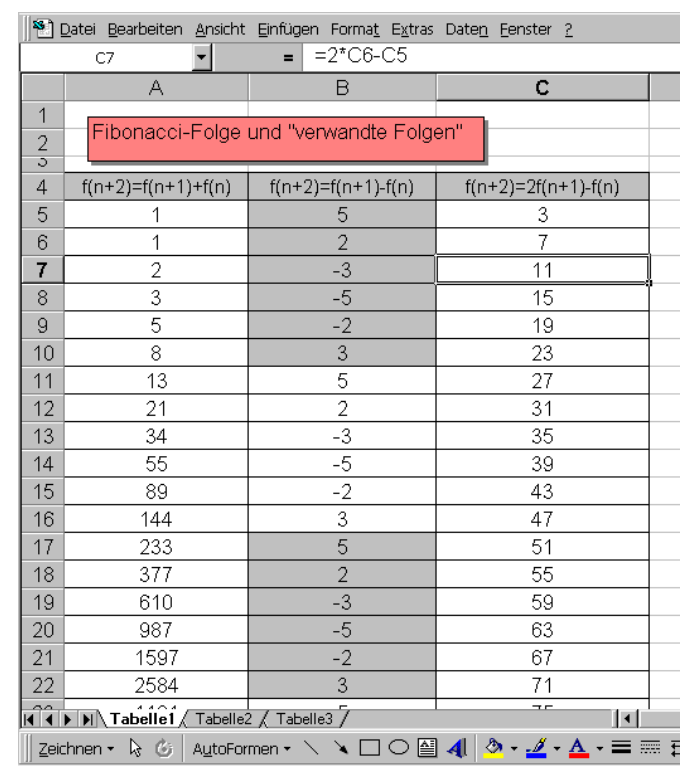

Abb. 30: Fibonacci-Folge und ,verwandte“ Folgen

Diese überraschende Beobachtung kann mit dem Verfahren der „Homogenisierung“ erklärt werden. Man versteht hierunter das Überführen einer inhomogenen linearen Differenzengleichung in eine „homogenisierte“ Differenzengleichung höherer Ordnung: Man schreibt die Differenzengleichung für verschiedene Werte des Laufindex $k$ auf und eliminiert die Inhomogenität durch ein Gleich-, Subtraktions- oder Einsetzungsverfahren (vgl. Dürr \& Ziegenbalg 1989, S. 82 f). In diesem konkreten Fall betrachtet man die einer arithmetischen Folge zugrunde liegenden Differenzengleichungen $f(n+1)=f(n)+a$ und $f(n+2)=f(n+1)+a$. Subtrahiert man die erste Gleichung von der zweiten, erhält man die obige Differenzengleichung zweiter Ordnung.

Es ist festzuhalten, dass das experimentelle Arbeiten bei den hier beschriebenen Aktivitäten insbesondere dem Aufstellen von Vermutungen im Hinblick auf Begriffseigenschaften bzw. dem Finden einer Problemstellung diente. Zum Begründen und Beweisen von Phänomenen ist es im Allgemeinen notwendig, wie etwa im zuletzt genannten Fall, auch (formale) logischdeduktive Schlussweisen hinzuzuziehen.

\subsubsection{Experimentelles Arbeiten im Problemlöseprozess}

Problemlösen gestaltet sich in der Regel als ein Wechselspiel zwischen experimentellinduktiven und systematisch-deduktiven Methoden und sollte in diesem Sinne auch im Mathematikunterricht vermittelt werden. Zur Entwicklung eines Lösungsansatzes bzw. zum Finden einer (plausiblen) Lösung können häufig verschiedene heuristisch-experimentelle Strategien angewendet werden (vgl. Kap. 2.2). Die Verwendung eines TKP unterstützt in diesem Zusammenhang insbesondere das systematische Probieren bzw. das Variieren von Bedingungen. 


\section{Beispiel: Experimenteller Zugang zu Zählproblemen}

Zählprobleme, wie etwa das in Kapitel 2.2.1 vorgestellten Problem der Dreieckszahlen, haben häufig das Finden und Aufstellen einer expliziten Zählfolge in expliziter Darstellung zum Gegenstand. Steht ein TKP zur Verfügung, bietet es sich an, eine explizite Formel zunächst durch graphisches und numerisches Experimentieren zu gewinnen. Hierzu stellt man die Folge der Dreieckszahlen bzw. die ersten empirisch ermittelten Folgenglieder numerisch dar und versucht die Folge durch eine zweite Testfolge ,,anzunähern“ (vgl. Abb. 31).

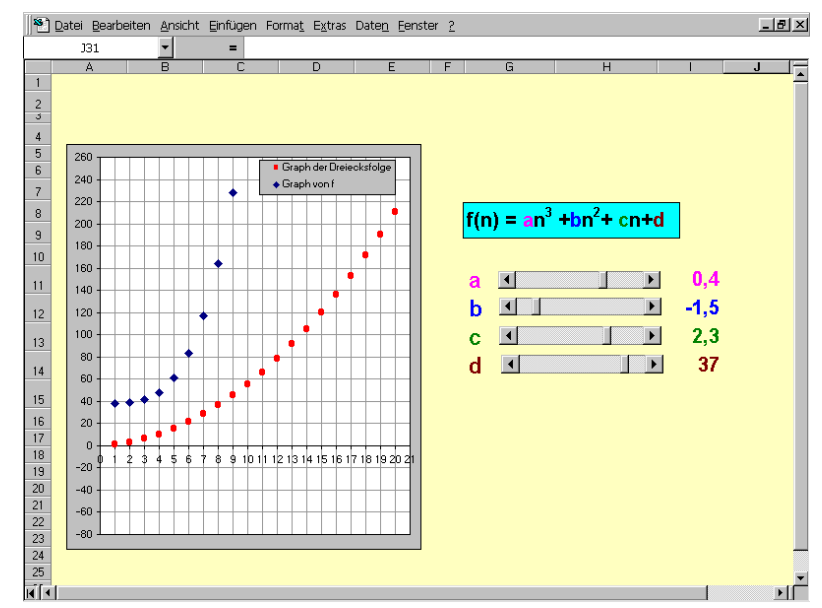

Abb. 31: Anpassen einer Testfolge an die Folge der Dreieckszahlen

Damit diese Strategie Erfolg hat und nicht in ein unsystematisches Versuch-und-IrrtumVerfahren mündet, ist es erforderlich, zunächst anhand der Darstellungen Vermutungen über Eigenschaften der „Zielfolge“ aufzustellen und insbesondere eine Beziehung zwischen Term und Graph herzustellen. Zur Bestimmung des Funktionstyps, der die Dreieckszahlen angemessen beschreibt, kann es hilfreich sein, die Differenzenfolge(n) zu betrachten. ${ }^{166}$ Eine auf experimentellem Wege induktiv gefundene Formel muss noch im Hinblick auf ihre Allgemeingültigkeit begründet werden. In diesem Zusammenhang kann z. B. das Beweisverfahren der vollständigen Induktion hinzugezogen werden.

\section{Beispiel: Experimentelles Lösen von Gleichungen}

Ein experimentelles Arbeiten, insbesondere ein numerisches Experimentieren, stellt auch beim Lösen komplexer Gleichungen, für die (zunächst noch) keine Lösungsformel zur Verfügung steht oder aus pragmatischen Gründen eine numerische Näherungslösung völlig ausreichend ist, eine wichtige Lösungsstrategie dar. So lassen sich z. B. Fragestellungen im Umfeld der „Tilgungsgleichung“ durch Verändern der Startwerte bzw. der Parameter und durch sukzessives Einschachteln des Zielwerts näherungsweise lösen (vgl. Abb. 32).

\footnotetext{
${ }^{166}$ Vgl. hierzu die Ausführungen zur Behandlung der „Kreisaufgabe“ in Kapitel 2.2.1, insbesondere Strategie IV.
} 


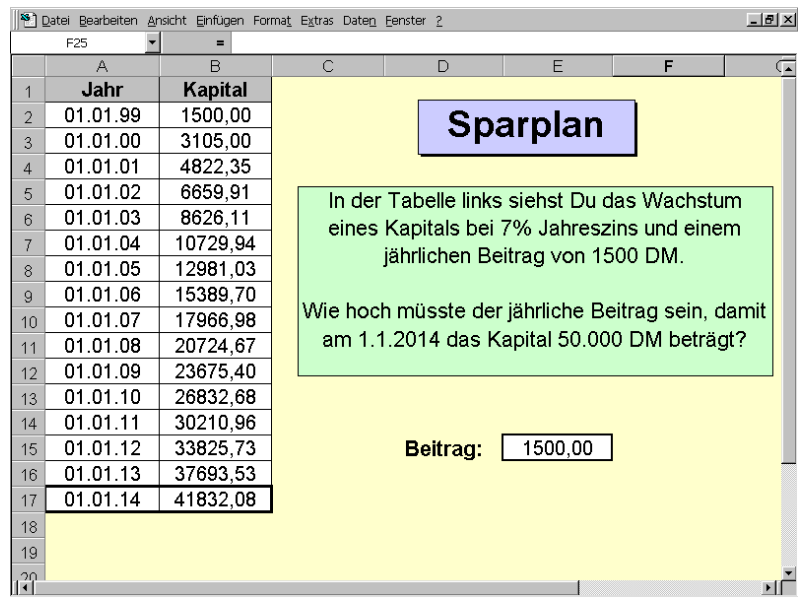

Abb. 32: Experimentelles Lösen einer lokalen Problemstellung

Darüber hinaus kann durch ein experimentelles Lösen von Gleichungen bzw. ein experimentelles Annähern eines Werts, eine Grundlage für das Verständnis von Intervallschachtelungen gelegt werden. Im Gegensatz zu dem vorstehend beschriebenen Zählproblem, bei der eine Kurve ,als Ganzes“ an eine Reihe vorgegebener Daten angepasst werden sollte, handelt es sich hierbei um lokale Problemstellungen, bei denen nur ein bestimmter Wert bzw. ein bestimmtes Folgenglied gesucht ist. Mit verschiedenen globalen und lokalen Problemstellungen sowie den damit verbundenen Schülerarbeitsweisen beschäftigte sich das DFG-Projekt „Arbeitsweisen von Lernenden beim computerunterstützten Problemlösen mit Hilfe des Folgenbegriffs“" (vgl. Weigand 1999b, Weigand \& Thies 2001).

\subsubsection{Modellbildung und Simulation}

Häufig interessiert man sich in der empirischen Forschung für den funktionalen Zusammenhang zweier beobachtbarer Variablen. Eine Analyse beginnt zunächst mit einer Datenerhebung und der Darstellung der Messpaare in einer Tabelle oder einem Koordinatensystem. Dabei können Störungen bzw. Messfehler die Daten unregelmäßig streuen lassen. Ist man an einer übersichtlicheren, aussagekräftigen Darstellung oder auch an Prognosen für die Zukunft interessiert, wird man nach einer zugrunde liegenden Gesetzmäßigkeit, einem funktionalen Zusammenhang suchen, der gegebenenfalls in Form eines möglichst einfachen Funktionsterms oder Funktionsgraphen wiedergegeben werden kann. Bei der Suche nach einer geeigneten Anpassungsfunktion beschränkt man sich häufig auf eine bestimmte Funktionenklasse. ${ }^{167}$ Dabei fließen Vorkenntnisse oder zumindest Annahmen über den Sachzusammenhang mit ein. Es ist nun das Ziel, unter dieser Modellannahme die am besten geeignete Funktion zu bestimmen. Diese Modellbildung ist Teil eines umfassenden Modellbildungsprozesses (vgl. Kap. 2.4). Dieser Prozess umfasst sowohl eine kritische Modellbewertung, also einen Ver-

${ }^{167}$ Häufig spiegelt eine parametrische Modellannahme die Beobachtungen nur unzureichend wider, bzw. sie stellt eine zu starke Vereinfachung der Ausgangssituation dar. In diesen Fällen stellt das Arbeiten mit so genannten ,gleitenden Mittelwerten“ eine Alternative dar (vgl. hierzu etwa Engel 1999). 
gleich mit alternativen, möglicherweise ,angemesseneren“ Modellen, als auch zielgerichtete Untersuchungen mit dem mathematischen Modell (Simulationsstudien), bei denen untersucht wird, wie sich das Modell unter bestimmten Bedingungen verhält.

Bei diesen Aktivitäten erweist sich ein TKP als ein wichtiges Experimentierwerkzeug. Es übernimmt zum einen aufwändige und umfangreiche algorithmische Berechungen und ermöglicht zum anderen durch die interaktive Verknüpfung der verschiedenen Darstellungsformen eine direkte Beobachtung der durch Parametervariation bewirkten Veränderungen. Schüler können somit ohne großen operativen Aufwand verschiedene parametrische Modelle „testen“, indem sie durch zielgerichtetes Variieren der Parameterwerte eine geeignete Anpassungsfunktion bestimmen und anschließend die „Güte“ der Anpassung an die Beobachtungsdaten vergleichen und beurteilen. Daneben wird auch das Arbeiten mit bestehenden Modellen, insbesondere die Simulation verschiedener Szenarien und das experimentelle Lösen von Problemen, unterstützt.

\section{Beispiel: Diskret modellierte Wachstumsprozesse}

Bei einer TKP-unterstützten Behandlung von Wachstumsprozessen wird durch die zelluläre Struktur des TKP eine diskrete Modellierung unterstützt. ${ }^{168}$ Durch die Möglichkeit der komfortablen Parametervariation mit Hilfe von Rollbalken sowie des einfachen Wechselns zwischen verschiedenen Darstellungen kann in diesem Zusammenhang etwa untersucht werden, ob die Entwicklung einer Population geeigneter durch ein diskretes exponentielles, gebremstes oder logistisches Wachstum beschrieben wird, und es können aufgrund des favorisierten Modells Prognosen für die zukünftige Entwicklung angestellt bzw. kritisch diskutiert werden. ${ }^{169}$ Neben derartigen Modellbildungsaktivitäten werden auch Simulationsstudien durch den Einsatz eines TKP unterstützt. So können bei vernetzten Systemen, wie etwa dem Räuber-Beute-System (vgl. Kap. 2.4.1), die Einflüsse verschiedener Parameter, wie der Wachstums- und der Interaktionsfaktoren sowie der Anzahl der Räuber- und Beutetiere zum Zeitpunkt $t=0$, untersucht und anhand verschiedener graphischer Darstellungen interpretiert werden (vgl. Abb. 33). ${ }^{170}$

\footnotetext{
${ }^{168}$ Die Entscheidung für ein diskretes Modell sollte jedoch im Rahmen der Modellbewertung kritisch reflektiert werden (vgl. hierzu Kap. 2.4.1).

169 Ein computerunterstütztes Unterrichtsprojekt zur Entwicklung der Weltbevölkerung findet sich in Prugger, Rauniak \& Schneider 1997, S. 62 f. Hierbei werden die oben beschriebenen Modelltypen einbezogen. Im Rahmen dieses Projekts wird jedoch kein TKP, sondern der symbolische Taschencomputer TI-92 verwendet.

${ }^{170}$ Umfassend mit dem Thema Populationsentwicklungen und Vernetzung - jedoch unter Berücksichtigung spezieller, systemdynamischer Software - haben sich u. a. Ossimitz $(1990,1994)$ und Winkelmann $(1992,1994)$ beschäftigt.
} 

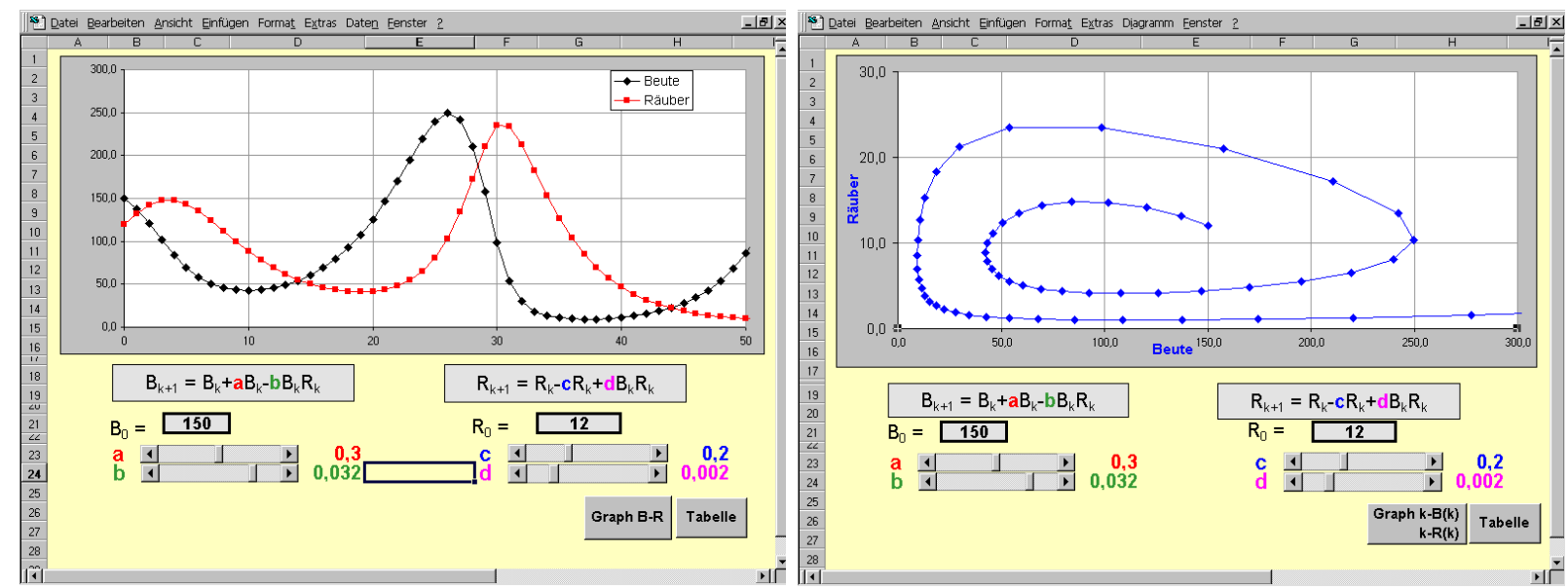

Abb. 33: Simulation des Räuber-Beute-Systems ${ }^{171}$

Umgekehrt lassen sich durch Parametervariation auch Szenarien mit bestimmten Eigenschaften konstruieren. So können Schüler der Frage nachgehen, unter welchen Bedingungen eine der beiden Populationen ausstirbt. Weiterhin können sie untersuchen, wie ein System abgeändert werden muss, damit es weniger oszilliert bzw. sich weniger stark aufschaukelt.

\section{Beispiel: Flugkurven von Basketball und Federball - ,,diskret“" betrachtet}

In dem von Weigand (1999c) beschriebenen Unterrichtsprojekt zum Thema „Sport“ wurden die Flugkurven eines Basketballs beim Freiwurf von der 4,60-m-Linie und eines BadmintonFederballs zunächst mit einer Videokamera aufgezeichnet und anschließend durch ein diskretes Modell beschrieben. Dazu wurde die Flugkurve des Basketballs mit Hilfe eines TKP schrittweise aus Anfangsgeschwindigkeit und Abflugwinkel durch Zerlegung der Gesamtbewegung in eine gleichbleibende (ungebremste) Bewegung in $x$ - und beschleunigte Bewegung in $y$-Richtung berechnet. Die Vernachlässigung des Reibungseinflusses war hierbei zulässig, musste jedoch bei der Beschreibung des Badmintonwurfs angemessen berücksichtigt werden. ${ }^{172}$ Nach Festlegung der jeweiligen Modelle wurden die speziellen Bahnkurven experimentell durch Parametervariation ermittelt.

Im Rahmen des Unterrichtsprojekts wurden darüber hinaus weitere Simulationsstudien durchgeführt. So wurden unterschiedliche Flugkurven erfolgreicher Korbwürfe mit dem Basketball untersucht und graphisch dargestellt (vgl. Abb. 34).

\footnotetext{
${ }^{171} \mathrm{Zu}$ Vergleichzwecken ist in der Graphik der Maßstab der Population der Räuber um den Faktor 10 gegenüber den der Hasen gestreckt.

${ }^{172}$ Zur Modellierung des Reibungseinflusses gibt es verschiedene Möglichkeiten (vgl. etwa Greiner 1989, S. 182f.). Welches Modell die reale Situation aber am besten beschreibt, muss letztlich durch eine Analyse oder Diskussion der jeweiligen Situation erfolgen. Bei der beschriebenen Einheit wurde die Newton'sche Reibung zugrunde gelegt.
} 

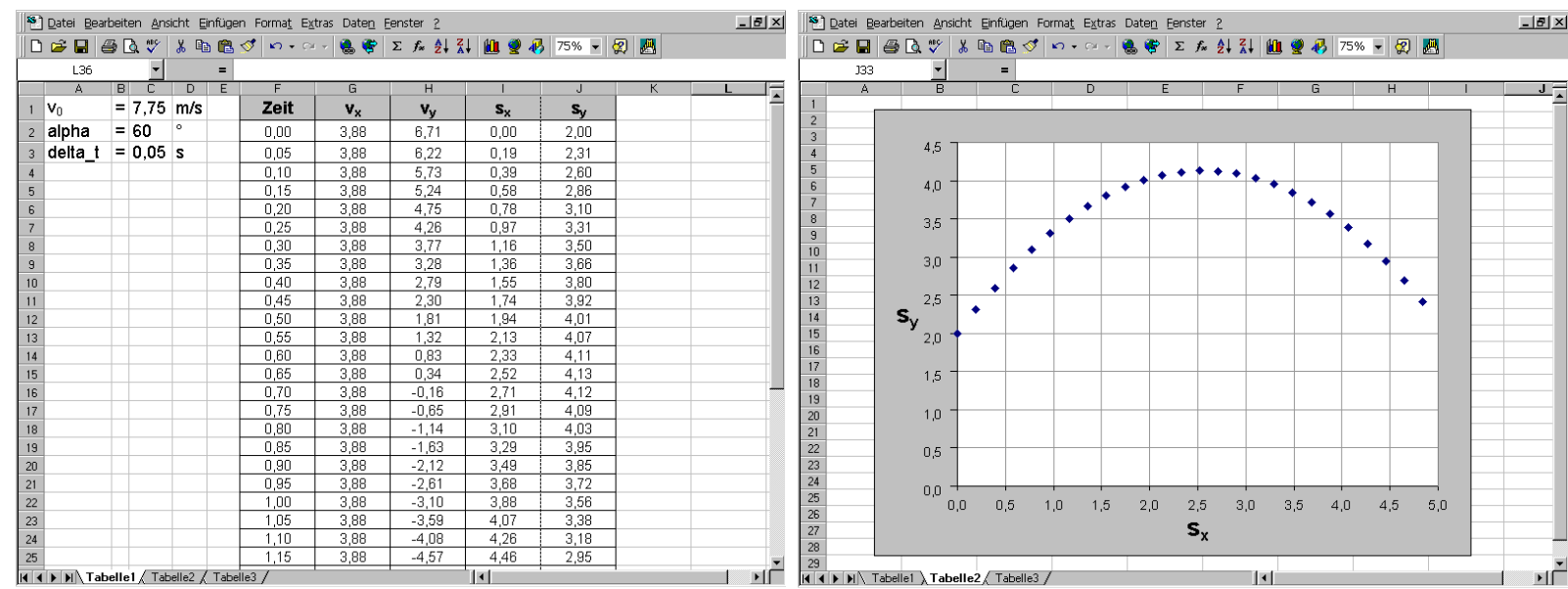

Abb. 34: Experimentierumgebung zur Ermittlung erfolgreicher Korbwürfe ${ }^{173}$

Bei der Simulation des Badmintonschlags war es möglich, verschiedene Abflugwinkel bei hart geschlagenen Schmetterbällen ${ }^{174}$ unter taktischen Aspekten zu analysieren: Bei welchem Abflugwinkel fliegt der Ball am weitesten? Wie lange ist die entsprechende Flugdauer? Wie wirkt sich ein veränderter Abflugwinkel auf Flugweite und Flugdauer aus?

Dieser experimentelle Zugang wurde durch algebraische Überlegungen ergänzt. So konnte die Vermutung, dass sich der Basketball auf einer parabelförmigen Flugkurve bewegt, formal begründet werden. Weiterhin ließen sich anhand der deduktiv abgeleiteten Formel für die Wurfparabel Probleme lösen, denen auf experimentellem Wege nur unzureichend nachgegangen werden konnte: Wie groß ist der Abwurfwinkel der niedrigsten Wurfparabel, bei der der Ball ohne Berührung in den Korb fliegt? Eine theoretische Durchdringung des Sachverhalts ermöglichte auch das Aufdecken von Zusammenhängen zwischen Abwurfwinkel und Abwurfgeschwindigkeit, die durch das alleinige Variieren der Parameterwerte nur schwer zugänglich gewesen wären. ${ }^{175}$ Diese theoretischen Resultate ließen sich anschließend noch experimentell und anschaulich nachvollziehen.

\subsection{Tabellenkalkulationsprogramme als Lernsysteme}

Computerlernsysteme gibt es bereits seit Anfang der 60er Jahre. ${ }^{176}$ Die damaligen Lernumgebungen der so genannten „Programmierten Unterweisung“ (PU) basierten auf dem lernpsychologischen Konzept des Behaviorismus (vgl. Kap. 3.1). So wurde im Rahmen der PU der

\footnotetext{
${ }^{173}$ Es wird hierbei von einer Abwurfhöhe von $2 \mathrm{~m}$ ausgegangen. Die Höhe des Basketballkorbs beträgt 3,05 m.

${ }^{174}$ Man ging hier von einer Anfangsgeschwindigkeit von etwa $130 \mathrm{~km} / \mathrm{h}$ aus.

${ }^{175}$ Bei einem erfolgreichen Korbwurf beträgt die minimale Abwurfgeschwindigkeit etwa 7,8 m/s. Der Abwurf muss dabei in einem Winkel von $51^{\circ}$ erfolgen. Wird der Abwurfwinkel in einem Bereich zwischen $30^{\circ}$ und $70^{\circ}$ variiert, sind nur geringfügige Änderungen der Abwurfgeschwindigkeit erforderlich. Dies erklärt die Bedeutung einer Technik zum Erzeugen stabiler Anfangsgeschwindigkeiten beim Korbwurf.

${ }^{176}$ Einen Überblick über die Entwicklung von Computerlernsystemen geben Weidemann \& Paechter 1997.
} 
Lernstoff in kleine Einheiten aufgeteilt. Jede Lerneinheit wurde durch Kontrollfragen abgeschlossen, deren Schwierigkeitsgrad bewusst gering gehalten wurde, um den Lernenden möglichst oft eine positive Verstärkung geben zu können. Die gegebenen Antworten wurden unmittelbar überprüft, das Feedback beschränkte sich jedoch in der Regel auf „richtig“ oder „falsch“. Selbstgesteuertes und aktives Lernen war im Rahmen der PU selten möglich: Die Interaktion mit dem Programm umfasste lediglich die Auswahl des jeweiligen Kapitels oder Moduls, das Vor- und Zurückblättern innerhalb der Kapitel, das Aufnehmen von Texten oder graphisch aufbereiteten Informationen und das Überprüfen des dabei gelernten Wissens. Im Sinne moderner konstruktivistischer Ansätze ${ }^{177}$ sollten jedoch Lernumgebungen geschaffen werden, die dem Lernenden eine hohes Maß an Eigentätigkeit belassen. Im Rahmen so genannter „Interaktiver Lernumgebungen“ (vgl. Weidemann \& Paechter 1997, S. 136 ff.) sollen Lernende insbesondere die Möglichkeit erhalten, sich aktiv mit den Lerninhalten auseinander $\mathrm{zu}$ setzen und individuelle Problemlösestrategien zu entwickeln und zu verfolgen

Als Basis interaktiver Lernumgebungen im Mathematikunterricht eignen sich insbesondere Tabellenkalkulationsprogramme. So lässt sich ein Tabellenblatt eines TKP auf vielfältige Weise mit Hilfe von Textboxen, Eingabeüberprüfungen, gestuften Hilfen und Formatierungsfunktionen inhaltlich funktionell und visuell ansprechend als elektronisches Arbeitsblatt gestalten. Da verschiedene Arbeitsblätter durch Schaltflächen miteinander verknüpft werden können, lassen sich Lernumgebungen zu einem größeren Themenbereich konstruieren. Indem ein TKP nun vielfältige Möglichkeiten zum Arbeiten mit tabellarischen und graphischen Darstellungen bietet und experimentelle Herangehensweisen an Problemstellungen unterstützt, kann es als eine geeignete offene - d. h. vom Lernenden selbst zu gestaltende und flexibel einsetzbare - Lernumgebung angesehen werden, die verschiedene Werkzeuge zur Bearbeitung der spezifischen Arbeitsaufträge bereitstellt. Es ist dabei jedoch zu berücksichtigen, dass es zur Konstruktion etwas fortgeschrittener Konzepte, wie etwa des Rollbalkens und dessen Formatierung, eines grundlegenden technischen Wissens im Umgang mit dem TKP bedarf. Daneben ist auch der Zeitbedarf nicht zu unterschätzen, der zwar beim Erstellen eines einzigen Diagramms (inklusive der Wahl einer geeigneten Skalierung) noch unerheblich und auch didaktisch zu rechtfertigen ist, der aber bei einer häufigen Wiederholung derselben Aktivität unverhältnismäßig groß werden kann. Dabei besteht die Gefahr, dass von der eigentlichen

\footnotetext{
${ }^{177}$ Wie bereits Piaget gehen Vertreter moderner konstruktivistischer Ansätze (vgl. etwa Glasersfeld 1991) davon aus, dass Lernen nicht als passiv und rezeptiv charakterisiert werden kann, sondern als aktiver konstruktiver Prozess gesehen werden muss. Wissen und Erkenntnis werden dabei als grundsätzlich subjektabhängig betrachtet. Die Annahme der Subjektabhängigkeit allen Erkennens basiert auf neurobiologischen Theorien. So gehen die Neurobiologen Maturana und Varela (1987) davon aus, dass das Nervensystem ein in sich abgeschlossenes Netzwerk ist. Umwelteinwirkungen haben keinen direkten Einfluss auf dessen innere Struktur und erfolgen lediglich als so genannte Perturbationen (Störungen). Damit werden individuelle kognitive Aktivitäten des Individuums ausgelöst. Hier zeigen sich Parallelen zur genetischen Erkenntnistheorie Piagets, wonach das Individuum seine kognitive Struktur in Wechselwirkung mit der Umwelt selbst generiert. Vgl. hierzu auch die Ausführungen von v. Hofe (2001), der in den Annahmen und Ergebnissen der konstruktivistischen Richtung der Lernpsychologie neue Argumente für das Konzept des „Entdeckenden Lernens“ sieht. Vgl. aber auch die kritischen Bemerkungen von Hoffmann 2001.
} 
Problemstellung abgelenkt wird. Bei dem Konzept, das der von Weigand (1999b) entwickelten Lernumgebung und auch dem im Rahmen dieser Arbeit entwickelten Lernprogramm „Differenzenfunktionen“"178 zugrunde liegt, wurde daher ein Kompromiss eingegangen. So sollten zentrale Darstellungen (wie in der Regel die Tabelle und in Ausnahmefällen der Graph) selbst konstruiert werden. Bestimmte technische Hilfsmittel zum Arbeiten mit Darstellungen (Rollbalken) wurden dagegen zur Verfügung gestellt. Da im Rahmen der Programme neue Inhalte erarbeitet werden sollten, war eine gewisse Strukturierung und Sequenzierung des Stoffs notwendig. Auf eine allzu enge Führung wurde jedoch größtenteils verzichtet. ${ }^{179}$ Dabei wurde darauf Wert gelegt, dass Aufgaben, zu denen Lösungsverfahren bekannt waren, und komplexere Probleme in einem angemessenen Verhältnis standen. Um den Schülern individuelle Lernstrategien zu eröffnen, wurde ihnen innerhalb der Module die Möglichkeit gegeben, zwischen der tabellarischen und graphischen Darstellung zu wählen. Im Rahmen einiger Aufgaben, so etwa bei dem Modul „Dreieckszahlen“, wurden darüber hinaus experimentelle Lernumgebungen geschaffen.

\section{Beispiel: Das Modul „Dreieckszahlen““}

Das Modul „Dreieckszahlen“ ist ein Teil des Lernprogramms „Differenzenfunktionen“. Die Schüler sollen im Rahmen dieser Aufgabe zunächst die rekursive Darstellung für die Dreieckszahlen bestimmen und anhand dieser Formel mit Hilfe des TKP die ersten 20 Folgenglieder berechnen. Anschließend wird das Problem aufgeworfen, eine explizite Formel zur Beschreibung dieser Zahlen zu finden. Hierzu werden den Schülern drei verschiedene Hilfestellungen gegeben, die zu individuellen Lösungsstrategien ausgebaut werden können: die Bleistift-und Papier-Methode, die Differenzenmethode und die experimentelle Methode (vgl. Abb. 35).

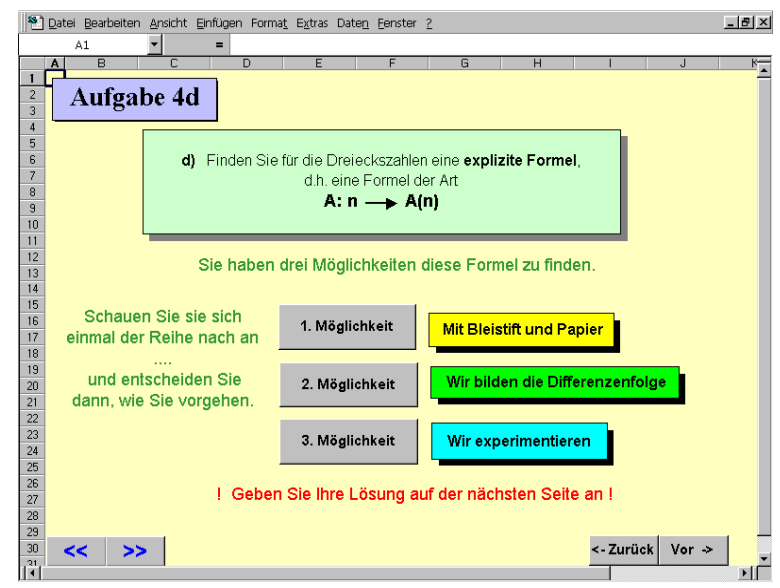

Abb. 35: Experimentierumgebung „Dreieckszahlen“

\footnotetext{
${ }^{178}$ Dieses Programm ist Grundlage der im Rahmen dieser Arbeit durchgeführten empirischen Untersuchung und wird in Kapitel 5.6 ausführlich vorgestellt.

${ }^{179}$ Im Rahmen des in dieser Arbeit entwickelten Programms war eine engere Führung stellenweise notwendig, da es in erster Linie zu Test- und Untersuchungszwecken diente und auf Interaktionsprozesse zwischen den Schülern und der Untersuchungsleiterin bewusst verzichtet wurde.
} 
Durch Betätigen der jeweiligen Schaltflächen ${ }^{180}$ verzweigt der Schüler zu entsprechend aufbereiteten Experimentier- bzw. Arbeitsblättern (vgl. Abb. 36).
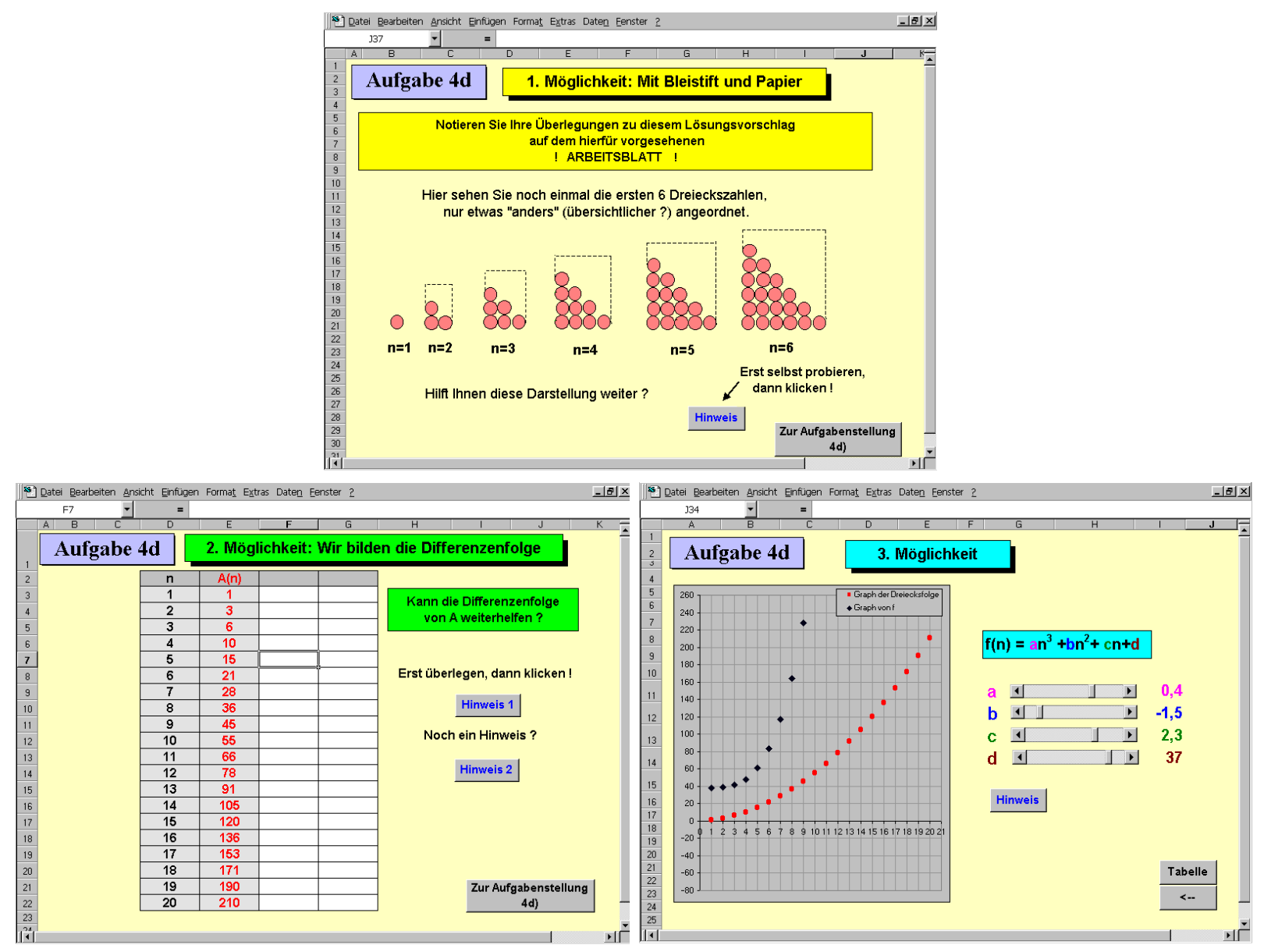

Abb. 36: Experimentier- und Arbeitsblätter zum Modul „Dreieckszahlen“

Mit der Schaltfläche „1. Möglichkeit“ gelangt man zu einem Arbeitsblatt, dass es nahe legt, die Formel im Rahmen eines inhaltlich-anschaulichen Beweises zu erfassen. Folgt man dem Lösungshinweis „2. Möglichkeit“ wird eine TKP-Tabelle zur Verfügung gestellt, die das Berechnen der 1. und 2. Differenzenfolge ermöglicht. Die Intention dieses Lösungshinweises ist es, auf die Differenzenmethode zur Bestimmung des Funktionstyps aufmerksam zu machen. Mit der Wahl der 3. Schaltfläche verzweigt man schließlich zu Arbeitsblättern, die es ermöglichen, eine Anpassungsfunktion für die ersten 20 empirisch dargestellten Dreieckszahlen durch graphisches oder numerisches Experimentieren zu finden.

\footnotetext{
${ }^{180}$ Hinter den Buttons stecken kleine Programme (Makros) in Visual Basic. Einfache Makros lassen sich ohne Programmierkenntnisse erstellen: Analog dem Erzeugen von Makros bei dynamischer Geometrie-Software werden die einzelnen Befehle schrittweise direkt in der TKP-Umgebung durchgeführt und dabei durch einen Recorder aufgezeichnet und abgespeichert.
} 
Neben diesen grundsätzlichen Hilfestellungen kann der Schüler durch das Betätigen spezieller Schaltflächen („Hinweis-Buttons“) weitere gestufte Lösungshinweise erhalten. So wird man in Verbindung mit dem TKP-Blatt „Differenzenfolge“ (vgl. Abb. 37) aufgefordert, erneut die Beziehung zwischen Funktion und Differenzenfunktion zu reflektieren. In einem zweiten Hinweis wird auf das Modul „Quadratische Z-Funktionen“ zurückverwiesen, in dem die Beziehung zwischen quadratischen Z-Funktionen und deren Differenzenfunktionen ausführlich lokal und global im Sinne eines operativen Durcharbeitens beleuchtet wird.

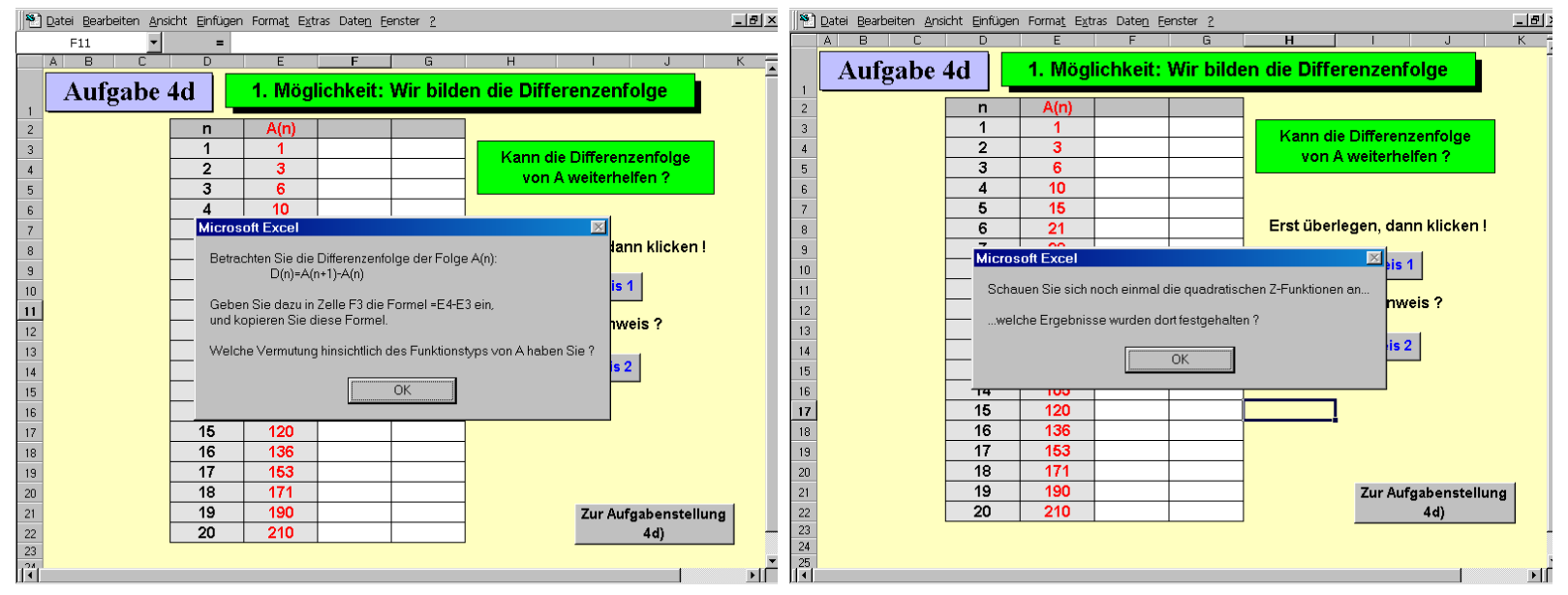

Abb. 37: Hilfetexte zur Lösungsstrategie „Differenzenfolge“

\subsection{Diskrete Arbeitsweisen in einer TKP-gestützten Lernumgebung}

Zur Einschätzung der Möglichkeiten und Grenzen eines TKP im Mathematikunterricht reicht eine didaktische Analyse der softwaretechnischen Komponenten nicht aus. Geht man von einem konstruktivistischen Ansatz aus, ist es erforderlich, die individuellen Lernprozesse der Schüler zu berücksichtigen und näher zu analysieren. Ein Ausgangspunkt kann hierbei das Beobachten und Analysieren der Arbeitsweisen im Sinne der in Kapitel 3.3 beschriebenen Dimensionen sein, die nun - insbesondere im Hinblick auf das in Kapitel 4.3 vorgestellte Lernumgebungskonzept - konkretisiert werden können:

\section{- Arbeiten auf der Werkzeugebene}

Beim Arbeiten mit dem Werkzeug TKP geht es um die Verwendungsweise spezifischer softwaretechnischer Elemente, wie der verschiedenen Hinweis-, Lösungs- und NavigationsSchaltflächen, sowie um das Arbeiten mit dem Rollbalkens, der im Rahmen der Lernumgebung ein wichtiges Experimentierwerkzeug darstellt. Daneben sind auch Schwierigkeiten im Umgang mit einer TKP-gestützten Lernumgebung $\mathrm{zu}$ untersuchen. Dies können TKPspezifische Probleme sein, wie das Verwenden einer fehlerhaften Syntax oder Probleme beim Kopieren und Verschieben von Formeln. Bei einer Analyse der Arbeitsweisen auf der Werkzeugebene sind aber auch allgemeine, im Zusammenhang mit Computerlernumgebungen be- 
obachtete ,problematische“ Handlungsweisen, wie ein Vermeiden mathematischer Analyse, ein unreflektiertes Benutzen oder Umgehen von Werkzeugen und ein Abweichen vom Ziel, zu berücksichtigen. ${ }^{181}$

\section{- Arbeiten auf der Darstellungsebene}

Das Arbeiten mit Darstellungen umfasst im Rahmen der hier vorgestellten Lernumgebung insbesondere die Auswahl von und den Wechsel zwischen den Darstellungen Graph und Tabelle. Beim Arbeiten mit der Tabelle kann dabei noch zwischen der numerischen und symbolischen Ebene unterschieden werden.

\section{- Arbeiten auf der Objektebene}

Ein TKP unterstützt insbesondere das Arbeiten mit diskreten Funktionen und Folgen (bzw. deren Darstellungen). Bei einer Analyse der Arbeitsweisen mit diesen Objekten kann die in Kapitel 3.3 beschriebene Einteilung ,,algorithmisch“, iterativ“ und „funktional“ zugrunde gelegt werden. So können durch Anklicken und Verknüpfen geeigneter in Beziehung stehender Zellen iterative Gesetzmäßigkeiten erfasst werden. Indem eine Folge durch Kopieren der entsprechenden Zellbezüge mit Hilfe eines TKP tabellarisch dargestellt wird, wird algorithmisch gearbeitet. Funktionales Arbeiten umfasst zum einen das TKP-unterstützte Ablesen aus graphischen oder tabellarischen Darstellungen, wobei lokale Aspekte betont werden. ${ }^{182}$ Zum anderen bezieht sich funktionales Arbeiten auf Funktionen als „Ganzes“, indem der Einfluss von Parametern auf graphische oder tabellarische Darstellungen analysiert wird.

In den obigen Ausführungen zeigt sich eine enge Verzahnung der drei Dimensionen des Arbeitens. Da Objekte bzw. deren Darstellungen im Rahmen unserer Untersuchung durch das Werkzeug TKP erzeugt werden, kann das Arbeiten mit dem Objekt nicht unabhängig vom Arbeiten mit Darstellungen und dem Werkzeug gesehen werden. Dies steht im Einklang mit den Grundthesen des semiotischen Ansatzes, nach denen es „kein Denken ohne Zeichen“ und damit keine Objekte ohne Darstellungen geben kann (vgl. Hoffmann 2001). ${ }^{183}$

\footnotetext{
${ }^{181}$ Vgl. Hölzl 1994, S. 224 ff. Es handelt sich hierbei um Handlungsweisen, die - wie Hölzl betont - auch im traditionellen Mathematikunterricht beobachtbar sind, die aber in Verbindung mit dem neuen Lernkontext möglicherweise deutlicher hervortreten.

182 Das Ablesen von Funktionswerten aus graphischen Darstellungen wird unterstützt, indem einzelne Koordinaten eines Funktionsgraphen durch das Deuten mit dem Cursor auf den entsprechenden Koordinatenpunkt automatisch angezeigt werden.

${ }^{183}$ Bei der im Rahmen dieser Arbeit durchgeführten Untersuchung (vgl. Kap. 5) wird allerdings der Versuch unternommen, jeweils eine der Dimensionen in den Vordergrund zu stellen und zu betonen. Dabei werden dann die Parameter dieser Dimension variiert, wohingegen die der anderen Dimensionen möglichst konstant gehalten werden.
} 
Computerlernumgebungen, die durch einen Lernenden in der Interaktion selbstständig erschlossen werden können, werden in der didaktischen Literatur auch als Mikrowelten bezeichnet. ${ }^{184}$ Diese Mikrowelten ermöglichen wichtige situative Erfahrungen mit spezifischen Prototypen und können das Bilden von „situated abstractions“185 (Hoyles \& Noss 1992) unterstützen. Berücksichtigt man jedoch die Bereichsgebundenheit des Lernens, so kann nicht davon ausgegangen werden, dass Wissen, das in diesem Kontext erworben wurde, einfach in andere Welten (wie etwa die traditionellen „Bleistift-und-Papier-Welt“) übertragen werden kann. Daher muss dem Transfer zwischen diesen Welten eine besondere Beachtung geschenkt werden. Im Rahmen dieser Arbeit interessieren insbesondere Übersetzungsprozesse zwischen der formalen algebraischen und der TKP-spezifischen Notation.

\subsection{Zusammenfassung}

TKP bieten vielfältige didaktisch-methodische Möglichkeiten im Umgang mit diskreten Objekten. So stellen TKP gegenständliche Prototypen („Darstellungen“) als kognitive und am Computer ,ausgelagerte“ Werkzeuge für den Umgang mit Begriffen zur Verfügung und betonen ein Arbeiten auf einer ,,vorformalen“ numerischen und graphischen Ebene. Darüber hinaus eignen sie sich in besonderer Weise zur Realisierung eines experimentellen Mathematikunterrichts im Sinne des operativen Prinzips sowie zur Entwicklung von Lernumgebungen, die Lernenden die Möglichkeit bieten, sich aktiv und indiviuell mit den Lerninhalten auseinander zu setzen. $\mathrm{Ob}$ und wie Schüler diese Möglichkeiten nutzen, welches Verständnis und welche Vorstellungen sie entwickeln und inwiefern es zu Problemen beim Transfer zwischen TKP-Mikrowelt und traditioneller „Bleistift-und-Papier-Welt“ kommt, lässt sich letztendlich nur empirisch klären. Zur Analyse des Schülerhandelns kann dabei das in Kapitel 3 vorgestellte Konzept der Arbeitsweisen verwendet werden.

Ziel einer empirischen Untersuchung ist es nun, Arbeitsweisen von Schülern sowie deren Begriffsverständnis beim Umgang mit einer speziellen inhaltlichen Thematik (Differenzenfolgen) konkret zu untersuchen. Diese Untersuchung wird im nächsten Kapitel vorgestellt.

\footnotetext{
${ }^{184}$ Vgl. Papert 1980. Der Begriff Mikrowelt ist jedoch keineswegs eindeutig. Zu einem kurzen geschichtlichen Abriss der Entwicklung dieses Begriffs im Kontext der Programmiersprache Logo vgl. Hoyles 1993.

${ }^{185}$ Unter dem Begriff „situated abstraction“ verstehen Hoyles und Noss ,[...] the first step in constructing a mathematical generalisation. It is situated in that the knowledge is defined by actions within a context but it is an abstraction in that the description is not a routinised report of action but exemplifies the students' reflections on their actions as they strive to communicate with each other and with the computer" (Hoyles 1994, S. 174). Eine derartige situative Abstraktion zeigt sich etwa in dem von Hoyles, Sutherland und Healy (1991) beschriebenen Unterrichtsversuch zur Behandlung der Polygonalzahlen (vgl. Kap. 4.1.1). So konnten die beiden Schülerinnen die rekursive Gesetzmäßigkeit zwar nicht formal algebraisch, wohl aber mit Gesten und mit der „Excel-Sprache“ erfassen.
} 


\section{Planung und Durchführung der empirischen Untersuchung}

Das Ziel der im Rahmen dieser Arbeit durchgeführten empirischen Untersuchung ist es, die Arbeitsweisen und das Verständnis von Schülern im Umgang mit einer auf dem Tabellenkalkulationsprogramm (TKP) Excel basierenden Lernumgebung zu einem ausgewählten diskreten Lerngegenstand zu erfassen und zu analysieren. Die mit der Untersuchung verbundenen Fragestellungen, die zugrunde liegende Methode und die Überlegungen zu Datenerhebung und -auswertung werden im Folgenden erläutert, und es werden die beteiligten Lerngruppen vorgestellt. Im Zentrum der empirischen Untersuchung stand der Begriff der „Differenzenfunktion“ (vgl. Kap. 2.3.3). Das zu diesem Zweck konzipierte Lern- und Versuchsprogramm wird ausführlich in Kapitel 5.6 vorgestellt. Abschließend wird über die Durchführung der Untersuchung berichtet.

\subsection{Fragestellungen}

Anschließend an die Überlegungen aus Kapitel 4.4 interessieren drei verschiedene Ebenen des Arbeitens:

- Objektebene: Arbeiten mit den Differenzenfolgen/-funktionen;

- Darstellungsebene: Arbeiten mit den Darstellungen Graph und Tabelle;

- Werkzeugebene: Arbeiten mit dem TKP Excel.

Diese drei Ebenen sollen im Hinblick auf das erreichte bzw. zugrunde liegende Verständnis, d. h. die Problemlösefähigkeit sowie die Kenntnisse über bzw. Vorstellungen von Eigenschaften der Folgen/Z-Funktionen und Differenzenfolgen/-funktionen, interpretiert werden. Insbesondere sollen Beziehungen zwischen den Arbeitsweisen und dem Verständnis der Schüler aufgedeckt werden. Diese Interpretation erfolgt auf der Basis der Verständnismodelle von Vollrath und Skemp (vgl. Kap. 3.4) und berïcksichtigt neben den Arbeitsweisen auch verbale, schriftlich fixierte Äußerungen der Schüler. Das folgende Modell gibt die Interessensschwerpunkte der empirischen Untersuchung schematisch wieder:

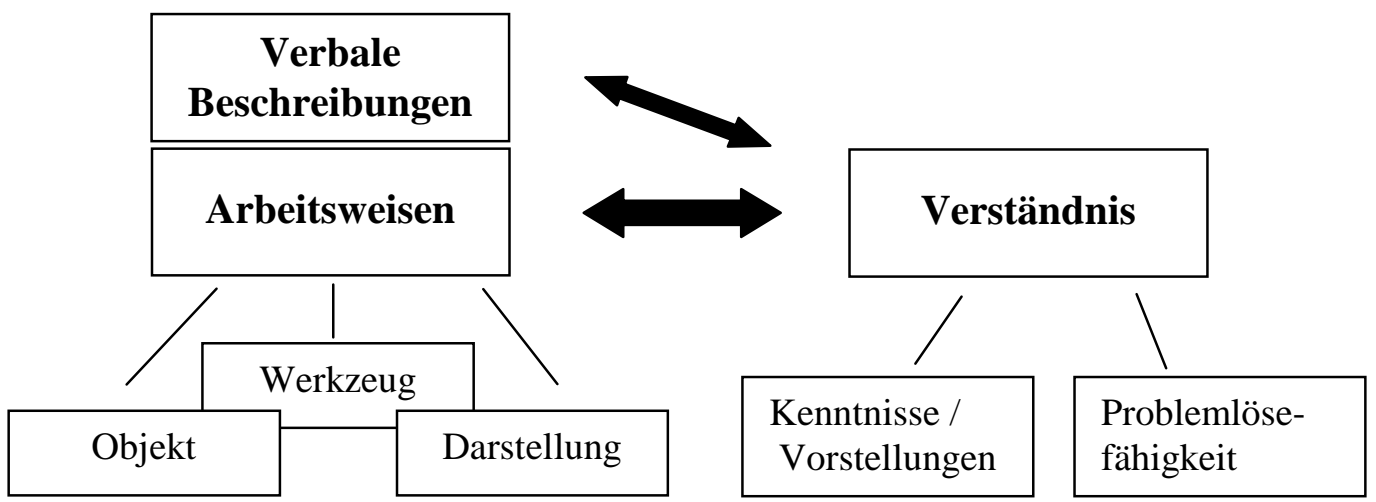

Abb. 38: Interessensschwerpunkte der empirischen Untersuchung 
Vor dem Hintergrund dieses Modells lassen sich die Fragestellungen der Untersuchung weiter präzisieren:

Im Hinblick auf die Arbeitsweisen:

- Wie arbeiten die Schüler mit den mathematischen Objekten Folge/Z-Funktion, Differenzenfolge/-funktion? Welche inhaltlichen Probleme ergeben sich beim algorithmischen, iterativen und funktionalen Arbeiten?

- Wie arbeiten die Schüler mit den Darstellungen? Gibt es bevorzugte Darstellungen? Lassen sich Strategien beim Verwenden der Darstellungen feststellen?

- Nutzen die Schüler die technischen Bedienungselemente des Werkzeugs (Rollbalken, Hilfe-Schaltflächen etc.) und wie nutzen sie diese? Welche Probleme ergeben sich dabei?

Im Hinblick auf das Verständnis:

- Können Schüler das Änderungsverhalten einer Folge/Z-Funktion mit Hilfe der Differenzenfolge/-funktion beschreiben?

- Erkennen Schüler Beziehungen zwischen (lokalen, globalen) Eigenschaften von Folgen/Z-Funktionen und deren Differenzenfolgen/-funktionen, und können sie diese begründen?

- Können Schüler Beziehungen zwischen einer Folge/Z-Funktion und deren Differenzenfolge/-funktion zum Lösen einfacher Probleme einsetzen?

- Wie begründen die Schüler Beziehungen zwischen Eigenschaften von Folgen/Z-Funktionen und deren Differenzenfolgen/-funktion? Argumentieren sie darstellungsabhängig oder eher darstellungsunabhängig? Welche Sichtweisen auf die Eigenschaften der Funktionen zeigen sich?

- Erkennen Schüler die heuristische Bedeutung des Differenzenfolgenkonzepts beim Lösen von Zählproblemen?

- Auf welche Problemlösefähigkeiten deuten die Strategien der Schüler im Rahmen von Problemlöseprozessen hin?

\subsection{Zur Methode der empirischen Untersuchung}

Es gibt bisher nur wenige empirische Untersuchungen, die sich mit Denk- und Handlungsprozessen von Schülern beim computerunterstützten Lernen auseinander setzen. Die vorliegenden Arbeiten unterscheiden sich sowohl im Hinblick auf die verwendeten Computerprogramme und die behandelten mathematischen Inhalte als auch hinsichtlich der theoretischen Orientierung und dem methodischen Vorgehen. So befasste sich Hölzl (1994) mit der Analyse von Lernprozessen beim Einsatz dynamischer Geometrie-Software im Bereich der Mittelstufengeometrie. Verschiedene Untersuchungen beschäftigten sich mit dem Umgang von Schülern mit Computeralgebrasystemen im Rahmen des Analysisunterrichts bzw. beim Umgang 
mit dem Funktionsbegriff, wobei den begrifflichen und technischen Schwierigkeiten der Lernenden besondere Beachtung geschenkt wurde (vgl. v. Hofe 1998, 1999, Warmuth 1995, Weigand 1999a). In jüngerer Zeit findet man erste umfassendere Untersuchungen zu Arbeitsweisen von Schülern beim Problemlösen mit einem TKP (vgl. Weigand 1999b, Weigand \& Thies 2001). Während es sich bei den meisten der vorstehend genannten Untersuchungen um qualitative Fallstudien handelt, die durch Videoaufzeichnungen dokumentiert wurden, ${ }^{186}$ verwendete Weigand (1999a, 1999b) erstmals ein Aufzeichnungsinstrument ${ }^{187}$, das es mit einem vertretbaren Zeitaufwand erlaubte, Daten bei einer größeren Schülergruppe zu erheben. Damit waren sowohl qualitative als auch quantitative Aussagen über die Arbeitsweisen der Probanden möglich.

Die Ergebnisse der bisherigen Studien können aufgrund der heterogenen Untersuchungsschwerpunkte nur m. E. zur Beantwortung der im Rahmen dieser empirischen Untersuchung interessierenden Fragestellungen hinzugezogen werden und als Ausgangspunkt von Hypothesenbildung und -tests dienen. Wir verstehen die Untersuchung daher in erster Linie als eine explorative Studie. Damit ist das „,mehr oder weniger systematische Sammeln von Informationen über einen Untersuchungsgegenstand gemeint, das die Formulierung von Hypothesen und Theorien vorbereitet" (Bortz \& Döring 1995, S. 330). Explorative Untersuchungen können sich zum Zweck der Hypothesenbildung sowohl mit vorhandenen Theorien (theoriebasierte Exploration) als auch mit bekannten Methoden (methodenbasierte Exploration) auseinander setzen. Weiterhin - und diese Strategie steht bei dieser Untersuchung im Vordergrund können explorative Untersuchungen auch bei der eingehenden Beobachtung des Untersuchungsgegenstands und der Erhebung quantitativen und qualitativen Datenmaterials anknüpfen (empiriebasierte Exploration). ${ }^{188}$ Durch eine Analyse quantitativen Datenmaterials lassen sich in diesem Zusammenhang bisher unberücksichtigte Muster aufdecken und durch eine geeignete tabellarische oder graphische Darstellung sichtbar machen. Qualitatives Datenmaterial $^{189}$ kann mit dem Ziel aufbereitet werden, wichtige Aspekte oder typische Merkmalskombinationen (Strukturen) aufzudecken, Zusammenhänge zwischen Ereignissen und Phänomenen festzustellen sowie die Veränderung von Merkmalskonfigurationen im Verlauf der Zeit im Rahmen von Längsschnittstudien zu beschreiben.

Indem qualitativ erhobenes Material im Rahmen einer umfassenden Datenanalyse quantifiziert und quantitativ weiterverarbeitet wird, können qualitative und quantitative Methoden miteinander verbunden werden (vgl. auch Kap. 5.4). So können Texte hinsichtlich einiger

\footnotetext{
${ }^{186}$ In diesem Zusammenhang wurden insbesondere der sozialen Interaktion und der Kommunikation der Schüler besondere Aufmerksamkeit geschenkt.

${ }^{187}$ Es handelt sich hierbei um das Programm ScreenCam der Firma Lotus (vgl. auch Kap. 5.3.2).

${ }^{188} \mathrm{Zu}$ einer umfassenden Behandlung des Themas „Hypothesengewinnung und Theoriebildung“ vgl. Bortz \& Döring 1995, S. 327 ff.

189 Bortz \& Döring (1995, S. 272) verstehen hierunter nicht-numerisches Material, insbesondere „Texte“ wie Beobachtungsprotokolle, Interviewtexte etc.
} 
ausgewählter Merkmale ausgezählt und damit statistischen Vergleichsmethoden zugänglich gemacht werden. ${ }^{190}$ Eine andere Möglichkeit zur Quantifizierung qualitativ erhobener Daten besteht darin, das Material in bereits feststehende oder im Verlauf der Datenanalyse erst entwickelte Kategorien einzusortieren. Die Analyse der Häufigkeitsverteilungen über diesen Kategorien ermöglicht dann einen ersten quantitativen Gesamteindruck über das Datenmaterial. Wenngleich explorative Untersuchungen in erster Linie der Hypothesengewinnung und Theoriebildung dienen, verzichten sie dabei keineswegs auf eine theoretische (Vorab-)Analyse des Forschungsgegenstands. „Allein die Auswahl derjenigen Variablen, die in den explorierten Datensatz aufgenommen werden, die Art und Weise ihrer Operationalisierung und Messung oder die Selektion von Untersuchungsobjekten ist von teils impliziten, teils expliziten Vorannahmen und Theorien geleitet. Der Unterschied besteht allerdings darin, dass dieses theoretische Vorverständnis noch nicht so weit elaboriert und fokussiert ist, dass sich operationale und schließlich auch statistische Hypothesen formulieren lassen, die einer Signifikanzprüfung unterzogen werden könnten“ (Bortz \& Döring 1995, S. 332).

Auch in die hier vorgestellte empirische Untersuchung fließen theoretische Überlegungen ein. So begründet sich das Interesse für die Arbeitsweisen von Schülern im Umgang mit TKP in der Bedeutung von Handlungen für die kognitive Konstruktion von Begriffen und Operationen (vgl. Kap. 3) sowie den didaktisch-methodischen Möglichkeiten beim Einsatz dieses neuen Werkzeugs (vgl. Kap. 4). Statt des Begriffs „Handlung“ verwenden wir in diesem Zusammenhang den Begriff „Arbeitsweisen“, der stärker an tatsächlich beobachtbare Handlungen gebunden ist und im Hinblick auf die Untersuchungsfragen in Kapitel 5.1 noch weiter operationalisiert werden kann (vgl. Tab. 4).

\begin{tabular}{|l|l|l|l|l|l|}
\hline \multicolumn{2}{|c|}{$\begin{array}{c}\text { Arbeitsweisen } \\
\text { Objekten auf der }\end{array}$} & \multicolumn{2}{|c|}{$\begin{array}{c}\text { Arbeiten auf der } \\
\text { Darstellungsebene }\end{array}$} & \multicolumn{2}{c|}{$\begin{array}{c}\text { Arbeiten auf der } \\
\text { Werkzeugebene }\end{array}$} \\
\hline $\begin{array}{l}\text { Algorithmisches Arbeiten } \\
\text { Funktionales Arbeiten } \\
\text { Iteratives Arbeiten }\end{array}$ & $\begin{array}{l}\text { Inhaltliche } \\
\text { Probleme }\end{array}$ & $\begin{array}{l}\text { Anzahl und Art } \\
\text { der gewählten } \\
\text { Darstellungen }\end{array}$ & $\begin{array}{l}\text { Utrategien im } \\
\text { Umgang mit den } \\
\text { Darstellungen }\end{array}$ & $\begin{array}{l}\text { Verwenden der } \\
\text { (technischen) Be- } \\
\text { dienungselemente } \\
\text { des Werkzeugs }\end{array}$ & $\begin{array}{l}\text { Technische } \\
\text { Probleme }\end{array}$ \\
\hline
\end{tabular}

Tab. 4: Analyse der Arbeitsweisen auf drei Ebenen

Auch bei der Analyse des Verständnisses werden mit den Modellen von Skemp und Vollrath theoretische Konzepte hinzugezogen, die - wie in Kap. 3.4 herausgestellt - eine Beschreibung des Verstehens durch gewisse nachprüfbare Fähigkeiten und Kenntnisse erlauben. Diese Fähigkeiten und Kenntnisse lassen sich durch die eingangs dieses Kapitels beschriebenen Fragen konkretisieren und damit bestimmten Stufen des Verstehens zuordnen.

\footnotetext{
${ }^{190}$ Hierzu wird meist eine quantitative Inhaltsanalyse eingesetzt (vgl. Bortz \& Döring 1995, S. 138 ff.).
} 


\section{3 Überlegungen zur Datenerhebung}

Im Zentrum der Untersuchung steht die Analyse der Arbeitsweisen der Probanden beim Umgang mit einer TKP-gestützten Lernumgebung. Es stellt sich somit zunächst die Frage, wie diese Daten erhoben und aufgezeichnet werden können.

\subsubsection{Beobachtungsform}

Eine der wichtigsten Grundtechniken zur Erhebung qualitativen Datenmaterials stellt neben der nicht-standardisierten oder halb-standardisierten Befragung die Methode der Beobachtung dar. ${ }^{191}$ Beobachtungsmethoden werden insbesondere zur Erfassung von Verhaltensweisen eingesetzt und bilden daher im Hinblick auf unsere Untersuchungsziele einen geeigneten Rahmen.

Innerhalb dieses Rahmens sind weitere Entscheidungen hinsichtlich der Form der Beobachtung notwendig. ${ }^{192}$ Grundsätzlich gibt es hierbei verschiedene Alternativen. So ist es möglich, die Untersuchung in der natürlichen Umgebung der Schüler, also im Klassenraum, durchzuführen (Feldbeobachtung). In Form einer Unterrichtssequenz werden die Schüler durch die Versuchsleiterin mit dem Thema „Differenzenfunktionen“ vertraut gemacht. Ausgewählte Problemstellungen oder Module werden mit Hilfe des Rechners bearbeitet, wobei es das umfassende und komplexe Thema nahe legt, die individuelle Arbeit am Computer immer wieder durch alternative Arbeits- und Sozialformen abzulösen. Bei diesem methodischen Vorgehen ist jedoch zu bedenken, dass durch die aktive Teilnahme der Versuchsleiterin am Unterrichtsgeschehen Sicht- und Arbeitsweisen der Schüler beeinflusst werden können. Da man aber an individuellen und „spontanen“ Arbeitsweisen, Lösungsstrategien und Argumentationen interessiert ist, stößt die obige Methode an Grenzen. Eine Beobachtung im schulischen Umfeld wirft darüber hinaus auch organisatorische Probleme auf, da die Computerräume der meisten Schulen nur für kleinere Gruppen eine ausreichende Anzahl an Rechnern bieten. Das Interesse an individuellen Arbeitsweisen macht es jedoch erforderlich, dass für jeden Schüler ein eigener Computer bereitsteht. Aus diesen Gründen ist es sinnvoll, die Untersuchung in Räumlichkeiten wie etwa die öffentlichen Computerräume einer Universität zu verlagern, die diese technischen Voraussetzungen erfüllen. Um die Beeinflussung durch die Versuchsleiterin zu minimieren, empfiehlt es sich, das Untersuchungsprogramm sowohl als Test- als auch als Lernprogramm zu konzipieren, so dass die gesamte Untersuchung durch das Arbeiten am

\footnotetext{
${ }^{191}$ Einen Überblick über die verschiedenen qualitativen Datenerhebungsmethoden geben Bortz \& Döring 1995, S. $282 \mathrm{ff}$.

${ }^{192} \mathrm{Zu}$ den verschiedenen Formen der Beobachtung vgl. z. B. Lamnek 1993, S. 247 ff. Bei den unterschiedlichen Beobachtungsformen orientiert man sich im Rahmen der Differenzierungsmethoden Wissenschaftlichkeit, Standardisierung, Transparenz, Beobachterrolle, Partizipationsgrad, Realitätsbezug und Natürlichkeit der Situation unterschiedlich stark an qualitativen bzw. quantitativen Forschungsmethoden. Diese Unterscheidungsmerkmale
} 
Rechner realisiert werden kann. Dabei kann das Thema „Differenzenfunktionen“ nur exemplarisch behandelt werden, wobei jedoch durch eine geeignete Auswahl der Aufgaben erste Eindrücke über die Arbeits- und Sichtweisen der Schüler gewonnen werden können. Diese Untersuchungsmethode geht zurück auf einen Vorschlag von Weigand (1989), der in einer empirischen Untersuchung das Verständnis von Schülern im Umgang mit Iterationen analysierte und dabei ein speziell zu diesem Zweck konzipiertes Computerprogramm verwendete.

Das Versuchsdesign lässt sich somit folgendermaßen beschreiben: Die Beobachtung erfolgt in einem künstlich geschaffenen Umfeld (Laborbeobachtung), in der die individuellen Arbeitsweisen der Schüler erfasst werden. ${ }^{193}$ Die Versuchsleiterin greift dabei soweit möglich nicht in die Bearbeitungsphase der Schüler ein. Sie ist jedoch in einer allgemeinen Einleitungs- und Instruktionsphase explizit in die Situation eingebunden und darüber hinaus während der gesamten Bearbeitungsphase im Untersuchungsraum anwesend, um auf mögliche Fragen eingehen zu können. Damit handelt es sich um eine Form der teilnehmenden Beobachtung, bei der der Partizipationsgrad der Teilnahme zwischen aktiver und passiver Teilnahme variiert. Diese Beobachtung verläuft offen, d. h. den Schülern wird zu Beginn der Untersuchung mitgeteilt, dass ihre Arbeitsweisen am Computer aufgezeichnet werden.

\subsubsection{Datenfixierung}

Die Aufzeichnung der Arbeitsweisen erfolgt über das im Hintergrund mitlaufende und für den Probanden „unsichtbare“ Programm „ScreenCam“ der Firma Lotus. Mit diesem Programm können alle Aktivitäten des Computerbenutzers, d. h. alle Eingaben über Tastatur und Maus in Form eines „Computerprotokolls“ aufgezeichnet und abgespeichert werden. Für die Auswertung lässt sich dann analysieren, ob und wie ein Proband eine Aufgabe gelöst hat, wie lange er für eine Aufgabe benötigt hat, welche und wie viele Darstellungen er betrachtet hat, wie häufig und wie lange er mit welchen Parametern experimentiert hat, wie und wann ein Wechsel zwischen den Darstellungen erfolgte. Mit solchen Computerprotokollen ist es möglich, Daten bei einer größeren Gruppe von Probanden zu erheben. Verglichen mit den Möglichkeiten einer Datenaufzeichnung mit Videokameras, bei deren Einsatz man in der Regel nur wenige Probanden beobachten kann und darüber hinaus Probleme beim genauen Positionieren der Videokamera entstehen können, stellen Computerprotokolle einen wesentlichen

und damit auch die Extremtypen qualitativer und quantitativer Beobachtung sind jedoch - wie Lamnek hervorhebt - analytischer Natur. In der Realität sind Kombinationen sowie differenziert-nuancierte Formen denkbar.

${ }^{193}$ Das Arbeiten am Computer kann prinzipiell einzeln oder in Partnerarbeit erfolgen. Bei dieser Untersuchung stehen individuelle Arbeitsweisen im Zentrum des Forschungsinteresses. Deshalb sind kooperative Bearbeitungen weniger geeignet. Dabei ist jedoch zu berücksichtigen, dass ein individuelles Arbeiten die Rekonstruktion der Verständnis- und Denkprozesse der Schüler erschweren kann. Hier wären verbale Äußerungen, wie sie bei der Bearbeitung in Paaren zu erwarten wären, hilfreich. Dennoch meinen wir, dass sich individuelle Denkprozesse und unterschiedliche Verständnisniveaus (in einem gewissen Rahmen) auch in den sichtbaren Arbeitsweisen äußern bzw. in sie hinein interpretiert werden können und sich ferner in den unterschiedlichen verschriftlichten Argumentationen widerspiegeln. 
organisatorischen Vorteil dar. Durch den Einbau eines Mikrofons ließen sich auch verbale Äußerungen der Probanden festhalten. Da wir uns in dieser Untersuchung für Einzelbearbeitungen entschieden haben, erscheint jedoch eine zusätzliche Sprachaufzeichnung nicht notwendig.

Um die erhobenen Daten interpretativ auswerten zu können, muss das Computerprotokoll zunächst in eine verschriftlichte Form überführt werden. Während es für sprachliche Äußerungen verschiedene Transkriptionssysteme ${ }^{194}$ unterschiedlicher Genauigkeit gibt, sind Kriterien zur Analyse nonverbalen Verhaltens erst zu entwickeln. Diese Kriterien sollen einerseits die Arbeitsweisen der Probanden möglichst umfassend wiedergeben, zum anderen aber das komplexe Geschehen soweit reduzieren und vorstrukturieren, dass das verschriftlichte Verlaufsprotokoll anschließend als Basis für die Auswertung und Interpretation dienen kann. Ausgehend von der Fragestellung und den verschiedenen Dimensionen des Arbeitens, bietet es sich zunächst an, sämtliche eindeutig zu identifizierenden Aktivitäten wie Tastatureingaben, Wechsel zwischen Darstellungen und Wechsel zwischen den einzelnen Aufgaben sowie das Betätigen der Hinweis- oder Lösungsschaltflächen zu notieren. Darüber kann das Bedienen des Rollbalkens kenntlich gemacht werden. Hier ist eine erste Entscheidung notwendig, wie genau das Arbeiten mit dem Rollbalken protokolliert wird. Sicher ist es nicht sinnvoll, alle gewählten Zahlenwerte zu notieren, da zum Teil damit zu rechnen ist, dass der Rollbalken sehr ausgiebig und möglicherweise auch unreflektiert bedient wird. In einem ersten Schritt wird daher zunächst nur die Art bzw. der Name des Parameters notiert, der mit dem Rollbalken verändert wird. In Verbindung mit einigen Fragestellungen wird es darüber hinaus erforderlich sein, auch die tatsächlich gewählten Zahlenwerte näher zu analysieren, sowie mögliche (Reflexions-)Pausen des Schülers während des Experimentierens mit den Parametern näher zu untersuchen. In diesen Fällen werden neben der Reihenfolge der variierten Parameter auch die jeweiligen „Zwischenwerte“ notiert, die sich nach dem Experimentieren mit den betreffenden Rollbalken ergeben. Zu den komplexeren Aktivitäten gehören die Cursorbewegungen, die durch die Steuerung mit der Computermaus entstehen. Die Positionierung des Cursors auf dem Bildschirm kann ein Indiz dafür sein, auf welchen Bildausschnitt die Aufmerksamkeit des Betrachters gerade gerichtet ist. So ist zum einen denkbar, dass mit dem Cursor der gerade gelesene Aufgabentext „,nachgefahren“ wird. Zum anderen kann das Anklicken einzelner Tabellenwerte bzw. das Deuten mit dem Cursor auf bestimmte Koordinatenpunkte, einen Hinweis auf die zugrunde liegenden funktionalen Betrachtungen geben. Nicht alle Cursorbewegungen spiegeln zielgerichtetes und aufgabenbezogenes Handeln wider. Ziellose Bildschirmbewegungen, Scrollen mit den Bildlaufleisten oder auch der Aufruf eines an-

\footnotetext{
${ }^{194}$ Ein Standard zur Verschriftlichung hat sich bisher aber hier noch nicht durchgesetzt. Statt dessen scheint sich der Fokus häufig zu stark auf eine möglichst genaue Klassifikation von Äußerungen zu richten. Der damit verbundene Aufwand steht oft in keinem Verhältnis mehr zur interessierenden Fragestellung (vgl. Flick 1991, S. 161 f.).
} 
deren Programms sind denkbar. In dem Verlaufsprotokoll wird daher eine Kommentarspalte eingefügt, die Raum für zusätzliche Bemerkungen bzw. ausführliche Beschreibungen bietet. Die folgende Tabelle gibt einen Transkriptausschnitt im Sinne der oben beschriebenen Kriterien wieder. ${ }^{195}$

\begin{tabular}{|c|c|c|c|c|c|c|}
\hline Zeit & Aug. & $\begin{array}{l}\text { Aufg.- } \\
\text { stellung }\end{array}$ & Tabelle & Graph & Kommentar & Eingaben über Tastatur \\
\hline 6.29 & $3 b$ & $\mathrm{x}$ & & & & \\
\hline 6.31 & & & $\mathrm{x}$ & & $\begin{array}{l}\text { Der Cursor zeigt auf das Intervall } \\
{[5,6] \text { und anschließend auf den }} \\
\text { Funktionswert } D(5) \text {. }\end{array}$ & \\
\hline 6.38 & & $\mathrm{x}$ & & & & \\
\hline 6.52 & & & & $\mathrm{X}$ & $\begin{array}{l}\text { Der Cursor zeigt auf die Funkti- } \\
\text { onswerte } D(5), D(6) \text {. }\end{array}$ & \\
\hline 7.05 & & $\mathrm{x}$ & & & $\begin{array}{l}\text { Lösungsüberprüfung mit dem } \\
\text { „OK-Button“. Es wird auf einen } \\
\text { Fehler hingewiesen. }\end{array}$ & $\begin{array}{l}\text { Eingabe des Änderungs- } \\
\text { werts } 2 \\
\text { Markieren des Kontroll- } \\
\text { kästchens „steigt““ }\end{array}$ \\
\hline 7.20 & & & $\mathrm{x}$ & & & \\
\hline 7.27 & & $\mathrm{x}$ & & & & $\begin{array}{l}\text { Eingabe des Änderungs- } \\
\text { werts } 7\end{array}$ \\
\hline 7.32 & & & $\mathrm{x}$ & & $\begin{array}{l}\text { Der Cursor zeigt auf die Funkti- } \\
\text { onswerte } D(5), D(4), D(3)\end{array}$ & \\
\hline 7.37 & & $\mathrm{x}$ & & & $\begin{array}{l}\text { Lösungsüberprüfung mit dem } \\
\text { „OK-Button“. Die Eingabe wird } \\
\text { bestätigt. }\end{array}$ & \\
\hline
\end{tabular}

Tab. 5: Transkriptausschnitt

\section{4 Überlegungen zur Auswertung der erhobenen Daten}

Im Rahmen der Auswertung und Interpretation des verschriftlichten Datenmaterials können verschiedene Methoden Anwendung finden. Beck und Maier (1994) beschreiben vier Typen der Textinterpretation innerhalb der mathematikdidaktischen Forschung, die sich zum einen in der Rolle der Theorie im Forschungsprozess und zum anderen in den zugrunde liegenden Zielen der Untersuchung unterscheiden. Das Ziel kategoriengeleiteter und kategorienentwickelnder Interpretationsansätze ist in der Regel eine Beschreibung des gesamten Untersuchungsfelds. Bei der kategoriengeleiteten Interpretation „legt der Forscher vor Eintritt in die Textanalyse begriffliche Kategorien fest, auf welche die im Text vorfindbaren Sinneinheiten kodiert werden. Diese Kategorien werden also nicht aus dem Text gewonnen, sondern entspringen theoretischen Vorannahmen und bleiben während des gesamten Kodiervorgangs unverändert. So lässt sich das Ergebnis dieser Interpretation anschließend in einer Häufigkeitsverteilung über die Kategorien quantifizieren“ (a. a. O., S. 45). Auf der Basis dieser Häu-

\footnotetext{
${ }^{195}$ Dieser Transkriptausschnitt wird im Rahmen der Auswertung (vgl. Kap. 6.3.2.3) genauer beschrieben.
} 
figkeitsverteilung können Strukturen beschrieben oder zuvor aufgestellte Hypothesen mit statistischen Mitteln geprüft werden. Bei der Methode der kategorienentwickelnden Interpretation ,wird versucht, ohne explizite Vorgaben Sinneinheiten im Text zu isolieren und zu klassifizieren“ (a. a. O., S. 47). Dabei wird zwar nicht völlig theoriefrei vorgegangen, aber man versucht, das theoretische Vorwissen bei der Kategorienfindung möglichst zurückzustellen. Einer kategorienentwicklenden Interpretation kann eine kategoriengeleitete Interpretation nachfolgen, indem das Datenmaterial mit dem entwickelten Kategoriensystem erneut analysiert und gegebenenfalls auch quantitativ ausgewertet wird. Während die kategorienentwickelnde und die kategoriengeleitete Interpretation auf die Beschreibung des gesamten Datenmaterials ausgerichtet sind, steht bei der explorativ-paraphrasierenden und der systematischextensionalen Textinterpretation die Charakterisierung einzelner Phänomene und Strukturen im Zentrum des Forschungsinteresses (Fallstudien). Dabei bezieht man sich bei der explorativ-paraphrasierenden Textinterpretation in der Regel auf mathematische oder mathematikdidaktische Vorstellungen und Theorien, wohingegen man beim systematisch-extensionalen Vorgehen versucht, sich einer theoriegeleiteten Interpretation weitestgehend zu enthalten, und bewusst mehrere Interpretationsmöglichkeiten anstrebt.

Im Rahmen unserer Untersuchung werden alle vier Methoden berücksichtigt. So wird beim Feststellen der Art und Häufigkeit der gewählten Darstellungen und der Art des gewählten Rollbalkens (bzw. Parameters) kategoriengeleitet vorgegangen. Diese Kategorien entstammen weniger theoretischen Modellen, sondern ergeben sich unmittelbar aus der Fragestellung und der Konzeption des Untersuchungsprogramms. ${ }^{196}$ Daneben interessieren wir uns für Arbeitsweisen im Umgang mit den diskreten Objekten Folge/Z-Funktion und Differenzenfolge/-funktion, die durch die Kategorien algorithmisch, iterativ und funktional beschrieben werden können. Auch bei der Interpretation des Verständnisses beziehen wir uns mit den Modellen von Vollrath und Skemp auf theoretische Konzepte, die gewisse Kategorien für verschiedene Verständnisstufen bzw. -ausprägungen angeben (Vollrath.: intuitiv, inhaltlich, integriert; formal; Skemp: instrumentell, relational, logisch). Ein kategorienbildendes Vorgehen ist in verschiedener Hinsicht erforderlich. So interessiert beim Arbeiten mit den verschiedenen Darstellungen neben der Art und der Anzahl der gewählten Darstellungen weiterhin, in welcher Reihenfolge die Darstellungen im Problemlöseprozess eingesetzt werden und ob und wann ein Strategiewechsel erfolgt. Zur Analyse der Strategien im Umgang mit Darstellungen und damit auch der Strategien im Umgang mit den mathematischen Objekten stehen keine Kategoriensysteme zur Verfügung. Sie werden erst anhand der Daten entwickelt. Insbesondere wird genauer zu untersuchen sein, wie ein zielgerichtetes Experimentieren von einem unreflektierten Vorgehen nach „Versuch und Irrtum“ abgegrenzt werden kann. Steht die techni-

\footnotetext{
${ }^{196}$ Flick (1991, S. 165) unterscheidet drei Quellen der Kategorienbildung. Erstens können Kategorien aus den Daten gewonnen werden, zum Zweiten ergeben sie sich aus einem (vorab formulierten) theoretischen Modell. Eine dritte Quelle für Kategorien bildet die Fragestellung, die der Ausgangspunkt der Untersuchung ist.
} 
sche Komponente, das Arbeiten mit dem TKP, im Vordergrund, kann analysiert werden, ob und wie die Bedienungselemente von Excel und die speziellen Hilfefunktionen des Lernprogramms genutzt werden und welche Schwierigkeiten sich im Umgang mit dem neuartigen Werkzeug ergeben. Auch hier werden Kategorien zu bilden sein. Ähnliches gilt bei der Analyse der inhaltlichen Probleme sowie bei der Suche nach typischen Argumentationsmustern in den verbalen Äußerungen der Probanden.

Neben diesen Analysen, die das gesamte Datenmaterial betreffen, sollen interessante Fälle detaillierter anhand von Transkriptausschnitten analysiert werden. Dabei wird man sich z. B. beim Beschreiben ausgezeichneter Problemlösestrategien auf mathematische bzw. mathematikdidaktische Vorstellungen bzw. Theorien und bereits vorhandene Kategorien beziehen können (explorativ-paraphrasierende Interpretation). Dagegen wird eine sinnvolle Analyse der Cursorbewegungen generell nur in Einzelfällen möglich sein, wobei man sich bei der Interpretation bewusst sein muss, dass sich durchaus verschiedene Absichten und Vorstellungen hinter den Aktivitäten der Probanden verbergen können (systematisch-extensionale Interpretation).

\subsection{Versuchsteilnehmer}

Das Forschungsinteresse richtet sich in erster Linie auf die Arbeitsweisen von Schülern. Um erste Erfahrungen über Arbeitsweisen von Lernenden mit dem Lernprogramm „Differenzenfunktionen“ zu sammeln und auch um die Qualität des Lernprogramms besser einschätzen zu können, wurde im Sommersemester 1999 zunächst eine Voruntersuchung mit Studierenden durchgeführt. Die Beobachtungen sollten dazu genutzt werden, das Programm zu überarbeiten und zu verbessern. Daneben war es ein Anliegen der Voruntersuchung, einen Überblick über die Arbeitsweisen der Studierenden zu erhalten. Damit sollte ein Vergleich mit den Arbeitsweisen von Schülern ermöglicht und insbesondere der Einfluss unterschiedlicher Vorkenntnisse ${ }^{197}$ untersucht werden.

\subsubsection{Studierende}

Die Probandengruppe der Studierenden setzte sich aus Teilnehmern der 2-stündigen Veranstaltung „Computer im Mathematikunterricht“ zusammen. Es handelte sich hierbei um 59 Studierende des Fachs Mathematik für die Lehrämter Grundschule, Haupt- und Realschule, Gymnasium sowie Sonderschule. Die Teilnahme an der Untersuchung war ein Teil des in diesem Semester zu erbringenden Leistungsnachweises. In der Vorlesung wurde zunächst ein Einblick in die technische Struktur und die wesentlichen Befehle des TKP Excel gegeben und

\footnotetext{
${ }^{197}$ So war zu erwarten, dass die Studierenden im Umgang mit Funktionen (insbesondere auch mit Folgen) und deren Darstellungen vertrauter waren als die Schüler. Im Gegensatz zu den Schülern besaßen sie zudem Kennt-
} 
das Programm unter didaktischen Gesichtspunkten diskutiert. Zum Erlernen und Üben des praktischen Umgangs mit Excel stand den Studierenden ein Lernprogramm zur Verfügung, das die gleiche Benutzeroberfläche wie das Untersuchungsprogramm besaß. Im Rahmen einer Übungsstunde hatten diese Studierenden anschließend die Aufgabe, das Lernprogramm „Differenzenfunktionen" zu bearbeiten.

\subsubsection{Schüler}

Der Hauptuntersuchung wurde mit drei Klassen der 11. Jahrgangsstufe aus drei verschiedenen Gießener Schulen durchgeführt. Insgesamt waren 53 Schüler an der Untersuchung beteiligt:

Schule A - 14 Schüler - Integrierte Gesamtschule;

Schule B - 22 Schüler - Additive Gesamtschule;

Schule C - 17 Schüler - Additive Gesamtschule (Informatikkurs).

Alle drei Klassen hatten zum Zeitpunkt der Untersuchung die Differenzialrechnung noch nicht behandelt. Während die Schüler der Schule C - nach Angaben des Fachlehrers - bereits vertiefte Kenntnisse in Excel besaßen, hatte nur ein Teil der Schüler der Schulen A und B im Rahmen von Informatikkursen erste Erfahrungen im Umgang mit einem Tabellenkalkulationsprogramm gesammelt. Diese Klassen wurden an ihren jeweiligen Schulen von der Versuchsleiterin eine Woche vor der Untersuchung in die grundlegende Bedienung des TKP Excel eingeführt. Im Rahmen einer eigenen Unterrichtseinheit wurden dazu zunächst die Programmoberfläche und die wesentlichen Befehle anhand von Beispielen vorgestellt. Im Anschluss konnten die Schüler mit Hilfe des bereits bei den Studierenden eingesetzten Lernprogramms noch einmal den technischen Umgang mit Excel üben und sich mit der Programmoberfläche und Steuerung vertraut machen. Bei den Schülern der Schule C wurde auf eine eigene Wiederholung verzichtet. Die Schüler hatten aber die Gelegenheit, sich auf freiwilliger Basis mit dem Lernprogramm zu Excel zu beschäftigen.

\subsection{Lern- und Versuchsumgebung}

Im Hinblick auf die Bedeutung des Folgenbegriffs und der Begriffe Differenzenfolge/Differenzenfunktion sowohl beim Problemlösen als auch in Verbindung mit einer Vorbereitung kontinuierlicher Konzepte aus dem Bereich der Differenzialrechnung (vgl. Kap. 2.2.1 und 2.3.3), wurde auf der Basis des TKP Excel ein Lern- und Versuchsprogramm zum Thema „Differenzenfunktionen“ konzipiert. Der Begriff der Differenzenfolge war den Probanden aus ihrem bisherigen Unterricht bzw. ihrem bisherigen Studium nicht bekannt. Das Programm stellte insofern ein Lernprogramm dar, als es die Probanden zunächst schrittweise in den in- 
haltlich neuen Themenkomplex eingeführte. Darüber hinaus diente es als Versuchsumgebung, in der das Arbeiten auf der Objekt-, Darstellungs- und Werkzeugebene untersucht werden konnte. Das Programm kann in einer ersten Übersicht wie folgt beschrieben werden:

\section{Teil: Einführung (Aufgabe 1)}

Die Schüler lernen den Begriff der Folge und deren Differenzenfolge kennen und werden in die Bedienung des Lernprogramms eingeführt.

\section{Teil: Arbeiten mit Darstellungen von Folgen/Z-Funktionen und Differenzenfolgen/- funktionen (Aufgabe 2, 3, 5)}

Das Konzept der Differenzenfolgen wird um das der Differenzenfunktionen erweitert. Die Schüler sollen Differenzenfolgen/-funktionen berechnen sowie Beziehungen zwischen Eigenschaften von Differenzenfolgen/-funktionen und deren Ausgangsfolgen/-funktionen anhand verschiedener Darstellungen erkennen und beschreiben.

\section{Teil: Lösen eines Zählproblems (Aufgabe 4)}

Die Schüler sollen ein Zählproblem lösen, d. h. eine rekursive und explizite Darstellung der Zählfolge angeben. In Zusammenhang mit der Suche nach einer expliziten Formel werden verschiedene Impulse zur Lösungsfindung gegeben. Dabei können die Schüler ihre Kenntnisse über Z-Funktionen und Differenzenfunktionen anwenden.

Da die Ergebnisse des Vortests mit den Studierenden - wie geplant - zu einer Überarbeitung des Versuchsprogramms führten, unterscheiden sich Umfang und auch die Formulierungen der einzelnen Aufgaben bei der Programmversion der Studierenden von denen der Schüler. Die Testversionen der Schülergruppen A/B und der Schülergruppe C sind im Wesentlichen vergleichbar. Es wurden beim Testprogramm der Gruppe $\mathrm{C}$ jedoch noch einige geringfügige Änderungen vorgenommen. Die Hintergründe für die Modifizierung der Programme und die Art der vorgenommenen Änderungen werden in Kapitel 5.7 erläutert. Im Folgenden werden die Untersuchungsfragen und -ziele in Zusammenhang mit den einzelnen Aufgaben diskutiert. Es handelt sich dabei um die Aufgaben, die dem Test mit den Schülergruppen A und B zugrunde lagen. 


\subsubsection{Aufgabe 1: Die Streichholzfolge}

Die erste Aufgabe dient dazu, die Probanden in die inhaltlich neue Thematik einzuführen und die grundlegenden Elemente des Werkzeugs Excel vorzustellen. Im Mittelpunkt der Aufgabe steht die „Streichholzfolge“, ein einfaches Zählproblem, dem ein linearer Zusammenhang zugrunde liegt. Diese Aufgabe erlaubt bewusst wenig Variationsmöglichkeiten, damit sichergestellt ist, dass die Probanden die Aufmerksamkeit auf die relevanten (neuen) Inhalte richten. Aus diesem Grund werden die Lösungen dieser Aufgabe in der Auswertung nur zum Teil und hauptsächlich nur unter werkzeugspezifischen Aspekten berücksichtigt. Im Folgenden werden die einzelnen Aufgabenteile sowie die damit verbundenen Ideen und Ziele detailliert beschrieben.

\section{a) Beschreiben Sie in Worten, wie sich die Anzahl der Streichhölzer von Stufe zu Stufe ändert.}

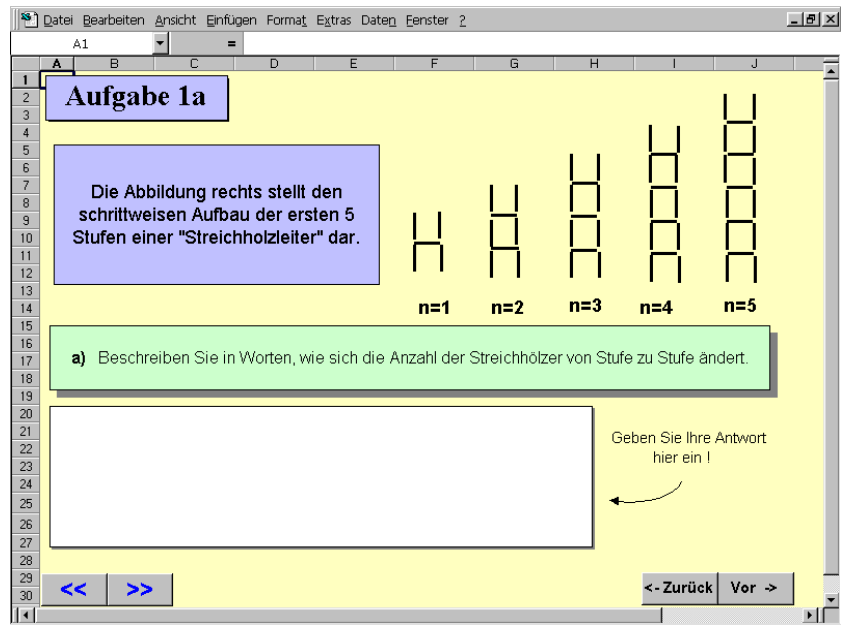

Abb. 39: Aufgabe 1a

Im Rahmen des ersten Aufgabenteils lernen Probanden die Streichholzfolge als Figurenmuster kennen. Die Probanden sollen die zugrunde liegende Gesetzmäßigkeit auf der ikonischen Ebene erfassen und verbal formulieren. 
b) Berechnen Sie in der Tabelle die Anzahl der Streichhölzer in Stufe 1, 2, 3, ... mit einer Formel.

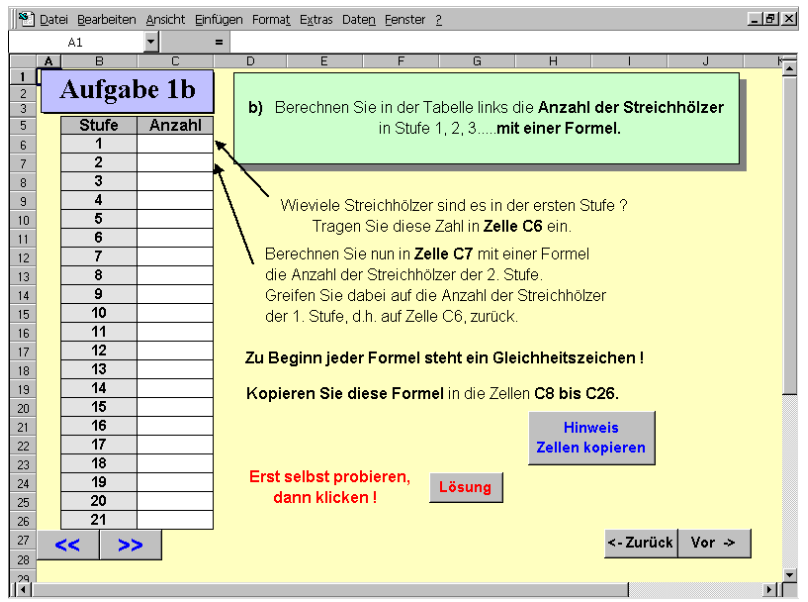

Abb. 40: Aufgabe 1b

Die Probanden sollen hier die rekursive Gesetzmäßigkeit der Streichholzfolge mit Hilfe relativer Zellbezüge erfassen. Die Formel soll zur Berechnung der ersten 21 Folgenglieder genutzt werden, indem sie in die entsprechenden Zellen kopiert wird. Die Aufgabenstellung wird durch einen Informationstext sowie einen „Hinweis-Button“ ergänzt. Damit werden die beiden technischen Besonderheiten „relative Zellbezüge“ und „Zellen kopieren“ erläutert. Diese Informationen dienen als Hilfestellungen und sollen die Probanden mit der Verwendung des Werkzeugs TKP (wieder) vertraut machen.

c) Wie viele Streichhölzer braucht man für eine Leiter mit 12 Sprossen?

d) Wie viele Sprossen hat eine Leiter, für die 60 Streichhölzer zur Verfügung stehen?

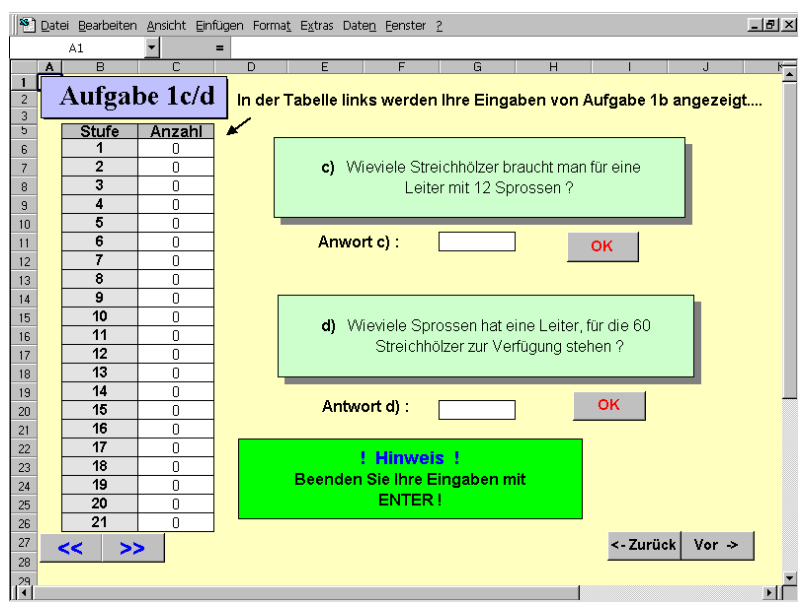

Abb. 41: Aufgabe 1c und 1d

Die Aufgabenteile 1c und 1d dienen dazu, das Arbeiten mit Tabellen zu üben. In diesem $\mathrm{Zu}-$ sammenhang sollen ,einfache“ Informationen über die Streichholzfolge aus der Tabelle ent- 
nommen werden. Die Folgenglieder müssen hierzu nicht mehr neu berechnet werden, sie werden automatisch von der Tabelle in Aufgabenteil 1b übernommen. Dabei prüft das Programm jedoch nicht, ob alle Folgenglieder richtig bestimmt wurden. Somit kann gegebenenfalls auch ein richtiges Lesen der Tabelle zu einem fehlerhaften Ergebnis führen. Dies wird bei einer Lösungsüberprüfung mit dem „Ok-Button“ angezeigt und durch einen Hinweis, noch einmal die Tabelle in $1 \mathrm{~b}$ zu überprüfen, ergänzt.

Im Sinne einer „Rückschau“ werden auf den nächsten beiden Blättern die wesentlichen Aspekte der bisherigen Aufgaben hervorgehoben. Dabei wird insbesondere das Änderungsverhalten der Folge und dessen Bedeutung bei der iterativen Berechnung der Folgenglieder betont. Diese Blätter dienen zur Vorbereitung der folgenden Aufgaben und sollen von den Probanden aufmerksam gelesen werden. ${ }^{198}$

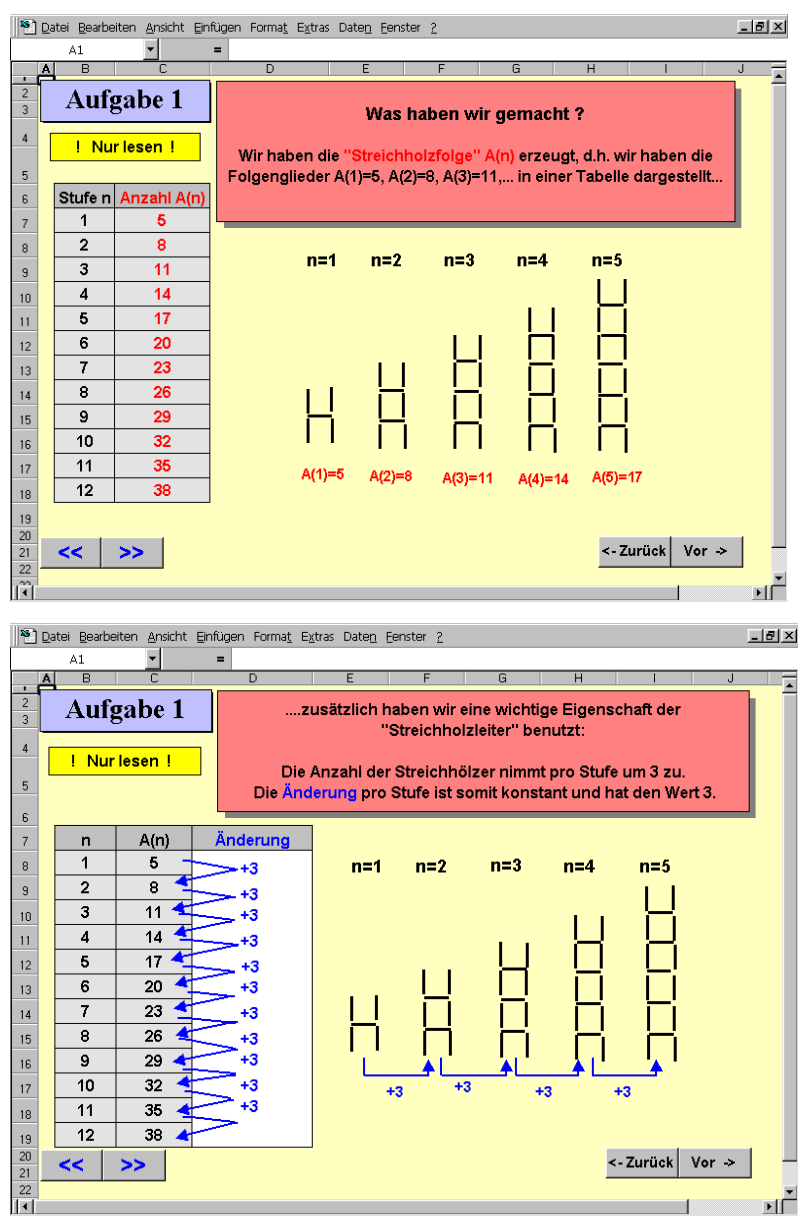

Abb. 42: Informationsblätter: Vorbereitung von Aufgabe 1e

\footnotetext{
${ }^{198}$ Im gesamten Programm werden immer wieder solche Rück- und Vorschauen eingebaut. Man kann sie in Beziehung sehen zu den von Ausubel vorgeschlagenen Organisationshilfen, den so genannten ,,advanced organizers“. „Hauptfunktion einer Organisationshilfe ist, die Kluft zu überbrücken zwischen dem, was der Lernende bereits weiß und dem, was er wissen muss, bevor er erfolgreich die jeweilige Aufgabe lernen kann." (Ausubel, zit. nach Weidenmann, Krapp, Hofer, Huber \& Mandl 1993, S. 521)
} 
e) Berechnen Sie die Änderung der Anzahl der Streichhölzer von Stufe zu Stufe mit einer Formel.

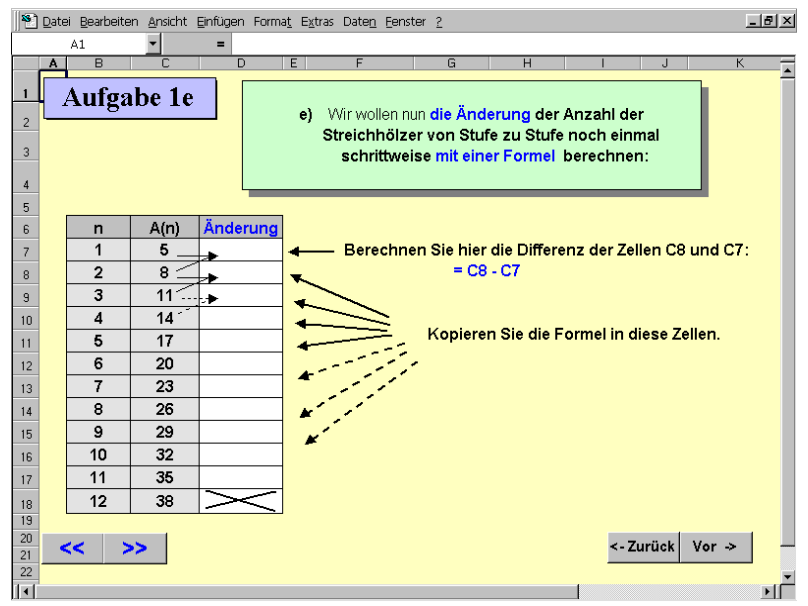

Abb. 43: Aufgabe 1e

Die Aufgabenteile 1e und 1f dienen dazu, den Begriff der Differenzenfunktion formal vorzubereiten. In Aufgabenteil 1e sollen zunächst alle Änderungen zweier aufeinander folgender Folgenglieder unter Verwendung relativer Zellbezüge berechnet werden.

Im Anschluss an Aufgabenteile 1e wird im Rahmen von Informationsblättern zunächst die Excel-Syntax der formalen algebraischen Syntax gegenübergestellt. Damit soll ein Verwechseln der beiden formalen Sprachen vermieden werden.
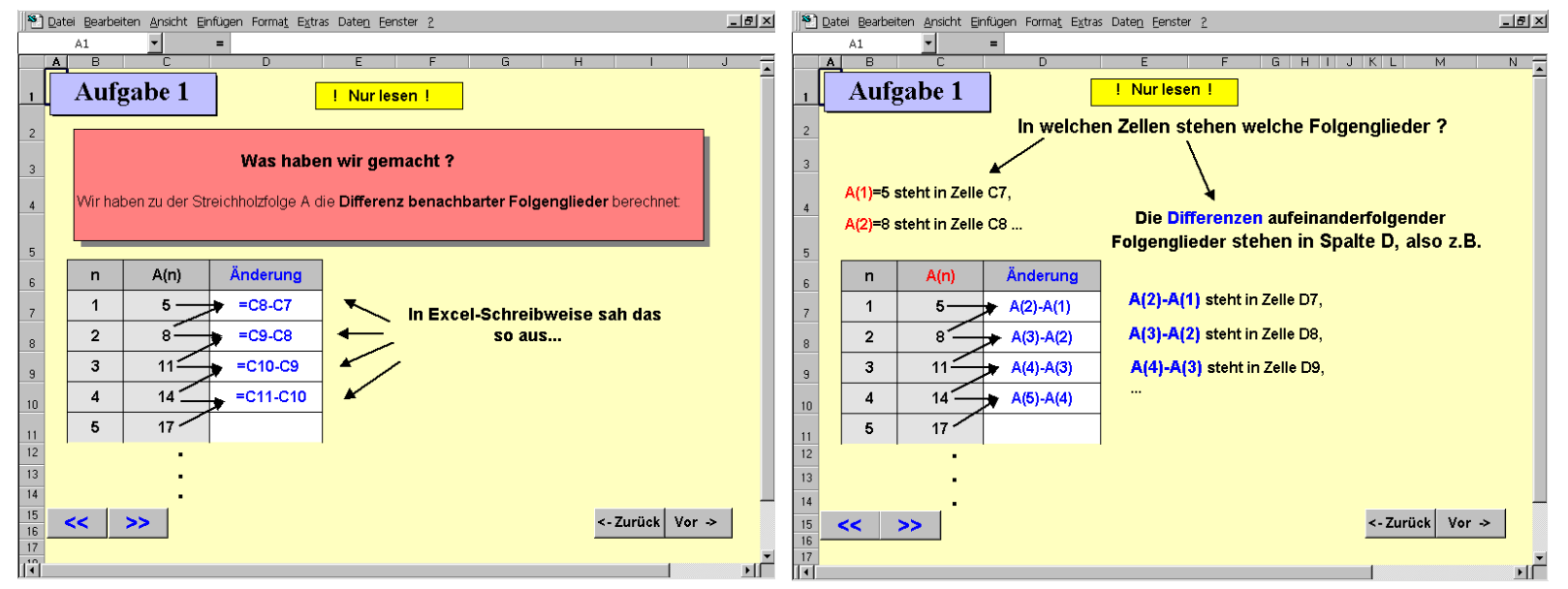

Abb. 44: Informationsblätter:

Vorbereitung von Aufgabe 1f 
f) Wir erhalten eine zweite Folge, deren Folgenglieder sich aus den Folgengliedern von $A$ berechnen lassen. Ergänzen Sie die fehlenden Lücken.

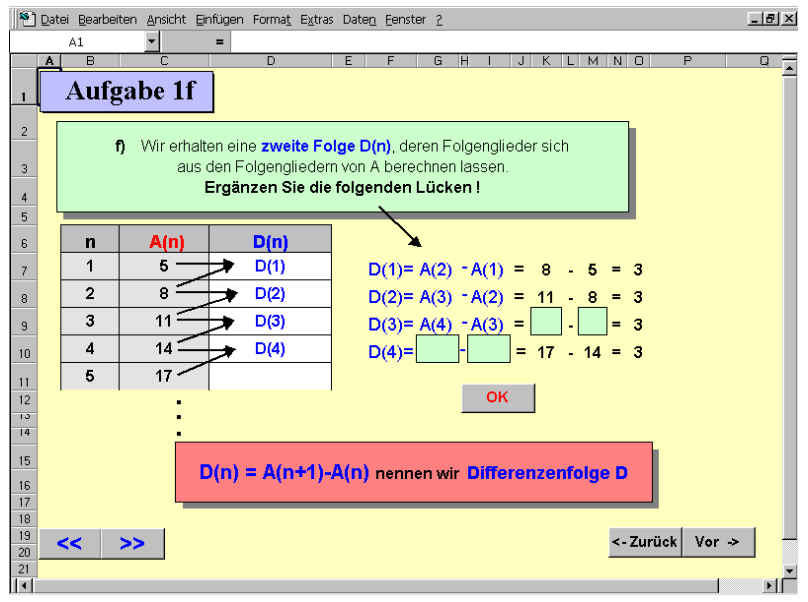

Abb. 45: Aufgabe 1f

Ziel von Aufgabenteil 1f ist es, die Beziehung zwischen einer Folge und deren Differenzenfolge exemplarisch zu verdeutlichen. Zu diesem Zweck sollen die Probanden das dritte bzw. vierte Glied der Differenzenfolge als numerische bzw. formale Beziehung der entsprechenden Ausgangsfolgenglieder ausdrücken. Im Rahmen dieser Aufgabe wird der Begriff „Differenzenfolge" eingeführt.

g) Hier sind die Streichholzfolge und deren Differenzenfolge graphisch dargestellt. Beschreiben Sie die beiden Funktionen.

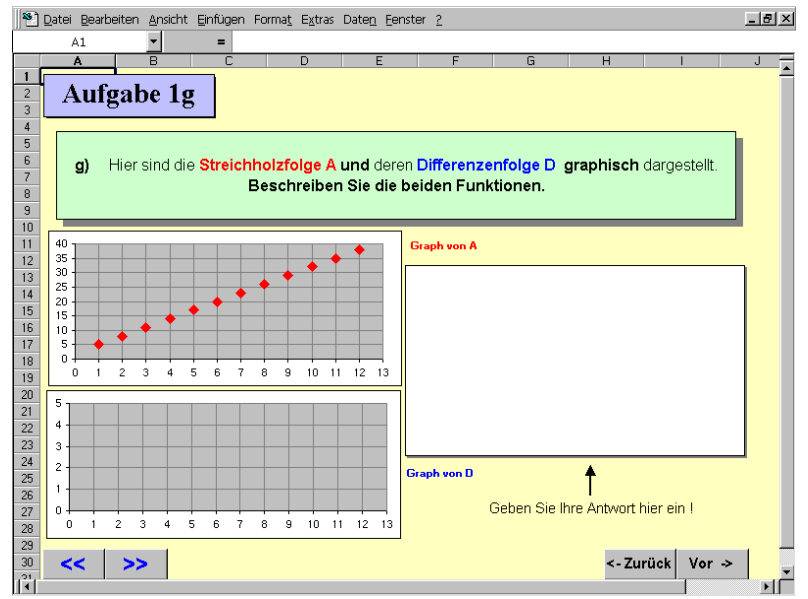

Abb. 46: Aufgabe 1g

Aufgabenteil 1g zeigt die graphischen Darstellungen der Streichholzfolge und der zuvor berechneten Differenzenfolge. Die Probanden sollen hier die beiden Folgen beschreiben. Es ergibt sich die Frage, welche Eigenschaften die Probanden erkennen bzw. welche Sicht oder Vorstellungen sich in den Beschreibungen widerspiegeln. Insbesondere interessiert hierbei, ob 
die Probanden den Zusammenhang zwischen Folge und Differenzenfolge erkennen oder ob sie beide Graphen isoliert betrachten.

h) Hier sehen Sie eine abgeänderte Version der Streichholzfolge. Berechnen Sie in der Tabelle die Differenzenfolge.

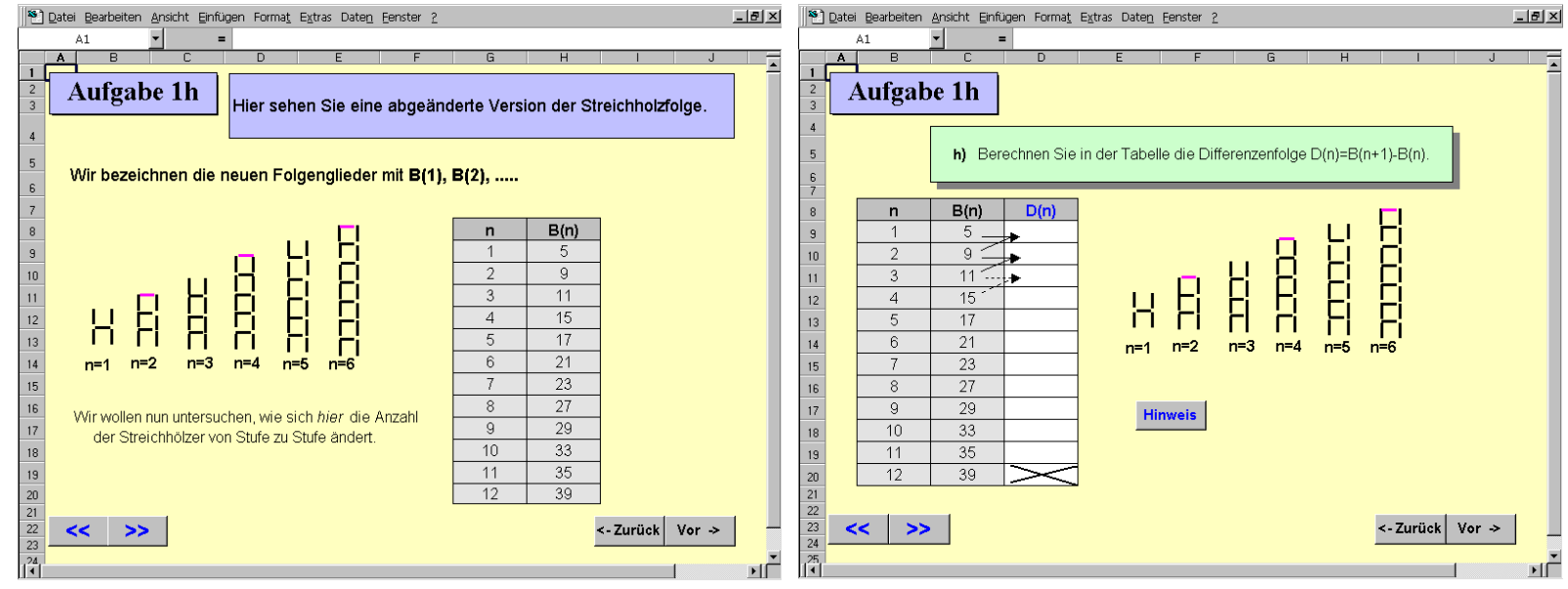

Abb. 47: Aufgabe 1h

Zur Festigung der Beziehung zwischen Folge und Differenzenfolge wird in den Aufgabenteilen $1 \mathrm{~h}$ und $1 \mathrm{j}$ eine abgeänderte Streichholzfolge betrachtet. Die Probanden sollen im Aufgabenteil $1 \mathrm{~h}$ zunächst die Differenzenfolge berechnen, indem sie die Formel $D(n)=B(n+1)-B(n)$ in die TKP-Syntax übersetzen und die entsprechende Excel-Formel in die übrigen Zellen kopieren.

\section{j) Wie lautet $D(9)$ und was bedeutet dieser Wert?}

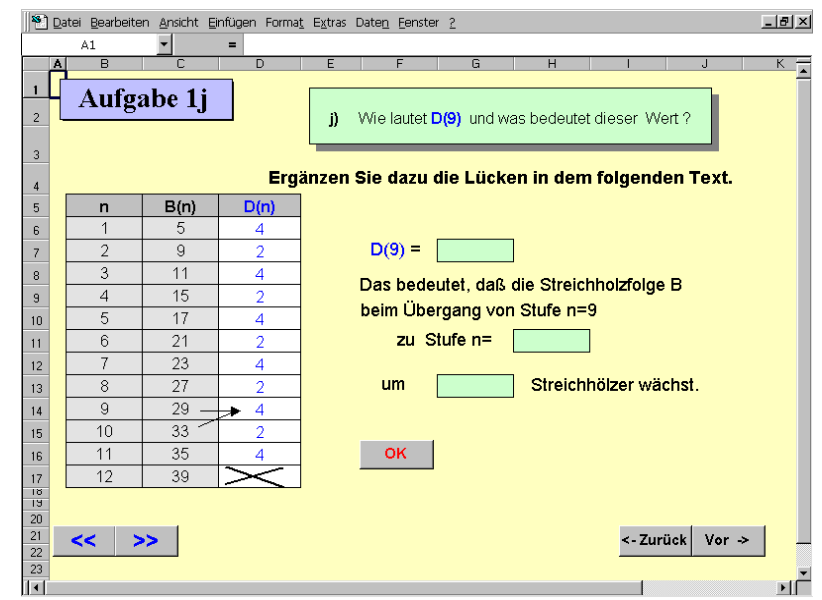

Abb. 48: Aufgabe 1j

In Aufgabe $1 \mathrm{j}$ soll das Folgenglied $D(9)$ inhaltlich interpretiert werden. Hiermit soll das Verständnis des (lokalen) Zusammenhangs von Folge und Differenzenfolge überprüft werden. 


\subsubsection{Aufgabe 2: Durchschnittstemperaturen}

Um die Bedeutung der Differenzenfolge als Folge von Änderungen hervorzuheben und inhaltlich einsichtig zu machen, werden in der zweiten Aufgabe zwei empirische Folgen von Durchschnittstemperaturen betrachtet. Es handelt sich zum einen um die Durchschnittstemperaturen in den Sommermonaten der Jahre 1972-1992 auf der Nordseeinsel Föhr (Aufgabenteile $2 \mathrm{a}$ und $2 \mathrm{~b}$ ) und zum anderen um die Temperaturen in Gießen in den Sommermonaten der Jahre 1985-1996 (Aufgabenteile 2c und 2d).

a) Auf der Sommerinsel Föhr wurden in der Sommersaison der Jahre 1979-1992 folgende Durchschnittstemperaturen gemessen. [...] Wir interessieren uns dafür, wie sich die Sommertemperaturen in diesen Jahren verändert haben.

Bilden Sie dazu in der Tabelle die Differenzenfunktion $D(n)=T(n+1)-T(n)$.

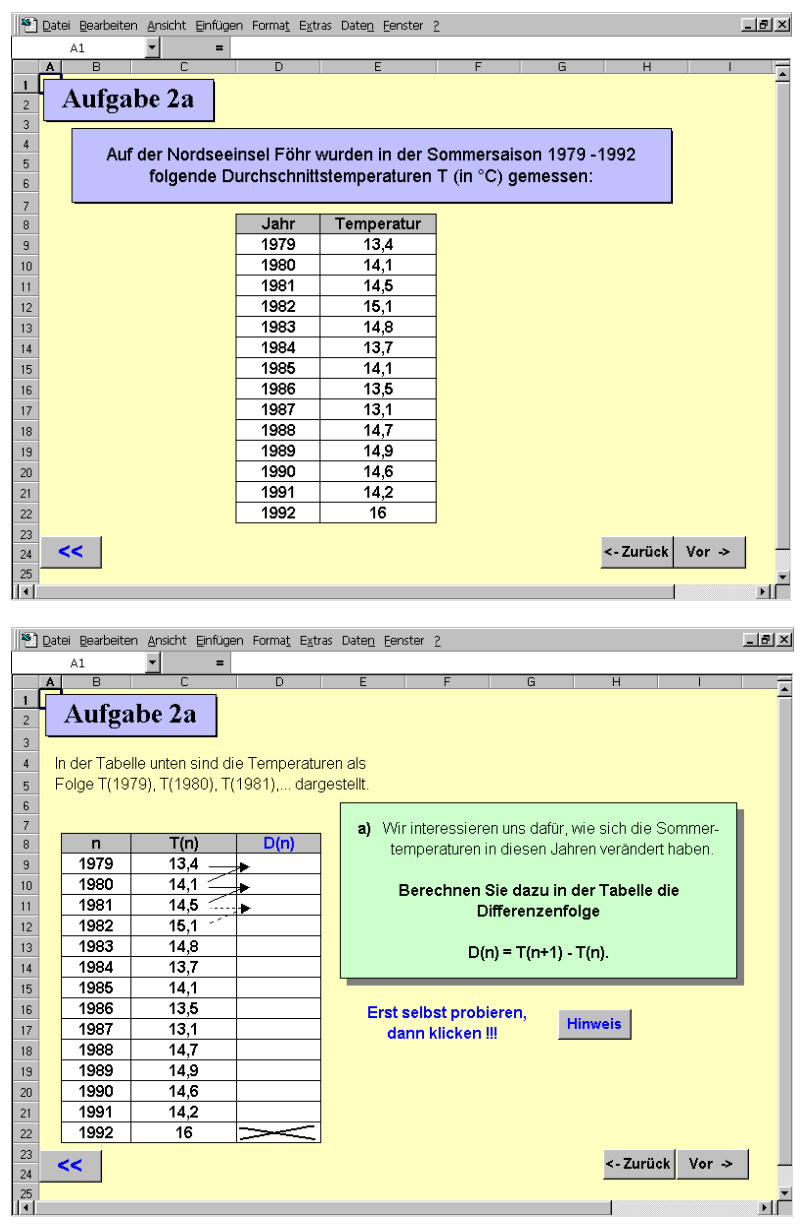

Abb. 49: Aufgabe 2a

Ähnlich wie bei Aufgabe 1h sollen die Probanden hier zunächst die Differenzenfolge berechnen und tabellarisch darstellen. Dabei soll untersucht werden, ob sich die Fertigkeiten der Probanden im Umgang mit Excel, d. h. insbesondere das Arbeiten mit relativen Zellbezügen und das Verwenden der Kopieroption, im Rahmen der Bearbeitung weiterentwickelt haben bzw. welche Schwierigkeiten auftreten. 
b) Um wie viel veränderte sich die Temperatur des Sommers 1984 gegenüber der des Sommers 1983?
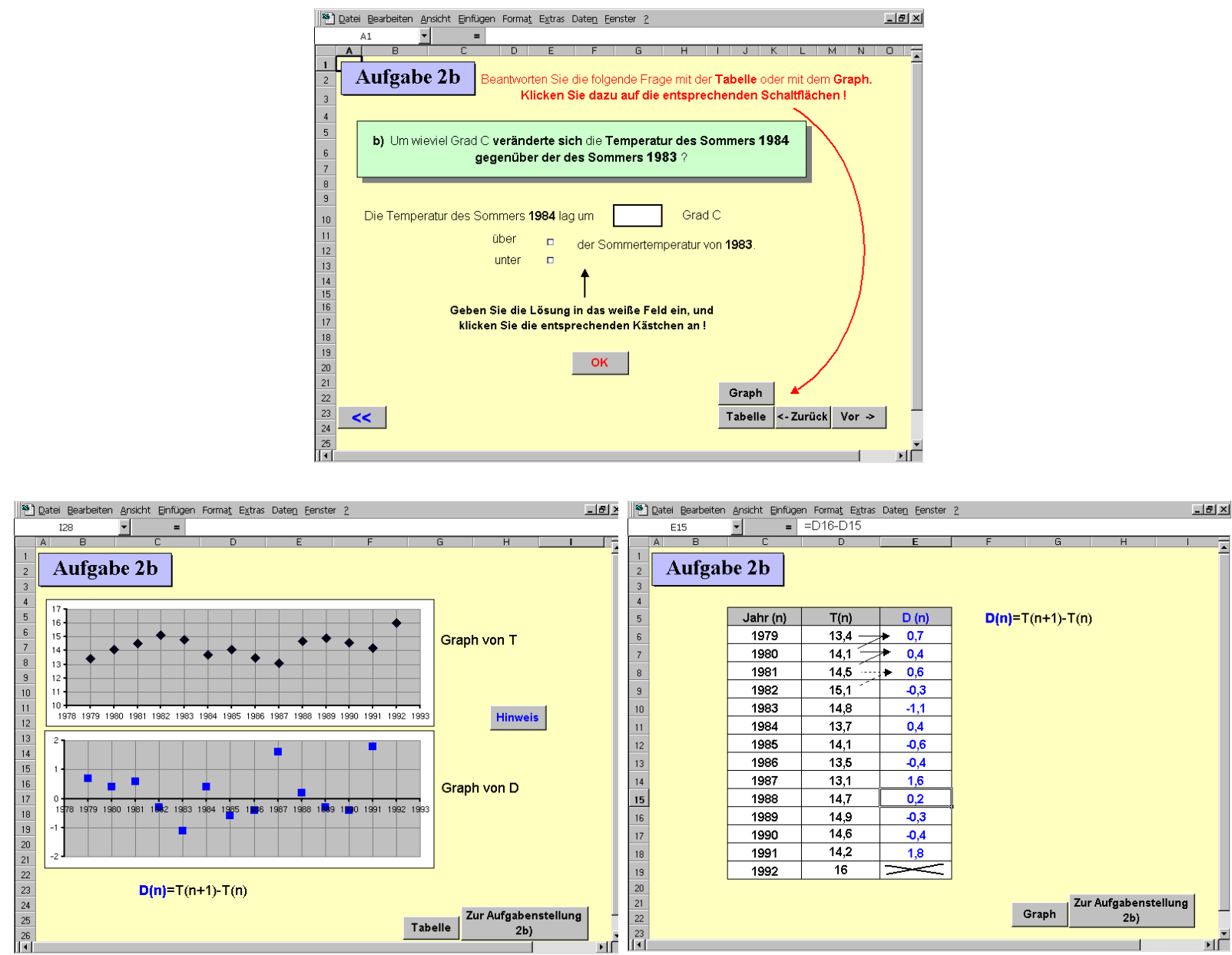

Abb. 50: Aufgabe 2b

Mit dieser Aufgabe soll überprüft werden, ob die Probanden die inhaltliche Bedeutung der Differenzenfolge erfasst haben, d. h. insbesondere eine Frage zum lokalen Änderungsverhalten der Ausgangsfolge beantworten können. Zur Beantwortung dieser Fragestellung können die Probanden die Darstellungen Tabelle und Graph hinzuziehen. Um angeben zu können, wie sich die Durchschnittstemperatur der Sommersaison 1984 gegenüber der der Sommersaison 1983 geändert hat, müssen die Probanden den Wert $D(1983)=-1,1$ aus der Tabelle oder dem Graph ablesen und inhaltlich interpretieren, d. h. das negative Vorzeichen als „Temperaturrückgang“ deuten. Während der numerische Wert $-1,1$ in der Tabelle direkt ersichtlich ist, ist es beim Arbeiten mit dem Graphen erforderlich, die Werte $D(1983)$ bzw. $T(1983)$ und $T(1984)$ zunächst durch ein Deuten mit dem Cursor auf die entsprechenden Koordinatenpunkte zu ,visualisieren“. 199

\footnotetext{
${ }^{199}$ Wenn der Cursor in die Nähe eines Koordinatenpunkts bewegt wird, werden die Koordinaten dieses Punkts automatisch angezeigt.
} 
Die Tabelle bietet somit gegenüber dem Arbeiten mit dem Graph einen Vorteil. Es ergibt sich die Frage, mit welcher Darstellung die Probanden die Aufgabe beantworten, ob sie den Vorteil des Arbeitens mit der Tabelle nutzen und ob sie mit Hilfe einer oder mehrerer Darstellungen arbeiten. Hier ist insbesondere ein Vergleich der Arbeitsweisen der Schüler und der Studierenden interessant.

c) Wir betrachten nun die Durchschnittstemperaturen in Gießen [...]. Welche durchschnittliche Sommertemperatur war im Jahr 1989 zu verzeichnen? Tragen Sie den Wert in die Tabelle ein!

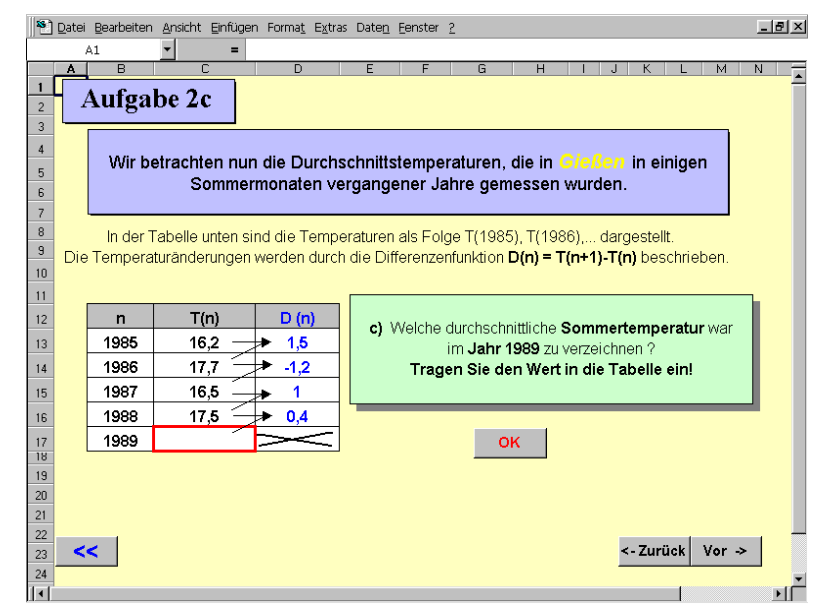

Abb. 51: Aufgabe 2c

Die Differenzenfolge ergibt sich aus der Folge $T$ durch Differenzenbildung. Umgekehrt lassen sich Glieder der Ausgangsfolge $T$ durch eine geeignete Summenbildung berechnen. Konkret erhält man den Wert $T(n)$ als Summe der Terme $T(n-1)$ und $D(n-1)$. Bei dieser Aufgabe soll überprüft werden, ob die Probanden die bisherige Operation „Bestimmen der Differenzenfolge“" lokal mit Hilfe der Tabelle umkehren können.

d) Hier sind die Gießener Temperaturen und ihre jährlichen Änderungen in den Jahren 1985- 1996 in einer Graphik dargestellt. Es fehlen jedoch zwei Werte.

Ergänzen Sie die fehlenden Werte [...]: [T(1992), $D(1994)]$.

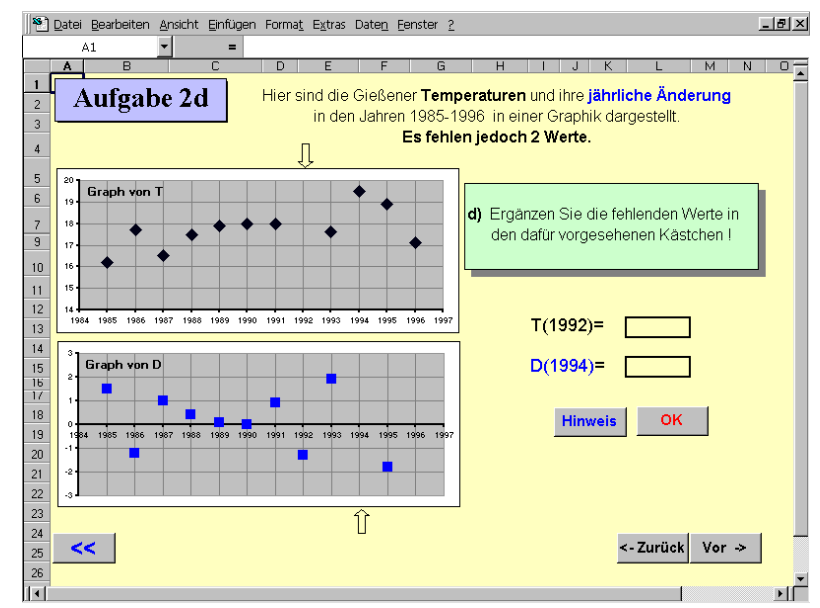

Abb. 52: Aufgabe 2d 
Bei dieser „Interpolationsaufgabe“ sind die Temperaturfolge und deren Differenzenfolge bis auf zwei Folgenglieder graphisch dargestellt. Die fehlenden Folgenglieder sollen mit Hilfe bereits dargestellter Folgenglieder der Folge $T$ bzw. $D$ bestimmt werden. Anders als bei der Tabelle in der vorhergehenden Aufgabe können die entsprechend zu verknüpfenden Funktionswerte der Folgen erst durch ein Deuten mit dem Mauszeiger auf den jeweiligen Koordinatenpunkt abgelesen werden. Hier ist die Frage, ob die Probanden auf der Basis der lokalen Beziehung zwischen Folge und Differenzenfolge Interpolationsprobleme lösen können..

\section{e) In welchen Zeitintervallen nahmen die durchschnittlichen Sommertemperaturen zu?}
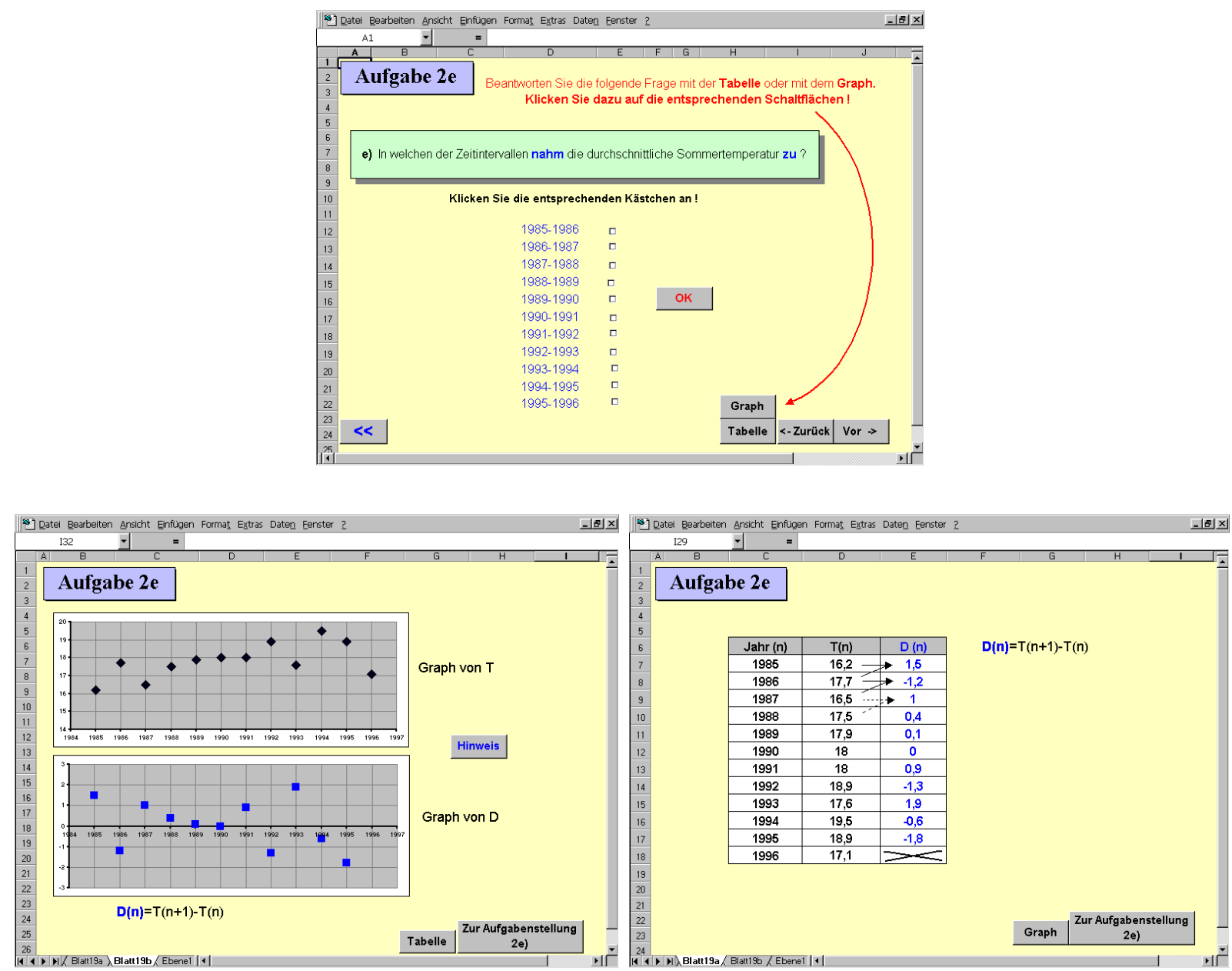

Abb. 53: Aufgabe 2e

Die Schüler sollen bei dieser Fragestellung das globale Änderungsverhalten der Temperaturfolge untersuchen, d. h. angeben, in welchen Zeitintervallen die durchschnittliche Temperatur ab- bzw. zugenommen hat. Zur Beantwortung dieser Frage können die Probanden die Tabelle oder den Graphen verwenden. Die graphischen Darstellungen von Folge und Differenzenfolge bieten den Vorteil, dass sich die Bereiche, in denen die durchschnittliche Sommertemperatur zunimmt, besser ,auf einen Blick“ erkennen lassen. So kann man anhand des Graphen von $T$ die Bereiche streng monotonen Wachstums schnell erfassen oder anhand des Graphen von 
$D$ feststellen, welche Funktionswerte größer Null sind bzw. oberhalb der $x$-Achse liegen. Ziel dieser Aufgabe ist es festzustellen, ob die Probanden die Vorteile des Arbeitens mit dem Graph erkennen und diese Darstellung zur Lösung dieses Problems verwenden. Weiterhin kann man in diesem Zusammenhang fragen, inwieweit Kenntnisse über den Zusammenhang zwischen Folge und Differenzenfolge zur Lösung dieses Problems eingesetzt werden, d. h. ob z. B. das Änderungsverhalten von $T$ mit Hilfe des Graphen von $T$ oder von $D$ analysiert wird.

\subsubsection{Aufgabe 3: Quadratische Z-Funktionen}

In dieser Aufgabe wird das Konzept der Differenzenfolge um das der Differenzenfunktion erweitert. In diesem Zusammenhang sollen quadratische Z-Funktionen und deren lokales und globales Änderungsverhalten analysiert werden.

a) Hier ist die Z-Funktion $f(n)=n^{2}-2 n+3(n \in Z)$ tabellarisch dargestellt. Berechnen Sie in der Tabelle $D(n)=f(n+1)-f(n)$.

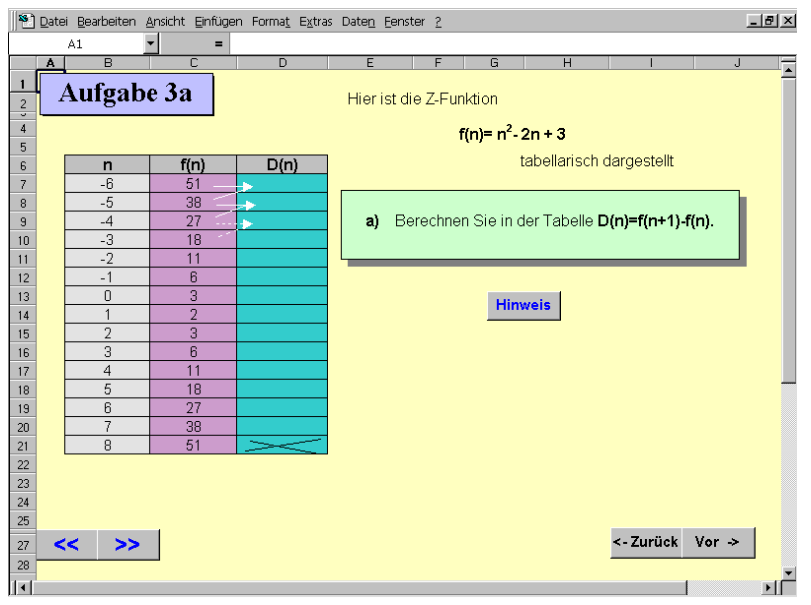

Abb. 54: Aufgabe 3a

Der Aufgabenteil 3a sowie die folgenden beiden Aufgabenteile ähneln den Aufgaben 2a bzw. $2 \mathrm{~b}$ und $2 \mathrm{~d}$. Da bei der empirischen Untersuchung mit den Schülern nach den ersten beiden großen Aufgabenblöcken eine Pause eingeplant war, sollte mit diesen „strukturähnlichen“ Aufgaben zum einen ein behutsamer Wiedereinstieg in die Thematik ermöglicht werden. Zum anderen erlauben die Formulierungen der Aufgaben einen Vergleich mit den entsprechenden Aufgabenteilen aus Aufgabe 2, d. h. insbesondere eine Aussage darüber, ob bei den Probanden ein Lerneffekt bzw. ein Verständniszuwachs zu verzeichnen ist. So soll bei dieser Aufgabe - ähnlich wie Aufgabe 2a - überprüft werden, ob die Schüler die technische Unterstützung von Excel zur Berechnung der Differenzenfolge nutzen, d. h. die erste Differenz mit Hilfe relativer Zellbezüge bilden und dann die restlichen Folgenglieder durch Kopieren der Formel berechnen. Dabei interessiert, ob es beim Übersetzen der Formel $D(n)=f(n+1)-f(n)$ in die 
TKP-Syntax zu Problemen kommt und in welcher Beziehung diese Probleme zu den technischen Schwierigkeiten bei Aufgabe 2a stehen.

Der Begriff der Differenzenfolge wird auf einem Informationsblatt um den Begriff der Differenzenfunktion erweitert und anhand des Graphen lokal als Änderung gedeutet.

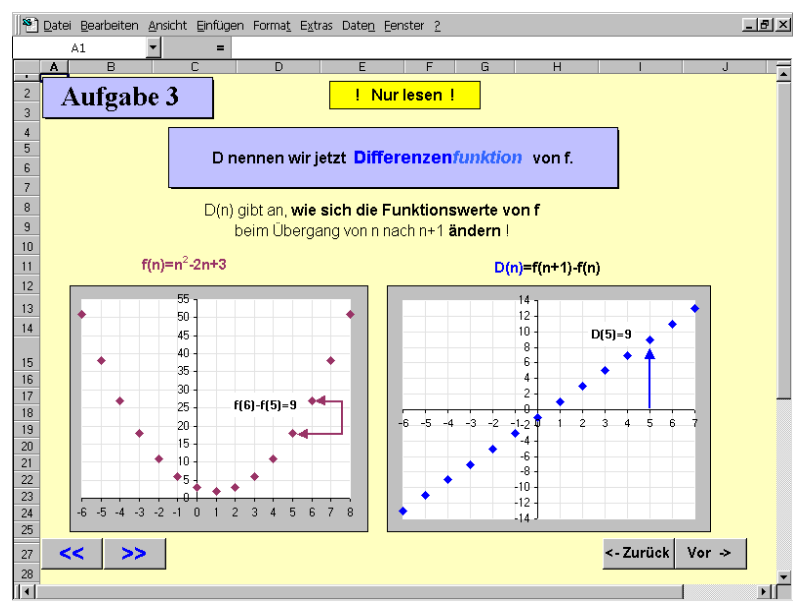

Abb. 55: Informationsblatt: Vorbereitung Aufgabe 3b

b) Wir betrachten nun eine andere Z-Funktion: $f(n)=n^{2}-4 n-13$. Wie ändert sich die Funktion beim Übergang von $n=5$ nach $n=6$ ?

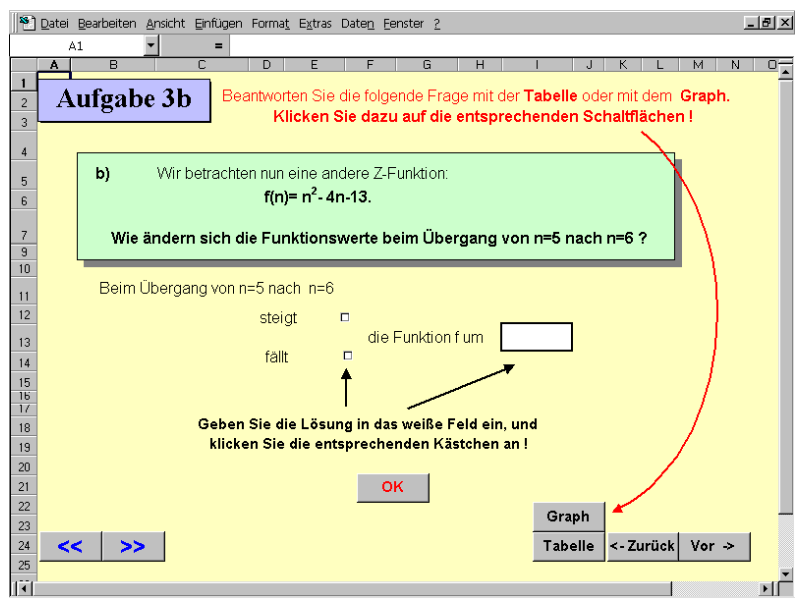

Abb. 56: Aufgabe 3b - Fragestellung 


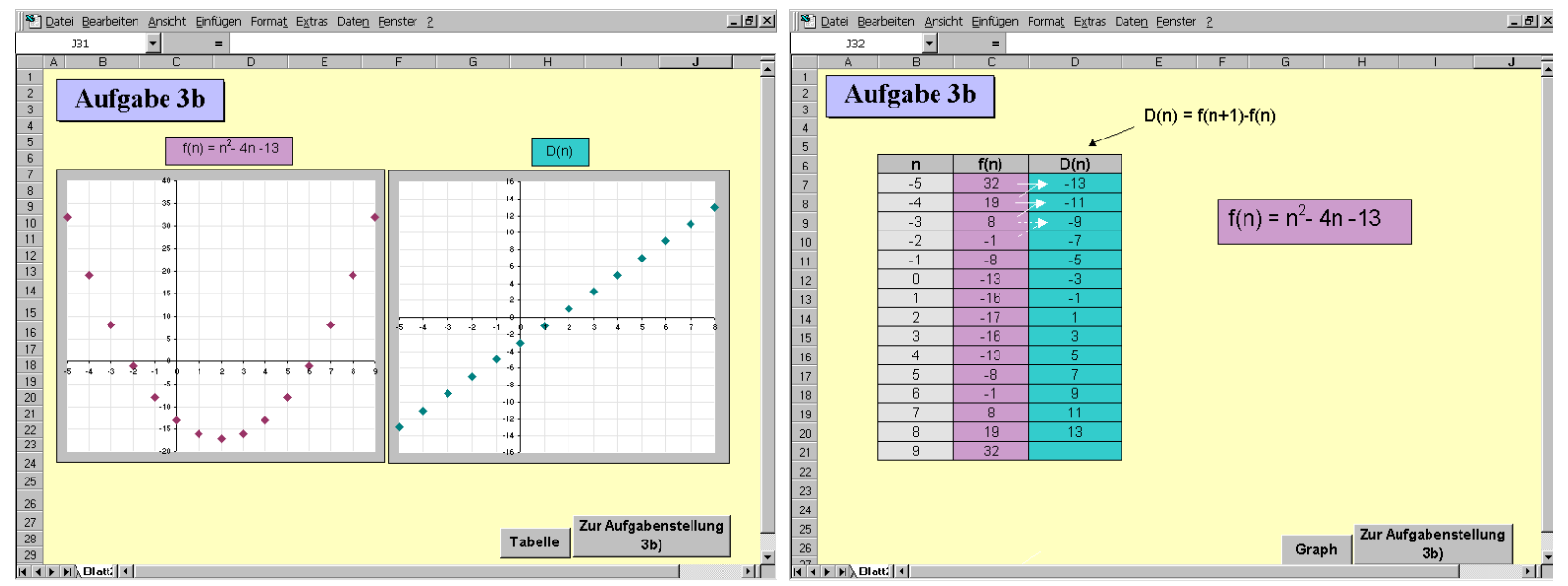

Abb. 57: Aufgabe 3b - Graph und Tabelle

Bei dieser Aufgabe sollen die Probanden ähnlich wie bei Aufgabe $2 \mathrm{~b}$ die Differenzenfunktion und ihre Beziehung zur Ausgangsfunktion zur Beschreibung des Änderungsverhaltens der Ausgangsfunktion nutzen. Dabei können sie sowohl den Graph als auch die Tabelle verwenden. Hier kann man fragen, mit welcher Darstellung die Probanden die Aufgabe beantworten und ob sich die Arbeitsweisen von denen bei Aufgabe $2 b$ unterscheiden. Ein inhaltliches Verständnis von Differenzenfunktionen zeigt sich hierbei darin, dass der positive Funktionswert $D(5)=7$ als ,Zuwachs“ gedeutet werden kann und dass - sofern die Probanden mit dem Graph arbeiten - der Wert 7 nicht durch einen Vergleich der Funktionswerte $f(5)$ und $f(6)$ berechnet, sondern direkt an der graphischen Darstellung der Differenzenfunktion abgelesen wird.

c) Wir betrachten wieder eine quadratische Z-Funktion $f$. Ergänzen Sie in der Tabelle die fehlenden Funktionswerte $f(-4)$ und $D(2)$.

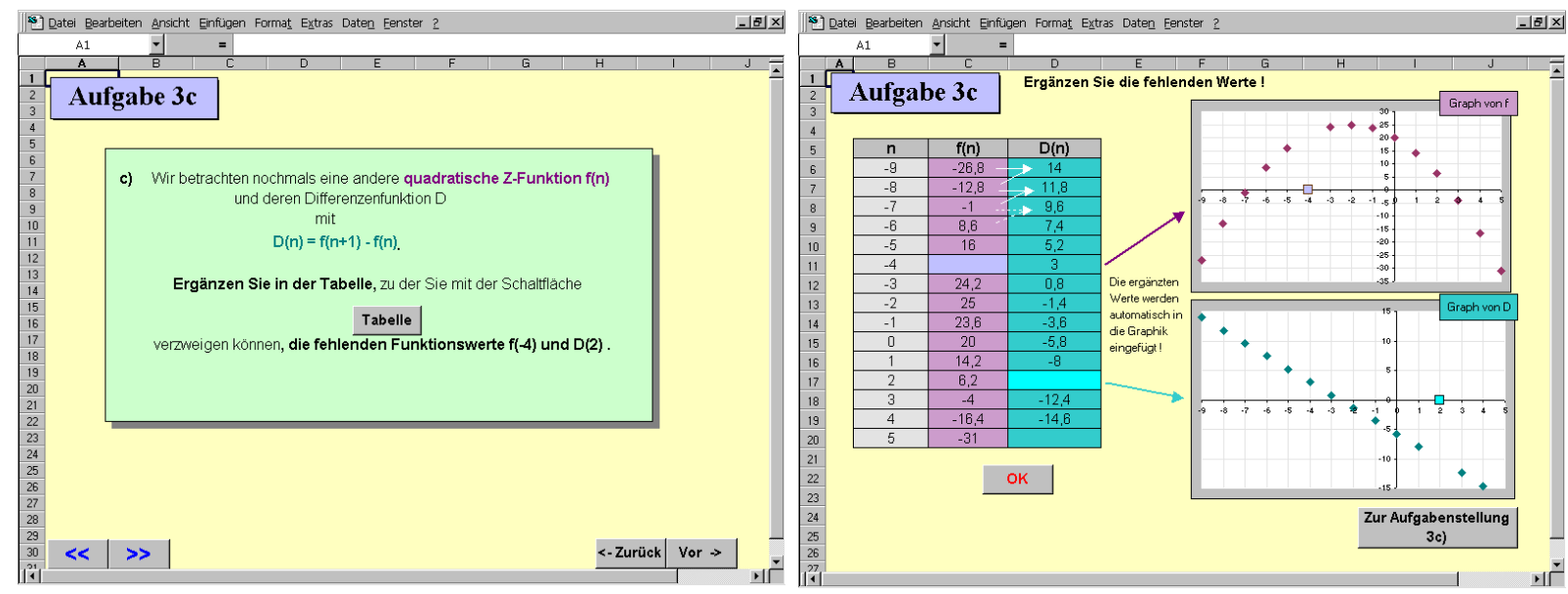

Abb. 58: Aufgabe 3c 
Gegenstand dieser „Interpolationsaufgabe“ ist die quadratische Z-Funktion $f$ mit $f(n)=-1,1 n^{2}-4,7 n+20$ sowie deren Differenzenfunktion, die bis auf die Funktionswerte $f(-4)$ und $D(2)$ tabellarisch und graphisch dargestellt sind. Die formale Darstellung ist den Probanden nicht bekannt. Die Probanden sollen die fehlenden Funktionswerte ähnlich wie bei Aufgabe $2 \mathrm{~d}$ mit Hilfe bereits dargestellter Funktionswerte der Funktion $f$ bzw. $D$ bestimmen und in die Tabelle eintragen. Die Funktion $f$ ist so gewählt, dass die zugehörige Differenzenfunktion monton fallend ist und die absolute Änderung beim Übergang von $n$ nach $n+1$ den konstanten Wert $-2,2$ besitzt. Damit erscheint es unwahrscheinlich, dass in der tabellarischen Darstellung von $D$ ein „Muster“ erkannt wird, das ohne Bezug zu $f$ in die Tabellenlücke an der Stelle von $D(2)$ fortgesetzt wird. Die in die Tabelle eingetragenen Funktionswerte werden interaktiv in die graphische Darstellung der jeweiligen Funktionen übertragen. Zur Ergebniskontrolle steht den Probanden ein „Ok-Button“ zur Verfügung und sie können zudem das Ergebnis anhand des Graphen ,visuell“ überprüfen. Hier ist die Frage, ob die Probanden auf der Basis der lokalen Beziehung zwischen einer Z-Funktion und deren Differenzenfunktion Interpolationsprobleme lösen können und ob - im Vergleich mit Aufgabe 2d - die Art der Darstellung einen Einfluss auf die entsprechenden Bearbeitungen besitzt.

Auf einem Informationsblatt werden die wesentlichen Aspekte der Aufgaben 3a-3c - d. h. die Bedeutung der Differenzenfunktion zur Beschreibung eines lokalen Änderungsverhaltens der Ausgangsfunktion - noch einmal zusammengefasst.

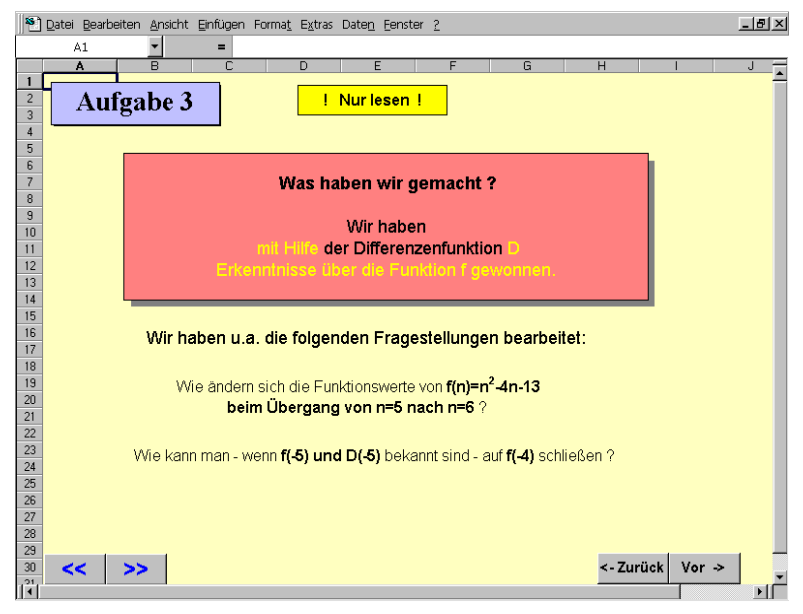

Abb. 59: Informationsblatt:

Rückschau auf die Aufgaben 3a-3c 
Die folgenden Aufgabenteile haben die Funktionenklasse der quadratischen Z-Funktionen und deren Differenzenfunktionen zum Gegenstand. Vor der Bearbeitung der eigentlichen Testaufgaben haben die Probanden die Möglichkeit, diese Funktionenklasse zunächst experimentell kennen zu lernen und zu untersuchen. Zum Variieren der Parameter stehen den Probanden Rollbalken zur Verfügung.
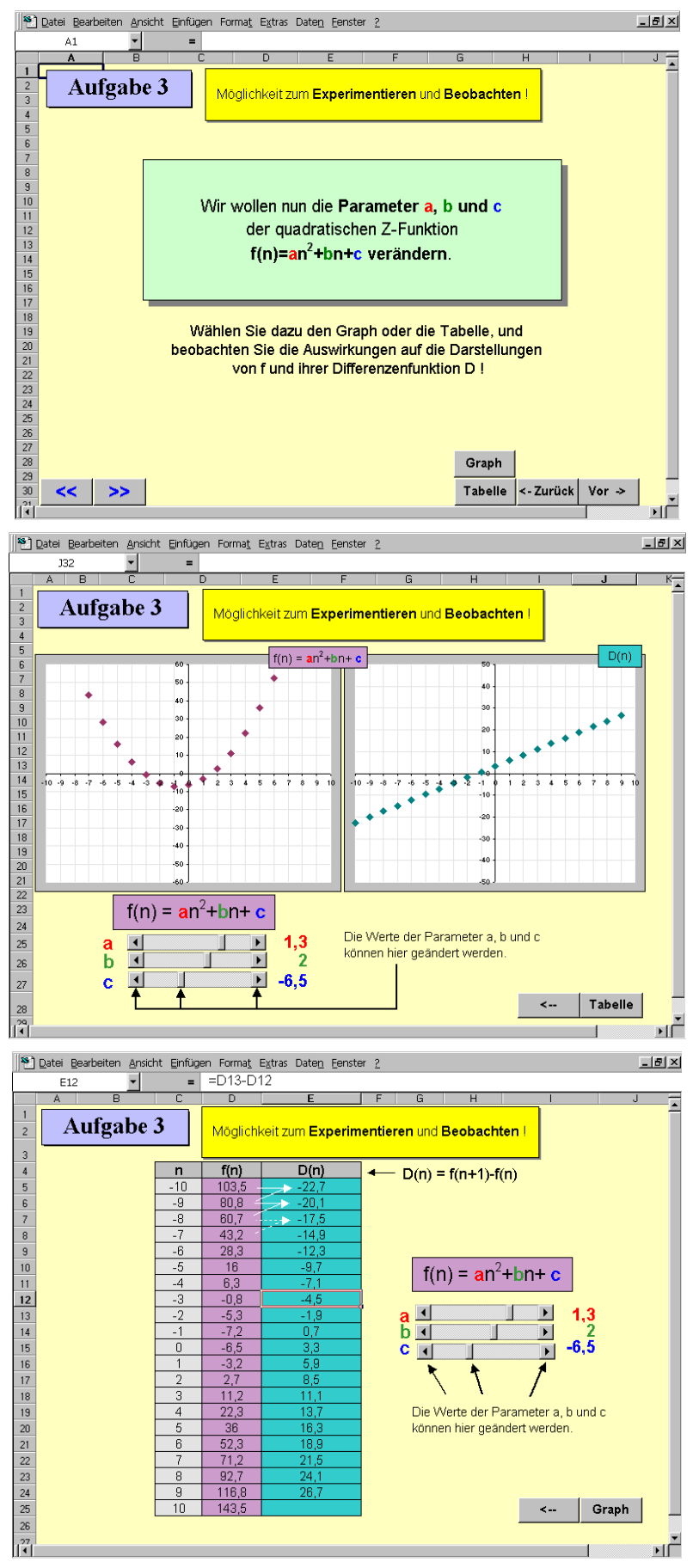

Abb. 60: Aufgabe 3:

Möglichkeit zum Experimentieren mit der Funktionenklassen $f(n)=a n^{2}+b n+c$ 
d) Verändern Sie den Parameter $c$ in $f(n)=2 n^{2}-8 n+c$. Begründen Sie das ,,seltsame“ Verhalten der Differenzenfunktion $D$.
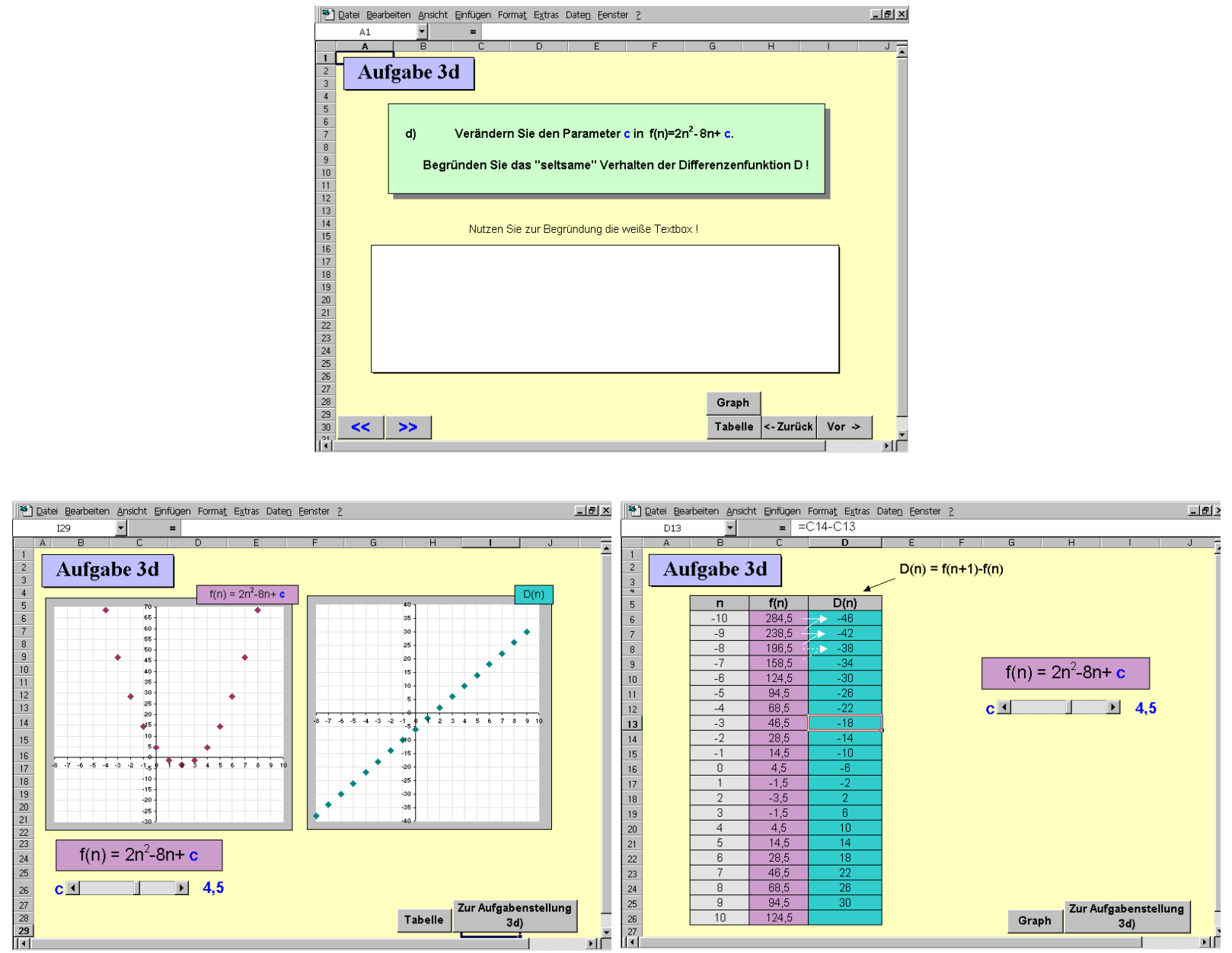

Abb. 61: Aufgabe 3d

Das Variieren des Parameters $c$ in $f(n)=2 n^{2}-8 n+c$ hat keinen Einfluss auf die Differenzenfunktion $D$. Ziel dieser Aufgabe ist es herauszufinden, ob die Probanden dieses Phänomen erkennen und wie sie es begründen, d. h. welche Sichtweisen und welche Vorkenntnisse sich in ihren Argumentationen widerspiegeln. Da die Schüler den Ableitungsbegriff noch nicht kennen, interessiert hier insbesondere ein Vergleich der Argumentationen der Studierenden und der Schüler. Zur Fixierung ihrer Antworten steht den Probanden auf dem ExcelAufgabenblatt ein Textfeld zur Verfügung. Bei dieser Fragestellung soll weiterhin untersucht werden, welche Darstellungen bevorzugt gewählt werden und inwiefern von der Möglichkeit des Experimentierens mit dem Parameter $c$ Gebrauch gemacht wird. 
e) Wir betrachten eine weitere Z-Funktion $f(n)=2 n^{2}-9 n-8$.

Geben Sie [in dem folgenden ,Lückentext"] möglichst genau die Bereiche an, in denen die Funktionswerte der Differenzenfunktion $D$ ihre Vorzeichen wechseln. Welche Bedeutung hat dies für die Funktion $f$ ?
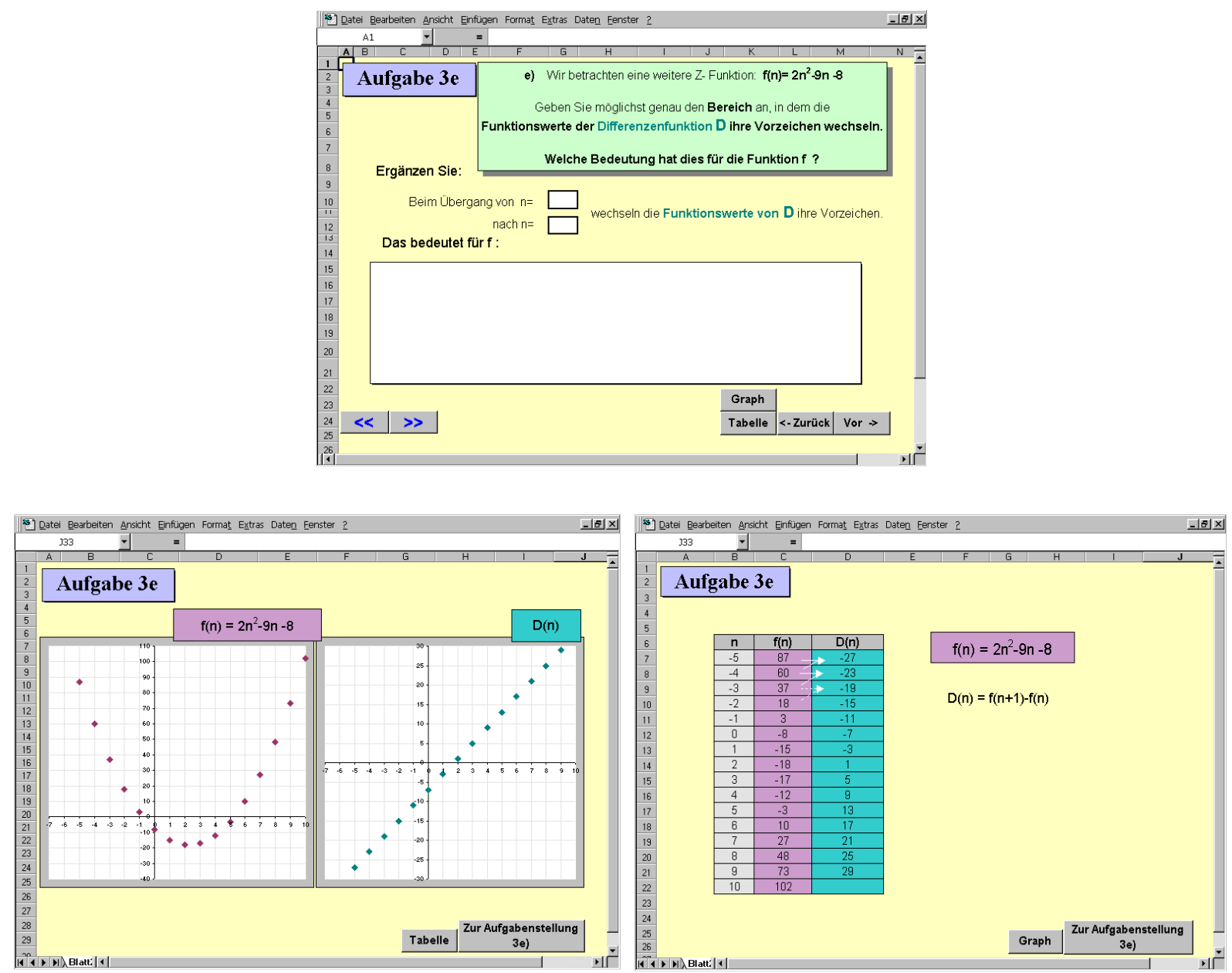

Abb. 62: Aufgabe 3e

In dem Intervall, in dem die Werte der Differenzenfunktion ihr Vorzeichen wechseln, nimmt die Ausgangsfunktion ihren minimalen Wert an. Die Probanden können zur Beantwortung der Frage sowohl mit dem Graph als auch mit der Tabelle arbeiten. Zur Beantwortung der beiden Teilaufgaben eignet sich aus „Expertensicht“ eher die graphische Darstellung von $D$, da sie das Erfassen sowohl lokaler als auch globaler Eigenschaften ,auf einen Blick“ ermöglicht. Jedoch bietet die Tabelle zumindest im Hinblick auf das Erkennen des Vorzeichenwechsels eine Alternative, da der Tabellenbereich überschaubar groß ist und sämtliche Funktionswerte ganze Zahlen sind. Hier ist die Frage, welche Darstellungen die Probanden wählen und ob ihre Wahl das Erkennen der Beziehung zwischen Eigenschaften der Funktion und ihrer Differenzenfunktion beeinflusst. Darüber hinaus können die Argumentationen dahingehend analysiert werden, ob eher lokale oder (auch) globale Beziehungen zwischen den beiden Funktionen erkannt werden. 
f) Berechnen Sie $D(n)=f(n+1)-f(n)$, wobei $f(n)=a n^{2}+b n+c$.

[Bearbeiten Sie diese Aufgabe mit Bleistift und Papier auf dem hierfür vorgesehenen Arbeitsblatt.]

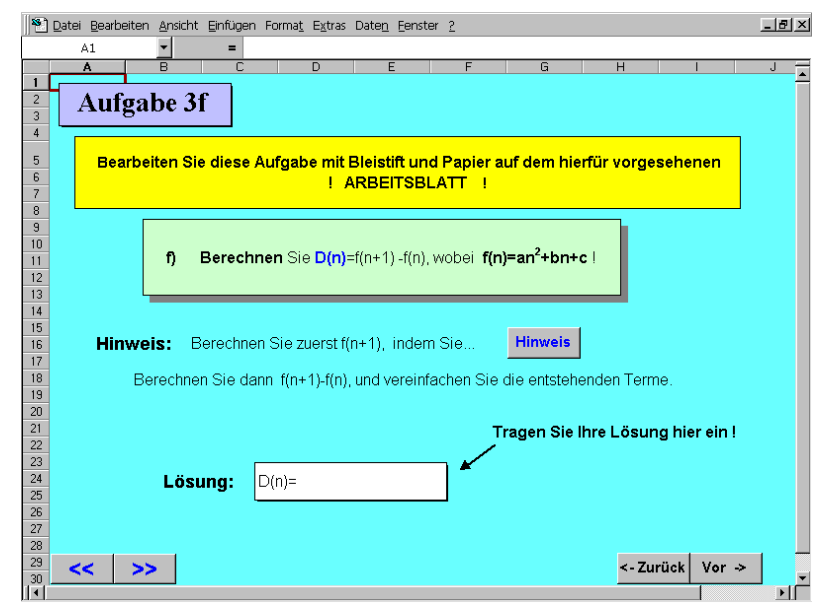

Abb. 63: Aufgabe 3f

In dieser Aufgabe wird die numerische und graphische Ebene des TKP vorübergehend verlassen. Ziel dieser Aufgabe ist es herauszufinden, ob die Probanden mit dem Begriff der Differenzenfunktion auch formal umgehen können bzw. welche Schwierigkeiten sich dabei ergeben. Dazu soll die Differenzenfunktion der allgemeinen quadratischen Funktion $f$ mit $f(n)=a n^{2}+b n+c$ bestimmt werden.

Die Kenntnis der formalen Beziehung zwischen Funktion und Differenzenfunktion kann bei der Beantwortung der folgenden Aufgabenstellung und auch im Rahmen der Bearbeitung der Problemstellung 4d hilfreich sein. Das Ergebnis von Aufgabe 3f wird daher auf einem „Lösungsblatt" festgehalten, welches in die lineare Abfolge des Programms integriert ist.

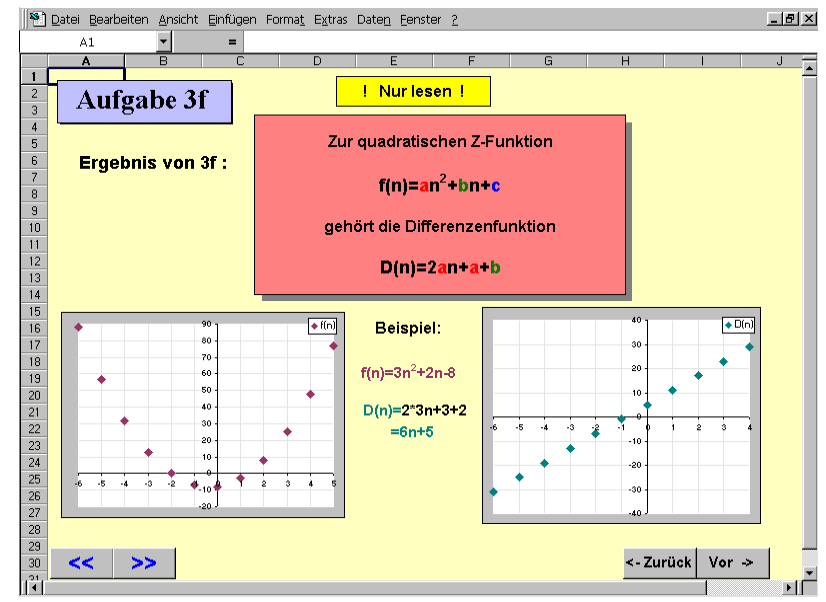

Abb. 64: Informationsblatt:

Lösung Aufgabe 3f 
g) Verändern Sie den Parameter $b$ in $f(n)=3 n^{2}+b n+40$.

\section{Beschreiben und begründen Sie das Verhalten der Differenzenfunktion $D$.}
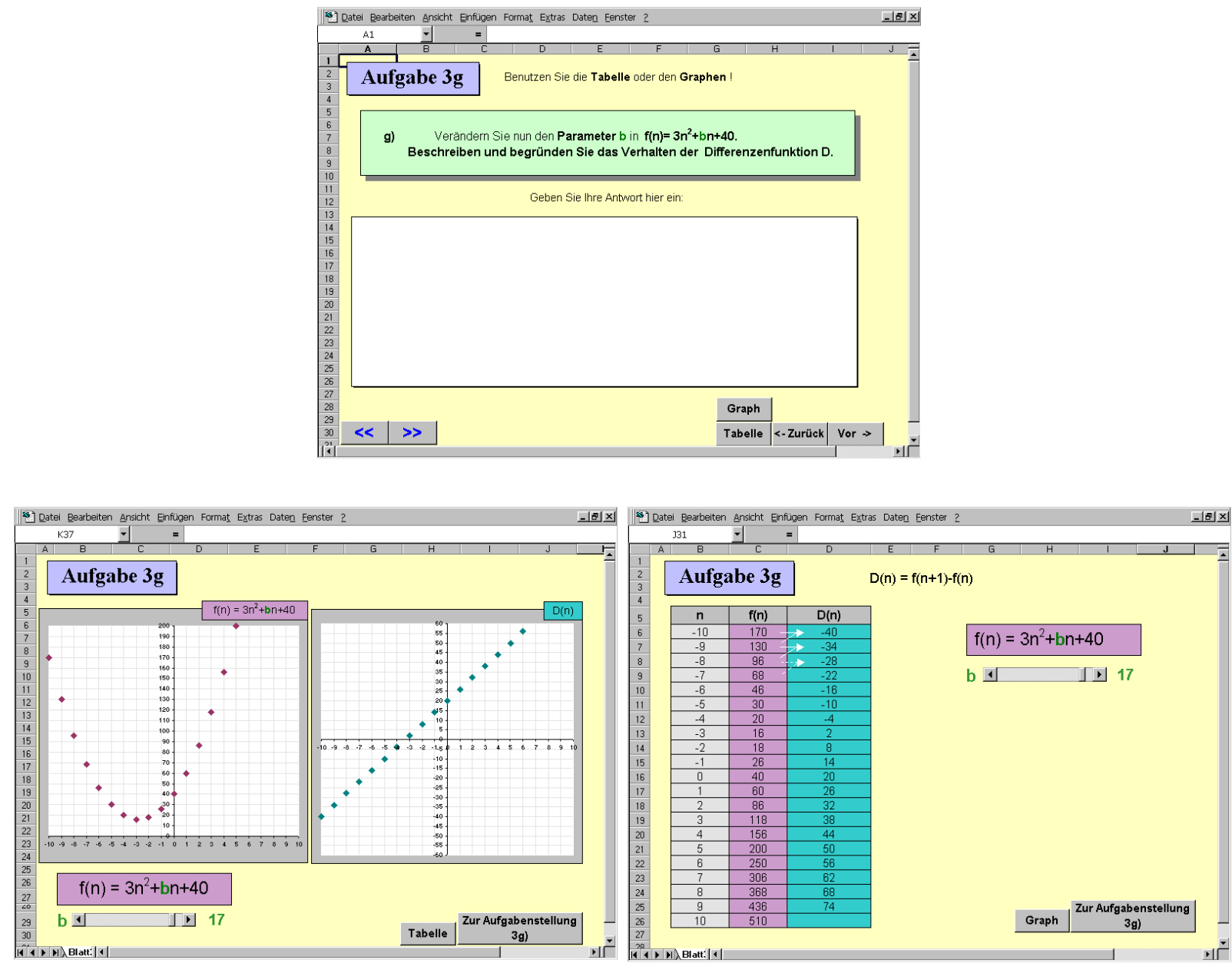

Abb. 65: Aufgabe 3g

Ziel dieser Aufgabe ist es herauszufinden, wie die Probanden das Verhalten der Differenzenfunktion begründen. Zwei Erklärungsansätze erscheinen hierbei möglich. Eine graphischgeometrische Argumentation stellt einen Bezug zwischen dem Graph von $f$ und $D$ her. Durch Variation von $b$ wird der Graph von $f$ scheinbar ,als Ganzes“ verschoben. Tatsächlich handelt es sich jedoch nicht um eine Verschiebung des Graphen von $f$, sondern von dessen kontinuierlichem Pendant, einer Parabel (vgl. auch Kap. 2.3.3). Das lineare Änderungsverhalten ändert sich dabei nicht. Die absolute Änderung bzw. Änderungsrate der Differenzenfunktion bleibt daher konstant. Die Gerade, auf der die Koordinatenpunkte der Differenzenfunktion liegen, wird daher nur parallel verschoben. Der zweite Erklärungsansatz basiert auf der algebraischen Beziehung zwischen $f$ und $D$. Dabei werden die Parameter der Formel $D(n)=2 a n+a+b$ im Hinblick auf die graphische Darstellung von $D$ interpretiert. Da der Parameter $a$ konstant ist, ändert sich nicht die Änderungsrate von $D$, sondern nur der Achsenabschnitt. 
Es ist hier die Frage, ob eine Beziehung zur vorherigen Aufgabe gesehen wird und dadurch häufiger formale Begründungen - wie mit dem zweiten Erklärungsansatz angedeutet - initiiert werden. Weiterhin kann gefragt werden, ob diese Sichtweise in Verbindung zu bestimmten Vorkenntnissen zu sehen ist und welche Darstellungen zur Beantwortung dieser Frage bevorzugt gewählt werden. Insbesondere interessiert in diesem Zusammenhang ein Vergleich zwischen Studierenden und Schülern.

\subsubsection{Aufgabe 4: Dreieckszahlen}

Gegenstand dieser Aufgabe ist das Zählproblem „Dreieckszahlen“. Es soll der rekursive Zusammenhang der Folgenglieder numerisch und symbolisch erfasst werden sowie eine explizite Formel zum Berechnen der $n$-ten Dreieckszahl gefunden werden. Damit wird zum einen das iterative Arbeiten mit dem TKP betont, zum anderen können im Rahmen der expliziten Beschreibung der Dreieckszahlen Kenntnisse über quadratische Z-Funktionen und deren Differenzenfunktionen als heuristische Strategie angewendet werden. Im Folgenden werden die einzelnen Aufgabenteile sowie die damit verbundenen Fragestellungen beschrieben.

\section{a) Die folgende Abbildung zeigt den schrittweisen Aufbau der ersten sechs Stufen der sogenannten ,Dreieckszahlen“". Übertragen Sie die ersten 6 Dreieckszahlen in die Tabelle.}

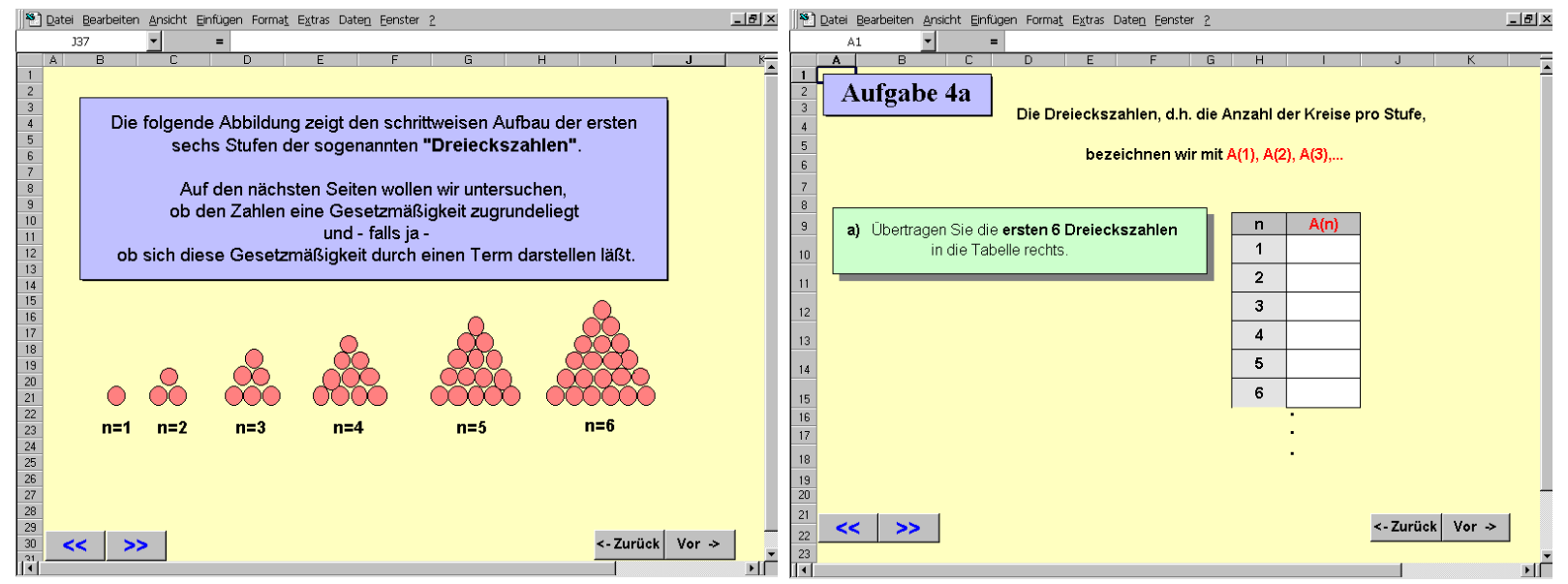

Abb. 66: Aufgabe 4a

Mit dieser Aufgabe werden die Probanden in die Thematik „Dreieckszahlen“ inhaltlich eingeführt. Sie sollen zunächst die zeichnerische Darstellung analysieren und anschließend die ermittelten Folgenglieder in eine Tabelle eintragen. Da sich die Tabelle auf einem separaten Blatt befindet, kann in einem gewissen Sinne überprüft werden, inwiefern die Probanden den iterativen Aufbau der Dreieckszahlen numerisch erfasst haben. So sprechen ein schrittweises, tabellarisches Auflisten der Folgenglieder und ein Verzicht auf ein Zurückblättern zu der zeichnerischen Darstellung dafür, dass die iterative Gesetzmäßigkeit bei der ersten Analyse der Zeichnung erkannt wurde und die Folgenglieder nun „aus dem Gedächtnis“ numerisch 
rekonstruiert werden. Es ergibt sich somit die Frage, wie die Probanden mit der zeichnerischen und tabellarischen Darstellung umgehen und welche Probleme beim numerischen Erfassen der Folgenglieder auftreten.

b) Nach welchem ,Muster“ ist die Folge aufgebaut? Ergänzen Sie dazu die fehlenden Werte!

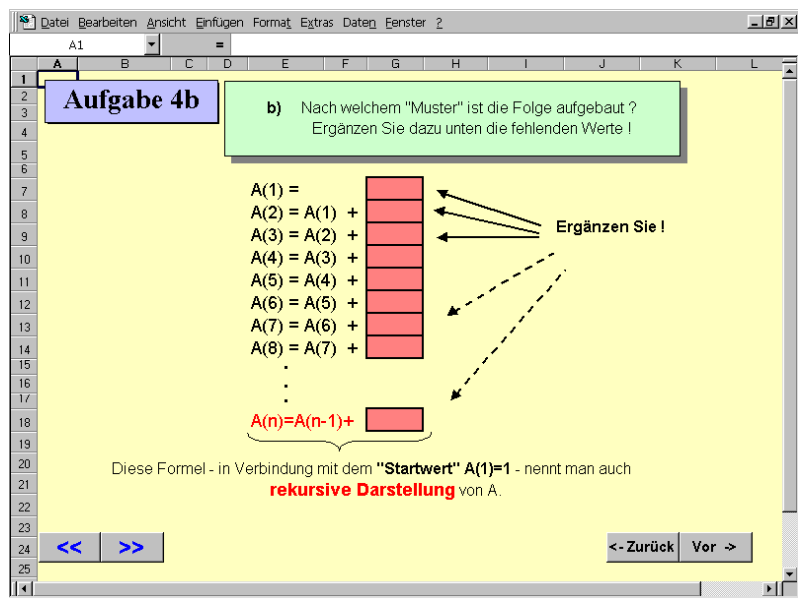

Abb. 67: Aufgabe 4b

Das Ziel dieser Aufgabe ist das formelmäßige Erfassen der Gesetzmäßigkeit und das Aufstellen der allgemeinen rekursiven Formel. Hier interessiert, ob die Probanden den Transfer von der numerischen zur formalen Ebene leisten können und welche Probleme dabei auftreten.

c) Berechnen Sie in der Tabelle mit einer Formel die ersten 16 Dreieckszahlen.

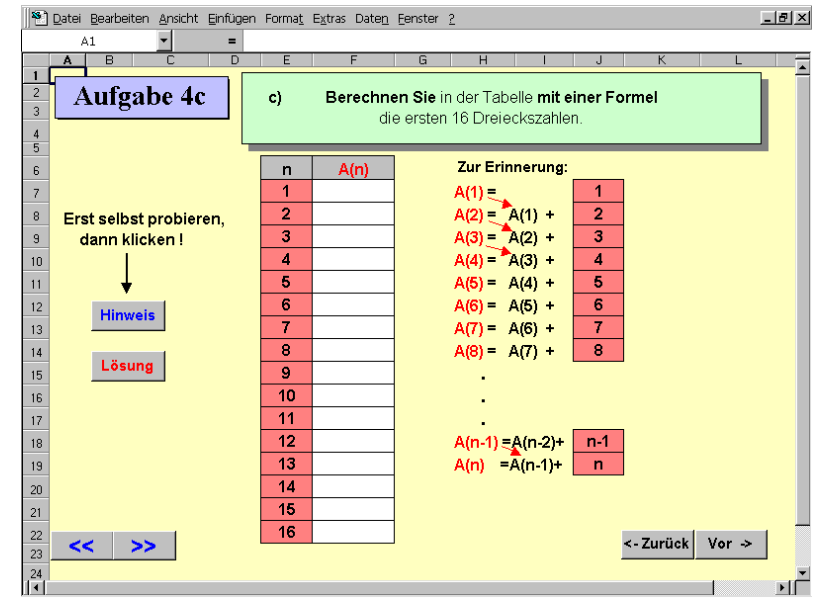

Abb. 68: Aufgabe 4c

Das Formulieren der rekursiven Gesetzmäßigkeit unter Verwendung von Zellen (bzw. Zellennamen) als Variablen, also unter Ausnutzung der TKP-Syntax, stellt eine Möglichkeit dar, das Formulieren der entsprechenden allgemeinen algebraischen Formel vorzubereiten. Umge- 
kehrt kann durch das Übersetzen der Formel $A(n)=A(n-1)+n$ in die TKP-Syntax die rekursive Gesetzmäßigkeit noch einmal auf eine anschauliche Weise operativ erfahrbar gemacht werden (vgl. Kap. 4.1.1). Diese Vermutung soll im Rahmen dieser Aufgabenstellung überprüft werden. Insbesondere soll im Vergleich mit Aufgabe $4 \mathrm{~b}$ untersucht werden, inwiefern sich durch das Arbeiten in den zwei verschiedenen Mikrowelten (,Algebraisch-formale Welt“ und „Excel-Welt“") Probleme oder Chancen im Hinblick auf das Erfassen rekursiver Gesetzmäßigkeiten ergeben.

Die folgenden beiden Informationsblätter haben das Ziel, den zentralen Inhalt der letzten drei Aufgabenteile zusammenfassend zu erläutern und die Probanden auf die Aufgabe $4 \mathrm{~d}$ - das Finden einer expliziten Formel für die Dreieckszahlen - vorzubereiten. Dazu wird die Struktur und Bedeutung einer rekursiven Formel sowie die einer expliziten Formel am Beispiel der Dreieckszahlen aufgezeigt.
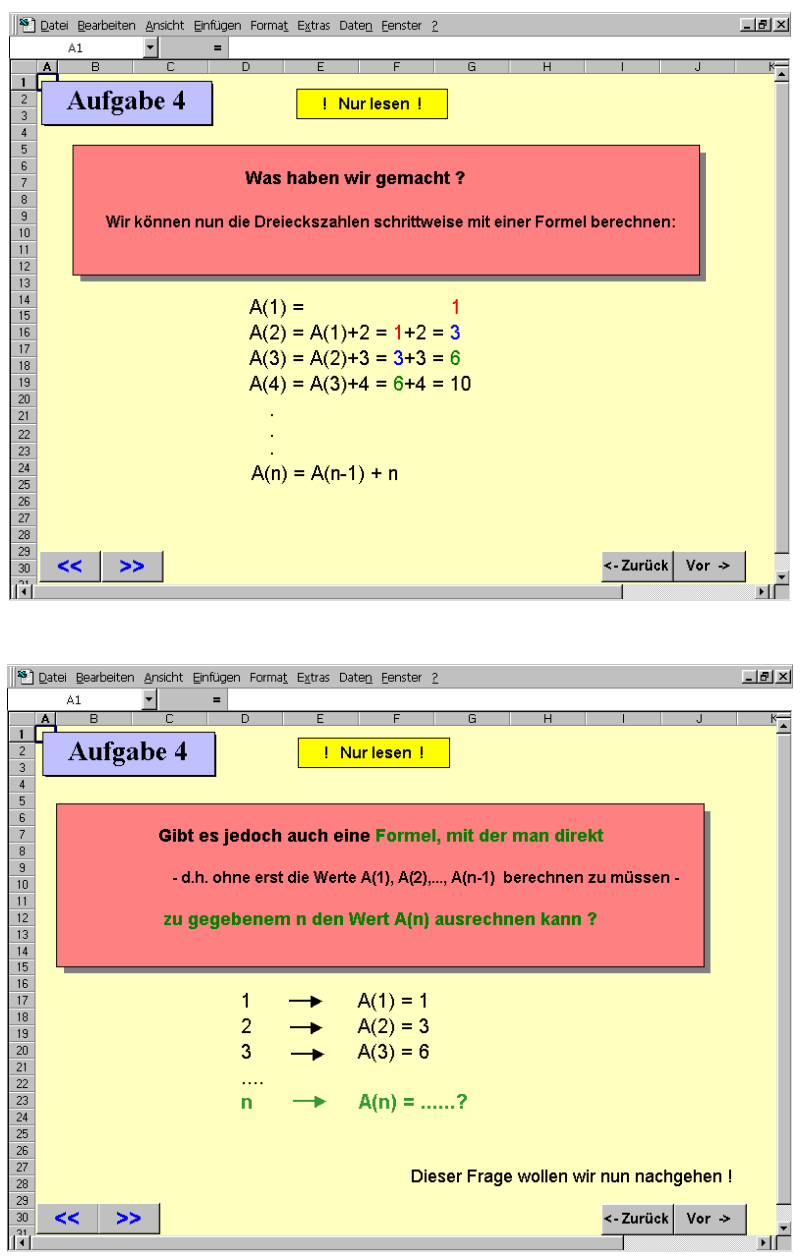

Abb. 69: Informationsblätter: Struktur und Bedeutung einer rekursiven und expliziten Formel 
d) Finden Sie für die Dreieckszahlen eine explizite Formel, d.h. eine Formel der Art $A: n \rightarrow A(n)$.

Sie haben drei Möglichkeiten diese Formel zu finden:

1. Möglichkeit: Mit Bleistift und Papier

2. Möglichkeit: Wir bilden die Differenzenfunktion

\section{Möglichkeit: Wir experimentieren}
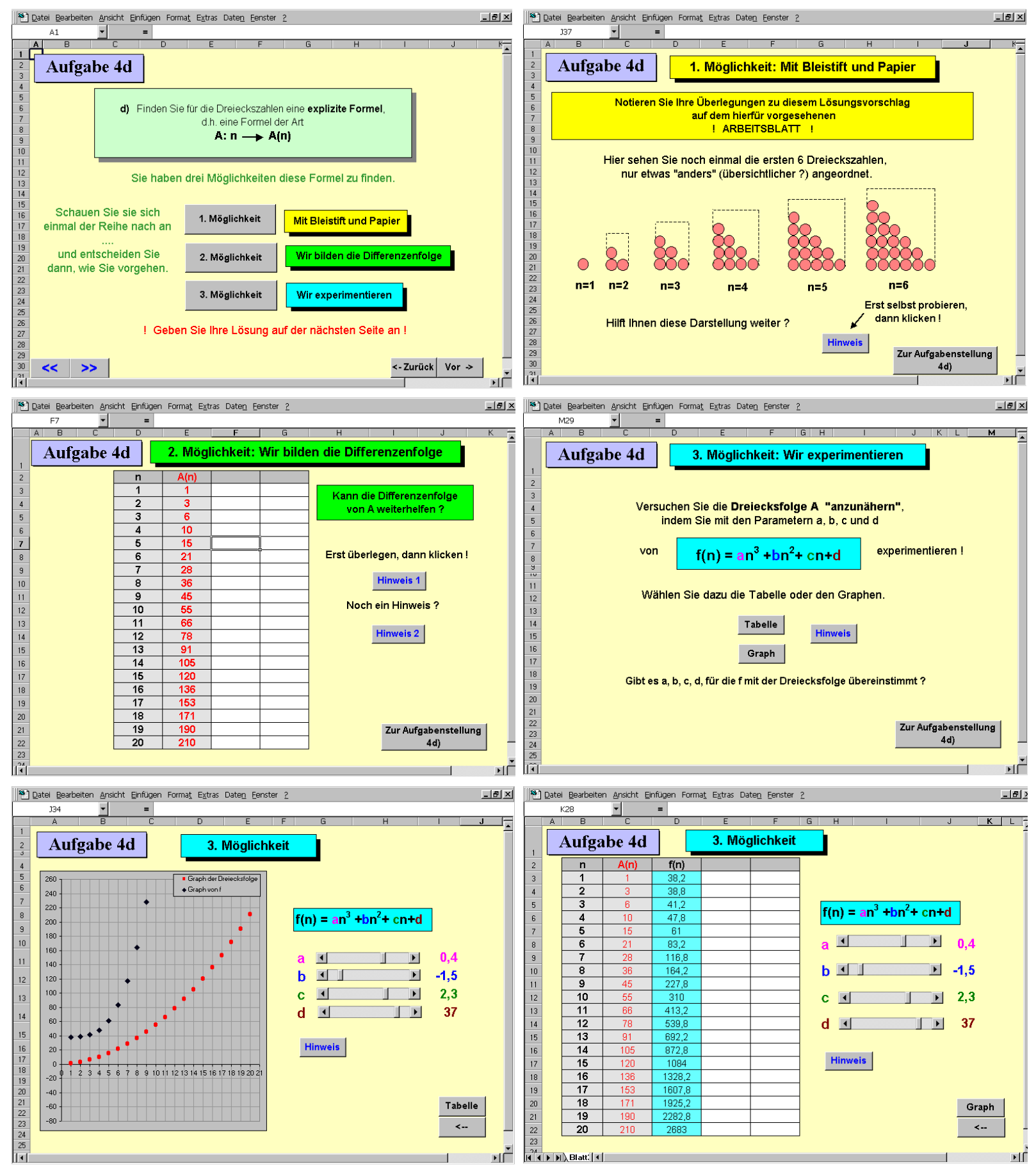

Abb. 70: Aufgabe 4d 
Den Probanden werden zur Lösung dieser Aufgabe drei Hinweise gegeben. Ausgehend von diesen Hinweisen lassen sich verschiedene Strategien zum Lösen des Zählproblems verfolgen. So gibt es die Möglichkeit, die explizite Formel mit Hilfe einer Zeichnung zu gewinnen (inhaltlich-anschaulicher Beweis). Verfolgt man den zweiten Hinweis, können Kenntnisse über Differenzenfunktionen gewinnbringend angewendet werden: Wenn die Probanden die numerisch berechnete Differenzenfolge als arithmetische Folge $D(n)=n+1$ identifizieren, kann dies zur Vermutung führen, dass die Ausgangsfolge durch ein quadratisches Polynom beschrieben wird. Die Probanden haben dann zum einen die Möglichkeit, die explizite Formel über den in Aufgabe $3 \mathrm{~g}$ gefundenen algebraischen Zusammenhang und durch Vergleich der entsprechenden Parameter zu gewinnen. Zum anderen können sie dem Experimentierhinweis folgen und eine geeignete quadratische Anpassungsfunktion für die graphisch und tabellarisch dargestellte Dreiecksfolge bestimmen. Es besteht weiterhin die Möglichkeit, ausschließlich mit den Parametern der „Testfolge“ $f$ mit $f(n)=a n^{3}+b n^{2}+c n+d$ zu experimentieren. Dabei können die gesuchten Parameter $a=0, b=0,5, c=0,5$ und $d=0$ sukzessive eingeschachtelt werden. Im Rahmen dieser Aufgabe kann man fragen, welche Strategien die Probanden im Lösungsprozess verfolgen. Insbesondere interessiert, inwiefern Kenntnisse über Differenzenfunktionen angewendet werden und welche Bedeutung die experimentelle Strategie für die Probanden besitzt.

\subsubsection{Aufgabe 5: Noch eine Z-Funktion}

\section{Die Graphik zeigt die Darstellung einer Z-Funktion $f$. Skizzieren Sie auf dem Arbeitsblatt den Verlauf der Differenzenfunktion $D$.}

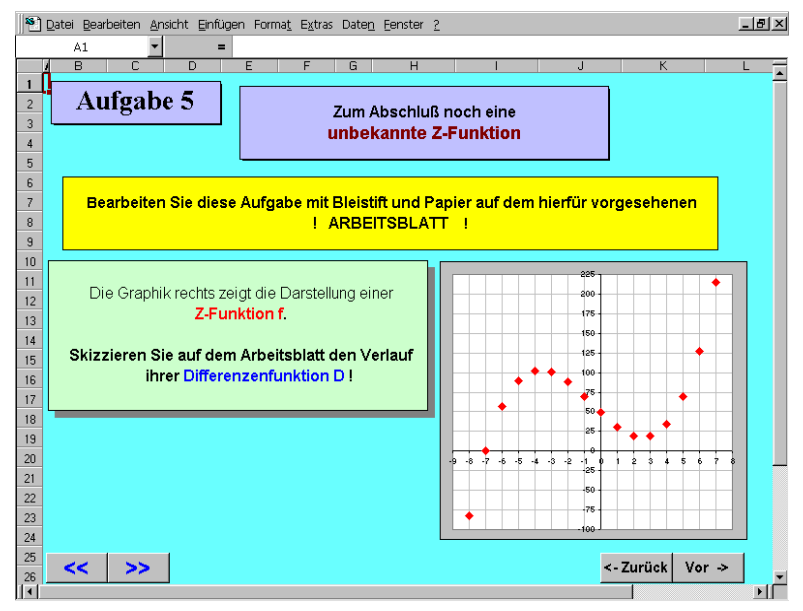

Abb. 71: Aufgabe 5

Gegenstand dieser Aufgabe ist eine spezielle kubische Funktion, deren Differenzenfunktion durch „graphisches Differenzenbilden“ (als Analogie zum graphischen Differenzieren) gefunden werden soll. Die Termdarstellung der Funktion ist nicht gegeben, um zu vermeiden, dass die Differenzen numerisch-algorithmisch mit Hilfe der Formel $D(n)=f(n+1)-f(n)$ be- 
rechnet und erst nachträglich in die Zeichnung eingetragen werden. Die Möglichkeit des numerischen Berechnens ist jedoch prinzipiell auch beim Arbeiten mir dem Graph gegeben, indem die Probanden die Funktionswerte durch ein Deuten auf die jeweiligen Koordinatenpunkte ablesen und anschließend miteinander verknüpfen. Dieses Arbeiten spiegelt jedoch inhaltliches Wissen über die Bedeutung der Differenzenfunktion wider und soll daher als Alternative zum „Abschätzen“ der Änderungen und dem Anfertigen einer ,echten“ Skizze berücksichtigt werden. Mit dieser Aufgabe soll überprüft werden, ob die Probanden ihre Kenntnisse von quadratischen Differenzenfunktionen auch auf eine kubische Funktion übertragen können. Es soll dabei insbesondere untersucht werden, wie sie die Skizze anfertigen, welche Eigenschaften von Differenzenfunktionen sie dabei ausnutzen und welche Sichtweisen ihr Vorgehen widerspiegelt.

\subsection{Versuchsdurchführung}

Die gesamte Untersuchung wurde in öffentlichen Rechnerräumen der Universität Gießen durchgeführt. Die Studierenden bearbeiteten das Testprogramm im Rahmen einer Übung zur Vorlesung „Computer im Mathematikunterricht“, die Schüler wurden zum Zwecke der Untersuchung für jeweils einen Vormittag vom Schulunterricht befreit. Im Folgenden werden der Ablauf der Untersuchung sowie die aus den Ergebnissen der Voruntersuchung resultierenden Veränderungen des Programms erläutert.

\subsubsection{Voruntersuchung mit den Studierenden}

Der Vortest mit den Studierenden fand in einem Rechnerraum der Universitätsbibliothek statt. Auf eine ausführliche inhaltliche Einführung in die Thematik „Differenzenfunktionen“ wurde verzichtet. Die Studierenden bearbeiteten das Untersuchungsprogramm von Anfang an eigenständig und ohne Unterbrechung. Abschließend hatten die Studierenden die Möglichkeit, eine Zusatzaufgabe (Extrapolationsaufgabe) zu lösen. Die insgesamt benötigte Arbeitszeit lag in einem Bereich von $1 \mathrm{~h}$ und $1 \mathrm{~h} 45 \mathrm{~min}$.

Überraschend viele der Studierenden hatten große inhaltliche und technische Probleme bei der Bearbeitung des Lernprogramms. Diese Schwierigkeiten lassen sich zum einen auf die mangelnden Excel-Kenntnissen der Studierenden ${ }^{200}$, zum andern auch auf die Programmkonstruktion zurückführen. Auf der Basis dieser Beobachtungen konnte das Lernprogramm wie geplant im Hinblick auf die Hauptuntersuchung überarbeitet werden (vgl. Kap. 5.7.2). Ein weiteres Ziel der Voruntersuchung - das Erfassen von Datenmaterial, das den Vergleich der Arbeitsweisen von Studierenden und Schülern ermöglicht - konnte nur bedingt erreicht werden. Die Arbeitsweisen vieler Studierenden ließen bereits im Rahmen der Bearbeitung der ersten

\footnotetext{
${ }^{200}$ Diese Studierenden hatten es offensichtlich trotz ausdrücklichen Hinweises seitens der Versuchsleiterin versäumt, sich vor der Untersuchung mit den grundlegenden Eigenschaften von Excel vertraut zu machen.
} 
Aufgabe (die nur wenige technische Kenntnisse voraussetzte und zahlreiche Hilfestellungen zum Arbeiten mit dem TKP gab) mangelhafte Grundfertigkeiten im Umgang mit Excel erkennen. Da zu erwarten war, dass ein inhaltliches Arbeiten durch das zu geringe technische Grundlagenwissen beeinträchtigt und möglicherweise sogar verhindert werden würde, wurden diese Studierenden im Rahmen der Auswertung nicht berücksichtigt. Insgesamt wurde somit eine Auswahl von 21 Studierenden in den Vergleich einbezogen.

\subsection{2 Überarbeitung des Versuchsprogramms}

Im Hinblick auf die Hauptuntersuchung wurde das Programm der Studierenden unter inhaltlichen Gesichtspunkten verändert. Indem einige kleinere Teilaufgaben ${ }^{201}$ hinzugefügt und verbindende Informationsblätter ${ }^{202}$ ergänzt wurden, wurde das Programm etwas kleinschrittiger. Mit den zusätzlichen Aufgaben sollten zuvor behandelte Inhalte gefestigt und der Übergang zu komplexeren Aufgaben vereinfacht werden. Zwei weitere Aufgaben der Studierenden aus dem Themenkomplex 2 („,Durchschnittstemperaturen“) wurden umformuliert zu Aufgabe 2e. Aufgrund der veränderten Aufgabenstellung können die beiden ursprünglichen Aufgaben der Studierenden aber nicht beim Vergleich der Arbeitsweisen der Schüler und der Studierenden berücksichtigt werden.

Aufgabe 5 wurde gekürzt und inhaltlich verändert. Gegenstand der ursprünglichen Aufgabe, die aus drei Aufgabenteilen bestand, waren polynomiale Z-Funktionen dritten und vierten Grades. Die Studierenden sollten im ersten Aufgabenteil das Änderungsverhalten einer kubischen Z-Funktion mit Hilfe deren Differenzenfunktion beschreiben. Gegenstand des zweiten Aufgabenteils war die Analyse des Einflusses der Parameter $a, b, c$ und $d$ auf die Klasse der kubischen Z-Funktionen $f(n)=a n^{3}+b n^{2}+c n+d$ und deren Differenzenfunktionen. Eine inhaltliche Deutung ihrer Beobachtungen bereitete den Studierenden große Schwierigkeiten, so dass im Rahmen der Hauptuntersuchung auf eine explizite Behandlung kubischer Z-Funktionen verzichtet wurde. Es wurde lediglich die Idee des dritten Aufgabenteils - das graphische Differenzenbilden - übernommen. Die Studierenden hatten hierbei die Aufgabe, die Differenzenfunktion der Z-Funktion $f(n)=-0,072 n^{4}+0,03 n^{3}+5,2 n^{2}-26 n-65,5$ graphisch zu bestimmen. Im Rahmen des Versuchsprogramms der Schüler wurde anstelle der polynomialen Z-Funktion vierten Grades eine kubische Z-Funktion vorgegeben. Somit ermöglicht diese Aufgabe auch einen bedingten Vergleich der Sicht- und Arbeitsweisen der Studierenden und der Schüler. ${ }^{203}$

\footnotetext{
${ }^{201}$ Es handelt sich hierbei um die Aufgaben 1f, 1h, 1j, 2c, 2d, 3a, 3b, 3c, 3e.

${ }^{202}$ Diese Informationsblätter sind in den Abbildungen 42, 44, 55, 59 und 67 dargestellt.

${ }^{203}$ Es sollte sich sowohl bei den Studierenden als auch bei den Schülern um eine Funktion handeln, die zuvor noch nicht im Hinblick auf ihr Änderungsverhalten analysiert wurde.
} 
Neben den mathematischen Inhalten wurde auch das methodische Vorgehen überdacht. Das Konzept der passiven Teilnahme der Untersuchungsleiterin sollte grundsätzlich beibehalten werden. Jedoch wurde eine kleine gemeinsame Einführungsphase eingeplant, in der die Beschäftigung mit Differenzenfunktionen über die Bedeutung von Funktionsänderungen in Umweltsituationen im Unterrichtsgespräch motiviert wurde. Zu diesem Zweck wurden vier Excel-Tabellenblätter vorbereitet, die von der Versuchsleiterin vor dem eigentlichen Testbeginn mit den Schülern gemeinsam besprochen wurden. Weiterhin wurde noch einmal auf die wesentlichen Steuerungs- und Kontrollelemente des Programms hingewiesen.
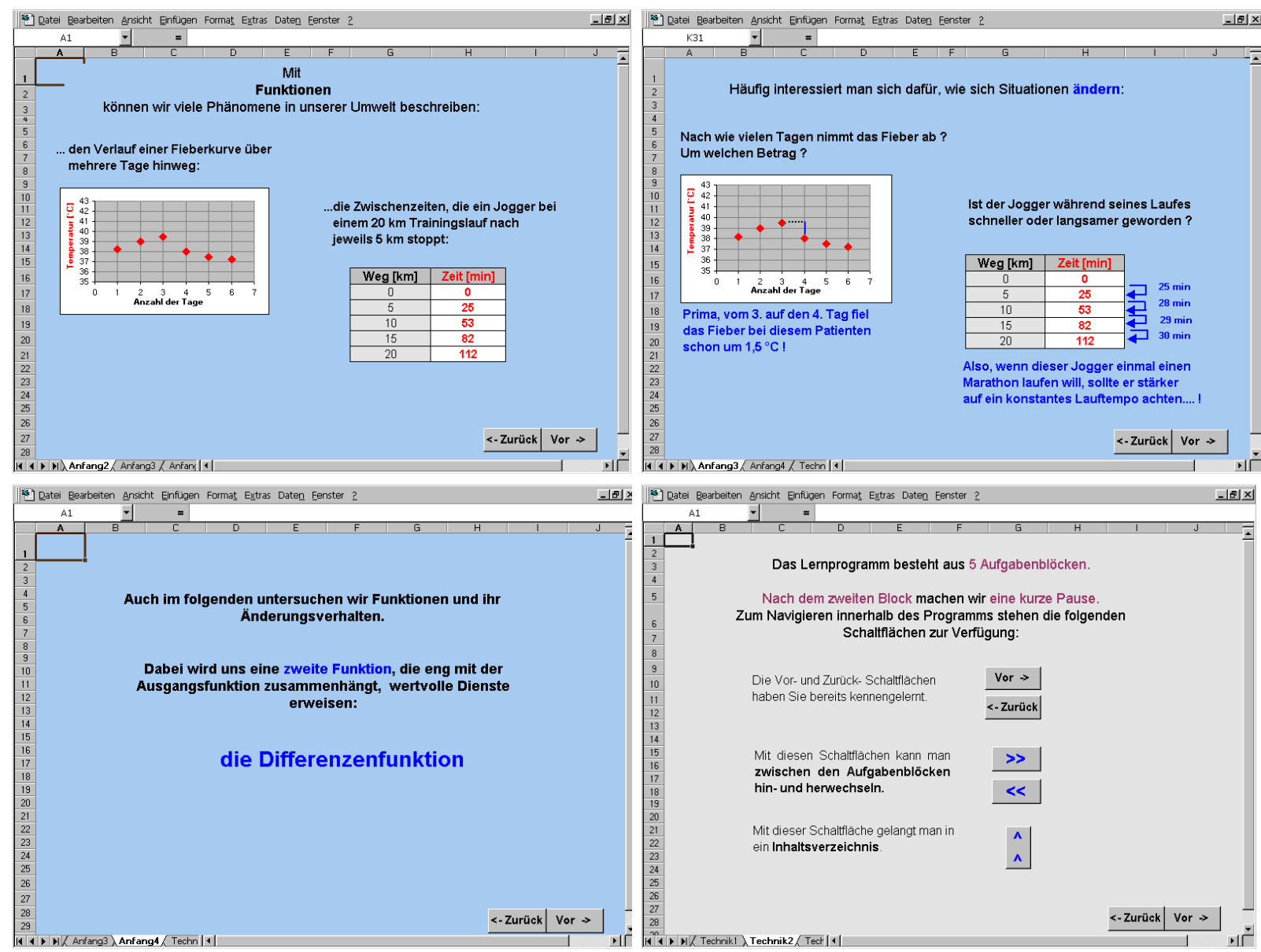

Abb. 72: Einführung in die Thematik „Differenzenfunktionen“

Da das Programm der Schüler deutlich umfangreicher als das der Studierenden ist, wurde nach den ersten beiden Aufgaben eine Pause eingeplant. Vor Beginn der zweiten Testphase wurden auf vier Excel-Blättern noch einmal die wesentlichen Aspekte der bisher bearbeiteten Aufgabenteile herausgestellt und die neue Fragestellung, d. h. die Analyse einer ganzen Funktionenklasse, motiviert. Diese Informationsblätter wurden von der Untersuchungsleiterin nicht weiter erläutert. 


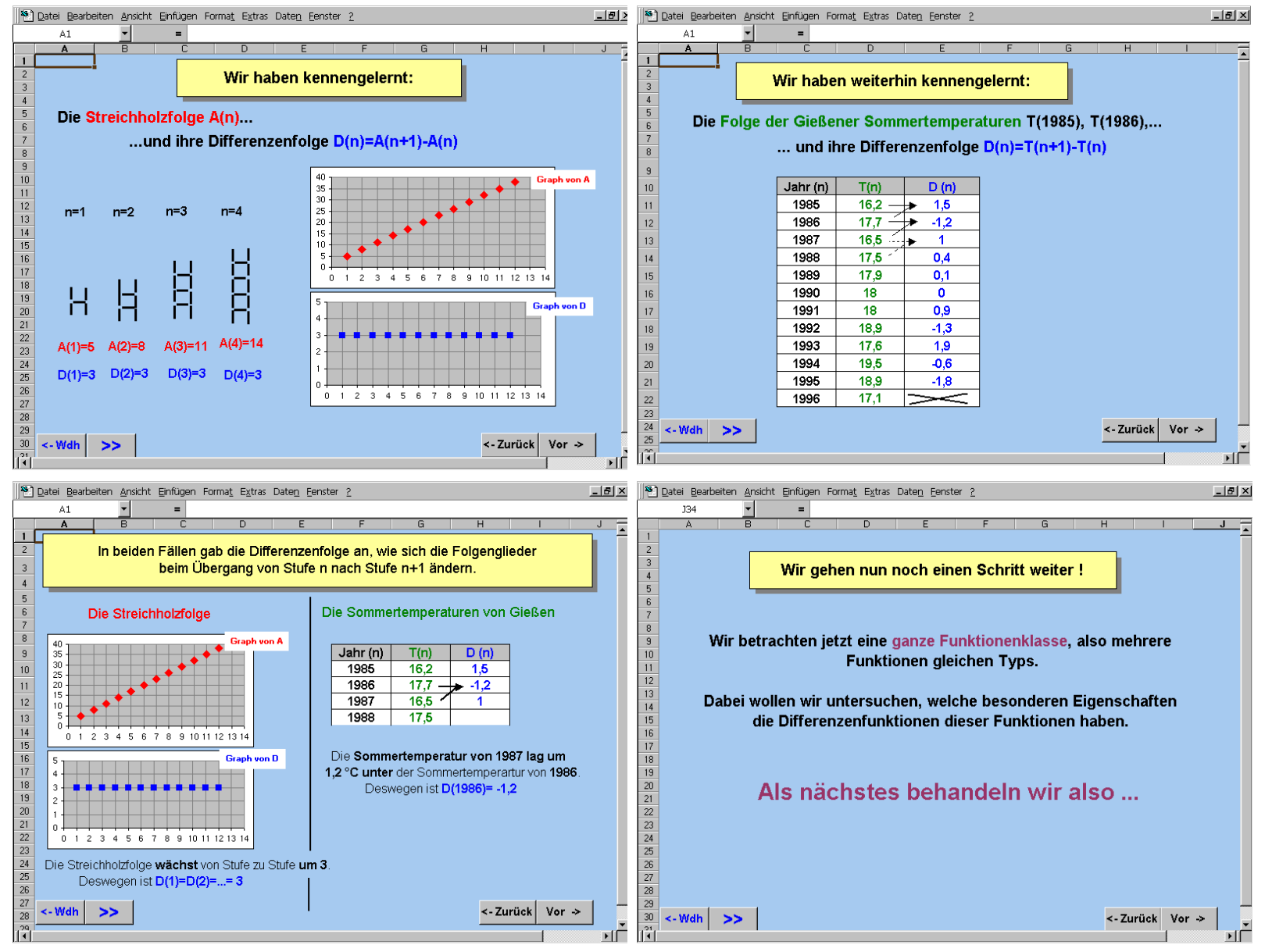

Abb. 73: Wiederholung vor Beginn der zweiten Testphase

\subsubsection{Hauptuntersuchung mit den Schülern}

\section{Untersuchung mit der Schülergruppe A und B}

Die Hauptuntersuchung mit den Schülern der Schulen A und B fand an jeweils einem Vormittag in den Rechnerräumen des Hochschulrechenzentrums der Universität Gießen statt. Nach der Einführungsphase, in der die Versuchsleiterin die inhaltlichen Ziele des Programms herausstellte sowie einige technische Aspekte näher erläuterte, bearbeiteten die Schüler die Testaufgaben selbstständig ohne weitere Instruktionen durch die Versuchsleiterin. Nach den ersten beiden Aufgabenblöcken fand wie vorgesehen eine Pause statt. Im Anschluss an die Untersuchung hatten die Schüler die Gelegenheit, einige Zusatzaufgaben zu dem Themenkomplex „Quadratische Z-Aufgaben“ zu bearbeiten. Die Gesamtarbeitszeit der einzelnen Schüler lag in einem Bereich von $1 \mathrm{~h} 30$ min und $2 \mathrm{~h} 20 \mathrm{~min}$.

Bei der Durchführung der Untersuchung mit den Schülern der Gruppe B trat während der Bearbeitung der dritten Aufgabe ein Problem mit dem lokalen Netzwerk des Hochschulrechenzentrums auf, wodurch etwa $75 \%$ der Probanden von einem Rechnerabsturz betroffen waren. Die ab Beginn der zweiten Testphase erhobenen ScreenCam-Aufzeichnungen waren 
dadurch verloren. Die betroffenen Schüler wurden gebeten, die dritte Aufgabe noch einmal von Anfang an zu bearbeiten. Dadurch erhofften wir uns, im Rahmen der Auswertung zumindest einen Eindruck über die ursprünglichen Lösungsergebnisse und die verbalen Beschreibungen zu gewinnen. Die Arbeitsweisen lassen sich jedoch durch eine wiederholte Bearbeitung einer Aufgabe nicht oder nur bedingt rekonstruieren. Sie werden daher in der nachfolgenden Auswertung nur zum Teil mitberücksichtigt.

\section{Zweite Überarbeitung des Versuchsprogramms}

Eine dritte Schülergruppe war in der ursprünglichen Versuchsplanung nicht vorgesehen und wurde nur aufgrund der Datenverluste im Rahmen der Gruppe B zusätzlich hinzugezogen. In diesem Zusammenhang wurden am Untersuchungsprogramm noch einige wenige Änderungen vorgenommen. So wurden zwei Teilaufgaben (Aufgabe 3c und 3e) aus zeitlichen Gründen entfernt. Darüber hinaus wurde die Tabelle aus Aufgabe $1 \mathrm{~h}$ und $1 \mathrm{j}$ um drei Spalten nach rechts verschoben. Damit wurde vermieden, dass der Spaltenname und der Name der in dieser Spalte berechneten Differenzenfunktion $D$ übereinstimmen. Diese „Identität“ hatte in den Gruppen A und B bei der Bearbeitung von Aufgabe 1j zu Fehlinterpretationen geführt, indem anstelle des Wertes $D(9)$ der Wert aus Zelle D9 abgelesen wurde. Diese Änderung wird in der Auswertung berücksichtigt.

Da sich bei einigen der Schüler der Gruppen A und B aufgrund der ungewohnt langen selbstständigen Arbeitsphasen Konzentrationsschwächen zeigten, die sich in dem Überlesen bzw. schnellen Überblättern der Informationsblätter äußerten, wurde das methodische Vorgehen erneut überdacht. In diesem Zusammenhang wurden einige der Informationsblätter aus dem Programm entfernt. ${ }^{204}$ Die Inhalte der ursprünglichen Blätter wurden stattdessen gemeinsam mit der Versuchsleiterin erörtert. Darüber hinaus wurden auch die Aufgaben if und $3 \mathrm{f}$ gemeinsam im Unterrichtsgespräch gelöst. Die besondere Behandlung dieser beiden Aufgaben wird im Rahmen der Auswertung berücksichtigt.

\section{Untersuchung mit der Schülergruppe C}

Die Durchführung der Untersuchung mit den Schülern der Gruppe C verlief im Sinne der oben beschriebenen Planung. Die Arbeitszeit betrug zwischen 1 h 30 min und 1 h und 55 min.

\footnotetext{
${ }^{204}$ Es handelte sich hierbei um die in den Abbildungen 42, 44 und 59 dargestellten Informationsblätter.
} 


\subsubsection{Vergleich der drei Testversionen: Übersicht}

Die folgende Tabelle zeigt die Inhalte der Testprogramme der Studierenden und der Schüler der Gruppe C im Vergleich zu dem Programm der Schülergruppen A und B.

\begin{tabular}{|c|c|c|c|}
\hline & Studierende & Schüler A/B & Schüler C \\
\hline \multicolumn{4}{|l|}{ Aufgabe 1} \\
\hline $\mathrm{a}$ & $\mathrm{X}$ & $\mathrm{X}$ & $\mathrm{X}$ \\
\hline $\mathrm{b}$ & $\mathrm{X}$ & $\mathrm{X}$ & $\mathrm{X}$ \\
\hline $\mathrm{c}$ & $\mathrm{X}$ & $\mathrm{X}$ & $\mathrm{X}$ \\
\hline $\mathrm{d}$ & $\mathrm{X}$ & $\mathrm{X}$ & $\mathrm{X}$ \\
\hline $\mathrm{e}$ & $\mathrm{X}$ & $\mathrm{X}$ & $\mathrm{X}$ \\
\hline f & 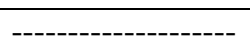 & $\mathrm{X}$ & Klassengespräch \\
\hline $\mathrm{g}$ & $\mathrm{X}$ & $\mathrm{X}$ & $\mathrm{X}$ \\
\hline $\mathrm{h}$ & ---------------------- & $\mathrm{X}$ & - \\
\hline $\mathrm{j}$ & --------------- & $\mathrm{X}$ & 0 \\
\hline \multicolumn{4}{|l|}{ Aufgabe 2} \\
\hline $\mathrm{a}$ & $\mathrm{X}$ & $\mathrm{X}$ & $\mathrm{X}$ \\
\hline $\mathrm{b}$ & $\mathrm{X}$ & $\mathrm{X}$ & $\mathrm{X}$ \\
\hline $\mathrm{c}$ & - & $\mathrm{X}$ & $\mathrm{X}$ \\
\hline d & 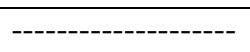 & $\mathrm{X}$ & $\mathrm{X}$ \\
\hline $\mathrm{e}$ & $\mathrm{O}$ & $\mathrm{X}$ & $\mathrm{X}$ \\
\hline Aufgabe 3 & & $\mathrm{X}$ & $\mathrm{X}$ \\
\hline $\mathrm{a}$ & ---------- & $\mathrm{X}$ & $\mathrm{X}$ \\
\hline $\mathrm{b}$ & - - & $\mathrm{X}$ & $\mathrm{X}$ \\
\hline $\mathrm{c}$ & ------------------ & $\mathrm{X}$ & ------------------ \\
\hline d & $\mathrm{X}$ & $\mathrm{X}$ & $\mathrm{X}$ \\
\hline $\mathrm{e}$ & -------------- & $\mathrm{X}$ & ------------- \\
\hline $\mathrm{f}$ & $\mathrm{X}$ & $\mathrm{X}$ & Klassengespräch \\
\hline $\mathrm{g}$ & $\mathrm{X}$ & $\mathrm{X}$ & $\mathrm{X}$ \\
\hline \multicolumn{4}{|l|}{ Aufgabe 4} \\
\hline $\mathrm{a}$ & $\mathrm{X}$ & $\mathrm{X}$ & $\mathrm{X}$ \\
\hline $\mathrm{b}$ & $\mathrm{X}$ & $\mathrm{X}$ & $\mathrm{X}$ \\
\hline c & $\mathrm{X}$ & $\mathrm{X}$ & $\mathrm{X}$ \\
\hline $\mathrm{d}$ & $\mathrm{X}$ & $\mathrm{X}$ & $\mathrm{X}$ \\
\hline Aufgabe 5 & - & $\mathrm{X}$ & $\mathrm{X}$ \\
\hline
\end{tabular}

Tab. 6: Vergleich der drei Testversionen

$\mathrm{X}=$ Gleiche Aufgabe

= Aufgabe bedingt vergleichbar

$O=$ Aufgabe nicht vergleichbar

--- = Keine vergleichbare Aufgabe vorhanden 


\section{Ergebnisse der empirischen Untersuchung}

Die Auswertung der empirischen Untersuchung stützt sich auf die Computerprotokolle und schriftlichen Aufzeichnungen von 53 Schülern und 21 Studierenden. Aufgrund von Problemen mit der Datenspeicherung und den damit verbundenen Datenverlusten insbesondere bei der Schülergruppe B (vgl. Kap. 5.7.3), kann die Anzahl der in die Auswertung einbezogenen Probanden von Aufgabe zu Aufgabe variieren. Ein Vergleich zwischen der Gruppe der Schüler und der Studierenden ist aufgrund des für die Schüler erweiterten und abgeänderten Testprogramms nicht bei allen Aufgaben möglich (vgl. Kap. 5.7.4). Im Folgenden werden nun die Ergebnisse der fünf Testaufgaben analysiert.

\subsection{Ergebnisse zu Aufgabe 1}

Gegenstand dieser Aufgabe ist die „Streichholzfolge“, ein einfaches Zählproblem, dem eine lineare Gesetzmäßigkeit zugrunde liegt. Das iterative Aufbauprinzip der Streichholzfolge sollte zunächst verbal und anschließend mit Hilfe des Tabellenkalkulationsprogramms (TKP) Excel tabellarisch erfasst werden. Mit dem Begriff der Differenzenfolge wurde den Probanden im Anschluss ein Werkzeug bereitgestellt, das es erlaubt, die absoluten Änderungen bzw. die mittlere Änderungsrate von Zählfolgen im „Intervall“ $[n, n+1]$ sowohl lokal als auch global zu beschreiben. Die Probanden sollten in diesem Zusammenhang die Differenzenfolge mit Hilfe des TKP berechnen und Eigenschaften der Differenzenfolge untersuchen bzw. zur Lösung einfacher Problemstellungen einsetzen. Die Aufgabe hatte das Ziel, die Schüler vorrangig mit den technischen Bedienungselementen des Programms vertraut $\mathrm{zu}$ machen. In der folgenden Auswertung wird daher ein Schwerpunkt auf die Analyse der Arbeitsweisen mit dem Werkzeug Excel gelegt.

\subsubsection{Ergebnisse zu Aufgabe 1a}

Die Probanden sollten im Rahmen dieser Aufgabe das iterative Aufbauprinzip der Streichholzfolge verbal beschreiben. Sämtliche Studierende sowie $94 \%$ der Schüler erkannten das iterative Aufbauprinzip der Streichholzfolge, also die Änderung um 3 Streichhölzer von Stufe zu Stufe. Dabei hoben zusätzlich $38 \%$ der Studierenden aber nur 10\% der Schüler das Anfangsfolgenglied 5 explizit hervor. Dies ist wohl darauf zurückzuführen, dass die Schüler bisher wenig bzw. gar nicht mit rekursiven Folgen bzw. Figurenmustern vertraut waren. Letzteres mag auch ein Grund dafür sein, dass $6 \%$ der Schüler die Aufgabe fehlerhaft bearbeiteten, indem sie die Anzahl der Streichhölzer, die von Stufe zu Stufe hinzukommen, mit 1 bzw. 2 bezifferten. 


\subsubsection{Ergebnisse zu Aufgabe 1b}

Die Probanden hatten hier die Aufgabe, mit Hilfe einer rekursiven Formel die ersten 21 Folgenglieder der Streichholzfolge zu berechnen. Eine Unterscheidung in richtige und falsche Bearbeitungen erwies sich hinsichtlich der Beurteilung der Leistungen und des Lernfortschritts der Schüler und Studierenden als zu grob. Daher wird hier danach klassifiziert, ob die Probanden die Aufgabe beim ersten oder erst beim zweiten „Lösungsversuch“ richtig bearbeiteten, ob sie mehr als zwei Anläufe benötigten oder ob sie die Aufgabe fehlerhaft lösten. Als „Lösungsversuch“ oder „Lösungsanlauf“ gilt dabei ein fehlerfreier Bearbeitungsabschnitt, bei dem keine technischen oder inhaltlichen Probleme auftreten und der entweder zur Lösung der Aufgabe oder zu einer fehlerhaften Eingabe führt. Es ergibt sich die folgende Häufigkeitsverteilung:

\begin{tabular}{|l|c|c|}
\hline & Schüler (49) & Studierende (21) \\
\hline \hline 1 Lösungsversuch & $53 \%(26)$ & $38 \%(8)$ \\
\hline 2 Lösungsversuche & $20 \%(10)$ & $52 \%(11)$ \\
\hline >2 Lösungsversuche & $23 \%(11)$ & $10 \%(2)$ \\
\hline Fehlerhafte Lösung & $4 \%(2)$ & $0 \%(0)$ \\
\hline
\end{tabular}

Tab. 7: Aufgabe 1b - Lösungsversuche

Auch wenn prozentual weniger Studierende als Schüler die Aufgabe im ersten Lösungsanlauf lösten, berechneten insgesamt $90 \%$ der Studierenden aber nur $73 \%$ der Schüler die 21 Folgenglieder der Streichholzfolge nach maximal zwei Lösungsanläufen erfolgreich. Möglicherweise hatte ein Teil der Studierenden die Aufgabe im ersten Anlauf „unterschätzt“ und erst im zweiten Versuch die nötige Konzentration zur Bearbeitung aufgebracht. Die Arbeitsweisen und Schwierigkeiten der Probanden sollen im Folgenden noch einmal genauer analysiert werden.

\subsubsection{Erstellen der Tabelle}

Die Arbeitsweisen der Probanden beim Erstellen der Tabelle lassen sich wie folgt klassifizieren:

- Technisch: Es wird versucht, die Tabelle ausschließlich unter Verwendung relativer Zellbezüge und durch Kopieren der entsprechenden Formel zu erstellen. Zum Teil werden dabei die Folgenglieder zunächst Schritt für Schritt in jeder Zelle neu berechnet, wobei auf die Kopieroption verzichtet wird.

- Traditionell - technisch: Die ersten Folgenglieder werden zunächst numerisch eingetragen. Anschließend werden relative Zellbezüge verwendet und die entsprechende Formel in die übrigen Zellen der Tabelle kopiert. 
- Technisch - traditionell: Es wird versucht, die Tabelle zunächst durch Kopieren bestimmter Zellinhalte zu erstellen. Gelöst wird die Aufgabe jedoch letztendlich durch das Eintragen der numerischen Folgenglieder.

- Traditionell: Der Wert jedes Folgenglieds wird numerisch bestimmt und in die Tabelle eingetragen.

Tabelle 8 gibt die entsprechende Häufigkeitsverteilung an:

\begin{tabular}{|l||c|c|}
\hline & Schüler (51) & Studierende (21) \\
\hline \hline Technisch & $80 \%(41)$ & $71 \%(15)$ \\
\hline Traditionell - technisch & $18 \%(9)$ & $24 \%(5)$ \\
\hline Technisch - traditionell & $2 \%(1)$ & $0 \%(0)$ \\
\hline Traditionell & $0 \%(0)$ & $5 \%(1)$ \\
\hline
\end{tabular}

Tab. 8: Aufgabe 1b - Arbeitsweisen beim Erstellen der Tabelle

Die Probanden versuchten bis auf eine Ausnahme, die technischen Möglichkeiten zur (effektiven) Berechnung einer rekursiven Folge zu nutzen. Dabei nahmen jedoch etwa $20 \%$ beider Probandengruppen zunächst die Unterstützung des neuen Werkzeuges nur zum Teil in Anspruch. Der Strategiewechsel „,traditionell - technisch“ kann darauf zurückgeführt werden, dass erkannt wurde, dass das Verwenden der technischen Möglichkeiten tatsächlich mit einer operative Entlastung verbunden ist. Lediglich ein Studierender löste die Aufgabe durch schrittweises Eintragen der numerischen Folgenglieder, und ein Schüler wechselte vom technisch unterstützten zum „traditionellen“ Arbeiten. Dieser Strategiewechsel ist insofern nachvollziehbar, als dieser Schüler erhebliche Schwierigkeiten im Umgang mit der TKP-Syntax hatte.

\subsubsection{Inhaltliche/technische Probleme}

Beim Lösen dieser Aufgabe zeigten sich sowohl inhaltliche Probleme im Umgang mit der rekursiven Folge als auch technische Probleme, also Probleme beim Arbeiten mit dem Werkzeug. Die Fehler inhaltlicher Art lassen sich danach unterteilen, ob ein falsches Anfangsfolgenglied oder ein falscher Änderungswert verwendet wurde. Die folgende Tabelle gibt an, wie viele der Probanden jeweils einer der beiden Fehlerkategorien zugeordnet werden können, wobei ein Proband auch hinsichtlich beider Kategorien berücksichtigt werden kann .

\begin{tabular}{|l||c|c|}
\hline & Fehlerhafter Folgenanfang & Falscher Änderungswert \\
\hline Schüler (51) & $20 \%(10)$ & $8 \%(4)$ \\
\hline Studierende (21) & $29 \%(6)$ & $0 \%(0)$ \\
\hline
\end{tabular}

Tab. 9: Aufgabe 1b - Inhaltliche Fehlertypen 
Bis auf 4 Schüler verwendeten sämtliche Probanden in der Excel-Formel zur Berechnung der ersten 21 Folgenglieder den richtigen Änderungswert 3. Die verbale Formulierung der der Streichholzfolge zugrunde liegenden Gesetzmäßigkeit hatte offensichtlich das Formalisieren des Sachverhalts unterstützt. Das Benennen des ersten Folgenglieds bereitetet dagegen beiden Probandengruppen größere Schwierigkeiten. Diese Schwierigkeiten können jedoch zum Teil auf Konzentrationsschwächen zurückgeführt werden. So hatten 3 der 6 Studierenden, die ein fehlerhaftes Anfangsfolgenglied angaben, dieses im Rahmen von Aufgabenteil 1a richtig benannt.

Auf der Werkzeugebene bereitete den Schülern das Arbeiten mit Zellnamen als Variablennamen (technische) Schwierigkeiten. So traten syntaktische Fehler auf, indem das „,=“-Zeichen in Formeln vergessen wurde, es wurden falsche Zellbezüge bzw. Zirkelbezüge hergestellt oder es wurde auf Zellbezüge ganz verzichtet bzw. versucht, das Problem des Formulierens einer Gesetzmäßigkeit an das TKP zu „delegieren“. In dem letztgenannten Fall wurden die ersten beiden Folgenglieder 5 und 8 in die ersten beiden Zellen numerisch eingegeben. Diese Zellinhalte wurden anschließend in die übrigen Zellen der entsprechenden Spalte kopiert, wobei sich nur die numerischen Werte 5 und 8 zyklisch wiederholten. Es ergeben sich also die folgenden drei Fehlerkategorien:

I. Vergessen des ,=“-Zeichens;

II. Fehlerhafte Zellbezüge;

III. Fehlende Zellbezüge.

Die Tabelle 10 zeigt die entsprechende Häufigkeitsverteilung, wobei wieder ein Proband mehreren Kategorien zugewiesen werden kann.

\begin{tabular}{|l||c|c|c|}
\hline & I & II & III \\
\hline \hline Schüler (51) & $6 \%(3)$ & $20 \%(10)$ & $12 \%(6)$ \\
\hline Studierende (21) & $24 \%(5)$ & $24 \%(5)$ & $14 \%(3)$ \\
\hline
\end{tabular}

Tab. 10: Aufgabe 1b - Technische Fehlertypen

Während hinsichtlich der Fehlertypen II und III keine großen Unterschiede zwischen den Probandengruppen feststellbar sind, traten bei den Schülern prozentual weniger syntaktische Fehler auf als bei den Studierenden (Fehlertyp I). Dieses zunächst überraschende Ergebnis kann darauf zurückgeführt werden, dass in dem Testprogramm, das von den Schülern bearbeitet wurde, auf die Notwendigkeit des „,=“-Zeichens in Formeln noch einmal explizit hingewiesen wurde. 
Wie aus einer weiteren (ganzheitlichen) Analyse ${ }^{205}$ der Arbeitsweisen hervorgeht, überlagerten bei beiden Probandengruppen technische Probleme die inhaltlichen Schwierigkeiten. So zeigten $35 \%$ der Schüler (bzw. $48 \%$ der Studierenden) fehlerhafte Arbeitsweisen bei der Verwendung des TKP, wohingegen sich nur bei $22 \%$ der Schüler (bzw. $29 \%$ der Studierenden) Schwierigkeiten beim Arbeiten mit dem Folgenbegriff beobachten ließen. Die technischen Probleme können zum Teil auf die noch mangelnde Vertrautheit mit dem Werkzeug zurückgeführt werden.

\subsubsection{Ergebnisse zu Aufgabe 1c}

Bei dieser Aufgabe sollte mit Hilfe der in Aufgabe 1b berechneten Tabelle der Wert des 12. Folgengliedes bestimmt werden. Zur Lösungsüberprüfung stand ein „Ok-Button“ zur Verfügung, der angab, ob das Ergebnis richtig oder falsch war. Die meisten Probanden hatten mit dieser Aufgabe keine Schwierigkeiten und gaben den Funktionswert bereits nach einmaligem Lesen der Tabelle richtig an (vgl. Tab. 11).

\begin{tabular}{|l||c|c|}
\hline & Schüler (49) & Studierende (21) \\
\hline \hline 1 Lösungsversuch & $78 \%(38)$ & $86 \%(18)$ \\
\hline 2 Lösungsversuche & $10 \%(5)$ & $9 \%(2)$ \\
\hline >2 Lösungsversuche & $10 \%(5)$ & $5 \%(1)$ \\
\hline Fehlerhafte Lösung & $2 \%(1)$ & $0 \%(0)$ \\
\hline
\end{tabular}

Tab. 11: Aufgabe 1c - Lösungsversuche

\subsubsection{Lesen der Tabellendarstellung}

Beim Lesen der Tabellendarstellung traten im Wesentlichen zwei Fehlertypen auf:

- Ignorieren der Tabelle: Es werden Werte als Lösung angegeben, die keinen Bezug zur Tabelle erkennen ließen, wie z. B. 34, 40, 36.

- Ablesen aus falscher Tabelle: Das Lesen der Tabelle bereitet keine Schwierigkeiten, doch ist der in der Tabelle angegebene Wert falsch, da die Streichholzfolge bereits in Aufgabe $1 \mathrm{~b}$ fehlerhaft erzeugt wurde.

In der folgenden Tabelle 12 ist die entsprechende Häufigkeitsverteilung angegeben. Dabei kann ein Proband beiden Fehlerkategorien zugeordnet werden.

\footnotetext{
205 Es wurden hierbei die Probanden dahingehend klassifiziert, ob ein technisches bzw. inhaltliches Problem auftrat oder nicht, ohne Berücksichtigung der speziellen Ausprägung dieses Fehlers.
} 


\begin{tabular}{|l||c|c|}
\hline & Ignorieren der Tabelle & Ablesen aus falscher Tabelle \\
\hline \hline Schüler (51) & $16 \%(8)$ & $4 \%(2)$ \\
\hline Studierende (21) & $19 \%(4)$ & $5 \%(1)$ \\
\hline
\end{tabular}

Tab. 12: Aufgabe 1c - Inhaltliche Fehlertypen

Dass $16 \%$ der Schüler und $19 \%$ der Studierenden Werte angaben, die keinen Bezug zur Tabelle erkennen ließen, lässt sich darauf zurückführen, dass möglicherweise (fehlerhafte) numerische Operationen im Kopf durchgeführt wurden. Hiermit wäre etwa der Wert $36=12 \cdot 3$ zu erklären, bei dessen Berechnung das Anfangsfolgenglied 5 offensichtlich nicht berücksichtigt wurde. Das Ablesen eines richtigen Werts aus einer falschen Tabelle kann nicht als ,echter" Fehler des entsprechenden Probanden gewertet werden, sondern ist auf die Konstruktion des Lernprogramms bzw. die Kopplung von Aufgabe 1b und 1c zurückzuführen.

\subsubsection{Verwenden des ,Ok-Buttons“}

Fast alle Probanden nutzten die Möglichkeit der Lösungsüberprüfung mit Hilfe des „OkButtons“. Dadurch konnten Fehler entdeckt anschließend nach maximal 4 Lösungsanläufen korrigiert werden. Dass einer der beiden Schüler, die eine falsche Lösung lieferten, sein Ergebnis nicht korrigierte, ist dabei darauf zurückzuführen, dass er die Lösung gerade nicht mit Hilfe des Buttons überprüfte. Nur in zwei Ausnahmefällen zeigte sich ein unreflektiertes Vorgehen, das zum Teil auf die Präsenz des „Ok-Buttons“ zurückgeführt werden kann. So benötigte der Studierende III ${ }^{206} 5$ und der Schüler C $8^{207} 7$ Lösungsanläufe, die jeweils mit dem Button überprüft wurden. Dabei zeugen die eingegebenen Werte (z. B. 29, 30, 32, 40, 50, 41) davon, dass die Tabelle nicht gelesen, sondern fiktive Werte ausgedacht wurden, in der Hoffnung, durch ein Vorgehen nach „Versuch und Irrtum“ die richtige Lösung zu erraten.

\subsubsection{Ergebnisse zu Aufgabe 1d}

Bei dieser Aufgabe sollten die Probanden - ausgehend von einer vorgegebenen Streichhölzeranzahl - die Anzahl der möglichen Stufen einer entsprechenden „Streichholzleiter“ bestimmen, d. h. die Tabelle zum Ablesen eines Werts des Definitionsbereichs der Streichholzfolge verwenden. Hierbei mussten inhaltliche Gesichtspunkte beachtet werden. So wurde der als Anzahl der verfügbaren Streichhölzer angegebene Zahlenwert 60 nicht als Funktionswert angenommen. Es musste zunächst ein Bezug zur Sachsituation hergestellt werden und anschlieBend der zum nächst kleinere Funktionswert gehörende Wert des Definitionsbereichs abgelesen werden. Als Lösung ergibt sich in diesem Fall $n=19$, denn $A(19)=59$. Wie die folgende

\footnotetext{
${ }^{206}$ Vgl. die ScreenCam-Datei II1.scm.

${ }^{207}$ Vgl. die ScreenCam-Datei C8.scm.
} 
Tabelle zeigt, haben die Studierenden diese Aufgabe - erwartungsgemäß - etwas besser gelöst als die Schüler.

\begin{tabular}{|l||c|c|}
\hline & Schüler (49) & Studierende (21) \\
\hline 1 Lösungsversuch & $59 \%(29)$ & $76 \%(16)$ \\
\hline 2 Lösungsversuche & $21 \%(10)$ & $19 \%(4)$ \\
\hline >2 Lösungsversuche & $16 \%(8)$ & $0 \%(0)$ \\
\hline Fehlerhafte Lösung & $4 \%(2)$ & $5 \%(1)$ \\
\hline
\end{tabular}

Tab. 13: Aufgabe 1d -Lösungsversuche

\subsubsection{Lesen der Tabellendarstellung}

Die (inhaltlichen) Fehler beim Lesen der Tabellendarstellung lassen sich wie folgt klassifizieren:

I. Fehlinterpretation der diskreten Sachsituation.

(Lösung: $n=20 ; n=19,5 ; n=19,3$ ).

II. Aufgabenstellung falsch verstanden: Es wird das 60. Folgenglied bestimmt.

(Lösung: $n=182 ; n=170 ; n=200$ ).

III. Sonstige Werte (Lösung: $n=60$ ).

Die Tabelle 14 zeigt die entsprechende Häufigkeitsverteilung. Ein Proband kann dabei hinsichtlich mehrerer Fehlertypen berücksichtigt werden.

\begin{tabular}{|c||c|c|c|}
\hline & I & II & III \\
\hline \hline Schüler (49) & $24 \%(12)$ & $14 \%(7)$ & $2 \%(1)$ \\
\hline Studierende (21) & $14 \%(3)$ & $5 \%(1)$ & $5 \%(1)$ \\
\hline
\end{tabular}

Tab. 14: Aufgabe 1d - Inhaltliche Fehlertypen

Diese Umkehraufgabe bereitete den Probanden größere Schwierigkeiten als die vorhergehende Aufgabe, wobei der Unterschied bei den Studierenden weniger deutlich ist. Insgesamt $24 \%$ der Schüler hatten die diskrete Sachsituation offenbar fehlerhaft interpretiert bzw. die Aufgabe ohne Berücksichtigung inhaltlicher Gesichtspunkte gelöst. Dabei zeigt sich eine mangelnde Vertrautheit mit Folgen bzw. diskreten Funktionen und - betrachtet man Lösungen wie etwa $n=19,3$ - eine starke Fixierung auf stetige Funktionen. Die Lösungen der Probanden, die der Fehlerkategorie II zugeordnet werden können, sind ein Indiz dafür, dass der Aufgabentext nicht gründlich gelesen wurde. Dies scheint eine generelle Gefahr beim Arbeiten mit elektronischen Arbeitsblättern darzustellen, insbesondere wenn die entsprechenden Blätter eine zu hohe Informationsdichte aufweisen. 


\subsubsection{Nutzen des ,Ok-Buttons“}

Fast alle Probanden nutzten wie in Aufgabe 1c die Möglichkeit der Lösungsüberprüfung mit Hilfe des „Ok-Buttons“ und lösten die Aufgabe nach maximal 4 Lösungsversuchen. Nur bei dem Schüler A $17^{208}$ bewirkte die Präsenz dieses Buttons einen extremen Handlungsaktivismus und eine Lösungssuche nach dem Verfahren „Versuch und Irrtum“ (15 Lösungsanläufe). Die beiden Schüler, welche die Aufgabe fehlerhaft bearbeiteten, verwendeten den „OkButton" nicht.

\subsubsection{Ergebnisse zu Aufgabe 1e}

Ziel dieser Aufgabe war es, den Begriff der Differenzenfolge vorzubereiten. Hierzu sollten die Probanden zunächst mit Hilfe des TKP die Folge der Änderungen der Streichholzfolge beim Übergang von einem zum nächsten Folgenglied berechnen. Die entsprechende Formel war im Testprogramm vorgegeben, um technische Schwierigkeiten zu vermeiden und die Aufmerksamkeit der Probanden auf die inhaltliche Beziehung zwischen Folge und Differenzenfolge zu richten. Wie die folgende Tabelle verdeutlicht, haben Schüler und Studierende die Aufgabe etwa gleich gut bearbeitet.

\begin{tabular}{|l|c|c|}
\hline & Schüler (49) & Studierende (21) \\
\hline \hline 1 Lösungsversuch & $72 \%(35)$ & $76 \%(16)$ \\
\hline 2 Lösungsversuche & $18 \%(9)$ & $14 \%(3)$ \\
\hline >2 Lösungsversuche & $10 \%(5)$ & $5 \%(1)$ \\
\hline Fehlerhafte Lösung & $0 \%(0)$ & $5 \%(1)$ \\
\hline
\end{tabular}

Tab. 15: Aufgabe 1e - Lösungsversuche

\subsubsection{Erstellen der Tabelle}

Beim Erstellen der Tabelle lassen sich zwei verschiedene Vorgehensweisen unterscheiden, die bereits bei Aufgabe $1 \mathrm{~b}$ auftraten:

\begin{tabular}{|l||c|c|}
\hline & Schüler (49) & Studierende (21) \\
\hline \hline Technisch & $90 \%(44)$ & $100 \%(21)$ \\
\hline Traditionell-technisch & $10 \%(5)$ & $0 \%(0)$ \\
\hline
\end{tabular}

Tab. 16: Aufgabe 1e - Arbeitsweisen beim Erstellen der Tabelle

Sämtliche Studierenden und die meisten Schüler verwendeten zum Erstellen der Tabelle die Kopierfunktion des TKP. Dabei umging ein Schüler das Arbeiten mit relativen Zellbezügen

\footnotetext{
${ }^{208}$ Vgl. die ScreenCam-Datei A17.scm.
} 
und kopierte lediglich den numerischen Wert 3. Nur $10 \%$ der Schüler gingen zunächst traditionell vor und gaben schrittweise den Wert 3 in die entsprechenden Zellen ein.

\subsubsection{Inhaltliche/technische Probleme}

Beim Lösen dieser Aufgabe zeigten sich - wie bereits bei Aufgabe $1 \mathrm{~b}$ - sowohl inhaltliche als auch technische Probleme. Inhaltliche Schwierigkeiten äußerten sich bei $10 \%$ der Schüler und $14 \%$ der Studierenden insofern, als die eigentliche Aufgabenstellung aus den Augen verloren und versucht wurde - in Analogie zu Aufgabe 1b -, eine arithmetische Folge $A(n+1)=A(n)+d$ mit Änderung $d=3$ zu erzeugen.

Unter technischen Gesichtspunkten lassen sich wie in Aufgabe 1b drei Fehlertypen unterscheiden:

I. Vergessen des ,,"-Zeichen;

II. Fehlerhafte Zellbezüge;

III. Fehlende Zellbezüge.

Es ergibt sich die folgende Verteilung, wobei wieder ein Proband mehreren Kategorien zugeordnet werden kann.

\begin{tabular}{|l||c|c|c|}
\hline & I & II & III \\
\hline Schüler (49) & $8 \%(4)$ & $10 \%(5)$ & $0 \%(0)$ \\
\hline Studierende (21) & $10 \%(2)$ & $5 \%(1)$ & $0 \%(0)$ \\
\hline
\end{tabular}

Tab. 17: Aufgabe 1e - Technische Fehlertypen

Da die einzelnen Lösungsschritte und auch die einzugebende Formel bei dieser Aufgabe angegeben waren, lassen die Arbeitsweisen der Probanden mit fehlerhaften Bearbeitungen darauf schließen, dass der Aufgabentext nicht sorgfältig genug gelesen wurde.

\subsubsection{Ergebnisse zu Aufgabe 1f}

Aufgabe 1f diente zur Einführung des Begriffs der Differenzenfolge. Das Ausfüllen des dort angegebenen „Lückentexts“, mit Hilfe dessen die lokale Beziehung zwischen Folge und Differenzenfolge noch einmal deutlich herausstellt werden sollte, bereitete den Schülern keine größeren Probleme. Bei einer genaueren Untersuchung der Arbeitsweisen der Probanden müsste berücksichtigt werden, dass diese Aufgabe im Rahmen der Untersuchungsdurchführung mit der Schülergruppe C aus dem Testprogramm entfernt und in einem Lehrer-SchülerGespräch behandelt wurde. Da die Aufgabe hauptsächlich zur Festigung des bisher Gelernten diente, soll auf eine solche Analyse hier verzichtet werden. 


\subsubsection{Ergebnisse zu Aufgabe 1g}

Im Rahmen der bisherigen Aufgaben wurde die Streichholzfolge und deren Differenzenfolge ausschließlich anhand ihrer zeichnerischen bzw. tabellarischen Darstellung untersucht. Diese Aufgabe hatte erstmals die graphische Darstellung beider Folgen zum Gegenstand. Die Schüler und die Studierenden sollten hierbei die Eigenschaften beider Graphen verbal beschreiben. Die Antworten der Probanden lassen sich danach unterscheiden, ob geometrische oder funktionale Eigenschaften der jeweiligen Darstellungen hervorgehoben werden oder ob beim Beschreiben beider Graphen ein Bezug zum (iterativen) Aufbau der Ausgangsfolge hergestellt wird. Nachfolgend sind einige für die jeweiligen Kategorien prototypische Beschreibungen aufgeführt.

\section{Der Graph von A}

A Geometrische Sichtweise

- Der Graph ist eine Gerade.

B Funktionale Sichtweise

- Die Streichholzfolge $A$ ist eine lineare Funktion.

- Die Funktion steigt konstant an.

- Die Funktion ist stetig.

- Die Folge wächst monoton.

- $\quad A(n)=3 n+2$.

- Pro $x$-Wert Zunahme um 1 nimmt der $y$-Wert um $3 \mathrm{zu.}$

- Graph steigt konstant an (mit konstanter Steigung), nämlich um 3.

C Beziehung zum iterativen Aufbau der Folge wird hergestellt

- $\quad$ Funktion steigt konstant an, weil bei den Streichhölzern immer 3 hinzukommen.

- $\quad$ Differenz ist 3.

- Steigt linear, da immer die gleich Anzahl an Streichhölzern hinzukommen.

- Die Streichholzfolge beschreibt eine lineare Funktion, die beim Ausgangswert 5 beginnt und deren Werte jeweils um 3 zunehmen.

K Keine einzuordnende Antwort

- Streichholzfolge stellt Anzahl der Streichhölzer graphisch dar.

\section{Der Graph von D}

A Geometrische Sichtweise

- $\quad$ Der Graph ist eine Parallele zur $x$-Achse.

- Der Graph ist eine Gerade.

B Funktional-ganzheitliche Sichtweise

- Lineare Funktion.

- D zeigt keine Steigung.

- Der Graph hat einen konstanten y-Wert. 
C Beziehung zur Folge A wird hergestellt

- $\quad$ Es wird der Wert 3 beschrieben, der immer dazukommt.

- $\quad D$ zeigt die Anzahl der Streichhölzer an, die hinzugefügt werden.

- Da immer die gleiche Anzahl an Streichhölzern dazukommt, bilden die Werte eine Parallele zur $x$-Achse.

K Keine einzuordnende Antwort / keine Bearbeitung

- $\quad$ Differenz ist 0.

- $\quad$ Geringfügig und gleichbleibend.

Bei dieser Unterscheidung wird berücksichtigt, dass eine Beschreibung verschiedene Sichtweisen widerspiegeln kann. Daher können die Antworten einzelner Teilnehmer auch mehreren Kategorien zugeordnet werden. Die folgenden beiden Tabellen geben die entsprechenden Häufigkeitsverteilungen wieder.

\begin{tabular}{|l||c|c|c|c|}
\hline Schüler (49) & A & B & C & K \\
\hline \hline Graph $A$ & $14 \%(7)$ & $84 \%(41)$ & $22 \%(11)$ & $2 \%(1)$ \\
\hline Graph $D$ & $29 \%(14)$ & $73 \%(36)$ & $18 \%(9)$ & $10 \%(5)$ \\
\hline
\end{tabular}

Tab. 18: Aufgabe 1g - Sichtweisen der Schüler

\begin{tabular}{|l||c|c|c|c|}
\hline Studierende (21) & A & B & C & K \\
\hline \hline Graph $A$ & $19 \%(4)$ & $95 \%(20)$ & $29 \%(6)$ & $0 \%(0)$ \\
\hline Graph $D$ & $62 \%(13)$ & $57 \%(12)$ & $29 \%(6)$ & $0 \%(0)$ \\
\hline
\end{tabular}

Tab. 19: Aufgabe 1g - Sichtweisen der Studierende

Beim Beschreiben des Graphen von A argumentierte nur ein geringer Anteil der Schüler und Studierenden geometrisch (Kategorie A), wohingegen die funktionale Sicht bei beiden Probandengruppen deutlich überwog (Kategorie B). Wie eine weitere Analyse der Probanden, die Kategorie B zugeordnet werden können, zeigt, beschrieben $20 \%$ aller Schüler und $24 \%$ aller Studierenden explizit das Änderungsverhalten der Funktion („Pro $x$-Wert Zunahme um 1 nimmt der $y$-Wert um 3 zu."). Die Betonung dieses funktionalen Aspekts kann möglicherweise auf die schrittweise dargestellte Ausgangssituation zurückgeführt werden, zu der $22 \%$ der Schüler und $29 \%$ der Studierenden einen Bezug herstellten (Kategorie C).

Die Differenzenfolge D war im Rahmen dieser Aufgabe insbesondere den Schülern sowohl in der Interpretation als Folge der Änderungen der Streichholzfolge $A$ als auch in der Darstellung als Graph einer konstanten Folge bisher noch nicht bzw. nur auf einer intuitiven Ebene bekannt. So ist es wohl zu erklären, dass sich in den Antworten häufiger eine geometrische Sicht widerspiegelt als bei der Beschreibung des Graphen von A (Kategorie A). Andererseits betonte mit $73 \%$ immer noch die Mehrheit der Schüler funktionale Aspekte (Kategorie B). Insgesamt $18 \%$ der Schüler waren darüber hinaus in der Lage, eine Beziehung zur Ausgangsfolge herzustellen (Kategorie C). Bei der Gruppe der Studierenden überwog überraschenderweise eine geometrische Sicht auf den Graph der Differenzenfolge (Kategorie A). Dieses Er- 
gebnis lässt sich insofern etwas relativieren, als nur 2 der 13 Probanden, deren Antworten der Kategorie A zugeordnet werden können, ausschließlich geometrisch argumentierten, wohingegen die Beschreibungen der anderen 11 Probanden noch andere Sichtweisen widerspiegeln. Trotz dieser sehr visuell geprägten Sicht haben $29 \%$ der Studierenden den globalen Zusammenhang zwischen Folge und Differenzenfolge erkannt (Kategorie C). Dieser im Vergleich zu den Schülern etwas höhere Prozentsatz lässt sich auf Vorkenntnisse der Studierenden aus der Differenzialrechnung zurückführen.

\subsubsection{Ergebnisse zu Aufgabe 1h}

Das Testprogramm der Studierenden wurde für die Schüler um die beiden Aufgabenteile 1h und $1 \mathrm{j}$ erweitert. Im Aufgabenteil $1 \mathrm{~h}$ sollten die Schüler die Differenzenfolge einer geringfügig gegenüber Aufgabe 1a veränderten Streichholzfolge $B$ berechnen. Anders als bei Aufgabe 1e werden bei dieser Aufgabe keine direkt visuell zugänglichen Lösungshinweise und Hilfestellungen gegeben. Dies kann ein Grund dafür sein, dass - betrachtet man den Anteil derjenigen Probanden, welche die jeweiligen Aufgabenteile im ersten Lösungsanlauf gelöst haben- diese Aufgabe im Vergleich zu Aufgabe 1e deutlich schlechter bearbeitet wurde (vgl. Tab. 20).

\begin{tabular}{|l||c|c|c|c|}
\hline & $\begin{array}{c}\text { 1 Lösungs- } \\
\text { versuch }\end{array}$ & $\begin{array}{c}\text { 2 Lösungs- } \\
\text { versuche }\end{array}$ & $\begin{array}{c}\text { >2 Lösungs- } \\
\text { versuch }\end{array}$ & $\begin{array}{c}\text { Fehlerhafte } \\
\text { Lösung }\end{array}$ \\
\hline $\begin{array}{l}\text { Aufgabe 1e } \\
\text { (49 Schüler) }\end{array}$ & $72 \%(35)$ & $18 \%(9)$ & $10 \%(5)$ & $0 \%(0)$ \\
\hline $\begin{array}{l}\text { Aufgabe 1h } \\
\text { (49 Schüler) }\end{array}$ & $41 \%(20)$ & $31 \%(15)$ & $22 \%(11)$ & $6 \%(3)$ \\
\hline
\end{tabular}

Tab. 20: Lösungsversuche:

Vergleich: Aufgabe 1e - Aufgabe 1h

\subsubsection{Verwenden des, „Hinweis-Buttons“}

Um technischen Schwierigkeiten bei dieser Aufgabe entgegenzusteuern, hatten die Schüler hier die Möglichkeit, die entsprechende Formel über einen „Hinweis-Button“ abzurufen. Ingesamt $44 \%$ der Schüler nutzten dieses Angebot. Dabei gingen $22 \%$ der Probanden reflektiert vor, indem sie den Hinweis erst nach dem ersten Lösungsversuch zur Überprüfung bzw. Korrektur der eingegebenen Formel einsetzten. Dagegen verwendeten die übrigen $22 \%$ der Schüler den Button ohne eine vorhergehende Lösungsaktivität und umgingen damit die eigentliche Aufgabenstellung. Etwa $40 \%$ der Schüler, die den „Hinweis-Button“ einsetzten, bearbeiteten die Aufgabe anschließend (zunächst) fehlerhaft. Dies ist wieder ein Indiz dafür, dass der angezeigte Text der Hilfestellung offenbar nicht konzentriert gelesen wurde. 


\subsubsection{Inhaltliche/technische Probleme}

Diese Aufgabe bereitete den Schülern keine größeren inhaltlichen Schwierigkeiten. Lediglich $4 \%$ der Schüler berechneten anstelle der Differenzenfolge eine arithmetische Folge $A$ mit $A(n+1)=A(n)+d$ mit $d=3$ bzw. $d=4$. Dagegen hatten $59 \%$ der Probanden Probleme bei der (technischen) Verwendung des TKP. Wie bei der Bearbeitung von Aufgabe 1e wurde zum einen das ,=“-Zeichen in der entsprechenden Excel-Formel vergessen, zum anderen wurden fehlerhafte bzw. falsche Zellbezüge hergestellt. Darüber hinaus zeigten sich aber auch erhebliche Probleme beim Übersetzen der Formel $D(n)=B(n+1)-B(n)$ in die TKP-Syntax. Dabei wurden in der Regel beide Schreibweisen miteinander „vermischt", indem etwa die Formeln , $=B(n+1)-B(n) “,,,=B 9+1-B 9$ “ oder , $=(C 9+1)-C 9$ “ eingegeben wurden. Somit lassen sich die folgende Fehlertypen unterscheiden:

I. Vergessen des ,=“-Zeichens;

II. Fehlerhafte Zellbezüge;

III. Fehlende Zellbezüge;

IV. Vermischen der algebraischen Syntax mit der TKP-Syntax.

Die folgende Tabelle zeigt die Häufigkeitsverteilung über diesen Kategorien, wobei wieder ein Proband hinsichtlich mehrerer Fehlertypen berücksichtigt werden kann.

\begin{tabular}{|l||c|c|c|c|}
\hline & I & II & III & IV \\
\hline \hline Schüler (49) & $14 \%(7)$ & $14 \%(7)$ & $2 \%(1)$ & $39 \%(19)$ \\
\hline
\end{tabular}

Tab. 21: Aufgabe 1h - Technische Fehlertypen

Die Schwierigkeiten bei der Bearbeitung dieser Aufgabe - insbesondere die Ausprägung des Fehlertyps IV - lassen darauf schließen, dass die Schüler im Umgang mit dem TKP offenbar weniger gut vertraut waren als angenommen. Dabei deuten die Arbeitsweisen darauf hin, dass die Schüler offensichtlich die numerische und formale Beziehung zwischen Folge und Differenzenfolge erfasst hatten, eine operative Umsetzung mit dem TKP jedoch aufgrund mangelnder technischer Grundfertigkeiten behindert wurde.

\subsubsection{Ergebnisse zu Aufgabe 1j}

Die Schüler sollten bei dieser Aufgabe aus der Wertetabelle der Funktionen $B$ und $D$ den Wert des 9. Gliedes der Differenzenfolge $D(9)=4$ entnehmen und inhaltlich deuten, d. h. in dem vorbereiteten Lückentext das Intervall $[9,10]$ angeben und den Änderungswert 4 ergänzen. Aufgrund einer ungünstigen Positionierung ${ }^{209}$ der Tabelle im Testprogramm der Schü-

\footnotetext{
${ }^{209}$ Bei diesem Programm stimmten der Funktionsname der Differenzenfolge und der Name der Spalte, in der diese Folgenglieder numerisch aufgelistet waren, überein.
} 
lergruppen $\mathrm{A}$ und $\mathrm{B}$ gaben $65 \%$ dieser Schüler zunächst anstelle des Funktionswerts $D(9)=4$ den Inhalt der Zelle D9, also den Funktionswert $D(4)=2$, an. Dieser Fehler setzte sich zum Teil in einer fehlerhaften Interpretation dieses Werts als Änderung der Folge $B$ fort. Im Rahmen der Untersuchung mit der Schülergruppe $C$ wurde die Tabelle auf dem ExcelBlatt zwei Spalten weiter nach rechts platziert, d. h. die Glieder der Folge $D$ standen nun in Spalte G. Dies führte zu einer signifikanten Reduktion der Ablesefehler (ChiquadratVierfeldertest, $p=0,001$ ) und zu einer insgesamt besseren Bearbeitung der Aufgabe (vgl. Tab. 22 und 23).

\begin{tabular}{|l||c|c|}
\hline & $\begin{array}{c}\text { Gruppe A/B } \\
\text { (34 Schüler) }\end{array}$ & $\begin{array}{c}\text { Gruppe C } \\
\text { (15 Schüler) }\end{array}$ \\
\hline \hline 1 Lösungsversuch & $32 \%(11)$ & $46 \%(7)$ \\
\hline 2 Lösungsversuche & $27 \%(9)$ & $20 \%(3)$ \\
\hline >2 Lösungsversuche & $35 \%(12)$ & $27 \%(4)$ \\
\hline Fehlerhafte Lösung & $6 \%(2)$ & $7 \%(1)$ \\
\hline
\end{tabular}

Tab. 22: Aufgabe 1j - Lösungsversuche

\begin{tabular}{|c||c|c|}
\hline \multicolumn{1}{|c||}{} & \multicolumn{2}{c|}{ Fehlertyp ,D(9)=2 “ } \\
\hline & Ja & Nein \\
\hline $\begin{array}{c}\text { Gruppe A/B } \\
\text { (34 Schüler) }\end{array}$ & $65 \%(22)$ & $35 \%(12)$ \\
\hline $\begin{array}{c}\text { Gruppe C } \\
\text { (15 Schüler) }\end{array}$ & $13 \%(2)$ & $87 \%(13)$ \\
\hline
\end{tabular}

Tab. 23: Aufgabe 1j - Auftreten des Fehlertyps „,D(9)=2“

\subsection{Ergebnisse zu Aufgabe 2}

Im Rahmen dieser Aufgabe sollten die Probanden das Änderungsverhalten einer empirischen Folge untersuchen, d. h. einer Folge, die nicht durch eine einfache Gesetzmäßigkeit und damit auch nicht durch eine einfache algebraische Formel beschrieben werden kann. Aufgabe 2 umfasst Fragen zu lokalen und globalen Eigenschaften zweier Temperaturfolgen, die mit Hilfe der entsprechenden Differenzenfolgen auf der numerischen bzw. graphischen Ebene beantwortet werden sollten. Bei zwei Aufgabenteilen konnten die Probanden zwischen der tabellarischen und der graphischen Darstellung wählen.

\subsubsection{Ergebnisse zu Aufgabe 2a}

Bei dieser Aufgabe, die strukturell mit Aufgabe 1h übereinstimmt, war zunächst die Differenzenfolge der Folge der Durchschnittstemperaturen zu berechnen. Obwohl $12 \%$ der Schüler die Aufgabe fehlerhaft lösten, kann man - betrachtet man die Anzahl der Probanden, die nur 
einen bzw. zwei Lösungsanläufe benötigten - eine Verbesserung gegenüber Aufgabe 1h feststellen (vgl. Tab. 24). Die Studierenden haben diesen Aufgabenteil fehlerfrei bearbeitet. Dabei lösten nur geringfügig mehr Studierende als Schüler die Aufgabe bereits im Rahmen des ersten Lösungsversuchs.

\begin{tabular}{|c||c|c|c|c|}
\hline & $\begin{array}{c}\text { 1 Lösungs- } \\
\text { versuch }\end{array}$ & $\begin{array}{c}\text { 2 Lösungs- } \\
\text { versuche }\end{array}$ & $\begin{array}{c}\text { >2 Lösungs- } \\
\text { versuch }\end{array}$ & $\begin{array}{c}\text { Fehlerhafte } \\
\text { Lösung }\end{array}$ \\
\hline \hline $\begin{array}{c}\text { Aufgabe 1h } \\
\text { 49 Schüler) }\end{array}$ & $41 \%(20)$ & $31 \%(15)$ & $22 \%(11)$ & $6 \%(3)$ \\
\hline $\begin{array}{c}\text { Aufgabe 2a } \\
\text { 50 Schüler) }\end{array}$ & $60 \%(30)$ & $18 \%(9)$ & $10 \%(5)$ & $12 \%(6)$ \\
\hline $\begin{array}{c}\text { Aufgabe 2a } \\
\text { (21 Studierende) }\end{array}$ & $67 \%(14)$ & $14 \%(3)$ & $19 \%(4)$ & $0 \%(0)$ \\
\hline
\end{tabular}

Tab. 24: Lösungsversuche:

Vergleich: Aufgabe 1h - Aufgabe 2a

Wie eine weitere Analyse der Arbeitsweisen zeigt, überlagern bei beiden Probandengruppen die technischen Schwierigkeiten im Umgang mit dem TKP Probleme, die auf ein mangelndes inhaltliches Verständnis des Begriffs Differenzenfolge hindeuten. So berechneten lediglich zwei Studierende und ein Schüler anstelle der Glieder der Differenzenfolge $D(n)=T(n+1)-T(n)$ Glieder einer arithmetischen Folge oder einer Folge $D$ mit $D(n)=T(n)-T(n-1)$. Dagegen bereitete der Umgang mit dem Werkzeug TKP $36 \%$ der Schüler und $29 \%$ der Studierenden Probleme, wenngleich bei den Schülern im Vergleich zu Aufgabe $1 \mathrm{~h}$ ein Lernfortschritt feststellbar ist. Die technischen Schwierigkeiten der Probanden werden im Folgenden im Hinblick auf die bereits in Aufgabe 1h gebildeten Fehlerkategorien analysiert:

I. Vergessen des ,=“-Zeichens;

II. Fehlerhafte Zellbezüge;

III. Fehlende Zellbezüge;

IV. Vermischen der algebraischen Syntax mit der TKP-Syntax.

Die Tabelle 25 zeigt die Häufigkeitsverteilung über diesen Kategorien. Ein Proband kann dabei mehreren Fehlertypen zugeordnet werden:

\begin{tabular}{|c||c|c|c|c|}
\hline & I & II & III & IV \\
\hline \hline $\begin{array}{c}\text { Aufgabe 1h } \\
\text { 49 Schüler) }\end{array}$ & $14 \%(7)$ & $14 \%(7)$ & $2 \%(1)$ & $39 \%(19)$ \\
\hline $\begin{array}{c}\text { Aufgabe 2a } \\
\text { 50 Schüler) }\end{array}$ & $6 \%(3)$ & $10 \%(5)$ & $0 \%(0)$ & $30 \%(15)$ \\
\hline $\begin{array}{c}\text { Aufgabe 2a } \\
\text { (21 Studierende) }\end{array}$ & $0 \%(0)$ & $14 \%(3)$ & $0 \%(0)$ & $19 \%(4)$ \\
\hline
\end{tabular}

Tab. 25: Technische Fehlertypen:

Vergleich: Aufgabe 1h - Aufgabe 2a 
Im Vergleich zu Aufgabe 1h traten bei den Schülern weniger häufig rein syntaktische Fehler und fehlerhafte Zellbezüge auf (Kategorie I und II). Dagegen bereitete bei der Berechnung der Differenzenfolge $D(n)=T(n+1)-T(n)$ die Abgrenzung der Excel-Synatx von der algebraischen Syntax immer noch die größten Schwierigkeiten (Kategorie IV). So gaben $30 \%$ der Schüler in der Zelle D9, welcher das erste Differenzenfolgenglied zugewiesen werden sollte,

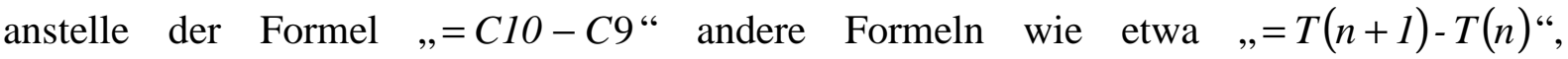
, $=(C 10+1)-C 9$ “ oder ,=T10-T9“ ein. Vier Schüler, die der Fehlerkategorie IV zugeordnet werden können, hatten bereits in Aufgabe 1h die beiden formalen Sprachen miteinander vermischt, wohingegen bei den übrigen Schülern dieser Fehler hier zum ersten Mal auftrat.

Die Studierenden beachteten in ihren Formeln durchgängig das ,=“-Zeichen; jedoch verwendeten $14 \%$ der Probanden zunächst fehlerhafte Zellbezüge. Im Vergleich zu den Schülern bereitete den Studierenden die Abgrenzung der Excel-Syntax von der algebraischen Syntax weniger Schwierigkeiten. Nur $19 \%$ der Probanden wiesen in ihren ersten Lösungsanläufen der Zelle D9 die falsche Formel „, $=T(n+1)-T(n) “$ zu.

\subsubsection{Ergebnisse zu Aufgabe 2b}

Die Probanden sollten bei dieser Aufgabe bestimmen, wie sich die Durchschnittstemperatur des Jahres 1984 gegenüber der des Jahres 1983 veränderte. Der entsprechende Wert konnte sowohl aus der tabellarischen als auch aus der graphischen Darstellung der Temperaturfolge und deren Differenzenfolge entnommen werden. Die Aufgabe wurde nur von $26 \%$ der Schüler und $33 \%$ der Studierenden im ersten Versuch richtig gelöst, wurde aber von über der Hälfte der Probanden nach höchstens zwei Lösungsversuchen erfolgreich bearbeitet (vgl. Tab. 26). Während die Ergebnisse aus Aufgabe 2a für ein instrumentelles Verständnis im Umgang mit Differenzenfolgen sprechen, zeigt sich hier, dass das Erkennen und Interpretieren funktionaler Beziehungen bzw. Eigenschaften offensichtlich höhere Anforderungen an die Probanden stellt.

\begin{tabular}{|c|c|c|}
\hline & Schüler (50) & Studierende (21) \\
\hline \hline 1 Lösungsversuch & $26 \%(13)$ & $33 \%(7)$ \\
\hline 2 Lösungsversuche & $26 \%(13)$ & $33 \%(7)$ \\
\hline >2 Lösungsversuche & $44 \%(22)$ & $33 \%(7)$ \\
\hline Fehlerhafte Lösung & $4 \%(2)$ & $0 \%(0)$ \\
\hline
\end{tabular}

Tab. 26: Aufgabe 2b - Lösungsversuche

\subsubsection{Auswahl der Darstellungen}

Die folgende Tabelle zeigt, wie oft die einzelnen Darstellungsformen Tabelle und Graph von den Schülern bzw. den Studierenden gewählt wurden. 


\begin{tabular}{|l||c|c|}
\hline & Tabelle & Graph \\
\hline \hline Schüler (50) & 171 & 61 \\
\hline Studierende (21) & 63 & 12 \\
\hline
\end{tabular}

Tab. 27: Aufgabe 2b - Absolute Häufigkeit der betrachteten Darstellungen

Die Schüler arbeiteten mit tendenziell mehr Darstellungen als die Studierenden, wenngleich kein signifikanter Unterschied feststellbar ist (Wilcoxon-Test, $p=0,1833$ ). Im Mittel betrachteten die Schüler 4,6 Darstellungen (Standardabweichung 3,1) und die Studierenden 3,6 Darstellungen (Standardabweichung 2,1). Beide Probandengruppen setzten dabei signifikant häufiger die Tabelle als den Graph ein (Wilcoxon-Test für Paardifferenzen, $p<0,001$ ). Um eine detailliertere Aussage über die Verwendung der offensichtlich von beiden Gruppen bevorzugten Darstellung Tabelle treffen zu können, werden relative Maßzahlen für deren Auswahlhäufigkeit betrachtet. Man stellt dabei fest, dass die Studierenden die tabellarische Darstellung tendenziell (wenn auch nicht signifikant) häufiger einsetzen als die Schüler (Wilcoxon-Test, $p=0,0549$ ). Dieses Ergebnis kann darauf zurückgeführt werden, dass die Studierenden durch ihre Schul- und Universitätsausbildung mit den Darstellungen Graph und Tabelle besser vertraut sind als die Schüler und daher die - im Rahmen dieser Aufgabe - vorteilhaftere tabellarische Darstellung dem Graph vorzogen.

\subsubsection{Strategien beim Arbeiten mit Darstellungen}

Die Analyse der Anzahl der ausgewählten Darstellungen ermöglicht eine erste Interpretation der Arbeitsweisen der Probanden. Dabei wird jedoch die jeweilige Zugriffsdauer nicht berücksichtigt, die zum Teil so kurz (wenige Sekunden) ist, dass es unwahrscheinlich erscheint, dass die entsprechende Darstellung auch (konzentriert) gelesen wurde. Im Folgenden soll daher das Augenmerk auf die tatsächlich zur Lösung der Aufgabenstellung eingesetzten Darstellungen gerichtet werden. Als lösungsrelevant werden dabei solche Darstellungen bezeichnet, die der Proband näher analysiert hat (erkennbar etwa an Cursorbewegungen, d. h. an dem Deuten mit dem Cursor auf bestimmte Funktionswerte bzw. Koordinatenpunkte, oder an einer längeren Zugriffszeit) und die somit offensichtlich eine Grundlage für einen Lösungsversuch waren. Dabei werden nur die Probanden berücksichtigt, welche die Aufgabe erfolgreich bearbeiteten. Es ergeben sich die folgenden Kategorien:

\section{I. $\quad$ Arbeiten mit der Tabelle}

Die Probanden arbeiten ausschließlich mit der Tabelle bzw. die Auswahl und das Arbeiten mit dem Graph kann als nicht lösungsrelevant angesehen werden.

\section{Arbeiten mit dem Graph}

Die gesamte Lösung der Fragestellung ist auf das Arbeiten mit dem Graph von $T$ bzw. $D$ zurückzuführen. Die Tabelle wird zwar auch betrachtet, sie spielt im Lösungsprozess aber eine untergeordnete Rolle. 


\section{Arbeiten mit Tabelle und Graph}

Die Aufgabe wird sowohl mit dem Graph als auch mit der Tabelle bearbeitet. Eine Bevorzugung einer der beiden Darstellungen ist nicht erkennbar. Die Arbeitsweisen lassen sich (m. E.) ${ }^{210}$ hier weiter danach unterscheiden, wie der Graph im Lösungsprozess eingesetzt wird:

Analyse des Graphenverlaufs

Der Graph von $T$ dient der Entscheidungsfindung, ob die Temperatur des Jahres 1984 über oder unter der Temperatur des Jahres 1983 liegt. Die Probanden analysieren hierbei die „Umgebung“ der beiden Koordinatenpunkte (1983,T(1983)) und (1984,T(1984)). Die Markierung des Kontrollkästchens zu „unter“ auf dem Lösungsblatt erfolgt unmittelbar im Anschluss an die Auswahl des Graphen.

\section{Analyse von Koordinatenpunkten}

Die Probanden entnehmen dem Graph von $T$ bzw. $D$ die numerischen Werte $T(1983)=14,8$ und $T(1984)=13,7$ bzw. $D(1983)=-1,1$, indem sie mit dem Mauszeiger auf die entsprechenden Koordinatenpunkte deuten.

Obwohl versucht wurde, die obigen Kategorien bestmöglich voneinander abzugrenzen, ist eine gewisse „Unschärfe“ und „Subjektivität“ bei der Zuordnung der einzelnen Probanden zu den Kategorien nicht ausgeschlossen. Die folgende Tabelle gibt die entsprechende Häufigkeitsverteilung wieder.

\begin{tabular}{|l||c|c|c|}
\hline & I & II & III \\
\hline Schüler (48) & $73 \%(35)$ & $6 \%(3)$ & $21 \%(10)$ \\
\hline Studierende (21) & $90 \%(19)$ & $0 \%(0)$ & $10 \%(2)$ \\
\hline
\end{tabular}

Tab. 28: Aufgabe 2b - Strategien beim Arbeiten mit Darstellungen

Wie bereits bei der Analyse der bevorzugt gewählten Darstellungen werden hier (nicht signifikante) Unterschiede in den Arbeitsweisen der Schüler und der Studierenden deutlich (Chiquadrat-Mehrfeldertest, $p=0,227)$. So haben die Studierenden fast ausschließlich mit der Tabelle gearbeitet (Kategorie I). Lediglich $10 \%$ der Probanden haben beide Darstellungen zur Lösung hinzugezogen (Kategorie III). Dagegen haben „nur“ 73 \% der Schüler die Aufgabe mit Hilfe der Tabelle gelöst. Insgesamt $21 \%$ der Schüler arbeiteten sowohl mit der Tabelle als auch mit dem Graph. Interessant sind in diesem Zusammenhang die Arbeitsweisen von sieben Probanden ${ }^{211}$, die den numerischen Wert 1,1 (letztendlich) mit Hilfe der Tabelle bestimmten, den Graph von $T$ jedoch zur Analyse des Änderungsverhaltens einsetzen. Die Darstellungen Tabelle und Graph wurden somit reflektiert und problemadäquat verwendet. Fünf

\footnotetext{
${ }^{210}$ In einem Ausnahmefall ist eine Zuordnung zu einer dieser beiden Unterkategorien nicht möglich, da der entsprechende Proband keine Bildschirmaktivitäten zeigte.

${ }^{211}$ Diese Probanden lassen sich der ersten Unterkategorie von Kategorie III zuordnen.
} 
Schüler $^{212}$, die sowohl mit der Tabelle als auch mit dem Graph arbeiteten, verwendeten den Graph zum Bestimmen der Funktionswerte T(1983) und T(1984) bzw. D(1983). Zwei Probanden beschränkten sich auf das Ablesen der Werte T(1983) und T(1984). Dagegen analysierten drei Probanden alle drei in Beziehung stehenden Funktionswerte. Dies spricht dafür, dass diese Schüler den lokalen Zusammenhang zwischen $T$ und $D$ erkannt hatten. Drei Schüler verwendeten ausschließlich den Graph. Anhand der Cusorbewegungen dieser Probanden lässt sich feststellen, dass ein Schüler lediglich die Temperaturfolge betrachtete und den Wert des Temperaturabfalls berechnete, indem er die Differenz $T(1984)-T(1983)$ „im Kopf“ bestimmte. Zwei Schüler arbeiteten sowohl mit der graphischen Darstellung der Temperaturfolge als auch mit deren Differenzenfolge und ermittelten die numerischen Werte $T(1983)$, $T(1984)$ und $D(1983)$ durch das Deuten mit dem Cursorzeiger auf die entsprechenden Koordinatenpunkte. Dieses Vorgehen weist darauf hin, dass auch diese beiden Probanden anhand der graphischen Darstellung die lokale Beziehung zwischen Folge und Differenzenfolge erkannt haben. Das Vorgehen eines der beiden Probanden soll im Folgenden noch einmal illustriert werden.

\begin{tabular}{|c|c|c|c|c|c|c|}
\hline Zeit & Aufg. & $\begin{array}{l}\text { Aufg.- } \\
\text { stellung }\end{array}$ & Tabelle & Graph & Kommentar & Eingaben über Tastatur \\
\hline 17.52 & $2 b$ & $\mathrm{x}$ & & & & \\
\hline 17.55 & & & & $\mathrm{x}$ & $\begin{array}{l}\text { Der Cursor deutet auf die } \\
\text { Funktionswerte } \\
T(1983), T(1984), \\
D(1983), D(1984), D(1983) .\end{array}$ & \\
\hline 18.16 & & $\mathrm{x}$ & & & & $\begin{array}{l}\text { Eingabe des Änderungs- } \\
\text { werts }-1,1 \text {. }\end{array}$ \\
\hline 18.43 & & & & $\mathrm{x}$ & $\begin{array}{l}\text { Der Cursor deutet auf die } \\
\text { Funktionswerte } \\
T(1983), T(1984) \text {. }\end{array}$ & \\
\hline 18.51 & & $\mathrm{x}$ & & & & $\begin{array}{l}\text { Markieren des Kontrollkäst- } \\
\text { chens ,,unter“. }\end{array}$ \\
\hline 18.57 & & & & & $\begin{array}{l}\text { Lösungsüberprüfung mit dem } \\
\text { „OK-Button“. Es wird auf } \\
\text { einen Fehler hingewiesen. }\end{array}$ & \\
\hline 19.01 & & & & & $\begin{array}{l}\text { Lösungsüberprüfung mit dem } \\
\text { „OK-Button“. Es wird auf } \\
\text { einen Fehler hingewiesen. } \\
\text { Lesen des Aufgabentexts. }\end{array}$ & $\begin{array}{l}\text { Markieren des Kontrollkäst- } \\
\text { chens ,,̈̈ber“ } \\
\text { Löschen des Werts -1,1; } \\
\text { Deaktivieren des Kontroll- } \\
\text { kästchens ,über“. }\end{array}$ \\
\hline 19.57 & & & & & & $\begin{array}{l}\text { Eingabe des Änderungs- } \\
\text { werts } 1,1 ; \text { Markieren des } \\
\text { Kontrollkästchens „unter“. }\end{array}$ \\
\hline
\end{tabular}

Tab. 29: Aufgabe 2b - Transkriptausschnitt ${ }^{213}$ (Schüler A23)

\footnotetext{
${ }^{212}$ Diese Probanden lassen sich der zweiten Unterkategorie von Kategorie III zuordnen. Es werden hierbei auch zwei Probanden einbezogen, die bereits der ersten Unterkategorie III zugeordnet werden konnten.

${ }^{213}$ Vgl. die ScreenCam-Datei A23.scm.
} 
Der betreffende Schüler deutete - um den Wert der Temperaturänderung zu bestimmen - mit dem Cursor auf die Koordinatenpunkte (1983,T(1983)), (1984,T(1984)) und (1983, D(1983)). Danach wechselte er zum Aufgabenblatt und gab den Wert -1,1 in das entsprechende Lösungsfeld ein. Im Anschluss analysierte der Schüler anhand der graphischen Darstellung von $T$ die Art des Änderungsverhaltens, markierte das Kontrollkästchen zu „unter“ und überprüfte das Ergebnis mit dem „OK-Button“. Die negative Rückmeldung führte zu einer weiteren (fehlerhaften) Lösungseingabe: Der Schüler markierte (offensichtlich unreflektiert) zusätzlich das alternative Kontrollkästchen „über“. Nachdem sich auch dieses Ergebnis im Rahmen der Lösungsüberprüfung als falsch herausstellte, folgte eine etwa einminütige Reflektionsphase und anschließend die korrekte Lösungseingabe.

Der hier beschriebene Schüler nutzte offensichtlich sowohl den Graph von $T$ als auch den der Differenzenfolge $D$ zum Lösen der Aufgabe. Während er zum Ablesen des numerischen Änderungswerts den Graph von $D$ verwendete, erfolgte die Interpretation des Änderungsverhaltens anhand des Graphen von $T$. Dabei beachtete der Schüler zunächst nicht die Bedeutung des negativen Vorzeichens, indem er anstelle des (absoluten) Änderungswerts 1,1 den Funktionswert $D(1983)=-1,1$ angab.

\subsubsection{Inhaltliche Probleme}

Bei der Analyse der inhaltlichen Probleme der Probanden lassen sich drei Fehlertypen unterscheiden:

\section{I. $\quad$ Annahme einer falschen lokalen Beziehung zwischen $T$ und $D$}

Es wird mit der Beziehung $D(n)=T(n)-T(n-1)$ operiert, d. h. es wird der Wert $D(1984)=0,4$ anstelle des Werts $D(1983)=-1,1$ abgelesen.

\section{Angabe eines Funktionswerts von T}

Es werden Werte angegeben wie etwa $14,8=T(1983), 13,7=T(1984)$.

III. Fehlerhafte inhaltliche Deutung:

Es erfolgen Eingaben im Sinne von:

„Temperatur von 1984 liegt -1,1 unter der Temperatur von 1983“,

„Temperatur von 1984 liegt 1,1 über der Temperatur von 1983“.

Die Probanden werden nun den entsprechenden Fehlerkategorien zugewiesen, wobei ein Proband auch in verschiedenen Kategorien berücksichtigt werden kann (vgl. Tab. 30). Die Fehler von sechs Schülern lassen sich nicht einem der beschriebenen Typen zuordnen. Diese Schüler werden in der folgenden Tabelle im Rahmen einer weiteren Kategorie IV berücksichtigt.

\begin{tabular}{|l||c|c|c|c|}
\hline & I & II & III & IV \\
\hline \hline Schüler (50) & $34 \%(17)$ & $26 \%(13)$ & $38 \%(19)$ & $12 \%(6)$ \\
\hline Studierende (21) & $33 \%(7)$ & $14 \%(3)$ & $29 \%(6)$ & $0 \%(0)$ \\
\hline
\end{tabular}

Tab. 30: Aufgabe 2b - Inhaltliche Fehlertypen 
Die Probanden, die dem Fehlertyp II zugeordnet werden können, hatten offensichtlich den Aufgabentext nicht richtig gelesen bzw. hatten Probleme, Darstellungen „relativ zu lesen“, d. h. eine Beziehung zwischen zwei Funktionswerten herzustellen. Fehlertyp III weist dagegen darauf hin, dass beim Arbeiten mit der Differenzenfunktion die inhaltliche Bedeutung negativer Funktionswerte nicht erkannt wurde. Beiden Fehlertypen lassen sich prozentual mehr Schüler als Studierende zuweisen. Das bessere Abschneiden der Studierenden kann auf Vorkenntnisse aus der Differenzialrechnung zurückgeführt werden. Dagegen bereitete das Arbeiten mit der lokalen Beziehung $D(n)=T(n+1)-T(n)$ beiden Probandengruppen vergleichbar große Schwierigkeiten (Fehlertyp I). Diese Beziehung wurde von etwa $35 \%$ der Probanden mit der ebenfalls „,naheliegenden“ aber im Rahmen des Untersuchungsprogramms nicht vereinbarten Beziehung $D(n)=T(n)-T(n-1)$ verwechselt. Dieser Fehler kann darauf zurückgeführt werden, dass sich zum einen der Wert eines bestimmten Differenzenfolgenglieds auf zwei aufeinander folgende Glieder der Folge $T$ bezieht, dass die Tabellenstruktur es zum anderen jedoch erforderlich macht, das entsprechende Glied der Folge $D$ nicht der „Intervallmitte“, sondern einer der beiden Grenzen zuzuordnen. Im Rahmen des Testprogramms wurde - in Anlehnung an den aufsteigenden Differenzenoperator in der Theorie der Finiten Mathematik (vgl. Kap. 2.1.4.2, Tab. 1) - die Festlegung $D(n)=T(n+1)-T(n)$ getroffen. Die optische Anordnung der Zellen bzw. der graphischen Darstellung der Funktionswerte ließ diese Beziehung jedoch nicht, ,auf einen Blick“ erkennen, sondern erforderte ein äußerst konzentriertes Arbeiten mit den entsprechenden Darstellungen.

\subsubsection{Ergebnisse zu Aufgabe 2c}

Die Aufgabenteile 2c, 2d und 2e wurden zusätzlich in das Testprogramm der Schüler aufgenommen. Ein Vergleich mit den Studierenden ist daher bei dieser und den folgenden Aufgaben nicht möglich. Gegenstand von Aufgaben $2 \mathrm{c}$ ist eine von Aufgabe $2 \mathrm{a}$ und $2 \mathrm{~b}$ verschiedene Temperaturfolge. Während die vorhergehende Aufgabe den Probanden die Möglichkeit bot, zwischen den Darstellungen Tabelle und Graph zu wählen, ist hier lediglich die tabellarische Darstellung gegeben, anhand derer die Schüler den Wert $T(1989)=T(1988)+D(1988)=17,5+0,4=17,9$ bestimmen sollten. Da die Schüler offensichtlich mit der Tabelle mittlerweile hinreichend vertraut waren, wurde die Aufgabe von der Mehrzahl der Schüler fehlerfrei nach wenigen Lösungsanläufen gelöst (vgl. Tab. 31).

\begin{tabular}{|l|c|}
\hline & Schüler (50) \\
\hline \hline 1 Lösungsversuch & $80 \%(40)$ \\
\hline 2 Lösungsversuche & $14 \%(7)$ \\
\hline >2 Lösungsversuche & $4 \%(2)$ \\
\hline Falsche Lösung & $2 \%(1)$ \\
\hline
\end{tabular}

Tab. 31: Aufgabe 2c - Lösungsversuche 
Insgesamt 28 der 40 Schüler, welche die Aufgabe im ersten Lösungsversuch lösten, führten die Rechnung im Kopf aus und übertrugen lediglich den numerischen Wert in die entsprechende Zelle. Diese Schüler konzentrierten sich offensichtlich auf den lokalen und im Lernprogramm durch „Pfeile“ visualisierten Zusammenhang zwischen $T(1988)$ und $T(1989)$, wobei nicht zweifelsfrei festgestellt werden kann, ob auch der allgemeine Zusammenhang $D(n)=T(n+1)-T(n)$,,mitbedacht“" wurde. Dagegen verwendeten 12 Schüler zum Berechnen des numerischen Werts T(1989) die TKP-Adressen der in Beziehung stehenden Zellen. Damit übertrugen sie zum einen die Ausführung der arithmetischen Rechenoperation an das TKP. Zum anderen weist dieses Vorgehen darauf hin, dass die optische Anordnung der in Beziehung stehenden Zellen erkannt und die Zellen somit als „Vergegenständlichung“ von Variablen verwendet wurden. Die tabellarische Darstellung von Folge und Differenzenfolge war offensichtlich für diese Schüler als kognitiver Prototyp (im Sinne Dörflers) wirksam.

\subsubsection{Ergebnisse zu Aufgabe 2d}

Diese Aufgabe schließt inhaltlich an Aufgabe 2c an. Die dort betrachtete Temperaturfolge ist hier - bis auf die Folgenglieder $T(1992)=18,9$ und $D(1994)=-0,6$ - graphisch dargestellt. Die fehlenden Werte sollen durch Verknüpfen geeigneter Folgenglieder bestimmt werden.

\subsubsection{Arbeiten mit dem Graph}

Im Gegensatz zu Aufgabe 2c, bei der die Mehrheit der Schüler mit der Berechnung des Werts $T(1989)$ keine Schwierigkeiten hatten, lösten hier nur 16 \% bzw. $18 \%$ der Probanden die beiden Aufgabenteile im ersten Lösungsanlauf fehlerfrei. Die meisten Schüler benötigten mehrere Lösungsanläufe, wobei das Berechnen des Werts $D(1994)$ die Probanden vor ähnlich große Probleme stellte wie beim Berechnen des Werts T(1992) (vgl. Tab. 32).

\begin{tabular}{|l|c|c|}
\hline Schüler (50) & $T(1992)$ & $D(1994)$ \\
\hline 1 Lösungsversuch & $16 \%(8)$ & $18 \%(9)$ \\
\hline 2 Lösungsversuche & $32 \%(16)$ & $24 \%(12)$ \\
\hline >2 Lösungsversuche & $42 \%(21)$ & $48 \%(24)$ \\
\hline Falsche Lösung & $10 \%(5)$ & $10 \%(5)$ \\
\hline
\end{tabular}

Tab. 32: Aufgabe 2d - Lösungsversuche

Ein Grund hierfür ist darin zu sehen, dass die Schüler mit der graphischen Darstellung der Folge und deren Differenzenfolge bisher nur wenig vertraut waren und dass die Aufgabe darüber hinaus eine große Konzentrationsleistung erforderte. So mussten die Schüler zum einen die lokal in Beziehung stehenden Folgenglieder bzw. die entsprechenden Koordinatenpunkte erkennen. Zum anderen war es erforderlich, die zunächst „unsichtbaren“ numerischen Funktionswerte der betrachteten Folgenglieder durch Deuten auf die jeweiligen Koordinatenpunkte 
zu visualisieren. Da die Koordinaten der graphisch dargestellten Folgenglieder nur für die Dauer des „Hindeutens“ sichtbar sind, mussten sich die Schüler die entsprechenden Funktionswerte merken und anschließend numerisch miteinander verknüpfen. Viele Probanden brachten diese Ruhe und Konzentration nicht auf und wechselten in kurzer Zeit häufig zwischen den Koordinatenpunkten. Ein Beispiel soll dies verdeutlichen. So visualisierte ein Schüler ${ }^{214}$ zur Berechnung des Werts $T(1992)=18,9$ mit Hilfe des Cursors innerhalb von drei Minuten die folgenden 20 Folgenglieder: $T(1992), D(1992), D(1991), T(1993), D(1992)$, $D(1993), D(1994), T(1992), T(1991), D(1991), T(1992), D(1992), T(1991), T(1993)$, $D$ (1993), $T(1994), T(1993), D(1991), T(1991), T(1992)$.

\subsubsection{Inhaltliche Probleme}

Bei der Analyse der inhaltlichen Probleme der Schüler lassen sich die folgenden Fehlertypen unterscheiden:

\section{Fehlerhaftes Berechnen des Werts T(1992)}

I. Die Beziehung $D(n)=T(n)-T(n-1)$ wird zur Berechnung verwendet.

II. Die lokale Beziehung zwischen $T$ und $D$ wird nicht erkannt.

Als Werte für $T(1992)$ werden z. B. angegeben:

$17,1=T(1991)-D(1991)$ oder $16,3=T(1993)+D(1992)$.

IIIa. Es wird auf die falsche Folge zugegriffen (Temperaturfolge aus Aufgabe 2a).

Fehlerhaftes Berechnen des Werts D(1994)

I. Die Beziehung $D(n)=T(n)-T(n-1)$ wird zur Berechnung verwendet.

II. $\quad$ Die lokale Beziehung zwischen $T$ und $D$ wird nicht erkannt.

Als Werte für $D(1994)$ werden z. B. angegeben:

$21,3=T(1994)-D(1995)$ oder $17,6=T(1994)+D(1993)$.

IIIb. Die Beziehung $D(n)=T(n)-T(n+1)$ liegt der Berechnung zugrunde,

d. h. es wird die Reihenfolge der zu verknüpfenden Folgenglieder vertauscht.

Man erhält die folgende Häufigkeitsverteilung:

\begin{tabular}{|c||c|c|}
\hline Schüler (50) & $T(1992)$ & $D(1994)$ \\
\hline \hline I & $56 \%(28)$ & $56 \%(26)$ \\
\hline II & $52 \%(26)$ & $42 \%(21)$ \\
\hline IIIa & $8 \%(4)$ & ----- \\
\hline IIIb & ----- & $34 \%(17)$ \\
\hline
\end{tabular}

Tab. 33: Aufgabe 2d - Inhaltliche Fehlertypen

\footnotetext{
${ }^{214}$ Vgl. die ScreenCam-Datei A21.scm.
} 
Über die Hälfte der Schüler hat beim Berechnen der Funktionswerte $T(1992)$ und $D(1994)$ die Beziehung $D(n)=T(n)-T(n-1)$ verwendet (Fehlertyp I). Während die Tabelle offenbar insbesondere durch die visuelle Unterstützung der Pfeile - das Erkennen der jeweils in Beziehung stehenden Zellen bzw. Variablen erleichtert, scheint es anhand des Graphen schwieriger zu sein, lokale Zusammenhänge zwischen Folge und Differenzenfolge zu erkennen. Überraschend viele Probanden haben zunächst unreflektierte Berechnungen angestellt. Dabei weisen die angegebenen Werte für $D(1994)$ darauf hin, dass die veränderte Fragestellung nicht erkannt, d. h. die vorher angewandte Operation nicht umgekehrt wurde (Fehlertyp II). Insgesamt $8 \%$ der Schüler umgingen bei der Berechnung von T(1992) die Aufgabenstellung, indem sie den entsprechenden Wert aus der Tabelle zu Aufgabe 2a entnahmen, in der jedoch eine andere Temperaturfolge dargestellt war (Fehlertyp IIIa). Da der Funktionswert D(1994) in dieser Tabelle nicht aufgeführt war, konnte ein ähnlicher Fehler bei der Bearbeitung des zweiten Teils von Aufgabe 2d nicht auftreten. Statt dessen vertauschten $34 \%$ der Schüler bei der Berechnung des (negativen) Werts $D(1994)=-0,6$ die Reihenfolge der Folgenglieder $T(1995)=18,9$ und $T(1994)=19,5$, vergaßen jedoch das Ergänzen des negativen Vorzeichens (Fehlertyp IIIb).

\subsubsection{Ergebnisse zu Aufgabe 2e}

Bei dieser Aufgabe sollten die Schüler diejenigen Zeitintervalle angeben, in denen die durchschnittlichen Sommertemperaturen zugenommen haben. Zur Beantwortung dieser Frage konnten die Schüler, wie bereits in Aufgabe 2b, sowohl mit der Tabelle als auch mit dem Graph arbeiten. Sämtliche im Rahmen des Aufgabe möglichen Zeitintervalle waren auf dem TKP-Aufgabenblatt angegeben. Die Auswahl erfolgte durch das Markieren des jeweiligen Kontrollkästchens. Die Schüler lösten diese Aufgabe im Vergleich zu Aufgabe $2 b$ besser, betrachtet man den Anteil der Probanden, welche zur Lösung der Aufgabe einen, zwei bzw. mehrere Lösungsanläufe benötigten (vgl. Tab. 34).

\begin{tabular}{|l||c|c|}
\hline & $\begin{array}{c}\text { Aufgabe 2b } \\
\text { (50 Schüler) }\end{array}$ & $\begin{array}{c}\text { Aufgabe 2e } \\
\text { (50 Schüler) }\end{array}$ \\
\hline \hline 1 Lösungsversuch & $26 \%(13)$ & $40 \%(20)$ \\
\hline 2 Lösungsversuche & $26 \%(13)$ & $20 \%(10)$ \\
\hline >2 Lösungsversuche & $44 \%(22)$ & $36 \%(18)$ \\
\hline Fehlerhafte Lösung & $4 \%(2)$ & $4 \%(2)$ \\
\hline
\end{tabular}

Tab. 34: Lösungsversuche: Vergleich: Aufgabe 2b - Aufgabe 2e 


\subsubsection{Auswahl der Darstellungen}

Die folgende Tabelle gibt an, wie oft die einzelnen Darstellungsformen Tabelle und Graph von den Schülern gewählt wurden.

\begin{tabular}{|c||c|c|}
\hline & Tabelle & Graph \\
\hline \hline $\begin{array}{c}\text { Aufgabe 2b } \\
\text { (50 Schüler) }\end{array}$ & 171 & 61 \\
\hline $\begin{array}{c}\text { Aufgabe 2e } \\
\text { (50 Schüler) }\end{array}$ & 274 & 177 \\
\hline
\end{tabular}

Tab. 35: Absolute Häufigkeit der betrachteten Darstellungen: Vergleich: Aufgabe 2b - Aufgabe 2e

Wie in Aufgabe $2 \mathrm{~b}$ arbeiteten die Schüler häufiger mit der Tabelle als mit dem Graph, wenngleich es sich um keinen signifikanten Unterschied handelt (Wilcoxon-Test für Paardifferenzen, $p=0,0585)$. Betrachtet man relative Maßzahlen für die Auswahlhäufigkeit des Graphen, stellt man fest, dass der Graph signifikant häufiger betrachtet wurde als noch in Aufgabe $2 b$ (Wilcoxon-Test für Paardifferenzen, $p=0,0168$ ). Ob die Schüler auch tatsächlich häufiger mit dem Graph gearbeitet haben, d. h. ihn tatsächlich zur Lösung der Aufgabenstellung eingesetzt haben, soll im Folgenden analysiert werden.

\subsubsection{Strategien beim Arbeiten mit Darstellungen}

Beim Arbeiten mit den Darstellungen lassen sich - in Anlehnung an das Kategoriensystem in Kapitel 6.2.2.2 - vier Strategien unterscheiden:

\section{Arbeiten mit der Tabelle}

Die Schüler arbeiten ausschließlich mit der Tabelle bzw. das Arbeiten mit dem Graph kann als nicht lösungsrelevant bezeichnet werden. Sofern der Graph ausgewählt wird, ist die Zugriffsdauer nur kurz oder die Auswahl dient lediglich der Kontrolle der getätigten Eingaben.

II. Arbeiten mit dem Graph

Die gesamte Lösung ist hauptsächlich auf das Arbeiten mit dem Graph zurückzuführen. Man erkennt dies zum einen an den Bewegungen des Cursors, der den globalen Verlauf von $D$ bzw. $T$, nachzeichnet"; zum anderen wird unmittelbar im Anschluss an das Arbeiten mit dem Graph zum Lösungsblatt gewechselt und eine Eingabe getätigt. Die Tabelle spielt - sofern sie ausgewählt wird - im Lösungsprozess keine Rolle.

III. Arbeiten mit Graph und Tabelle

Die Aufgabe wird sowohl mit dem Graph als auch mit der Tabelle bearbeitet. Eine Bevorzugung einer der beiden Darstellungen ist nicht erkennbar.

IV. Strategiewechsel Graph => Tabelle

Teile der Aufgabe werden zunächst mit dem Graph gelöst, die restliche Bearbeitung erfolgt mit der Tabelle.

V. $\quad$ Strategiewechsel Tabelle $=>$ Graph

Teile der Aufgabe werden zunächst mit der Tabelle gelöst, die restliche Bearbeitung erfolgt mit dem Graph. 
Die Tabelle 36 zeigt die entsprechende Häufigkeitsverteilung. Wie bei Aufgabe $2 \mathrm{~b}$ werden hier nur die Probanden berücksichtigt, die die Aufgabe erfolgreich bearbeiteten.

\begin{tabular}{|c||c|c|c|c|c|}
\hline & I & II & III & IV & V \\
\hline \hline $\begin{array}{c}\text { Aufgabe 2b } \\
\text { (48 Schüler) }\end{array}$ & $73 \%(35)$ & $6 \%(3)$ & $21 \%(10)$ & $0 \%(0)$ & $0 \%(0)$ \\
\hline $\begin{array}{c}\text { Aufgabe 2e } \\
\text { (48 Schüler) }\end{array}$ & $48 \%(23)$ & $25 \%(12)$ & $8 \%(4)$ & $15 \%(7)$ & $4 \%(2)$ \\
\hline
\end{tabular}

Tab. 36: Strategien beim Arbeiten mit Darstellungen:

Vergleich: Aufgabe 2b - Aufgabe 2e

Wie es sich bereits bei der Analyse der relativen Häufigkeiten der ausgewählten Darstellungen abzeichnete, kommt der graphischen Darstellung in diesem Lösungsprozess im Vergleich zu Aufgabe 2b eine größere Bedeutung zu. Während in Aufgabe 2b 27 \% der Schüler mit dem Graph arbeiteten, wählten diese Darstellung hier - der globalen Fragestellung angemessen $52 \%$ der Probanden (Kategorie II-V). Insgesamt $25 \%$ der Schüler verwendeten zur Lösung der Aufgabe sogar ausschließlich den Graph (Kategorie II). Um feststellen zu können, ob eher mit dem Graph der Folge oder mit dem der Differenzenfolge gearbeitet wurde, werden die Bildschirmaktivitäten, d. h. insbesondere die Bewegung des Cursors der Probanden noch weiter analysiert. Bei dieser zusätzlichen Analyse zeigt sich, dass $60 \%$ der Schüler, die zur Lösung der Aufgabe den Graph hinzuzogen, ausschließlich mit der Folge $T$ arbeiteten, d. h. die Intervalle betrachteten, in denen der Graph von $T$ monoton steigt. Dagegen arbeiteten $20 \%$ dieser Schüler mit dem Graph von $D$ und betrachteten die Koordinatenpunkte, die oberhalb der $x$-Achse liegen. Diese Probanden hatten offensichtlich die globale Beziehung zwischen der Temperaturfolge und deren Differenzenfolge erkannt. Weitere $8 \%$ arbeiteten sowohl mit dem Graph von $T$ als auch von $D$. Die übrigen Schüler zeigten keine Bildschirmaktivitäten beim Betrachten der graphischen Darstellungen, so dass in diesen Fällen keine Aussagen über die von ihnen analysierten Funktionen gemacht werden kann.

Insgesamt $19 \%$ der Probanden änderten im Laufe der Bearbeitung ihre Strategie hinsichtlich der Auswahl von Darstellungen (Kategorie IV und V). Von den sieben Probanden, die von dem Graph zur Tabelle wechselten, hatten vier Probanden Teile der Aufgabe zuvor erfolgreich mit dem Graph bearbeitet. Ein Grund für ihren Strategiewechsel ist daher nicht direkt ersichtlich. Möglicherweise wurde im Laufe der Bearbeitung doch die vertrautere Darstellung Tabelle bevorzugt. Die restlichen drei Probanden sowie die Schüler, die der Kategorie V zugeordnet werden können, änderten ihre Strategie aufgrund von Schwierigkeiten im Umgang mit der erstgewählten Darstellung. Das folgende Fallsbeispiel verdeutlicht das Vorgehen eines dieser Probanden. 


\begin{tabular}{|c|c|c|c|c|c|c|}
\hline Zeit & Aufg. & $\begin{array}{l}\text { Aufg.- } \\
\text { stellung }\end{array}$ & Tabelle & Graph & Kommentar & Eingaben über Tastatur \\
\hline .. & $2 \mathrm{e}$ & & & & & \\
\hline 41.22 & & & $\mathrm{x}$ & & & \\
\hline 41.30 & & $\mathrm{x}$ & & & & 1990-1991 (FEHLER) ${ }^{215}$ \\
\hline 41.35 & & & $\mathrm{x}$ & & & \\
\hline 41.39 & & $\mathrm{x}$ & & & OK (FEHLER) $)^{216}$ & 1992-1993 (FEHLER) \\
\hline 41.52 & & & & $\mathrm{x}$ & & \\
\hline 42.05 & & $\mathrm{x}$ & & & & 1985-1986 \\
\hline 42.13 & & & & $\mathrm{x}$ & Cursor auf $D^{217}$ & \\
\hline 42.26 & & $\mathrm{x}$ & & & $\begin{array}{l}\text { OK (FEHLER) } \\
\text { OK (FEHLER) } \\
\text { OK (FEHLER) }\end{array}$ & $\begin{array}{l}\text { Löschen 1990-1991 } \\
\text { Löschen 1992-1993 } \\
\text { 1991-1992 } \\
\text { 1993-1994 } \\
\text { Löschen 1988-1989 (FEHLER) } \\
\text { 1989-1990 } \\
\text { Löschen 1989-1990 (FEHLER) } \\
\text { 1988-1989 }\end{array}$ \\
\hline 43.09 & & & & $\mathrm{x}$ & Cursor auf $D$ & \\
\hline 43.49 & & $\mathrm{x}$ & & & & $\begin{array}{l}\text { 1989-1990 } \\
\text { Löschen 1986-1987 }\end{array}$ \\
\hline$\ldots$ & & & & & & \\
\hline
\end{tabular}

Tab. 37: Aufgabe 2e - Transkriptausschnitt ${ }^{218}$ (Schüler A11)

Der Schüler A11 wechselte zunächst schrittweise zwischen Tabelle und Lösungsblatt hin- und her und markierte mit jedem Wechsel zum Lösungsblatt nur eines der Kontrollkästchen zu den vorgegebenen Antworten. Die (fehlerhaften) Teillösungen wurden anschließend mit dem „OK-Button“ überprüft. Nach der negativen Rückmeldung des Lernprogramms änderte der Schüler seine Strategie und arbeitete bevorzugt mit dem Graph. Es fällt auf, dass unmittelbar nach der Analyse der Folge $D$ (Cursorbewegung) auf dem Lösungsblatt mehrere Eingaben auf einmal getätigt bzw. korrigiert wurden. Der Schüler hatte offensichtlich durch das Arbeiten mit dem Graph Bereiche monotonen Wachstums besser ,auf einen Blick“ erkennen können als durch das Arbeiten mit der Tabelle. Der Strategiewechsel führte also in diesem Fall zu einem effizienteren und problemadäquateren Arbeiten (wenngleich das weitere Vorgehen des

\footnotetext{
${ }^{215}$ Mit dieser Kurzform wird ausgedrückt, dass der betreffende Proband das Kontrollkästchen zu dem Intervall 1990-1991 markierte. Diese Eingabe ist jedoch falsch, was entsprechend im Transkript vermerkt wurde.

${ }^{216}$ Diese Kurzform kennzeichnet, dass der betreffende Proband die Lösung mit dem „OK-Button“ überprüfte. Da die Aufgabe jedoch noch nicht vollständig bearbeitet war bzw. eine falsche Eingabe getätigt wurde, wies das Programm auf einen Fehler hin.

${ }^{217}$ Die Bezeichnung „Cursor auf $D^{“}$ kennzeichnet, dass der betreffende Proband mit dem Cursor auf die Funktionswerte der Funktion $D$ deutet.

${ }^{218}$ Vgl. ScreenCam-Datei A11a.scm.
} 
Schülers durch das wiederholte Klicken auf den „OK-Button“ auch Kennzeichen eines Versuch-und-Irrtum-Vorgehens aufweist).

\subsection{Ergebnisse zu Aufgabe 3}

Gegenstand der dritten Aufgabe ist die Funktionenklasse der quadratischen Z-Funktionen, d. h. Funktionen des Typs $f(n)=a n^{2}+b n+c(n \in \boldsymbol{Z})$. Da im Rahmen der Versuchsdurchführung technische Probleme mit dem Netzwerk des Hochschulrechenzentrums auftraten, waren einige Schüler der Gruppen A und B gezwungen, die Aufgabenteile 3a-3d ein zweites Mal zu bearbeiten (vgl. Kap. 5.7.3). Dadurch ist ein Vergleich mit den Arbeitsweisen und den Ergebnissen der anderen Schüler bei diesen Aufgaben nur bedingt und an ausgezeichneten Stellen möglich.

\subsubsection{Ergebnisse zu Aufgabe 3a}

Gegenstand dieser Aufgabe war die Z-Funktion $f$ mit $f(n)=n^{2}-2 n+3$. Die Schüler sollten - ähnlich wie in Aufgabe 2a - die Funktion $D$ mit $D(n)=f(n+1)-f(n)$ berechnen und tabellarisch darstellen. Diese Funktion wurde im Folgenden als Differenzenfunktion bezeichnet. In der folgenden Tabelle werden die Probanden hinsichtlich ihrer in beiden Aufgaben benötigten Lösungsanläufe unterschieden. In der dritten Zeile sind dabei nur diejenigen Probanden aufgeführt, die in Aufgabe 3a nicht von Netzwerkproblemen betroffen waren und die Aufgabe daher nur einmal gelöst hatten.

\begin{tabular}{|c||c|c|c|c|}
\hline & $\begin{array}{c}\text { 1 Lösungs- } \\
\text { versuch }\end{array}$ & $\begin{array}{c}\text { 2 Lösungs- } \\
\text { versuche }\end{array}$ & $\begin{array}{c}\text { >2 Lösungs- } \\
\text { versuch }\end{array}$ & $\begin{array}{c}\text { Fehlerhafte } \\
\text { Lösung }\end{array}$ \\
\hline \hline $\begin{array}{c}\text { Aufgabe 2a } \\
\text { (50 Schüler) }\end{array}$ & $60 \%(30)$ & $18 \%(9)$ & $10 \%(5)$ & $12 \%(6)$ \\
\hline $\begin{array}{c}\text { Aufgabe 3a } \\
\text { (52 Schüler) }\end{array}$ & $65 \%(34)$ & $21 \%(11)$ & $8 \%(4)$ & $6 \%(3)$ \\
\hline $\begin{array}{c}\text { Aufgabe 3a } \\
\text { (37 Schüler) }\end{array}$ & $62 \%(23)$ & $27 \%(10)$ & $8 \%(3)$ & $3 \%(1)$ \\
\hline
\end{tabular}

Tab. 38: Lösungsversuche:

Vergleich: Aufgabe 2a - Aufgabe 3a

Berücksichtigt man bei der Analyse der Lösungsanläufe auch diejenigen Schüler, die die Aufgabe $3 \mathrm{a}$ ein zweites Mal bearbeiteten, so stellt man eine geringfügige Verbesserung gegenüber Aufgabe 2a fest. Prozentual mehr Probanden lösten die Aufgabe im ersten bzw. zweiten Lösungsanlauf und weniger Probanden lieferten eine fehlerhafte Lösung. Vergleicht man nur die 37 Schüler, welche bei der Bearbeitung beider Aufgabenteile von Netzwerkproblemen nicht betroffen waren und welche die Aufgaben daher nur ein einziges Mal bearbeiteten, ist ein ähnlicher Lernfortschritt zu beobachten. 
Die meisten der 15 Schülern, welche die Aufgabe ein zweites Mal bearbeiteten, lösten die Aufgabe erwartungsgemäß fehlerfrei und im ersten Lösungsanlauf. Vier Schüler benötigten mehr als zwei Lösungsanläufe. Möglicherweise hatten diese Probanden bereits bei der ersten Bearbeitung Schwierigkeiten bei der Berechnung der Differenzenfunktion. Dieser Vermutung kann aufgrund der fehlenden Aufzeichnungen jedoch nicht weiter nachgegangen werden.

Bei dieser Aufgabe traten wie bereits bei den Aufgaben 1b, 1h und 2a nur bei wenigen Probanden inhaltliche Probleme auf. Auf eine detaillierte Analyse soll daher an dieser Stelle verzichtet werden. Die technischen Schwierigkeiten der Probanden werden im Folgenden im Hinblick auf die im Rahmen der Bearbeitung von Aufgabe 2a beobachteten Fehlermuster analysiert:

I. Vergessen des ,=“-Zeichens;

II. Fehlerhafte Zellbezüge;

III. Vermischen der algebraischen Syntax mit der TKP-Syntax.

Die Tabelle 39 zeigt die Häufigkeitsverteilung über diesen Kategorien. Dabei werden im Rahmen von Aufgabe 3a nur die Schüler berücksichtigt, die diese Aufgabe nur einmal bearbeitet haben. Diese Schüler können wieder mehreren Fehlertypen zugeordnet werden.

\begin{tabular}{|c||c|c|c|}
\hline & I & II & III \\
\hline $\begin{array}{c}\text { Aufgabe 2a } \\
\text { (50 Schüler) }\end{array}$ & $6 \%(3)$ & $10 \%(5)$ & $30 \%(15)$ \\
\hline $\begin{array}{c}\text { Aufgabe 3a } \\
\text { (37 Schüler) }\end{array}$ & $5 \%(2)$ & $14 \%(5)$ & $11 \%(4)$ \\
\hline
\end{tabular}

Tab. 39: Technische Fehlertypen:

Vergleich: Aufgabe 2a - Aufgabe 3a

Gegenüber Aufgabe 2a hatten prozentual deutlich weniger Probanden Schwierigkeiten im Umgang mit der TKP-Syntax und deren Abgrenzung zur „traditionellen“ algebraischen Syntax (Fehlertyp III). Das „,=“-Zeichen wurde - ähnlich wie in Aufgabe 2a - lediglich von etwa $5 \%$ der Probanden nicht beachtet (Fehlertyp I). Dieser Fehler kann als „Flüchtigkeitsfehler“ gewertet werden. Insgesamt $14 \%$ der Probanden verwendeten in Aufgabe 3a zunächst fehlerhafte Zellbezüge (Fehlertyp II). Der Anteil der Probanden, der diesem Fehlertyp zugeordnet werden kann, ist damit zwar etwas höher als in Aufgabe 2a. Es ist jedoch zu berücksichtigen, dass sich das Verwenden fehlerhafter Zellbezüge im Rahmen der Bearbeitung von Aufgabe 3a lediglich durch ein Vertauschen der in Beziehung stehenden Zellen äußerte, ${ }^{219}$ wohingegen bei Aufgabe 2a auch technisch „gravierendere“ Fehler wie „Zirkelschlüsse“ auftraten.

\footnotetext{
${ }^{219}$ Anstelle der Formel ,,=C8-C7“ wurde die Formel ,=C7-C8“ eingegeben.
} 


\subsubsection{Ergebnisse zu Aufgabe 3b}

Bei dieser Aufgabe, die in ihrer Struktur Aufgabe 2b ähnelt, sollte das Änderungsverhalten der Funktion $f$ mit $f(n)=n^{2}-4 n-13$ beim Übergang von $n=5$ nach $n=6$ mit Hilfe der Differenzenfunktion analysiert werden. Die Schüler konnten hierbei zwischen den Darstellungen Tabelle und Graph wählen. Die Analyse der Arbeitsweisen der 15 Schüler, die von Netzwerkproblemen betroffen waren und die Aufgabe ein zweites Mal bearbeiten mussten, ist im Rahmen dieser Aufgabe nicht sinnvoll, da insbesondere die Auswahl und das Arbeiten mit den beiden Darstellungen das ursprüngliche Vorgehen der Probanden nur unzureichend widerspiegeln. Diese Schüler werden daher hier nicht berücksichtigt.

Anhand der folgenden Tabelle erkennt man, dass die Aufgabe im Vergleich zu Aufgabe 2b deutlich besser gelöst wurde. Insbesondere haben $63 \%$ der Schüler die Aufgabe bereits im ersten Lösungsanlauf fehlerfrei gelöst.

\begin{tabular}{|c||c|c|c|c|}
\hline & $\begin{array}{c}\text { 1 Lösungs- } \\
\text { versuch }\end{array}$ & $\begin{array}{c}\text { 2 Lösungs- } \\
\text { versuche }\end{array}$ & $\begin{array}{c}\text { >2 Lösungs- } \\
\text { versuch }\end{array}$ & $\begin{array}{c}\text { Fehlerhafte } \\
\text { Lösung }\end{array}$ \\
\hline \hline $\begin{array}{c}\text { Aufgabe } 2 b \\
\text { (50 Schüler) }\end{array}$ & $26 \%(13)$ & $26 \%(13)$ & $44 \%(22)$ & $4 \%(2)$ \\
\hline $\begin{array}{c}\text { Aufgabe 3b } \\
\text { (38 Schüler) }\end{array}$ & $63 \%(24)$ & $21 \%(8)$ & $16 \%(6)$ & $0 \%(0)$ \\
\hline
\end{tabular}

Tab. 40: Lösungsversuche:

Vergleich: Aufgabe 2b - Aufgabe 3b

\subsubsection{Auswahl der Darstellungen}

Die folgende Tabelle gibt die absolute Häufigkeit der von den Schülern betrachteten Darstellungen in den Aufgaben 2b bzw. 3b wieder. ${ }^{220}$

\begin{tabular}{|c||c|c|}
\hline & Tabelle & Graph \\
\hline \hline $\begin{array}{l}\text { Schüler 2b } \\
\text { (36 Schüler) }\end{array}$ & 134 & 46 \\
\hline $\begin{array}{l}\text { Schüler 3b } \\
\text { (36 Schüler) }\end{array}$ & 65 & 33 \\
\hline
\end{tabular}

Tab. 41 Absolute Häufigkeit der betrachteten Darstellungen: Vergleich: Aufgabe 2b - Aufgabe 3b

Verglichen mit Aufgabe $2 b$ betrachteten die Schüler im Rahmen des Lösungsprozesses zu Aufgabe $3 \mathrm{~b}$ signifikant weniger Darstellungen (Wilcoxon-Test für Paardifferenzen, $p<0,001$ ). Während sie in Aufgabe $2 \mathrm{~b}$ im Mittel 5 Darstellungen (Standardabweichung 3,3) auswählten, wurden bei dieser Aufgabe nur durchschnittlich 2,7 Darstellungen (Standardabweichung 1,6) betrachtet. Dieses Vorgehen weist darauf hin, dass die Probanden das neue

\footnotetext{
${ }^{220}$ In den Vergleich werden nur die 36 Schüler einbezogen, die beide Aufgabenteile bearbeitet haben und nicht von Netzwerkproblemen betroffen waren.
} 
Werkzeug und insbesondere die Darstellungen im Laufe der Bearbeitungszeit reflektierter und zielgerichteter einsetzten. Dabei wurde wie in Aufgabe 2b die Tabelle tendenziell häufiger gewählt als der Graph, wobei es sich auch bei dieser Aufgabe um einen signifikanten Unterschied handelt (Wilcoxon-Test für Paardifferenzen, $p=0,0016$ ).

Auf einen Vergleich der relativen Maßzahlen für die Auswahlhäufigkeit der Tabelle wird an dieser Stelle verzichtet. Stattdessen konzentrieren wir uns im Folgenden auf die Analyse der (aussagekräftigeren) lösungsrelevanten Hauptstrategien im Umgang mit den Darstellungen.

\subsubsection{Strategien beim Arbeiten mit Darstellungen}

Klassifiziert man die Arbeitsweisen der Schüler, die die Aufgabe erfolgreich berabeiteten, nach den lösungsrelevanten Hauptstrategien im Umgang mit den Darstellungen, lassen sich wie in Aufgabe $2 b$ folgende Vorgehensweisen unterscheiden:

I. Arbeiten mit der Tabelle;

II. Arbeiten mit dem Graph;

III. Arbeiten mit Tabelle und Graph.

Mit Einschränkungen ${ }^{221}$ können bei Kategorie III wie bei Aufgabe $2 \mathrm{~b}$ folgende Unterkategorien unterschieden werden:

- Analyse des Graphenverlaufs;

- Analyse von Koordinatenpunkten.

In der folgenden Tabelle sind die Häufigkeitsverteilungen über diesen Kategorien aufgeführt (Aufgabe $2 b$ und $3 b$ im Vergleich).

\begin{tabular}{|c||c|c|c|}
\hline & I & II & III \\
\hline \hline $\begin{array}{c}\text { Aufgabe 2b } \\
\text { (48 Schüler) }\end{array}$ & $73 \%(35)$ & $6 \%(3)$ & $21 \%(10)$ \\
\hline $\begin{array}{c}\text { Aufgabe 3b } \\
\text { (38 Schüler) }\end{array}$ & $74 \%(28)$ & $5 \%(2)$ & $21 \%(8)$ \\
\hline
\end{tabular}

Tab. 42: Strategien beim Arbeiten mit Darstellungen: Vergleich Aufgabe 2b - Aufgabe 3b

Die im Rahmen dieser Aufgabe beobachteten Strategien der Schüler im Umgang mit den Darstellungen differieren kaum von den Strategien bei der Bearbeitung von Aufgabe 2b. Insgesamt $74 \%$ der Probanden verwendeten ausschließlich die Tabelle (Kategorie I). Zwei Schüler (5\%) arbeiteten mit dem Graph (Kategorie II). Einer dieser beiden Probanden analysierte sowohl die Funktion als auch deren Differenzenfunktion. Dagegen visualisierte der andere Schüler mit Hilfe des Cursors lediglich die Funktionswerte $f(5)$ und $f(6)$, berechnete deren

\footnotetext{
${ }^{221}$ In zwei Ausnahmefällen ist eine Zuordnung zu einer dieser beiden Unterkategorien nicht möglich, da bei den entsprechenden Probanden keine Bildschirmaktivitäten feststellbar sind.
} 
Differenz und berücksichtigte damit nicht, dass derselbe Wert anhand des Funktionswerts $D(5)$,einfacher“ bestimmt werden kann. Acht Probanden (21\%) betrachteten den Graph und die Tabelle (Kategorie III). Dabei untersuchten zwei Schüler ${ }^{222}$, das Änderungsverhalten des Graphen von $f$ und beschrieben die Funktion im Anschluss (korrekterweise) als „monoton fallend“ im „Intervall“ $[5,6]$. Sechs Probanden ${ }^{223}$, die sowohl mit der Tabelle als auch mit dem Graph arbeiteten, verwendeten den Graph, indem sie - zum Bestimmen der Funktionswerte $f(5)$ und $f(6)$ bzw. $D(5)$ und $D(6)$ - mit dem Cursor auf die entsprechenden Koordinatenpunkte deuteten. Drei dieser Probanden beschränkten sich auf das Ablesen der Werte $f(5)$ und $f(6)$. Ein anderer Proband analysierte alle drei in Beziehung stehenden Funktionswerte. Während dieser Schüler den lokalen Zusammenhang zwischen den Funktionen $f$ und $D$ offensichtlich erkannt hatte, verloren zwei weitere Schüler die eigentliche Fragestellung aus den Augen und analysierten anstelle des Änderungsverhaltens der Funktion das Änderungsverhalten der Differenzenfunktion (vgl. auch Kap. 6.3.2.3).

\subsubsection{Inhaltliche Probleme}

Die Fehler der Probanden lassen sich in ein gegenüber Aufgabe 2b leicht modifiziertes Kategoriesystem einordnen. Wir betrachten dazu die folgenden Fehlertypen:

I. Annahme einer falschen Beziehung zwischen $f$ und $D$

Es wird mit der Beziehung $D(n)=f(n)-f(n-1)$ operiert,

d. h. es wird der Wert $D(6)=9$ anstelle des Werts $D(5)=7$ abgelesen.

II. Fehler beim relativen Lesen der Darstellung

a) Angabe eines Funktionswerts von $f$.

b) Angabe der Änderung von $D$.

\section{Fehlerhafte inhaltliche Deutung}

Die Funktion $f$ wird als „fallend“ beschrieben.

Die Probanden werden nun den entsprechenden Kategorien zugewiesen, wobei ein Proband auch hinsichtlich verschiedener Fehlertypen berücksichtigt werden kann (vgl. Tab. 43). Die Fehler von vier Schülern lassen sich nicht in das Kategoriensystem einordnen. Diese Schüler werden wie in Aufgabe 2b im Rahmen einer weiteren Kategorie IV berücksichtigt.

\footnotetext{
${ }^{222}$ Diese Probanden lassen sich der ersten Unterkategorie von Kategorie III zuordnen.

${ }^{223}$ Diese Probanden lassen sich der zweiten Unterkategorie III zuordnen. Es werden hierbei auch die beiden Probanden einbezogen, die bereits der ersten Unterkategorie III zugeordnet werden konnten.
} 


\begin{tabular}{|c||c|c|c|c|c|}
\hline & I & IIa & Ilb & III & IV \\
\hline $\begin{array}{c}\text { Aufgabe 2b } \\
\text { 50 Schüler) }\end{array}$ & $34 \%(17)$ & $26 \%(13)$ & $0 \%(0)$ & $38 \%(19)$ & $12 \%(6)$ \\
\hline $\begin{array}{c}\text { Aufgabe 3b } \\
\text { (38 Schüler) }\end{array}$ & $0 \%(0)$ & $0 \%(0)$ & $24 \%(9)$ & $13 \%(5)$ & $11 \%(4)$ \\
\hline
\end{tabular}

Tab. 43: Inhaltliche Fehlertypen:

Vergleich: Aufgabe 2b - Aufgabe 3b

Überraschenderweise bereitete den Schülern im Rahmen dieser Aufgabe der Umgang mit der formalen Begriffsbestimmung der Differenzenfolge keine Schwierigkeiten. So lässt sich kein Fehler auf ein Operieren mit der (falschen) Beziehung $D(n)=f(n)-f(n-1)$ zurückführen (Fehlertyp I). Dies kann als Lernfortschritt gewertet werden, wobei jedoch berücksichtigt werden muss, dass sicher auch die „,schönen“ ganzen Zahlen das Ablesen des (korrekten) Änderungswerts erleichtert haben. Mit der Interpretation des positiven Werts $D(5)=7$ als Kennzeichen monotonen Wachstums hatten erwartungsgemäß prozentual weniger Probanden Schwierigkeiten als bei der inhaltlichen Deutung des entsprechenden negativen Werts in Aufgabe $2 \mathrm{~b}$ (Fehletyp III). Die Fehler von $24 \%$ der Probanden, die der Kategorie IIb zugeordnet werden können, lassen sich darauf zurückführen, dass der Aufgabentext nicht richtig gelesen wurde bzw. die falsche Darstellung betrachtet wurde. Diese Probanden analysierten nicht das Änderungsverhalten der Funktion $f$, sondern das der Differenzenfunktion $D$ beim Übergang von $n=5$ nach $n=6$ und gaben den Änderungswert $2=D(6)-D(5)$ an. Dies soll im Folgenden an einem Beispiel illustriert werden.

\begin{tabular}{|c|c|c|c|c|c|c|}
\hline Zeit & Aug. & $\begin{array}{l}\text { Aufg.- } \\
\text { stellung }\end{array}$ & Tabelle & Graph & Kommentar & $\begin{array}{l}\text { Eingaben über } \\
\text { Tastatur }\end{array}$ \\
\hline 6.29 & $3 b$ & $\mathrm{x}$ & & & & \\
\hline 6.31 & & & $\mathrm{x}$ & & $\begin{array}{l}\text { Cursor auf Intervall }[5,6] \text { und auf } \\
\text { Funktionswert } D(5)\end{array}$ & \\
\hline 6.38 & & $\mathrm{x}$ & & & & \\
\hline 6.52 & & & & $\mathrm{x}$ & Cursor auf $D(5), D(6)$ & \\
\hline 7.05 & & $\mathrm{x}$ & & & OK (FEHLER) & $\begin{array}{l}\text { Änderung } 2 \\
\text { „steigt“" }\end{array}$ \\
\hline 7.20 & & & $\mathrm{x}$ & & & \\
\hline 7.27 & & $\mathrm{x}$ & & & & Änderung 7 \\
\hline 7.32 & & & $\mathrm{x}$ & & $\begin{array}{l}\text { Cursor auf } \\
D(5), D(4), D(3) \\
\end{array}$ & \\
\hline 7.37 & & $\mathrm{x}$ & & & OK & \\
\hline
\end{tabular}

Tabelle 44: Aufgabe 3b - Transkriptausschnitt ${ }^{24}$

(Schüler A4)

Anhand dieses Transkriptausschnitts wird deutlich, dass der betreffende Schüler zunächst mit der Tabelle arbeitete und offensichtlich auch den lösungsrelevanten Wert $D(5)$ in der Tabelle

\footnotetext{
${ }^{224}$ Vgl. die ScreenCam-Datei A4.scm.
} 
erkannte (der Cursor deutete auf die Zelleninhalte $n=5$ und $n=6$ sowie auf den Funktionswert $D(5))$. Anstatt die Lösung auf dem Lösungsblatt zu notieren, wechselte der Proband jedoch zunächst zum Graph. Die Cursorbewegungen weisen darauf hin, dass der Schüler die Koordinatenpunkte $(5, D(5))$ und $(6, D(6))$ und damit das Änderungsverhalten von $D$ analysierte. Dafür spricht auch die anschließende Eintragung auf dem Lösungsblatt: Es wurde der Wert 2 eingegeben sowie das Kontrollkästchen zu „steigt“ markiert. Aufgrund der negativen Rückmeldung im Rahmen der Lösungsüberprüfung mit dem „OK-Button“ korrigierte der Proband anschließend sein Ergebnis. Dabei verwendete er jedoch wieder die tabellarische Darstellung.

Da dieser spezielle Fehlertyp im Rahmen von Aufgabe $2 b$ nicht auftrat, kann angenommen werden, dass die Aufmerksamkeit der Schüler, die der Kategorie Ilb zugeordnet werden können, durch die „schöne“ Eigenschaft „Linearität“ bzw. „konstante Änderung(srate)“ auf das Änderungsverhalten von $D$ gelenkt wurde. Dagegen war offensichtlich keine der beiden empirischen Funktionen $T$ und $D$ in Aufgabe $2 \mathrm{~b}$ unter rein visuellen Gesichtspunkten ,,ausgezeichnet“. Möglicherweise wurde hier ein derartiger Fehler zudem durch die Interpretation in dem situativen Kontext „Jahresdurchschnittstemperaturen“ verhindert.

\subsubsection{Ergebnisse zu Aufgabe 3c}

Gegenstand dieser „Interpolationsaufgabe“, die in ihrer Struktur den Aufgaben 2c und 2d ähnelt, ist die quadratische Z-Funktion $f(n)=-1,1 n^{2}-4,7 n+20$ und deren Differenzenfunktion. Die Schüler sollten hierbei die nicht in der tabellarischen Darstellung aufgeführten Funktionswerte $f(-4)=21,2$ und $D(2)=-10,2$ durch Verknüpfen geeigneter „,benachbarter“ Funktionswerte bestimmen. Die mit der Tabelle interaktiv verbundene graphische Darstellung dient im Rahmen dieser Aufgabe zur visuellen Kontrolle der beiden gesuchten Funktionswerte.

Aufgabe 3c ist kein Bestandteil des Versuchsprogramms der Gruppe C, so dass lediglich die Schülergruppen A und B in die folgende Auswertung einbezogen werden. Da über die Arbeitsweisen und inhaltlichen Schwierigkeiten der Probanden, welche die Aufgabe mehrfach bearbeitet haben, keine Aussagen gemacht werden kann, werden weiterhin nur die Schüler berücksichtigt, die nicht von Netzwerkproblemen betroffen waren.

\subsubsection{Arbeiten mit der Tabelle}

Im Vergleich zu Aufgabe 2d, die ausschließlich mit Hilfe des Graphen bearbeitet werden sollte, lösten die Schüler dieses tabellarisch dargestellte Interpolationsproblem tendenziell besser (vgl. Tab. 45). Während in Aufgabe 2d insgesamt $16 \%$ bzw. $18 \%$ der Probanden die beiden Teilaufgaben im ersten Lösungsversuch lösten, hatten bei der Bearbeitung dieser Aufgabe $52 \%$ der Probanden hinsichtlich der Berechnung von $f(-4)$ und $38 \%$ der Probanden hin- 
sichtlich der Berechnung von $D(2)$ keinerlei Schwierigkeiten. Dies bestätigt die in 6.2.4.2 geäußerte Vermutung, dass Schüler eine lokale Beziehung zwischen Folge und Differenzenfolge anhand der Tabelle (zunächst) besser erkennen als anhand des Graphen, dass also die Tabelle für die Probanden intuitiv zugänglicher zu sein scheint.

\begin{tabular}{|l|c|c||c|c|}
\hline \multirow{2}{*}{} & \multicolumn{2}{|c||}{$\begin{array}{c}\text { Aufgabe } 2 \mathrm{~d} \\
\text { (50 Schüler) }\end{array}$} & \multicolumn{2}{c|}{$\begin{array}{c}\text { Aufgabe 3c } \\
\text { (21 Schüler) }\end{array}$} \\
\cline { 2 - 5 } & $T(1992)$ & $D(1994)$ & $f(-4)$ & $D(2)$ \\
\hline \hline \multirow{2}{*}{ 1 Lösungsversuch } & $16 \%(8)$ & $18 \%(9)$ & $52 \%(11)$ & $38 \%(8)$ \\
\hline 2 Lösungsversuche & $32 \%(16)$ & $24 \%(12)$ & $24 \%(5)$ & $33 \%(7)$ \\
\hline >2 Lösungsversuche & $42 \%(21)$ & $48 \%(24)$ & $14 \%(3)$ & $19 \%(4)$ \\
\hline Falsche Lösung & $10 \%(5)$ & $10 \%(5)$ & $0 \%(0)$ & $0 \%(0)$ \\
\hline Keine Bearbeitung & $0 \%(0)$ & $0 \%(0)$ & $10 \%(2)$ & $10 \%(2)$ \\
\hline
\end{tabular}

Tab. 45: Lösungsversuche:

Vergleich: Aufgabe 2d - Aufgabe 3c

Im Unterschied zu der auch auf der tabellarischen Ebene dargestellten Interpolationsaufgabe $2 c$ traten jedoch bei der Bearbeitung der mit dieser Aufgabe strukturell vergleichbaren ersten Teilaufgabe von 3c, d. h. beim Bestimmen des Werts $f(-4)$ größere Schwierigkeiten auf. Dies kann auf die unterschiedliche Positionierung der „Interpolationslücken“ in der tabellarischen Darstellung zurückgeführt werden. Während der $\mathrm{zu}$ berechnende Funktionswert $T(1989)$ in Aufgabe 2c in eine Zelle am Ende der Wertetabelle eingetragen werden sollte, ist die „Position“ der Funktionswerte $f(-4)$ und $D(2)$ im Rahmen dieser Aufgabe ,innerhalb“ der vorgegebenen Tabelle. Damit ist es hier schwieriger als bei Aufgabe 2c die adäquaten, zu verknüpfenden Funktionswerte zu erkennen.

\subsubsection{Inhaltliche Probleme}

Bei der Analyse der Fehler der Schüler inhaltlicher Art lassen sich die folgenden Kategorien unterscheiden:

\section{Fehlerhaftes Berechnen des Werts $f(-4)$}

I. Die lokale Beziehung zwischen $f$ und $D$ wird nicht erkannt. Als Werte für $f(-4)$ werden z. B. angegeben: $27,2=f(-3)+D(-4)$ oder $10,8=f(-5)-D(-5)$.

II. Es wird mit Hilfe des Graphen ein „Näherungswert“ bestimmt.

Fehlerhaftes Berechnen des Werts D(2)

I. Die lokale Beziehung zwischen $T$ und $D$ wird nicht erkannt. Als Werte für $D(2)$ werden z. B. angegeben: $2,2=f(3)+f(2)$ oder $6,2=T(2)$.

Ib Die Beziehung $D(n)=T(n)-T(n+1)$ liegt der Berechnung zugrunde, d. h. es wird die Reihenfolge der zu verknüpfenden Folgenglieder vertauscht.

II. Es wird mit Hilfe des Graphen ein „Näherungswert“ bestimmt. 
Man erhält die folgende Häufigkeitsverteilung:

\begin{tabular}{|c||c|c|}
\hline Schüler (21) & $f(f-4)$ & $D(2)$ \\
\hline \hline I & $24 \%(5)$ & $19 \%(4)$ \\
\hline Ib & --- & $24 \%(5)$ \\
\hline II & $19 \%(4)$ & $14 \%(3)$ \\
\hline
\end{tabular}

Tab. 46: Aufgabe 3c - Inhaltliche Fehlertypen

Das Erkennen der lokalen Beziehung zwischen $f$ und $D$ bereitete den Probanden in Verbindung mit der Berechnung des Werts $D(2)$ erwartungsgemäß weniger Schwierigkeiten (wenngleich der Unterschied sehr gering ist) als bei der Berechnung des Werts $f(-4)$. Überraschenderweise traten bei keinem der Probanden Fehler auf, die sich auf das Verwenden der Formel $D(n)=f(n)-f(n-1)$ zurückführen lassen. Statt dessen zeugen die angegebenen Werte wie etwa 27,2; 10,8; 2,2 (Fehlertyp I) davon, dass die inhaltliche Bedeutung der Berechnungen und der entsprechenden numerischen Beziehungen nicht ausreichend reflektiert, sondern Werte in der „Umgebung“ der beiden gesuchten Funktionswerte scheinbar willkürlich miteinander verknüpft wurden. Problematisch erwies sich in Verbindung mit der Berechnung von $D(2)$ darüber hinaus - wie bereits bei Aufgabe $2 \mathrm{~d}$ - der korrekte Umgang mit dem Vorzeichen bzw. das Beachten der Reihenfolge der zu verknüpfenden Funktionswerte (Fehlertyp $\mathrm{Ib}$ ). So berechneten $24 \%$ der Probanden anstelle der ,unkomfortablen“ Differenz $D(2)=f(3)-f(2)=-4-6,2$ die Summe $4+6,2 \quad$ (bzw. die Differenz $f(2)-f(3)=6,2-(-4))$, berücksichtigten beim Notieren des Ergebnisses jedoch nicht, dass das negative Vorzeichen ergänzt werden musste.

Eine weitere Fehlerquelle stellte überraschenderweise die graphische Darstellung der Z-Funktion $f$ und deren Differenzenfunktion dar (Fehlertyp II). Obwohl er als Instrument für eine visuelle Lösungskontrolle vorgesehen war, wurde der Graph von $f$ von $19 \%$ und der Graph von $D$ von $14 \%$ der Schüler in den Lösungsprozess miteinbezogen. Dabei analysierten die Probanden - wie anhand ihrer Cursorbewegungen deutlich wird - zunächst die ,ungefähre“ Lage der in beiden graphischen Darstellungen fehlenden Koordinatenpunkte. Auf der Basis dieser Beobachtungen wurden anschließend die Ergebnisse $f(-4)=21$ und $D(2)=-10$ in der Tabelle notiert. Indem diese Schüler die Funktionen $f$ und $D$ isoliert betrachteten und die fehlenden Funktionswerte lediglich ,per Augenmaß“ interpolierten, umgingen sie die eigentliche Aufgabenstellung. Für die Behandlung derartiger Interpolationsaufgaben im Rahmen von TKP-Lernumgebungen erscheint es somit geboten, die Funktion graphischer Kontrollinstrumente deutlicher hervorzuheben oder ganz auf zusätzliche Darstellungen zu verzichten. 


\subsubsection{Ergebnisse zu Aufgabe 3d}

Im Rahmen dieser Aufgabe sollte die Funktionenklasse $f(n)=2 n^{2}-8 n+c$ untersucht und der Einfluss des Parameters $c$ auf die entsprechenden Differenzenfunktionen beschrieben und begründet werden. $\mathrm{Zu}$ Analysezwecken konnten die Probanden zwischen den Darstellungen Tabelle und Graph wählen und den Parameter $c$ mit Hilfe eines Rollbalkens variieren. Die Aufgabe wurde sowohl von den Schülern als auch von den Studierenden bearbeitet und soll insbesondere Aufschluss über den Einfluss unterschiedlicher Vorkenntnisse auf die Argumentationen beider Gruppen geben. Im Folgenden werden zunächst die verbalen Beschreibungen der Probanden analysiert. Hierbei werden auch diejenigen Schüler berücksichtigt, welche die Aufgabe ein zweites Mal bearbeitet haben. Es ist davon auszugehen, dass sich ihre Argumentationen gegenüber der ersten Bearbeitung dieser Aufgabe nicht geändert haben. Aussagen über die Art und Weise ihres Umgangs mit den Darstellungen Graph und Tabelle sind jedoch nur bedingt möglich. Daher werden diese Probanden im Rahmen der darauf folgenden Analysen der Arbeitsweisen nicht berücksichtigt.

\subsubsection{Verbale Beschreibung der Differenzenfunktion}

Die verbalen Beschreibungen der Schüler und Studierenden lassen sich danach unterscheiden, ob sich Beziehungen zu einer bestimmten Darstellung erkennen lassen oder ob darstellungsunabhängig bzw. formal argumentiert wird. Dabei werden zunächst alle Antworten berücksichtigt, unabhängig davon, ob eine Beziehung zwischen Funktion und Differenzenfunktion hergestellt oder ob nur eine der beiden Funktionen isoliert betrachtet wird. Insbesondere werden auch solche Beschreibungen einbezogen, die sich ausschließlich (und entgegen der Aufgabenstellung) auf die Funktion $f$ beziehen.

\section{A Darstellungsabhängige Beschreibungen (Graph)}

Das $c$ verschiebt nur die Parabel. Sie verändert sie nicht. Die Differenz zwischen den Punkten bleibt deshalb gleich und die Differenzenfunktion kann sich nicht ändern.

Die Parabel wird nur in ihrer Position, nicht in ihrer Form verändert.

Es verändert sich nur der Achsenabschnitt, nicht die Form des Graphen.

$D$ bewegt sich auf der $y$-Achse im positiven und negativen Bereich, auf der $x$-Achse hat sie einen festen Punkt.

$D$ gibt den Schnittpunkt mit der $y$-Achse an.

- Graph von $D$ ist konstant.

\section{B Darstellungsabhängig Beschreibungen (Tabelle)}

Der Wert $c$ erhöht bzw. senkt den Wert von $f$ in der Tabelle. Je größer $c$, desto höher der $y$-Wert.

\section{Darstellungsunabhängig Beschreibungen}

Wenn man den Parameter $c$ verändert, bleibt die Differenz trotzdem dieselbe.

Die Differenz $f(n+1)-f(n)$ ändert sich nicht.

Es ist egal, wie groß oder klein $c$ ist, da $D$ nur von $n$ abhängt. 
Wenn man $c$ verändert, verändern sich auch alle $f(n)$-Werte.

Der Parameter ändert sich nicht.

Die Tabelle 47 zeigt die Häufigkeitsverteilung über den vorstehend aufgeführten Kategorien. Bis auf einen Schüler, der die Aufgabe nicht bearbeitet hat, werden alle Probanden in genau einer Kategorie berücksichtigt.

\begin{tabular}{|l||c|c|c|}
\hline & A & B & C \\
\hline Schüler (52) & $85 \%(44)$ & $2 \%(1)$ & $13 \%(7)$ \\
\hline Studierende (21) & $52 \%(11)$ & $0 \%(0)$ & $48 \%(10)$ \\
\hline
\end{tabular}

Tab. 47: Aufgabe 3d - Verbale Beschreibungen

Die Schüler argumentierten signifikant häufiger mit Bezug auf den Graph als die Studierenden (Chiquadrat-Vierfeldertest, $p=0,002) .{ }^{225}$ So beschrieben $85 \%$ der Schüler aber nur $52 \%$ der Studierenden Eigenschaften der graphischen Darstellungen von $f$ bzw. $D$ oder verwendeten im Rahmen ihrer Argumentationen geometrische Begriffe wie etwa „Form des Graphen“ oder „Parabel“ (Kategorie A). Insgesamt 48 \% der Studierenden sahen offensichtlich keine Notwendigkeit darin, sich in ihren Beschreibungen auf die inhaltlich-anschauliche Ebene des Graphen zu beziehen, und argumentierten formal bzw. darstellungsunabhängig (Kategorie C). Dies kann auf ihre stark formal geprägte Mathematikausbildung zurückgeführt werden, deutet aber auch - insbesondere bei den Probanden, die auch eine adäquate Begründung angaben (vgl. Kap. 6.3.4.2) - auf ein „höheres“ mathematisches Begriffsverständnis hin. Bei einer detaillierteren Analyse der verbalen Beschreibungen fällt auf, dass $58 \%$ der Schüler (und auch $28 \%$ der Studierenden) entgegen der Fragestellung ausschließlich das Verhalten der Z-Funktion $f$ analysierten. Offensichtlich wurde ihre Aufmerksamkeit im Umgang mit den dynamisierten Funktionen stärker auf die tatsächlich „,bewegten“ Objekte und auf veränderliche Aspekte wie den sich ändernden Scheitelpunkt und weniger auf die „statische Darstellung“ der Differenzenfunktion gelenkt.

\subsubsection{Begründung der Eigenschaften der Differenzenfunktion}

Wenngleich sich fast alle Schüler zum Teil sehr ausgiebig mit den einzelnen Darstellungen befassten und den Einfluss des Parameters $c$ analysiert (vgl. hierzu auch Kap. 6.3.4.3) sowie ihre Beobachtungen verbal formulierten, konnten doch nur $34 \%$ eine angemessene Begründung für die Invarianz der Differenzenfunktion angeben (vgl. Tab. 48). Die Studierenden haben diese Aufgabe signifikant besser gelöst (Chiquadrat-Vierfeldertest, $p=0,01$ ).

\footnotetext{
${ }^{225}$ Es werden hierbei nur die Hauptkategorien A und C berücksichtigt.
} 


\begin{tabular}{|l||c|c|}
\hline & Begründung & Keine (adäquate) Begründung \\
\hline \hline Schüler (53) & $34 \%(18)$ & $66 \%(35)$ \\
\hline Studierende (21) & $67 \%(14)$ & $33 \%(7)$ \\
\hline
\end{tabular}

Tab. 48: Aufgabe 3d - Qualität der verbalen Begründungen

Bei den Begründungsansätze der Probanden lassen sich darstellungsabhängige und darstellungsunabhängige Sichtweisen unterscheiden. Im Folgenden sind einige prototypische Argumentationen aufgeführt.

\section{A Darstellungsabhängige Argumentationen (Graph)}

- Die Differenzenfunktion gibt die Steigung der Funktion $f$ an. Da sich bei einer Änderung des Parameters $c$ die Steigung der Funktion nicht ändert, ändert sich $D$ nicht.

- Das $c$ verschiebt nur die Parabel. Sie verändert sie nicht. Die Differenz zwischen den Punkten bleibt deshalb gleich und die Differenzenfunktion kann sich nicht ändern.

- Man verschiebt die Parabel nur und so entstehen keine Unterschiede bei dem Wert $n$.

- $\quad c$ verschiebt die Parabel nur nach oben, verändert aber nicht das Verhältnis der Werte zueinander.

- Jeder einzelne Funktionswert wird um den gleichen Abstand verschoben, was die Differenz natürlich gleich bleiben lässt.

\section{B Darstellungsunabhängige Argumentationen}

- $\quad D$ verändert sich nicht, da für $f(n+1)$ und $f(n)$ der Wert $c$ jeweils gleich ist.

- $\quad D$ beschreibt die Differenz $D(n)=f(n+1)-f(n)$, und da $c$ in $f(n+1)$ und $f(n)$ genauso groß ist, fällt das $c$ weg.

- $\quad$ Zu jedem Funktionswert wird der gleiche Wert $c$ addiert bzw. subtrahiert. Also bleibt die Differenz gleich.

Man erhält die folgende Häufigkeitsverteilung:

\begin{tabular}{|l||c|c|}
\hline \multicolumn{1}{|c||}{} & A & B \\
\hline \hline Schüler (18) & $94 \%(17)$ & $6 \%(1)$ \\
\hline Studierende (14) & $36 \%(5)$ & $64 \%(9)$ \\
\hline
\end{tabular}

Tab. 49: Aufgabe 3d - Argumentationsmuster bei adäquaten Begründungen

Während die Schüler hauptsächlich geometrisch mit Bezug auf die graphischen Darstellungen der Funktion und deren Differenzenfunktion argumentierten, begründeten die Studierenden die Invarianz von $D$ signifikant häufiger darstellungsunabhängig bzw. formal (ChiquadratVierfeldertest, $p=0,001)$. Bei einer genaueren Analyse der geometrischen Argumentationen fällt auf, dass nur zwei Schüler und ein Studierender den kontinuierlichen Begriff „Steigung“ verwendet haben. Die meisten Probanden benutzten dagegen Begriffe wie „Abstand“, „Differenz“ oder „Verhältnis“, die auf eine diskrete Sicht auf die Eigenschaften der untersuchten Funktionen hindeuten. Berücksichtigt man, dass die Schüler bis zu diesem Zeitpunkt noch keine Kenntnisse der Differenzialrechnung erworben hatten, überrascht diese Beobachtung 
nicht. Andererseits hatten offensichtlich auch umfassendere Vorkenntnisse, wie sie den Studierenden zugesprochen werden können, keinen Einfluss auf eine stärker von „kontinuierlichen“ analytischen Begriffen geprägte Sicht auf die Funktionseigenschaften. Dies lässt jedoch nicht den Schluss zu, dass die Studierenden Analogien zwischen den Begriffen Differenzenfunktion und Ableitungsfunktion nicht erkannt haben, sondern spricht vielmehr für die steuernde Wirkung der diskreten (graphischen) Darstellung.

\subsubsection{Arbeiten mit Darstellungen}

Die folgende Tabelle zeigt, wie oft die einzelnen Darstellungsformen Tabelle und Graph von den Schülern bzw. den Studierenden gewählt wurden. ${ }^{226}$

\begin{tabular}{|l||c|c|}
\hline & Tabelle & Graph \\
\hline \hline Schüler (36) & 39 & 65 \\
\hline Studierende (21) & 30 & 32 \\
\hline
\end{tabular}

Tab. 50 Aufgabe 3d - Absolute Häufigkeit der betrachteten Darstellungen

Hinsichtlich der Anzahl der ausgewählten Darstellungen sind keine signifikanten Unterschiede zwischen beiden Probandengruppen feststellbar (Wilcoxon-Test, $p=0,6523$ ). Im Mittel betrachteten die Schüler 2,9 Darstellungen (Standardabweichung 1,9) und die Studierenden 3 Darstellungen (Standardabweichung 1,6). Die Schüler arbeiteten dabei signifikant häufiger mit dem Graph als mit der Tabelle (Wilcoxon-Test für Paardifferenzen, $p=0,0019$ ), wohingegen sich aus der absoluten Häufigkeit der von den Studierenden betrachteten Darstellungen zunächst auf keine (siginfikant) bevorzugte Darstellungsart schließen lässt (Wilcoxon-Test für Paardifferenzen, $p=0,8160$ ).

$\mathrm{Zu}$ einer weiteren Charakterisierung der Arbeitsweisen soll nun die Analyse der Anzahl der ausgewählten Darstellungen durch eine Klassifikation der Probanden nach der von ihnen bevorzugten Darstellungsart ergänzt werden. Es lassen sich dabei drei verschiedene Typen unterscheiden: ${ }^{227}$

I. Es wird ausschließlich mit dem Graph gearbeitet.

II. Es wird ausschließlich mit der Tabelle gearbeitet.

III. Es wird sowohl mit dem Graph als auch mit der Tabelle gearbeitet.

\footnotetext{
${ }^{226}$ Bei der Gruppe der Schüler werden die 15 Probanden, die die Aufgabe wegen Netzwerkproblemen ein zweites Mal lösten, nicht berücksichtigt. Ebenfalls nicht mit in die Auswertung einbezogen werden zwei Probanden, die die Aufgabe nicht bzw. ohne mit den Darstellungen zu experimentieren lösten.

${ }^{227}$ Bei der Klassifikation der Probanden nach den von ihnen verwendeten Darstellungen werden - im Unterschied zu Aufgabe $2 b$ - neben der Art der gewählten Darstellungen keine weiteren Kriterien wie etwa „Dauer der Analyse der Darstellung“ oder „Lösungsrelevanz der Darstellung“ berücksichtigt.
} 
Man erhält die folgende Häufigkeitsverteilung:

\begin{tabular}{|l||c|c|c|}
\hline & I & II & III \\
\hline Schüler (36) & $44 \%(16)$ & $0 \%(0)$ & $56 \%(20)$ \\
\hline Studierende (21) & $10 \%(2)$ & $14 \%(3)$ & $76 \%(16)$ \\
\hline
\end{tabular}

Tab. 51: Aufgabe 3d - Strategien beim Arbeiten mit Darstellungen

Der Unterschied zwischen den Arbeitsweisen der Schüler und der Studierenden, der in der Tabelle 50 bereits erkennbar ist, tritt hier noch deutlicher hervor (Chiquadrat-Mehrfeldertest, $p=0,004)$. So haben $44 \%$ der Schüler aber nur $10 \%$ der Studierenden ausschließlich mit dem Graph gearbeitet (Kategorie I). Dagegen haben $90 \%$ der Studierenden im Rahmen ihrer Funktionsuntersuchungen die tabellarische Darstellung verwendet, wobei $76 \%$ der Probanden die Tabelle in Verbindung mit dem Graph nutzten und $14 \%$ der Probanden sogar ausschließlich mit der Tabelle arbeiteten (Kategorie II und III). Die Schüler und die Studierenden haben dabei die Möglichkeit des Experimentierens umfassend genutzt. Sämtliche Probanden, die mit dem Graph arbeiteten, betätigten mindestens einmal den entsprechenden Rollbalken und analysierten bzw. beobachteten den Einfluss des Parameters $c$ auf die Funktionenklasse $f(n)=2 n^{2}-8 n+c$ und auf die entsprechenden Differenzenfunktionen. Auch die Tabelle wurde von $90 \%$ der Probanden, die diese Darstellung auswählten, zum numerischen Experimentieren genutzt.

Die große Bedeutung, die der (im Hinblick auf eine inhaltliche Begründung der Invarianz der Differenzenfunktion eher ungeeigneten) Tabelle von den Studierenden beigemessen wurde, und das Ignorieren dieser Darstellung von einem großen Teil der Schülergruppe lässt sich möglicherweise weniger auf unterschiedliche fachmathematische als vielmehr auf unterschiedliche fachdidaktische Kenntnisse zurückführen. So wurde insbesondere im Rahmen der Veranstaltung „Computer im Mathematikunterricht“ die Bedeutung des dynamischen Visualisierens für das Entdecken von Eigenschaften und für das Generieren von Vermutungen thematisiert. Es ist anzunehmen, dass sich die Studierenden daher anhand aller in das Lernprogramm eingebundenen Darstellungen und damit auch anhand der Tabelle einen Erkenntnisgewinn erhofften.

\subsubsection{Beziehung: Arbeiten mit Darstellungen - Argumentationen}

Während die Argumentationen derjenigen Schüler, welche die Invarianz von $D$ geometrisch begründeten, auf ein umfassendes Experimentieren mit der graphischen Darstellung zurückgeführt werden können, lässt sich ein derartiger Einfluss bei den Studierenden nur bedingt feststellen. Obwohl auch die meisten Studierenden die graphische Darstellung im Rahmen ihrer Funktionsanalysen einsetzten, argumentierten 9 der 14 Studierenden, welche die beobachtete Eigenschaft von $D$ angemessen begründeten, formal. Insbesondere beeinflusste auch 
ein (fast) ausschließliches Arbeiten mit dem Graph nicht die Sichtweise der Probanden auf die Funktionseigenschaften, wie der folgende Transkriptausschnitt verdeutlicht.

\begin{tabular}{|c|c|c|c|c|c|c|c|}
\hline Zeit & Aufg. & $\begin{array}{c}\text { Aufg.- } \\
\text { stellung }\end{array}$ & Tabelle & Graph & $\begin{array}{c}\text { Excel- } \\
\text { Blatt Nr. }\end{array}$ & Kommentar & Eingaben über Tastatur \\
\hline 28.07 & $3 \mathrm{~d}$ & $\mathrm{x}$ & & & 18 & & \\
\hline 28.53 & & & & $\mathrm{x}$ & & Experimentieren mit $c$ & \\
\hline 29.56 & & $\mathrm{x}$ & & & & & \\
\hline 30.06 & & & & $\mathrm{x}$ & & Experimentieren mit $c$ & \\
\hline 30.37 & & $\mathrm{x}$ & & & & & \\
\hline 30.39 & & & & & 17 & & $\begin{array}{l}\text { Experimentieren mit } c \\
\text { Experimentieren mit } \\
\text { man }\end{array}$ \\
\hline 30.40 & Exp & & & $\mathrm{x}$ & 16 & & \\
\hline 31.12 & & & $\mathrm{x}$ & & 15 & & $\begin{array}{l}\text { Die Veränderung des } \\
\text { Parameters verändert die } \\
\text { Differenzenfunktion nicht, da } \\
\text { zu jedem Funktionswert der } \\
\text { gleiche Wert } c \text { addiert und } \\
\text { subtrahiert wird. Also bleibt } \\
\text { die Differenz gleich. }\end{array}$ \\
\hline 32.18 & & & & $\mathrm{x}$ & 16 & & \\
\hline
\end{tabular}

Tabelle 52: Aufgabe 3d - Transkriptausschnitt ${ }^{228}$

(Studierender I36)

\subsubsection{Ergebnisse zu Aufgabe 3e}

Diese Aufgabe gliedert sich in zwei Teilaufgaben. Die Schüler sollten zunächst das Intervall $^{229}$ angegeben, in dem die Funktionswerte der Differenzenfunktion das Vorzeichen ändern. In einem zweiten Aufgabenteil sollte dieses Ergebnis mit Blick auf die Eigenschaften der Ausgangsfunktion $f$ mit $f(n)=2 n^{2}-9 n-8$ interpretiert werden. Bei dieser Fragestellung konnten die Schüler zwischen den Darstellungen Graph und Tabelle wählen. Ein Vergleich mit der Gruppe der Studierenden entfällt, da diese Aufgabe nicht Teil ihres Testprogramms war.

\subsubsection{Aufgabenteil 1: Lesen von Darstellungen}

Von den 53 Probanden haben 3 Schüler den ersten Teil der Aufgabe nicht bearbeitet. Weitere 10 Schüler gaben ein Intervall an, ohne zuvor eine Darstellung betrachtet zu haben. Es ist anzunehmen, dass diese Schüler die Aufgabe nicht selbstständig gelöst, sondern das Ergebnis unzulässigerweise von ihren Mitschülern übernommen haben. Die Antworten dieser Proban-

\footnotetext{
${ }^{228}$ Vgl. die ScreenCam-Datei I36.scm.

${ }^{229}$ Die Funktionswerte von $D$ wechseln beim Übergang von $n=1$ nach $n=2$ das Vorzeichen.
} 
den werden als „,nicht bearbeitet“ gewertet. In der folgenden Tabelle sind die Schüler danach klassifiziert, ob sie die Aufgabe richtig, falsch oder gar nicht bearbeitet haben.

\begin{tabular}{|l||c|c|c|}
\hline & Richtig & Falsch & Keine Bearbeitung \\
\hline Schüler (53) & $64 \%(34)$ & $11 \%(6)$ & $25 \%(13)$ \\
\hline
\end{tabular}

Tab. 53: Aufgabe 3e - Qualität der Lösungen

Insgesamt $64 \%$ der Schüler haben die erste Teilaufgabe richtig gelöst. Dass etwa ein Viertel der Probanden das Ergebnis von ihren jeweiligen Nachbarn übernommen haben bzw. nicht bearbeitet haben, deutet nicht zwangsläufig auf ein unzureichendes Verständnis der Aufgabenstellung bzw. Schwierigkeiten im Umgang mit den Darstellungen hin. Vielmehr lässt sich diese Beobachtung auf die insgesamt recht lange Testdauer, die unterschiedlichen Arbeitsgeschwindigkeiten der Schüler und den damit verbundenen Wunsch der „langsameren“ Schüler, mit ihren schneller arbeitenden Mitschülern aufzuschließen, zurückführen.

\subsubsection{Aufgabenteil 2: Argumentieren über Eigenschaften}

Insgesamt 10 der 53 Schüler haben den zweiten Teil nicht bearbeitet. Weitere 5 Schüler haben die gesamte Aufgabe gelöst, ohne zuvor eine Darstellung betrachtet zu haben. Da somit anzunehmen ist, dass diese Schüler auch die zweite Teilaufgabe nicht selbstständig gelöst haben, werden ihre Antworten hier nicht berücksichtigt und die Aufgabe als „nicht bearbeitet“ gewertet. Im Folgenden werden zunächst die schriftlichen Äußerungen der Probanden hinsichtlich der beschriebenen Eigenschaften klassifiziert. Anschließend werden die dabei gebildeten Kategorien detaillierter im Hinblick auf „diskrete“ bzw. „kontinuierliche“ Sichtweisen analysiert.

\section{Klassifikation}

Die Beschreibungen der Schüler lassen sich in einem ersten Schritt danach klassifizieren, ob eine Beziehung zwischen dem Vorzeichenwechsel der Differenzenfunktion $D$ und Eigenschaften der Ausgangsfunktion $f$ erkannt wurde oder ob das Intervall nur unzureichend bzw. fehlerhaft im Hinblick auf Eigenschaften von $f$ interpretiert wurde. Darüber hinaus lassen sich die mathematisch adäquaten ${ }^{230}$ verbalen Formulierungen danach unterscheiden, ob die Schüler lokale oder globale Eigenschaften von $f$ erkannt haben. Es ergeben sich die folgenden vier Kategorien:

\footnotetext{
${ }^{230}$ Es werden auch umgangssprachliche Formulierungen akzeptiert bzw. Verwechslungen von Begriffen wie „Scheitelpunkt" und „Wendepunkt" sowie der Funktionsbezeichnungen $f$ und $D$ toleriert.
} 


\section{A Lokal}

- Bei $n=2$ ist der Tiefstpunkt erreicht (Wendepunkt).

- Bei dem Punkt 2 hat die Funktion ihren Tiefpunkt.

- Die Kurve $f$ hat ihren tiefsten Punkt erreicht.

- Der Scheitel ist erreicht.

- Zwischen $n=1$ und $n=2$ liegt der Scheitelpunkt.

B Global

In diesem Bereich fällt bzw. steigt die Funktion wieder.

- Der Graph fällt bis ca. -1 und beginnt wieder gen $0 \mathrm{zu}$ steigen.

- Die Steigung ändert sich zwischen den Werten 1 und 3 von negative in positive Steigung.

- Die Funktion verändert ihre Steigung.

- Wenn der Graph fällt, entstehen negative $f$-Werte, wenn er steigt positive. ${ }^{231}$

C Lokal und Global

- Ist die Differenz zwischen 2 Stufen positiv, so steigt der Graph, ist die Differenz negativ, fällt der Graph. Der Scheitelpunkt ist der 0-Wert.

- Bei negativen Werten fällt der Graph, bei positiven steigt er, d. h. der Tiefpunkt der Funktion liegt zwischen den Differenzenfunktionswerten von $D$, bei denen sich das Vorzeichen ändert.

- Die Funktion ist am Scheitelpunkt angelangt, ab hier steigt die Funktion wieder.

- Bis $(1,-3)$ fällt $f$, von $(2,1)$ an steigt $f$, und dazwischen liegt der Scheitelpunkt.

D Bedeutung des Vorzeichenwechsels wurde nicht erkannt

- Die Funktionswerte gehen nun in den negativen Bereich und beim Übergang von $n=5$ nach $n=6$ gelangen sie wieder in den positiven Bereich mit Vorzeichen ,plus“. ${ }^{232}$

- Es ändert sich.

Der Funktionswert wechselt von $n=1$ nach $n=2$ vom negativen ins positive. ${ }^{233}$

- Die Werte von $f$ und $D$ steigen.

Es ergibt sich die folgende Häufigkeitsverteilung:

\begin{tabular}{|l||c|c|c|c|c|}
\hline & A & B & C & D & Keine Bearbeitung \\
\hline Schüler (53) & $17 \%(9)$ & $9 \%(5)$ & $23 \%(12)$ & $23 \%(12)$ & $28 \%(15)$ \\
\hline
\end{tabular}

Tab. 54: Aufgabe 3e - Argumentationsmuster

Etwa $50 \%$ der Probanden haben eine Beziehung zwischen dem Vorzeichenwechsel der Differenzenfunktion $D$ und den Eigenschaften der Ausgangsfunktion $f$ erkannt. Dabei haben nur $17 \%$ der Schüler den Blick ausschließlich auf den Tiefpunkt von $f$ gerichtet (Kategorie A). Insgesamt $32 \%$ der Schüler haben im Hinblick auf das Intervall [1,2] - obwohl die Fragestellung nur implizit in dieses Richtung deutete - (auch) das Montonieverhalten der Funktion $f$ und damit globale Eigenschaften beschrieben (Kategorie B und C). Dabei wurde zum Teil

\footnotetext{
${ }^{231}$ Es ist anzunehmen, dass hier an ,negative $D$-Werte“ gedacht wurde.

${ }^{232}$ Es wird beschrieben, in welchem Intervall die Funktionswerte von $f$ ihr Vorzeichen wechseln.

${ }^{233}$ Es wird keine Beziehung zur Funktion f hergestellt.
} 
auch ein Bezug zu globalen Eigenschaften der Differenzenfunktion hergestellt, indem auf den Zusammenhang zwischen dem Vorzeichen der Funktionswerte der Differenzenfunktion und dem Monotonieverhalten der Ausgangsfunktion verwiesen wurde. Wie bereits beim ersten Aufgabenteil lässt sich der hohe Anteil der Schüler, welche die Aufgabe nicht bearbeiteten, weniger auf inhaltliche als auf zeitliche Probleme der Probanden zurückführen.

\section{Beziehung diskret-kontinuierlich}

Interessant sind die lokalen Argumente der Schüler, die den Tiefpunkt beschreiben (Kategorien A und C). Sie lassen sich anhand der folgenden prototypischen Aussagen klassifizieren:

I. Tiefpunkt der diskreten Funktion wird beschrieben:

„Tiefpunkt liegt bei $n=2$ “.

II. Tiefpunkt der „gedachten“ kontinuierlichen Funktion wird beschrieben:

„Tiefpunkt liegt zwischen den Differenzenfunktionswerten von $D$, bei denen sich das Vorzeichen ändert“.

III. Keine genaue Aussage:

„Funktion ist am Scheitelpunkt angelangt, danach steigt die Funktion wieder“.

Die Tabelle 55 gibt die entsprechende Häufigkeitsverteilung wieder:

\begin{tabular}{|l||c|c|c|}
\hline & I & II & III \\
\hline \hline Schüler (21) & $9 \%(2)$ & $43 \%(9)$ & $48 \%(10)$ \\
\hline
\end{tabular}

Tab. 55: Aufgabe 3e - Sichtweise Extremstelle

Nur $9 \%$ der Schüler, die eine Beziehung zwischen dem Vorzeichenwechsel der Funktionswerte der Differenzenfunktion und der Extremstelle der Funktion $f$ erkannt haben, verwiesen auf den diskreten Tiefpunkt bei $n=2$ (Kategorie I). Die verbalen Formulierungen der anderen Schüler deuten auf ein Funktionsverständnis hin, dass stark von einer kontinuierlichen Sicht auf lokale Eigenschaften geprägt ist. So ist anzunehmen, dass die Probanden, die der Kategorie II zugeordnet werden können, sich anstelle der diskreten Funktion $f$ eine reellwertige quadratische Interpolationsfunktion zu den durch die Koordinaten dieser Z-Funktion vorgegebenen „Stützpunkten“ vorstellten und deren Scheitelpunkt bestimmten. Insgesamt $48 \%$ der Probanden umgingen offenbar eine Auseinandersetzung mit der diskreten Situation, indem sie den Scheitelpunkt nur vage beschrieben aber keine genauen Koordinaten angaben (Kategorie III).

\subsubsection{Strategien beim Arbeiten mit Darstellungen}

Während sich zur Beantwortung der ersten Teilaufgabe beide Darstellungen eignen, erscheint im Hinblick auf die zweite Teilaufgabe - aus „Expertensicht“ - das Arbeiten mit dem Graph vorteilhafter. Die Arbeitsweisen der Probanden werden daher im Folgenden aufgabenspezi- 
fisch analysiert. ${ }^{234}$ Um mögliche Unterschiede im strategischen Vorgehen feststellen zu können, werden nur die 35 Schüler berücksichtigt, die beide Teile der Aufgabe bearbeitet haben. Zur Charakterisierung der Arbeitsweisen werden die Probanden wie in Aufgabe 3d nach der bzw. den von ihnen bevorzugten Darstellungsart(en) klassifiziert. Es werden die folgenden Kategorien unterschieden:

A Es wird ausschließlich mit dem Graph gearbeitet.

B Es wird ausschließlich mit der Tabelle gearbeitet.

C Es wird sowohl mit dem Graph als auch mit der Tabelle gearbeitet.

Die Tabelle 56 gibt die entsprechende Häufigkeitsverteilung an.

\begin{tabular}{|l||c|c|c|}
\hline Schüler (35) & A & B & C \\
\hline 1. Teilaufgabe & $14 \%(5)$ & $43 \%(15)$ & $43 \%(15)$ \\
\hline 2. Teilaufgabe & $23 \%(8)$ & $28 \%(10)$ & $49 \%(17)$ \\
\hline
\end{tabular}

Tab. 56: Aufgabe 3e - Strategien beim Arbeiten mit Darstellungen

Insgesamt $57 \%$ der Schüler betrachteten zur Bestimmung des Intervalls den Graph (Kategorie A und C), wohingegen $43 \%$ der Probanden ausschließlich mit der Tabelle arbeiteten. Der Anteil der Schüler, die zur Beschreibung der Bedeutung des Vorzeichenwechsels der Differenzenfunktion mit der graphischen Darstellung arbeiteten, ist mit $72 \%$ deutlich, wenn auch nicht signifikant höher (McNemar-Test, $p=0,059$ ). Damit arbeitete die Mehrheit der Schüler mit der zur Beantwortung dieser Teilaufgabe adäquaten Darstellung. Es ist auffällig, dass dennoch ein relativ großer Prozentsatz von $28 \%$ der Schüler ausschließlich die Tabelle betrachtete. Bis auf eine Ausnahme haben diese Schüler bereits in der ersten Teilaufgabe die tabellarische Darstellung bevorzugt. Im Folgenden soll untersucht werden, ob das Festhalten an der im Rahmen der ersten Teilaufgabe adäquaten Strategie einen Einfluss auf die Qualität der verbalen Formulierungen bei der zweiten Teilaufgabe hatte.

\subsubsection{Einfluss der Darstellungswahl auf das Lösen der Aufgabe}

Es wird nun untersucht, inwiefern die Darstellungswahl der Probanden die Qualität der jeweiligen Lösungen beeinflusste. Dabei betrachten wir nur die 35 Schüler, die beide Teile der Aufgabe bearbeitet haben. Die Probanden werden zunächst dahingehend unterschieden, ob sie die graphische Darstellung genutzt oder ausschließlich mit der Tabelle gearbeitet haben. An-

\footnotetext{
${ }^{234}$ Dabei wird die erste Teilaufgabe mit dem Notieren des gesuchten Intervalls auf dem Lösungsblatt als beendet angesehen. Alle Aktivitäten, d. h. insbesondere das Auswählen und Betrachten von Darstellungen, die sich an die erste Teilaufgabe anschließen, werden im Rahmen der zweiten Teilaufgabe berücksichtigt. Werden beide Aufgaben gleichzeitig beantwortet, d. h. sind keine Aktivitäten nach der Bearbeitung der ersten Teilaufgabe feststellbar, werden die zuvor beobachteten Arbeitsweisen des Probanden im Rahmen beider Teilaufgaben berücksichtigt.
} 
schließend wird untersucht, ob die jeweilige Teilaufgabe richtig bzw. falsch bearbeitet wurde. Es ergeben sich die folgenden Häufigkeitsverteilungen:

\begin{tabular}{|l||c|c|}
\hline \multicolumn{1}{|c||}{} & \multicolumn{2}{c|}{ 1.Teilaufgabe } \\
\cline { 2 - 3 } & Richtig & Falsch \\
\hline $\begin{array}{l}\text { Arbeiten mit Graph } \\
\text { (20 Schüler) }\end{array}$ & $90 \%(18)$ & $10 \%(2)$ \\
\hline $\begin{array}{l}\text { Arbeiten mit Tabelle } \\
(15 \text { Schüler })\end{array}$ & $80 \%(12)$ & $20 \%(3)$ \\
\hline
\end{tabular}

Tab. 57: Aufgabe 3e - Einfluss der Darstellung auf das Benennen des Intervalls

\begin{tabular}{|l|c|c|}
\hline \multicolumn{1}{|c||}{} & \multicolumn{2}{c|}{ 2. Teilaufgabe } \\
\cline { 2 - 3 } & Richtig & Falsch \\
\hline \hline $\begin{array}{l}\text { Arbeiten mit Graph } \\
\text { (25 Schüler) }\end{array}$ & $84 \%(21)$ & $16 \%(4)$ \\
\hline $\begin{array}{l}\text { Arbeiten mit Tabelle } \\
\text { (10 Schüler) }\end{array}$ & $30 \%(3)$ & $70 \%(7)$ \\
\hline
\end{tabular}

Tab. 58: Aufgabe 3e - Einfluss der Darstellung auf die verbale Beschreibung

Die erste Teilaufgabe wurde von der Mehrzahl der Probanden richtig gelöst, unabhängig davon, ob sie ausschließlich mit der Tabelle arbeiteten oder im Lösungsprozess den Graph hinzuzogen. Durch die freie Darstellungswahl war es den Schülern in diesem Zusammenhang offensichtlich möglich, ihren individuellen Lösungsweg mit der von ihnen bevorzugten Darstellungsform gewinnbringend zu verfolgen. Im zweiten Aufgabenteil führte das Arbeiten mit dem Graph zu signifikant besseren Ergebnissen (Chiquadrat-Vierfeldertest, $p=0,002$ ). So haben $84 \%$ der Probanden, welche die graphische Darstellung hinzugezogen haben, aber nur $30 \%$ der Probanden, die ausschließlich mit der Tabelle gearbeitet haben, die Beziehung zwischen dem Vorzeichenwechsel der Funktionswerte der Differenzenfunktion und den Eigenschaften der Ausgangsfunktion adäquat beschrieben.

Wie eine weitere Analyse zeigt, wirkte sich das ausschließliche Arbeiten mit der Tabelle auch auf die Qualität der verbalen Formulierungen aus. So argumentierten diese Probanden global aber recht ungenau (,In diesem Bereich fällt bzw. steigt die Funktion wieder“, „Der Graph fällt bis ca. -1 und beginnt wieder gen 0 zu steigen“). Insbesondere wurde der Scheitelpunkt in allen Fällen nicht explizit benannt. Das Festhalten an der Strategie „Tabelle“ erwies sich somit im Rahmen dieser Aufgabe als ungeeignet. Die Möglichkeit der freien Darstellungswahl birgt daher auch die Gefahr, dass der Beitrag spezifischer Darstellungsformen zur Lösung von Problemen nicht erkannt und inadäquate Wege beschritten werden. 


\subsubsection{Ergebnisse zu Aufgabe 3f}

Die Schüler und die Studierenden sollten im Rahmen dieser Aufgabe die Termdarstellung der Differenzenfunktion der allgemeinen quadratischen Z-Funktion $f$ mit $f(n)=a n^{2}+b n+c$ bestimmen. Die Bearbeitung dieser Aufgabe erfolgte auf einem separaten Arbeitsblatt. Da sich bei den Schülern der Gruppen A und B erhebliche Schwächen im Umgang mit Termen beobachten ließen, war diese Aufgabe im Lernprogramm der Schüler der Gruppe C nicht mehr vorgesehen. Die Termdarstellung der Differenzenfunktion wurde stattdessen im Rahmen eines Unterrichtsgesprächs hergeleitet und diskutiert. In der folgenden Tabelle werden die Schüler der Gruppe A und B sowie die Studierenden danach unterschieden, ob die Aufgabe richtig, fehlerhaft oder gar nicht bearbeitet wurde.

\begin{tabular}{|l||c|c|c|}
\hline & Richtig & Fehlerhaft & Keine Bearbeitung \\
\hline Schüler (36) & $39 \%(14)$ & $19 \%(7)$ & $42 \%(15)$ \\
\hline Studierende (21) & $81 \%(17)$ & $9,5 \%(2)$ & $9,5 \%(2)$ \\
\hline
\end{tabular}

Tab. 59: Aufgabe 3f - Ergebnisse

Die Studierenden haben die Aufgabe erwartungsgemäß signifikant besser gelöst als die Schüler (Chiquadrat-Vierfeldertest, $p=0,008$ ). Insgesamt $81 \%$ der Studierenden aber nur $39 \%$ der Schüler gaben die richtige Termdarstellung an. Fehlerhaft bearbeitet haben diese Aufgabe $19 \%$ der Schüler und 9,5\% der Studierenden. Um die Schwierigkeiten der Probanden analysieren zu können, werden auf dem Weg zur Berechnung der Termdarstellung der Differenzenfunktion vier Lösungsschritte unterschieden:

$$
\begin{aligned}
D(n) & =f(n+1)-f(n) & & \\
& =a(n+1)^{2}+b(n+1)+c-\left(a n^{2}+b n+c\right) & & \text { (Einsetzen der Funktionsterme) } \\
& =a\left(n^{2}+2 n+1\right)+b(n+1)+c-\left(a n^{2}+b n+c\right) & & \text { (Anwenden der binomischen Formel) } \\
& =a n^{2}+2 a n+a+b n+b+c-a n^{2}-b n-c & & \text { (Auflösen der Klammern) } \\
& =2 a n+a+b & & \text { (Zusammenfassen der Terme) }
\end{aligned}
$$

Schwierigkeiten im Umgang mit der Binomischen Formel hatten drei Schüler und ein Studierender. Dabei wurde der Term $(n+1)^{2}$ entweder nicht aufgelöst oder die binomische Formel fehlerhaft angewendet (Beispiel: $(n+1)^{2}=n^{2}+2 n+2$ oder $\left.(n+1)^{2}=n^{2}+1\right)$. Beim Auflösen der Klammer berücksichtigten drei Schüler ${ }^{235}$ und ein Studierender nicht das negative Vorzeichen. Zwei Schüler hatten zwar mit den ersten drei Lösungsschritten keine Schwierigkeiten, waren jedoch nicht in der Lage, die Terme angemessen zusammenzufassen. Auffallend hoch ist der Anteil der Schüler, die ein leeres Arbeitsblatt ablieferten und im Rahmen der

\footnotetext{
${ }^{235}$ Einer dieser drei Schüler hatte bereits Schwierigkeiten bei der Anwendung der binomischen Formel.
} 
obigen Klassifikation der Kategorie „Keine Bearbeitung“ zugewiesen wurden. Aus den Untersuchungsbeobachtungen wurde jedoch deutlich, dass ein Teil dieser Schüler auf einem eigenen Lösungsblatt einen Lösungsversuch unternommen hatten, aber dieses Schriftstiuck am Ende der Untersuchung nicht abgaben. Es ist anzunehmen, dass auch diese Schüler aufgrund formaler Schwächen die Aufgabe nicht fehlerfrei lösen konnten.

Während die Fehler der zwei Studierenden, die die Aufgabe fehlerhaft lösten, auf Konzentrationsschwächen zurückgeführt werden können, weist die große Zahl an Schülern, welche die Aufgabe nicht oder nur fehlerhaft bearbeiteten, auf schwache algebraische Leistungen insbesondere bei elementaren Termumformungen hin.

\subsubsection{Ergebnisse zu Aufgabe 3g}

Gegenstand dieser Aufgabe ist die Funktionenklasse $f(n)=1,5 n^{2}+b n-8$. Die Schüler und Studierenden sollten den Einfluss des Parameters $b$ auf die graphische bzw. tabellarische Darstellung der entsprechenden Differenzenfunktion untersuchen und begründen. Der Parameter $b$ konnte mit Hilfe eines Rollbalkens variiert werden.

\subsubsection{Verbale Beschreibung der Differenzenfunktion}

Die verbalen Beschreibungen der Schüler und Studierenden lassen sich danach klassifizieren, ob Eigenschaften der Differenzenfunktion $D$ (und möglicherweise auch Eigenschaften der Ausgangsfunktion $f$ ) oder ausschließlich Eigenschaften von der Funktion $f$ beschrieben werden. Innerhalb dieser beiden Hauptkategorien sind weitere Unterscheidungen hinsichtlich der von den Probanden benannten Funktionseigenschaften möglich. Die Antworten der Probanden können dabei auch mehreren Unterkategorien zugeordnet werden. Wie in Aufgabe 3c werden zunächst alle Antworten der Schüler und Studierenden berücksichtigt, unabhängig davon, ob eine Beziehung zwischen den Funktionen $f$ und $D$ hergestellt bzw. der Einfluss des Parameters $b$ auf die Differenzenfunktion begründet wurde.

\section{A Beschreibung von Eigenschaften von D}

\section{Al Parallelverschiebung}

- Die Differenzenfunktion wandert an der y-Achse nach unten.

- Die Differenzenfunktion wird wie die Parabel nur hin- und her verschoben.

- $\quad b$ verschiebt die Kurve $D$ nach oben.

Alle Differenzenfunktionen sind parallel.

\section{A2 Änderung des Achsenabschnitts}

- Der $y$-Achsenabschnitt wandert auf der $y$-Achse rauf und runter.

- Es ändert sich nur der Achsenabschnitt.

Je größer $b$, desto größer wird der Achsenabschnitt von $D$.

A3 Steigung konstant

- Die Steigung bleibt konstant.

- Die Differenz der $D$-Werte zueinander ändert sich nicht. 
Es verändert sich die Lage von $D$. Die Steigung bleibt gleich.

A4 Lokale numerische Eigenschaften

- Wenn man den Parameter $b$ um 0,1 ändert, ändert sich auch $D$ um 0,1.

Wird $b$ größer, werden auch $D(0)$ und alle anderen Punkte größer und umgekehrt.

Je größer $b$, desto größer $D$.

\section{B Beschreibung von Eigenschaften von $f$ (ausschließlich)}

B4 Formgleichheit, Symmetrie

Wenn $b=0$ ist, dann teilt die $x$-Achse den Graph in genau 2 Hälften. Wenn $b$ positiv ist, dann bewegt sich die Parabel in den negativen Bereich.

- Der Graph verschiebt sich auf der $x$-Achse.

B2 Konstanter Achsenabschnitt

- Der Scheitelpunkt verändert sich. Die Schnittstelle mit der $y$-Achse bleibt gleich.

- Auf der $y$-Achse bleibt ein Punkt ständig an einem Punkt $(0,40)$. Um diesen Punkt dreht sich (rotiert, rollt) die Parabel.

B3 Tiefpunkt ändert sich

Je höher der Parameter $b$ ist, umso geringer ist der Tiefpunkt.

Der Scheitelpunkt verändert sich.

\section{K Keine Zuordnung möglich}

- Um so positiver die Zahl in der Funktion wird, um so positiver werden auch die Differenzen.

- Die Werte von $D$ ändern sich.

- Wenn die Werte steigen, dann fällt der Graph.

Die folgenden Tabellen 60 und 61 geben die Häufigkeitsverteilungen über den oben genannten Kategorien wieder. Dabei werden 6 Schüler, welche die Aufgabe nicht bearbeiteten bzw. die Bearbeitung ohne Fixierung einer verbalen Beschreibung abbrachen, nicht berücksichtigt.

\begin{tabular}{|l||c|c|c|}
\hline & A & B & K \\
\hline Schüler (47) & $49 \%(23)$ & $32 \%(15)$ & $19 \%(9)$ \\
\hline Studierende (21) & $86 \%(18)$ & $0 \%(0)$ & $14 \%(3)$ \\
\hline
\end{tabular}

Tab. 60: Aufgabe 3g -Verbale Beschreibungen

(Hauptkategorien)

\begin{tabular}{|l||c|c|c|c||c|c|c|}
\hline \multicolumn{1}{|c||}{} & \multicolumn{4}{c||}{} & \multicolumn{3}{c|}{ B } \\
\cline { 2 - 8 } & A1 & A2 & A3 & A4 & B1 & B2 & B3 \\
\hline \hline Schüler (47) & $32 \%(15)$ & $13 \%(6)$ & $18 \%(8)$ & $0 \%(0)$ & $11 \%(5)$ & $23 \%(11)$ & $9 \%(4)$ \\
\hline Studierende (21) & $33 \%(7)$ & $33 \%(7)$ & $19 \%(4)$ & $24 \%(5)$ & $0 \%(0)$ & $0 \%(0)$ & $0 \%(0)$ \\
\hline
\end{tabular}

Tab. 61. Aufgabe 3g - Verbale Beschreibungen (Unterkategorien)

Insgesamt $49 \%$ der Schüler beschrieben den Einfluss des Parameters $b$ auf das Verhalten der Differenzenfunktion (Kategorie A). Der größte Teil der Schüler (32\%) betonte dabei die Parallelität der entsprechenden Graphen (Kategorie A1), was für eine geometrische Sicht auf die 
Eigenschaften der Differenzenfunktion spricht. Vergleichsweise weniger Probanden (13\% bzw. $18 \%$ ) verwendeten algebraische Begriffe, die auf eine funktionale Sicht auf die Eigenschaften hindeuten (Kategorie A2 und A3). Auffällig groß (32\%) ist der Anteil der Schüler, welche die Funktion $D$ unberücksichtigt ließen und sich ausschließlich auf das Beschreiben der Funktion $f$ konzentrierten (Kategorie B). Zieht man zur genaueren Analyse die Tabelle 61 hinzu, fällt auf, dass die meisten Schüler eine Eigenschaften von $f$ analysierten, die im Rahmen dieser Aufgabenstellung von untergeordneter Relevanz ist: der konstante Achsenabschnitt (Kategorie B2). Dies scheint ein für diese Aufgabe typisches „diskretes“ Problem zu sein, welches sich auf ein ausgiebiges Experimentieren mit der graphischen Darstellung zurückführen lässt: Die diskreten Koordinatenpunkte erschweren offensichtlich die Konzentration auf die kontinuierliche „Gestalt“ der Parabel und steuern vielmehr die Aufmerksamkeit auf den einzigen „,statischen“ Punkt - den Achsenabschnitt $(\emptyset, 40)$. Während sich die Studierenden diesem visuellen Einfluss entziehen konnten, wurden die Schüler durch dieses Phänomen möglicherweise von der eigentlichen Aufgabenstellung abgelenkt.

Im Gegensatz zu den Schülern bearbeiteten alle Studierenden die Aufgabe im Sinne der Fragestellung und betrachteten die Funktion $f$ nicht isoliert. Insgesamt $33 \%$ der Studierenden und damit ein etwa gleich großer Prozentsatz wie bei den Schülern, betonten die Parallelität der Graphen der Differenzenfunktionen (Kategorie A1). Im Unterschied zu den Schülern weisen die Äußerungen der Studierenden jedoch auf eine ausgeprägte funktionale Sicht auf die Eigenschaften der Differenzenfunktionen - insbesondere auf den sich ändernden Achsenabschnitt - hin (Kategorie A2). Auffallend ist die starke Konzentration der Studierenden (24\%) auf irrelevante numerische Eigenschaften (Kategorie A4). Es handelt sich auch hierbei um ein (offensichtlich) diskretes Phänomen, welches nur in der Gruppe der Studierenden nicht aber bei den Schülern zu beobachten war: Eine Differenzenfunktion wird nicht als ,ganzheitliches“ Objekt angesehen, sondern - im Sinne der Formel $D_{n}(b)=2 a n+a+b$ mit $n$ fest aber beliebig - als „Aneinanderreihung“ isolierter, diskreter Funktionswerte, deren Änderung in Abhängigkeit von $b$ beschrieben wird. Es ist zu vermuten, dass das Arbeiten mit der Tabelle bzw. eine verengte Sicht auf die tabellarische Darstellung die verbalen Beschreibungen dieser Studierenden beeinflusst hat (vgl. auch Kap. 6.3.7.4).

\subsubsection{Begründung der Eigenschaften der Differenzenfunktion}

Insgesamt $62 \%$ der Studierenden aber nur $8 \%$ der Schüler haben den Einfluss des zu variierenden Parameters $b$ auf die Differenzenfunktionen adäquat begründet (vgl. Tab. 62). Damit wurde diese Aufgabe im Vergleich zu Aufgabe 3d von signifikant weniger Schülern zufriedenstellend gelöst (McNemar-Test, $p=0,001$ ). Dagegen erhält man für die Studierenden ein ähnliches Ergebnis wie bei Aufgabe $3 \mathrm{~d}$ bzw. es sind keine signifikante Unterschiede feststellbar (McNemar-Test, $p=0,705)$. 


\begin{tabular}{|l|c|c|}
\hline & Begründung & Keine (adäquate) Begründung \\
\hline \hline Schüler (53) & $8 \%(4)$ & $92 \%(49)$ \\
\hline Studierende (21) & $62 \%(13)$ & $38 \%(8)$ \\
\hline
\end{tabular}

Tab. 62: Aufgabe 3g - Qualität der verbalen Begründung

Bei den Argumentationen der Probanden, die die Eigenschaften der Differenzenfunktionen adäquat begründen, lassen sich folgende Kategorien unterscheiden:

\section{A Graphisch-geometrische Argumentation}

Es wird eine Beziehung zwischen der graphischen Darstellung der Differenzenfunktion $D$ und der entsprechenden Ausgangsfunktion $f$ hergestellt. Die Eigenschaften von $D$ werden in Bezug auf die Eigenschaften von $f$ begründet.

- $\quad f$ ändert die Form nicht, nur die Lage. Die Differenzen liegen nur auf einem anderen Niveau.

- Da sich der Scheitelpunkt von $f$ verändert, verändert sich auch der Schnittpunkt von $D$ mit der $x$-Achse.

\section{B Algebraische Argumentation}

Es wird eine Beziehung zwischen der graphischen Darstellung von $D$ und der Termdarstellung $D(h)=2 a n+a+b$ hergestellt. Die Ausgangsfunktion wird dabei nur implizit (über den formalen Zusammenhang der allgemeinen quadratischen Z-Funktion und deren Differenzenfunktion) in die Analysen mit einbezogen.

- Beim Verändern von $b$ wird der Achsenabschnitt der Funktion $D$ verändert. Da der Wert $b$ in der abgeleiteten Formel für $b$ in den Achsenabschnitt miteinfließt, wird nur dieser verändert und die Steigung bleibt konstant, denn $b$ hat mit dieser nichts zu tun.

- Die Abstände bleiben gleich, da das $b$ nur den Achsenabschnitt beschreibt und das $a$ konstant ist.

$D(n)=2 a n+a+b, \mathrm{~d}$. h. ändert man $b$, so ändert sich der Achsenabschnitt, die Steigung bleibt aber erhalten.

$(a+b)$ ist der Achsenabschnitt einer linearen Funktion, d. h. dieser ändert sich, wenn man $b$ ändert.

Man erhält die folgende Häufigkeitsverteilung:

\begin{tabular}{|l||c|c|}
\hline & A & B \\
\hline \hline Schüler (4) & $0 \%(0)$ & $100 \%(4)$ \\
\hline Studierende (13) & $38 \%(5)$ & $62 \%(8)$ \\
\hline
\end{tabular}

Tab. 63: Aufgabe 3g - Argumentationsmuster bei adäquaten Begründungen

Diejenigen Schüler, welche die Aufgabe zufriedenstellend lösten, argumentierten ausschließlich formal in Bezug auf die allgemeine Termdarstellung der Differenzenfunktion. Offensichtlich wurde die Möglichkeit des Arbeitens auf einer rein inhaltlich-anschaulichen Ebene und die Analyse der Graphen $f$ und $D$ nicht genutzt bzw. dieses Arbeiten trug nicht zum Verständnis der Beziehung zwischen Funktion und Differenzenfunktion bei (vgl. auch Kap. 6.3.7.4). 
Andererseits stellte auch der formale Weg keine echte Alternative dar, wie die geringe Anzahl von insgesamt 4 Schülern, die der Kategorie B zugeordnet werden können, verdeutlicht. Die Herleitung und Diskussion der allgemeinen Termdarstellung $D(n)=2 a n+a+b$ im Unterrichtsgespräch, das im Rahmen der Untersuchung mit den Schülern der Gruppe C durchgeführt wurde, hatte insofern einen Einfluss auf das Ergebnis, als 3 Schüler dieser Probandengruppe die Aufgabe zufriedenstellend lösten, aber nur ein Schüler der Gruppen A/B.

Auch die Studierenden bevorzugten - wie bereits bei Aufgabe 3d - eine algebraische Erklärung. Dagegen argumentierten $38 \%$ der Probanden graphisch-geometrisch. Diese Argumentationen unterscheiden sich qualitativ insofern, als 3 der 5 Probanden, die der Kategorie A zugeordnet werden können, lediglich die lokale Beziehung zwischen dem „Tiefpunkt von $f$ “ und dem ,Schnittpunkt von $D$ mit der $x$-Achse“ beschrieben, wohingegen die beiden anderen Probanden ,ganzheitlich“ argumentierten. Die graphisch-geometrischen Formulierungen aller 5 Studierenden lassen sich nicht auf Schwierigkeiten im Umgang mit der Termdarstellung der Differenzenfunktion bzw. Probleme bei deren Interpretation zurückführen. So hatten 3 dieser Probanden im Rahmen von Aufgabe $3 \mathrm{~d}$ formal und darstellungsunabhängig argumentiert und sämtliche 5 Probanden hatten die allgemeine Formel $D(n)=2 a n+a+b$ im Rahmen von Aufgabe $3 \mathrm{f}$ richtig abgeleitet. Es liegt vielmehr die Vermutung nahe, dass das Arbeiten mit der nicht-formalen Darstellung offensichtlich zu einer Erweiterung des (inhaltlichen) Begriffsverständnisses beitrug.

\subsubsection{Arbeiten mit Darstellungen}

Die folgende Tabelle zeigt, wie oft die Darstellungsformen Tabelle und Graph von den Schülern bzw. den Studierenden gewählt wurden. Es werden hierbei nur die Probanden berücksichtigt, welche die Aufgabe vollständig bearbeiteten.

\begin{tabular}{|l||c|c|}
\hline & Tabelle & Graph \\
\hline \hline Schüler (47) & 49 & 103 \\
\hline Studierende (21) & 31 & 43 \\
\hline
\end{tabular}

Tab. 64 Aufgabe 3g - Absolute Häufigkeit der betrachteten Darstellungen

Die Anzahl der betrachteten Darstellungen ist in beiden Probandengruppen in etwa gleich bzw. es ist kein signifikanter Unterschied feststellbar (Wilcoxon-Test, $p=0,3056$ ). Im Mittel betrachteten die Schüler 3,2 Darstellungen (Standardabweichung 1,9) und die Studierenden 3,5 Darstellungen (Standardabweichung 1,6). Die Schüler arbeiteten dabei signifikant häufiger mit dem Graph als mit der Tabelle (Wilcoxon-Test, $p<0,001$ ), wohingegen bei den Studierenden lediglich ein nicht signifikanter Unterschied feststellbar ist (Wilcoxon-Test, $p=0,1124)$. Damit ist auch die relative Maßzahl für die Häufigkeit der von den Schülern 
betrachteten graphischen Darstellungen signifikant höher als der entsprechende Anteil bei den Studierenden (Wilcoxon-Test, $p=0,0419$ ).

$\mathrm{Zu}$ einer weiteren Charakterisierung der Arbeitsweisen soll nun wie bei Aufgabe 3d die Analyse der Anzahl der ausgewählten Darstellungen durch eine Klassifikation der Probanden nach der von ihnen bevorzugten Darstellungsart ergänzt werden. Es werden wieder die folgenden Kategorien unterschieden:

I. Es wird ausschließlich mit dem Graph gearbeitet.

II. Es wird ausschließlich mit der Tabelle gearbeitet.

III. Es wird sowohl mit dem Graph als auch mit der Tabelle gearbeitet.

Man erhält die folgende Häufigkeitsverteilung:

\begin{tabular}{|l||c|c|c|}
\hline & I & II & III \\
\hline \hline Schüler (47) & $36 \%(17)$ & $2 \%(1)$ & $62 \%(29)$ \\
\hline Studierende (21) & $9,5 \%(2)$ & $9,5 \%(2)$ & $81 \%(17)$ \\
\hline
\end{tabular}

Tab. 65: Aufgabe 3g - Strategien beim Arbeiten mit Darstellungen

Wie bereits bei Aufgabe 3d maßen die Schüler dem Graph bei der Analyse der Eigenschaften der Differenzenfunktion eine größere Bedeutung bei als die Studierenden. So haben $36 \%$ der Schüler, aber nur 9,5\% der Studierenden, ausschließlich mit dem Graph gearbeitet (Kategorie I). Dagegen haben etwa $90 \%$ der Studierenden im Rahmen ihrer Funktionsuntersuchungen die tabellarische Darstellung verwendet; 9,5 \% der Probanden sogar ohne zusätzlich den Graph zu betrachten (Kategorie II und III). Auch hierbei scheint für die Studierenden das Angebot, sowohl den Graph als auch die Tabelle wählen zu können, einen Anreiz geschaffen zu haben, nicht nur den Graph, sondern auch die Tabelle umfassend im Hinblick auf interessante Eigenschaften bzw. numerische Muster zu analysieren.

Beiden Probandengruppen nutzen die Möglichkeit der Parametervariation ausgiebig. So haben bis auf einen Schüler und einen Studierenden alle Probanden, die mit dem Graph gearbeitet haben, mindestens einmal den entsprechenden Rollbalken betätigt und somit den Einfluss des Parameters $b$ auf die Funktionenklasse $f(n)=1,5 n^{2}+b n-8$ und die entsprechenden Differenzenfunktionen analysiert. Auch die Tabelle wurde von sämtlichen Studierenden und von $90 \%$ der Schüler, die diese Darstellung auswählten, zum numerischen Experimentieren genutzt.

\subsubsection{Beziehung: Arbeiten mit Darstellungen - Argumentationen}

Wie aus der Klassifikation der verbalen Beschreibungen (vgl. Kap. 6.3.7.1, Tab. 61) ersichtlich ist, haben $23 \%$ der Schüler (Kategorie B2) und $24 \%$ der Studierenden (Kategorie A4) ihre Aufmerksamkeit auf irrelevante Eigenschaften der betrachteten Funktionen gerichtet. 
Eine Analyse der entsprechenden Bildschirmaktivitäten bestätigt die Vermutung, dass insbesondere die Beschreibung des Achsenabschnitts und der um diesen Koordinatenpunkt „herumrollenden Parabel“" durch eine starke Fixierung auf das Arbeiten mit dem Graph beeinflusst wurde. Eine mögliche Ursache für die unzureichenden, lokal-numerischen Antworten der Studierenden, ist dagegen, wie bereits angenommen, in einem umfangreichen und zum Teil ausschließlichen Experimentieren mit der Tabelle zu sehen.

Während in den vorstehend beschriebenen Fällen das Experimentieren mit den Darstellungen offensichtlich zu fehlerhaften und inadäquaten Argumentationen führte, haben $49 \%$ der Schüler und $86 \%$ der Studierenden jedoch die neuen technischen Möglichkeiten nutzen können, um den Einfluss des Parameters $b$ auf das Verhalten der Differenzenfunktion im Sinne der Fragestellung zu beschreiben. ${ }^{236}$ Die Analyse der beobachteten Eigenschaften bzw. der Beziehung von Funktion und Differenzenfunktion mit Hilfe des Graphen führte jedoch - wie bereits bei Aufgabe 3d festgestellt - nicht zwangsläufig auch zu einer geometrischgraphischen Begründung für das Verhalten der Differenzenfunktion. So hatten die meisten der Schüler und Studierenden, die das Verhalten der Differenzenfunktion algebraisch begründeten, zuvor zum Teil ausgiebig mit dem Graph experimentiert. Es ist zu vermuten, dass das Ziel des Experimentierens nicht nur das Entdecken von Eigenschaften war, sondern dass die Probanden zunächst auch bemüht waren, eine Beziehung zwischen der graphischen Darstellung der Differenzenfunktion $D$ und der entsprechenden Ausgangsfunktion $f$ herzustellen. Dieser Versuch wurde jedoch abgebrochen und die (naheliegendere, einfachere) algebraische Begründung angegeben. Da somit die inhaltlich-anschauliche Ebene keine echte Alternative im Lösungsprozess darstellte, ist es verständlich, dass diese Aufgabe die Mehrheit der Schüler aufgrund ihrer algebraischen Schwächen überforderte.

\subsection{Ergebnisse zu Aufgabe 4}

Das Aufgabenmodul „Dreieckszahlen“ gliedert sich in vier Teile. Gegenstand der ersten drei Teile ist die rekursive Darstellung des Zählproblems. Im vierten Aufgabenteil wird eine Arbeitsumgebung zum Finden einer expliziten Formel zur Beschreibung der Dreieckszahlen bereitgestellt.

\subsubsection{Ergebnisse zu Aufgabe 4a}

Bei dieser Aufgabe sollten die Probanden die ersten 6 Folgenglieder der auf dem vorhergehenden Excel-Blatt zeichnerisch dargestellten Dreieckszahlen angeben. Die Aufgabe wurde von den Studierenden besser gelöst als von den Schülern, betrachtet man die relative Häufigkeit der Probanden, welche die Aufgabe im ersten Lösungsanlauf lösten (vgl. Tab. 66). Als

\footnotetext{
${ }^{236}$ Vgl. Tab. 60, Kategorie A.
} 
fehlerhafter Lösungsversuch gilt hierbei die Angabe mindestens eines falschen Folgenglieds. Das bessere Abschneiden der Studierenden kann auf eine im Rahmen ihrer fachwissenschaftlichen Ausbildung gewonnene größere Erfahrung im Umgang mit rekursiven Folgen zurückgeführt werden.

\begin{tabular}{|l||c|c|c|c|c|}
\hline & $\begin{array}{c}\text { 1 Lösungs- } \\
\text { versuch }\end{array}$ & $\begin{array}{c}\text { 2 Lösungs- } \\
\text { versuche }\end{array}$ & $\begin{array}{c}\text { >2 Lösungs- } \\
\text { versuch }\end{array}$ & $\begin{array}{c}\text { Fehlerhafte } \\
\text { Lösung }\end{array}$ & $\begin{array}{c}\text { Keine } \\
\text { Bearbeitung }\end{array}$ \\
\hline \hline Schüler (53) & $66 \%(35)$ & $23 \%(12)$ & $4 \%(2)$ & $7 \%(4)$ & $0 \%(0)$ \\
\hline Studierende (21) & $81 \%(17)$ & $4,5 \%(1)$ & $0 \%(0)$ & $10 \%(2)$ & $4,5 \%(1)$ \\
\hline
\end{tabular}

Tab. 66: Aufgabe 4a - Lösungsversuche

Die Arbeitsweisen der Probanden lassen sich danach unterscheiden, ob während des Lösungsprozesses noch einmal zu dem Excel-Blatt mit der zeichnerischen Darstellung der Dreieckszahlen zurückgeblättert wurde oder eine einmalige Analyse der Zeichnung zur Wiedergabe der ersten 6 Folgenglieder ausreichte. Wie eine detailliertere Analyse der Arbeitsweisen zeigt, wechselten die Probanden, welche die Aufgabe erst nach mehreren Lösungsanläufen lösten, bis auf einen Fall im Laufe der Bearbeitung noch einmal zu der zeichnerischen Darstellung zurück. Hier diente das Zurückblättern der Überprüfung und Korrektur der numerischen Eingaben. Ein wiederholtes Analysieren der zeichnerischen Darstellung während des Lösungsprozesses ließ sich jedoch auch bei Probanden beobachten, welche die Aufgabe im ersten Lösungsanlauf lösten (vgl. Tab 67).

\begin{tabular}{|l||c|c|}
\hline & Einmalige Analyse & Mehrmalige Analyse \\
\hline Schüler (35) & $31 \%(11)$ & $69 \%(24)$ \\
\hline Studierende (17) & $82 \%(14)$ & $18 \%(3)$ \\
\hline
\end{tabular}

Tab. 67: Aufgabe 4a - Arbeiten mit der zeichnerischen Darstellung der Dreieckszahlen

So wechselten $69 \%$ der Schüler, die die Aufgabe im ersten Lösungsanlauf lösten, mindestens einmal zum vorhergehenden Excel-Blatt zurück. Dieses Verhalten deutet darauf hin, dass die zeichnerische Darstellung zuvor nur unzureichend analysiert wurde bzw. das entsprechende Excel-Blatt einfach „überblättert“ wurde. Während die meisten dieser Schüler das iterative Aufbauprinzip im Laufe der Bearbeitung erkannt haben, legt - wie der folgende Transkriptausschnitt verdeutlicht (vgl. Tab. 68) - die Arbeitsweise anderer Probanden die Vermutung nahe, dass eine iterative Gesetzmäßigkeit nicht gesehen wurde und die einzelnen Folgenglieder schrittweise durch Abzählen der die Dreieckszahlen darstellenden Kreise ermittelt wurden. 


\begin{tabular}{|c|c|c|c|c|}
\hline Zeit & Aufg. & $\begin{array}{c}\text { Excel- } \\
\text { Blatt Nr. }\end{array}$ & Kommentar/Beschreibung der Aktivität & Eingaben über Tastatur \\
\hline 29.57 & 4 & Aufg 4 & & \\
\hline 30.06 & & 31 & Cursor deutet auf Dreieckszahlen & \\
\hline 30.29 & $4 a$ & 32 & & \\
\hline 30.41 & & 31 & & \\
\hline 30.43 & $4 a$ & 32 & & $A(1)=1$ \\
\hline 30.49 & & 31 & & \\
\hline 30.52 & $4 \mathrm{a}$ & 32 & & $A(2)=3$ \\
\hline 30.55 & & 31 & Cursor deutet auf 3. Dreieckszahl & \\
\hline 30.59 & $4 \mathrm{a}$ & 32 & & $A(3)=6$ \\
\hline 31.03 & & 31 & Cursor deutet auf 4. Dreieckszahl & \\
\hline 31.09 & $4 \mathrm{a}$ & 32 & & $A(4)=10$ \\
\hline 31.13. & & 31 & Cursor deutet auf 5. Dreieckszahl & \\
\hline 31.22 & $4 \mathrm{a}$ & 32 & & $A(5)=15$ \\
\hline 31.26 & & 31 & Cursor deutet auf 6. Dreieckszahl & \\
\hline 31.34 & $4 \mathrm{a}$ & 32 & & $A(6)=21$ \\
\hline
\end{tabular}

Tab. 68: Aufgabe 4a - Transkriptausschnitt ${ }^{237}$ (Schüler C1)

Im Vergleich zu den Schülern waren signifikant weniger Studierende, die die Aufgabe im ersten Lösungsanlauf lösten, auf eine wiederholte Beschäftigung mit der zeichnerischen Darstellung angewiesen (Chiquadrat-Vierfeldertest, $p=0,001$ ). Offensichtlich hatte die Mehrheit dieser Probanden (82\%) - wie auch der folgende Transkriptausschnitt verdeutlicht - den Text des entsprechenden Excel-Blatts vor der Bearbeitung von Aufgabe 2a (also vor der Eingabe der ersten Folgenglieder) konzentriert gelesen und den iterativen Aufbau der Dreieckszahlen nach sorgfältiger Analyse erkannt.

\begin{tabular}{|c|c|c|l|l|}
\hline Zeit & Aufg. & $\begin{array}{c}\text { Excel- } \\
\text { Blatt Nr. }\end{array}$ & Kommentar & Eingaben über Tastatur \\
\hline 43.22 & 4 & 30 & & \\
\hline 43.26 & & 31 & Cursor deutet auf Text & \\
\hline 43.48 & $4 \mathrm{a}$ & 32 & Cursor deutet auf Text & \\
\hline 43.56 & & 31 & Cursor deutet auf Dreieckszahlen & \\
\hline 44.29 & & 32 & & $\begin{array}{l}\text { Cursor wird über die einzelnen „Lagen“ } \\
\text { der Dreieckszahlen bewegt }\end{array}$ \\
\hline 44.33 & & 31 & & $\begin{array}{l}\text { Eingabe der Folgenanfangs- } \\
\text { glieder 1, 3, 6, .., 21 }\end{array}$ \\
\hline 45.17 & & 32 & & \\
\hline
\end{tabular}

Tab. 69: Aufgabe 4a - Transkriptausschnitt ${ }^{238}$ (Studierender II35)

\footnotetext{
${ }^{237}$ Vgl. ScreenCam-Datei C1.scm.

${ }^{238}$ Vgl. ScreenCam-Datei II35.scm.
} 


\subsubsection{Ergebnisse zu Aufgabe 4b}

Bei dieser Aufgabe sollte die rekursive Folge der Dreieckszahlen auf induktivem Weg symbolisch erfasst werden. Das Formulieren der rekursiven Gesetzmäßigkeit bereitete beiden Probandengruppen größere Schwierigkeiten als das numerische Fortführen der Folge in Aufgabe 2a. Insgesamt $62 \%$ der Studierenden, aber nur $45 \%$ der Schüler lösten diese Aufgabe im ersten Lösungsanlauf (vgl. Tab. 70). Der relativ hohe Prozentsatz an fehlerhaften Lösungen ist darauf zurückzuführen, dass es im Rahmen dieser Aufgabe keine Möglichkeit zur eigenständigen Lösungskontrolle (etwa mit Hilfe eines „Ok-Buttons“) gab.

\begin{tabular}{|l||c|c|c|c|c|}
\hline & $\begin{array}{c}\text { 1 Lösungs- } \\
\text { versuch }\end{array}$ & $\begin{array}{c}\text { 2 Lösungs- } \\
\text { versuche }\end{array}$ & $\begin{array}{c}\text { >2 Lösungs- } \\
\text { versuch }\end{array}$ & $\begin{array}{c}\text { Fehlerhafte } \\
\text { Lösung }\end{array}$ & $\begin{array}{c}\text { Keine } \\
\text { Bearbeitung }\end{array}$ \\
\hline \hline Schüler (53) & $45 \%(24)$ & $34 \%(18)$ & $4 \%(2)$ & $17 \%(9)$ & $0 \%(0)$ \\
\hline Studierende (21) & $62 \%(13)$ & $9 \%(2)$ & $5 \%(1)$ & $19 \%(4)$ & $5 \%(1)$ \\
\hline
\end{tabular}

Tab. 70: Aufgabe 4b - Lösungsversuche

Beim Lösen dieser Aufgabe traten im Wesentlichen vier Fehlertypen auf. Drei Fehlertypen lassen sich auf inhaltliche Schwierigkeiten beim Erfassen der rekursiven Struktur bzw. beim induktiven Schluss auf die allgemeine rekursive Darstellung der Dreieckszahlen zurückführen. Daneben waren auch Fehler zu beobachten, deren Ursache in einer inadäquaten Verwendung des technischen Werkzeugs TKP zu suchen ist.

\section{I. $\quad$ Numerischer Fehler}

Beispiel:

$$
\begin{aligned}
& A(3)=A(2)+4 \\
& A(4)=A(3)+6 \\
& A(5)=A(4)+9
\end{aligned}
$$

\section{Bezug auf ein falsches Folgenglied, Selbstbezug}

Beispiele:

$$
\begin{aligned}
& A(3)=A(2)+A(2) \\
& A(3)=A(2)+A(3) \\
& A(3)=A(1)+A(1)+1
\end{aligned}
$$

\section{Fehlerhafter induktiver Schluss}

Beispiele:

$$
\begin{aligned}
& A(n)=A(n-1)+(n-1) \\
& A(n)=A(n-1)+A(n)
\end{aligned}
$$

\section{Technische Probleme: Fehlende Zellbezüge}

Die folgende Tabelle zeigt die Häufigkeitsverteilungen über diesen Kategorien. Dabei kann ein Proband hinsichtlich mehrerer Fehlertypen berücksichtigt werden. 


\begin{tabular}{|l||c|c|c|c|}
\hline & I & II & III & IV \\
\hline \hline Schüler (53) & $21 \%(11)$ & $9 \%(5)$ & $28 \%(15)$ & $9 \%(5)$ \\
\hline Studierende (21) & $9,5 \%(2)$ & $19 \%(4)$ & $0 \%(0)$ & $9,5 \%(2)$ \\
\hline
\end{tabular}

Tab. 71: Aufgabe 4b - Inhaltliche/technische Fehlertypen

Das Erfassen der rekursiven Gesetzmäßigkeit, d. h. insbesondere das Angeben der ersten 8 Folgenglieder in rekursiver Struktur, stellte beide Probandengruppen vor nicht unerhebliche Probleme. Während bei den Schülern die Fehlertypen I und III überwogen, dominierte bei den Studierenden Fehlertyp II. So gaben $21 \%$ der Schüler falsche (numerische) Änderungswerte an (Fehlertyp I). Dieser Fehler trat nur bei 9,5 \% der Studierenden auf. Größere Schwierigkeiten bereitete es den Studierenden dagegen, zwischen der Folge der Dreieckszahlen $A$ und der Folge der Änderungen zu differenzieren (Fehlertyp II). Anstelle der Folgenglieder $A(1)$, $A(2)=A(1)+2, A(3)=A(2)+3, \ldots$ gaben $19 \%$ der Probanden z. B. die Folgenglieder $A(1)=A(1), A(2)=A(1)+A(2), A(3)=A(2)+A(3)$ an. Beide Fehlertypen lassen sich möglicherweise darauf zurückführen, dass die Probanden das iterative Aufbauprinzip der Dreieckszahlen im Rahmen der Bearbeitung von Aufgabe 2a nicht erkannt hatten und die Folgenglieder zum Teil isoliert betrachteten. Eine weitere Ursache insbesondere des Fehlertyps II liegt möglicherweise in einer Verwechslung der beiden in ihrer Struktur ,ähnlichen“ Gleichungen $A(n)=A(n-1)+A(n)$ und $A(n)=A(n-1)+D(n)$ begründet, wobei der Term $D(n)$ das $n$-te Glied der Folge der Differenzen $A(n)-A(n-1)$ angibt.

Insgesamt $28 \%$ der Schüler gaben die ersten 8 Folgenglieder richtig an, hatten jedoch Schwierigkeiten, von diesen Anfangsfolgengliedern induktiv auf die allgemeine rekursive Formel zu schließen (Fehlertyp III). Dabei wurde in 10 Fällen die Beziehung $A(n)=A(n-1)+A(n)$ und in 4 weiteren Fällen die Beziehung $A(n)=A(n-1)+(n-1)$ angegeben. Ein Proband brach die Aufgabe an dieser Stelle ab. Bei den Studierenden konnte dieser Fehlertyp nicht beobachtet werden. Alle Probanden gaben die allgemeine rekursive Formel unter Berücksichtigung der (zum Teil fehlerhaften) Struktur der Anfangsfolgenglieder an.

Neben diesen inhaltlichen Schwierigkeiten waren bei etwa $9 \%$ der Probanden beider Gruppen auch technische Schwierigkeiten zu beobachten (Fehlertyp IV). Wie eine detaillierte Analyse der Arbeitsweisen zeigt, waren 7 Schüler und 3 Studierende darum bemüht, die Folge der Änderungen 2, 3, 4, 5, 6, 7, 8 mit Hilfe einer Formel in Excel algorithmisch und möglicherweise auch zeitlich effektiv zu „,berechnen“. Bei diesem Vorgehen erkannten jedoch 5 Schüler $(9 \%)$ und 2 Studierende $(9,5 \%)$ nicht die Notwendigkeit relativer Zellbezüge und erzeugten auf diese Weise fehlerhafte numerische Werte. Diese Probanden bearbeiteten die Aufgabe daraufhin im „traditionellen“ Sinne und gaben die jeweiligen numerischen Werte schrittweise ein. Diese Beobachtung macht deutlich, dass die Bereitstellung eines Werkzeugs wie Excel u. U. die Gefahr in sich birgt, dass das technische Hilfsmittel unreflektiert und probleminadä- 
quat auch dort eingesetzt wird, wo ein traditionelles Vorgehen einfacher, schneller und „sicherer" zum Ziel führt.

\subsubsection{Ergebnisse zu Aufgabe 4c}

Bei dieser Aufgabe sollten die Probanden die ersten 16 Folgen der Dreieckszahlen mit Hilfe des TKP berechnen. Wie die folgende Tabelle 72 zeigt, haben die Studierenden diesen Aufgabenteil deutlich besser gelöst als die Schüler. Hierbei beziehen wir uns auf die relative Häufigkeit der Probanden, die nur einen Lösungsanlauf benötigten sowie den Anteil der Probanden, welche die Aufgabe fehlerhaft lösten. Es fällt weiterhin auf, dass die Schüler die Aufgabe schlechter gelöst haben als die „Streichholzaufgabe“ 1 b. Dies kann zum einen auf die einfachere mathematische Struktur der „Streichholzfolge“ zurückgeführt werden. Zum anderen hat möglicherweise das sprachliche Formulieren der Gesetzmäßigkeit im Rahmen von Aufgabe 1a das Erkennen der in Beziehung stehenden Zellen unterstützt. Die Studierenden haben Aufgabe 4c etwas besser bearbeitet als Aufgabe 1b, was auf eine zunehmende Vertrautheit mit dem Lernprogramm und auf ein im Vergleich zu Aufgabe 1b konzentrierteres Arbeiten zurückgeführt werden kann.

\begin{tabular}{|l|l|c|c|c|c|}
\hline & $\begin{array}{c}\text { 1 Lösungs- } \\
\text { versuch }\end{array}$ & $\begin{array}{c}\text { 2 Lösungs- } \\
\text { versuche }\end{array}$ & $\begin{array}{c}\text { >2 Lösungs- } \\
\text { versuch }\end{array}$ & $\begin{array}{c}\text { Fehlerhafte } \\
\text { Lösung }\end{array}$ \\
\hline \hline \multirow{2}{*}{ Aufgabe 1b } & Schüler (49) & $53 \%(26)$ & $20 \%(10)$ & $23 \%(11)$ & $4 \%(2)$ \\
\cline { 2 - 6 } & Studierende (21) & $38 \%(8)$ & $52 \%(11)$ & $10 \%(2)$ & $0 \%(0)$ \\
\hline \multirow{2}{*}{ Aufgabe 4c } & Schüler (53) & $34 \%(18)$ & $30 \%(16)$ & $15 \%(8)$ & $21 \%(11)$ \\
\cline { 2 - 6 } & Studierende (21) & $57 \%(12)$ & $33 \%(7)$ & $5 \%(1)$ & $5 \%(1)$ \\
\hline
\end{tabular}

Tab. 72: Lösungsversuche:

Vergleich: Aufgabe 1b-4c

Der hohe Prozentsatz der Schüler, die eine fehlerhafte Lösung angaben und nicht mehr korrigierten, überrascht insofern, als im Rahmen dieser Aufgabe sowohl ein „Hinweis“- als auch ein „Lösungs-Button“ zur Überprüfung des eigenen Ergebnisses zur Verfügung stand. Aufgrund der fortgeschrittenen Testdauer kann vermutet werden, dass diese Probanden kein Interesse mehr an einer weiteren Bearbeitung hatten.

\subsubsection{Inhaltliche/technische Probleme}

In Anlehnung an bereits bestehende Fehlerkategorien (vgl. Kap. 6.2.1) lassen sich die inhaltlichen und technischen Schwierigkeiten der Probanden im Umgang mit dieser Aufgabe wie folgt klassifizieren: 
I. Vermischen der algebraischen Syntax mit der TKP-Syntax;

II. Fehlerhafte Zellbezüge;

III. Fehlende Zellbezüge;

IV. Numerisches Erzeugen einer fehlerhaften Folge.

Die folgende Tabelle spiegelt die entsprechende Häufigkeitsverteilung wider, wobei wieder ein Proband mehreren Kategorien zugeordnet werden kann.

\begin{tabular}{|l||c|c|c|c|}
\hline & I & II & III & IV \\
\hline Schüler (53) & $2 \%(1)$ & $58 \%(31)$ & $4 \%(2)$ & $11 \%(6)$ \\
\hline Studierende (21) & $10 \%(2)$ & $23 \%(5)$ & $10 \%(2)$ & $0 \%(0)$ \\
\hline
\end{tabular}

Tab. 73: Aufgabe 4c - Inhaltliche/technische Fehlertypen

Verglichen mit Aufgaben, welche die Berechnung der Differenzenfolge oder der Differenzenfunktion zum Gegenstand hatten, ${ }^{239}$ traten im Rahmen dieser Aufgabe weniger Fehler auf, die auf einem Vermischen der algebraischen Sprache mit der Syntax des TKP beruhen (Fehlertyp I). Rein syntaktische Fehler, wie etwa das Vergessen des „=“-Zeichens, waren nicht zu beobachten und auch die Notwendigkeit relativer Zellbezüge wurde im Allgemeinen beachtet (Fehlertyp III). Während bei der Fragestellung zu Aufgabe 3a auf die zu berechnende Formel $D(n)=f(n+1)-f(n)$ explizit hingewiesen worden war, war bei dieser Aufgabe die allgemeine rekursive Darstellung in algebraischer Form nur zur zusätzlichen Illustration am unteren Tabellenrand angegeben. Offensichtlich waren die meisten Probanden dadurch in der Lage, sich stärker auf die „TKP-Welt“ zu konzentrieren. Es ist jedoch auch möglich, dass den Probanden die Excel-Syntax mittlerweile hinreichend vertraut war, so dass weitestgehend problemlos zwischen dieser Syntax und der algebraischen Sprache differenziert werden konnte.

Insgesamt $11 \%$ der Schüler umgingen das Werkzeug Excel zunächst und gaben stattdessen die numerischen Folgenglieder schrittweise ein bzw. wechselten zur numerischen Ebene, nachdem das Arbeiten mit der Excel-Syntax nicht zum Ziel führte (Fehlertyp IV). In allen Fällen traten jedoch beim Bestimmen der Folgenglieder (Rechen-)Fehler auf, die durch den Einsatz des TKP an dieser Stelle hätten vermieden werden können. Die größten Schwierigkeiten bereitete den Probanden - insbesondere den Schülern - das korrekte Benennen und InBeziehung-Setzen der entsprechenden Zellen, also das Arbeiten mit Zellen als Variablen (Fehlertyp II). Im Folgenden soll durch einen Vergleich mit den Ergebnissen aus Aufgabe 4b analysiert werden, ob das Erfassen einer rekursiven Gesetzmäßigkeit ein generelles Problem in Verbindung mit dieser Fragestellung darstellt oder ob hinsichtlich des Arbeitens auf verschiedenen Darstellungsebenen Unterschiede feststellbar sind.

\footnotetext{
${ }^{239} \mathrm{Vgl}$ etwa Aufgabe 2a (Tab. 25) und Aufgabe 3a (Tab. 39).
} 


\subsubsection{Vergleich: Aufgabe 4b - Aufgabe 4c}

Zur Beurteilung und zum Vergleich des Lösungserfolgs in Aufgabe 4b und 4c werden die Probanden hinsichtlich der folgenden Kriterien differenziert: Die Probanden, welche die Aufgaben $4 \mathrm{~b}$ und $4 \mathrm{c}$ im ersten Lösungsversuch lösten und jene Probanden, die mehrere Anläufe zur Lösung benötigten bzw. die Aufgaben nicht oder falsch lösten (vgl. Tab. 74 und 75).

Unter Berücksichtigung dieser Kriterien haben die Schüler Aufgabe 4c etwas schlechter gelöst, wenngleich die Unterschiede nicht signifikant sind (McNemar-Test, $p=0,239$ ). Bei den Studierenden lassen sich keine deutlichen (signifikanten) Unterschiede feststellen (McNemarTest, $p=0,739$ ).

\begin{tabular}{|l||c|c|}
\hline Schüler (53) & 1 Lösungsversuch & $>1$ Lösungsversuch \\
\hline \hline Aufgabe 4b & $45 \%(24)$ & $55 \%(29)$ \\
\hline Aufgabe 4c & $34 \%(18)$ & $66 \%(35)$ \\
\hline
\end{tabular}

Tab. 74: Lösungsversuche Schüler: Vergleich Aufgabe 4b - Aufgabe 4c

\begin{tabular}{|l||c|c|}
\hline Studierende (21) & 1 Lösungsversuch & $>1$ Lösungsversuch \\
\hline Aufgabe 4b & $62 \%(13)$ & $38 \%(8)$ \\
\hline Aufgabe 4c & $57 \%(12)$ & $43 \%(9)$ \\
\hline
\end{tabular}

Tab. 75 Lösungsversuche Studierende:

Vergleich Aufgabe 4b - Aufgabe 4c

Legt man die Tabellen 74 und 75 in einer anderen Form an, so dass der Fokus auf die Veränderungen der Leistungen der Probanden gerichtet ist (vgl. Tab. 76 und 77), so fällt auf, dass sich $30 \%$ der Schüler gegenüber Aufgabe 4b verschlechtert haben, wohingegen $19 \%$ der Schüler eine bessere Leistung erbracht haben. Bei den Studierenden haben nur $24 \%$ der Probanden ein schlechteres Ergebnis geliefert als bei Aufgabe 4b, dagegen haben sich $19 \%$ der Probanden verbessert.

\begin{tabular}{|c|l||c|c|}
\hline \multicolumn{2}{|c||}{ Schüler (53) } & \multicolumn{2}{c|}{ Aufgabe 4c } \\
\cline { 3 - 4 } \multicolumn{2}{|c|}{} & 1 Lösungsversuch & $>1$ Lösungsversuche \\
\hline \hline \multirow{2}{*}{ Aufgabe 4b } & 1 Lösungsversuch & $15 \%(8)$ & $30 \%(5)$ \\
\cline { 2 - 4 } & $>1$ Lösungsversuche & $19 \%(10)$ & $36 \%(19)$ \\
\hline
\end{tabular}

Tab. 76: Leistungsveränderung Schüler:

Vergleich: Aufgabe 4b - Aufgabe 4c 


\begin{tabular}{|l|l||c|c|}
\hline \multicolumn{2}{|l||}{ Studierende (21) } & \multicolumn{2}{c|}{ Aufgabe 4c } \\
\cline { 3 - 4 } \multicolumn{2}{|c|}{} & 1 Lösungsversuch & $>1$ Lösungsversuche \\
\hline Aufgabe 4b & 1 Lösungsversuch & $38 \%(8)$ & $24 \%(5)$ \\
\cline { 2 - 4 } & >1 Lösungsversuche & $19 \%(4)$ & $19 \%(4)$ \\
\hline
\end{tabular}

Tab. 77: Leistungsveränderung Studierende: Vergleich: Aufgabe 4b - Aufgabe 4c

Eine Verbesserung der Leistungen von Aufgabe $4 \mathrm{~b}$ zu Aufgabe 4c bestätigt die Vermutung, dass die ,Zellensprache“ bzw. das Arbeiten mit Zellen als prototypische Darstellungen für Variablen insbesondere für algebraisch schwächere Schüler eine gute Möglichkeit bietet, rekursive Zusammenhänge auf einer „,vorformalen“ Ebene zu erfassen. Dagegen deutet eine Verschlechterung darauf hin, dass das Arbeiten mit Zellen als Variablen nicht per se einfacher ist, sondern im Unterricht längerfristig entwickelt werden muss. Aufgrund der Bereichsspezifität des Denkens (vgl. Kap. 3.2.3), auf die beide Ergebnisse zurückgeführt werden können, sind somit - wie hier deutlich wird - prinzipielle Chancen, aber auch Schwierigkeiten mit dem Wechsel zwischen verschiedenen Darstellungsebenen verbunden.

\subsubsection{Ergebnisse zu Aufgabe 4d}

Ziel dieser Aufgabe ist das Finden einer expliziten Formel für die Dreieckszahlen. Das Lernprogramm zeigt zur Lösung des Zählproblems drei grundsätzliche Wege auf: die Lösungsstrategien „Bleistift und Papier“, „Differenzenfolge“ und „Experimentieren“. Die Probanden hatten die Möglichkeit, zwischen diesen Wegen zu wählen, die jeweiligen Hilfen (,HinweisButtons") in Anspruch zu nehmen sowie die angebotenen Hilfsmittel beliebig miteinander zu kombinieren. Wie die Tabelle 78 verdeutlicht, lösten die Studierenden die Aufgabe nicht signifikant besser als die Schüler (Chiquadrat-Vierfeldertest, $p=0,966$ ). Insgesamt $57 \%$ beider Probandengruppen fanden die explizite Darstellung $A(n)=0,5 n^{2}+0,5 n$. Die anderen Probanden gaben ein falsches Ergebnis an oder bearbeiteten die Aufgabe überhaupt nicht. Dieses zunächst überraschende Ergebnis kann darauf zurückgeführt werden, dass der Themenkomplex „Dreieckszahlen“ beiden Probandengruppen noch nicht bekannt war und das Finden der expliziten Formel somit für Schüler und Studierende ein ,echtes“ und ähnlich schwieriges Problem darstellte.

\begin{tabular}{|l||c|c|c|}
\hline & Erfolgreich & Nicht erfolgreich & Keine Bearbeitung \\
\hline \hline Schüler (53) & $57 \%(30)$ & $41 \%(22)$ & $2 \%(1)$ \\
\hline Studierende (21) & $57 \%(12)$ & $38 \%(8)$ & $5 \%(1)$ \\
\hline
\end{tabular}

Tab. 78: Aufgabe 4d - Ergebnisse 
Im Folgenden sollen die Lösungsstrategien der Probanden genauer analysiert werden. Dabei bezeichnen wir die Probanden, welche die explizite Formel für die Dreieckszahlen richtig bestimmten, als „erfolgreiche“ Probanden und nennen die anderen ,erfolglose“ Probanden. Es wird zunächst eine Übersicht über die Hauptstrategien der erfolgreichen und der erfolglosen Probanden gegeben. Anschließende werden die Arbeitsweisen der Probanden noch einmal detailliert analysiert. Dabei berücksichtigen wir im Rahmen der Strategien „Bleistift und Papier“ und „Differenzenfolge“ nur die erfolgreichen Probanden. Im Hinblick auf das experimentelle Arbeiten werden die Arbeitsweisen sämtlicher Probanden genauer untersucht.

\subsubsection{Lösungsstrategien}

\section{Erfolgreiche Probanden}

Bei den erfolgreichen Probanden lassen sich fünf verschiedene Lösungsstrategien unterscheiden (vgl. Tab. 79). Die ersten drei Strategien entsprechen den im Rahmen des Lernprogramms aufgezeigten Wegen zur Lösung des Zählproblems. Ein Teil der Probanden kombinierte zwei der aufgezeigten Lösungsstrategien. Einige Schüler und Studierende lösten die Aufgabe auf zwei verschiedenen Wegen.

\begin{tabular}{|l||c|c|}
\hline & Schüler (30) & Studierende (12) \\
\hline \hline Bleistift und Papier & $33 \%(10)$ & $50 \%(6)$ \\
\hline Differenzenfolge & $7 \%(2)$ & $17 \%(2)$ \\
\hline Experimentieren & $53 \%(16)$ & $8 \%(1)$ \\
\hline Mischstrategie Diff. und Exp. & $0 \%(0)$ & $17 \%(2)$ \\
\hline Zwei verschiedene Lösungen & $7 \%(2)$ & $8 \%(1)$ \\
\hline
\end{tabular}

Tab. 79: Aufgabe 4d - Strategien erfolgreicher Probanden

Wie eine weitere Analyse der Arbeitsweisen zeigt, setzten sich alle erfolgreichen Studierenden und $93 \%$ der erfolgreichen Schüler mit dem Lösungsvorschlag „Differenzenfolge“ auseinander und berechneten zum Teil auch die Differenzenfolge der Dreieckszahlen ( $77 \%$ der Schüler, $50 \%$ der Studierenden). Dennoch verwendeten - wie die obige Tabelle deutlich macht - nur $7 \%$ der Schüler und $34 \%$ der Studierenden Eigenschaften der Differenzenfolge zum Lösen der Aufgabe und wandten damit Kenntnisse über Beziehungen zwischen Folgen und Differenzenfolgen an. Dagegen bevorzugten $33 \%$ der Schüler und $50 \%$ der Studierenden die Lösung mit Bleistift und Papier.

Die Möglichkeit des experimentellen Problemlösens wurde von den Schülern signifikant häufiger als von den Studierenden in Anspruch genommen (Chiquadrat-Vierfeldertest, $p=0,04$ ). Während die Schüler jedoch rein experimentell arbeiteten und eine Anpassungsfunktion für die Dreieckszahlen bestimmten, kombinierten 17 \% der Studierenden die Strategie „Differenzenfolge“ und „Experimentieren“. Diese Probanden berechneten zunächst die erste und zweite 
Differenzenfolge der Dreieckszahlen und der „Testfolge“ $f$ und passten dann durch Variieren der Parameter sowohl $f$ als auch deren Differenzenfolgen an die Dreieckszahlen und deren Differenzenfolgen an (vgl. auch Kap. 6.4.4.4). Drei Probanden verfolgten beim Lösen der Aufgabe zwei verschiedene Wege. Zwei Schüler lösten die Aufgabe zunächst experimentell und ergänzten anschließend noch eine Lösung mit Bleistift und Papier. Ein Studierender löste die Aufgabe zunächst mit Bleistift und Papier und überprüfte seine Lösung danach auf experimentellem Wege.

\section{Erfolglose Probanden}

Die erfolglosen Probanden beschäftigten sich bis auf eine Ausnahme mit mindestens zwei Lösungsvorschlägen, d. h. sie klickten die entsprechenden Seiten an und lasen die dortigen Informationen. Die Lösungsansätze lassen sich nach drei wesentlichen Hauptstrategien klassifizieren (vgl. Tab. 80). So versuchte lediglich ein Studierender, die explizite Formel mit Hilfe der zeichnerischen Darstellung der Dreieckszahlen zu finden. Insgesamt 50 \% der Studierenden, aber nur $14 \%$ der Schüler können der Strategie „Differenzenfolge“ zugeordnet werden. Diese Probanden berechneten mit Hilfe der Tabelle die Differenzenfolge der Dreieckszahlen, nahmen zum Teil die Hilfestellungen des Lernprogramms („Hinweis“-Button) in Anspruch, konnten diese Hilfen aber nicht gewinnbringend zur Lösung des Problems einsetzen. Die anderen Lösungsvorschläge wurden zwar zum Teil zur Kenntnis genommen, es war hier jedoch keine weitere Aktivität beobachtbar. Nur $5 \%$ der Studierenden, aber $68 \%$ der Schüler näherten sich der Fragestellung auf experimentellem Wege. Die Schüler unternahmen damit signifikant häufiger den Versuch, das Problem experimentell zu lösen (Chiquadrat-Vierfeldertest, $p=0,007)$. Bei $18 \%$ der Schüler und $25 \%$ der Studierenden waren keine Bildschirmaktivitäten zu beobachten. Es ist davon auszugehen, dass diese Probanden sich nicht oder nur sehr oberflächlich mit der Problemstellung beschäftigten.

\begin{tabular}{|l||c|c|}
\hline & Schüler (22) & Studierende (8) \\
\hline \hline Bleistift und Papier & $0 \%(0)$ & $12 \%(1)$ \\
\hline Differenzenfolge & $14 \%(3)$ & $50 \%(4)$ \\
\hline Experimentieren & $68 \%(15)$ & $5 \%(1)$ \\
\hline Keine Aktivität & $18 \%(4)$ & $25 \%(2)$ \\
\hline
\end{tabular}

Tab. 80: Aufgabe 4d - Strategien erfolgloser Probanden

\subsubsection{Strategie „Bleistift und Papier“6}

Insgesamt $33 \%$ der Schüler und $50 \%$ der Studierenden bevorzugten den Lösungsweg „Bleistift und Papier“. Ein großer Teil dieser Probanden hatte sich zuvor ausführlich mit den anderen Lösungsvorschlägen beschäftigt, die Bearbeitung jedoch in allen Fällen erfolglos abgebrochen. Während die Schüler ihren Lösungsweg zum Teil ausführlich dokumentierten 
(vgl. Abb. 74), gaben die meisten Studierenden ihre Lösung auf dem entsprechenden Arbeitsblatt ohne weitere Erläuterungen an.

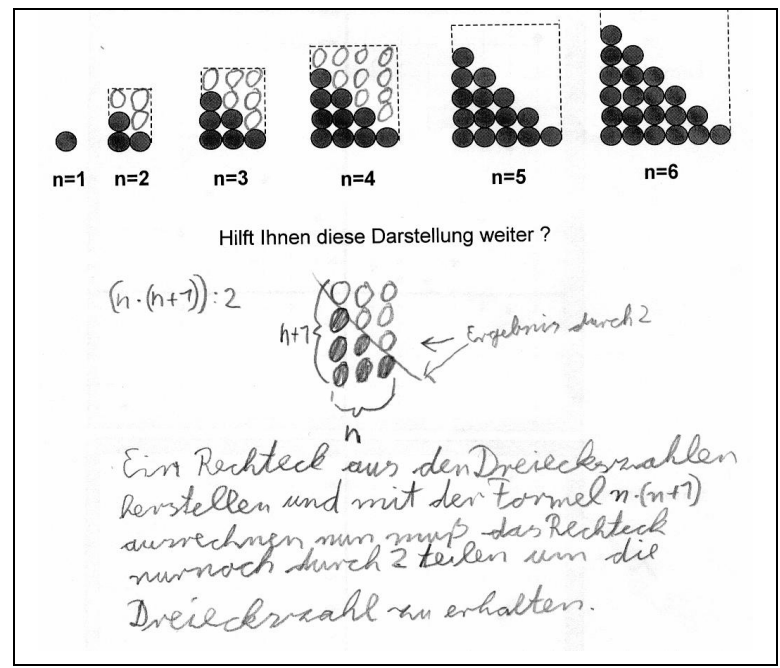

Abb. 74: Aufgabe 4d - Strategie „Mit Bleistift und Papier““

(Schüler B15)

Die große Bedeutung, die diesem Lösungsweg von den Schülern und insbesondere den Studierenden beigemessen wurde, kann insofern erklärt werden, als zum Erfassen der expliziten Formel auf der Grundlage der vorgegebenen zeichnerischen Darstellung keine umfangreichen algebraischen Vorkenntnisse erforderlich sind. Insbesondere werden keine Kenntnisse über Differenzenfolgen oder über den Einfluss von Parametern auf den Verlauf des Funktionsgraphen allgemeiner quadratischer und kubischer Folgen benötigt, und es müssen keine algebraischen Termumformungen durchgeführt werden. Darüber hinaus führt dieser Weg - sofern die Lösungsidee erkannt ist - sehr schnell und elegant zum Ziel, d. h. zur gesuchten expliziten Formel. Während die ersten drei „Kennzeichen“ dieses Lösungswegs die Vorgehensweisen eines großen Teils der (algebraisch schwachen) Schülern erklären könnte, lässt sich die Entscheidung der Studierenden für die Strategie „Bleistift und Papier“ (vermutlich) auf das vierte Argument zurückführen. Ein weiterer Grund für das Vorgehen dieser Studierenden kann darin gesehen werden, dass sie dem experimentellen Weg offensichtlich nur eine geringe Bedeutung beimaßen (vgl. auch die Kap. 6.4.4.4).

\subsubsection{Strategie, „Differenzenfolge“}

Zwei Schüler der Gruppe C und zwei Studierende konnten ihre im Rahmen von Aufgabe 3f erworbenen Kenntnisse über die formale Beziehung zwischen der allgemeinen quadratischen Z-Funktion $f$ mit $f(n)=a n^{2}+b n+c$ und deren Differenzenfunktion $D$ mit $D(n)=2 a n+a+b$ zur Lösung des Zählproblems gewinnbringend einsetzen. Der Einfluss der besonderen Thematisierung von Aufgabe 3f im Rahmen eines Klassengesprächs mit den Schülern der Gruppe C zeigt sich somit auch bei dieser Aufgabe. Die vier Probanden berech- 
neten zunächst mit Hilfe der Tabelle die Anfangsfolgenglieder der ersten Differenzenfolge, untersuchten deren Eigenschaften und identifizierten diese Folge als arithmetische Folge $D$ mit $D(n)=n+1$. Die Parameter $a$ und $b$ der quadratischen Ausgangsfolge $A$ mit $A(n)=a n^{2}+b n+c$ wurden im Anschluss durch Parametervergleich der Terme $n+1$ und $2 a n+a+b$ bestimmt. Mit der Nebenbedingung $A(1)=1$ fanden die Probanden abschließend den Parameter $c=0$. Die einzelnen Lösungsschritte sind in den Aufzeichnungen eines der beiden Schüler deutlich zu erkennen (vgl. Abb. 75).

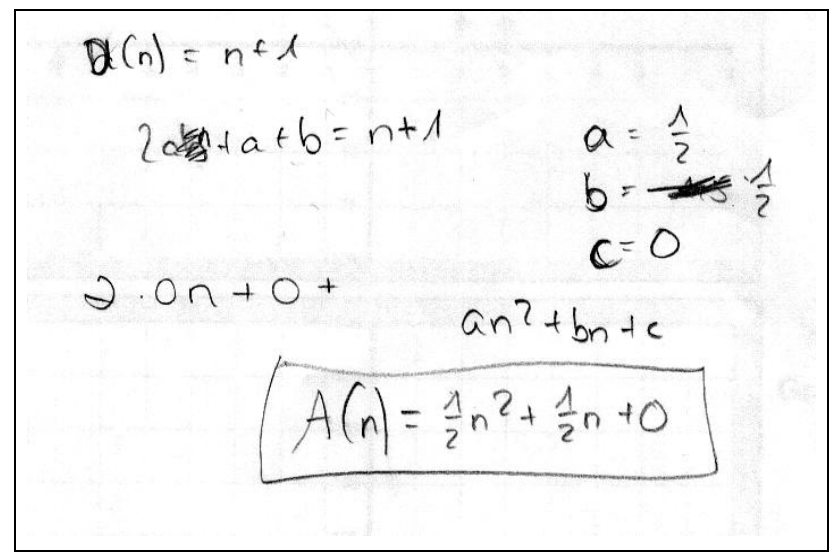

Abb. 75: Aufgabe 4d - Strategie „Differenzenfolge“ (Schüler C14)

\subsubsection{Experimentelles Problemlösen}

Es werden nun die Arbeitsweisen der erfolgreichen Schüler und Studierenden sowie der erfolglosen Probanden beim experimentellen Problemlösen genauer analysiert. Insbesondere soll untersucht werden, wie sich die Lösungsstrategien der einzelnen Probanden qualitativ unterscheiden. Da die Experimentierphase der meisten Schüler und Studierenden sowohl systematische als auch unsystematische Komponenten aufweist, muss eine andere Möglichkeit zur Unterscheidung der Arbeitsweisen gefunden werden. Im Folgenden erfolgt eine erste Differenzierung der Probanden auf der Basis des von ihnen im ersten Experimentierschritt ausgewählten Rollbalkens und des Werts, der dem entsprechenden Parameter nach diesem Experimentierschritt zugewiesen wurde. ${ }^{240}$ Insbesondere soll analysiert werden, welchen Parametern der Anpassungsfunktion direkt zu Beginn des Experimentierens der Wert Null zugewiesen wurde. Hierbei gehen wir davon aus, dass bereits der erste Experimentierschritt Rückschlüsse auf das Verständnis des Probanden zulässt. Wird etwa durch ein Betätigen des entsprechenden Rollbalkens dem Parameter $a$ der Anpassungsfolge $f$ mit

\footnotetext{
${ }^{240}$ Als Experimentierschritt bezeichnen wir die Auswahl eines bestimmten Parameters (Rollbalkens) und dessen Variation. Dabei wird nicht berücksichtigt, wie häufig ein bestimmter Rollbalken beim Experimentieren hin- und herbewegt wird. Es interessiert lediglich dessen „Endstellung“, also der Wert der dem Parameter letztendlich (im Rahmen dieses Experimentierschritts) zugewiesen wird.
} 
$f(n)=a n^{3}+b n^{2}+c n+d$ der Wert Null zugewiesen, kann davon ausgegangen werden, dass erkannt oder zumindest vermutet wurde, dass den Dreieckszahlen eine quadratische Gesetzmäßigkeit zugrunde liegt. Das Variieren des Parameters $d$ mit dem „Zielwert“ $d=0$ spricht dafür, dass die Folge der Dreieckszahlen offensichtlich gedanklich zu $A(0)=0$ extrapoliert und der Parameter $c$ als „Achsenabschnitt“ der Folge $f$ identifiziert wurde. Weist ein Proband allen Parametern zu Beginn den Wert Null zu, kann dies ebenfalls als strategisches Vorgehen gewertet werden: Ausgehend von der Funktion $f$ mit $f(n)=0$ ist es nun möglich, zunächst nur den Einfluss eines einzigen Parameters zu untersuchen. Wir erhalten damit das folgende Kategoriensystem:

I. $\quad$ Parameter $a=0$

Dem führenden Parameter des kubischen Polynoms $f$ mit $f(n)=a n^{3}+b n^{2}+c n+d$ wird im ersten Experimentierschritt der Wert Null zugewiesen.

II. $\quad$ Parameterd $=0$

Dem konstanten Parameter $d$ in $f(n)=a n^{3}+b n^{2}+c n+d$ wird im ersten Experimentierschritt der Wert Null zugewiesen.

III. Parameter $a=b=c=d=0$

$\mathrm{Zu}$ Beginn wird allen Parametern der Wert Null zugewiesen.

IV. Keine Einordnung möglich

Im Rahmen des ersten Experimentierschritts werden keinerlei inhaltliche oder strategische Überlegungen deutlich.

Bei der folgenden Analyse der Arbeitsweisen der Schüler und Studierenden wird sich zeigen, dass diese Klassifikation nicht nur eine Möglichkeit zur Beschreibung des ersten Experimentierschritts, sondern auch eine Ausgangsbasis zur Untersuchung des gesamten (teilweise auch unsystematischen) Lösungsprozesses darstellt.

\section{Arbeitsweisen der ,,erfolgreichen“ Schüler}

Die Arbeitsweisen und insbesondere der erste Experimentierschritt von $50 \%$ der 18 Schüler, welche die Aufgabe experimentell lösten, zeigen systematische Züge im Sinne der Kategorien I-III. Die übrigen Schüler arbeiteten weitestgehend unsystematisch (vgl. Tab. 81).

\begin{tabular}{|l||c|c|c|c|}
\hline & I & II & III & IV \\
\hline \hline Schüler (18) & $28 \%(5)$ & $11 \%(2)$ & $11 \%(2)$ & $50 \%(9)$ \\
\hline
\end{tabular}

Tab. 81: Aufgabe 4d - Experimentelles Arbeiten der erfolgreichen Schüler 
- Kategorie I

Insgesamt $28 \%$ der Schüler wählten zu Beginn der ersten bzw. zweiten Experimentierpha$\mathrm{se}^{241}$ für den Parameter $a$ den Wert Null. Dies weist darauf hin, dass diese Probanden die der Dreiecksfolge zugrunde liegende quadratische Gesetzmäßigkeit erkannt oder zumindest eine entsprechende Hypothese aufgestellt hatten. Wenngleich der erste Experimentierschritt bei allen Probanden identisch ist und das Experimentieren mit den Parametern weitestgehend als systematisch (mit unsystematischen Komponenten) bezeichnet werden kann, unterscheiden sich die Arbeitsweisen im Detail.

So variierte der Schüler B20 - wie der folgende Transkriptausschnitt ${ }^{242}$ verdeutlicht (vgl. Tab. 82) - die Parameter $a, b, c$ und $d$ in einer ersten Experimentierphase (Experimentierschritt 1-4) zunächst unsystematisch. Die den Parametern zugewiesenen Werte deuten nicht auf inhaltliche Überlegungen hin. Der betreffende Proband änderte daraufhin seine Strategie und berechnete zunächst die Differenzenfolge der Dreieckszahlen. In einer zweiten sich daran anschließenden Experimentierphase mit dem Graph, wies der Schüler dem führenden Parameter der Anpassungsfolge den Wert Null zu und beschränkte sich im Folgenden auf das Experimentieren mit den Parametern der quadratischen Testfolge $f$ mit $f(n)=b n^{2}+c n+d$. Dieses Vorgehen deutet darauf hin, dass die Differenzenfolge der Dreieckszahlen als eine arithmetische Folge identifiziert worden war und auf der Basis dieser Beobachtung auf eine quadratische Gesetzmäßigkeit der Dreieckszahlen geschlossen wurde. Der betreffende Schüler fand die gesuchte Folge im Rahmen der zweiten Experimentierphase nach 8 Experimentierschritten.

\begin{tabular}{|c||c|c|c|c|c|c|c|c|c|c|c|c|c|}
\hline \multicolumn{1}{|c||}{} & \multicolumn{9}{c||}{1. Experimentierphase } & \multicolumn{7}{c|}{ 2. Experimentierphase } \\
& \multicolumn{9}{|c|}{ Graph } & \multicolumn{7}{c|}{ Graph } \\
\hline \hline Exp.schritt & 0 & 1 & 2 & 3 & 4 & 5 & 6 & 7 & 8 & 9 & 10 & 11 & 12 \\
\hline \hline$a$ & 0,4 & 0,7 & & & & 0 & & & & & & & \\
\hline$b$ & $-1,5$ & & 1,1 & & & & 0,5 & & & & 0,5 & & \\
\hline$c$ & 2,3 & & & $-1,9$ & & & & 0,7 & & 0,6 & & 0,5 & \\
\hline$d$ & 37 & & & & 27 & & & & -1 & & & & 0 \\
\hline
\end{tabular}

Tab. 82: Aufgabe 4d - Experimentierschritte (Schüler B20) ${ }^{243}$

\footnotetext{
241 Als „Experimentierphase“ bezeichnen wir einen Lösungsversuch im Rahmen der Strategie „Experimentieren“, der nicht durch einen Wechsel zu einem anderen Excel-Blatt unterbrochen wurde bzw. bei dem die Probanden ihre Lösungsstrategie nicht grundsätzlich änderten.

${ }^{242}$ In diesem tabellarischen Transkript ist die Anzahl der Experimentschritte sowie der Wert des Parameters am Ende eines Experimentierschritts aufgeführt. In der zweiten Spalte der Tabelle (Experimentierschritt 0) sind die der Anpassungsfolge $f$ am Anfang zugewiesenen Parameter aufgelistet.
}

${ }^{243}$ Vgl. die ScreenCam-Datei B20.scm. 
Zwei weitere Schüler (A11 und B22) ${ }^{244}$ bestimmten zunächst die Anfangsfolgenglieder der Differenzenfolge der Dreieckszahlen mit Hilfe der Tabelle numerisch und nutzten die Möglichkeit des Experimentierens erst anschließend. Die offensichtlich bewusste Entscheidung für die Experimentier-Strategie sowie die Wahl des Parameters $a=0$ deutet (ähnlich wie bei den vorstehend beschriebenen Probanden) auf Kenntnisse über Eigenschaften von Folgen und deren Differenzenfolgen hin. Dennoch kann bei einem der beiden Probanden nicht ausgeschlossen werden, dass ein weiterer Impuls durch das Betätigen des „Hinweis-Buttons“6245 ausging. Die folgenden Experimentierschritte beider Probanden lassen zunächst keine strategischen oder inhaltlichen Überlegungen erkennen. So wies der Schüler A11 in einem weiteren Schritt dem führenden Parameter $b$ der (quadratischen) Anpassungsfolge $f$ mit $f(n)=b n^{2}+c n+d$ den negativen Wert $-0,7$ zu. Eine Korrektur des qualitativ vom gesuchten Ergebnis völlig abweichenden Graphen erfolgte erst im übernächsten Experimentierschritt, bei dem $b=0,5$ gefunden wurde. Der andere Schüler (B22) wählte zunächst $b=0$ und variierte die Parameter $c$ und $d$ in $f(n)=c n+d$. Nach einem bzw. 4 weiteren Experimentierschritten nahm das experimentelle Vorgehen beider Probanden jedoch wieder systematische Züge an: Beide Schüler schachtelten die gesuchten Parameter $b, c$ und $d$ sukzessive und reflektiert ein.

Während die oben beschriebenen Schüler ausschließlich mit dem Graph experimentierten, arbeiteten zwei Schüler (C2 und C10) zunächst mit der Tabelle und wechselten erst nach 3 bzw. 4 Experimentierschritten zum Graph. Dabei betrachtete einer der beiden Schüler $(\mathrm{C} 10)^{246}$ zunächst die quadratische Anpassungsfolge $f$ mit $f(n)=-n^{2}+c n+d$ und variierte nur die Parameter $c$ und $d$, ungeachtet der von der gesuchten Parabelform abweichenden geometrischen Gestalt des Graphen von $f$. Diese Experimentierphase erstreckte sich jedoch lediglich über zwei Experimentierschritte; der Proband war im Anschluss in der Lage, im Rahmen von 4 weiteren Experimentierschritten die Parameter $b$ und $c$ und $d$ durch systematisches Suchen zu finden. Auch das strategische Vorgehen des anderen Schülers (C2) verbesserte sich, nachdem er zum Graphen wechselte (vgl. Tab. 83). So wurden die Parameter $b$ und $c$ sukzessive innerhalb von 6 Experimentierschritten eingeschachtelt.

\footnotetext{
${ }^{244}$ Vgl. die ScreenCam-Dateien A11b.scm und B22.scm.

${ }^{245}$ Der „Hinweis-Button“ sollte die Probanden zu einem zielgerichteten Experimentieren anleiten. Indem die Frage aufgeworfen wird, ob bestimmten Parametern der Wert Null zugewiesen werden kann, sollte die Aufmerksamkeit der Probanden auf Eigenschaften der Folgen $A$ und $f$ gerichtet und die Anzahl der von ihnen betrachteten Parameter eingeschränkt werden.

${ }^{246}$ Vgl. die ScreenCam-Datei C10.scm.
} 


\begin{tabular}{|c||c|c|c|c||c|c|c|c|c|c|}
\hline \multicolumn{1}{|c||}{} & \multicolumn{3}{c||}{$\begin{array}{l}\text { 1. Exp.phase } \\
\text { Tabelle }\end{array}$} & \multicolumn{5}{c|}{$\begin{array}{c}\text { 2. Exp.phase } \\
\text { Graph }\end{array}$} \\
\hline \hline Exp.schritt & 0 & 1 & 2 & 3 & 4 & 5 & 6 & 7 & 8 & 9 \\
\hline \hline$a$ & 0,4 & 0 & & & & & & & & \\
\hline$b$ & $-1,5$ & & $-0,5$ & & 0,4 & & 0,6 & & 0,5 & \\
\hline$c$ & 2,3 & & & & & 1,7 & & 0 & & 0,5 \\
\hline$d$ & 37 & & & 0 & & & & & & \\
\hline
\end{tabular}

Tab. 83: Aufgabe 4d - Experimentierschritte (Schüler C2) ${ }^{247}$

Beide Probanden hatten sich vor dem experimentellen Lösen der Aufgabe mit dem Lösungsvorschlag „Differenzenfolge“ beschäftigt. Im Unterschied zum Schüler C10 hatte der Schüler C2 jedoch die Differenzenfolge tatsächlich berechnet, so dass angenommen werden kann, dass beim Variieren der Parameter und insbesondere bei der Wahl des Werts für den Parameter $a$ Kenntnisse über die Beziehung zwischen Folgen und Differenzenfolgen einflossen. Die Hintergründe für das Vorgehen des Probanden C10 sind dagegen weniger offensichtlich. Möglicherweise lag dem ersten Experimentierschritt die allgemeine strategische Überlegung, die Anzahl der zu variierenden Parameter einzuschränken, zugrunde.

\section{- Kategorie II}

Zwei Schüler (A9 und A12) variierten zu Beginn der Experimentierphase zunächst den Parameter $d$ in $f(n)=a n^{3}+b n^{2}+c n+d$ und setzten $d=0$. Da beide Probanden von Anfang an mit den graphischen Darstellungen der Folgen $f$ und $A$ arbeiteten, ist anzunehmen, dass $d$ als „Achsenabschnitt“ von $f$ identifiziert und dem ,fiktiven“ Achsenabschnitt der Folge der Dreieckszahlen, d. h. den Koordinaten (0, 0), angepasst wurde. Die folgende Experimentierphase beider Schüler lässt keine Rückschlüsse auf inhaltliche oder strategische Überlegungen zu. Wie der folgende Transkriptausschnitt (vgl. Tab. 84) verdeutlicht, wählte der Schüler A9 erst im 7. Experimentierschritt für den Parameter $a$ den Wert Null. Erst in den darauf folgenden Experimentierschritten wurden die Parameter $b$ und $c$ durch systematisches Suchen gefunden.

\begin{tabular}{|c||c|c|c|c|c|c|c|c|c|c|c|c|}
\hline \multicolumn{1}{|c||}{} & \multicolumn{10}{c|}{ Graph } \\
\hline \hline Exp.schritt & 0 & 1 & 2 & 3 & 4 & 5 & 6 & 7 & 8 & 9 & 10 & 11 \\
\hline \hline$a$ & 0,4 & & & & & & & 0 & & & & \\
\hline$b$ & $-1,5$ & & & $-0,9$ & & & 0,1 & & 0,5 & & & \\
\hline$c$ & 2,3 & & 0 & & & 0 & & & & 0,4 & & 0,5 \\
\hline$d$ & 37 & 0 & & & 2 & & & & & & 0 & \\
\hline
\end{tabular}

Tab. 84: Aufgabe 4d - Experimentierschritte (Schüler A9) ${ }^{248}$

\footnotetext{
${ }^{247}$ Vgl. die ScreenCam-Datei C2.scm.

${ }^{248}$ Vgl. die ScreenCam-Dateien A9.scm.
} 
Ein ähnliches Vorgehen ist bei dem Schüler A12 ${ }^{249}$ erkennbar. Obwohl beide Probanden zuvor die Differenzenfolge berechnet hatten, ist anzunehmen, dass kein Transfer von Kenntnissen über die Eigenschaften einer quadratischen Folge und deren Differenzenfolge erfolgte. Vielmehr liegt die Vermutung näher, dass beide Schüler offensichtlich im Laufe des Experimentierens „erkannten“, dass der absolute Wert des Parameters $a$ so klein wie möglich sein sollte, um die Auswirkung auf den Verlauf des Graphen soweit wie möglich zu reduzieren. Dabei erhielt der Schüler A9 durch das Betätigen des „Hinweis-Buttons“ offensichtlich einen zusätzlichen Impuls, dem Parameter $a$ den Wert Null zuzuweisen.

\section{- Kategorie III}

Zwei Schüler (A5 und C17) ${ }^{250}$ wiesen bereits zu Beginn der Experimentierphase allen Parametern $a, b, c, d$ der Folge $f$ mit $f(n)=a n^{3}+b n^{2}+c n+d$ den Wert Null zu. Beide Schüler hatten zuvor die Differenzenfolge der Dreieckszahlen bestimmt. Das Vorgehen des Schülers A5 lässt jedoch nur bedingt auf Transferleistungen schließen. So ist der erste Experimentierschritt in erster Linie auf das Betätigen des Hinweis-Buttons zurückzuführen. Dagegen weist der sich daran anschließende Suchprozess darauf hin, dass der Proband bei der Folge der Dreieckszahlen eine quadratische Gesetzmäßigkeit vermutete (vgl. Tab. 85).

\begin{tabular}{|c||c|c|c|c|c|c|c|c|c|}
\hline \multicolumn{1}{|c||}{} & \multicolumn{10}{|c|}{ Graph } \\
\hline \hline Exp.schritt & 0 & 1 & 2 & 3 & 4 & 5 & 6 & 7 & 8 \\
\hline \hline$a$ & 0,4 & 0 & & & 0 & & & & \\
\hline$b$ & $-1,5$ & 0 & & 0,4 & & 0,4 & & 0,5 & \\
\hline$c$ & 2,3 & 0 & 2,3 & & & & 1,8 & & 0,5 \\
\hline$d$ & 37 & 0 & & & & & & & \\
\hline
\end{tabular}

Tab. 85: Aufgabe 4d - Experimentierschritte (Schüler A5)

Der Schüler C17 hatte den „Hinweis-Button“ nicht in Anspruch genommen. Im Unterschied zu Proband A5 arbeitete er zunächst mit der Tabelle und wechselte erst im dritten Experimentierschritt zum Graph. Die Experimentierphase mit der graphischen Darstellung der Anpassungsfunktion kann insofern als systematisch bezeichnet werden, als die gesuchten Parameter $b, c$ und $d$ sukzessive im Rahmen von 15 Experimentierschritten eingeschachtelt wurden.

\section{- Kategorie IV}

Die Arbeitsweisen von $50 \%$ der Schüler können insofern als unsystematisch bezeichnet werden, als in weiten Teilen der Experimentierphase keinerlei Rückschlüsse auf inhaltliche oder strategische Überlegungen gezogen werden können. Die Experimentierphasen umfassten da-

\footnotetext{
${ }^{249} \mathrm{Vgl}$ die ScreenCam-Datei A12.scm.

${ }^{250}$ Vgl. die ScreenCam-Dateien A5.scm und C17.scm.
} 
bei bis zu 32 Experimentierschritte. Bis auf zwei Probanden experimentierten die Schüler zum Teil ausgiebig mit der inadäquaten Darstellung Tabelle und auch das Arbeiten mit dem Graph ließ bei allen Probanden wenig Systematik erkennen. Zwei Beispiele sollen dies verdeutlichen.

\section{Beispiel 1:}

Der Schüler C11 arbeitete - wie der folgende Transkriptausschnitt (vgl. Tab. 86) verdeutlicht - zweimal mit der Tabelle. Sowohl in der zweiten als auch in der dritten Experimentierphase wurden alle Parameter unreflektiert und in schneller zeitlicher Abfolge variiert. ${ }^{251}$ Eine „Systematik“ zeigt sich nur insofern, als die Rollbalken konsequent in der Reihenfolge ihrer Auflistung betätigt wurden, also zunächst von „oben“ nach „unten“ und dann in der umgekehrten Richtung. Erst das Betätigen des „Hinweis-Buttons“ zwischen den Experimentierschritten 21 und 22 beeinflusste das Vorgehen des Probanden. So wurde zunächst allen Parametern der Wert Null zugewiesen. Anschließend war der Schüler in der Lage, die Werte für die Parameter $b$ und $c$ durch systematisches Suchen zu bestimmen.

\begin{tabular}{|c||c|c||c|c|c|c||c|c|c|c|c|c|c|}
\hline \multicolumn{1}{|c||}{} & \multicolumn{1}{|c||}{$\begin{array}{l}\text { 1. Exp.phase } \\
\text { Tabelle }\end{array}$} & \multicolumn{3}{c||}{$\begin{array}{c}\text { 2. Exp.phase } \\
\text { Graph }\end{array}$} & \multicolumn{7}{c|}{ 3. Exp.phase } \\
& \multicolumn{2}{|c|}{} & \multicolumn{7}{c|}{ Tabelle } \\
\hline \hline Exp.schritt & 0 & 1 & 2 & 3 & 4 & 5 & 6 & 7 & 8 & 9 & 10 & 11 & 12 \\
\hline$a$ & 0,4 & -1 & & & & 0 & & & & 0,4 & & & \\
\hline$b$ & $-1,5$ & & 1,4 & & & & & & $-1,8$ & & $-1,1$ & & \\
\hline$c$ & 2,3 & & & 0,6 & & & & 3,2 & & & & 3,8 & \\
\hline$d$ & 37 & & & & 33 & & -37 & & & & & & -30 \\
\hline
\end{tabular}

\begin{tabular}{|c||c|c|c|c|c|c|c|c|c|c|c|c|c|}
\hline \multicolumn{1}{|c||}{} & \multicolumn{3}{c|}{ Tabelle } & \multicolumn{10}{c|}{ Gxperimentierphase } \\
\hline Exp.schritt & 13 & 14 & 15 & 16 & 17 & 18 & 19 & 20 & 21 & 22 & 23 & 24 & 25 \\
\hline$a$ & & & 0,7 & & & & 0,2 & & 0 & & & & \\
\hline$b$ & & 0 & & & & $-1,9$ & & & 0 & 0,4 & & 0,5 & \\
\hline$c$ & 4,9 & & & & 3,2 & & & & 0 & & 0,4 & & 0,5 \\
\hline$d$ & & & & -37 & & & & -36 & 0 & & & & \\
\hline
\end{tabular}

Tab. 86: Aufgabe 4d - Experimentierschritte (Schüler C11) $)^{252}$

${ }^{251}$ Insbesondere wurde der Parameter $d$ im Rahmen der Experimentierphase mit der graphischen Darstellung dreimal variiert. Dabei wurden ihm völlig inadäquate Werte zugewiesen.

${ }^{252}$ Vgl. die ScreenCam-Datei C11.scm. 


\section{Beispiel 2:}

Der Schüler B26 arbeitete zwar ausschließlich mit dem Graph, fand aber - wie der folgende Transkriptausschnitt (vgl. Tab. 87) verdeutlicht - zunächst keine sinnvolle Experimentierstrategie, obwohl er zuvor die Differenzenfolge der Dreieckszahlen bestimmt hatte. So wurde dem führenden Parameter der Anpassungsfolge $f$ mit $f(n)=a n^{3}+b n^{2}+c n+d$ im ersten Experimentierschritt der Wert 0,2 zugewiesen. Auch wenn der Schüler möglicherweise erkannt hatte, dass der Einfluss des Parameters $a$ auf den Verlauf des Graphen von $f$ soweit wie möglich reduziert werden musste, fehlte ihm dennoch der „Mut“, diesem Parameter den Wert Null zuzuweisen. Im Folgenden variierte der Schüler B26 ähnlich wie der vorstehend beschriebene Schüler C11 zunächst alle Parameter unreflektiert und in schneller zeitlicher Abfolge in der Reihenfolge ihrer Auflistung. Auch hier wurde das Experimentieren erst mit dem Aufruf des „Hinweis-Buttons“ (Experimentierschritt 6) systematischer.

\begin{tabular}{|c||c|c|c|c|c|c|c|c|c|c|c|c|c|}
\hline \multicolumn{1}{|c||}{} & \multicolumn{10}{c|}{ Graph } \\
\hline \hline Exp.schritt & 0 & 1 & 2 & 3 & 4 & 5 & 6 & 7 & 8 & 9 & 10 & 11 & 12 \\
\hline \hline$a$ & 0,4 & 0,2 & & & & & 0 & 0 & & & & & \\
\hline$b$ & $-1,5$ & & $-1,6$ & & & & 0 & & 0,5 & & & & \\
\hline$c$ & 2,3 & & & 2,2 & & 0,4 & 0 & & & 0,2 & & 0,5 & \\
\hline$d$ & 37 & & & & -8 & & 0 & & & & 2 & & 0 \\
\hline
\end{tabular}

Tab. 87: Aufgabe 4d - Experimentierschritte (Schüler B26) ${ }^{253}$

\section{Arbeitsweisen der ,erfolgreichen“"Studierenden}

Von den 12 Studierenden, welche die Aufgabe erfolgreich lösten, arbeiteten vier Probanden mit der experimentellen Versuchsumgebung. Ein Studierender überprüfte auf diesem Wege seine mit Bleistift und Papier gefundene Lösung. Ein weiterer Proband fand die explizite Darstellung durch ein im Sinne von Kategorie IV unsystematisches Versuch-und-IrrtumVerfahren. Das Vorgehen der übrigen beiden Studierenden, welche die Strategien „Experimentieren“ und „Differenzenfolge“ kombinierten, weist dagegen auf ein instrumentelles Verständnis im Umgang mit Differenzenfolgen hin. Dies soll am Beispiel des Studierenden I20 verdeutlicht werden (vgl. Tab. 88):

\footnotetext{
${ }^{253}$ Vgl. die ScreenCam-Datei B26.scm.
} 


\begin{tabular}{|c||c|c||c|c|c|c|c|c||c|}
\hline & \multicolumn{2}{|c||}{$\begin{array}{c}\text { 1. Ex.phase } \\
\text { Tabelle }\end{array}$} & \multicolumn{2}{c||}{$\begin{array}{c}\text { 2. Exp.phase } \\
\text { Tabelle }\end{array}$} & \multicolumn{2}{c|}{$\begin{array}{c}\text { 3. Exp.phase } \\
\text { Tabelle }\end{array}$} & $\begin{array}{c}\text { 4. Exp.phase } \\
\text { Tabelle }\end{array}$ \\
\hline \hline Exp.schritt & 0 & 1 & 2 & 3 & 4 & 5 & 6 & 7 \\
\hline \hline$a$ & 0,4 & 0,02 & 0 & & & & & & \\
\hline$b$ & $-1,5$ & & & 0,5 & & & & & \\
\hline$c$ & 2,3 & & & & 4,9 & 0,5 & & & \\
\hline$d$ & 37 & & & & & & -30 & & 0 \\
\hline
\end{tabular}

Tab. 88: Aufgabe 4d - Experimentierschritte (Studierender I20)

Der Studierende I20 arbeitete zunächst in der „Experimentierumgebung“. Nach einem ersten Experimentierschritt mit der tabellarischen Darstellung änderte der Proband seine Strategie und berechnete unter Berücksichtigung des Lösungsvorschlags „Differenzenfolge“ zunächst die Anfangsfolgenglieder der ersten und zweiten Differenzenfolge der Dreiecksfolge. Auf der Basis der dabei gemachten Beobachtungen ${ }^{254}$ wechselte er erneut zur „Experimentierumgebung“, berechnete in der Tabelle die erste und zweite Differenzenfolge der Anpassungsfolge $f$ und variierte im Anschluss die Parameter $a$ und $b$ in $f(n)=a n^{3}+b n^{2}+c n+d$ so, dass die Glieder der 2. Differenzenfolge der Folge $f$ zunächst den konstanten Wert -3 und dann den konstanten Wert 1 annahmen (Experimentierschritt 2 und 3, vgl. auch Abb. 76).

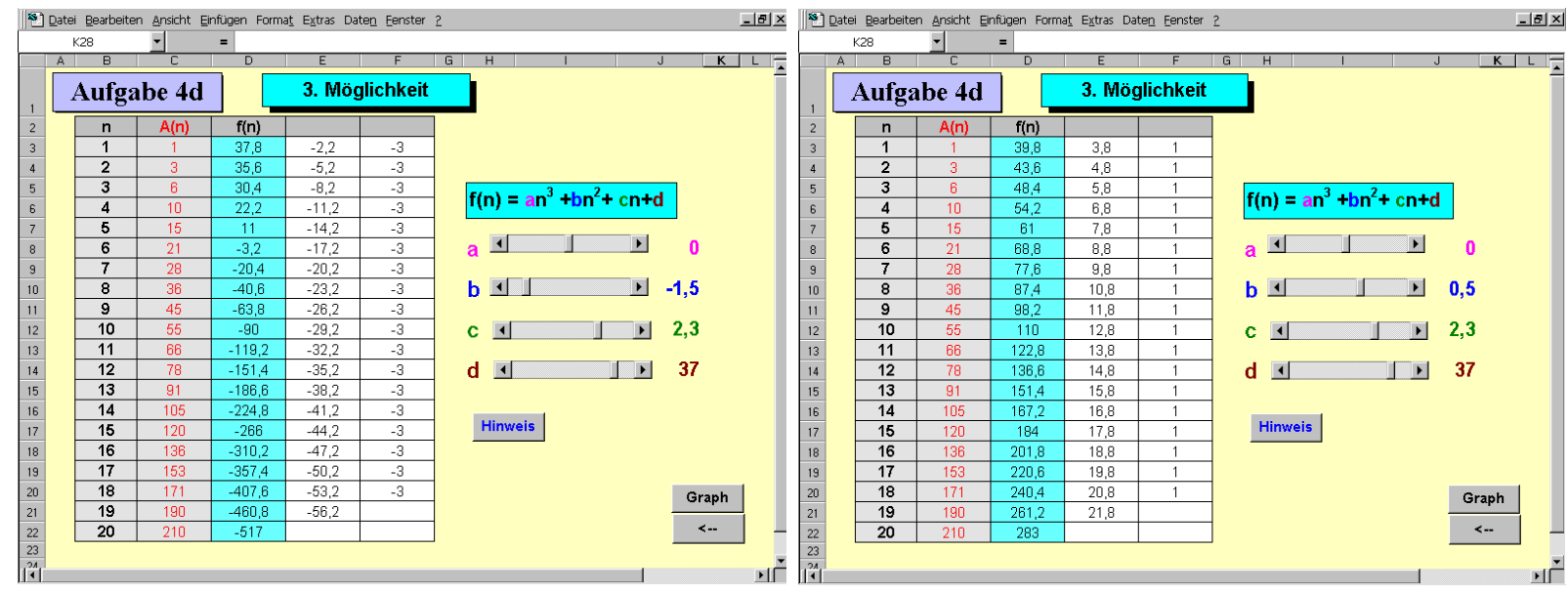

Abb. 76: Aufgabe 4d - 2. und 3. Experimentierschritt des Studierenden $\mathbf{I 2 0}^{255}$

Der Studierende brach die zweite Experimentierphase ab, nachdem er im Rahmen des 4. Experimentierschritts erfolglos mit dem Parameter $c$ experimentiert hatte, und wechselte zurück zum Lösungsvorschlag „Differenzenfolge“. Dabei konzentrierte sich der Proband auf den Folgenanfang der 1. Differenzenfolge der Dreieckszahlen. Auf der Basis dieser Beobachtung passte der Proband im Rahmen eines 5. Experimentierschritts durch Variieren des Parameters

\footnotetext{
${ }^{254}$ Die 2. Differenzenfolge der Folge der Dreieckszahlen ist konstant und die Folgenglieder haben den Wert 1.

${ }^{255}$ Vgl. die ScreenCam-Datei I20.scm.
} 
$c$ die 1. Differenzenfolge der Folge $f$ an die 1. Differenzenfolge der Dreiecksfolge an und fand damit $c=0,5$ (vgl. Abb. 77).

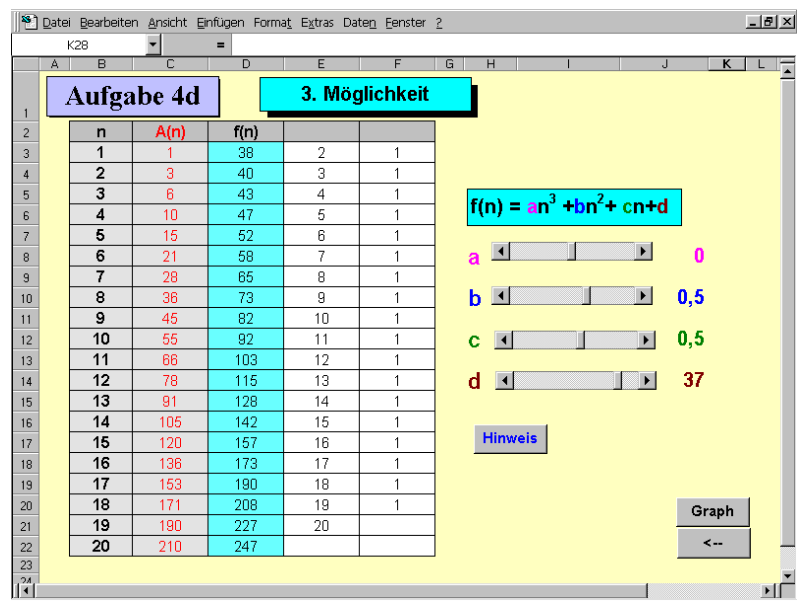

Abb. 77: Aufgabe 4d - 5. Experimentierschritt des Studierenden I20

Wie bereits in der vorhergehenden Experimentierphase wurde der als nächstes zu bestimmende Parameter (in diesem Fall der Parameter $d$ ) zunächst nur unsystematisch variiert (Experimentierschritt 6). Nachdem der Proband ein weiteres Mal zum Lösungsvorschlag „Differenzenfolge“ und wieder zurück zur „Experimentierumgebung“ gewechselt war, konnte er auch die Folge $f$ selbst an die Folge der Dreieckszahlen anpassen und $d=\theta$ bestimmen (vgl. Abb. 78).

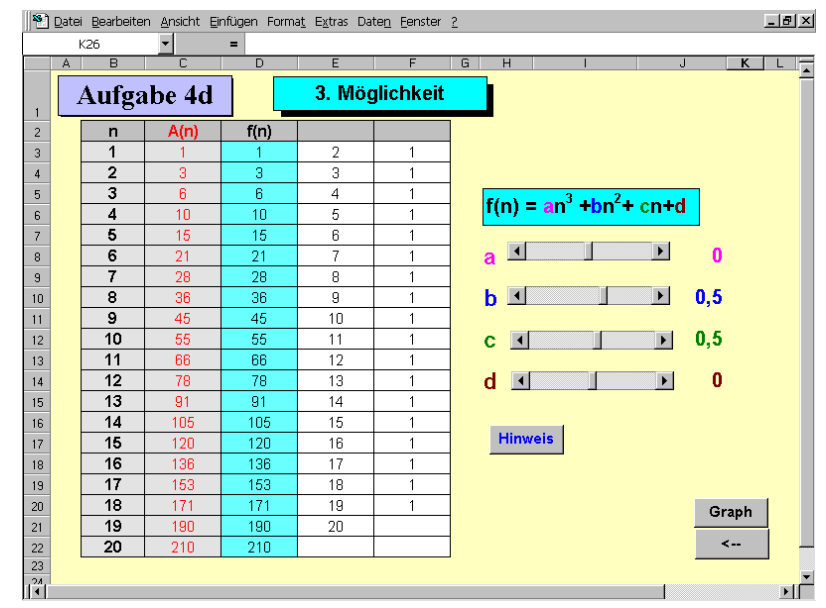

Abb. 78: Aufgabe 4d - 7. Experimentierschritt des Studierenden I20

\section{Erfolglose Probanden}

Wie bereits in Kap. 6.4.4.1 herausgestellt, haben die erfolglosen Schüler signifikant häufiger als die Studierenden den Versuch unternommen, eine explizite Darstellung für die Dreieckszahlen auf experimentellem Wege zu bestimmen. Dabei lassen sich auch im Umgang mit der „Experimentierumgebung“ deutliche Unterschiede zwischen den Probandengruppen feststellen. So experimentierten die Schüler unsystematisch und zum Teil ausschließlich mit der in- 
adäquaten Darstellung Tabelle. Die Experimentierphasen waren dabei vergleichsweise lang: Insgesamt 9 der 15 erfolglos experimentierenden Schüler tätigten mehr als 7 Experimentierschritte; zwei Schüler brachen die Bearbeitung sogar erst nach 20 Schritten ab. Einen Einblick in das experimentelle Arbeiten eines dieser Probanden gibt der folgende Transkriptausschnitt:

\begin{tabular}{|c||c|c|c|c|c|c|c|c|c|}
\hline \multicolumn{1}{|c||}{} & \multicolumn{5}{|c|}{$\begin{array}{c}\text { Exp.schritt } \\
\text { Tabelle }\end{array}$} & \multicolumn{5}{c|}{$\begin{array}{c}\text { Exp.schritt } \\
\text { Graph }\end{array}$} \\
\hline \hline Exp.schritt & 0 & 1 & 2 & 3 & 4 & 5 & 6 & 7 & 8 \\
\hline \hline$a$ & 0,4 & 0,6 & & & & & & & 1 \\
\hline$b$ & $-1,5$ & & $-1,3$ & & & & & $-0,5$ & \\
\hline$c$ & 2,3 & & & 2,5 & & & -5 & & \\
\hline$d$ & 37 & & & & 40 & 50 & & & \\
\hline
\end{tabular}

Tab. 89: Aufgabe 4d - Experimentierschritte (Schüler A20) ${ }^{256}$

Im Gegensatz zu den Schülern brach der einzige Studierende, der eine experimentelle Lösung in Betracht zog, die Bearbeitung bereits nach einem Experimentierschritt mit der Tabelle ab. Das Experimentieren schien für diesen aber auch für die anderen Probanden, die einen formalen Lösungsversuch bevorzugten, offensichtlich keine Methode zur Lösungsgewinnung darzustellen.

\subsection{Antworten zu Aufgabe 5}

Bei dieser Aufgabe sollten die Schüler auf einem separaten Arbeitsblatt die Differenzenfunktion einer kubischen Z-Funktion graphisch ermitteln. Die Termdarstellung $f(n)=0,72 n^{3}+1,2 n^{2}-20 n+49$ der Ausgangsfunktion war den Schülern dabei nicht bekannt. ${ }^{257}$ Die Studierenden bearbeiteten eine ähnliche Fragestellung. Ihre Aufgabe war es, die Differenzenfunktion einer polynomialen Funktion vierten Grades zu bestimmen. Auch ihnen war die Termdarstellung der Ausgangsfunktion ${ }^{258}$ nicht bekannt.

\subsubsection{Ergebnisse der Schüler}

Die Schüler lassen sich bei dieser Aufgabe danach unterscheiden, ob sie eine qualitativ ,,richtige“ oder „falsche“ Differenzenfunktion skizzierten oder ob sie die Aufgabe nicht bearbeiteten. Die folgende Tabelle gibt die Häufigkeitsverteilung an:

\footnotetext{
${ }^{256}$ Vgl. die ScreenCam-Datei A20.scm.

${ }^{257} \mathrm{Zu}$ den Hintergründen für diese Art der Aufgabenpräsentation vgl. Kap. 5.6.5.

${ }^{258}$ Es handelt sich hierbei um die Funktion $f(n)=-0,072 n^{4}+0,03 n^{3}+5,2 n^{2}-26 n-65,5$.
} 


\begin{tabular}{|l||c|c|c|}
\hline & Richtig & Falsch & Keine Bearbeitung \\
\hline \hline Schüler (53) & $51 \%(27)$ & $30 \%(16)$ & $19 \%(10)$ \\
\hline
\end{tabular}

Tab. 90: Aufgabe 5 - Ergebnisse Schüler

Insgesamt $51 \%$ der Schüler skizzierten einen parabelförmigen Graph für $D$, der in einem weiteren Sinne als „richtige“ Lösung bezeichnet werden kann. Die Skizzen dieser Probanden geben die Bereiche positiven und negativen monotonen Wachstums, den Extremwert sowie die Bereiche, in denen die Funktionswerte der Differenzenfunktion ihr Vorzeichen wechseln, qualitativ richtig wieder. Einzelnen „Unregelmäßigkeiten“ (vgl. Abb. 79) und einer Verschiebung des Graphen um eine Einheit nach rechts, was auf die Verwendung der (falschen) Formel $D(n)=f(n)-f(n-1)$ hindeutet, werden im Rahmen dieser Klassifizierung vernachlässigt.

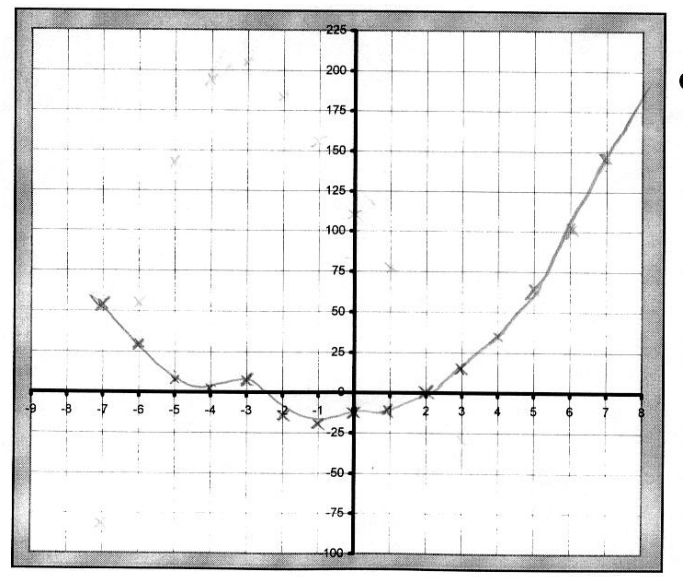

Abb. 79: Aufgabe 5 - Qualitativ richtige Lösung (Schüler A5)

Eine qualitativ falsche Differenzenfunktion (vgl. Abb. 80 und 81) gaben $30 \%$ der Probanden an.

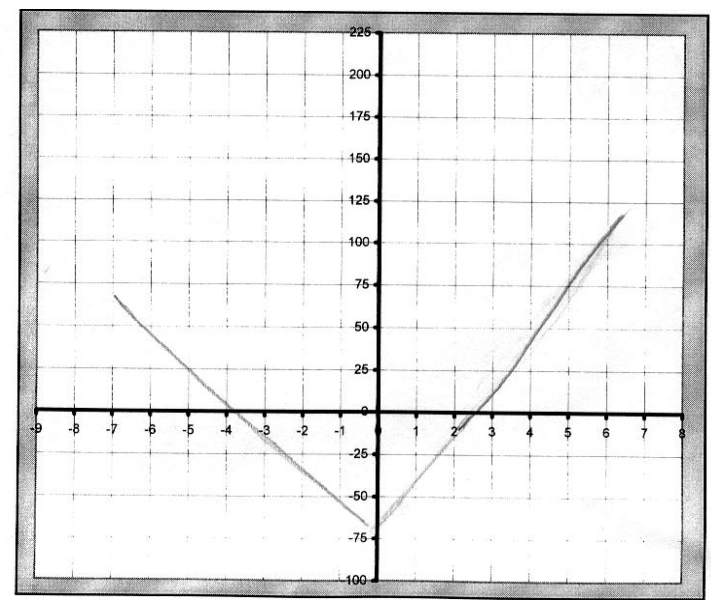

Abb. 80: Aufgabe 5 - Qualitativ falsche Lösung (Schüler B15)

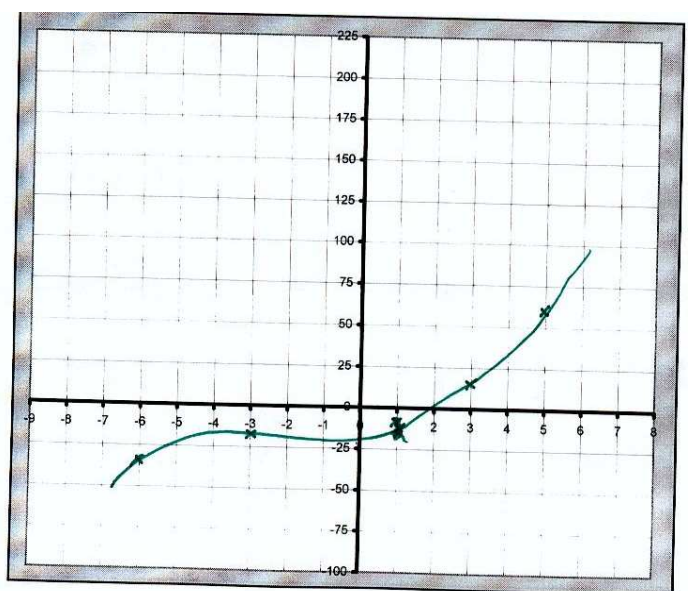

Abb. 81: Aufgabe 5 - Qualitativ falsche Lösung (Schüler A11) 
Insgesamt $19 \%$ der Probanden bearbeiteten die Aufgabe nicht. Offensichtlich hatten diese Probanden keine Zeit oder kein Interesse mehr, sich mit dieser letzten Aufgabe des Versuchsprogramms auseinander zu setzen.

Hinsichtlich der Art des skizzierten Graphen können drei Kategorien unterschieden werden, die im Folgenden mit jeweils einem prototypischen Beispiel angegeben werden:

I. Diskret (vgl. Abb. 82)

II. Kontinuierlich mit diskreten Stützstellen (vgl. Abb. 83)

III. Kontinuierlich (vgl. Abb. 84)

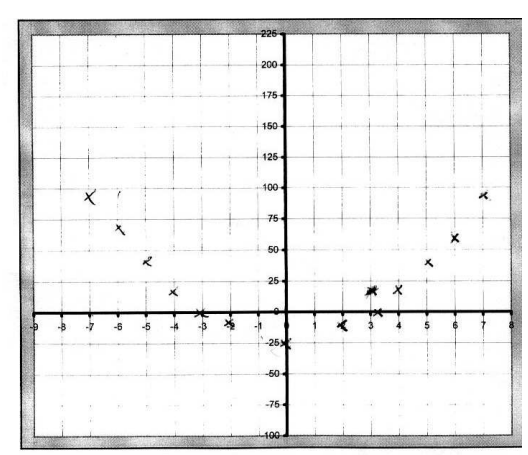

Abb. 82: Aufgabe 5 Skizzentyp I

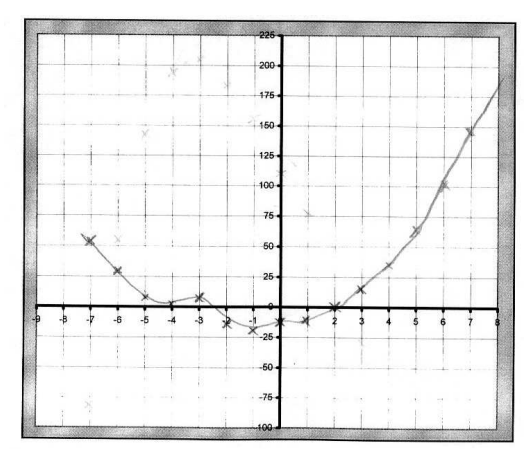

Abb. 83: Aufgabe 5 Skizzentyp II

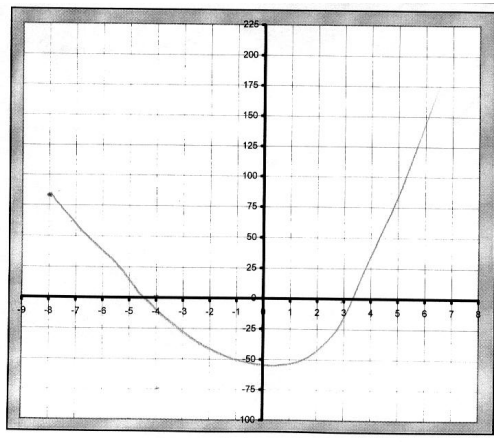

Abb. 84: Aufgabe 5 Skizzentyp III

Die folgende Tabelle gibt die Häufigkeitsverteilung an:

\begin{tabular}{|l||c|c|c|}
\hline & I & II & III \\
\hline $\begin{array}{l}\text { Richtige Lösungen } \\
\text { (27 Schüler) }\end{array}$ & $37 \%(10)$ & $33 \%(9)$ & $30 \%(8)$ \\
\hline $\begin{array}{l}\text { Falsche Lösungen } \\
\text { (16 Schüler) }\end{array}$ & $25 \%(4)$ & $19 \%(3)$ & $56 \%(9)$ \\
\hline
\end{tabular}

Tab. 91: Aufgabe 5 - Art des skizzierten Graphen

Insgesamt $37 \%$ der Schüler, die eine qualitativ richtige Lösung angaben, ,skizzierten“ die Differenzenfunktion im Sinne der Fragestellung als diskrete Funktion. Weitere $33 \%$ ermittelten zunächst die Funktionswerte der Funktion $D$, interpolierten jedoch im Anschluss zwischen diesen diskreten „Stützstellen“. Die restlichen Probanden skizzierten einen kontinuierlichen Graphen, was auf eine kontinuierliche Sicht auf die Eigenschaften der Ausgangsfunktion $f$ hindeutet. Interessant sind die Arbeitsweisen der ,erfolgreichen“ Probanden, die den Kategorien I und II zugeordnet werden können. So orientierte sich die Mehrheit dieser Schüler offensichtlich nicht an ausgezeichneten Eigenschaften der Ausgangsfunktion, sondern analysierte den Graph der Ausgangsfunktion $f$ schrittweise von „links nach rechts“. Diese Probanden bestimmten durch Deuten mit dem Cursor auf jeweils „benachbarte“ Koordinatenpunkte die Funktionswerte der Funktion $f$ und berechneten anschließend deren Differenz. Den Berechnungen wurde dabei zum Teil die falsche Formel $D(n)=f(n)-f(n-1)$ zugrunde gelegt. 
Die Mehrheit der Probanden (56 \%), die eine qualitativ falsche Lösung angaben, „skizzierte“ einen kontinuierlichen Graphen. Die meisten Schüler beschrieben dabei die Differenzenfunktion durch zwei stückweise lineare Funktionen (vgl. Abb. 80). Dieses überraschende Fehlermuster kann auf einen fehlerhaften Transfer von Kenntnissen über quadratische Z-Funktionen zurückgeführt werden. So wurde die kubische Z-Funktion offensichtlich als eine aus zwei stückweise quadratischen Z-Funktionen zusammengesetzte Funktion aufgefasst, deren Differenzenfunktionen lineare Z-Funktionen darstellen.

\subsubsection{Ergebnisse der Studierenden}

Auch die Studierenden werden hinsichtlich qualitativ richtiger und falscher Lösungen (vgl. Abb. 85 und 86) unterschieden.

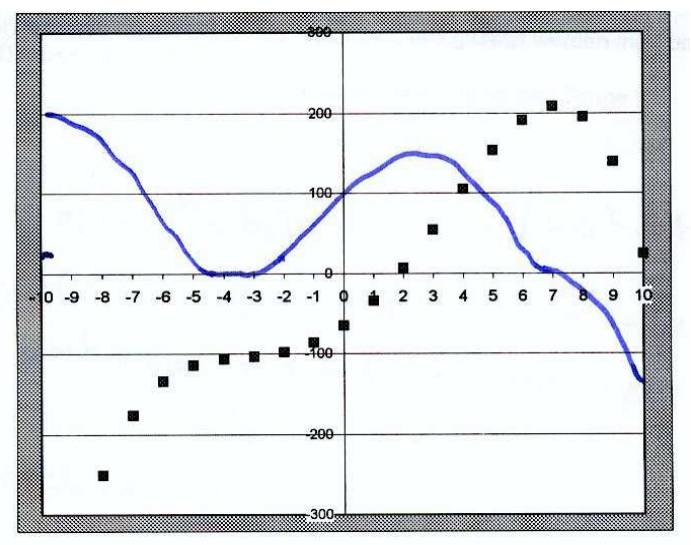

Abb. 85: Aufgabe 5 - Qualitativ richtige Lösung (Studierender II6)

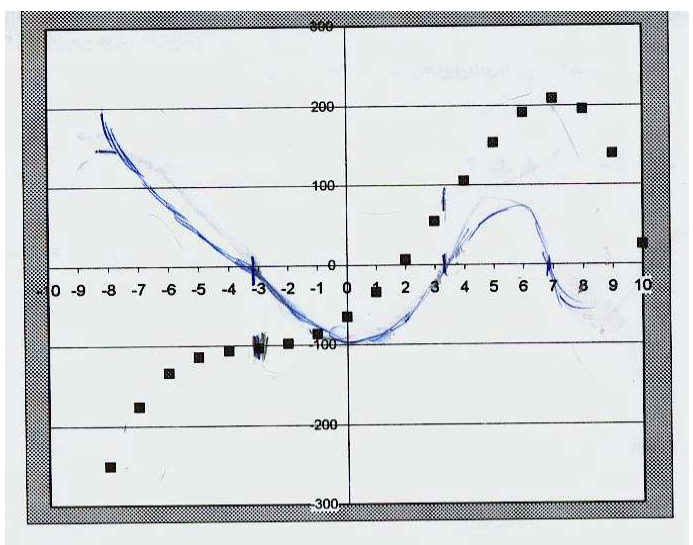

Abb. 86 Aufgabe 5 - Qualitativ falsche Lösung (Studierender I20)

Die folgende Tabelle gibt die entsprechende Häufigkeitsverteilung an.

\begin{tabular}{|l||c|c|c|}
\hline & Richtig & Falsch & Keine Bearbeitung \\
\hline \hline Studierende (21) & $33 \%(7)$ & $43 \%(9)$ & $24 \%(5)$ \\
\hline
\end{tabular}

Tab. 92: Aufgabe 5 - Ergebnisse Studierende

Ein statistischer Vergleich der Gruppe der Studierenden und der Schüler ist aufgrund der unterschiedlichen zugrunde liegenden Funktionen nicht sinnvoll. Dennoch sollte festgehalten werden, dass überraschenderweise prozentual mehr Schüler als Studierende die Aufgabe des graphischen „Differenzenbildens“ richtig lösten. Dies kann zum einen auf die geringere Komplexität der Z-Funktion $f$ zurückgeführt werden. Zum anderen erwies sich das schrittweise Vorgehen der Schüler und das Berechnen einzelner Funktionswerte der Differenzenfunktion unter Verwendung der graphischen Darstellung der Ausgangsfunktion als wichtige Strategie zur Lösungsgewinnung. Im Unterschied zu den Schülern haben die Studierenden ausschließlich kontinuierliche Graphen skizziert. Hier ist der Einfluss einer durch die Differenzialrechnung geprägten kontinuierlichen Sichtweise erkennbar. Das Durcharbeiten des Testpro- 
gramms reichte offensichtlich nicht aus, um einen Sichtwechsel herbeizuführen und um - insbesondere bei dieser Aufgabe - „diskretes“ Arbeiten zu initiieren, ist offensichtlich ein alleiniges Durcharbeiten des Testprogramms nicht ausreichend. 


\section{Diskussion der Ergebnisse}

Die Ergebnisse der empirischen Untersuchung zum Themenkomplex „Differenzenfunktionen" werden in diesem Kapitel hinsichtlich der in Kap. 5.1 aufgeführten Interessensschwerpunkte - die Arbeitsweisen der Probanden und das zugrunde liegende bzw. erreichte Begriffsverständnis - zusammengefasst und diskutiert. Die gewonnenen Ergebnisse lassen sich an einigen Stellen zu verallgemeinerten Aussagen verdichten, die als Hypothesen für zukünftige Untersuchungen dienen können.

\subsection{Diskrete Arbeitsweisen}

Bei der Diskussion der Arbeitsweisen beziehen wir uns auf die verschiedenen Arbeitsebenen: die Objektebene, die Darstellungsebene und die Werkzeugebene (vgl. Kap. 3.3 und Kap. 4.4). Da sowohl die drei Arbeitsebenen untereinander als auch die Arbeits- und Verständnisebenen miteinander in Beziehung stehen, ist eine trennscharfe Analyse der einzelnen Ebenen nicht möglich. Wir werden jedoch im Folgenden versuchen, die jeweilige Analysedimension durch eine geeignete Betonung der relevanten Aspekte in den Vordergrund zu stellen.

\subsubsection{Arbeiten auf der Objektebene}

Hinsichtlich des Arbeitens auf der Objektebene kann zwischen algorithmischem, iterativem und funktionalem Arbeiten unterschieden werden (vgl. Kap. 3.3).

\subsubsection{Algorithmisches Arbeiten}

Ein TKP unterstützt algorithmisches Arbeiten, indem Formeln unter Verwendung relativer Zellbezüge in andere Zellen kopiert werden können. Damit lassen sich - sofern die explizite oder rekursive Berechnungsvorschrift einer Folge bekannt ist - beliebig viele Folgenglieder ohne großen Zeit- und Rechenaufwand numerisch und tabellarisch darstellen. Die Ergebnisse zeigen, dass die Probanden diese technische Möglichkeit nach einer „Eingewöhnungsphase“ und einem Abwägen mit traditionellen Mitteln auch nutzten. Dabei weist die Analyse der Arbeitsweisen darauf hin, dass die Probanden - sieht man von anfänglichen technischen Problemen im Umgang mit der Excel-Syntax ab - Funktionswerte von Folgen und Differenzenfolgen bzw. von Z-Funktionen und Differenzenfunktionen mit Hilfe der Kopierfunktion des TKP berechnen konnten, also ein instrumentelles Begriffsverständnis entwickelt haben.

\subsubsection{Iteratives Arbeiten}

Im Rahmen der „Streichholzaufgabe“ und in Verbindung mit den „Dreieckszahlen“ sollte die den Zählproblemen zugrunde liegende rekursive Gesetzmäßigkeit bzw. das iterative Aufbau- 
prinzip der jeweiligen Folge erfasst werden. Iteratives Arbeiten wurde dabei auf insgesamt vier verschiedenen Ebenen untersucht: der sprachlichen Ebene, der numerischen Ebene und der symbolischen Ebene, die in eine algebraische Ebene und eine TKP-Ebene unterschieden wurde. $^{259}$

\section{Sprachliche Ebene}

Im Rahmen der „Streichholzaufgabe“ sollte die iterative Gesetzmäßigkeit ausgehend von einer ikonischen Darstellung zunächst verbal formuliert und erst danach mit Hilfe relativer Zellbezüge in der Excel-Syntax erfasst werden. Diese Aufgabe wurde von den Probanden gut gelöst. Die meisten Probanden beschrieben jedoch im Wesentlichen das Änderungsverhalten der Folge; nur wenige gaben explizit das Anfangsfolgenglied an.

\section{Numerische Ebene}

Das Erfassen der Folgenglieder der Dreieckszahlen auf der numerischen Ebene bereitete beiden Probandengruppen kaum Schwierigkeiten, wenngleich diese Aufgabe von den Studierenden etwas besser gelöst wurde. Wie die Arbeitsweisen der Probanden verdeutlichen, hatten die meisten Studierenden das iterative Aufbauprinzip nach einer einmaligen Analyse der entsprechenden zeichnerischen Darstellung der Dreieckszahlen erkannt. Die Schüler stützten sich dagegen beim numerischen Fortführen der Folge stärker auf das entsprechende Figurenmuster.

\section{Symbolisch-algebraische Ebene}

Das Erfassen des Aufbauprinzips der Dreieckszahlen auf der symbolisch-algebraischen Ebene stellte die Probanden vor größere Probleme als erwartet. So gingen beide Probandengruppen beim induktiven Fortführen der formalen rekursiven Darstellung zum Teil nicht regelgerecht vor. Während die Schüler insbesondere falsche numerischen Zuwächse beim Übergang von einer Stufe zur nächsten benannten, gaben viele Studierende anstelle der numerischen $\mathrm{Zu}$ wächse die Folgenglieder der Ausgangsfolge in formaler Darstellung an, also z. B. $A(4)=A(3)+\boldsymbol{A}(4), A(n)=A(n-1)+\boldsymbol{A}(\boldsymbol{n})$. Den Schülern bereitete darüber hinaus - erwartungsgemäß - der induktive Schluss auf die allgemeine rekursive Darstellung größere Probleme, wobei sich hierbei allerdings ähnliche Fehlermuster wie vorstehend bei den Studierenden beschrieben zeigten. Die Ursachen für diese Fehler sind möglicherweise in einer Verwechslung der beiden in ihre formalen Strukturen „ähnlichen“ Gleichungen $A(n)=A(n-1)+A(n)$ und $A(n)=A(n-1)+D(n)$ zu suchen, wobei der Term $D(n)$ das $n$-te Glied der Folge der Differenzen $A(n)-A(n-1)$ bezeichnet. Damit ergibt sich für den Mathe-

\footnotetext{
${ }^{259}$ Bei der „Streichholzaufgabe“ wurde lediglich die sprachliche Ebene und die TKP-Ebene betrachtet. Das Arbeiten mit den Dreieckszahlen wurde auf der numerischen und der algebraischen Ebene sowie der TKP-Ebene untersucht.
} 
matikunterricht die Aufgabe, das Konzept der Differenzenfolgen in Verbindung mit rekursiven Folgen erneut zu thematisieren sowie rekursive und explizite Darstellungen der Folgen bewusst gegenüberzustellen.

\section{TKP-Ebene}

Das Formulieren der rekursiven Gesetzmäßigkeit unter Verwendung der Excel-Syntax bereitete den Schülern tendenziell größere Schwierigkeiten als das Arbeiten auf den beiden zuvor beschriebenen Ebenen, wohingegen bei den Studierenden keine signifikante Verschlechterung zu beobachten war. Beim Erfassen der rekursiven Darstellung der Dreieckszahlen wurden häufig falsche Zellbezüge hergestellt. Das Erfassen der und Arbeiten mit rekursiven Beziehungen in Form von Zellen bereitete offensichtlich den meisten Probanden Probleme. Dass das formelmäßige Erfassen der „Streichholzfolge“ zu besseren Ergebnissen geführt hatte, kann zum einen auf die einfachere mathematische Struktur zurückgeführt werden. Ein anderer Grund kann aber auch in der dort vorgeschalteten sprachlichen Formulierung der GesetzmäBigkeit gesehen werden, die im weiteren Aufgabenverlauf möglicherweise das Erkennen der in Beziehung stehenden Zellen unterstützte. Dies weist auf die Notwendigkeit hin, zum einen beim Arbeiten mit einem TKP die Beziehung zwischen Zellen bzw. Zellennamen und dem Variablenbegriff im Unterricht deutlicher herauszustellen und zum anderen dabei den begleitenden oder vorgeschalteten sprachlichen Kommentar stärker zu berücksichtigen.

Trotz der tendenziell schlechteren Ergebnisse auf der TKP-Ebene hatte jedoch ein Teil der Schüler beim Erfassen des iterativen Zusammenhangs mit Hilfe der Excel-Syntax weniger Schwierigkeiten als beim Formulieren der Gesetzmäßigkeit auf der algebraischen Ebene. Ein ähnliches Bild vermittelt die Analyse der Arbeitsweisen der Studierenden. Unter Berücksichtigung der Bereichsgebundenheit des Lernens ${ }^{260}$ sowie der oben aufgezeigten Probleme und Möglichkeiten zu deren Bewältigung ergibt sich somit eine Chance, auf der Basis einer TKPLernumgebung, den individuellen und unterschiedlichen Schülerdenkweisen gerecht zu werden und damit einen Beitrag zum Formalisieren von Gesetzmäßigkeiten zu leisten.

\subsubsection{Funktionales Arbeiten}

Die Untersuchungsergebnisse machen deutlich, dass ein algorithmisches Berechnen von Differenzenfolgen bzw. Differenzenfunktionen - wie es insbesondere durch das Kopieren von Formeln in Excel unterstützt wird - nicht automatisch ein Verständnis der zugrunde liegenden funktionalen Beziehungen nach sich zieht. So konnten zwar die meisten Probanden die Differenzenfolge einer Ausgangsfolge berechnen; das elementare Arbeiten auf den nicht-formalen Ebenen „Graph“ und „Tabelle“ erwies sich jedoch bei beiden Probandengruppen als inhaltlich anspruchsvoll und fehleranfällig.

\footnotetext{
${ }^{260}$ Damit ist hier gemeint, dass Übersetzungs- oder Transferleistungen zwischen der „TKP-Welt“ und der „Bleistift-und-Papier-Welt" offensichtlich nicht automatisch ablaufen.
} 


\section{Lokale Funktionsuntersuchungen}

Bei der Bearbeitung von Problemstellungen, die sich auf lokale Funktionseigenschaften ${ }^{261}$ beziehen, benötigten die meisten Probanden mehrere Lösungsanläufe. Ein Hauptproblem stellte dabei das Erkennen und Benennen der in Beziehung stehenden Folgenglieder bzw. Funktionswerte und insbesondere das Arbeiten mit der lokalen Beziehung $D(n)=f(n+1)-f(n)$ dar, die häufig mit der inhaltlich ebenfalls sinnvollen, aber im Rahmen des Untersuchungsprogramms nicht vereinbarten Beziehung $D(n)=f(n)-f(n-1)$ verwechselt wurde. Als problematisch erwies sich herbei, dass sowohl die optische Anordnung der Tabellenzellen als auch die graphische Darstellung keine der beiden Möglichkeiten auszeichnet und somit von den Probanden eine große Konzentrationsleistung gefordert wurde. Hier ergibt sich die Frage, ob eine tabellarische Darstellung etwa der folgenden Art die Beziehung zwischen den einzelnen Funktionswerten besser verdeutlichen kann: ${ }^{262}$

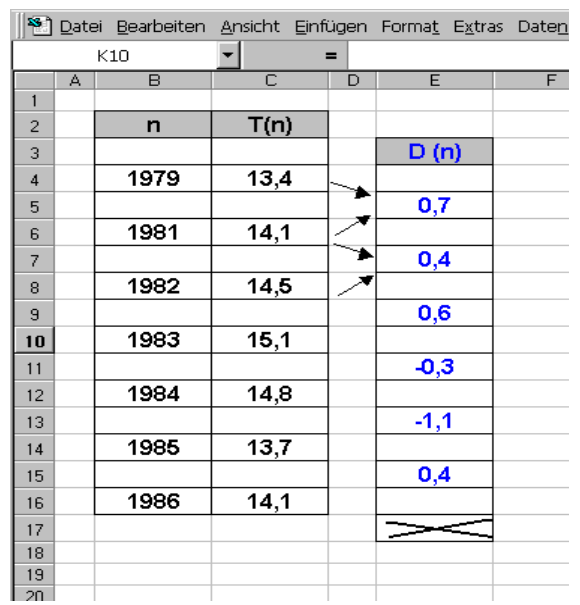

Abb. 87: Alternative tabellarische Darstellung einer Differenzenfunktion

Die Ergebnisse weisen weiterhin auf Schwierigkeiten beim Lösen von Interpolationsaufga$b e n^{263}$ hin. Dabei erwies sich das Arbeiten mit der Tabelle als weniger fehleranfällig als das Arbeiten mit dem Graph. Es ist zu vermuten, dass beim Arbeiten mit tabellarischen Darstellungen das Erkennen der numerischen Beziehung zwischen den Funktionswerten und das Entwickeln einer prototypischen Vorstellung von den Begriffen Folge und Differenzenfolge bzw. Z-Funktion und Differenzenfunktion durch die in das Lernprogramm integrierte visuelle Hilfestellung (Pfeile) unterstützt wurde. Bei dem Graph wurde dagegen auf derartige Visualisierungen aus Gründen der Übersichtlichkeit verzichtet. Möglicherweise kann jedoch ein wie

\footnotetext{
${ }^{261} \mathrm{Vgl}$. Kap. 2.3.3.

${ }^{262}$ Auch bei dieser Darstellung muss jedoch letztendlich eine Zuordnung des Funktionswerts $D(n)$ zu einer der beiden Intervallgrenzen $n$ bzw. $n+1$ erfolgen.

${ }^{263}$ Als Interpolationsaufgaben bezeichnen wir in diesem Zusammenhang Aufgaben, bei denen fehlende Funktionswerte in der tabellarischen oder graphischen Darstellung einer Folge bzw. Z-Funktion ergänzt werden sollen (vgl. Kap. 5.6, Aufg. 2d und Aufg. 3c).
} 
oben bei der Tabelle angedeutetes „Gegeneinander Verschieben“ der beiden Funktionen auch in Verbindung mit deren graphischen Darstellungen realisiert und dabei die Aufmerksamkeit der Schüler stärker auf die in Beziehung stehenden Funktionswerte gerichtet werden. Dennoch erscheint es zudem empfehlenswert, für das Lösen von Interpolationsaufgaben eine eigene Strategie der Problembehandlung im Unterricht zu entwickeln.

Wie weiterhin deutlich wurde, differieren Fehlerquote und Fehlermuster der Probanden bei der Analyse lokaler Eigenschaften von Funktionen unterschiedlichen Typs. Dies zeigte sich vor allem bei Aufgaben, bei denen ein gegebener Funktionswert einer Differenzenfunktion als absolute Änderung zu interpretieren war (Aufg. 2b und 3b). Die Untersuchungsergebnisse zeigen, dass die Anzahl der benötigten Lösungsanläufe beim Arbeiten mit den quadratischen Z-Funktionen deutlich geringer war als beim Arbeiten mit der „unregelmäßige“ empirische Temperaturfolge. Insbesondere wurde bei der Analyse des Änderungsverhaltens in keinem Fall die (in unserer Untersuchung nicht vereinbarte und damit nicht korrekte) Beziehung $D(n)=f(n)-f(n-1)$ zugrunde gelegt. Andererseits trat bei den quadratischen Z-Funktionen eine neuer Fehlertyp auf, der in Verbindung mit der empirischen Temperaturfolge nicht beobachtet werden konnte: Anstelle des Änderungsverhaltens der Ausgangsfunktion wurde offensichtlich bedingt und gesteuert durch die „Regelmäßigkeit“ der Funktion $D$ und deren konstante Änderung als ausgezeichnete Eigenschaft - das Änderungsverhalten der Differenzenfunktion analysiert und als Änderung der Ausgangsfolge in dem gegebenen Intervall angesehen. Im Hinblick auf das Entwickeln einer angemessenen Vorstellung zur Änderung(srate $)^{264}$ einer Funktion, zeigt sich hier die Bedeutung des situativen Kontexts für die Behandlung diskreter Funktionen. Der inhaltliche Bezug zu einer außermathematischen Situation erleichtert offensichtlich eine Differenzierung zwischen der Ausgangsfolge und deren Differenzenfolge bzw. das Erkennen der Differenzenfunktion als Änderungsfunktion.

Eine weitere Schwierigkeit beim Lösen lokaler Problemstellungen stellte die Interpretation des Vorzeichens eines Funktionswerts der Differenzenfolge bzw. -funktion als Kennzeichen für das lokale Wachstumsverhalten der Ausgangsfunktion dar. Dabei erwies sich insbesondere die Deutung einer negativen Änderung im Rahmen des situativen Kontexts „Durchschnittstemperaturen“ (Aufg. 2b) als fehleranfällig. Überraschenderweise zeigen die Testergebnisse im Hinblick auf diesen Fehlertyp nur ein geringfügig besseres Abschneiden der Studierenden gegenüber den Schülern. Offensichtlich konnten entsprechende Kenntnisse aus der Infinitesimalrechnung hier nicht gewinnbringend eingebracht werden. Diese Ergebnisse weisen darauf hin, dass derartige Interpretationsaktivitäten im Unterricht stärker gefördert werden müssen.

\footnotetext{
${ }^{264}$ Im Rahmen des Konzepts der Z-Funktionen wird nur der Begriff der „Änderung“ thematisiert. Da der Definitionsbereich jedoch $\mathbf{Z}$ ist, kann die absolute Änderung auch als mittlere Änderungsrate im Intervall $[n, n+1]$ und damit die Differenzenfunktion als (spezielle) Änderungsratenfunktion bezeichnet werden (vgl. auch Kap. 2.3.3).
} 


\section{Globale Funktionsuntersuchungen}

Globale Funktionsuntersuchungen beziehen sich zum einen auf empirische oder durch einen Term darstellbare Funktionen als „Ganzes“ (Aufg. 2d und 5) zum anderen auf (parameterabhängige) Funktionenklassen wie $f(n)=2 n^{2}-8 n+c$ oder $f(n)=3 n^{2}+b n+40$ (Aufg. 3d und 3g). Während sich das Arbeiten mit Funktionenklassen als inhaltlich äußerst anspruchsvoll erwies (vgl. auch Abschnitt 7.1.2 und 7.2.1), bereitete das globale Analysieren von und das Arbeiten mit ausgezeichneten Funktionen den Schülern weniger Schwierigkeiten als die entsprechenden lokalen Fragestellungen. So benötigten die Schüler zur Analyse des globalen Änderungsverhaltens der empirischen Temperaturfolge weniger Lösungsanläufe. Darüber hinaus erzielten die Schüler beim graphischen Differenzenbilden gute Ergebnisse, die sich unter anderem auf das schrittweise „Abarbeiten“ und Analysieren des Graphen in Richtung der positiven $x$-Achse zurückführen lassen. Die Studierenden hatten dagegen bei der Bearbeitung dieser Aufgabe größere Schwierigkeiten als erwartet. Ihr offensichtlich durch die Differenzialrechnung geprägtes kontinuierliches Denken, d. h. ihr vorrangig qualitatives Vorgehen, das bei einer globalen Analyse des Änderungsverhaltens der diskreten Funktion ansetzte, führte bei der Mehrheit der Probanden zu einer fehlerhaften Lösung. Offensichtlich waren diese Studierenden einerseits nicht in der Lage, sich an markanten Punkten wie Extremwerten oder Bereichen monotonen Wachstums zu orientieren. Andererseits wurde ein diskretes, schrittweises Vorgehen, das bei den Schülern dominierte, von den Studierenden nicht als Alternative zur Problemlösung in Betracht gezogen. Wie das Vorgehen der Schüler zeigt, gibt es für ein diskretes Arbeiten eine intuitive Grundlage, auf der im Mathematikunterricht aufgebaut werden kann. Die Untersuchungsergebnisse weisen aber auch darauf hin, dass derartige Denk- und Arbeitsweisen bewusst langfristig aufrecht erhalten und regelmäßig geschult werden müssen.

Die Untersuchungsergebnisse lassen weiterhin insbesondere bei den nicht mit den Begriffen Ableitung und Ableitungsfunktion vertrauten Schülern eine grundsätzliche Problematik bei globalen Problemstellungen erkennen: Ausgangsfolge/-funktion und Differenzenfolge/ -funktion werden häufig nur isoliert betrachtet. So untersuchte über die Hälfte der Schüler das Änderungsverhalten der Temperaturfolge anhand der Ausgangsfunktion selbst und verwendete nicht die Differenzenfolge als Analyseinstrument. Darüber hinaus stellten viele Schüler beim Arbeiten mit Funktionenklassen keinen Bezug zwischen den Eigenschaften von Ausgangs- und Differenzenfunktion her und beschrieben die beiden Funktionen unabhängig voneinander. Dennoch weisen der reflektierte Umgang mit der Differenzenfolge und die qualitativ guten verbalen Argumentationen einiger Schüler darauf hin, dass ein Verständnis für die globale Beziehung zwischen Funktion und Differenzenfunktion mit der hier verwendeten Lernumgebung entwickelt werden kann. 


\subsubsection{Arbeiten auf der Darstellungsebene}

Bei zahlreichen Aufgaben ${ }^{265}$ konnten die Probanden zwischen den Darstellungen Graph und Tabelle wählen. Zur Beantwortung einiger Fragestellungen konnten die Probanden darüber hinaus mit Hilfe eines Rollbalkens bestimmte Parameter einer Z-Funktion variieren und die Auswirkungen auf die graphische und tabellarische Darstellungen beobachten. Letzteres bezeichnen wir im Folgenden als „Arbeiten mit dynamisierten Darstellungen“. In allen anderen Fällen verwenden wir den Ausdruck „Arbeiten mit nicht-dynamisierten Darstellungen“.

\subsubsection{Arbeiten mit nicht-dynamisierten Darstellungen}

Wie die Untersuchungsergebnisse zeigen, bevorzugten beide Probandengruppen beim Arbeiten mit nicht-dynamisierten Darstellungen die Tabelle, wobei die Studierenden tendenziell etwas häufiger mit der tabellarischen Darstellung arbeiteten als die Schüler. Dieses Auswahlverhalten kann in Verbindung mit lokalen Problemstellungen als problemadäquat bezeichnet werden. Bei globalen Problemstellungen bietet jedoch das Arbeiten mit dem Graphen insofern Vorteile, als globale Eigenschaften, wie Bereiche monotonen Wachstums oder Bereiche, in denen Funktionswerte einen positiven Wert annehmen, auf einen Blick erkannt werden können. Dieser Vorteil wurde von den meisten Schülern nicht genutzt und führte insbesondere beim Analysieren des Zusammenhangs zwischen dem Vorzeichenwechsel der Differenzenfunktion und den Eigenschaften der Ausgangsfunktion (Aufgabe 3e) zu deutlich schlechteren Ergebnissen, als bei jenen Schülern, die bevorzugt oder ergänzend mit dem Graph arbeiteten. Beim Zugang zu Differenzenfolgen und -funktionen erwies sich somit die tabellarische Darstellung als intuitiv zugänglicher. Zur Förderung eines problemadäquaten Arbeitens mit Darstellungen und zur Vermeidung von Fehlern erscheint es jedoch geboten, dass die durch die einzelnen Darstellungsformen (insbesondere durch den Graphen) verdeutlichten Begriffsaspekte stärker herausgestellt werden.

Wenngleich sich bei den meisten Aufgabentypen eine bevorzugte Darstellungsart identifizieren lässt, unterscheiden sich die Arbeitsweisen der Schüler jedoch hinsichtlich der Reihenfolge der betrachteten Darstellungen bzw. der in Lösungsprozessen verfolgten Strategien auf vielfältige Weise. Das Ermöglichen eigenverantwortlicher Lern- und Lösungswege birgt - wie bereits oben angedeutet - die Gefahr, dass inadäquate Wege beschritten werden, d. h. Darstellungen gewählt und Eigenschaften betrachtet werden, die keinen oder nur einen geringen Beitrag zur Lösung liefern. Andererseits ist jedoch zu berücksichtigen, dass mit der freien Auswahl der Darstellungen Schülern die Möglichkeit geboten wurde, eigene Lösungsstrategien zu entwickeln und insbesondere bei Problemen im Umgang mit einer bestimmten Darstellung die alternative Darstellungsform zu wählen. Die Untersuchungsergebnisse unterstrei-

\footnotetext{
${ }^{265}$ So etwa bei Aufg. 2b, 2e, 3b, 3d, 3e, 3g (vgl. Kap. 5.6).
} 
chen damit die Bedeutung des Angebots mehrerer Darstellungen, weisen jedoch auch auf die Aufgabe hin, Schüler zu befähigen, Darstellungen immer wieder hinsichtlich ihres Beitrags zu Problemlösungen miteinander zu vergleichen, zu beurteilen und problemadäquat auszuwählen. Hier sind sicherlich auch die Grenzen des von uns verwendeten Lernprogramms erreicht, und es bedarf einer didaktisch durchdachten globalen Strategie zur Entwicklung eines adäquaten Arbeitens mit Darstellungen.

\subsubsection{Arbeiten mit dynamisierten Darstellungen}

Die Möglichkeit des graphischen und tabellarischen Experimentierens mit Parametern, so etwa beim Analysieren der Funktionenklassen $f(n)=2 n^{2}-8 n+c$ (Aufgabe 3d) oder $f(n)=3 n^{2}+b n+40$ (Aufgabe 3g), wurde von beiden Probandengruppen umfassend genutzt und führte insbesondere bei Aufgabe 3d bei der Mehrheit der Studierenden und einem zufriedenstellenden Anteil der Schüler (34\%) zu adäquaten verbalen Beschreibungen und Argumentationen (vgl. auch Kap. 7.2.1). Hinsichtlich der Auswahl der betrachteten Darstellungen zeigten sich jedoch sowohl innerhalb der Probandengruppen als auch im Vergleich der beiden Gruppen zueinander deutliche Unterschiede. So gab es Probanden, die ausschließlich mit einer Darstellung arbeiteten, während andere sowohl den Graphen als auch die Tabelle in ihre Untersuchungen mit einbezogen. Diejenigen Schüler, die sich auf das Arbeiten mit einer Darstellung beschränkten, bevorzugten fast ausschließlich den Graphen; die Studierenden maßen der Tabelle eine größere Bedeutung bei. Die Ergebnisse zeigen weiterhin, dass ein größerer Teil der Studierenden als der Schüler mit beiden Darstellungen arbeitete. Die Arbeitsweisen der Mehrheit der Studierenden, die sowohl die Tabelle als auch den Graph zum Experimentieren einsetzten, deuten darauf hin, dass der Versuch unternommen wurde, beide Darstellungen beim Analysieren der Eigenschaften der Z-Funktionen und deren Differenzenfunktionen angemessen zu berücksichtigen. Dies lässt sich auf Vorerfahrungen mit dem dynamischen Visualisieren als Methode zum Entdecken invarianter Eigenschaften oder interessanter Phänomene zurückführen. Dabei wurde jedoch offensichtlich durch die reine Präsenz der tabellarischen Darstellung ein Anreiz zum Experimentieren geschaffen und der Blick häufig auf irrelevante Eigenschaften gerichtet. So führt die Analyse der tabellarischen Darstellung in Zusammenhang mit Untersuchungen der Funktionenklasse $f(n)=3 n^{2}+b n+40$ nur in wenigen Fällen zum Entdecken der invarianten Eigenschaft „konstante Änderung“. Statt auf „vertikale Zusammenhänge“ zu achten, konzentrierten sich die Studierenden auf „horizontale Abhängigkeiten“ und analysierten weniger bedeutungsvolle lokale numerische Eigenschaften. Die Schüler nutzten ähnlich wie die Studierenden die Möglichkeiten des dynamischen Visualisierens ausgiebig. Auch sie hatten Schwierigkeiten, die relevanten Eigenschaften zu erkennen und $\mathrm{zu}$ beschreiben, wenngleich ihre verbalen Beschreibungen von denen der Studierenden differierten. So richteten die Schüler beim Arbeiten mit der Funktionenklasse 
$f(n)=2 n^{2}-8 n+c$ ihren Blick häufig auf das sich ändernde Objekt, also die Ausgangsfunktion, wohingegen die Invarianz der Differenzenfunktion nicht beachtet wurde. Beim Untersuchen der Funktionenklasse $f(n)=3 n^{2}+b n+40$ und der entsprechenden Differenzenfunktionen rückte für einige Schüler eine andere Eigenschaft in den Mittelpunkt des Untersuchungsinteresses: Sie konzentrierten sich beim Analysieren der beiden „dynamisierten“ graphischen Darstellungen auf den konstanten Achsenabschnitt der Ausgangsfunktion, also auf den einzigen fixen Punkt im Koordinatensystem. Somit erwies sich der diskrete Graph im Rahmen von Aufgabe $3 \mathrm{~g}$ als eine für die Schüler nur bedingt geeignete Darstellung für das Entdecken und Begründen globaler Eigenschaften von quadratischen Z-Funktionen und deren Differenzenfunktionen.

Diese Ergebnisse zeigen, dass dynamisches Visualisieren als Möglichkeit zum Entdecken von Eigenschaften erst gelernt werden muss, d. h. das insbesondere mit dieser Arbeitsform wenig vertraute Schüler zunächst angeleitet werden müssen, für die Fragestellung bedeutsame Invarianten und Eigenschaften in dynamisierten Objekten zu erkennen. Zum anderen wird deutlich, dass ein und dieselbe Darstellung die Aufmerksamkeit der Lernenden auf individuell unterschiedliche Begriffsaspekte bzw. -eigenschaften lenkt. Aufgrund dieser Ergebnisse erscheint insbesondere beim Arbeiten mit Funktionenklassen eine stärkere Steuerung durch entsprechend geplante Unterrichtseinheiten geboten, in denen die individuellen Sichtweisen der Schüler auf die Darstellungen kanalisiert und möglicherweise korrigiert werden sowie das Entwickeln defizitärer Begriffsvorstellungen verhindert wird. ${ }^{266}$ In Verbindung mit der tabellarischen Darstellung sollte dabei vor allem der Änderungsaspekt herausgestellt werden. Beim Arbeiten mit dem Graph ist es erforderlich, den Blick der Schüler auf die graphische Darstellung beider Funktionen zu lenken. Dabei sollte die ganzheitliche „Gestalt“ betont werden, ohne jedoch die Charakteristika der diskreten Begriffe außer Acht zu lassen.

\subsubsection{Arbeiten auf der Werkzeugebene}

Im Rahmen der Analyse der Arbeitsweisen auf der Werkzeugebene wurden zum einen Schwierigkeiten im Umgang mit der TKP-Syntax erfasst. Darüber hinaus galt das Untersuchungsinteresse der Art und Weise, wie die Bedienungs- und Steuerungselementen der TKPgestützten Lernumgebung verwendet wurden.

\subsubsection{Umgang mit der TKP-Syntax}

Die Ergebnisse zeigen, dass in der Anfangsphase der Versuchsdurchführung technische Probleme, insbesondere Probleme beim Verwenden der Excel-Syntax, inhaltliche Probleme beim Umgang mit den mathematischen Objekten „Folge und Differenzenfolge“ bzw. „Funktion

\footnotetext{
${ }^{266}$ Es zeigt sich somit hier die Bedeutung des Einsatzes geeigneter Darstellungen (Prototypen) sowie die Notwendigkeit einer Aufmerksamkeitsfokussierung (vgl. Kap. 3.1.4 und 3.2.3).
} 
und Differenzenfunktion“ überlagerten. Neben rein syntaktischen Fehlern, wie dem Vergessen des „=“-Zeichens, traten auch komplexere Fehlermuster auf. So verwendeten die Probanden in TKP-Formeln falsche Zellbezüge bzw. stellten Zirkelbezüge her oder sie verzichteten ganz auf die TKP-Syntax, indem sie anstelle der eigentlich zu berechnenden Formel lediglich die entsprechenden numerischen Werte eingaben. Darüber hinaus traten „Interferenzfehler“ auf, d. h. die TKP- und die algebraische Syntax wurden beim „Übersetzen“ einer Formel aus der traditionellen „Bleistift-und-Papier-Welt“ in die „TKP-Welt“ vermischt. Wenngleich mit fortschreitender Bearbeitungsdauer ein Lernfortschritt bei den Schülern und Studierenden zu verzeichnen war, machen die Ergebnisse deutlich, dass ein sinnvoller Einsatz eines TKP im Mathematikunterricht einer größeren Erfahrungsbasis bedarf. Die Sichtweise von Zellen als Variablen muss - wie bereits in Verbindung mit dem iterativen Arbeiten herausgestellt langfristig entwickelt werden. Die Interferenzfehler weisen darüber hinaus darauf hin, dass die beiden formalen Sprachen deutlicher zueinander in Beziehung gesetzt werden müssen, wobei Besonderheiten der Syntaxen herausgestellt und kontrastiert werden sollten.

\subsubsection{Bedienung und Steuerung des Lernprogramms}

Die Bedienung und Steuerung des Lernprogramms über die Aktionsbuttons bereitete den Probanden keine grundsätzlichen Schwierigkeiten. Die Auswahl der Darstellungen erfolgte größtenteils reflektiert, d. h. die Probanden betrachteten im Allgemeinen nicht unverhältnismäßig viele Darstellungen. Die Anzahl der von den Studierenden ausgewählten Darstellungen war bei lokalen Problemstellungen geringer als bei den Schülern. Beim Arbeiten mit Funktionenklassen bzw. dynamisierten Darstellungen unterschied sich das Verhalten von Schüler und Studierenden jedoch nur unwesentlich. Während bei den Studierenden aufgrund des gegenüber den Schülern reduzierten Lernprogramms keine Aussage über die Entwicklung des Auswahlverhaltens möglich ist, konnte bei den Schülern während der Versuchsdurchführung bei strukturähnlichen Aufgaben eine Verringerung der Anzahl der betrachteten Darstellungen festgestellt werden.

Der Rollbalken erwies sich im Rahmen des Untersuchungsprogramms als ein wichtiges Experimentierwerkzeug. Durch die komfortable Möglichkeit, Parameter zu variieren und deren Einfluss auf die dargestellten Funktionen beobachten zu können, konnten Begriffseigenschaften von Funktionenklassen wie $f(n)=2 n^{2}-8 n+c$ und $f(n)=3 n^{2}+b n+40$ analysiert werden. Darüber hinaus wurde ein experimenteller Zugang zum Lösen von Zählproblemen (Aufg. 4d) ermöglicht. Allerdings weisen die Ergebnisse auch auf Gefahren beim Einsatz solcher Bedienungselemente hin. So betätigten manche Schüler die Rollbalken häufig (unreflektiert) in der Reihenfolge ihrer Anordnung und weniger auf der Basis inhaltlicher Überlegungen. Die einfache Bedienung der Rollbalken behinderte in diesen Fällen offensichtlich auch kreative „Denkpausen“ und begünstigte ein zielloses Vorgehen im Sinne von „Versuch und Irrtum“. Damit ergibt sich die Aufgabe, die Bedeutung des Einsatzes von Rollbalken aufga- 
benspezifisch $\mathrm{zu}$ überdenken und gegebenenfalls das Variieren der Parameter durch das schrittweise (zeitverzögerte) Eingeben von numerischen Werten in eine Zelle bewusst zu verzögern.

Das Angebot der technischen Hilfestellungen wurde von den meisten Probanden zweckgebunden genutzt. Mit Hilfe des „Ok-Buttons“ konnten Lösungen überprüft sowie Fehler entdeckt und (in den meisten Fällen) korrigiert werden. Nur in Ausnahmefällen führte die Verfügbarkeit der Lösungsüberprüfung mit dem „Ok-Button“ zu einem Handlungsaktivismus, d. h. es wurden sehr viele (willkürliche) Eingaben in kurzer Zeit getätigt. Dagegen verleitete das Vorhandensein des „Hinweis-Buttons“ manche Probanden dazu, die angebotene Hilfe in Anspruch zu nehmen, bevor überhaupt ein eigener Lösungsversuch unternommen wurde.

Während der gesamten Versuchsdurchführung zeigte sich ein weiteres Problem: Die zusätzlichen Informations- und Hinweisblätter wurden wenig oder kaum beachtet, was sich im „Überblättern“ von Informationen oder nur kurzem Lesen von Hilfetexten äußerte. Dadurch wirkten sich Hilfen, die etwa durch „Hinweis-Buttons“ abgerufen werden konnten, nur marginal auf die Bearbeitung der Aufgaben aus; explizite Lösungshinweise wurden von den Probanden häufig nicht befolgt. Auch die im Lernprogramm der Schüler integrierten Informationsblätter, die Ergebnisse zusammenfassten bzw. neue Inhalte vorbereiteten, besaßen nur einen geringfügigen Einfluss auf die Arbeitsweisen und das Verständnis der Probanden. Aufgrund dieser Ergebnisse erscheint der Einsatz derartiger Informationsblätter nur bedingt geeignet. Hier sind für zukünftige Lernprogramme Alternativen zu überdenken. Ob die Vermittlung von Informationen im Rahmen eines Unterrichtsgesprächs ${ }^{267}$ realisiert werden sollte oder im Rahmen (kleinerer) Übungsaufgaben in einem Lernprogramm, bleibt eine offene Untersuchungsfrage.

\subsection{Lernerfolg und Verständniszuwachs}

Die Ergebnisse der Analyse der Arbeitsweisen auf den drei Arbeitsebenen sowie die verbalen Beschreibungen der Probanden werden nun im Hinblick auf das erreichte bzw. zugrunde liegende Verständnis der Probanden im Sinne der in Kapitel 3.4 vorgestellten Verständnismodelle interpretiert.

\subsubsection{Kenntnisse und Begriffsvorstellungen}

\section{Kenntnisse von Begriffseigenschaften}

Im Zentrum der empirischen Untersuchung stand der Begriff der Differenzenfolge bzw. Differenzenfunktion, ein für beide Probandengruppen bislang unbekannter Begriff. Im Laufe der

\footnotetext{
${ }^{267}$ Ein derartiges Konzept führte bei den Schülern der Gruppe C im Rahmen von Aufgabe 3g zu besseren Ergebnissen (vgl. Kap. 6.3.7.2).
} 
Versuchsdurchführung lassen sich in verschiedener Hinsicht Lernerfolge bzw. ein Verständniszuwachs nachweisen. Dieser Verständniszuwachs spiegelte sich zum einen in bestimmten Kenntnissen von Eigenschaften der betrachteten Objekte wider sowie in der Fähigkeit, die Begriffe bzw. deren Darstellungen zum Lösen einfacher Probleme einzusetzen. So waren die meisten Probanden - wenngleich nach einer unterschiedlichen Anzahl von Lösungsanläufen in der Lage, Funktionswerte der Differenzenfunktion als die absolute Änderung der Ausgangsfunktion in einem bestimmten Intervall zu interpretieren sowie Beziehungen zwischen lokalen Eigenschaften der Z-Funktion und deren Differenzenfunktion zu erkennen. Darüber hinaus entwickelte ein Teil der Probanden auch ein Verständnis für die Differenzenfunktion als Instrument zur globalen Analyse des Änderungsverhaltens der Ausgangsfunktion. Die Ergebnisse zeigen jedoch auch, dass die heuristische Bedeutung der Differenzenfolgen für das Lösen von Zählproblemen nur von wenigen Probanden erkannt wurde. Beide Probandengruppen benutzten in ihren Lösungen nur zum Teil Kenntnisse über Eigenschaften von Differenzenfolgen. Diese Ergebnisse weisen auf ein inhaltliches Verständnis der Probanden hin; ein relationales oder integriertes Verständnis konnte jedoch im Rahmen des Lernprogramms offensichtlich nur in wenigen Fällen erreicht werden und muss längerfristig entwickelt werden.

\section{Sichtweisen auf Begriffseigenschaften}

Neben der Analyse der Arbeitsweisen vermitteln auch die verbalen Beschreibungen ein Bild von den Vorstellungen bzw. dem Verständnis der Probanden. Hier zeigen sich deutliche Unterschiede zwischen den beiden Probandengruppen. So erzielten die Studierenden beim Beschreiben und Begründen des globalen Zusammenhangs zwischen der Folge/Z-Funktion und deren Differenzenfolge/-funktion -erwartungsgemäß - bessere Ergebnisse als die Schüler, die häufig nur eine der beiden Funktionen isoliert betrachteten. Das bessere Abschneiden der Studierenden ist sicherlich auf deren Kenntnisse in der Differenzialrechnung zurückzuführen. Darüber hinaus unterschieden sich die Argumentationsmuster der erfolgreichen Schüler und Studierenden. So begründeten die Schüler die Invarianz der Differenzenfunktion im Rahmen der Analyse der Funktionenklasse $f(n)=2 n^{2}-8 n+c$ fast ausschließlich graphischgeometrisch in Bezug zu den entsprechenden Darstellungen. Diese inhaltlich-anschauliche Ebene stellte somit für die - wie im Laufe der Versuchsdurchführung deutlich wurde - algebraisch schwachen Schüler eine alternative Arbeits- und Argumentationsebene dar. Hier können Anknüpfungspunkte für einen anschließenden Analysisunterricht gesehen werden. Die Studierenden argumentierten dagegen mehrheitlich formal ohne Bezug zur graphischen Darstellung der Ausgangsfunktion, obwohl auch sie zuvor von den Möglichkeiten des dynamischen Visualisieren zum Teil umfassend Gebrauch gemacht hatten. Die Begründungen dieser Studierenden sind natürlich einer höheren (formalen) Stufe des Begriffsverständnisses zuzuordnen. Im Unterricht ist aber darauf hinzuarbeiten, dass neben diesem formalen Begriffsverständnis auch die anderen Verständnisebenen angesprochen und entwickelt werden. Zum einen kann das Arbeiten auf der graphisch-anschaulichen Ebene im Sinne des Prinzips der Stu- 
fengemäßheit (vgl. Kap. 3.2.1) ein formales Arbeiten und Denken vorbereiten. Zum anderen ermöglicht ein inhaltliches Verständnis das Finden alternativer Lösungswege in Fällen, in denen formale Überlegungen (zunächst) nicht weiterführen.

Während die Schüler bei der Analyse der Funktionenklasse $f(n)=2 n^{2}-8 n+c$ noch vergleichsweise gute Ergebnisse erzielten, waren sie mit dem Untersuchen der Funktionenklasse $f(n)=3 n^{2}+b n+40$ und der entsprechenden Differenzenfunktionen sowohl auf einer inhaltlichen als auch auf einer formalen Ebene überfordert. Die Ergebnisse machen deutlich, dass - bei Variation des Parameters $b$ in $f(n)=a n^{2}+b n+c$ (mit $a$ und $c$ fest) und der damit verbundenen Änderung der Z-Funktion und deren Differenzenfunktion - ein „In-BeziehungSetzen“ beider graphischer Darstellungen und eine entsprechende geometrische Argumentation eine anspruchsvolle Aufgabe ist. Naheliegender sind Argumentationen, die sich auf die Analyse der graphischen Darstellung der Differenzenfunktion unter Berücksichtigung deren Termdarstellung $D(n)=2 a n+a+b$, also in diesem konkreten Fall $D(n)=6 n+3+b$, beziehen. Indem die invarianten Eigenschaften der Differenzenfunktion „nur“ anhand der Termdarstellung erklärt werden, wird jedoch - anders als bei einer geometrischen Argumentation keine „direkte“ (graphisch-anschauliche) Beziehung zwischen den Graphen der Ausgangsfunktion und der Differenzenfunktion hergestellt. Eine stärker auf algebraischen Überlegungen basierende Argumentation kann aber möglicherweise als Ausgangspunkt einer ergänzenden geometrischen Argumentation dienen.

Neben dem konkreten inhaltlichen Ziel, ein Verständnis für Differenzenfunktionen zu entwickeln, hatte das Lernprogramm die Aufgabe, die Lernenden zu einem diskreten elementaren Arbeiten und einer diskreten Sicht auf die verwendeten diskreten Begriffe bzw. deren Eigenschaften anzuleiten. Dieses Ziel wurde zumindest teilweise erreicht. So argumentierten die meisten der erfolgreichen Schüler beim Analysieren der Funktionenklasse $f(n)=2 n^{2}-8 n+c$ unter Verwendung von Begriffen wie „Abstand“ und „Differenz“, was auf eine diskrete Sicht auf die Eigenschaften der entsprechenden Funktionen schließen lässt. Darüber hinaus gingen sie beim graphischen Differenzenbilden schrittweise vor, indem sie den Graphen der Ausgangsfunktion in Richtung der positiven $x$-Achse sukzessive analysierten, die Differenz jeweils benachbarter Funktionswerte berechneten und graphisch als Funktionswerte der Differenzenfunktion darstellten. Die „diskreten“ Argumentationen der meisten Studierenden in Verbindung mit der Analyse der Funktionenklasse mit $f(n)=2 n^{2}-8 n+c$ deuten ebenfalls auf diskrete Denk- und Arbeitsweisen hin.

Die Analyse der Arbeitsweisen und der verbalen Argumentationen beider Probandengruppen zeigt aber auch, dass - bedingt durch Vorerfahrungen mit (meist reellen) Funktionen bzw. der Differenzialrechnung - eine diskrete Sicht häufig durch eine ergänzende kontinuierliche Sicht überlagert wird. Dies kann - sofern es sich um eine „,bewusste“ und „,zielgerichtete“ kontinuierliche Sichtweise handelt - das Erkennen von Eigenschaften unterstützen (vgl. Kap. 7.1.2.2). 
Werden die Charakteristika der diskreten Begriffe jedoch aus den Augen verloren, sind - wie bei dieser Untersuchung ebenfalls beobachtet - Fehler möglich. So gaben Schüler als Konsequenz einer „unzulässigen“ (gedanklichen) Interpolation den Extremwert einer „gedachten“ kontinuierlichen aber nicht den der „,realen“ diskreten Funktion an (vgl. Kap. 6.3.5.2), und die Studierenden skizzierten beim graphischen Differenzenbilden anstelle der diskreten Differenzenfunktion eine kontinuierliche Funktion. Dies macht deutlich, dass zwar intuitive Grundlagen für diskrete Sicht- und Arbeitsweisen vorhanden sind, dass aber die Vorerfahrungen der Schüler im Umgang mit kontinuierlichen Funktionen zu dieser „,neuen“ Sichtweise bewusst in Beziehung gesetzt werden müssen.

\subsubsection{Problemlösefähigkeit}

Die im Rahmen des Lernprogramms konzipierte Arbeitsumgebung zum Themenkomplex „Dreieckszahlen“ konnte von etwa $60 \%$ beider Probandengruppen erfolgreich zum Finden einer expliziten Darstellung für die Folge der Dreieckszahlen genutzt werden. Die meisten Probanden konzentrierten sich auf eine der angebotenen Hilfestellungen „Bleistift und Papier“, „Differenzenfolge“ und „Experimentieren“. Zum Teil wurden jedoch auch Lösungsansätze miteinander kombiniert, wodurch sich „,neuartige“ und interessante Lösungsstrategien, wie etwa das Experimentieren mit einer Testfolge und deren Differenzenfolgen, ergaben. Wie die Untersuchungsergebnisse zeigen, wurden Kenntnisse über Differenzenfolgen nur zum geringen Teil aufgegriffen. Insbesondere nutzte nur ein kleiner Teil der Probanden die formale Darstellung der Differenzenfolge und ihre Beziehung zur quadratischen Funktion zum Finden der expliziten Formel. Das Erkennen der heuristischen Bedeutung dieses Begriffs im Rahmen von Zählproblemen erfordert somit offensichtlich eine größere Erfahrung im Umgang mit Differenzenfolgen und -funktionen.

Die Ergebnisse zeigen weiterhin deutliche Unterschiede in den Arbeitsweisen der Schüler und der Studierenden. Die erfolgreichen Schüler bevorzugten den experimentellen Weg und damit das Arbeiten mit den graphischen und numerischen Darstellungen der Dreieckszahlen. Ein Teil der Schüler ging reflektiert und zielgerichtet vor, was auf Kenntnisse über den Einfluss der Parameter auf den Verlauf des entsprechenden Funktionsgraphen oder Kenntnisse über Eigenschaften von Folgen und Differenzenfolgen schließen lässt. Es zeigten sich aber auch unsystematische Vorgehensweisen, bei denen die Lösung nach „Versuch und Irrtum“ gefunden wurde. Auch die erfolglosen Schüler nahmen die Möglichkeit des experimentellen Arbeitens zum Teil ausgiebig in Anspruch, wenngleich dieser Weg letztendlich nicht zum Ziel führte. Bemerkenswert ist, dass die meisten erfolgreichen Schüler keine Notwendigkeit sahen, die auf experimentellem Wege gefundene Lösung (formal oder inhaltlich-anschaulich) zu verifizieren.

Im Gegensatz zu den Schülern leitete die Mehrheit der erfolgreichen Studierenden die explizite Formel auf der Basis der zeichnerischen Darstellung der Dreieckszahlen (inhaltlich- 
anschaulicher Beweis) her. Nur wenige Studierenden lösten die Aufgabe auf experimentellem Wege. Ein experimentelles Herangehen wurde auch dann nicht in Betracht gezogen, wenn andere (traditionelle) Wege nicht zum Ziel führten. Die Aufgabe wurde daher von den meisten erfolglosen Teilnehmern bereits nach einer kurzen Bearbeitungsphase abgebrochen. Die Studierenden sahen somit eine heuristisch-experimentelle Phase des Probierens offensichtlich nicht (mehr) als eine Möglichkeit zur Hypothesengewinnung an. Die Herangehensweisen beider Probandengruppen machen die Bedeutung und die Notwendigkeit einer frühzeitigen Förderung des experimentellen Arbeitens deutlich. Zur Vermeidung eines unsystematischen Vorgehens nach „Versuch und Irrtum“ muss jedoch immer wieder der Bezug zu theoretischen Überlegungen deutlich herausgestellt werden.

\subsection{Weiterführende Fragen}

Die hier durchgeführte Studie versteht sich auch als eine explorative Studie zur Hypothesengenerierung für weitere empirische Untersuchungen hinsichtlich der Arbeitsweisen mit und des Begriffsverständnisses von diskreten Begriffen. So schließen sich an die Ergebnisse der empirischen Untersuchung sowie die theoretischen Überlegungen der ersten vier Kapitel eine Reihe von Fragen und Problemstellungen an. Einige dieser Problemstellungen sollen abschließend aufgeführt werden:

- Das im Rahmen der empirischen Untersuchung eingesetzt Lernprogramm wurde speziell für diesen Testzweck konzipiert. Die Bearbeitung des Themenkomplexes „Differenzenfunktionen“"war daher auf die Untersuchungsdauer beschränkt. Kommunikations- und Interaktionsprozesse zwischen den Probanden wurden nicht berücksichtigt. Hinsichtlich der Behandlung dieses mathematischen Inhalts im Mathematikunterricht muss daher eine eigenständige Strategie entwickelt werden. Insbesondere ist zu überlegen, wie das Programm bzw. einzelne Module in den Mathematikunterricht integriert werden können.

- Das Bereitstellen verschiedener Darstellungen von Folgen und Differenzenfolgen bzw. Z-Funktionen und Differenzenfunktionen ermöglicht den Aufbau individueller Lösungsstrategien im Rahmen lokaler und globaler diskreter Funktionsuntersuchungen. Darüber hinaus können durch das Arbeiten mit dynamisierten Darstellungen Beziehungen zwischen Eigenschaften von Funktionenklassen analysiert werden. Es ist eine offene Frage, wie sich die Wechselbeziehung zwischen den individuell ausgebildeten Begriffsvorstellungen und Problemlösestrategien sowie den kanonisierten fachwissenschaftlichen Sichtweisen mathematischer Begriffe und Problemstellungen gestaltet.

- Eine TKP-basierte Lernumgebung im Sinne des im Rahmen dieser Arbeit vorgestellten Konzepts fördert heuristisches und experimentelles Arbeiten und kann zu einer Verbesserung der allgemeinen heuristischen Fähigkeiten beitragen. Dennoch ist die Gefahr eines unreflektierten Experimentierens groß. Es muss also das zielgerichtete Experimentieren 
angeleitet werden bzw. die dafür notwendigen Grundkenntnisse müssen vermittelt oder vom Schüler selbst reaktiviert werden. Darüber hinaus sind die auf experimentellem Wege gefundenen Lösungen von den Schülern durch theoretische Überlegungen auf Allgemeingültigkeit zu prüfen. Gerade der computerunterstützte Unterricht muss deshalb Problemlösen stärker als Zusammenspiel induktiver und deduktiver Methoden bewusst machen.

- Beim Umgang mit einem TKP-gestützten Lernprogramm stellt die meist nur flüchtige Informationsaufnahme beim Lesen von auf TKP-Blättern oder in Hilfe-Fenstern dargestellten Texten ein Problem dar. Für das Arbeiten mit dem Computer ist es eine wichtige und zentrale Aufgabe, Schüler zu einem konzentrierten und reflektierten Umgang mit einem Lernprogramm bzw. Lernmodulen und dessen Bedienungselementen anzuleiten. Es stellt sich die Frage, wie der offensichtlich durch das Werkzeug hervorgerufene Zeitdruck weggenommen und Schüler zum reflektierten Arbeiten mit diesem Werkzeug gebracht werden können.

- Durch den Einsatz eines TKP bzw. eines Lernprogramms auf der Basis eines TKP kann wie die Untersuchung zeigte - ein inhaltliches Verständnis für die Begriffspaare „Folge und Differenzenfolge“ bzw. „Z-Funktion und Differenzenfunktion“ entwickelt werden. Damit ist der Aufbau von Grundvorstellungen zum Begriff der absoluten Änderung bzw. der mittleren Änderungsrate im Sinne von Malle (1999) möglich und es kann auch eine wichtige Propädeutik für den Begriff der Ableitungsfunktion geleistet werden. Im Hinblick auf den Analysisunterricht ist dabei die Frage, ob die Schüler ihre Kenntnisse über Differenzenfunktionen gewinnbringend einsetzen können und ob sie durch die Behandlung dieses diskreten Begriffs ein besseres Verständnis der grundlegenden Begriffe der (kontinuierlichen) Analysis erwerben.

- Die Grundstruktur des im Rahmen der empirischen Untersuchung eingesetzten Lernprogramms kann als Basis von Lernumgebungen zu anderen diskreten Inhalten, etwa aus den Bereichen Kombinatorik und Differenzengleichungen, dienen. In diesem Zusammenhang erscheint es als ein wichtiges Anliegen, geeignete Aufgaben und Aufgabensequenzen zu finden und zu implementieren sowie die technischen Komponenten bzw. das Konzept der TKP-basierten Lernumgebung weiterzuentwickeln. Interessant wäre es hierbei zu untersuchen, inwiefern sich die im Rahmen der Untersuchung beobachteten diskreten Arbeitsweisen auch im Umgang mit anderen diskreten Inhalten zeigen. 
- Durch die Behandlung diskreter Mathematik kann - wie im theoretischen Teil dieser Arbeit herausgestellt - zu wichtigen Aspekten des Mathematikunterrichts wie Problemlösen, Anwenden und Begriffsbildung ein Beitrag geleistet werden. Damit erscheint es als eine wichtige Aufgabe, - eher unabhängig vom Einsatz eines TKP - ein globales Konzept zu entwickeln, wie Elemente der diskreten Mathematik in den bestehenden Mathematikunterricht integriert werden können, sowie entsprechende Unterrichtsvorschläge auszuarbeiten. 


\section{Literatur}

Abramovich, S. \& Pieper, A. (1996). Fostering Recursive Thinking in Combinatorics through the Use of Manipulatives and Computing Technology. The Mathematics Educator, Vol. 7, No. 1. 4-12.

Aebli, H. (1980). Denken: Das Ordnen des Tuns. Bd. 1. Stuttgart: Klett.

Aebli, H. (1983). Zwölf Grundformen des Lehrens. Stuttgart: Klett.

Aebli, H. (1985). Das operative Prinzip. ML 11. 4-6.

Aigner, M. (1990). Diskrete Mathematik. In: Fischer, G.; Hirzebruch, F.; Scharlau, W. \& Törnig, W. [Hrsg.]. Ein Jahrhundert Mathematik 1890-1990. Festschrift zum Jubiläum der DMV. Wiesbaden: Vieweg. 83-112.

Aigner, M. (1996). Diskrete Mathematik. Wiesbaden: Vieweg.

Aspetsberger, K. (2000). Der Einsatz von Computer-Algebra-Systemen zum Elementarisieren im Mathematikunterricht. In: Herget, W.; Weigand, H.-G. \& Weth, T. [Hrsg.]. Standardthemen des Mathematikunterrichts in moderner Sicht. Hildesheim: Franzbecker. 9-16.

Ausubel, D. P. u. a. (1973). Psychologische und pädagogische Grenzen des entdeckenden Lernens. In: Neber, H. [Hrsg.]. Entdeckendes Lernen. Weinheim u. a.: Beltz. 30-44.

Baptist, P. (1979). Optimale Wege in endlichen gerichteten Graphen. PM 21. 99-105.

Bauer, L. (1993). Das operative Prinzip als umfassendes, allgemeingültiges Prinzip für das Mathematiklernen? Didaktisch-methodische Überlegungen zum Mathematikunterricht in der Grundschule. ZDM 2. 76-83.

Bauersfeld, H. (1983). Subjektive Erfahrungsbereiche als Grundlage einer Interaktionstheorie des Mathematiklernens und -lehrens. In: Bauersfeld, H.; Bussmann, H.; Krummheuer, G.; Lorenz, J. H. \& Voigt, H. [Hrsg.]. Lernen und Lehren von Mathematik. Analysen zum Unterrichtshandeln. Köln: Aulis. 1-56.

Baumann, R. (1996). Informationssicherheit durch krytologische Verfahren. LOG IN 16, H. 5/6. 52-61.

Baumert, J.; Lehmann R.; Lehrke, M; Schmitz, B.; Clausen, M.; Hosenfeld, M.; Köller, O. \& Neubrand, J. (1997). TIMSS - Mathematisch-Naturwissenschaftlicher Unterricht im internationalen Vergleich. Opladen: Leske u. Budrich.

Beck, C. \& Maier, H. (1993). Das Interview in der mathematikdidaktischen Forschung. JMD 14, H. 2. 147-179.

Beck, C. \& Maier, H. (1994). Zu Methoden der Textinterpretation in der empirischen mathematikdidaktischen Forschung. In: Maier, H.; Voigt, J. [Hrsg.]. Verstehen und Verständigung. Arbeiten zur interpretativen Unterrichtsforschung. Bd. 19 der IDM-Reihe. Köln: Aulis. 43-76.

Besuden, H. (1979). Vollständige Induktion in der Grundschule? In: Dörfler, W. \& Fischer, R. [Hrsg.]. Beweisen im Mathematikunterricht. Wien: Hölder-Pichler-Tempsky. 83-91.

Beutelspacher, A. (1994). Kryptologie. Braunschweig u. a.: Vieweg.

Beutelspacher, A. \& Schulz, R.-H. [Hrsg.] (1987). Codieren und Chiffrieren. MU 33, H. 3. 
Beutelspacher, A.; Schwenk, J. \& Wolfenstetter, K.-D. (1995). Moderne Verfahren der Kryptographie. Braunschweig u. a.: Vieweg.

Biermann, M. \& Blum, W. (1998). Zur Rolle von Grundvorstellungen bei realitätsbezogenen Beweisen - Das Schorle-Beispiel. BzM. Hildesheim: Franzbecker. 121-124.

Biernoth, H. (1992). Der RSA-Algorithmus - Eine Einführung in die moderne Kryptographie. Pädagogische Prüfungsarbeit zur zweiten Staatsprüfung für das Lehramt an Gymnasien. Gießen. Unveröffentlicht.

Bigalke, H.-G.(1974). Graphentheorie im Mathematikunterricht? MU 20, H. 4, 5-10.

Blum, W. (1985). Anwendungsorientierter Mathematikunterricht in der didaktischen Diskussion. Math. Semesterberichte 32/2. 195-232.

Blum, W. (1991). Der (Taschen-)Computer als Werkzeug im Analysisunterricht - Möglichkeiten und Probleme. In: Postel, H.; Kirsch, A. \& Blum, W. [Hrsg.]. Mathematik lehren und lernen: Festschrift für Heinz Griesel. Hannover: Schroedel. 71-84.

Blum, W. (1996). Anwendungsbezüge im Mathematikunterricht - Trends und Perspektiven. In: Kadunz, G.; Kautschitsch, H.; Ossimitz, G. \& Schneider, E. [Hrsg.]. Trends und Perspektiven. Wien: Hölder-Pichler-Tempsky. 15-38.

Blum, W. (1998). Ursachen der TIMMS-Ergebnisse und Ansätze für Veränderungen im Mathematikunterricht. In: Blum, W. \& Neubrand, M. [Hrsg.]. TIMMS und der Mathematikunterricht. Hannover: Schroedel. 11-15.

Blum, W. (2000). Perspektiven für den Analysisunterricht. MU 46, H. 4/5. 5-17.

Blum, W. \& Kirsch, A. (1989). Warum haben nichttriviale Lösungen von $f^{\prime}=f$ keine Nullstellen? Beobachtungen und Bemerkungen zum ,inhaltlich-anschaulichen Beweisen“. In: Kautschitsch, H. \& Metzler, W. [Hrsg.]. Anschauliches Beweisen. Hölder-PichlerTempsky: Wien. 199-209.

Blum, W. \& Kirsch, A. (1991). Preformal proving - Examples and Reflections. Educational Studies in Mathematics. 22 (2). 183-203.

Blum, W. \& Törner, G. (1983). Didaktik der Analysis. Göttingen: Vandenhoeck und Ruprecht.

Bortz, J. \& Döring, N. (1995). Forschungsmethoden und Evaluation. Berlin u. a.: Springer.

Bovermann, K.; Danckwerts, R. \& Vogel, D. (1983). Der algorithmische Standpunkt in der elementaren Kombinatorik - Bemerkungen und Beispiele. MU 29, H. 5. 65-92.

Brockmeyer, H. (1996). Schüler entdecken Eigenschaften der Fibonaccischen Zahlen. PM 3/38. 108-109.

Bruner, J. S. (1971a). Über die Invarianz von Flüssigkeiten. In: Bruner, J. S.; Olver, R. R. \& Greenfield, P. M. [Hrsg.]. Studien zur kognitiven Entwicklung. Stuttgart: Klett. 223-249.

Bruner, J. S. (1971b). Über kognitive Entwicklung. In: Bruner, J. S.; Olver, R. R. \& Greenfield, P. M. [Hrsg.]. Studien zur kognitiven Entwicklung. Stuttgart: Klett. 21-96.

Burghes, D. (1995). The Introduction of Discrete Mathematics into the School Curriculum. Int. J. Math. Educ. Sci. Technol., Vol. 26, No. 3. 367-377.

Burghes, D. (1997). Ein Projekt zur Verbesserung des englischen Mathematikunterrichts. ML 84. 56-59. 
Claus, H. J. (1973). Relationen als Hilfsmittel zur Lösung kombinatorischer Aufgaben. BzM. Stuttgart: Schroedel. 66-73.

Coackley, P. (1992). Fibonacci with Spreadsheets. Micromath, Vol. 8, No. 1. 14-15.

Conway, J. H. \& Guy, R. K. (1997). Zahlenzauber. Basel: Birkhäuser.

Danckwerts, R. \& Vogel, D. [Hrsg.] (1983a). Kombinatorik. MU 29. H. 5.

Danckwerts, R. \& Vogel, D. (1983b). Algebra löst kombinatorische Probleme. MU 29, H. 5. $35-50$.

Danckwerts, R.; Deubner, W. \& Vogel, D. (1983). Anmerkungen zur Kombinatorik in Wissenschaft und Unterricht. MU 29. H. 5. 7-16.

Danckwerts, R. \& Vogel, D. (1992). Quo vadis Analysisunterricht? MNU 45, H. 6. 370-374.

Dieudonné, J. (1985). Geschichte der Mathematik 1700-1900. Wiesbaden: Vieweg.

Dion, G. S. (1995). Fibonacci Meets the TI-82. The Mathematics Teacher, Vol. 88, No. 2. 101-105.

Dörfler, W. (1985). Handlungen und Mathematiklernen - Vergleich von Ansätzen. BzM. Bad Salzdetfurth: Franzbecker. 94-97.

Dörfler, W. (1988a). Die Genese mathematischer Objekte und Operationen aus Handlungen als kognitive Konstruktion. In: Dörfler, W. [Hrsg.]. Kognitive Aspekte mathematischer Begriffsentwicklung. Wien: Hölder-Pichler-Tempsky. 55-125.

Dörfler, W. (1988b). Rolle und Mittel von Vergegenständlichung in der Mathematik. BzM. Bad Salzdetfurth: Franzbecker. 110-113.

Dörfler, W. (1989a). Didaktischer Einsatz mathematischer Software: Tabellenkalkulationsprogramme. In: Walsch, W. [Hrsg.]. Kleincomputer und Mathematikunterricht. Halle. 7885.

Dörfler, W. (1989b). Prototypen und Protokolle als kognitive Mittel gegen Bedeutungslosigkeit und Entfremdung im Mathematikunterricht. In: Landesinstitut für Schule und Weiterbildung, Soest [Hrsg.]. Die Zukunft des Mathematikunterrichts.

Dörfler, W. (1991). Der Computer als kognitives Werkzeug und kognitives Medium. In: Dörfler, W.; Peschek, W.; Schneider, E. \& Wegenkittl, K. [Hrsg.]. Computer-MenschMathematik. Wien: Hölder-Pichler-Tempsky. 51-75.

Dossey, J. A. (1991). Discrete Mathematics: The Math of Our Time. In: Kenney, M. J. \& Hirsch, C. R. [Hrsg.]. Discrete Mathematics across the Curriculum, K-12. Reston, Va.: NCTM. 1-9.

Dürr, R. \& Ziegenbalg, J. (1989). Mathematik für Computeranwendungen. Dynamische Prozesse und ihre Mathematisierung durch Differenzengleichungen. Paderborn: Schöningh.

Failing, M. (1998). Kryptologie. Ansätze offenen Unterrichts beim Einstieg in ein aktuelles Thema. Pädagogische Prüfungsarbeit zur zweiten Staatsprüfung für das Lehramt an Gymnasien. Gießen. Unveröffentlicht.

Fricke, A. (1970). Operative Lernprinzipien im Mathematikunterricht der Grundschule. In: Fricke, A. \& Besuden, A. [Hrsg.]. Mathematik: Elemente einer Didaktik und Methodik. Stuttgart: Klett. 79-116.

Engel, A. (1977). Elementarmathematik vom algorithmischen Standpunkt. Stuttgart: Klett.

Engel, A. [Hrsg.] (1979). Algorithmen. MU 25, H. 6. 
Engel, J. (1999). Von der Datenwolke zur Funktion. ML 97. 60-64.

Fey, J. T. [Hrsg.] (1984). Computing and Mathematics. The Impact on Secondary School Curricula. Reston, Va. : NCTM.

Fischer, R. (1976). Fundamentale Ideen bei reellen Funktionen. In: ZDM 8. 185-192.

Fischer, R. \& Malle, G. (1985). Mensch und Mathematik. Eine Einführung in didaktisches Denken und Handeln. Mannheim u. a.: BI-Wissenschaftsverlag.

Flachsmeyer, J. (1972). Kombinatorik. VEB Deutscher Verlag der Wissenschaften: Berlin.

Flick, U. (1991). Stationen des qualitativen Forschungsprozesses. In: Flick, U.; Kardorff, v. E.; Keupp, H.; Rosenstiel, v. L. \& Wolff, S. [Hrsg.]. Handbuch Qualitative Sozialforschung. Grundlagen, Konzepte, Methoden und Anwendungen. München: Psychologie Verlags Union. 147-173.

Floer, J. (1977). Optimierung von Netzwerken - Kürzeste Wege und größte Flüsse, I, II. PM 19. 1-6 und 40-44.

Franke, M. (1999). Didaktik der Geometrie. Heidelberg u. a.: Spektrum.

Freudenthal, H. (1973). Mathematik als pädagogische Aufgabe. Bd. 1 und 2. Stuttgart: Klett.

Fricke, A. (1970). Operative Lernprinzipien im Mathematikunterricht der Grundschule. In: Fricke, A. \& Besuden, A. [Hrsg.]. Mathematik: Elemente einer Didaktik und Methodik. Stuttgart: Klett. 79-116.

Fricke, A. (1984a) [Hrsg.]. Kombinatorik in der Wahrscheinlichkeitsrechnung. MU 30, H. 1.

Fricke, A. (1984b). Das stochastische Problem der vollständigen Serie. MU 30, H. 1. 79-85.

Fricke, A. (1984c). Wahrscheinlichkeit und Mittelwerte bei einem symmetrischen Irrfahrtproblem. MU 30, H.1. 65-78.

Fritsch, R. \& Fritsch, G. (1994). Der Vierfarbensatz: Geschichte, topologische Grundlagen und Beweisidee. Mannheim u. a.: BI-Wissenschaftsverlag.

Führer, L. (1997). Pädagogik des Mathematikunterrichts. Braunschweig u. a.: Vieweg.

Glaser, H. (1978). Symmetrieerfassung und das Lösen von Abzählproblemen. MU 24, H. 2. 76-104.

Glaserfeld, E. vom (1991). Radical Constructivsm in Mathematics Education. Dordrecht: Kluwer.

Gordon, S. P. (1979). A discrete Approach to the Calculus. Int. J. Math. Educ. Sci. Technol. Vol. 10, No. 1. 21-31.

Green, N. (1997). Unterrichtsvorschläge zur diskreten Mathematik. ML 84. 60-64.

Greiner, W. (1989). Mechanik. Teil 1. Thun u. a.: Verlag Harri Deutsch.

Haas, N. (2000). Das Extremalprinzip als Element mathematischer Denk- und Problemlöseprozesse - Untersuchungen zur deskriptiven, konstruktiven und systematischen Heuristik. Hildesheim: Franzbecker.

Hartmann, W. \& Streule, P. (2000). Ein Plädoyer für die Kurvendiskussion - aus der diskreten Sicht des Rechners! MNU 53, H. 4. 213-217.

Henn, H.-W. (1979). „Induktive“ und „Deduktive“ Mathematik im Unterricht. In: Dörfler, W. \& Fischer, R. [Hrsg.]. Beweisen im Mathematikunterricht. Wien: Hölder-Pichler-Tempsky. 209-216. 
Henn, H.-W. (2000). Änderungsraten als Zugang zu den zentralen Begriffen und Resultaten der Analysis. ISTRON - Materialien für einen realitätsbezogenen Mathematikunterricht Bd. 6. 1-13.

Herget, W. (1989). Prüfziffern und Strichcode - „Computer-Mathematik“ auch ohne Computer. ML 33. 19-28.

Herget, W. (1994). Artikelnummern und Zebrastreifen, Balkencode und Prüfziffern - Mathematik und Informatik im Alltag. In: Blum, W.; Henn, W.; Klika, M. \& Maaß, J. [Hrsg.]. Materialien für einen realitätsbezogenen Mathematikunterricht. Hildesheim: Franzbecker. 69-84.

Hering, H. (1985). Triangulationen vom Standpunkt des operativen Prinzips. MiSch 23, H. 12. 257-276.

Hering, H. (1986). Steuernde Einflüsse des operativen Prinzips auf den Analysisunterricht. MU 32, H. 6. 28-42.

Hering, H. (1988). Beispiele zur Steuerung mathematischer Aktivitäten durch das operative Prinzip in der Sekundarstufe I. MNU 41/2. 79-85.

Herscovics, N. \& Bergeron, J. C. (1983). Models of understanding. ZDM 15, H. 2. 75-83.

Hessisches Kultusministerium (2000). Lehrplan für das Fach Mathematik für die gymnasiale Oberstufe. Hannover: Schroedel.

Heuser, H. (1990). Lehrbuch der Analysis. Teil 2. Stuttgart: Teubner.

Hilgard, E. R. \& Bower, G. H. (1970). Theorien des Lernens. Bd. 1. Klett: Stuttgart.

Hölzl, R. (1994). Im Zugmodus der Cabri-Geometrie. Interaktionsstudien und Analysen zum Mathematiklernen mit dem Computer. Weinheim: Deutscher Studien Verlag.

Hofe, R. vom (1996). Über die Ursprünge des Grundvorstellungskonzepts in der deutschen Mathematikdidaktik. JMD 17, H. 3/4. 238-264.

Hofe, R. vom (1998). Probleme mit dem Grenzwert - Genetische Begriffsbildung und geistige Hindernisse - Eine Fallstudie aus dem computergestützten Analysisunterricht. JMD 19, H. 4. 257-291.

Hofe, R. vom (1999). Explorativer Umgang mit Funktionen - Interaktion und Kommunikation in selbstorganisierten Arbeitsphasen. Eine Fallstudie aus dem computergestützten Analysisunterricht. JMD 20, H. 2/3. 186-221.

Hofe, v. R. (2001). Mathematik entdecken. ML 105. 4-8.

Hoffmann, M. H.-G. (2001). Skizze einer semiotischen Theorie des Lernens. JMD 22, H. 3/4. 231-251.

Holland, G. (1975). Strategien zur Bildung geometrischer Begriffe. BzM. Hannover: Schroedel. 59-68.

Hoyles, C. (1993). Microworlds/Schoolworlds: The Transformation of an Innovation. In: Keitel, C. \& Ruthven, K. [Hrsg.]. Learning from Computers: Mathematics Education and Technology. Berlin u. a.: Springer. 1-17.

Hoyles, C. (1994). Computer-based Microworlds: A radical Vision or a Trojan mouse? In: Robiteille, D. F. u. a. [Hrsg.]. Selected Lectures from the $7^{\text {th }}$ International Congress on Mathematical Education. Canada. 171-182. 
Hoyles, C. \& Noss, R. (1992). Looking Back and Looking Forward. In: Hoyles, C. \& Noss, R. [Hrsg.]. Learning Mathematics and Logo. Cambridge: MIT Press. 431-468.

Hoyles, C.; Sutherland, R.\& Healy, L. (1991). Children Talking in Computer Environments: New Insights on the role of Discussion in Mathematics Learning. In: Durkin, K. \& Shire, B. [Hrsg.]. Language in Mathematical Education. Research and Practice. Milton Keynes: Open University. 162-175.

Ihringer, T. (1994). Diskrete Mathematik. Stuttgart: Teubner.

Jäger, J. \& Schupp, H. (1997). Das Problem des Handlungsreisenden. ML 81. 21-51.

Jahnke, H. N. (1990). Mathematik und Bildung in der Humboldtschen Reform. Göttingen: Vandenhoeck u. Ruprecht.

Jahnke, H. N. (1999). Geschichte der Analysis. Heidelberg: Spektrum.

Jeger, M. (1973). Einführung in die Kombinatorik. Bd. 1. Stuttgart: Klett.

Jungnickel, D. (1994). Graphen, Netzwerke und Algorithmen. Mannheim u.a.: BIWissenschaftsverlag.

Kaiser, G. (1997). Anregungen aus England. ML 84. 4-7.

Kapur, J. N. (1970). Combinatorial Analysis and School Mathematics. Educational Studies in Mathematics 3. 111-127.

Kautschitsch, H. (1999). Reaktivierung funktionalen Denkens durch computergestützte experimentelle Mathematik. In: Kadunz, G.; Ossimitz, G.; Peschek, W. \& Schneider, E. [Hrsg.]. Mathematische Bildung und neue Technologien. Leipzig: Teubner. 175-182.

Kautschitsch, H.; Keune, M. \& Henning, H. (1999). Operatives Prinzip - Funktionales Denken - Computer. Otto-von-Guericke-Universität Magdeburg. Preprint. Nr.8.

Keidel, K. \& Lorbeer, W. [Hrsg.] (1988). Informatik-Themen im Grundkurs Mathematik S II. München: Bayerischer Schulbuchverlag.

Kenney, M. J. \& Hirsch, C. R. [Hrsg.] (1991). Discrete Mathematics across the Curriculum, K-12. Reston, Va.: NCTM.

Kießwetter, K. \& Rosenkranz, R. (1982). Das Problem der verschneiten Straßen - minimale Gerüste. MU 28, H. 5. 5-26.

Kirsch, A. (1979). Vorschläge zur Behandlung von Wachstumsprozessen und Exponentialfunktionen im Mittelstufenunterricht. DdM 4, H. 4. 257-284.

Kirsch, A. (1979). Beispiele für ,prämathematische“ Beweise. In: Dörfler, W. \& Fischer, R. [Hrsg.]. Beweisen im Mathematikunterricht. Wien: Hölder-Pichler-Tempsky. 261-274.

Klingen, L. (1981). Algorithmen für die Analysis in der Schule. In: Bauersfeld, H.; Otte, M. \& Steiner, H.-G. [Hrsg.]. Schriftenreihe des IDM. Bd. 7. 19-38.

Klose, K.-D. [Hrsg.] (1978). Graphentheorie III. MU 24, H. 3.

König, D. (1936). Theorie der endlichen und unendlichen Graphen. Leipzig: Akademische Verlagsgesellschaft.

Koerber, B. \& Peters, I.-R. (1998). Informatische Bildung in Deutschland - Die Wurzeln der Zukunft. In: Koerber, B. \& Peters, I.-R. [Hrsg.]. Informatische Bildung in Deutschland Perspektiven für das 21. Jahrhundert. Berlin: LOG IN Verlag. 19-36. 
Körner, H. (2000). Populationsdynamik. In: Förster, F. u. a. [Hrsg.]. Materialien für einen realitätsbezogenen Mathematikunterricht. Bd. 6. Hildesheim: Franzbecker. 161-176.

Kroll, W. (1983). Tagung zur Situation des Mathematikunterrichts in der S II. PM 25. Nr. 7. 215-220.

Krüger, K. (2000). Erziehung zum funktionalen Denken. Zur Begriffsgeschichte eines didaktischen Prinzips. Berlin: Logos-Verlag.

Kütting, H. (1994). Didaktik der Stochastik. Mannheim u. a.: BI-Wissenschaftsverlag.

Lakatos, I. (1979). Beweise und Widerlegungen: die Logik mathematischer Entdeckungen. Braunschweig u. a.: Vieweg.

Lamnek, S. (1993). Qualitative Sozialforschung. Bd. 2: Methoden und Techniken. Weinheim: Beltz.

Laucken, U. (1984). Über den Wandel des Begriffs „Verstehen“ in verschiedenen psychologischen Denkformen. In: Engelkamp, J. [Hrsg.]. Psychologische Aspekte des Verstehens. Berlin: Springer. 231-254.

Lee, L. (1996). An Initiation into Algebraic Culture through Generalization Activities. In: Bednarz, N. \& Kieran, C.; Lee, L. [Hrsg.]. Approaches to Algebra. Perspectives for Research and Teaching. Dordrecht u. a.: Kluwer Academic Publishers. 87-106.

Leibniz, G. W. (1666). Dissertatio de Arte Combinatoria. In: Gerhardt, C. I. [Hrsg.] (1958). Leibniz, G. W., Mathematische Schriften. 7-132. Reprint: Hildesheim, New York 1971.

Löthe, H. (1997). Muster und Funktionen und ihre Rolle in Mathematik mit Informatik. BzM. Hildesheim: Franzbecker. 327-330.

Malle, G. (1986). Zur Rolle der Aufmerksamkeitsfokussierung in der Entwicklung mathematischen Denkens. In: Steiner, H.-G. [Hrsg.]. Grundfragen der Entwicklung mathematischer Fähigkeiten. Köln: Aulis. 28-35.

Malle, G. (1993). Didaktische Probleme der elementaren Algebra. Braunschweig u. a.: Wiesbaden.

Malle, G. (1999a). Grundvorstellungen zum Differenzen- und Differentialquotienten. Didaktikhefte der Österreichischen Mathematischen Gesellschaft. H. 30. 67-78.

Malle, G. (1999b). Minimale Netze. ML 96. 19-22.

Malmendier, N. \& Kaessler. P. (1985). Stochastik in der Primarstufe. Bericht über eine Unterrichtreihe. Sachunterricht und Mathematik in der Primarstufe 13. H. 10, 380-386, und H. $11,414-421$.

Mason, J. (1996). Expressing Generality and Roots of Algebra. In: Bednarz, N. \& Kieran, C.; Lee, L. [Hrsg.]. Approaches to Algebra. Perspectives for Research and Teaching. Dordrecht u. a.: Kluwer Academic Publishers. 65-86.

Maturana, H. \& Varela, F. (1984). Der Baum der Erkenntnis - Die biologischen Wurzeln des menschlichen Erkennens. Bern u. a.: Scherz.

Maurer, S. B. (1983). The Effects of a New College Mathematics Curriculum on High School Mathematics. In: Ralston, A. \& Young, G. S. [Hrsg.]. The Future of College Mathematics. New York u. a.: Springer. 153-176.

Müller, G. (1979). Das operative Prinzip in der linearen Algebra. BzM. Hannover u.a.: Schroedel. 287-290. 
Müller, G. \& Wittmann, E. C. (1984). Der Mathematikunterricht in der Primarstufe. Wiesbaden: Vieweg.

Müller-Philipp, S. (1994). Der Funktionsbegriff im Mathematikunterricht. Münster u.a.: Waxmann.

Nägler, G. \& Stopp, F. (1996). Graphen und Anwendungen. Stuttgart u. a.: Teubner.

National Council of Teachers of Mathematics (1989). Curriculum and evaluation standards for school mathematics. Reston, Va.: The Council.

National Council of Teachers of Mathematics (2000). Principles and Standards for School Mathematics. Reston, Va.: The Council.

Nelsen, R. B. (1993). Proofs without Words. Washington, DC: Mathematical Association of America.

Netto, E. (1901). Lehrbuch der Combinatorik. Leipzig: Teubner.

Neubert, B. (1998). Kombinatorische Aufgabenstellungen in der Grundschule. BzM. Hildesheim u. a.: Franzbecker. 479-482.

Neuwirth, E. (1995a). Spreadsheet Structures as a Model for Proving Combinatorial Identities. Journal of Computers in Mathematics and Science Education. 14 (3). 419-434.

Neuwirth, E. (1995b). Tabellenkalkulation als alternative Darstellungsform für formale Strukturen. In: Reichel, H.-C. [Hrsg.]. Computereinsatz im Mathematikunterricht. Mannheim u.a.: BI-Wissenschaftsverlag. 207-221.

Neuwirth, E. (2001). Kombinatorik, Rekursion und Tabellenkalkulation. MU 47, H. 3. 52-64.

Nussbaum, R. \& Stowasser, R. (1970). Färbungsprobleme. MU 16, H. 1. 35-52.

Ossimitz, G. (1990). Materialien zur Systemdynamik. Wien: Hölder-Pichler-Tempsky.

Ossimitz, G. (1994). Modellierung dynamischer Systeme im Mathematikunterricht - wozu? In: Hischer, H. [Hrsg.]. Mathematikunterricht und Computer - neue Ziele oder neue Wege zu alten Zielen? Hildesheim: Franzbecker. 72-78.

Palzkill, L. \& Schwirtz, W. (1971). Die Raumlehrestunde: eine Einführung in den operativen Geometrieunterricht der Hauptschule. Wuppertal u. a.: Henn.

Papert, S. (1980). Mindstorms. Children, Computers and Powerful Ideas. Brighton: Harvester Press.

Pascal, B. (1654). Traité du triangle arithmétique. In: Brunschvicg, L. \& Boutroux, P. [Hrsg.] (1908). Oevres de Blaise Pascal. Bd. III. Paris. 433-503. Reprint Vaduz 1965.

Peschek, W. (1989). Abstraktion und Verallgemeinerung im mathematischen Lernprozess. JMD 10, H. 3. 211-285.

Piaget, J. (1974). Psychologie der Intelligenz. Olten: Walter.

Polya, G. (1949). Schule des Denkens. Bern: Francke.

Prugger, E.; Rauniak, C. \& Schneider, E. (1997). Wachstums- und Abnahmeprozesse mit dem TI-92. Manuskript. Graz - Mürzzuschlag - Klagenfurt.

Pruzina, M. (1989). Numerisches Experimentieren und Begriffserarbeitung - dargestellt an Beispielen aus der Abiturstufe. MiSch 27, H. 6. 419-429.

Racke, U. \& Stein, G. (1995). Das logistische Wachstum als problematisches Beispiel mathematischer Modellbildung Teil 2. ZDM 26. H. 1. 1-5. 
Radfort, L. (1996). Some Reflections on Teaching Algebra through Generalization. In: Bednarz, N.; Kieran, C. \& Lee, L. [Hrsg.]. Approaches to Algebra. Perspectives for Research and Teaching. Dordrecht u. a.: Kluwer Academic Publishers. 107-111.

Ralston, A. (1979).The Twilight of The Calculus. Sea Bull Math 3. 49-56.

Ralston, A. (1981). Computer Science, Mathematics, and the Undergraduate Curricula in both. Amer. Math. Monthly, 88. 472-485.

Ralston, A. (1985). The Really New College Mathematics and its Impact on the High School Curriculum. In: Hirsch, C. R. \& Zweng, M. J. [Hrsg.]. The Secondary School Mathematics Curriculum. 29-42.

Ralston, A. (1992). The Impact of Computers and Computer Science on the Mathematics Curriculum. In: Cornu, B. \& Ralston, A. [Hrsg.]. The Influence of Computers and Informatics on Mathematics and its Teaching. Paris: UNESCO. 19-24.

Ralston, A. \& Young, G. S. (1983). The Future of College Mathematics. New York u.a.: Springer.

Rinkens, H. D. \& Schrage, G. (1974). Topologie in der Sekundarstufe I. MU 20, H. 1. 36-51.

Rosenstein, J. G.; Franzblau, D. S. \& Roberts, F. S. [Hrsg.] (1997). Discrete Mathematics in the Schools. DIMACS Series in Discrete Mathematics and Theoretical Computer Science. Vol. 36. American Mathematical Society.

Sandefur, J. T. (1985). Discrete Mathematics: A Unified Approach. In: Hirsch, C. R. \& Zweng, M. J. [Hrsg.]. The Secondary School Mathematics Curriculum. 90-106.

Sauer, M. (1997). „Algebra können“ - was heißt das. BzM. Hildesheim: Franzbecker. 435438.

Scheid, H. (1984). Ein Plädoyer für die Kombinatorik. MU 30, H. 1. 6-32.

Schoenfeld, A. H. (1985). Mathematical Problem Solving. Orlando u. a.: Academic Press.

Schornstein, J. (1996). Ein Vorschlag zur Stufung des Variablenbegriffs. BzM. Hildesheim: Franzbecker. 296-399.

Schmidt, G. (1981). Stichwörter zur Methodik des Mathematikunterrichts. In: Schmidt, G. [Hrsg.]. Methoden des Mathematikunterrichts in Stichwörtern und Beispielen 7/8. 1-84.

Schmidt, G. (1997). Die Tennisballpyramide. MU 43, H. 2. 38-53.

Schneider, E. (2000). Computeralgebrasysteme in einem allgemeinbildenden Mathematikunterricht. Didaktische Orientierungen. Praktische Erfahrungen. Habilitationsschrift. Klagenfurt.

Schreiber, A. (1983). Universelle Ideen im mathematischen Denken - ein Forschungsgegenstand der Fachdidaktik. In: mathematica didactica 2. 165-171.

Schupp, H. (1997). Optimieren ist fundamental. ML 81. 4-10.

Schuster, A. (2001). Diskrete Optimierungsprobleme als Unterrichtsgegenstand im Umfeld von Mathematik und Informatik. BzM. Hildesheim u. a.: Franzbecker. 552-555.

Schweiger, F. (1992). Fundamentale Ideen - Eine geisteswissenschaftliche Studie zur Mathematik-Didaktik. JMD 13, H. 2/3. 199-214.

Shell Center for Mathematical Education (1984). Problems with Patterns and Numbers. Manchester. 
Skemp, R. R. (1979). Goals of Learning and Qualities of Understanding. Math. Teaching, No. 88. 44-49.

Skemp, R. R. (1982). Symbolic Understanding. Math. Teaching, No. 99. 59-61.

Springnagel, P. (2000). Grundvorstellungen zum Differenzen- und Differentialquotienten. Eine empirische Untersuchung. Diplomarbeit Universität Wien. Unveröffentlicht.

Stacey, K. (1989). Finding and Using Patterns in Linear Generalising Problems. Educational Studies in Mathematics 20. 147-164.

Steiner, G. (1973). Mathematik als Denkerziehung. Stuttgart: Klett.

Stowasser, R. \& Mohry, B. (1978). Rekursive Verfahren. Ein problemorientierter Eingangskurs. Hannover. Schroedel.

Stowasser, R. \& Mohry, B. (1980). Didaktik und Lösungen zum Kursbuch Rekursive Verfahren. Hannover: Schroedel.

Tietze, U.-P.; Klika, M. \& Wolpers, H. (1997). Mathematikunterricht in der Sekundarstufe II. Bd. 1. Braunschweig u. a.: Vieweg.

Uhl, W. (1987). Kodierungen im Unterricht. MU 33, H. 3. 46-72.

Vogel, W. (1977). Optimierung von Netzwerken. MU 23, H. 4. 5-18.

Volkert, K. (1987). Geschichte der Analysis. Mannheim u. a.: BI-Wissenschaftsverlag.

Vollrath, H.-J. (1982). Funktionsbetrachtungen als Ansatz zum Mathematisieren in der Algebra. MU 28, H. 3. 5-27.

Vollrath, H.-J. (1984). Methodik des Begriffslehrens im Mathematikunterricht. Stuttgart: Klett.

Vollrath, H.-J. (1989). Funktionales Denken. JMD 10, H. 1. 3-37.

Vollrath, H.-J. (1992). The development of practical arithmetic in lower secondary schools. ZDM 24. 229-233.

Vollrath, H.-J. (1994). Algebra in der Sekundarstufe. Mannheim u. a.: BI-Wissenschaftsverlag.

Wagenschein, M. (1965). Ursprüngliches Verstehen und exaktes Denken. Bd. 1. Stuttgart: Klett.

Waimer, K. (1997). Das Wahlthema Kryptographie im Leistungskurs Klasse 13. Schriftliche Arbeit zur Zweiten Staatsprüfung für die Laufbahn des höheren Schuldienstes an Gymnasien. Tübingen. Unveröffentlicht.

Waits, B. K. \& Demana, F. (1997). Connections between Algebra and Calculus: Discrete and Continuous Models of Growth. The International Journal of Computer Algebra in Education. Vol. 4, No. 3. 239-251.

Warmuth, T. (1995). Untersuchungen zum Einsatz von Computeralgebrasystemen beim Bearbeiten realitätsorientierter Aufgaben im Analysisunterricht. Dissertation. Kassel.

Wefelscheid, H. [Hrsg.] (1974). Informationstheorie. MU 20, H. 3.

Weidenmann, B.; Krapp, A.; Hofer, M.; Huber, G. L. \& Mandl, H. [Hrsg.] (1993). Pädagogische Psychologie. Weinheim u. a.: Beltz.

Weidemann, B. \& Paechter, M. (1997). Design von multimedialen Lernumgebungen. In: Gross, G.; Langer, U. \& Seising, R. [Hrsg.]. Studieren und Forschen im Internet: Perspek- 
tiven für Wissenschaft, Wirtschaft, Kultur und Gesellschaft. Frankfurt a.M. u. a.: Lang. 125-148.

Weigand, H.-G. (1988). Zur Bedeutung der Darstellungsform für das Entdecken von Funktionseigenschaften. JMD 9, H. 4. 287-325.

Weigand, H.-G. (1989). Zum Verständnis von Iterationen im Mathematikunterricht. Bad Salzdetfurth: Franzbecker.

Weigand, H.-G. (1993). Didaktik des Folgenbegriffs. Mannheim u. a .: BI Wissenschaftsverlag.

Weigand, H.-G. (1995). Interpretatives oder normatives Paradigma? - Anmerkung zum Artikel von Chr. Beck. u. H. Maier: Das Interview in der mathematikdidaktischen Forschung (JMD 14 (1993), H. 2. 147-179). JMD 16, H. 1/2. 145-148.

Weigand, H.-G. (1999a). Eine explorative Studie zum computerunterstützten Arbeiten mit Funktionen. JMD 20, H. 1. 28-54.

Weigand, H.-G. (1999b). Evaluating Working Styles of Students in a Computer-Based Environment - A Comparative Study Between the USA and Germany - Proceedings of the 4th International Conference on Technology in Mathematics Teaching, Aug. 9 - 131999 in Plymouth.

Weigand, H.-G. (1999c). Wie fliegt eigentlich der Ball durch die Luft? Die Flugkurven von Basketball und Federball. ML 95. 53-57.

Weigand, H.-G. (2001a) [Hrsg.]. Diskrete Mathematik und Tabellenkalkulation. MU 47, H. 3.

Weigand, H.-G. (2001b) Tabellenkalkulation - ein schrittweise erweiterbares didaktisches Werkzeug. MU 47, H. 3. 16-27.

Weigand, H.-G. \& Thies, S. (2001). Arbeitsweisen von Lernenden beim Problemlösen mit einem Tabellenkalkulationsprogramm - Ergebnisse eines DFG-Projektes. BzM. Hildesheim: Franzbecker. 612-615.

Weigand, H.-G. \& Weller, H. (2001). Changes of Working Styles in a Computer Algebra Environment - the Case of Functions. International Journal of Computers for Mathematical learning 6. 87-111.

Wellstein, H. (1978). Abzählen von Gitterpunkten als Zugang zu Termen. DdM 6. 54-64.

Weth, T. (1999). Kreativität im Mathematikunterricht. Begriffsbildung als kreatives Tun. Hildesheim: Franzbecker.

Weth, T. (2001). Kreative Mathematik - Was ist das? ML 106. 4-8.

Winkelmann, B. (1982). Ansätze für den Computereinsatz im Analysisunterricht. LOG IN 2, H. 2. 22-26.

Winkelmann, B. (1992). Dynamische Systeme und rationales Verhalten. MU 38, H. 4. 46-61.

Winkelmann, B. (1994). Modellbildung und Simulation im Mathematikunterricht. In: Hischer, H. [Hrsg.]. Mathematikunterricht und Computer - neue Ziele oder neue Wege zu alten Zielen?. Hildesheim: Franzbecker. 79-83.

Winter, H. (1971). Geometrisches Vorspiel im Mathematikunterricht der Grundschule. MU 5. 40-66

Winter, H. (1976). Erfahrungen zur Stochastik in der Grundschule (Klasse 1-6). DdM 4. 2237. 
Winter, H. (1983). Über die Entfaltung begrifflichen Denkens im Mathematikunterricht. JMD 4, H. 1. 175-204.

Winter, H. (1984). Begriff und Bedeutung des Übens im Mathematikunterricht. ML 2. 4-16.

Winter, H. (1991). Entdeckendes Lernen im Mathematikunterricht. Braunschweig: Vieweg.

Winter, H. (1994). Über Wachstum und Wachstumsfunktionen. MNU 47/6. 330-339.

Witten, H. [Hrsg.] (1996). Kryptographie und Sicherheit in Netzen. LOG IN 16, H. 5/6.

Wittenberg, A. I. (1990). Bildung und Mathematik. Stuttgart: Klett.

Wittmann, E. Ch. (1976). Eine Erweiterung des operativen Prinzips. In: Winter, H. [Hrsg.]. Beiträge zur Mathematikdidaktik. Festschrift für Wilhelm Oehl. Hannover u. a .: Schroedel 167-177.

Wittmann, E. Ch. (1981). Grundfragen des Mathematikunterrichts. Braunschweig u.a.: Vieweg.

Wittmann, E. Ch. (1985a). Ein strategisches Zweipersonenspiel zum Spernerschen Lemma. ML 11. 54-55.

Wittmann, E. C. (1985b). Objekte - Operationen - Wirkungen: Das operative Prinzip in der Mathematikdidaktik. ML 11. 7-11.

Wittmann, E. C. (1985). Wider die Flut der „bunten Hunde“ und der „grauen Päckchen“: Die Konzeption des aktiv-entdeckenden Lernens und des produktiven Übens. In: Wittmann, E. C. \& Müller, N. M. [Hrsg.]. Handbuch produktiver Rechenübungen. Bd. 1. Klett: Stuttgart u. a. 152-166.

Worobjow, N. N. (1971). Die Fibonaccischen Zahlen. Berlin: Deutscher Verlag der Wissenschaften.

Wynands, A. (1991). Hilft der Computer im Umgang mit Variablen, Formeln, Funktionen und Gleichungen? JMD 12, H. 4. 347-366.

Zech, F. (1996). Grundkurs Mathematikdidaktik. Weinheim u. a.: Beltz Verlag.

Zimmermann, B. (1983). Problemlösen als eine Leitidee für den Mathematikunterricht. MU 3. $5-45$.

Zimmermann, B. (1991). Heuristik als ein Element mathematischer Denk- und Lernprozesse. Habilitationsschrift. Hamburg. 


\section{Verwendete Abkürzungen:}

BzM Beiträge zum Mathematikunterricht

DdM Didaktik der Mathematik

JMD Journal für Mathematikdidaktik

MiSch Mathematik in der Schule

ML Mathematik lehren

MNU Der mathematische und naturwissenschaftliche Unterricht

MU Der Mathematikunterricht

NCTM National Council of Teachers of Mathematics

PM Praxis der Mathematik

ZDM Zentralblatt für Mathematikdidaktik 


\section{Anhang}

Die beiliegende CD enthält die drei in Kapitel 5 beschriebenen Versionen des Lern- und Versuchprogramms „Differenzenfunktionen“:

\begin{tabular}{|l|l|}
\hline Version1.xls & Testprogramm der Studierenden \\
\hline Version2-Teil1.xls, & Testprogramm der Schülergruppen A und B \\
Version2-Teil2.xls & \\
\hline Version3-Teil1.xls, & Testprogramm der Schülergruppe C \\
Version3-Teil2.xls. & \\
\hline
\end{tabular}

Es handelt sich hierbei um Dateien für das Tabellenkalkulationsprogramm Microsoft Excel 97.

Darüber hinaus enthält die CD Ausschnitte aus den Computerprotokollen zu den im Rahmen dieser Arbeiten ausführlicher beschriebenen Fallstudien. Zur Ansicht der Dateien benötigt man das Programm Lotus ScreenCam 97. 


\section{Danksagung}

Herrn Prof. Dr. Hans-Georg Weigand möchte ich herzlich für die Idee zu diesem Thema sowie für die zahlreichen Anregungen und konstruktiven Hinweise danken. Auch nach seinem Wechsel an die Universität Würzburg hat er mit großem Interesse meine Arbeit verfolgt und mir mit manchen aufmunternden Worten über die eine oder andere „kritische Phase“ hinweggeholfen. Durch seine stete Diskussionsbereitschaft und sein unermüdliches Engagement bei der Durchsicht und Kommentierung meiner verschriftlichten und zum Teil zunächst noch nicht „,voll-strukturierten“ Gedanken zur „Diskreten Mathematik“ hat er das Entstehen dieser Arbeit wesentlich gefördert.

Herrn Prof. Dr. Rainer Danckwerts möchte ich für die Bereitschaft danken, diese Arbeit als Zweitgutachter anzunehmen.

Für die angenehme Arbeitsatmosphäre und die zahlreichen Freiräume für meine Untersuchungen sei allen Mitgliedern des Instituts für Didaktik der Mathematik der Justus-LiebigUniversität Gießen gedankt.

Bedanken möchte ich mich ebenfalls bei den an der empirischen Untersuchung beteiligten Lehrern, Schülern und Studierenden. Ohne ihre Kooperation wäre ein wesentlicher Teil dieser Arbeit nicht möglich gewesen.

Für Anmerkungen, Korrekturen, kritische Hinweise, technische Hilfen oder einfach nur für ein freundschaftliches „Kopf-Zurechtrücken“ danke ich Frau Dr. Katja Krüger, Herrn Dr. Georg Mogk, Herrn Dr. Bernd Neubert, Frau Katja Nießner und Frau Dr. Sandra Ziegler.

Ein besonderer Dank gilt meinen Eltern, für ihren steten Zuspruch und ihre uneingeschränkte Unterstützung. 